\title{
Der Ethanolmarkt der EU27 und der USA im Jahr 2023 -
}

Erstellung von Szenarien durch Anwendung der optimierten Szenario-Technik

\author{
Dissertation \\ zur Erlangung des Doktorgrades \\ der Fakultät für Agrarwissenschaften \\ der Georg-August-Universität Göttingen
}

vorgelegt von

Tillmann Anschütz

geboren in Göttingen

Göttingen, Mai 2014 
D7

1. Referent: Prof. Dr. Ludwig Theuvsen

2. Referent: Prof. Dr. Achim Loewen

Tag der mündlichen Prüfung: $\quad$ 22. Mai 2014 
Great job! See me in 2023 and buy me a steak dinner. Scenario \#4 is exactly what WILL happen. If I am wrong, I'll buy you a steak dinner! But, my predictions for this industry have been very right for 20 years. Thank you

Philip W. Madson; President, KATZEN International 


\section{Danksagung}

Ich möchte mich ganz herzlich bei meinem Doktorvater Prof. Dr. Ludwig Theuvsen für die Zeit während der Erstellung der Dissertation bedanken. Die Betreuung war geprägt von Kontinuität und dem nötigen Freiraum, neue Herangehensweisen zu entwickeln und umzusetzen.

Herrn Prof. Dr. Achim Loewen danke ich sowohl für die Übernahme des Korreferats als auch für die Beratung bei der Erstellung der Szenarien des Ethanolmarktes der USA. Meine Verbundenheit gilt darüber hinaus Herrn Prof. Dr. Ulrich Bodmer für die Übernahme der Drittprüfung sowie Herrn Dr. Albrecht Schaper von der Nordzucker AG und Herrn de Gooijer von der ScMI AG für deren hilfreiche Anmerkungen zu meiner Studie im Rahmen der Doktorandenseminare.

Mein großer Dank gilt des Weiteren der KWS SAAT AG und insbesondere Herrn Dr. Helge Beyer für die Freiheit, mich drei Jahre auf das Thema der Arbeit konzentrieren zu können, und die Teilnahme an zahlreichen Kongressen, um die Entscheidungsträger des europäischen und US-amerikanischen Ethanolmarktes zu treffen und zu befragen.

Den Entscheidungsträgern aus der Politik, der Ethanol-, Automobil- und Mineralölindustrie gilt meine Erkenntlichkeit für die Unterstützung bei der Erstellung der Szenarien. Ausdrücklich möchte ich mich in diesem Zusammenhang bei Herrn Dr. Christoph Berg von F.O. Licht bedanken.

Danken möchte ich des Weiteren meinen Kollegen vom Lehrstuhl für Betriebswirtschaftslehre des Agribusiness, die sich ebenfalls trotz enormer Hitze im Blauen Turm - die ersten Sonnenstrahlen waren ausreichend - in den Dienst der Wissenschaft gestellt haben. Außerdem gilt mein Dank Frau Dr. Melanie Brinkschulte vom Internationalen Schreibzentrum der Universität Göttingen, die mir mit zahlreichen Ratschlägen half, die Verständlichkeit des Textes zu optimieren.

Mein ganz besonderer Dank gilt meinen Eltern Julia und Martin, die mir immer die Liebe, Unterstützung und Kraft geben, Herausforderungen zu meistern. Überdies gilt mein Dank meiner Freundin Swaantje für die tolle Unterstützung bei der Fertigstellung der Dissertation und das Verständnis, wenn ich mal wieder das gesamte Wochenende in der Uni verbracht habe. Widmen möchte ich diese Arbeit meinem Bruder Robert Anschütz, der mich in dem gesamten Prozess der Erstellung der Studie begleitet und mir immer geholfen hat, wenn ich seine Hilfe brauchte. 


\section{Inhaltsverzeichnis}

Abbildungsverzeichnis........................................................................... VII

Tabellenverzeichnis......................................................................................... XIII



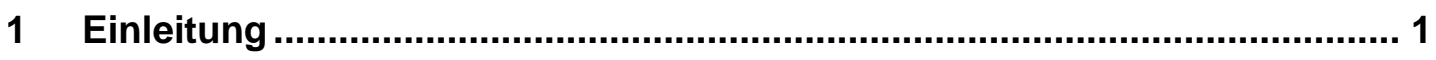

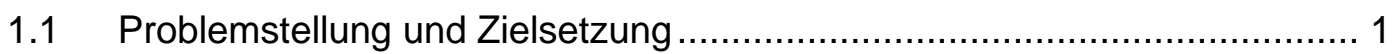

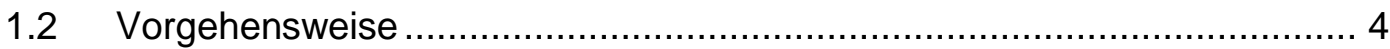

2 Beschreibung des Ethanolmarktes und Stand der Forschung .................. 6

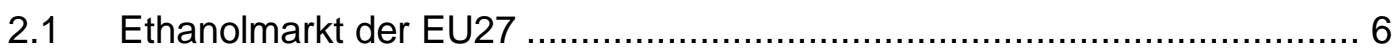

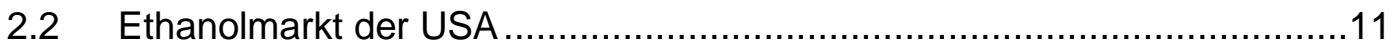

2.3 Stand der Forschung zur zukünftigen Entwicklung des Ethanolmarktes in der EU27 und den USA ......................................................................18

3 Szenario-Technik als Prognoseinstrument............................................27

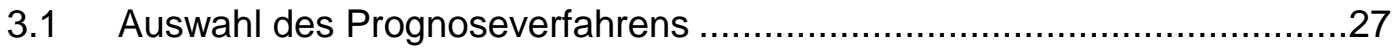

3.2 Geschichtliche Herleitung und Definition des Szenario-Begriffs ................31

3.3 Unterschiedliche Verfahren der Szenario-Technik ...............................32

3.4 Analyse des induktiven Ansatzes der Szenario-Technik ........................34

4 Methodische Beschreibung der Szenario-Technik .................................42

4.1 Formelle Ausrichtung der Szenario-Technik ........................................43

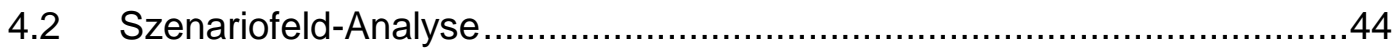

4.2.1 Bestimmung des Untersuchungsfeldes sowie der wirkenden und der beschreibenden Einflussfaktoren .....................................................45

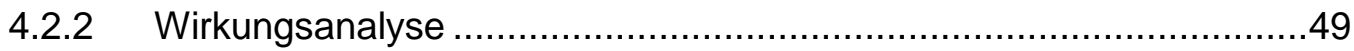

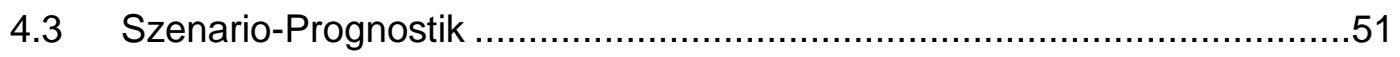

4.4 Szenario-Bildung und -Konsistenzprüfung …...................................54 
$5.1 \quad$ EU27 Szenariofeld-Analyse ......................................................61

5.1.1 Beschreibung des EU27-Szenariofeldes.....................................61

5.1.1.1 Abgrenzung des Untersuchungsfeldes und Bestimmung der beschreibenden Einflussfaktoren ........................................61

5.1.1.2 Bestimmung der wirkenden Einflussfaktoren .........................62

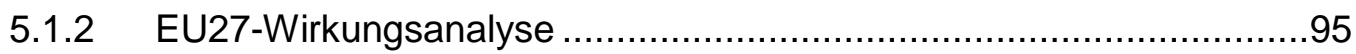

5.1.2.1 Durchführung der Wirkungsanalyse ....................................95

5.1.2.2 Auswertung der Wirkungsanalyse .........................................97

5.2 EU27 Szenario-Prognostik.....................................................104

5.2.1 Benzinnachfrage in der EU27 ...........................................105

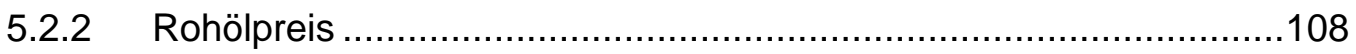

5.2.3 Rohstoffkosten der Ethanolproduktion der ersten Generation..........112

5.2.4 Anzahl benzinbetriebener Pkw, die mit mittleren Ethanolbeimischungen fahren können (E15 - E40) ......................116

5.2.5 Zölle auf Ethanolimporte in die EU27 ........................................121

5.2.6 Politische Förderung von Ethanol ...........................................124

5.2.7 Politische Vorgaben der Treibhausgasreduktion für Ethanol...........129



5.2.9 Ethanolproduktion........................................................... 138

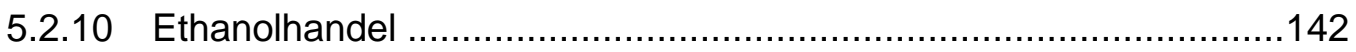



5.3 EU27 Szenario-Bildung und -Konsistenzprüfung .............................149

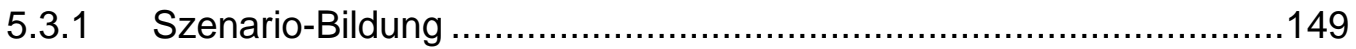

5.3.2 Durchführung der Konsistenzprüfung .....................................154

5.3.3 Auswertung der Konsistenzprüfung ........................................155

5.4 Ergebnisse der Betrachtung des Ethanolmarktes der EU27 ..................161 


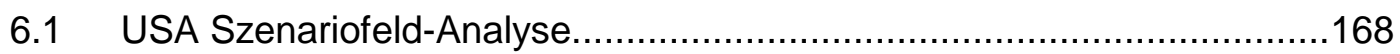

6.1.1 Beschreibung des USA-Szenariofeldes ....................................169

6.1.1.1 Abgrenzung des Untersuchungsfeldes und Bestimmung der beschreibenden Einflussfaktoren .......................................169

6.1.1.2 Bestimmung der wirkenden Einflussfaktoren .......................170



6.1.2.1 Durchführung der Wirkungsanalyse ..................................206

6.1.2.2 Auswertung der Wirkungsanalyse .......................................207

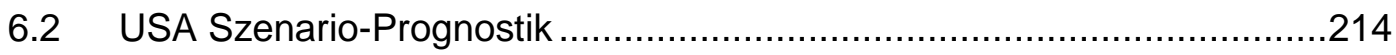

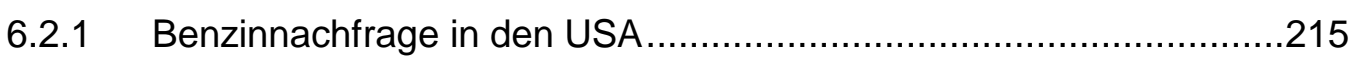

6.2.2 US-amerikanische Tankstelleninfrastruktur für den Verkauf von Benzin mit mittlerem Ethanolgehalt (E15 - E40) ..................................218

6.2.3 Rohstoffkosten bei der Produktion von Mais-Ethanol.....................220

6.2.4 Anzahl der Kraftfahrzeuge, die mit mittleren Ethanolbeimischungen zu Benzin (E15 - E40) fahren können ............................................223

6.2.5 Biokraftstoff-Verbrauchsmandate - Renewable Fuel Standard 2 (RFS

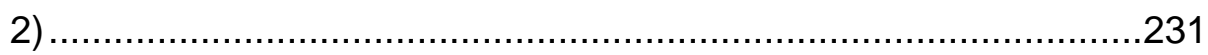

6.2.6 Höhe der genehmigten prozentualen Beimischung von Ethanol zu Benzin ..............................................................................238

6.2.7 Diskussion über die Energieunabhängigkeit der USA ....................241

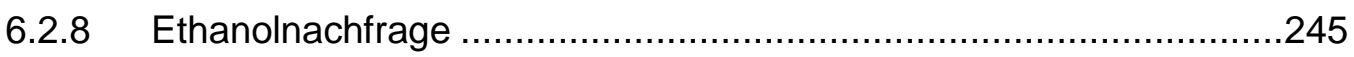

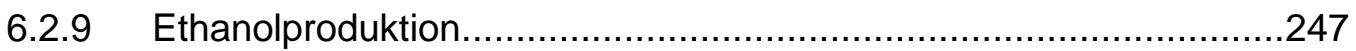

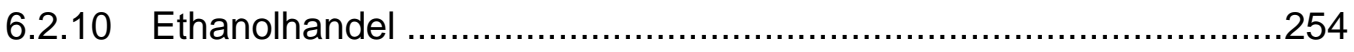

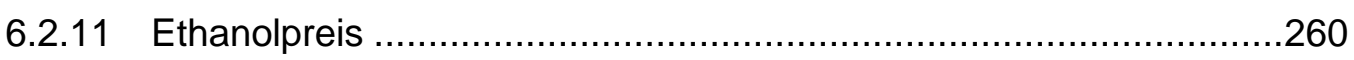

6.3 USA Szenario-Bildung und -Konsistenzprüfung .................................264

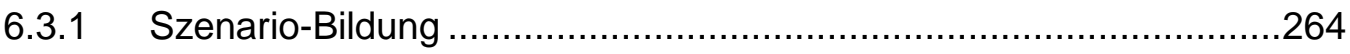

6.3.2 Durchführung der Konsistenzprüfung ....................................270

6.3.3 Auswertung der Konsistenzprüfung ........................................271

6.4 Ergebnisse der Betrachtung des Ethanolmarktes der USA ...................274 
7 Schlussfolgerungen und Handlungsempfehlungen für die

Ethanolwirtschaft der EU27 und der USA ..............................................283

7.1 Zusammenfassung der Untersuchung ...........................................283

7.2 Handlungsempfehlungen für die Ethanolwirtschaft ............................287

7.3 Ansätze für die weitere Forschung ..................................................293

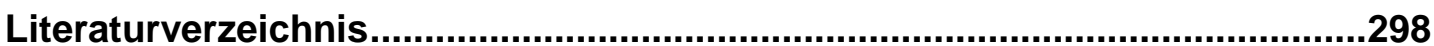

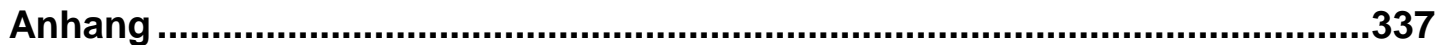




\section{Abbildungsverzeichnis}

Abbildung 1: Entwicklung der Produktion von Ethanol-Kraftstoff in der EU27, Brasilien, den USA und der Welt............................................ 2

Abbildung 2: Entwicklung des Ethanolmarktes der EU27 …........................... 6

Abbildung 3: Entwicklung des Ethanolmarktes der USA ..................................12

Abbildung 4: $\quad$ Auswirkungen der „Blend Wall“-Problematik auf die USamerikanische Ethanolnachfrage ...........................................13

Abbildung 5: Konflikt „RFS 2“ und „Blend Wall“ ...............................................

Abbildung 6: Entwicklung des Ethanolhandels in den USA............................17

Abbildung 7: Stand der Forschung: Entwicklung des Ethanolmarktes in der EU27

Abbildung 8: Stand der Forschung: Entwicklung des Ethanolmarktes in den USA

Abbildung 9: Klassifizierung der Prognoseverfahren .....................................28

Abbildung 10: Trichtermodell/Ansatz der „multiplen Zukunft“.............................33

Abbildung 11: Klassifizierung von Ansätzen der Szenario-Technik.......................33

Abbildung 12: Anstieg der Komplexität der Einflussanalyse ..............................37

Abbildung 13: Anstieg der Komplexität der Konsistenzmatrix .............................40

Abbildung 14: Schematische Darstellung - Optimierte Methodik der SzenarioTechnik ....................................................................... 42

Abbildung 15: Struktur zur Beschreibung eines Einflussfaktors ..........................46

Abbildung 16: Darstellung des Szenariofeldes mit beschreibenden und wirkenden

Einflussfaktoren

Abbildung 17: Ausschnitt des Fragebogens zur Durchführung der Wirkungsanalyse .50

Abbildung 18: Darstellung des Szenariofeldes nach Durchführung der

Wirkungsanalyse

Abbildung 19: Darstellung des Szenariofeldes nach Durchführung der SzenarioPrognostik....................................................................... 54

Abbildung 20: Matrix zur Durchführung der morphologischen Analyse .................56

Abbildung 21: Das 1. Szenario, dargestellt als Szenariofeld ................................58

Abbildung 22: Schematische Darstellung - Vorgehen zur Bildung der Szenarien des Ethanolmarktes der EU27 im Jahr 2023

Abbildung 23: Einfluss der Benzinnachfrage auf die Ethanolnachfrage in der EU27

Abbildung 24: Entwicklung der brasilianischen Ethanolexporte 
Abbildung 25: Entwicklung der US-amerikanischen Ethanolexporte

Abbildung 26: Absatz von Ethanol in Form von ETBE in der EU27

Abbildung 27: Höhere Ethanolnachfrage in der EU27 durch die Nutzung von E15

Abbildung 28: Herkunft der Rohölimporte in die EU27.

Abbildung 29: Zusammenfassung der beschreibenden und wirkenden

Einflussfaktoren des EU27-Szenariofeldes

Abbildung 30: Wirkungsanalyse EU27 - Herkunftsland der

Unternehmen/Organisationen der befragten Experten

Abbildung 31: Wirkungsanalyse EU27 - Herkunftsbranche der

Unternehmen/Organisationen der befragten Experten

Abbildung 32: Wirkungsanalyse EU27 - Vergleich der Mittelwerte der wirkenden

Einflussfaktoren

Abbildung 33: Wirkungsanalyse EU27 - Zusammenfassung der wirkenden

Schlüsselfaktoren

Abbildung 34: Entwicklung der Nachfrage nach Benzin und Diesel im

Transportbereich in der EU27 in den Jahren von 1990 bis 2010..105

Abbildung 35: Zukünftige Entwicklung der Nachfrage nach Benzin im

Straßenverkehr der EU27

Abbildung 36: Entwicklung der Nachfrage nach Benzin im Straßenverkehr in der

EU27 bis zum Jahr 2023...................................................108

Abbildung 37: Reale Entwicklung des Rohölpreises WTI 1980 - 2010 ...............109

Abbildung 38: Entwicklung des Rohölpreises (real 2010) $1980-2025 \ldots \ldots \ldots \ldots \ldots . . .111$

Abbildung 39: Rohstoffe zur Produktion von Ethanol in der EU27 .....................112

Abbildung 40: Entwicklung des (real 2005) Weizenpreises $1989-2010$...........113

Abbildung 41: Prognostizierte Entwicklung des (real 2005) Weizenpreises bis 2021

Abbildung 42: Prognostizierte Entwicklung des (real 2005) Weizenpreises bis 2023

Abbildung 43: Entwicklung der Anzahl benzinbetriebener Pkw in der EU27 und deren Ethanolverträglichkeit

Abbildung 44: Darstellung der Ergebnisse der Projektion „Hohe

Ethanolverträglichkeit der Pkw“

Abbildung 45: Darstellung der Ergebnisse der Projektion „Geringe

Ethanolverträglichkeit der Pkw“

Abbildung 46: ILUC-Werte für Ethanol aus Weizen, Mais, Zuckerrübe und

Zuckerrohr 130 
Abbildung 47: Entwicklung der Ethanolnachfrage in der EU27 im Zeitraum von

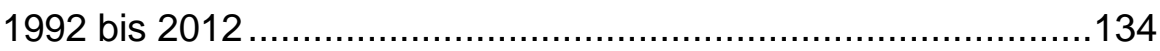

Abbildung 48: Zukünftige Entwicklung der Ethanolnachfrage in der EU27..........135

Abbildung 49: Zielvorgaben der Mitgliedsstaaten zur Nutzung von EthanolKraftstoff

Abbildung 50: Entwicklung der Ethanolproduktion in der EU27 139

Abbildung 51: Entwicklung der Ethanolimporte in die EU27 im Zeitraum von 2003 bis 2012

Abbildung 52: Entwicklung des (real 2005) Ethanol-Weltmarktpreises in den Jahren von 2001 bis 2011

Abbildung 53: Zukünftige Entwicklung des (real 2005) Ethanol-Weltmarktpreises bis zum Jahr 2023

Abbildung 54: Zusammenfassung der Zukunftsprojektionen der wirkenden und beschreibenden Schlüsselfaktoren des Ethanolmarktes der EU27

Abbildung 55: Morphologischer Kasten zur Bildung konsistenter Szenarien des Ethanolmarktes der EU27 im Jahr 2023 150

Abbildung 56: Konsistenzanalyse EU27 - Herkunftsbranche der Unternehmen/Organisationen der befragten Experten 156

Abbildung 57: Konsistenzanalyse EU27 - Herkunftsland der Unternehmen/Organisationen der befragten Experten 156

Abbildung 58: EU27: Vergleich der Szenarien der Studie mit dem Stand der Forschung

Abbildung 59: Schematische Darstellung - Vorgehen zur Bildung der Szenarien des Ethanolmarktes der USA im Jahr 2023 168

Abbildung 60: Einfluss der Benzinnachfrage auf die Ethanolnachfrage in den USA

Abbildung 61: Entwicklung der Ethanolimporte aus Brasilien in die USA 173

Abbildung 62: Entwicklung der US-amerikanischen Ethanolexporte nach Brasilien

Abbildung 63: Entwicklung der US-amerikanischen Ethanolexporte in die EU27

Abbildung 64: Entwicklung der US-amerikanischen Ethanolexporte ins Ausland 177

Abbildung 65: Standort der US-amerikanischen Ethanolanlagen.......................178

Abbildung 66: Standort der E85 Tankstellen in den USA.................................181

Abbildung 67: Umsatzanteil durch den Verkauf von DDGS .............................182 
Abbildung 68: Entwicklung der Anzahl an Flexible Fuel Vehicles in den USA.....187

Abbildung 69: Entwicklung der Anzahl an Elektrofahrzeugen in den USA ..........189

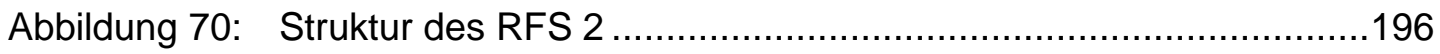

Abbildung 71: Einfluss des RFS 2 auf die Ethanolnachfrage ............................196

Abbildung 72: Entwicklung der Biokraftstoff-Verbrauchsmandate des RFS 2 .....197

Abbildung 73: Bedeutung der erlaubten Höhe der Beimischung von Ethanol zu Benzin, um die Verbrauchsmandate des RFS 2 zu erfüllen 199

Abbildung 74: Zusammenfassung der beschreibenden und wirkenden

Einflussfaktoren des USA-Szenariofeldes 205

Abbildung 75: Wirkungsanalyse USA - Herkunftsland der

Unternehmen/Organisationen der befragten Experten

Abbildung 76: Wirkungsanalyse USA - Herkunftsbranche der

Unternehmen/Organisationen der befragten Experten 208

Abbildung 77: Wirkungsanalyse USA - Darstellung der acht bedeutendsten

Einflussfaktoren für die langfristige Entwicklung des USamerikanischen Ethanolmarktes

Abbildung 78: Wirkungsanalyse USA - Zusammenfassung der wirkenden

Schlüsselfaktoren

Abbildung 79: Historische Entwicklung der Benzinnachfrage in den USA

Abbildung 80: Zukünftige Entwicklung der Benzinnachfrage in den USA bis zum Jahr 2023

Abbildung 81: Effizienzsteigerung von Kraftfahrzeugen in den USA bis zum Jahr 2023

Abbildung 82: Entwicklung des (real 2005) Maispreises 1989 - 2010 .220

Abbildung 83: Prognostizierte Entwicklung des (real 2005) Maispreises bis 2021

Abbildung 84: Prognostizierte Entwicklung des (real 2005) Maispreises bis 2023

Abbildung 85: Entwicklung der Anzahl an Kraftfahrzeugen in den USA in den Jahren von 1990 bis 2010.

Abbildung 86: Darstellung der Ergebnisse der Annahme „Hohe

Ethanolverträglichkeit von Kraftfahrzeugen" in den USA im Jahr 2023

Abbildung 87: Darstellung der Ergebnisse der Annahme „Geringe

Ethanolverträglichkeit von Kraftfahrzeugen" in den USA im Jahr 2023 230

Abbildung 88: Biokraftstoffverbrauchsmandate des RFS 2 bis zum Jahr 2022 ...232 
Abbildung 89: Verbrauchsmandate in Milliarden Litern im Jahr 2023 Option 1 Option 4

Abbildung 90: Ermittlung der „Blend Wall“ in Abhängigkeit von der maximal erlaubten Höhe der Ethanolbeimischung zu Benzin

Abbildung 91: Entwicklung der US-amerikanischen Rohölversorgung im Zeitraum von 1970 bis 2011

Abbildung 92: Entwicklung der US-amerikanischen Rohölversorgung im Zeitraum von 1993 bis 2023

Abbildung 93: Entwicklung der prozentualen nominalen Ausgaben für Rohöl und Rohölimporte am BIP der USA

Abbildung 94: Historische Entwicklung der Ethanolnachfrage in den USA .........245

Abbildung 95: Prognostizierte Verbrauchsmandate des RFS 2 -

Zukunftsprojektionen der Ethanolnachfrage

Abbildung 96: Historische Entwicklung der Ethanolproduktion in den USA

Abbildung 97: Zukunftsprojektion: „Sehr starker Anstieg der Produktion von Zellulose-Ethanol“

Abbildung 98: Zukunftsprojektion: „Moderater Anstieg der Produktion von Unspecified-Ethanol“

Abbildung 99: Zukunftsprojektion: „Starker Anstieg der Produktion von Mais-

Ethanol“"

Abbildung 100: Zukunftsprojektion: „Starker Anstieg der Produktion von Unspecified-Ethanol“"

Abbildung 101: Entwicklung des Ethanolhandels in den USA im Zeitraum von 2004 bis 2012 .

Abbildung 102: Ethanolexporte und -importe der USA im Jahr 2012

Abbildung 103: Entwicklung des (real 2005) Ethanol-Weltmarktpreises (2001 - 2011) und des (real 2010) US-amerikanischen Ethanolpreises (2009 2011)

Abbildung 104: Zukünftige Entwicklung des (real 2005) Ethanol-Weltmarktpreises und des (real 2010) US-amerikanischen Ethanolpreises

Abbildung 105: Zusammenfassung der Zukunftsprojektionen der wirkenden und beschreibenden Schlüsselfaktoren des Ethanolmarktes der USA

Abbildung 106: Morphologischer Kasten zur Bildung konsistenter Szenarien des US-amerikanischen Ethanolmarktes im Jahr 2023

Abbildung 107: Konsistenzanalyse USA - Herkunftsbranche der Unternehmen/Organisationen der befragten Experten 
Abbildung 108: Konsistenzanalyse USA - Herkunftsland der

Unternehmen/Organisationen der befragten Experten .272

Abbildung 109: USA: Vergleich der Szenarien der Studie mit dem Stand der

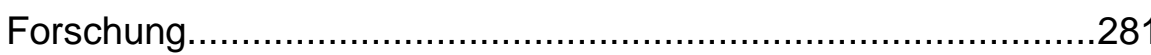

Abbildung 110: Szenarien des Ethanolmarktes der EU27 und der USA .286

Abbildung 111: Die optimierte Szenario-Technik als Instrument zur Beschreibung des Makroumfeldes des Ethanolmarktes der EU27 und der USA 


\section{Tabellenverzeichnis}

Tabelle 1: $\quad$ Übersicht über die wichtigsten politischen Richtlinien zur Förderung von Biokraftstoffen

Tabelle 2: $\quad$ Stand der Forschung I: Entwicklung des Ethanolmarktes in der EU27 und den USA

Tabelle 3: $\quad$ Stand der Forschung II: Entwicklung des Ethanolmarktes in der EU27 und den USA 20

Tabelle 4: $\quad$ Vergleich ausgewählter Prognoseverfahren................................29

Tabelle 5: $\quad$ Verfahren des induktiven Ansatzes der Szenario-Technik ..............35

Tabelle 6: Formelle Ausrichtung des optimierten Verfahrens der SzenarioTechnik

Tabelle 7: $\quad$ Maximale $\mathrm{CO}_{2}$-Gesamtemissionen von Biokraftstoffen in

Abhängigkeit von der politisch geforderten prozentualen $\mathrm{CO}_{2}^{-}$

Reduktion im Verhältnis zu fossilen Kraftstoffen .80

Tabelle 8: Kulturspezifische ILUC-Werte .88

Tabelle 9: $\quad$ Wirkungsanalyse EU27 - Anordnung der wirkenden

Einflussfaktoren hinsichtlich der Stärke des Einflusses auf den

Ethanolmarkt der EU27. .99

Tabelle 10: Wirkungsanalyse EU27 - Ergebnisse der Faktoranalyse 101

Tabelle 11: EU27 Szenario-Prognostik - Reihenfolge der Betrachtung der Schlüsselfaktoren 105

Tabelle 12: Anzahl der benzinbetriebenen Pkw in der EU27 im Jahr 2023.....118

Tabelle 13: Kulturspezifische ILUC-Werte bei der Produktion von Biokraftstoffen 131

Tabelle 14: $\quad$ Anlagen und Projekte zur Produktion von Zellulose-Ethanol in der EU27

Tabelle 15: Wirkungsanalyse USA - Anordnung der wirkenden Einflussfaktoren hinsichtlich der Stärke des Einflusses auf den Ethanolmarkt der USA.

Tabelle 16: USA Szenario-Prognostik - Reihenfolge der Betrachtung der Schlüsselfaktoren

Tabelle 17: $\quad$ Alter und Anzahl der Kraftfahrzeuge in den USA im Jahr 2023 ....225

Tabelle 18: Zitate von US-amerikanischen Präsidenten zum Thema Energieunabhängigkeit 


\section{Abkürzungsverzeichnis}

\begin{tabular}{|c|c|}
\hline ADM & Archer Daniels Midland \\
\hline$A G$ & Aktiengesellschaft \\
\hline $\mathrm{B} 100$ & reiner Biodieselkraftstoff \\
\hline $\mathrm{BDB}^{\mathrm{e}}$ & Bundesverband der deutschen Bioethanolwirtschaft e.V. \\
\hline BIP & Bruttoinlandsprodukt \\
\hline BtL & Biomass-to-Liquid \\
\hline BUND & Bund für Umwelt und Naturschutz Deutschland \\
\hline bzw. & beziehungsweise \\
\hline C5 & Zuckermolekül mit 5 Kohlenstoffatomen \\
\hline C6 & Zuckermolekül mit 6 Kohlenstoffatomen \\
\hline CAFE & Corporate Average Fuel Economy \\
\hline CARB & California Air Resources Board \\
\hline $\mathrm{CO}_{2}$ & Kohlenstoffdioxid \\
\hline CtL & Coal-to-Liquid \\
\hline DDGS & Distillers' Dried Grains with Solubles \\
\hline Dr. & Doktor \\
\hline$€$ & Euro (Währung der Europäischen Wirtschafts- und Währungsunion) \\
\hline E0 & Ottokraftstoff mit einem Ethanolanteil von $0 \%$ vol \\
\hline E5 & Ottokraftstoff mit einem Ethanolanteil von $5 \%$ vol \\
\hline E10 & Ottokraftstoff mit einem Ethanolanteil von $10 \%$ vol \\
\hline E15 & Ottokraftstoff mit einem Ethanolanteil von $15 \%$ vol \\
\hline E20 & Ottokraftstoff mit einem Ethanolanteil von $20 \%$ vol \\
\hline E25 & Ottokraftstoff mit einem Ethanolanteil von $25 \%$ vol \\
\hline E30 & Ottokraftstoff mit einem Ethanolanteil von $30 \%$ vol \\
\hline E40 & Ottokraftstoff mit einem Ethanolanteil von $40 \%$ vol \\
\hline E85 & Ottokraftstoff mit einem Ethanolanteil von $85 \%$ vol \\
\hline E90 & Ottokraftstoff mit einem Ethanolanteil von $90 \%$ vol \\
\hline EBA & Everything But Arms \\
\hline $\mathrm{EC}$ & European Commission \\
\hline$E G$ & Europäische Gemeinschaft \\
\hline EISA & Energy Independence and Security Act \\
\hline EPA & United States Environmental Protection Agency \\
\hline eq & Energieäquivalent \\
\hline ERW & Erwägungsgrund \\
\hline & et alii \\
\hline
\end{tabular}




\begin{tabular}{|c|c|}
\hline ETBE & Ethyl-Tertiär-Butyl-Ether \\
\hline EU15 & Die 15 Mitgliedsstaaten der Europäischen Union bis April 2004 \\
\hline EU27 & Die 27 Mitgliedsstaaten der Europäischen Union bis Juni 2013 \\
\hline f. & folgende \\
\hline $\mathrm{ff}$. & fortfolgende \\
\hline FAPRI & Food and Agricultural Policy Research Institute \\
\hline FFV & Flexible Fuel Vehicles \\
\hline F.O.B. & Free On Board \\
\hline$g$ & Gramm \\
\hline GSP & Generalized System of Preferences \\
\hline GtL & Gas-to-Liquid \\
\hline $\mathrm{hl}$ & Hektoliter \\
\hline ILUC & Indirekt Land Use Change \\
\hline Incoterm & International Commercial Term \\
\hline Ing. & Ingenieur \\
\hline $\mathrm{KN}$ & kombinierte Nomenklatur \\
\hline KWK & Kraft-Wärme-Kopplung \\
\hline $\mathrm{L}$ & Liter \\
\hline LCFS & Low Carbon Fuel Standard \\
\hline Lfd. Nr. & Laufende Nummer \\
\hline LLC & Limited Liability Company \\
\hline Ltd. & Limited Company \\
\hline MJ & Megajoule \\
\hline $\mathrm{n}$ & Stichprobenumfang \\
\hline NABU & Naturschutzbund Deutschland e.V. \\
\hline OECD/FAO & $\begin{array}{l}\text { Organisation for Economic Co-operation and Development/ Food } \\
\text { and Agriculture Organization of the United Nations }\end{array}$ \\
\hline OPEC & Organization of the Petroleum Exporting Countries \\
\hline plc. & public limited company \\
\hline Prof. & Professor \\
\hline $\mathrm{R}^{2}$ & Bestimmtheitsmaß \\
\hline RAC & Refiners Acquisition Cost \\
\hline RFS 1 & Renewable Fuel Standard 1 \\
\hline RFS 2 & Renewable Fuel Standard 2 \\
\hline SF & Schlüsselfaktor \\
\hline T1 & Thema 1 \\
\hline T2 & Thema 2 \\
\hline
\end{tabular}




$\begin{array}{ll}\text { UCO } & \text { Used Cooking Oil } \\ \text { US-\$ } & \text { US-amerikanischer Dollar (Währung in den USA) } \\ \text { USA } & \text { Vereinigte Staaten von Amerika } \\ \text { VEETC } & \text { Volumetric Ethanol Excise Tax Credit } \\ \text { vol } & \text { Volumen } \\ \text { WTI } & \text { West Texas Intermediate } \\ \text { z.B. } & \text { zum Beispiel }\end{array}$




\section{$1.1 \quad$ Problemstellung und Zielsetzung}

Die starke Abhängigkeit vom endlichen Rohstoff Rohöl für die Treibstoffproduktion und die Tatsache, dass sich der Großteil der weltweiten Erdölreserven in politisch instabilen Regionen befindet (EUROPEAN COMMISSION DIRECTORATEGENERAL FOR RESEARCH SUSTAINABLE ENERGY SYSTEMS 2006: 8), fördert das Bestreben der USA, dem Ziel der Energieunabhängigkeit näher zu kommen. Um Benzin zu substituieren und den Rohölverbrauch zu senken, setzen sie EthanolKraftstoff ein. Die Ankündigung des US-amerikanischen Präsidenten Barack Obama, die Abhängigkeit der USA von importiertem Rohöl in den nächsten 14 Jahren um ein Drittel zu senken (BEVILL 2011b), zeigt, welche Relevanz das Thema für die USA hat.

Auch für die EU27 $7^{1}$ ist das Thema Energieunabhängigkeit von Bedeutung, nimmt aber eine eher untergeordnete Rolle ein. Hier spielt die Senkung des Kohlenstoffdioxidausstoßes im Verkehrsbereich durch den Einsatz von Ethanol-Kraftstoff die entscheidende Rolle (RICHTLINIE 2009/28/EG ERW 1).

Der große Vorteil von Ethanol-Kraftstoff im Vergleich zu anderen Technologien ist seine Fähigkeit, die bereits bestehende Infrastruktur des Tankstellennetzes zu nutzen. Zudem ist die Technik, Ethanol als Kraftstoff einzusetzen, um Benzin zu substituieren, bereits in den zugelassenen Autos vorhanden.

In der vorliegenden Arbeit wird die zukünftige Entwicklung des EthanolKraftstoffmarktes in der EU27 und den USA betrachtet. Dies impliziert, dass die Verwendung von Ethanol in der chemischen Industrie sowie in der Nahrungsmittelindustrie in dieser Arbeit nicht behandelt wird. Der Schwerpunkt wird auf den Kraftstoffmarkt gelegt, da er mit Abstand der größte Ethanolmarkt und in den letzten zehn Jahren im Gegensatz zu den beiden anderen Märkten stark gewachsen ist (F.O. LICHT/AGRA INFORMA 2009: 19)².

Die weltweite Produktion von Ethanol-Kraftstoff ist im Zeitraum 2003 bis 2012 von 10,6 Milliarden Litern auf 82 Milliarden Liter gestiegen. Im Jahr 2012 sind die USA mit 52,5 Milliarden Litern (64\% der weltweiten Ethanolproduktion) der mit Abstand größte Ethanolproduzent der Welt, gefolgt von Brasilien mit 20,5 Milliarden Litern und der EU27 mit lediglich 4,3 Milliarden Litern (Abbildung 1).

\footnotetext{
${ }^{1}$ Die EU27 umfasst die 27 Mitgliedsstaaten der Europäischen Union vor dem Beitritt von Kroatien im Juli 2013 (EUROPÄISCHE UNION 2014).

${ }^{2}$ Der Begriff Ethanol bezieht sich in dieser Studie immer auf den Kraftstoffmarkt, somit sind die Begriffe Ethanol und Ethanol-Kraftstoff als Synonyme zu verstehen.
} 


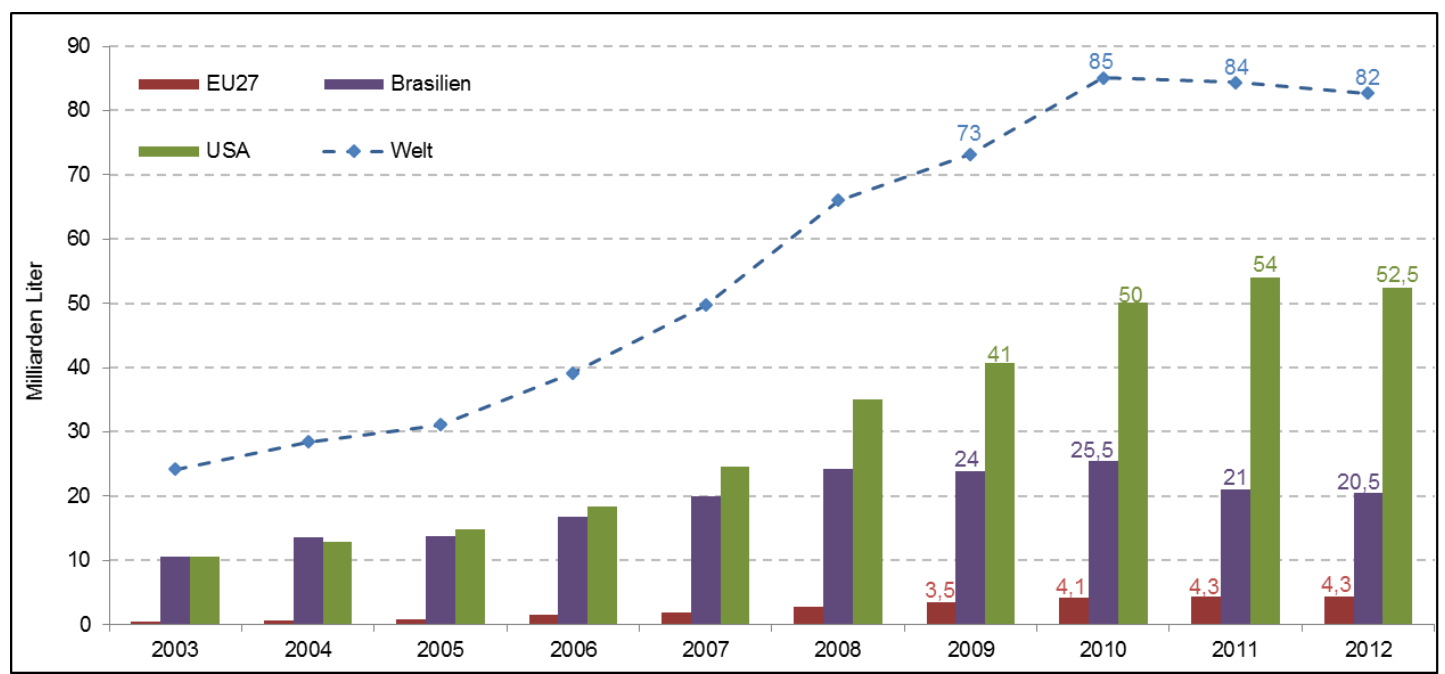

Abbildung 1: Entwicklung der Produktion von Ethanol-Kraftstoff in der EU27, Brasilien, den USA und der Welt

Quelle: In Anlehnung an F.O. LICHT 2012f: 74; F.O. LICHT 2011a: 68

Bei einer präziseren Betrachtung der Entwicklung fällt auf, dass sich die Situation auf dem Weltmarkt im Jahr 2011 grundlegend verändert hat. Zum ersten Mal seit über zehn Jahren ist die weltweite Ethanolproduktion gesunken (F.O. LICHT 2011a: 64). Verantwortlich hierfür ist der Einbruch der Produktion um 4,5 Milliarden Liter in Brasilien. Mit dem ersten Rückgang der US-amerikanischen Ethanolproduktion seit 1996 von 54 Milliarden Litern im Jahr 2011 auf 52,5 Milliarden Liter im Jahr 2012 und einer stagnierenden europäischen Ethanolproduktion setzte sich die Entwicklung 2012 fort (F.O. LICHT 2012f: 69). Die Jahre 2011 und 2012 haben gezeigt, dass der weltweite Ethanol-Boom der Jahre 2003 bis 2010 vorbei ist.

Die Zukunft des Ethanolmarktes ist von einer hohen Unsicherheit geprägt. Grund für die starke Verunsicherung in der EU27 ist das politische Vorhaben, den derzeitigen Biokraftstoffmarkt der ersten Generation ${ }^{3}$ zu einem Markt der zweiten Generation zu wandeln. Es wird zwar weiterhin an dem politischen Ziel festgehalten, im Jahr 2020 $10 \%$ der eingesetzten Energie im Verkehrssektor aus erneuerbaren Quellen zu beziehen (EUROPÄISCHE KOMMISSION 2012: 9), Biokraftstoffe der ersten Generation sollen aber nur noch zu maximal der Hälfte (5\%) dem Ziel angerechnet werden (EUROPÄISCHE KOMMISSION 2012:16). Im Zusammenhang mit dem angestrebten Wandel stehen darüber hinaus die folgenden zwei Punkte: (1) Die Ankündigung,

\footnotetext{
${ }^{3}$ Biokraftstoffe werden in eine erste Generation und eine zweite Generation unterteilt. Unterscheidungsmerkmal ist der Rohstoff, der zur Biokraftstoffproduktion genutzt wird. Zur eindeutigen Unterscheidung zwischen erster und zweiter Generation wird die Definition der INTERNATIONAL ENERGY AGENCY (2010b: 22) genutzt. Biokraftstoffe der ersten Generation werden aus Zucker, Stärke, Ölpflanzen, tierischen Fetten und Essensresten produziert, wohingegen Biokraftstoffe der zweiten Generation aus Zellulose, Hemizellulose und Lignin hergestellt werden.
} 
ab 2021 nur noch jene Biokraftstoffe politisch zu fördern, die eine hohe $\mathrm{CO}_{2}{ }^{-}$ Einsparung erreichen und nicht aus Lebens- und Futtermitteln produziert werden (EUROPÄISCHE KOMMISSION 2012: 3) und (2) die Berücksichtigung des Faktors Indirect Land Use Change (ILUC) bei der Ermittlung der $\mathrm{CO}_{2}$-Bilanz von Biokraftstoffen. Die Umsetzung beider Punkte hätte zur Folge, dass die politische Förderung für Biokraftstoffe der ersten Generation entfiele. Sollte es zu einem solchen Wandel kommen, ist die Ethanolindustrie der ersten Generation in ihrer Existenz bedroht.

In den USA sind es andere Einflüsse, die zu einer Unsicherheit bei der Prognose der langfristigen Entwicklung des Ethanolmarktes führen. Diese Unsicherheit ist sowohl auf die „Blend Wall“-Problematik als auch auf die wahrscheinliche Senkung des Verbrauchsmandats des Renewable Fuel Standard 2 (RFS 2) zurückzuführen.

Die „Blend Wall“ ist die maximal mögliche Menge Ethanol, die dem in den USA nachgefragten Benzin beigemischt werden kann. Die USA befinden sich seit 2011 an der "Blend Wall“, wodurch momentan kein weiteres Wachstum des USamerikanischen Ethanolmarktes möglich ist (CONGRESSIONAL RESEARCH SERVICE 2010a: 2f.). Darüber hinaus sorgt die anhaltende Diskussion über eine mögliche Kürzung des RFS 2 für weitere Verunsicherung. Der RFS 2 gibt bis zum Jahr 2022 jährlich ansteigende Verbrauchsmandate für Biokraftstoffe vor, die im USamerikanischen Transportbereich - trotz der bereits bestehenden „Blend Wall“Problematik - abgesetzt werden müssen. Bis zum Jahr 2022 steigt das Verbrauchsmandat für Biokraftstoffe aus Zellulose, Hemizellulose oder Lignin stark an (U.S. GOVERNMENT INFORMATION 2007: 212 STAT. 1523). Die Produktion von Zellulose-Ethanol war im Jahr 2012 allerdings nur minimal (OECD/FAO 2012: 224). Folglich ist die zukünftige Erfüllung des stetig steigenden Verbrauchsmandats für biogene Treibstoffe der 2. Generation unwahrscheinlich. Das könnte zu einer Reduktion des Mandats führen und damit ein weiteres Wachstum des Marktes deutlich erschweren.

Angesichts der vorherrschenden Unsicherheit hinsichtlich der langfristigen Entwicklung des Ethanolmarktes in der EU27 und den USA setzt sich die Dissertation zwei Ziele:

1. Die Faktoren zu identifizieren, die den Ethanolmarkt der EU27 und der USA bis zum Jahr 2023 entscheidend beeinflussen werden.

2. Die Erstellung unterschiedlicher Szenarien, die den Ethanolmarkt der EU27 und der USA im Jahr 2023 beschreiben. 
Die Faktoren, die die Zukunft des Ethanolmarktes in der EU27 und den USA entscheidend beeinflussen und im Folgenden als Schlüsselfaktoren bezeichnet werden, bilden die Grundlage für die Erstellung der Szenarien und dienen der Beobachtung des Marktes. Die gebildeten Szenarien, die jeweils eine mögliche Zukunft des Ethanolmarktes der EU27 und der USA beschreiben, stellen den realistischen Zukunftsraum des Marktes im Jahr 2023 dar. Der realistische Zukunftsraum ist der Bereich, in dem sich der Ethanolmarkt der EU27 und der USA im Jahr 2023 wahrscheinlich befinden wird.

Die Ergebnisse der Arbeit sollen Unternehmen dabei unterstützen, sich auf mögliche Veränderungen des Makroumfeldes des Ethanolmarktes frühzeitig vorzubereiten. Unternehmen können von den Ergebnissen profitieren, indem sie im Rahmen ihrer strategischen Planung den realistischen Zukunftsraum betrachten und Maßnahmen erarbeiten, um auf den Eintritt der unterschiedlichen Szenarien vorbereitet zu sein. Des Weiteren dienen die Schlüsselfaktoren dazu, frühzeitig zu erkennen, in Richtung welchen Szenarios sich der Markt bis zum Jahr 2023 entwickelt.

Aufgrund der starken Unterschiede zwischen dem Ethanolmarkt der EU27 und der USA werden die Regionen einzeln, aber nicht isoliert voneinander betrachtet. Das bedeutet, dass die Szenarien für den jeweiligen Ethanolmarkt zwar getrennt voneinander gebildet werden, dass aber der wechselseitige Einfluss bei der Erstellung der Szenarien berücksichtigt wird.

\section{$1.2 \quad$ Vorgehensweise}

Den einleitenden Ausführungen des ersten Kapitels folgend werden zu Beginn des zweiten Kapitels die historische Entwicklung und der Ist-Zustand des Ethanolmarktes der EU27 und der USA erläutert. Anschließend wird im zweiten Teil des Kapitels der Forschungsbedarf der Dissertation dargestellt, der sich aus einem Vergleich der bisherigen Forschung zur langfristigen Zukunft des Ethanolmarktes in der EU27 und den USA mit den momentanen Entwicklungen und möglichen, zukünftigen politischen Entscheidungen für die Ethanolmärkte ableitet.

Das dritte Kapitel dient der Begründung der Wahl der Szenario-Technik als Prognoseinstrument. Im Anschluss an die Definition des Szenario-Begriffs und einer Abgrenzung der verschiedenen Verfahren der Szenario-Technik wird in Kapitel 3.4 der induktive Ansatz der Szenario-Technik analysiert. 
In Kapitel 4 wird ein optimiertes Verfahren der Szenario-Technik beschrieben, da die Analyse in Kapitel 3.4 zeigt, dass sich kein etabliertes Verfahren findet, mit dem sich die Ziele dieser Arbeit erreichen lassen. Das optimierte methodische Vorgehen zur Bestimmung der Schlüsselfaktoren und zur Bildung der Szenarien unterteilt sich in die Kernschritte: „Szenariofeld-Analyse“ (Kapitel 4.2), „Szenario-Prognostik“ (Kapitel 4.3) und „Szenario-Bildung und -Konsistenzprüfung“ (Kapitel 4.4).

Das optimierte Verfahren der Szenario-Technik kommt in Kapitel 5 für den Ethanolmarkt der EU27 und in Kapitel 6 für den Ethanolmarkt der USA zur Anwendung. Der Aufbau beider Kapitel ist identisch und dementsprechend beziehen sich die folgenden Ausführungen auf beide Kapitel ${ }^{4}$.

Ziel der „Szenariofeld-Analyse“ (Kapitel 5.1/6.1) ist es, jene Schlüsselfaktoren zu bestimmen, die den Ethanolmarkt der EU27 und der USA bis zum Jahr 2023 entscheident beeinflussen werden. In der darauf folgenden „Szenario-Prognostik“ (Kapitel 5.2/6.2) wird für jeden erkannten Schlüsselfaktor mindestens eine Zukunftsprojektion gebildet, die eine Ausprägung des Schlüsselfaktors im Jahr 2023 beschreibt. Im letzten Kernschritt der „Szenario-Bildung und -Konsistenzprüfung“ (Kapitel 5.3/6.3) werden die einzelnen Zukunftsprojektionen durch die Nutzung eines morphologischen Kastens zu in sich konsistenten Szenarien verbunden und mittels einer Expertenbefragung auf ihre Konsistenz hin überprüft. Abschließend werden die Ergebnisse der Betrachtung des Ethanolmarktes der EU27 (Kapitel 5.4) und der USA (Kapitel 6.4) beschrieben.

Im siebten und letzten Kapitel werden, nach einer Zusammenfassung der Studie, Handlungsempfehlungen für Unternehmen, die im Ethanolmarkt der EU27 und/oder der USA tätig sind, abgeleitet und Ansätze für die weitere Forschung aufgezeigt.

\footnotetext{
${ }^{4}$ Aufgrund des bewusst gewählten, identischen Aufbaus der Kapitel 5 und 6 gibt es ab der dritten Gliederungsstufe zum Teil identische Überschriften.
} 
In den folgenden Kapiteln 2.1 und 2.2 werden der Ist-Zustand sowie die historische Entwicklung des Ethanolmarktes der EU27 und der USA beschrieben. Außerdem wird aufgezeigt, wie Überlegungen zur politischen Neuausrichtung der Förderung von Biokraftstoffen in der EU27 und den USA zu einer starken Verunsicherung der Märkte führen. Darauffolgend wird in Kapitel 2.3 der Stand der Forschung zur langfristigen Entwicklung des Ethanolmarktes in der EU27 und den USA dargestellt und der daraus resultierende Forschungsbedarf erläutert.

\subsection{Ethanolmarkt der EU27}

\section{Ethanolproduktion}

Die EU27 ist im Jahr 2012 mit 4,3 Milliarden Litern der drittgrößte EthanolKraftstoffproduzent der Welt hinter den USA mit 52,5 Milliarden Litern und Brasilien mit 20,5 Milliarden Litern (Abbildung 1). Gemeinsam produzieren diese drei Staaten/Regionen 92\% der weltweiten Ethanol-Kraftstoffproduktion in Höhe von 82 Milliarden Litern (F.O. LICHT 2012f: 74). Innerhalb der EU27 ist Frankreich, gefolgt von Deutschland und Belgien, der größte Ethanolproduzent (Abbildung 2).

Die Ethanolproduktion der EU27 ist im Zeitraum von 2003 bis 2012 von 0,4 Milliarden Litern auf 4,3 Milliarden Liter angestiegen (Abbildung 2), was einem durchschnittlichen jährlichen Wachstum von 30,2\% entspricht. Dieses enorme Marktwachstum ist auf die starke politische Förderung von Biokraftstoffen in der EU27 zurückzuführen. Die wichtigsten politischen Maßnahmen werden bei der Betrachtung der Entwicklung der Nachfrage erläutert, da sie diese beeinflussen.

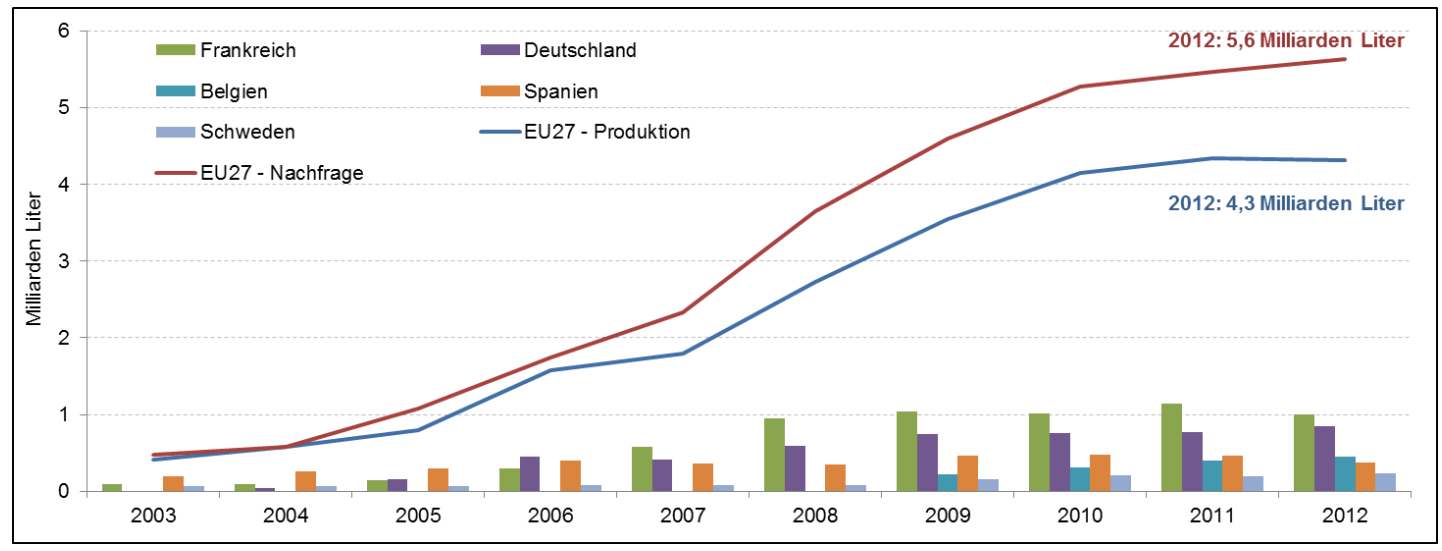

Abbildung 2: Entwicklung des Ethanolmarktes der EU27

Quelle: In Anlehnung an F. O. LICHT 2012f: 74; BERG 2012c: 19; EUROSTAT 2012

Nach mehreren Jahren des starken Produktionswachstums ist für das Jahr 2011 eine deutliche Abschwächung des Wachstums zu konstatieren, der ein geringer 
Rückgang der Ethanolproduktion im Jahr 2012 folgte. Diese rückläufige Entwicklung ist auf die günstigen Ethanolimporte aus Brasilien und den USA zurückzuführen, die den europäischen Ethanolpreis stark unter Druck setzten und etliche Ethanolproduzenten zwangen, die Produktion aufgrund fehlender Profitabilität auszusetzen (F.O. LICHT 2012a: 300; USDA FOREIGN AGRICULTURAL SERVICE 2012: 16f.; F.O. LICHT 2011a: 66).

In der EU27 wird beinahe ausschließlich Ethanol-Kraftstoff der ersten Generation produziert. Die wichtigsten Rohstoffe sind Weizen und Zuckerrüben (EUROPEAN COMMISSION JOINT RESEARCH CENTRE, INSTITUT FOR ENERGY ET AL. 2010: 74). Die Produktion von Ethanol der zweiten Generation aus Zellulose betrug im Jahr 2012 lediglich 0,03 Milliarden Liter - dies entspricht 0,7\% der gesamten Ethanolproduktion der EU275 (OECD/FAO 2012: 224).

\section{Ethanolnachfrage}

Die Nachfrage nach Ethanol-Kraftstoff ist in den Jahren von 2003 bis 2012 von 0,48 Milliarden Litern auf 5,6 Milliarden Liter angestiegen (Abbildung 2). Dies entspricht einem durchschnittlichen jährlichen Wachstum von 31,4\%, womit die Nachfrage in den letzten neun Jahren stärker als die Produktion gewachsen ist. Die EU27 ist folglich ein Ethanolimporteur. Maßgeblicher Treiber des starken Nachfragewachstums ist die politische Unterstützung für Biokraftstoffe in der EU27. In der folgenden Tabelle 1 sind die wichtigsten politischen Richtlinien zur Förderung von Biokraftstoffen in der Zeit von 2003 bis 2012 aufgelistet und die Kernpunkte jeder Richtlinie genannt.

\footnotetext{
${ }^{5}$ Eine genaue Auflistung der Kapazitäten zur Produktion von Zellulose-Ethanol befindet sich in Kapitel 5.2.9.
} 


\begin{tabular}{|c|c|c|c|}
\hline Richtlinie & Titel & Jahr & Kernpunkte \\
\hline 2003/30/EG & $\begin{array}{l}\text { Richtlinie des Europäischen } \\
\text { Parlaments und des Rates zur } \\
\text { Förderung der Verwendung von } \\
\text { Biokraftstoffen oder anderen } \\
\text { erneuerbaren Kraftstoffen im } \\
\text { Verkehrssektor }\end{array}$ & 2003 & $\begin{array}{l}\text { - Beimischungspflicht für die einzelnen } \\
\text { Mitgliedstaaten von Biokraftstoffen zu } \\
\text { Otto- und Dieselkraftstoffen. } \\
\text { Zielsetzung: } \\
\text { - 2005: } 2 \% \\
\text { - } 2010: 5,75 \%\end{array}$ \\
\hline 2003/96/EG & $\begin{array}{l}\text { Richtlinie des Rates zur Re- } \\
\text { strukturierung der gemein- } \\
\text { schaftlichen Rahmenvorschriften } \\
\text { zur Besteuerung von Energie- } \\
\text { erzeugnissen und elektrischem } \\
\text { Strom }\end{array}$ & 2003 & $\begin{array}{l}\text { - Mitgliedsstaaten können die Ver- } \\
\text { brauchssteuer für Biokraftstoffe sen- } \\
\text { ken oder diese vollständig von der } \\
\text { Steuer befreien, um den Absatz von } \\
\text { Biokraftstoffen zu fördern. }\end{array}$ \\
\hline 2009/28/EG & $\begin{array}{l}\text { Richtlinie des Europäischen } \\
\text { Parlaments und des Rates zur } \\
\text { Förderung der Nutzung von } \\
\text { Energie aus erneuerbaren Quel- } \\
\text { len und zur Änderung und an- } \\
\text { schließenden Aufhebung der } \\
\text { Richtlinie 2001/77/EG und } \\
\text { 2003/30/EG }\end{array}$ & 2009 & $\begin{array}{l}\text { - Im Jahr } 2020 \text { soll jedes Mitgliedsland } \\
\text { der EU27 10\% des Energie- } \\
\text { verbrauchs im Verkehrssektor aus } \\
\text { erneuerbaren Energiequellen bezie- } \\
\text { hen. } \\
\text { - Förderung von Biokraftstoffen der } 2 \text {. } \\
\text { Generation. } \\
\text { - Bestimmung von Nachhaltigkeits- } \\
\text { kriterien für Biokraftstoffe. }\end{array}$ \\
\hline
\end{tabular}

Tabelle 1: Übersicht über die wichtigsten politischen Richtlinien zur Förderung von Biokraftstoffen

Quelle: RICHTLINIE 2003/30/EG ARTIKEL 3 (1); RICHTLINIE 2003/96/EG; RICHTLINIE 2009/28/EG

Das Nachfragewachstum im Zeitraum von 2003 bis 2009 ist auf die Richtlinien 2003/30/EG und 2003/96/EG zurückzuführen. Die Beimischungsrichtlinie (2003/30/EG) verpflichtet die Mitgliedsstaaten sicherzustellen, dass am Ende des Jahres 2005 2\% des energetischen Kraftstoffverbrauchs (Otto- und Dieselkraftstoff) biogenen Ursprungs sind. Die Beimischungspflicht steigt bis zum Jahr 2010 auf 5,75\% an. Zur Förderung des Verbrauchs von Biokraftstoffen in der EU27 ermöglicht die Besteuerungsrichtlinie (2003/96/EG) den einzelnen Mitgliedsstaaten, die Verbrauchssteuer für Biokraftstoffe - und andere Bioenergieerzeugnisse - zu senken oder ganz aufzuheben.

Im Jahr 2009 wurde die Richtlinie 2009/28/EG verabschiedet, die die Richtlinie 2003/30/EG ersetzt. Kernpunkt der neuen und derzeit noch aktuellen Richtlinie ist das Ziel, dass in jedem Mitgliedsland im Jahr 2020 mindestens 10\% des Energieverbrauchs im Verkehrssektor aus erneuerbaren Energiequellen bezogen werden (RICHTLINIE 2009/28/EG ARTIKEL 3 (4)) (Tabelle 1). Im Gegensatz zu den in der Richtlinie 2003/96/EG formulierten Zielen fördert das 10\%-Ziel Biokraftstoffe nicht direkt. Die führenden Experten sind sich aber einig, dass Biokraftstoffe für den Kraftfahrzeugbereich den mit Abstand größten Beitrag zur Erreichung des 10\%-Ziels im Jahr 2020 leisten werden (EUROPÄISCHEN KOMMISSION 2012: 7; EUROPEAN COMMISSION JOINT RESEARCH CENTRE, INSTITUTE FOR ENERGY ET AL. 
2011: 46ff.; EUROPEAN COMMISSION JOINT RESEARCH CENTRE, INSTITUTE FOR PROSPECTIVE TECHNOLOGICAL STUDIES 2010a: 33).

Darüber hinaus fördert die RICHTLINIE 2009/28/EG (ARTIKEL 21 (2)) explizit Biokraftstoffe der zweiten Generation und die Nutzung von Elektrizität im Straßenverkehr durch die Mehrfachanrechnung des Energiegehalts dieser Energieträger auf das 10\%-Ziel (RICHTLINIE 2009/28/EG ARTIKEL 3 (4)). Um das politische Kernziel der Kohlenstoffdioxidreduktion zu erreichen, hat die EU27 in der RICHTLINIE 2009/28/EG (ARTIKEL 17 (1)) Nachhaltigkeitskriterien für Biokraftstoffe festgesetzt. So dürfen nur Biokraftstoffe, die eine im Vergleich zu fossilem Kraftstoff festgelegte $\mathrm{CO}_{2}$-Einsparung erreichen, dem 10\%-Ziel angerechnet werden ${ }^{6}$.

Zur Erreichung des Ziels der Treibhausgasreduktion und zur Überprüfung des jährlichen Fortschritts der Entwicklung sind die einzelnen Mitgliedsstaaten der EU27 in RICHTLINIE 2009/28/EG (ARTIKEL 4 (1)) verpflichtet, der EU-Kommission jährlich einen nationalen Aktionsplan für erneuerbare Energie vorzulegen, in dem sie die IstSituation erläutern und darlegen, wie sie in den nächsten Jahren das $10 \%$-Ziel erreichen wollen. Um den Absatz von Biokraftstoffen zu fördern und damit das Ziel zu erreichen, wird von den Mitgliedsstaaten die Beimischungspflicht von Biokraftstoff zu Fossilbrennstoff und/oder die Verbrauchssteuerbefreiung/Verbrauchssteuersenkung für Biokraftstoffe genutzt (F.O. LICHT 2012d: 420f.). Im Gegensatz zu den Jahren 2003 bis 2007, in denen hauptsächlich die Verbrauchssteuerbefreiung/Verbrauchssteuersenkung zur Absatzförderung genutzt wurde (HENNIGES 2006: 45; BECKER 2011: 13), setzen die meisten der Mitgliedsstaaten mittlerweile auf ein Quotensystem zur Zielerreichung (F.O. LICHT 2012d: 420f.).

\section{Ethanolhandel}

Bedeutend für den Ethanolmarkt der EU27 ist der Import, da die Ethanolnachfrage in den letzten Jahren stärker als die Produktion angestiegen ist (Abbildung 2). Im Jahr 2012 wurden 1,3 Milliarden Liter Ethanol-Kraftstoff in die EU27 eingeführt, womit die EU27 der weltweit wichtigste Importmarkt ist (F.O. LICHT 2012a: 302). Die Ethanolimporte stammen fast ausschließlich aus den wichtigen Ethanol produzierenden Staaten USA und Brasilien (F.O. LICHT 2012a: 301f.).

\footnotetext{
${ }^{6}$ Eine genaue Beschreibung der politischen Anforderungen an die Treibhausgasreduktion von Biokraftstoffen im Verhältnis zu fossilem Kraftstoff befindet sich in Kapitel 5.2.7.
} 
Die hohen Importströme kamen trotz des Außenschutzes in Form von Importzöllen, die in der Verordnung KN-Code $2207^{7}$ festgelegt sind, in die EU27 (F.O. LICHT 2012a: 300). Es ist aber wichtig zu erwähnen, dass in den letzten Jahren nicht unerhebliche Ethanolmengen als E90 (90\% Ethanol und 10\% Benzin) eingeführt wurden, ohne dass der reguläre Importzollsatz bezahlt wurde, da E90 bis März 2012 mit lediglich einem Wertzollsatz von 6,5\% verzollt wurde. Infolgedessen geriet der Ethanolpreis der EU27 stark unter Druck und etliche Ethanolproduzenten sahen sich gezwungen, die Produktion auszusetzen (F.O. LICHT 2012a: 300).

Wie bereits in der Einleitung erwähnt, ist der Ethanolmarkt der EU27 momentan stark verunsichert, da die Politik einen Wandel des derzeitigen Biokraftstoffmarktes der ersten Generation hin zu einem Markt der zweiten Generation anstrebt. Energiekommissar Günther Oettinger und Klimakommissarin Connie Hedegard empfehlen in ihrem Vorschlag vom 17. Oktober 2012 eine Neuausrichtung der politischen Förderung von Biokraftstoffen in der EU27 $7^{8}$ (EUROPÄISCHE KOMMISSION 2012). Sie halten zwar am 10\%-Ziel fest, wollen aber (1) die Anrechnung von Biokraftstoffen der ersten Generation auf das 10\%-Ziel auf die Hälfte begrenzen (5\%) und im Gegenzug Biokraftstoffe der zweiten Generation durch eine Vierfach-Anrechnung des Energiegehalts auf das 10\%-Ziel stärker fördern. (2) Schlagen die Kommissare ab dem Jahr 2021 eine ausschließliche politische Förderung solcher Biokraftstoffe vor, die nicht aus Lebens- und Futtermitteln produziert werden und (3) wollen sie den ILUC-Faktor ${ }^{9}$ bei der Berechnung der $\mathrm{CO}_{2}$-Bilanz von Biokraftstoffen berücksichtigen (EUROPÄISCHE KOMMISSION 2012: 3).

Die Vorschläge der Kommissare verunsichern den Markt, da der Anteil von Biokraftstoffen der ersten Generation am Gesamtenergieverbrauch im Verkehrssektor bereits $4,5 \%$ beträgt, wodurch die 5-prozentige Begrenzung nur sehr geringen Raum für weiteres Nachfragewachstum zulässt (F.O. LICHT 2012f: 70). Des Weiteren impliziert sowohl die Ankündigung ab 2021 nur noch solche Biokraftstoffe politisch zu

\footnotetext{
${ }^{7}$ In Kapitel 5.2.5 werden die Zollbestimmungen der EU27 im Detail erläutert.

$8 \mathrm{Da}$ sich der Vorschlag von EU-Energiekommissar Günther Oettinger und EUKlimakommissarin Connie Hedegaard auf die Zukunft bezieht, wird er bei der Bildung der Zukunftsprojektionen für den Ethanolmarkt der EU27 in den Kapiteln 5.2.6 und 5.2.7 ausführlich betrachtet.

${ }^{9}$ Der Begriff ILUC (Indirekt Land Use Change) beschreibt folgenden Sachverhalt: Landwirtschaftliche Kulturen werden für die Produktion von Biokraftstoffen auf Ackerland angebaut, welches für die Produktion von Nahrungsmitteln geeignet ist und dadurch die Nahrungsmittelproduktion auf Flächen verdrängt, die zuvor nicht zur landwirtschaftlichen Produktion genutzt wurden. Die Bewirtschaftung der zuvor ungenutzten Fläche z.B. zur Nahrungsmittelproduktion führt zu der Freisetzung von Treibhausgasen, die der Biokraftstoffproduktion angerechnet werden (USDA FOREIGN AGRICULTURAL SERVICE 2011: 9). Eine genaue Erläuterung des Themas ILUC befindet sich in Kapitel 5.1.1.2/Einflussfaktor 21.
} 
fördern, die nicht aus Lebens- und Futtermitteln produziert werden, als auch die Berücksichtigung eines ILUC-Faktors, wodurch Ethanol, hergestellt aus Weizen und Zuckerrüben, nicht mehr die politisch vorgegebenen prozentualen $\mathrm{CO}_{2}$ Reduktionswerte im Vergleich zu fossilem Kraftstoff erfüllt ${ }^{10}$, dass nur noch Biokraftstoffe der zweiten Generation politisch gestützt werden. Dies würde bedeuten, dass Ethanol der ersten Generation in direkter Konkurrenz zu fossilem Benzin stünde, infolgedessen es zu einem deutlichen Rückgang der Nachfrage nach EthanolKraftstoff der ersten Generation in der EU27 käme.

\section{$2.2 \quad$ Ethanolmarkt der USA}

\section{Ethanolproduktion}

Die US-amerikanische Ethanolproduktion ist im Zeitraum von 2004 bis 2011 stetig von 13 auf 54 Milliarden Liter angestiegen (Abbildung 3), was einem durchschnittlichen jährlichen Wachstum von 22,5\% entspricht. Allerdings kam es im Jahr 2012 erstmals seit 16 Jahren zu einem Rückgang der Ethanolproduktion auf 52,5 Milliarden Liter (F.O. LICHT 2013b: 212; F.O. LICHT 2012f: 69). Diese Produktionsabnahme ist auf die folgenden drei Einflüsse zurückzuführen: (1) Der USamerikanische Ethanolmarkt für Maisethanol (Conventional Biofuel) ist gesättigt. (2) Die Exportmengen für Ethanol sind im Vergleich zum Jahr 2011 deutlich zurückgegangen. (3) Die wegen der starken Trockenheit im Corn Belt im Sommer 2012 hohen Maispreise und die infolge des gesättigten Marktes im Verhältnis dazu geringen Ethanolpreise zwangen zahlreiche Ethanolproduzenten, die Produktion aufgrund fehlender Wirtschaftlichkeit auszusetzen ${ }^{11}$ (F.O. LICHT 2013b: 213; F.O. LICHT 2012f: 69).

\footnotetext{
${ }^{10}$ In Kapitel 5.1.1.2/Einflussfaktor 15a/15b befindet sich eine ausführliche Beschreibung der Auswirkungen des ILUC-Faktors auf die $\mathrm{CO}_{2}$-Bilanz von Ethanol-Kraftstoff.

${ }^{11}$ F.O. LICHT (2013b: 213) geht davon aus, dass 20 Anlagen in der zweiten Hälfte des Jahres 2012 und in den ersten Monaten des Jahres 2013 die Produktion aussetzten.
} 


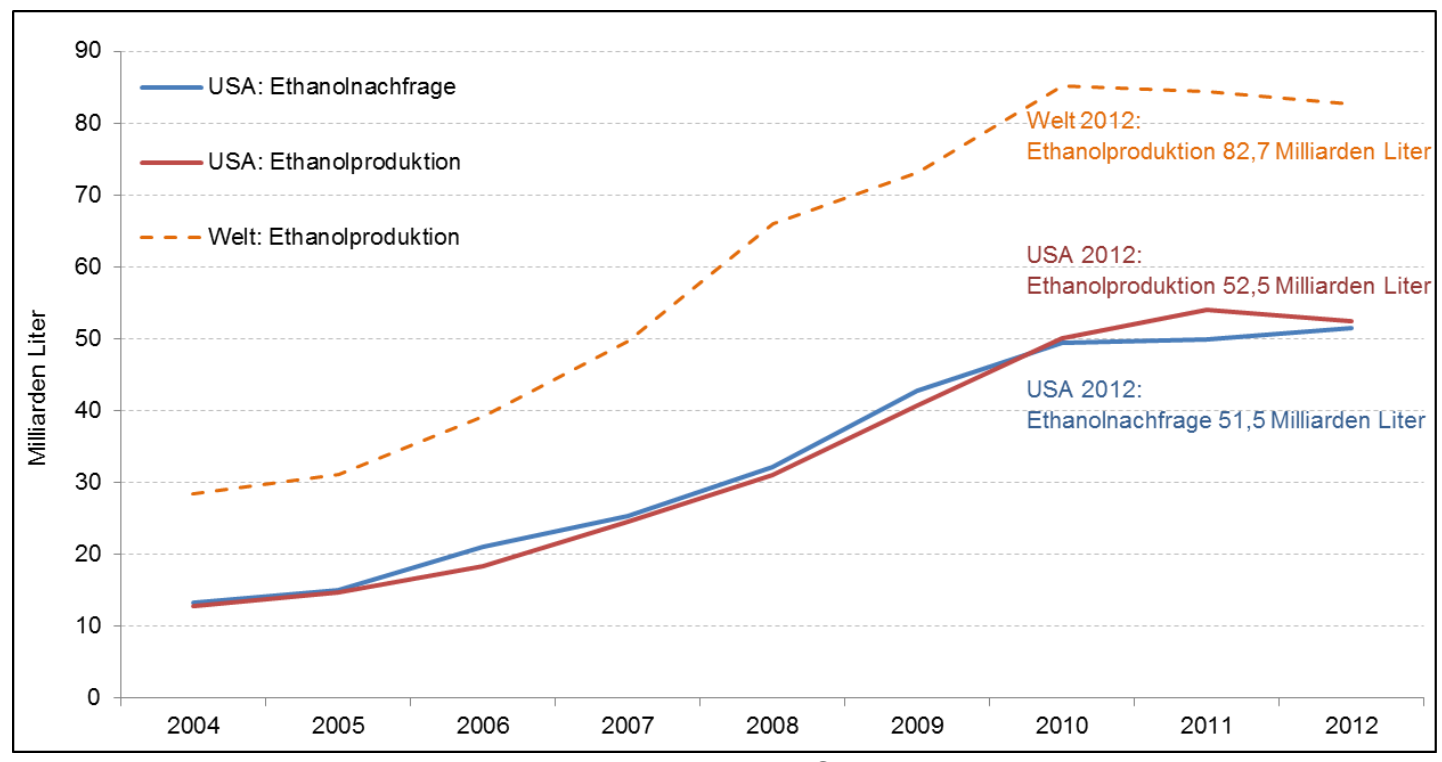

Abbildung 3: Entwicklung des Ethanolmarktes der USA

Quelle: In Anlehnung an F.O. LICHT 2012f: 73f.; BERG 2012c: 17; U.S. ENERGY INFORMATION ADMINISTRATION 2012b: 97

Die Ethanolproduktion in den USA erfolgt fast nur im Corn Belt und fast nur aus dem Rohstoff Mais (U.S. ENERGY INFORMATION ADMINISTRATION 2013b: 1; U.S. ENERGY INFORMATION ADMINISTRATION 2012j: 6ff.). Im Jahr 2012 wurden 115 Millionen Tonnen Mais - 42\% der gesamten US-amerikanischen Maisproduktion zu Ethanol fermentiert (U.S. DEPARTMENT OF ENERGY 2013b). In den USA bestehen lediglich Produktionskapazitäten von 0,53 Milliarden Litern für die Herstellung von Ethanol aus anderen Rohstoffen als Mais (RFA 2012c). Ferner gab es bis zum Anfang des Jahres 2013 keine kommerzielle Produktion von Zellulose-Ethanol (F.O. LICHT 2013a: 238). Die ersten kommerziellen Anlagen werden derzeitig zwar von Abengoa Bioenergy, DuPont Industrial Biosciences und im Rahmen des Gemeinschaftsprojekts „Project LIBERTY“ von POET und DSM im Corn Belt errichtet ${ }^{12}$, die Anlagen werden die Produktion aber nicht vor 2014/2015 aufnehmen (ABENGOA BIOENERGY 2012; DUPONT 2012; POET-DSM ADVANCED BIOFUELS 2012).

${ }^{12}$ Die folgenden drei Unternehmen errichten momentan kommerzielle Anlagen zur Herstellung von Zellulose-Ethanol in den USA:

- Abengoa Bioenergy baut eine Anlage zur jährlichen Produktion von 0,095 Milliarden Litern in Hugoton (Kansas) (ABENGOA BIOENERGY 2012).

- DuPont Industrial Biosciences erbaut eine 0,1-Milliarden-Liter-Anlage in Nevada (lowa) (DUPONT 2012).

- POET und DSM arbeiten gemeinsam am „Project LIBERTY“, um eine 0,095-MilliardenLiter-Anlage in Emmetsburg (lowa), zu errichten (POET-DSM ADVANCED BIOFUELS 2012). 


\section{Ethanolnachfrage}

Zwischen 2004 und 2012 ist die US-amerikanische Ethanolnachfrage von 13,3 Milliarden Litern auf 51,5 Milliarden Liter angestiegen (Abbildung 3). Maßgeblicher Treiber der US-amerikanischen Ethanolnachfrage ist der RFS 2, der jährlich ansteigende Verbrauchsmandate für im US-amerikanischen Transportbereich zu verbrauchende Biokraftstoffe bis zum Jahr 2022 vorgibt (U.S. GOVERNMENT INFORMATION 2007: 121 STAT. 1519). Abbildung 4 zeigt, wie sich die US-amerikanische Ethanolnachfrage in den Jahren von 2008 bis 2010 entsprechend den Verbrauchsvorgaben des RFS 2 entwickelt hat. Aufgrund der E10-,Blend Wall“, an der sich die USA seit 2011 befinden, blieb die Nachfrage seit 2010 nahezu konstant (F.O. LICHT 2013b: 213).

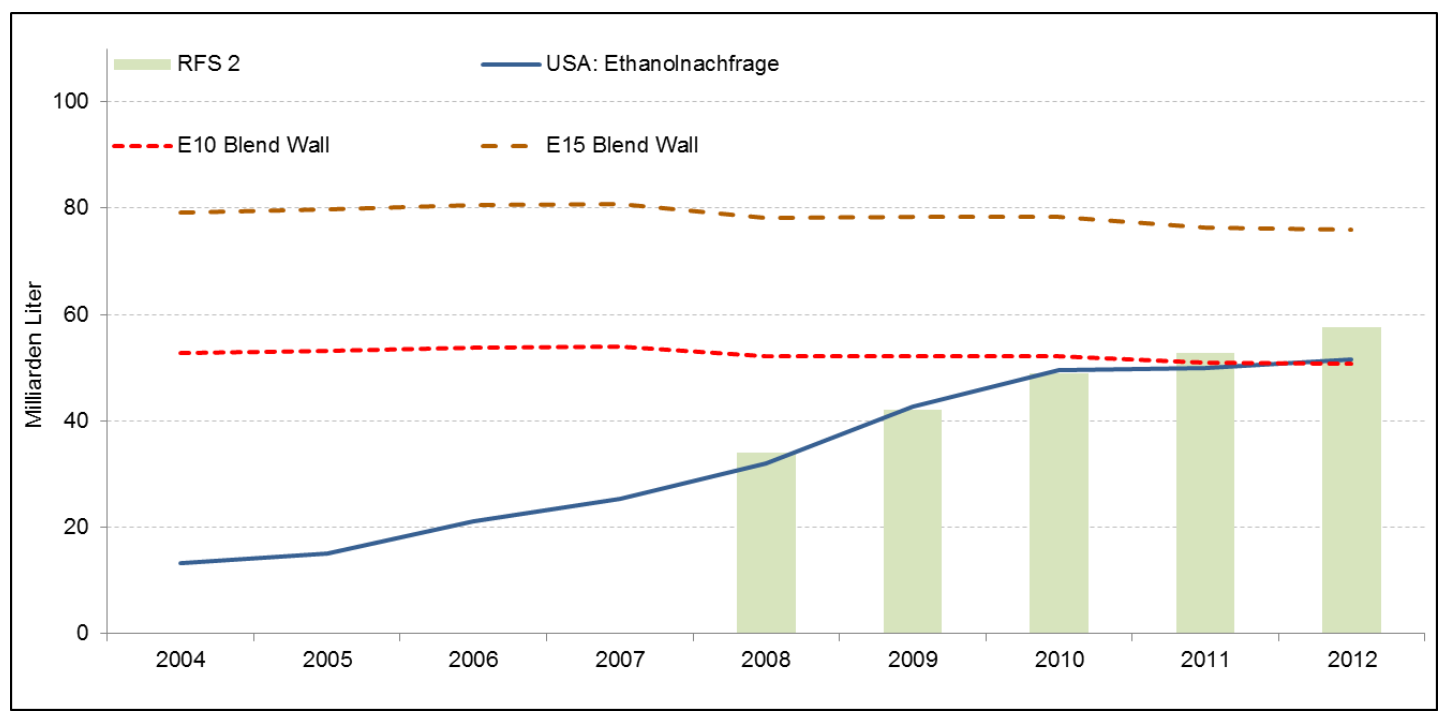

Abbildung 4: Auswirkungen der „Blend Wall“-Problematik auf die US-amerikanische Ethanolnachfrage

Quelle: In Anlehnung an F.O. LICHT 2012f: 73f.; U.S. GOVERNMENT INFORMATION 2007: 121 STAT. 1519; U.S. ENERGY INFORMATION ADMINISTRATION 2013a: 84; EIGENE BERECHNUNG

Die „Blend Wall” ist die maximale Menge Ethanol, die dem in den USA nachgefragten Benzin beigemischt werden kann (CONGRESSIONAL RESEARCH SERVICE 2010a: 5; OECD/FAO 2012: 110). Der Absatz von Ethanol als E85 an Flexible Fuel Vehicles (FFV) wird bei der Ermittlung der „Blend Wall“ nicht berücksichtigt. Da aber der Absatz von E85 minimal ist - lediglich 146 Millionen Liter/0,28\% der gesamten Ethanolnachfrage (U.S. ENERGY INFORMATION ADMINISTRATION 2012j: 29) ist die sich aus der "Blend Wall“ ergebende Beimischungsmenge mit der maximal möglichen Ethanolnachfrage in den USA gleichzusetzen. Aufgrund der Tatsache, dass die Binnennachfrage der entscheidende Treiber des US-amerikanischen Ethanolmarktes ist, ist ein weiteres Wachstum des Marktes nur möglich, wenn die E10„Blend Wall“ überwunden wird. Anzumerken ist, dass sich - sollte die „Blend Wall“- 
Problematik nicht zeitnah bewältigt werden - die Problematik in den nächsten Jahren weiter verschärfen wird, da die Benzinnachfrage in den USA nach Prognosen der U.S. ENERGY INFORMATION ADMINISTRATION (2013a: 84) weiter sinken wird (Abbildung 5).

Die United States Environmental Protection Agency (EPA) hat die Voraussetzungen für die Überwindung der „Blend Wall“ durch die Zulassung des Kraftstoffs E15 für alle Kraftfahrzeuge ab Baujahr 2001 geschaffen (U.S. GOVERNMENT INFORMATION 2011: 4662). Der Automobilbereich ist folglich nicht der verkaufshemmende Faktor, da der Großteil der US-amerikanischen Kraftfahrzeuge Baujahr 2001 und jünger ist (RFA 2012b). Verhindert wird die Nutzung von E15 durch das Fehlen einer Tankstelleninfrastruktur, die es ermöglicht, Benzin mit einem 15-prozentigen Ethanolanteil zu verkaufen (F.O. LICHT 2013c: 341). Die Zapfsäulen an den Tankstellen in den gesamten USA vertragen zwar E10, aber nicht E15 (NACS 2012b: 25), da sonst die Dichtungen erodieren und undicht werden (NACS 2012b: 30; U.S. ENERGY INFORMATION ADMINISTRATION 2011c).

Die „Blend Wall“-Problematik steht im Konflikt mit dem RFS 2, da die dort festgelegten jährlich ansteigenden Verbrauchsmandate für Biokraftstoffe nur dann im USamerikanischen Transportbereich abgesetzt werden können, wenn die „Blend Wall“ überwunden wird (Abbildung 5). Wird aber keine Lösung für die „Blend Wall“Problematik gefunden, so wird der politische Druck auf den RFS 2 weiter zunehmen, mit dem Ziel, eine Absenkung der Verbrauchsmandate zu erreichen.

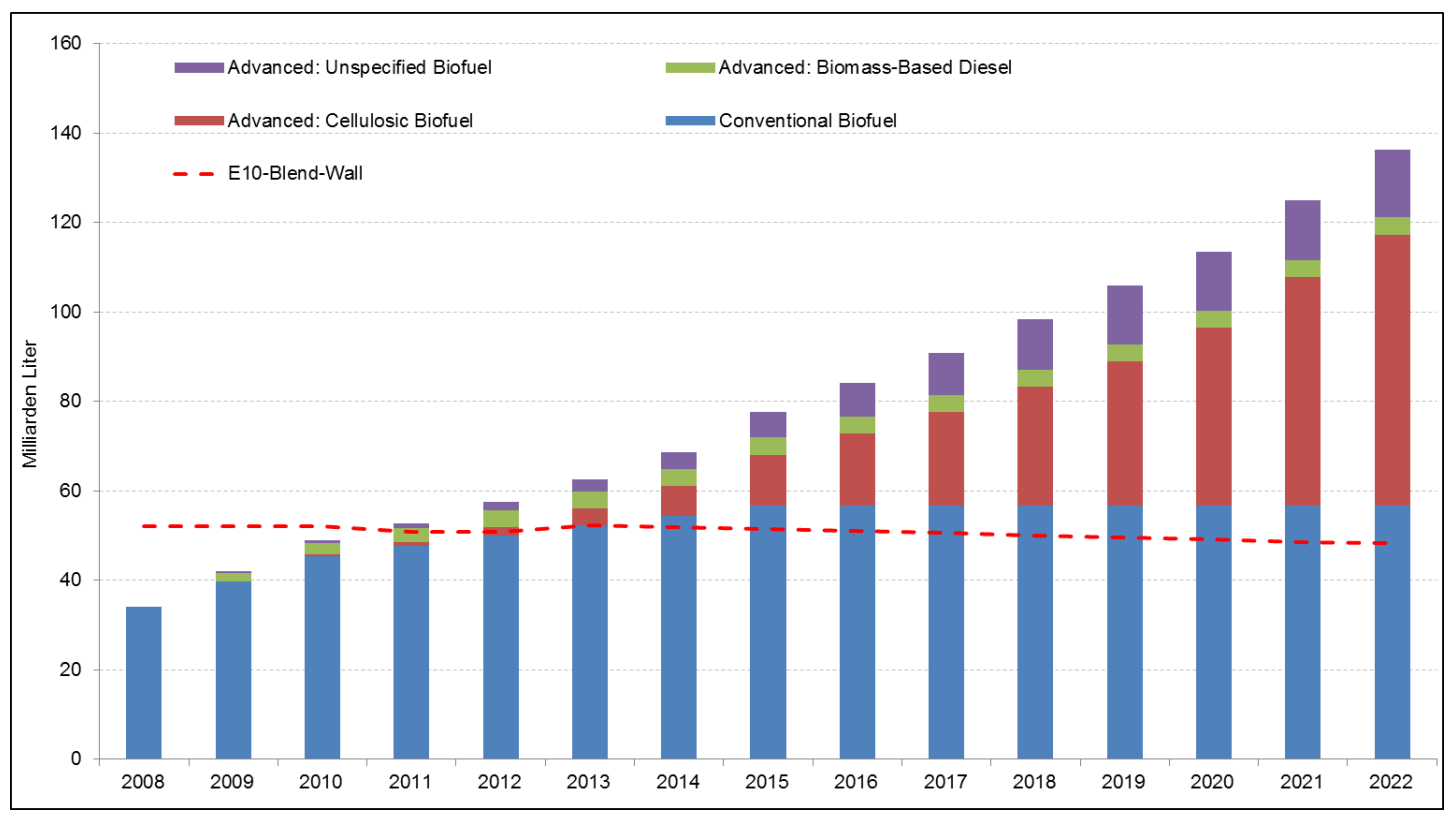

Abbildung 5: Konflikt „RFS 2“ und „Blend Wall“

Quelle: In Anlehnung an U.S. GOVERNMENT INFORMATION 2007: 121 STAT. 1522; U.S. ENERGY INFORMATION ADMINISTRATION 2013a: 84; U.S. ENERGY INFORMATION ADMINISTRATION 2013a: 84; EIGENE BERECHNUNG 
Im nächsten Abschnitt wird der RFS 2 vorgestellt und in diesem Zusammenhang die Problematik der ausreichenden Produktion von „Advanced: Cellulosic Biofuel“ erläutert.

Der RFS 2 ist Bestandteil des „Energy Independence Security Act“ (EISA) von 2007 (U.S. GOVERNMENT INFORMATION 2007: 121 STAT. 1519) und unterteilt die Biokraftstoffe in zwei Hauptgruppen: die „Conventional Biofuels“ und die „Advanced Biofuels“. Entscheidend für die Unterteilung sind der Rohstoff, der zur Produktion genutzt wird und die erzielte $\mathrm{CO}_{2}$-Reduktion des biogenen Kraftstoffs im Vergleich zu fossilem Kraftstoff. Ist der Rohstoff Mais und erreicht der Biokraftstoff eine 20prozentige Treibhausgasreduktion, so gehört der Kraftstoff der Gruppe der „Conventional Biofuels“ an; wird ein anderer Rohstoff wie z.B. Zellulose, Weizen, Zuckerrübe oder Zuckerrohr eingesetzt und eine $\mathrm{CO}_{2}$-Reduktion von mindestens $50 \%$ erreicht, so wird der Kraftstoff der Gruppe der „Advanced Biofuels“ angerechnet (U.S. GOVERNMENT INFORMATION 2007: 121 STAT. 1519). Die Gruppe „Advanced Biofuels“ unterteilt sich in die drei Unterkategorien „Advanced: Unspecified Biofuel“, „Advanced: Biomass-Based Diesel“ und „Advanced: Cellulosic Biofuel“ (U.S. GOVERNMENT INFORMATION 2007: 121 STAT. 1520) ${ }^{13}$.

Der Unterkategorie „Advanced: Unspecified Biofuel“ gehören biogene Kraftstoffe an, die eine 50-prozentige $\mathrm{CO}_{2}$-Reduktion im Vergleich zu fossilem Kraftstoff erreichen und beispielsweise aus Weizen, Sorghum, Zuckerrüben und Zuckerrohr produziert werden (CONGRESSIONAL RESEARCH SERVICE 2012: 4). Kraftstoffe, hergestellt aus pflanzlichen Fetten wie Sojaöl, Rapsöl, Palmöl oder Sonnenblumenöl, die ebenfalls eine im Vergleich zu fossilem Treibstoff 50-prozentige $\mathrm{CO}_{2}$-Einsparung erzielen, werden der Unterkategorie „Advanced: Biomass-Based Diesel“ angerechnet ${ }^{14}$ (CONGRESSIONAL RESEARCH SERVICE 2012: 4; U.S. GOVERNMENT INFORMATION 2007: 121 STAT. 1520). Der dritten Unterkategorie „Advanced: Cellulosic Biofuel" werden Biokraftstoffe angerechnet, die aus den Rohstoffen Zellulose, Hemizellulose und Lignin produziert werden und im Vergleich zu fossilem Kraftstoff eine 60-prozentige $\mathrm{CO}_{2}$-Ersparnis erzielen (CONGRESSIONAL RESEARCH SERVICE 2012: 4; U.S. GOVERNMENT INFORMATION 2007: 121 STAT. 1520).

Die Unterkategorie „Advanced: Cellulosic Biofuel“ ist für den zukünftigen USamerikanischen Ethanolmarkt von hoher Bedeutung, da die EPA im RFS 2 einen

\footnotetext{
${ }^{13}$ Eine Erläuterung bezüglich der hierarischen Struktur zwischen den beiden Gruppen und den Unterkategorien befindet sich in Kapitel 6.2.5.

${ }^{14} \mathrm{Da}$ diese Studie ausschließlich den Ethanolmarkt der EU27 und der USA betrachtet und sich nicht mit dem Biodieselmarkt befasst, wird die Unterkategorie „Advanced: BiomassBased Diesel“ nur am Rande diskutiert.
} 
starken Anstieg des Verbrauchsmandats von 0,38 Milliarden Litern im Jahr 2010 auf 61 Milliarden Liter im Jahr 2022 festgelegt hat (Abbildung 5) (U.S. GOVERNMENT INFORMATION 2007: 121 STAT. 1523). Problematisch ist allerdings, dass es in den USA bis Anfang 2013 noch keine kommerzielle Produktion von Biokraftstoffen aus Zellulose, Hemizellulose oder Lignin gab, sondern lediglich eine Herstellung in Pilot- und Demonstrationsanlagen. Folglich konnten die festgelegten Verbrauchsmandate nicht erfüllt werden und wurden von der EPA stark gekürzt (CONGRESSIONAL RESEARCH SERVICE 2012: 3; EPA 2010: 4; EPA 2011: 2): im Jahr 2010 von 0,38 Milliarden Litern auf 0,024 Milliarden Liter, im darauf folgenden Jahr 2011 von 0,95 Milliarden Litern auf nur noch 0,025 Milliarden Liter und von 1,9 Milliarden Litern auf lediglich 0,033 Milliarden Liter im Jahr 2012. Des Weiteren wird es bis 2015 zu keinem bedeutenden Anstieg der Produktion kommen, da die ersten, sich derzeit im Bau befindenden kommerziellen Anlagen von Abengoa Bioenergy, DuPont Industrial Biosciences und dem Gemeinschaftsprojekt von POET und DSM die Produktion nicht vor 2014/2015 aufnehmen werden. Dadurch wird die Differenz zwischen dem Verbrauchsmandat für „Advanced: Cellulosic Biofuel“ und der tatsächlichen Produktion immer weiter auseinander gehen, wodurch sich die Frage aufdrängt, ob die im RFS 2 festgelegten Verbrauchsmandate für „Advanced: Cellulosic Biofuel" noch realistisch sind oder gesenkt werden müssen.

Die mögliche Minderung der Verbrauchsmandate des RFS 2 verursacht eine starke Verunsicherung des US-amerikanischen Ethanolmarktes, da der RFS 2 das entscheidende politische Instrument zur Förderung von Biokraftstoffen in den USA ist. Darüber hinaus verunsichert den US-amerikanischen Markt der schwindende politische Rückhalt für Biokraftstoffe, der schon seit $2010 \mathrm{zu}$ erkennen ist (F.O. LICHT 2012c: 319). Beispielhaft für diese Entwicklung ist die Entscheidung, den Volumetric Ethanol Excise Tax Credit (VEETC) ${ }^{15}$ in Höhe von 0,45 US-\$ je Gallone auslaufen zu lassen, wodurch Ende 2011 der Ethanol-Importzoll in Höhe von 0,54 US-\$ je Gallone wegfiel. Des Weiteren ist der stärker werdende Einfluss der USamerikanischen Mineralöl- und Nahrungsmittelindustrie auf die Entscheidungsträger in Washington nicht zu unterschätzen, denn beide Industrien wollen eine Kürzung der Verbrauchsmandate erreichen (F.O. LICHT 2012c: 319; ETHANOL PRODUCER MAGAZINE 2012c; F.O. LICHT 2012g: 489f.).

\footnotetext{
${ }^{15}$ Durch den VEETC bekam die Mineralölindustrie eine Steuergutschrift von 0,45 US-\$ für jede beigemischte Gallone Ethanol zu fossilem Benzin (U.S. DEPARTMENT OF ENERGY 2013h).
} 


\section{Ethanolhandel}

In den Jahren von 2004 bis 2012 hat sich der US-amerikanische Ethanolmarkt von einem importierenden zu einem exportierenden Markt gewandelt (Abbildung 6) ${ }^{16}$. So waren die USA in den Jahren 2011 (5 Milliarden Liter) und 2012 (2,9 Milliarden Liter) der weltgrößte Ethanolexporteur vor Brasilien (BERG 2012c: 17; F.O. LICHT 2012a: 301).

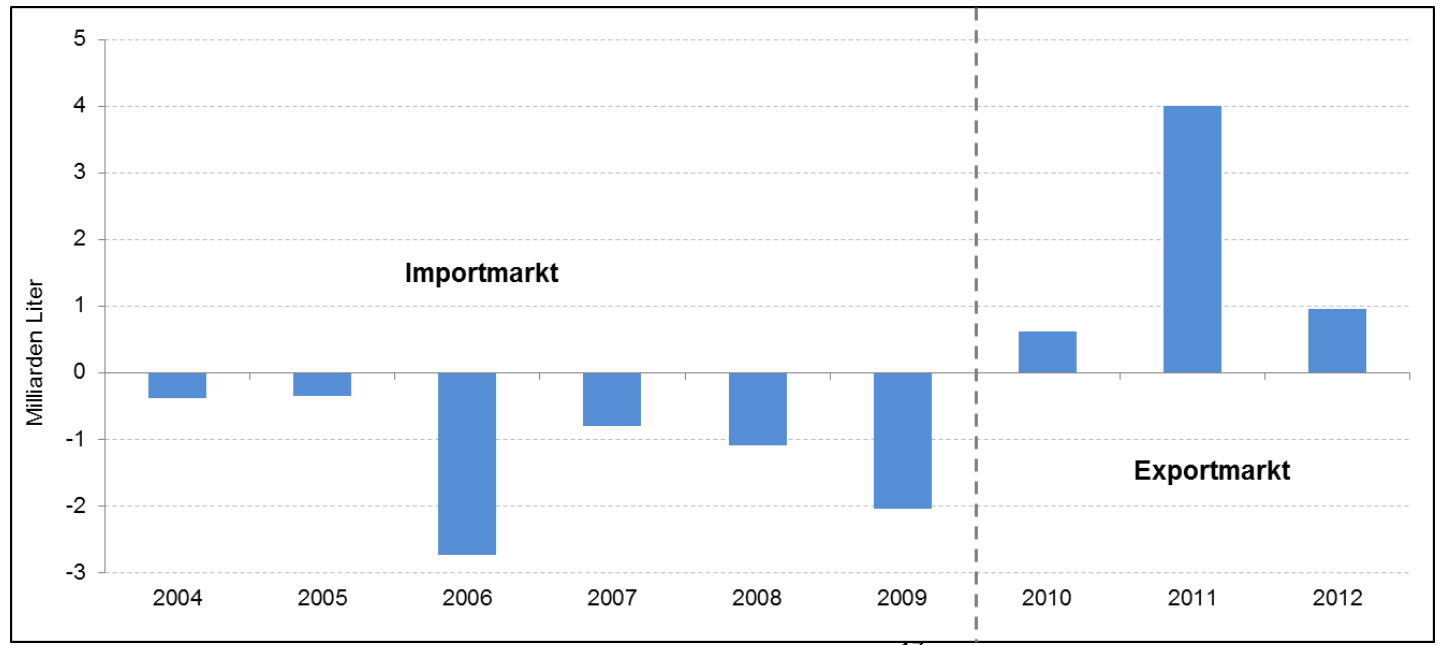

Abbildung 6: Entwicklung des Ethanolhandels in den USA ${ }^{1}$

Quelle: In Anlehnung an F.O. LICHT 2012f: 73f.; F.O. LICHT 2012a: 301ff.; BERG 2012c: 17; U.S. ENERGY INFORMATION ADMINISTRATION 2012b: 97

Dieser Wandel vom Nettoimporteur zum weltgrößten Ethanolexporteur ist auf die folgenden drei Kernpunkte zurückzuführen (F.O. LICHT 2012a: 302): (1) Aufgrund der „Blend Wall“-Problematik bei einem gleichzeitig starken Anstieg der Ethanolproduktion aus Mais war der US-amerikanische Markt gezwungen, die Ethanolüberproduktion auf dem Weltmarkt abzusetzen, um einen Wertverfall des Ethanols in den USA zu verhindern. (2) Die US-amerikanische Politik förderte bis zum Jahr 2011 noch den Export von Ethanol durch den VEETC in Höhe von 0,45 US-\$ je Gallone.

(3) Aufgrund des starken Rückgangs der Ethanolproduktion in Brasilien in Höhe von 4,5 Milliarden Litern im Jahr 2011 konnte Brasilien die Importmärkte nicht mehr ausreichend mit Ethanol versorgen und die USA nutzten die Situation und erschlossen diese Märkte für sich (F.O. LICHT 2012f: 73).

Im Jahr 2012 konnte der Trend der Jahre 2009 bis 2011 nicht fortgesetzt werden. Verantwortlich für den Rückgang der Ethanolexporte sind die geringere Ethanolproduktion im Vergleich zum Jahr 2011 (Abbildung 3), der Wegfall des VEETC zum Ende des Jahres 2011 (F.O. LICHT 2012a: 300; F.O. LICHT 2012f: 69) sowie die

\footnotetext{
${ }^{16}$ Eine genauere Beschreibung des US-amerikanischen Ethanolhandels befindet sich in Kapitel 6.2.10.

${ }_{17}$ In Abbildung 6 wird die Differenz zwischen dem Ethanolexport (Minuend) und dem Ethanolimport (Subtrahend) dargestellt.
} 
Tatsache, dass die für die USA wichtigen Exportregionen EU27 und Brasilien geringere Ethanolmengen auf dem Weltmarkt nachfragten als noch 2011 (F.O. LICHT 2012a: 300; BERG 2012c: 17). Darüber hinaus haben die USA im Jahr 2012 im Vergleich zu den Vorjahren größere Mengen Zuckerrohrethanol aus Brasilien importiert, um die Nachfrage nach „Advanced: Unspecified Biofuel“ zu befriedigen (OECD/FAO 2012: 94; F.O. LICHT2012a: 301).

\subsection{Stand der Forschung zur zukünftigen Entwicklung des Ethanol- marktes in der EU27 und den USA}

Die Tabelle 2 zeigt eine Aufstellung internationaler Studien - geordnet nach dem Veröffentlichungsjahr -, die sich mit der Entwicklung des Ethanolmarktes in einem oder in beiden Märkten beschäftigt haben. In der Darstellung werden Angaben zum Autor, zum Jahr der Veröffentlichung, zum Titel, zur Methodik und zu den Prognosen gemacht. Die Prognosen geben Aufschluss über die Produktion von und die Nachfrage nach sowie über den Handel mit Ethanol und werden getrennt für die beiden Märkte angegeben. Lediglich die renommiertesten aktuellen Studien, die sich mit der langfristigen Entwicklung des Ethanolmarktes in der EU27 und/oder den USA befasst haben, werden in der Aufstellung wiedergegeben, um den Umfang der Tabelle einzuschränken. 


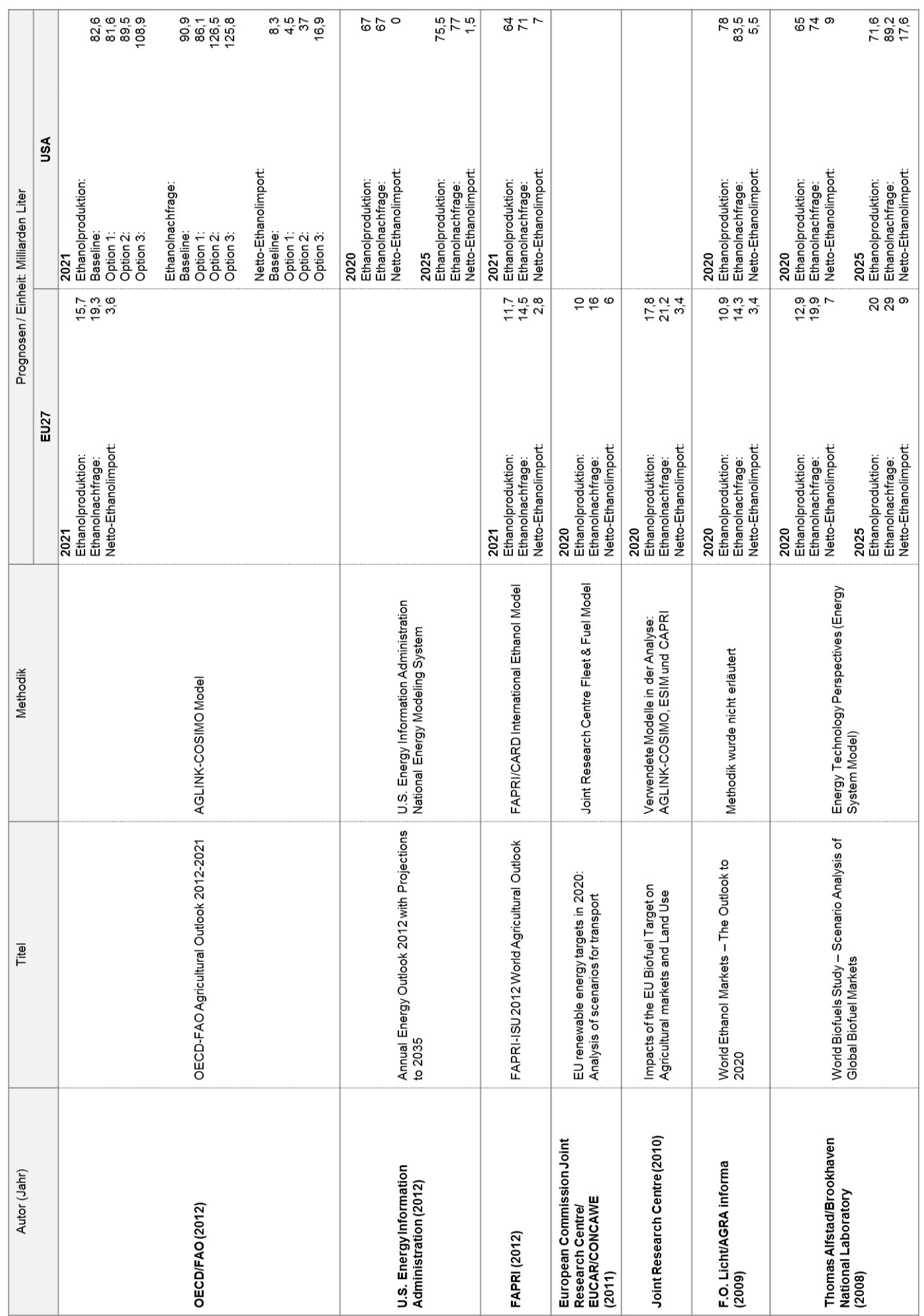

Tabelle 2: Stand der Forschung I: Entwicklung des Ethanolmarktes in der EU27 und den USA

Quelle: In Anlehnung an OECD/FAO 2012: 87ff.; U.S. ENERGY INFORMATION ADMINISTRATION 2012b: 97ff.; FAPRI 2012: 1ff.; EUROPEAN COMMISSION JOINT RESEARCH CENTRE, INSTITUT FOR ENERGY ET AL. 2011: 5ff.; EUROPEAN COMMISSION JOINT RESEARCH CENTRE, INSTITUT FOR PROSPECTIVE TECHNOLOGICAL STUDIES ET AL. 2010a: 9ff.; F.O. LICHT/AGRA INFORMA 2009: 133ff.; ALFSTAD 2008: $1 \mathrm{ff}$. 
Weitergehende Informationen zu den in Tabelle 2 dargestellten Studien sind dem Anhang $1 \mathrm{zu}$ entnehmen. Außerdem befinden sich dort Informationen zu den folgenden, in der Tabelle 3 genannten internationalen Studien. Auf diese wird in diesem Kapitel nicht genauer eingegangen, da sie nicht die aktuellsten Veröffentlichungen darstellen oder der Fokus der Untersuchungen nicht explizit auf der langfristigen Entwicklung des Ethanolmarktes liegt, sondern eher auf einer generellen Betrachtung von Biokraftstoffen.

\begin{tabular}{|c|c|c|c|c|}
\hline \multirow{2}{*}{ Autor } & \multirow{2}{*}{ Titel } & \multirow{2}{*}{ Jahr } & \multicolumn{2}{|c|}{ Region } \\
\hline & & & EU27 & USA \\
\hline $\begin{array}{l}\text { U.S. Energy Information } \\
\text { Administration }\end{array}$ & $\begin{array}{l}\text { Annual Energy Outlook } 2013 \text { with Projec- } \\
\text { tions to } 2040\end{array}$ & 2013 & & $x$ \\
\hline OPEC & World Oil Outlook & 2012 & $x$ & $x$ \\
\hline $\begin{array}{l}\text { International Energy } \\
\text { Agency }\end{array}$ & World Energy Outlook 2012 & 2012 & $\mathrm{X}$ & $x$ \\
\hline OECD/FAO & $\begin{array}{l}\text { OECD-FAO Agricultural Outlook } 2011 \text { - } \\
2020\end{array}$ & 2011 & $\mathrm{X}$ & $x$ \\
\hline $\begin{array}{l}\text { U.S. Energy Information } \\
\text { Administration }\end{array}$ & $\begin{array}{l}\text { Annual Energy Outlook } 2011 \text { with Projec- } \\
\text { tions to } 2035\end{array}$ & 2011 & & $x$ \\
\hline $\begin{array}{l}\text { International Energy } \\
\text { Agency }\end{array}$ & World Energy Outlook 2010 & 2010 & $\mathrm{X}$ & $x$ \\
\hline $\begin{array}{l}\text { Bloomberg New Energy } \\
\text { Finance }\end{array}$ & $\begin{array}{l}\text { Next-generation ethanol and biochemical: } \\
\text { What's in it for Europe }\end{array}$ & 2010 & $X$ & \\
\hline OPEC & World Oil Outlook & 2010 & $\mathrm{X}$ & $X$ \\
\hline $\begin{array}{l}\text { International Energy } \\
\text { Agency }\end{array}$ & Medium-Term Oil Market Report & 2009 & $\mathrm{X}$ & $x$ \\
\hline $\begin{array}{l}\text { De la Torre } \\
\text { Ugarte/English/Jensen }\end{array}$ & $\begin{array}{l}\text { Sixty billions gallons by } 2030 \text { : Economic } \\
\text { and agricultural impacts of ethanol and } \\
\text { biodiesel expansion }\end{array}$ & 2007 & & $x$ \\
\hline
\end{tabular}

Tabelle 3: Stand der Forschung II: Entwicklung des Ethanolmarktes in der EU27 und den USA

Quelle: U.S. ENERGY INFORMATION ADMINISTRATION 2013: 83ff.; OPEC 2012: 125f.; OECD/FAO 2011: 77ff.; U.S. ENERGY INFORMATION ADMINISTRATION 2011a: 23ff.; INTERNATIONAL ENERGY AGENCY 2010a: 355ff.; BLOOMBERG NEW ENERGY FINANCE 2010: 1ff.; OPEC 2010: 136f.; INTERNATIONAL ENERGY AGENCY 2009: 68ff.; DE LA TORRE UGARTE ET AL. 2007: $1290 \mathrm{ff}$.

Im Folgenden werden die Ergebnisse der in Tabelle 2 aufgelisteten Studien verglichen und der sich daraus ergebende Forschungsbedarf dargelegt.

Die Ergebnisse der Studien zum Ethanolmarkt der EU27, dargestellt in Abbildung 7, zeigen ein einheitliches Bild des zukünftigen EU27-Ethanolmarktes in den Jahren 2020/2021. Alle betrachteten Forschungseinrichtungen erwarten bis 2020/2021 einen starken Anstieg sowohl der Produktion als auch des Verbrauchs von Ethanol und gehen davon aus, dass die EU27 in den Jahren 2020/2021 als Ethanolimporteur am Markt auftreten wird. 


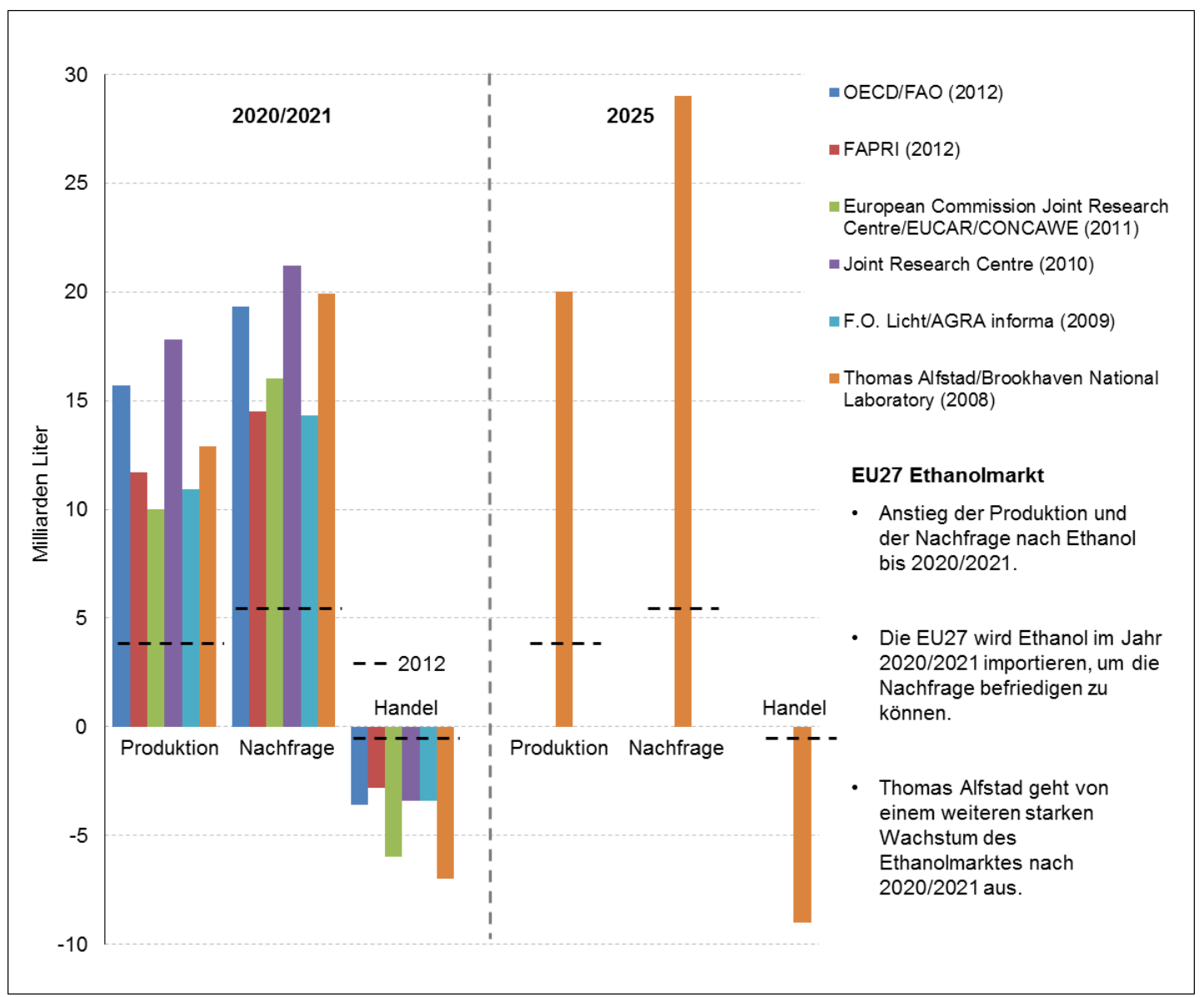

Abbildung 7: Stand der Forschung: Entwicklung des Ethanolmarktes in der EU27

Quelle: In Anlehnung an OECD/FAO 2012: 87ff.; FAPRI 2012: 1ff.; EUROPEAN COMMISSION JOINT RESEARCH CENTRE, INSTITUT FOR ENERGY ET AL. 2011: 5ff.; EUROPEAN COMMISSION JOINT RESEARCH CENTRE, INSTITUT FOR PROSPECTIVE TECHNOLOGICAL STUDIES ET AL. 2010a: 9ff.; F.O. LICHT/AGRA INFORMA 2009: 133ff.; ALFSTAD 2008: $1 \mathrm{ff}$.

Obgleich, wie bereits erwähnt und in der Grafik zu erkennen ist, übereinstimmend ein starkes Wachstum des Ethanolmarktes prognostiziert wird, bestehen dennoch Unterschiede in der erwarteten Stärke des Wachstums. Die OECD/FAO (2012) sowie das Joint Research Center (2010) erwarten ein stärkeres Wachstum der Ethanolproduktion als FAPRI (2012), Joint Research Center/EUCAR/CONCAWE (2011), F.O. Licht/AGRA informa (2009) und Thomas Alfstad/Brookhaven National Laboratory (2008).

In den Prognosen zur Entwicklung der Nachfrage zeigt sich ein ähnliches Bild, da auch hier ein genereller Anstieg bis zum Jahr 2020 erwartet wird. OECD/FAO (2012), Joint Research Centre (2010) und Thomas Alfstad/Brookhaven National Laboratory (2008) gehen im Vergleich zu den drei anderen Studien von einer höheren Nachfrage aus. Jedoch wird die erhöhte Nachfrage, die Thomas Alfstad/Brookhaven National Laboratory (2008) in seiner Studie ermittelte, im Gegensatz zu den Resultaten der Studien von OECD/FAO (2012) und Joint Research 
Centre (2010), maßgeblich durch Importe gedeckt. Trotz der genannten Unterschiede ist zu erkennen, dass die Forschungsinstitute ungeachtet der derzeitigen Unsicherheit des Marktes eine sehr ähnliche Entwicklung prognostizieren.

Lediglich die Studie von Thomas Alfstad/Brookhaven National Laboratory (2008) betrachtet die Entwicklung des Ethanolmarktes bis 2025. Die Studie kommt zu dem Ergebnis, dass der Ethanolmarkt im Zeitraum von 2020 bis 2025 weiterhin stark wachsen wird ${ }^{18}$.

In Abbildung 8 sind die Ergebnisse der wichtigsten Studien zur Entwicklung des USamerikanischen Ethanolmarktes dargestellt. Die Untersuchungen kommen übereinstimmend zu der Aussage, dass die US-amerikanische Ethanolproduktion und -nachfrage in den Jahren von 2012 bis 2020/2021 weiter wachsen werden. Darüber hinaus wird erwartet, dass die USA als Ethanolimporteur auf dem Markt auftreten werden.

\footnotetext{
${ }^{18}$ Es ist zu beachten, dass die Studie von Thomas Alfstad/Brookhaven National Laboratory (2008) nicht die politischen Vorgaben der Richtlinie 2009/28/EG berücksichtigt, da sie bereits 2008 veröffentlicht wurde.
} 




Abbildung 8: Stand der Forschung: Entwicklung des Ethanolmarktes in den USA Quelle: In Anlehnung an OECD/FAO 2012: 87ff.; U.S. ENERGY INFORMATION ADMINISTRATION 2012b: 97ff.; FAPRI 2012: 1ff.; F.O. LICHT/AGRA INFORMA 2009: 133ff.; ALFSTAD 2008: $1 \mathrm{ff}$.

Auffallend ist, dass die vier Prognosen der OECD/FAO (2012) $)^{19}$ und die Studie der F.O. Licht/AGRA informa (2009) eine höhere Produktion und Nachfrage prognostizieren als die drei anderen Studien, die von der U.S. Energy Information Administration (2012), dem FAPRI (2012) und dem Thomas Alfstad/Brookhaven National Laboratory (2008) herausgegeben wurden. In Bezug auf die Mengen des importierten Ethanols prognostizieren die Forschungsinstitute recht einheitliche Werte, lediglich die OECD/FAO (2012) erwartet in Option: 2 und Option: 3 deutlich höhere Ethanolimporte.

Die Forschungsinstitute gehen zwar einheitlich von einem Wachstum des Marktes aus, doch unterscheidet sich die Stärke des Wachstums deutlich in den einzelnen Studien. So prognostiziert die OECD/FAO (2012) in Option: 3 eine Ethanolproduktion von 109 Milliarden Litern, eine Ethanolnachfrage von 126 Milliarden Litern und einen Import von 17 Milliarden Litern, wohingegen das FAPRI (2012) eine Ethanolp-

\footnotetext{
${ }^{19}$ Die OECD/FAO (2012) beschreibt in der Studie „OECD-FAO Agricultural Outlook 20122021" vier unterschiedliche Szenarien - Baseline, Option: 1, Option: 2 und Option: 3 - die allesamt den US-amerikanischen Ethanolmarkt im Jahr 2020/2021 beschreiben.
} 
roduktion und -nachfrage von lediglich 67 Milliarden Litern für die Jahre 2020/2021 erwartet und die USA weder als Netto-Importeur noch als Netto-Exporteur betrachtet.

Ausschließlich die Studien der U.S. Energy Information Administration (2012) und von Thomas Alfstad/Brookhaven National Laboratory (2008) geben Auskunft über die Entwicklung des Marktes bis 2025. Beide Studien sehen ein weiteres Wachstum des Marktes im Vergleich zu ihren prognostizierten Werten für die Jahre 2020/2021. Die U.S. Energy Information Administration prognostiziert einen deutlichen Anstieg der Ethanolproduktion, um die gestiegene Nachfrage zu decken. Thomas Alfstad/Brookhaven National Laboratory (2008) prognostiziert hingegen einen deutlich höheren Ethanolimport, ebenfalls um die ansteigende Nachfrage befriedigen zu können.

Trotz der beschriebenen abweichenden Prognosewerte der einzelnen Forschungseinrichtungen - insbesondere in den USA - können identische Kernaussagen studienübergreifend für beide Märkte getroffen werden:

\section{EU27}

- Starker Anstieg der Produktion und der Nachfrage von Ethanol bis zu den Jahren 2020/2021 und dem Jahr $2025^{20}$

- Die EU27 wird als Importeur von Ethanol am Weltmarkt auftreten

\section{USA}

- Anstieg der Produktion und der Nachfrage von Ethanol bis zu den Jahren 2020/2021 und dem Jahr 2025.

- Die USA werden als Importeure von Ethanol am Weltmarkt auftreten.

Angesichts der Differenz in den genannten Zahlen ist anzumerken, dass die genauen Werte der Produktion, der Nachfrage und des Imports nicht von entscheidendem Interesse sind. Vielmehr sollte der zu erkennende langfristige Trend im Fokus stehen. Entscheidend - auch in Bezug auf das weitere Vorgehen - ist die fundierte Erkenntnis, dass alle Studien ein Wachstum des Marktes in der EU27 und den USA

\footnotetext{
${ }^{20}$ Die einzige Ausnahme dieser Entwicklung beschreibt das Counterfactual Scenario in der Studie des JOINT RESEARCH CENTRE (2010), das mithilfe des AGLINK-COSIMO-Modells erstellt wurde und bei dem ein auf jetzigem Niveau verharrender Entwicklungsstand des Ethanolmarktes der EU27 bis zum Jahre 2020 erwartet wird. Weitergehende Informationen zu dieser Studie befinden sich im Anhang 1.
} 
prognostizieren, wobei die Prognosen für den US-amerikanischen Markt deutlich heterogener ausfallen.

Die soeben dargelegten Ergebnisse stammen von renommierten Forschungsinstituten, die diese Werte mit Unterstützung etablierter Modelle ermittelt haben. Weder die Korrektheit noch die Relevanz der Ergebnisse sind in Zweifel zu ziehen. Gleichwohl resultiert aus der präziseren Betrachtung der Studien ein Forschungsbedarf.

Dieser Bedarf ergibt sich für den Ethanolmarkt der EU27 aus der Notwendigkeit einer Überprüfung, inwiefern die homogenen Ergebnisse der bereits bestehenden Studien das Feld der möglichen Entwicklungen vollständig abdecken. Obgleich Unterschiede - in der Produktion, der Nachfrage oder dem Handel - erkennbar sind, ist die Spannbreite möglicher Entwicklungen relativ eng gehalten, vor allem unter Berücksichtigung der Zeitspanne der Betrachtung und der derzeitigen hohen politischen Unsicherheit.

Es mag richtig sein, dass die Studien die zukünftige Entwicklung des Ethanolmarktes der EU27 darstellen; doch die homogene Sicht auf die langfristige Entwicklung des noch jungen, von einer hohen Unsicherheit geprägten Marktes erhöht die Wahrscheinlichkeit der Nichtbeachtung einer in Zukunft real eintretenden Entwicklung. Diese Nichtbeachtung verhindert ein frühzeitiges strategisches Planen und eine Ausrichtung der Marktteilnehmer auf diese Entwicklung und steigert ihr Risiko, in Zukunft an Wettbewerbsfähigkeit zu verlieren ${ }^{21}$.

Ein identisches Risiko durch Nichtbeachtung alternativer Zukünfte besteht ebenfalls für den Ethanolmarkt der USA, da auch für diesen Markt alle Studien trotz der großen Unsicherheit hinsichtlich der „Blend Wall“-Problematik und einer möglichen Kürzung des RFS 2 einen wachsenden Ethanolmarkt prognostizieren. Im Vergleich zur EU27 ist dieses Risiko aber geringer, da die Studien deutlich heterogenere Prognosen entwickelt haben, was maßgeblich auf die Veröffentlichung der OECD/FAO (2012) zurückzuführen ist.

Ein weiterer Punkt, der Forschungsbedarf erkennen lässt, ist die Ermittlung der entscheidenden quantitativen und qualitativen Faktoren, die ausschlaggebend für die zukünftige Entwicklung des Ethanolmarktes in der EU27 und den USA sind. Denn durch die Betrachtung dieser essentiellen Faktoren ist es möglich, frühzeitig zu erkennen, in welche Richtung sich der Markt in Zukunft entwickeln wird.

\footnotetext{
${ }^{21}$ Mit anderen Worten: Werden alternative Entwicklungen nicht in Erwägung gezogen, besteht die erhöhte Gefahr, dass Unternehmen bei Eintritt jener alternativen Entwicklungen infolge fehlender strategischer Planung Entscheidungen in kurzer Zeit und aufgrund einer geringeren Datenbasis treffen müssen, wodurch das Risiko eines wirtschaftlichen Schadens für die Unternehmen steigt.
} 
Ein zusätzliches Argument, das weitergehende Forschung begründet, ist die Notwendigkeit, die Entwicklung des Ethanolmarktes mit einer neuen Methodik zu betrachten, da in allen Studien der Forschungsinstitute, sowohl für die EU27 als auch für die USA, ein ähnliches methodisches Vorgehen in Form von modellbasierten Prognosen gewählt wurde (Anhang: 1). Aus diesem Grund ist ein methodisches Vorgehen sinnvoll, das sich von den verwendeten Modellen unterscheidet.

Die neue Methodik muss es ermöglichen, langfristig in die Zukunft zu schauen, qualitative Faktoren zu berücksichtigen und unterschiedliche Szenarien zu bilden. Somit erhält diese Methodik ihre Relevanz nicht nur als angewandtes Instrument, um die anderen Punkte, die den Forschungsbedarf ergeben, zu verwirklichen, sondern darüber hinaus auch dadurch, dass sie sich von den Methoden der genannten Studien unterscheidet. 
In Kapitel 3.1 wird erläutert, warum die Szenario-Technik als Prognoseinstrument ausgewählt wurde. Die Definition sowie die geschichtliche Herleitung des Begriffs „Szenario" erfolgen in Kapitel 3.2. In Kapitel 3.3 werden die unterschiedlichen Verfahren der Szenario-Technik voneinander abgegrenzt. Eine Analyse, inwiefern die bereits bestehenden Verfahren des induktiven Ansatzes der Szenario-Technik anwendbar sind, um die Ziele dieser Studie zu erreichen, folgt in Kapitel 3.4.

\subsection{Auswahl des Prognoseverfahrens}

Es gibt eine Vielzahl unterschiedlicher Verfahren zur Erstellung von Prognosen ${ }^{22}$ (FRERICHS KÜBLER 1980: 13ff.) und die Qualität der Prognosen wird erheblich durch die Wahl des Verfahrens beeinflusst (GÖTZE 1993: 30). GEORGOFF/MURDICK (1986: 3f.) erstellten die in Abbildung 9 dargestellte Klassifizierung von gängigen Prognoseverfahren.

22 „Prognosen sind Voraussagen über einen zukünftigen, realen Sachverhalt, die auf der Grundlage von praktischen Erfahrungen und/oder theoretischen Erkenntnissen getroffen werden" (GÖTZE 1993: 8). 




Abbildung 9: Klassifizierung der Prognoseverfahren

Quelle: In Anlehnung an GEORGOFF/MURDICK 1986: $3 f$.

Neben der Klassifizierung der gängigen Prognoseverfahren entwickelten GEORGOFF/MURDICK (1986: 1ff.) auch ein strukturiertes Vorgehen ${ }^{23}$, um die Auswahl eines geeigneten Prognoseverfahrens zu erleichtern. Als Ergebnis der Auswahl, bezogen auf die Zielsetzung der Arbeit, erscheinen die qualitativen Prognoseverfahren „Scenario Methods“ und „Delphi Technique“ - in Abbildung 9 grün hinterlegt der „Judgement Methods“ als geeignet. Die bestimmenden Faktoren, die zu dieser Einschätzung führen, werden im Folgenden erläutert. In Anbetracht der langfristigen ${ }^{24}$ Planungsdauer von über 10 Jahren - Prognosen für das Jahr 2023 sollen

23 Zur strukturierten Auswahl eines Prognoseverfahrens entwickelten GEORGOFF/MURDICK (1986: 1ff) ein System; in diesem sind 20 Prognoseverfahren in Spalten und 16 Auswahlkriterien in Zeilen angeordnet. Im Schnittpunkt von Spalte und Zeile erläutern Georgoff und Murdick ihre Einschätzung, inwieweit das jeweilige Prognoseverfahren fähig ist, das Auswahlkriterium zu erfüllen. Zur besseren Anwendung des Systems sind zum einen die Auswahlkriterien als Fragen formuliert, zum anderen besitzen die Zellen eine Hintergrundfarbe. Bei der Beurteilung der Erfüllung des Auswahlkriteriums durch das Prognoseinstrument repräsentiert die Hintergrundfarbe Gelb Stärke, Grau Schwäche und Weiß Neutralität. Wird ein Prognoseverfahren bei einem für die Forschungsarbeit bedeutenden Auswahlkriterium mit Grau (Schwäche) beurteilt, so sollte dieses Prognoseverfahren im anschließenden Auswahlprozess nicht weiter betrachtet werden.

${ }^{24}$ Differenzierung der Planungsdauer: kurzfristig ( 1 - 2 Jahre); mittelfristig (3 - 5 Jahre); langfristig (>5 Jahre) (GAUSEMEIER ET AL. 1995: 115). 
erstellt werden - können lediglich Methoden in Betracht gezogen werden, die eine solche „long-term projection“ ermöglichen. Ein weiteres Kriterium ist die Möglichkeit der Inklusion von sowohl qualitativen als auch quantitativen Daten in das Prognoseverfahren. Diese Flexibilität der Verfahren der „Scenario Methods“ und „Delphi Technique" verhindert einen systematischen Ausschluss von möglicherweise bedeutenden qualitativen oder quantitativen, den Ethanolmarkt beeinflussenden Faktoren. Darüber hinaus spricht das Fehlen von umfangreichen historischen Daten aufgrund des noch jungen Ethanolmarktes der EU27 und der USA gegen die alleinige Verwendung quantitativer Verfahren. Auch die geringe „external stability“ (Vorhandensein von Diskontinuitäten), verursacht durch den starken Einfluss der Politik auf beide Ethanolmärkte (OECD/FAO 2011: 80; F.O. LICHT/AGRA INFORMA 2009: 41), setzt der Anwendung quantitativer Verfahren Grenzen, jedoch nicht der von „Scenario Methods“ oder von „Delphi Technique“.

Zur weiteren Eingrenzung und Bestimmung des geeigneten Prognoseverfahrens wird die Tabelle $4^{25}$ von GÖTZE (1993: 31 ) genutzt, die unterschiedliche Prognoseverfahren miteinander vergleicht.

\begin{tabular}{|c|c|c|c|c|}
\hline \multirow[b]{2}{*}{ Verfahren } & \multicolumn{2}{|c|}{$\begin{array}{l}\text { Verwendung eines formalen } \\
\text { Modells }\end{array}$} & \multicolumn{2}{|c|}{$\begin{array}{l}\text { Berücksichtigung von Beziehungen zwischen } \\
\text { den zu prognostizierenden Größen }\end{array}$} \\
\hline & intuitiv & analytisch & Singulärprognose & Systemprognose \\
\hline Zeitreihenanalyse & & $x$ & $x$ & \\
\hline Leitindikatormethode & & $x$ & $x$ & \\
\hline Ein-Gleichungs-Modell & & $x$ & $x$ & \\
\hline Mehr-Gleichungs-Modell & & $x$ & & $x$ \\
\hline System-Dynamics-Methodik & & $x$ & & $x$ \\
\hline Einstufige Expertenbefragung & $x$ & & $x$ & \\
\hline Delphi-Methode & $x$ & & $x$ & \\
\hline Szenario-Technik & $x$ & & & $x$ \\
\hline
\end{tabular}

Tabelle 4: Vergleich ausgewählter Prognoseverfahren

Quelle: In Anlehnung an GÖTZE 1993: 31

${ }^{25}$ Die Tabelle von GÖTZE (1993: 31) wurde erstellt in Anlehnung an eine Veröffentlichung von FRERICHS/KÜBLER (1980: 6). 
Die analytischen Prognoseverfahren kommen bei der Analyse der Entwicklung des Ethanolmarktes nicht in Betracht, da sie ausschließlich quantitative Daten verarbeiten können, wodurch das Risiko der Nicht-Berücksichtigung bedeutender qualitativer Faktoren besteht. Ferner spricht das wahrscheinliche Auftreten von Diskontinuitäten bei langfristigen Betrachtungen gegen den Einsatz von analytischen Verfahren (GÖTZE 1993: 34).

Im Gegensatz zu den analytischen Verfahren sind die Voraussetzungen für den Einsatz von intuitiven Verfahren geringer. Es lassen sich sowohl quantitative als auch qualitative Daten in den Prozess integrieren (GÖTZE 1993: 34). Die Qualität der Ergebnisse der meisten intuitiven Prognoseverfahren ist aber stark abhängig von der Kompetenz der beteiligten Personen und deren Fähigkeit, wechselseitig aufeinander wirkende Variablen zu erkennen und zu berücksichtigen (BROCKHOFF 1977: 75). Dies trifft auch auf die Delphi-Methode und die einstufige Expertenbefragung zu, da in diesen Verfahren vernetzte Wirkungsbeziehungen nicht methodisch berücksichtigt werden; sie sind höchstens implizit im Ergebnis enthalten (Singulärprognose) (GESCHKA ET AL. 2006: 242; GÖTZE 1993: 35). Dieser methodische Mangel an fehlender Berücksichtigung der Interdependenzen der Faktoren zeigt sich bei der Szenario-Technik nicht (Systemprognose). Des Weiteren wird die Ungewissheit als Wesensmerkmal der Zukunft (KLEIN ET AL. 2006: 377) explizit in der Szenario-Technik durch die Bildung unterschiedlicher Szenarien berücksichtigt (GÖTZE 1993: 35; CORNELIUS ET AL. 2005: 94). Dies ist bedeutsam, da jede Zukunftsprognose - insbesondere solche mit langfristigem Betrachtungshorizont eine zu berücksichtigende Fehlerwahrscheinlichkeit besitzt (KLEIN ET AL. 2006: 377).

Nach dem Vergleich der Prognoseverfahren durch das System von Georgoff/Murdick und den tabellarischen Vergleich von Götze erscheint die SzenarioTechnik als die geeignete Prognosemethode, um das angestrebte Ziel der Arbeit zu erreichen. Die Szenario-Technik kann unter Berücksichtigung qualitativer und quantitativer Faktoren (MIBLER-BEHR 2006: 215) und deren Interdependenzen langfristige Projektionen erstellen. Auch der geringfügige Bestand historischer Daten, das mögliche Auftreten von Diskontinuitäten sowie die Möglichkeit der Erstellung unterschiedlicher Szenarien sprechen für die Anwendung der Szenario-Technik. 


\subsection{Geschichtliche Herleitung und Definition des Szenario-Begriffs}

Der Begriff „Szenario“ wird dem Zukunftsforscher HERMANN KAHN zugeschrieben (REIBNITZ 1992: 11; FINK/SIEBE 2006: 15; GAUSEMEIER ET AL. 1995: 91; HEINECKE 2006: 184; GRAF 2000: 14; VERITY 2003: 186; WEBER 1990: 120). Dieser entwickelte Anfang der 1950er Jahre an einem von der US Airforce und der Douglas Aircraft Company gegründeten Institut Planspiele, die er Szenarien nannte (REIBNITZ 1992: 12; BRADFIELD ET AL. 2005: 798; GRAF 2000: 14). 1961 wechselte Kahn zum Hudson Institute und veröffentlichte in Zusammenarbeit mit Anthony J. Wiener im Jahre 1967 die Studie „The Year 2000: A Framework for Speculation on the Next Thirty-Three Years“" (BRADFIELD ET AL. 2005: 798f.; GAUSEMEIER ET AL. 1995: 92; FINK/SIEBE 2006: 15). Die Studie wurde zur „landmark in the field of scenario planning“ (BRADFIELD ET AL. 2005: 799) und „gilt als Geburtsstunde der Szenario-Technik“ (GAUSEMEIER ET AL. 1995: 92).

Mit Beginn der Ölkrise und der wachsenden Unsicherheit auf den Märkten in den 1970er Jahren interessierten sich die ersten Unternehmen für ein Denken in Szenarien (GAUSEMEIER ET AL. 1995: 92; FINK/SIEBE 2006: 15). Dies waren vor allem international tätige Unternehmen mit langfristigen Planungshorizonten wie z.B. Royal Dutch Shell, GE, Lockheed und im deutschsprachigen Raum BASF, Daimler Benz und Volkswagen (BRADFIELD ET AL. 2005: 800; GAUSEMEIER ET AL. 1995: 92; FINK/SIEBE 2006: 15). Seit Mitte der 80er Jahre ist erkennbar, dass der Gebrauch von Szenarien in der strategischen Planung kontinuierlich ansteigt (FINK/SIEBE 2006: 15).

Eine genaue Definition des Szenario-Begriffs ist notwendig, da sich der Begriff zu einer Art Modewort mit breitem Eingang in die Journalismus- und Altagssprache entwickelt hat (KOSOW/GAßNER 2008: 9). Daher werden im Folgenden ausgewählte Definitionen des Szenario-Begriffs aus der wissenschaftlichen Literatur vorgestellt und anschließend bestimmt, welche Definition die Grundlage dieser Arbeit bildet.

In ihrem 1967 erschienen Werk „The Year 2000“ definieren KAHN/WIENER (1968:

6) Szenarien als „hypothetische Folgen von Ereignissen, die konstruiert werden, um die Aufmerksamkeit auf kausale Prozesse und Entscheidungspunkte zu lenken“. Das Frankfurter BATTELLE-INSTITUT versteht unter einem Szenario sowohl „die Beschreibung einer möglichen zukünftigen Situation als auch das Aufzeigen des Entwicklungsverlaufs, der zu dieser zukünftigen Situation hinführt" (GESCHKA/REIBNITZ 1986: 128). Ebenso definiert REIBNITZ (1992: 14) den SzenarioBegriff: „die Beschreibung einer zukünftigen Situation und die Entwicklung bzw. 
Darstellung des Weges, der aus dem Heute in die Zukunft hineinführt“. GAUSEMEIER ET AL. (2009: 59) definieren Szenarien als „eine allgemeinverständliche Beschreibung einer möglichen Situation in der Zukunft, die auf einem komplexen Netz von Einflussfaktoren beruht, sowie die Darstellung einer Entwicklung, die aus der Gegenwart zu dieser Situation führen könnte.“

Anlehnend an die eben beschriebenen Definitionen von Szenarien leitet sich die folgende Definition ab, die die Grundlage der vorliegenden Arbeit bildet: Ein Szenario beschreibt eine möglichst konsistente (widerspruchsfreie), zukünftige Situation, die auf der Verknüpfung von einzelnen Zukunftsprojektionen der Schlüsselfaktoren basiert. Es ist explizit anzumerken, dass in dieser Studie konsistente Szenarien ausschließlich für das Kalenderjahr 2023 entwickelt werden.

\subsection{Unterschiedliche Verfahren der Szenario-Technik}

Ein Instrument zur Erstellung von Szenarien ist die Szenario-Technik. Hierbei handelt es sich nicht um ein einheitliches Verfahren (GESCHKA/HAMMER 1986: 243) vielmehr wird in der Wissenschaft zwischen einer Vielzahl verschiedener Verfahren unterschieden (HUSS/HONTON 1987: 21). Aber alle Verfahren der SzenarioTechnik basieren auf den Ansätzen der „multiplen Zukunft“ und des „vernetzten Denkens“ (GAUSEMEIER ET AL. 2009: 60; HEES ET AL. 2006: 291).

Grundlage der „multiplen Zukunft“ ist die Annahme, dass sich die Zukunft nicht exakt prognostizieren lässt (GAUSEMEIER ET AL. 2009: 60). Daher werden mehrere alternative Zukünfte (Szenarien) entwickelt, um der Ungewissheit der Zukunft gerecht zu werden (FINK ET AL. 2002: 21). Das Trichtermodell ermöglicht die Visualisierung der Grundidee der „multiplen Zukunft“ (Abbildung 10). Der SzenarioTrichter (gestrichelte Linie) als Teil des Trichtermodells symbolisiert nur die ansteigende Unsicherheit und Komplexität des Themas mit fortlaufender Zeit, aber nicht die Grenzen möglicher Zukünfte. 


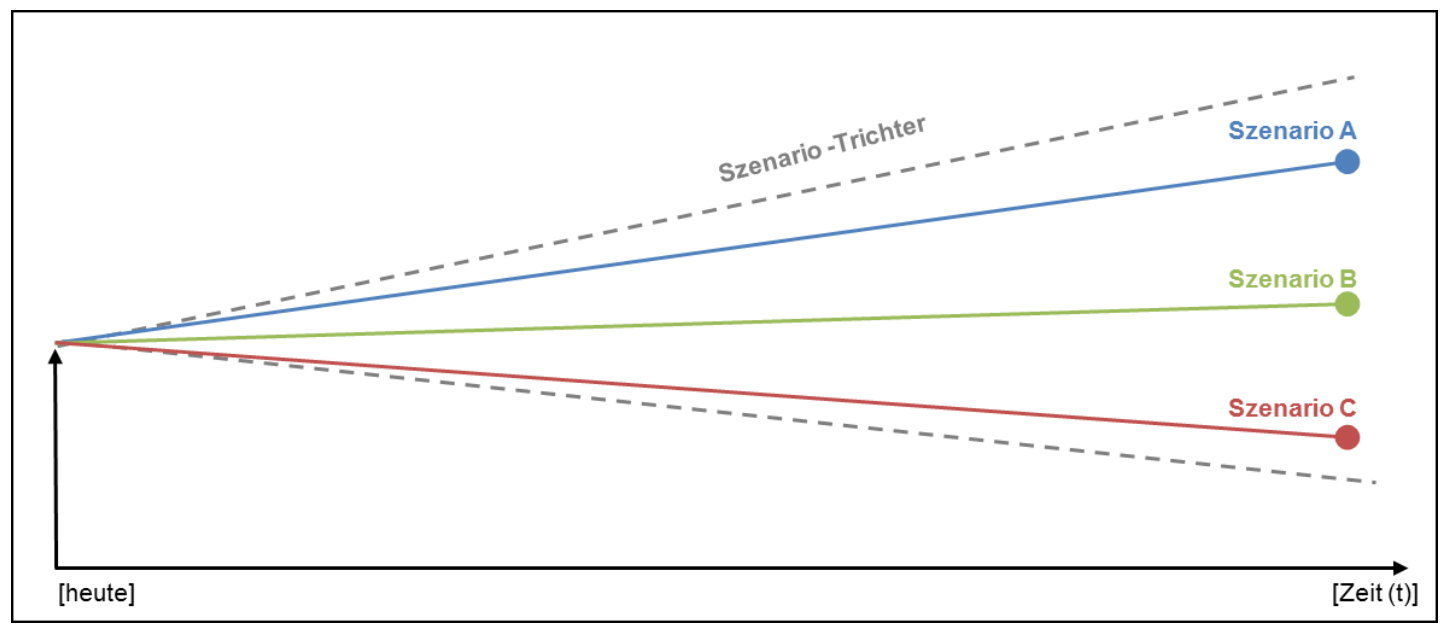

Abbildung 10: Trichtermodell/Ansatz der "multiplen Zukunft“

Quelle: In Anlehnung an GAUSEMEIER ET AL. 2009: 59; MIßLER-BEHR 1993: 4

Beim Ansatz des "vernetzten Denkens" werden die Faktoren des Szenariofeldes als ein verknüpftes Netzwerk betrachtet und nicht nur als monokausale Beziehungen (GAUSEMEIER ET AL. 1995: 89). Durch diese vernetzte Betrachtungsweise wird die Komplexität des Szenariofeldes in dem Instrument der Szenario-Technik berücksichtigt (FINK ET AL. 2002: 21).

GÖTZE unterteilt die Szenario-Technik in deduktive und induktive Ansätze (Abbildung 11).

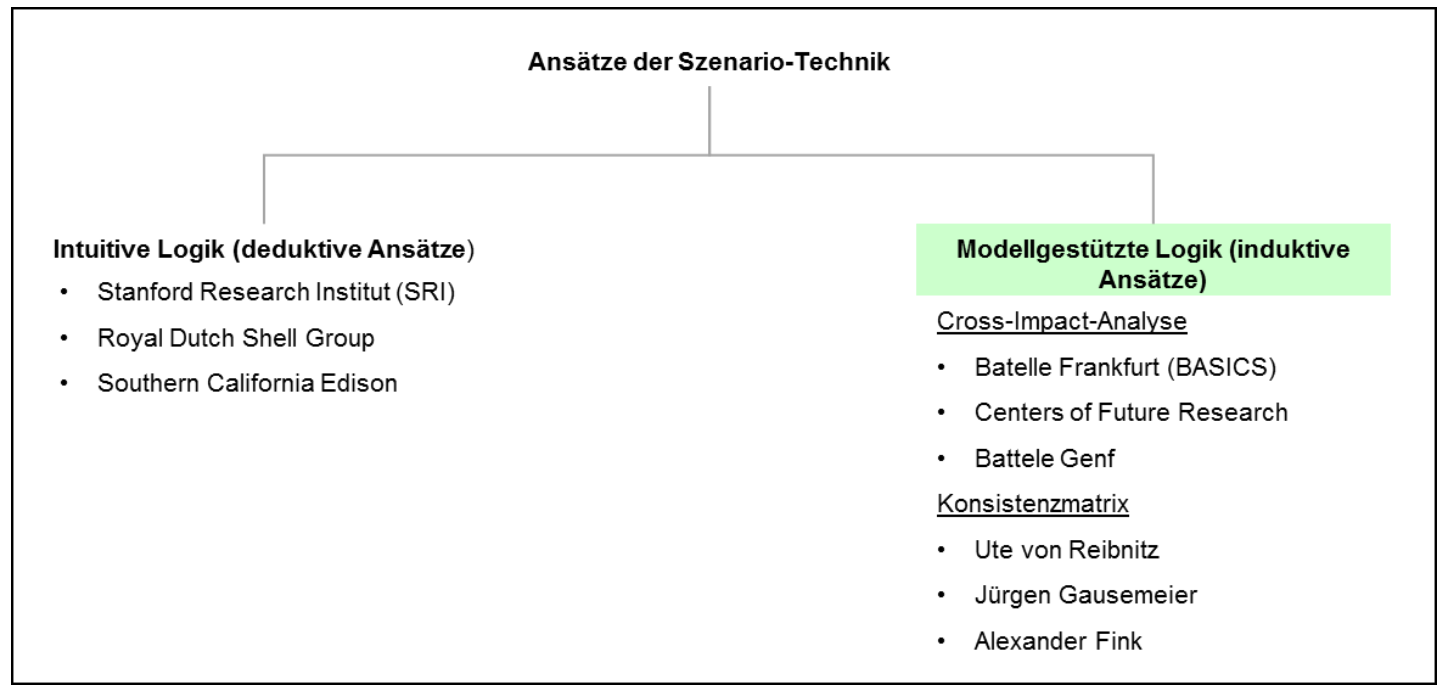

Abbildung 11: Klassifizierung von Ansätzen der Szenario-Technik

Quelle: In Anlehnung an GÖTZE 1993: 94

Zu den deduktiven Ansätzen zählen insbesondere die Szenario-Arbeiten des Stanford Research Institut, der Royal Dutch Shell Group sowie der Southern California Edison. Die „Intuitive Logik“ ist stark qualitativ orientiert und greift bei der Erstellung von Szenarien nicht auf einzelne Hilfsmittel zurück (MEYER-SCHÖNHERR 1992: 35; HEINECKE 2006: 183). Stattdessen wird intuitiv probiert, in sich konsistente und 
logische Szenarien zu bilden (MEYER-SCHÖNHERR 1992: 35). Die „Intuitive Logik“ wird vorrangig im angloamerikanischen Raum angewandt (FINK/SIEBE 2006: 18). Auf eine detaillierte Darstellung der deduktiven Methodik wird aus folgenden Gründen verzichtet: (1) Das intuitive Vorgehen erschwert dem unerfahrenen Nutzer die Planung und erhöht die Wahrscheinlichkeit unbefriedigender Ergebnisse (MEYER-SCHÖNHERR 1992: 42). (2) Zentrale Probleme des Vorgehens sind die schwierige Nachvollziehbarkeit der Ergebnisse und die Einschränkung, nur eine begrenzte Anzahl an Faktoren in den Prozess zu integrieren, was zu einer geringeren Eignung des Verfahrens bei komplexen Problemen führt (FINK ET AL. 2002: 22; GÖTZE 1993: 95). (3) Zudem besteht bei dieser Methodik die Gefahr, lediglich bereits absehbare oder auf Anhieb denkbare Zukünfte zu entwickeln, jedoch andere wesentliche Szenarien zu vernachlässigen (GÖTZE 1993: 95).

Der induktive Ansatz der Szenario-Technik hat sich hingegen vor allem in Kontinentaleuropa (Deutschland und Frankreich) etabliert (FINK/SIEBE 2006: 36; FINK ET AL. 2000: 4). Bekannte Vertreter der „Modellgestützten Logik“ sind das BATTELLEINSTITUT, das Centre of Future Research sowie die Autoren Ute von Reibnitz und Jürgen Gausemeier. Der induktive Ansatz greift bei der strukturierten Erstellung von Szenarien auf die Methoden der „Cross-Impact-Analyse“ und/oder der „Konsistenzmatrix“ zurück ${ }^{26}$ (MEYER-SCHÖNHERR 1992: 44). Der strukturierte Prozess des induktiven Ansatzes erleichtert die Anwendung der Methodik und die Nachvollziehbarkeit der Ergebnisse und ermöglicht darüber hinaus die Integration einer beachtlichen Zahl von Faktoren in den Prozess der Szenario-Erstellung. Die Gefahr, nur bereits absehbare oder auf Anhieb denkbare Szenarien zu entwickeln, besteht nicht (GÖTZE 1993: 95). Aufgrund dessen wird der induktive Ansatz der SzenarioTechnik gewählt, um das formulierte Ziel der Arbeit zu erreichen.

\subsection{Analyse des induktiven Ansatzes der Szenario-Technik}

Beim induktiven Ansatz der Szenario-Technik gibt es kein einheitliches Vorgehen zur Erstellung von Szenarien (HEES ET AL. 2006: 292). Es ist trotzdem ein gängiges, bei fast allen Autoren identisches Grundmuster zu erkennen (HEINECKE 2006: 185f.; GÖTZE 1993: 386ff.). In der folgenden Tabelle 5 werden beispielhaft die Vorgehensweisen von drei bedeutenden Autoren dargestellt. ${ }^{26}$ Die Verfahren "Cross-Impact-Analyse“ und „Konsistenzmatrix“ werden in Kapitel 3.4 be-
schrieben. 


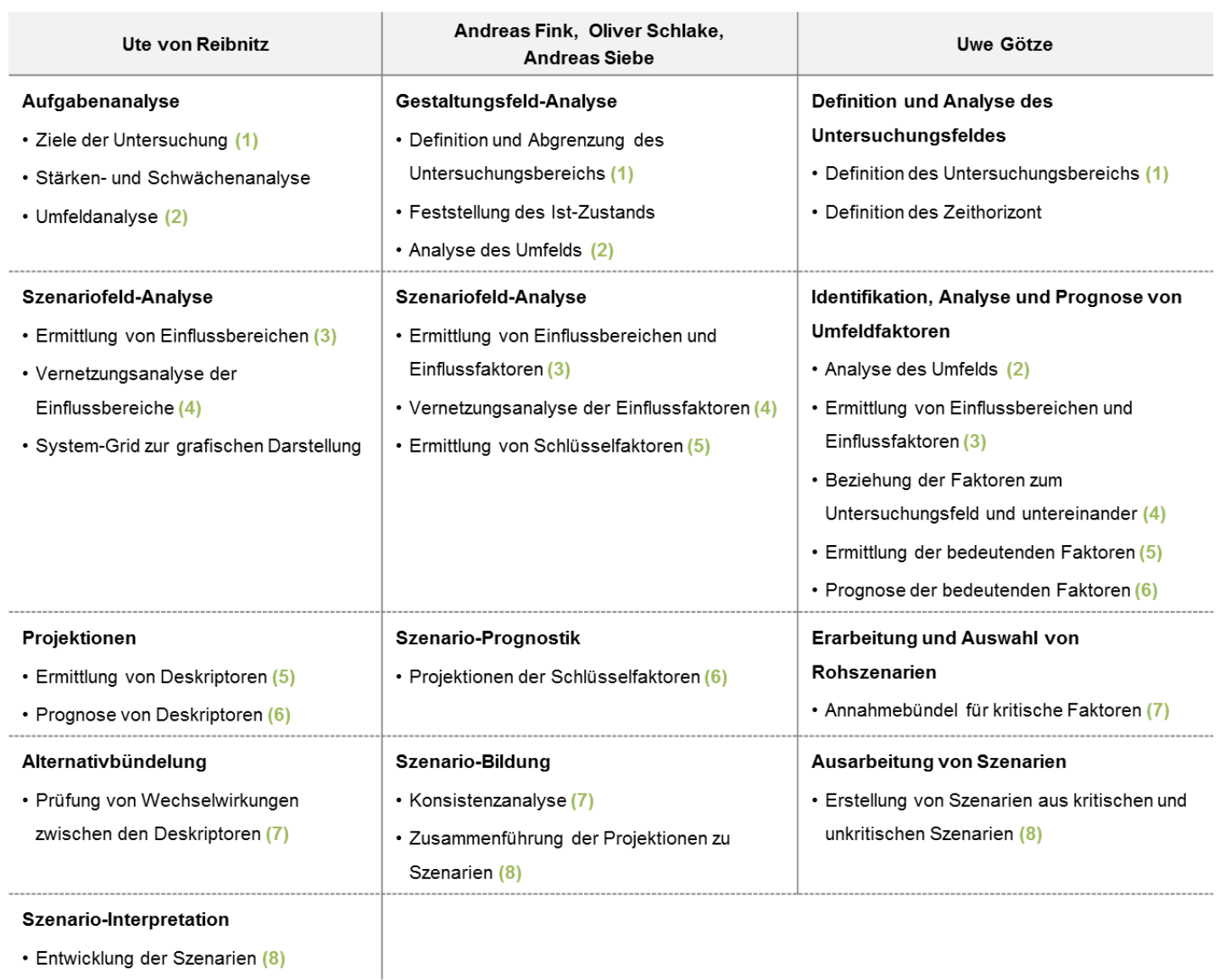

Tabelle 5: Verfahren des induktiven Ansatzes der Szenario-Technik

Quelle: In Anlehnung an AYE 2009: 120; REIBNITZ 1993; FINK ET AL. 2002; GÖTZE 1993

Obgleich sich die Verfahren von REIBNITZ (1993), FINK ET AL. (2002) und GÖTZE (1993) sowohl in Anzahl und Umfang der Schritte als auch in der Begrifflichkeit unterscheiden, ist die Grundstruktur des Vorgehens identisch.

Die induktive Szenario-Technik besitzt in der Regel folgende Kernpunkte des Vorgehens (WILMS 2006a: 399; AYE 2009: 120; WILMS 2006b: 50f.; HEINECKE 2006: 185f.; GÖTZE 1993: 386ff.; AXSON 2011: 23; ZERRES 1988; 73ff.):27

(1) Bestimmung des Untersuchungsbereichs

(2) Analyse des Umfeldes des Untersuchungsbereichs

(3) Identifizierung von Faktoren und Bereichen, die Einfluss auf den Untersuchungsbereich nehmen

(4) Ermittlung der Beziehungen zwischen den Faktoren oder den Bereichen

${ }^{27}$ Die Nummerierung der Kernpunkte ist identisch zu der Abfolge der in der Tabelle 5 dargestellten Verfahren und dient somit der Verdeutlichung der verfahrensübergreifenden Grundstruktur. 
(5) Bestimmung der bedeutenden Faktoren (Schlüsselfaktoren oder Deskriptoren)

(6) Bildung von Zukunftsprojektionen für die in (5) als bedeutend erkannten Faktoren

(7) Prüfung der Projektionen auf Widerspruchsfreiheit

(8) Zusammenführung der Projektionen zu Szenarien

Die Grundstruktur der induktiven Szenario-Technik ist dafür geeignet, das Ziel dieser Arbeit zu erreichen. Sie erlaubt ein strukturiertes und leicht nachvollziehbares Vorgehen, welches Ergebnisse produziert, die sowohl praktisch anwendbar als auch wissenschaftlich begründet sind. Aber trotz der Vielzahl an etablierten induktiven Verfahren der Szenario-Technik ${ }^{28}$, welche allesamt auf der oben beschriebenen Grundstruktur basieren, eignen sich die Verfahren selbst nicht, um das gesetzte Ziel der Arbeit zu erreichen. Als Gründe für die Untauglichkeit der etablierten Methoden sind die im Folgenden genannten Instrumente anzuführen, welche innerhalb der Grundstruktur zur Anwendung kommen: „Einflussanalyse“, „Konsistenzanalyse“ und „Cross-Impact-Analyse“. Diese Instrumente führen zu einer großen Komplexität (MIßLER-BEHR 2006: 215; BLASCHE 2006: 91) des induktiven Ansatzes und verlangen einen hohen Zeitaufwand (REIBNITZ 1992: 273), um die Szenarien zu erstellen.

Einflussanalyse: Das Instrument der Einflussanalyse ${ }^{29}$ ist fester Bestandteil der induktiven Szenario-Technik und kommt in Kernpunkt (4) der Grundstruktur (Ermittlung der Beziehungen zwischen den Faktoren oder den Bereichen) zum Einsatz. Ziel der Einflussanalyse ist es, die Bedeutung der einzelnen Faktoren, die Einfluss auf den Untersuchungsbereich nehmen, zu erkennen, um in Kernpunkt (5) die bedeutenden Faktoren zu bestimmen, die entscheidend die Zukunft des Untersuchungsbereichs beeinflussen. Das Instrument der Einflussanalyse wird bei den etablierten Verfahren in unterschiedlicher Form angewandt. Es wird zwischen dem Ansatz von GAUSEMEIER ET AL. (1995: 189ff.) und FINK ET AL. (2002: 189ff.) sowie dem Ansatz von REIBNITZ (1992: 35ff.) und PASTERNACK (2006: 105ff.) unterschieden. Beide Ansätze werden im Folgenden erläutert.

${ }^{28}$ Autoren, die etablierte Verfahren der induktiven Szenario-Technik beschrieben haben: REIBNITZ (1992); FINK ET AL. (2002); GÖTZE (1993); GAUSEMEIER ET AL. (1995); GAUSEMEIER ET AL. (2009) und PASTERNACK (2006)

${ }_{29}$ Der Begriff Einflussanalyse ist gleichzusetzen mit den Begriffen Vernetzungsanalyse und Interdependenzanalyse. 
Bei dem von GAUSEMEIER und FINK beschriebenen Instrument der Einflussanalyse wird die Stärke der direkten Beziehungen zwischen den einzelnen Faktoren, die Einfluss auf den Untersuchungsbereich nehmen, anhand einer festgelegten Skala beurteilt (GAUSEMEIER 1995: 191). Die Komplexität und der Arbeits- und Zeitaufwand der Einflussanalyse sind abhängig von der Anzahl der als relevant erkannten Einflussfaktoren $^{30}$. Während GAUSEMEIER ET AL. (1992: 187) 45 Einflussfaktoren in einer Beispielrechnung berücksichtigen, gehen FINK ET AL. (2002: 79) gar von 40 bis 90 Einflussfaktoren im Regelfall aus. Die durch die Integration einer solch hohen Zahl von Einflussfaktoren entstehende Problematik der ansteigenden Komplexität des Instruments wird in Abbildung 12 verdeutlicht. Die zunehmende Komplexität und der ansteigende Arbeits- und Zeitaufwand äußern sich in der Anzahl der zu beurteilenden direkten Beziehungen zwischen den Einflussfaktoren.

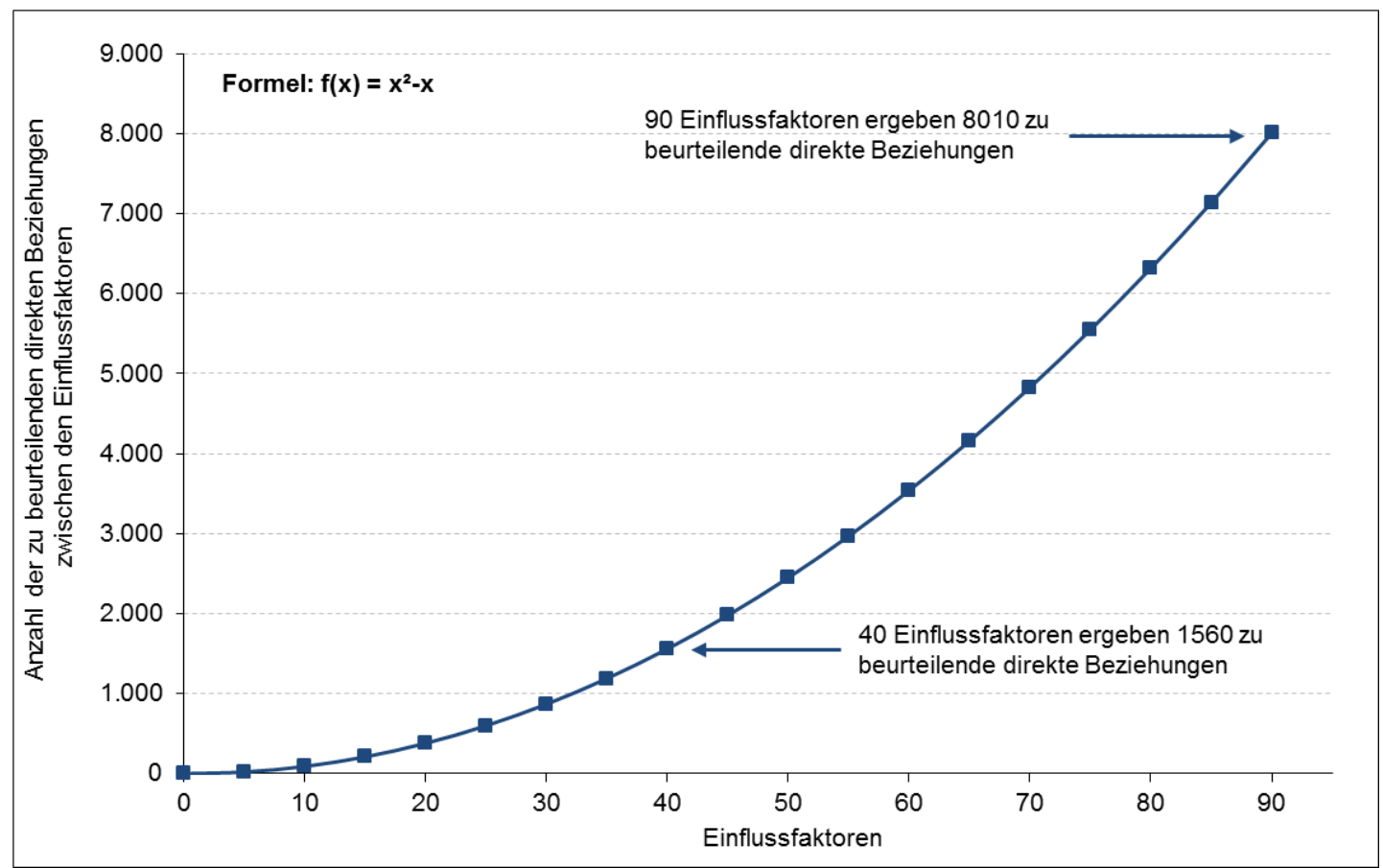

Abbildung 12: Anstieg der Komplexität der Einflussanalyse Quelle: EIGENE DARSTELLUNG

Bei 40 Einflussfaktoren muss der Anwender der Einflussanalyse 1560 direkte Beziehungen, bei 90 Einflussfaktoren gar 8010 direkte Beziehungen zwischen den Einflussfaktoren beurteilen. Die hieraus resultierende hohe Komplexität, der damit verbundene erhebliche Arbeits- und Zeitaufwand und die abschreckende Wirkung einer solch großen Matrix führen zu der Erkenntnis, dass das Instrument der Einflussanalyse, in der Form wie es GAUSEMEIER ET AL. (1995) und FINK ET AL. (2002) beschreiben, nicht geeignet ist, um Kernpunkt (4) der Grundstruktur zu un-

${ }^{30}$ Einflussfaktoren sind Faktoren, die Einfluss auf das Untersuchungsfeld - in dieser Arbeit der Ethanolmarkt der EU27 und der USA - nehmen. 
terstützen. Um eine Anwendbarkeit des Instruments zu ermöglichen, wäre eine deutliche Reduzierung der Zahl der Einflussfaktoren vonnöten. Dieser Schritt würde jedoch die Wahrscheinlichkeit der Nicht-Berücksichtigung von bedeutenden Einflussfaktoren in den Prozess der Szenario-Erstellung erhöhen. Ziel ist es daher, die Einflussanalyse zu optimieren, um sie in Kernpunkt (4) sinnvoll einsetzen zu können.

Das Instrument der Einflussanalyse, wie es REIBNITZ (1992: 35ff.) und PASTERNACK (2006: 105ff.) aufzeigen, ermittelt unter Zuhilfenahme einer festgelegten Skala nicht die Stärke der direkten Beziehungen zwischen Einflussfaktoren, sondern die Stärke der Beziehungen zwischen Einflussbereichen. Einflussbereiche werden definiert, um den Anwender bei der Bestimmung der Einflussfaktoren zu unterstützen. Typische Einflussbereiche sind Politik, Technologie, Wirtschaft und Gesellschaft. Sie sind sehr allgemein gehalten und erhalten ihre themenspezifische Charakterisierung erst durch die Ermittlung zugehöriger Einflussfaktoren (REIBNITZ 1992: 34f.). Durch die geringe Anzahl an Einflussbereichen besitzt das Instrument den Vorteil einer geringen Komplexität, einhergehend mit einer hohen Anwenderfreundlichkeit. Die unterstützende Wirkung des Instruments zur Erkennung der bedeutenden Faktoren in Kernpunkt (5) der Grundstruktur ist aber aufgrund der Allgemeinheit der Einflussbereiche nicht gegeben. Der Nutzer erhält mittels des Instruments nur eine Einschätzung der Bedeutung der einzelnen Einflussbereiche, nicht aber der Einflussfaktoren. Aufgrund dessen wird in der weiteren Arbeit auf die Berücksichtigung der Einflussanalyse, wie sie REIBNITZ und PASTERNACK darlegen, verzichtet.

Die Instrumente der Konsistenz- und Cross-Impact-Analyse können in den Kernpunkten (7) (Prüfung der Projektionen auf Widerspruchsfreiheit) und (8) (Zusammenführung der Projektionen zu Szenarien) der induktiven Szenario-Technik eingesetzt werden (GÖTZE 2006: 146f.). Kennzeichnendes Wesensmerkmal der CrossImpact-Analyse ist die Inklusion von Wahrscheinlichkeiten in den Prozess der induktiven Szenario-Technik, wodurch sich die Cross-Impact-Analyse eindeutig von der Konsistenzanalyse unterscheidet (GÖTZE 1993: 163; GÖTZE 2006: 146f.).

Aber ungeachtet der beachtlichen Komplexität der Cross-Impact-Analyse (MERCER 1995: 82) wird diese schon alleine aufgrund der Entscheidung gegen die Integration von Wahrscheinlichkeiten bei der Erstellung der Szenarien in dieser Studie nicht weiter berücksichtigt. Ausschlaggebend hierfür waren die Hinweise von REIBNITZ (1992: 49), HEES ET AL. (2006: 291f.) und VERITY (2003: 188), den Schwerpunkt auf alternative Entwicklungsmöglichkeiten der Zukunft zu legen und von Wahrscheinlichkeiten Abstand zu nehmen. Ferner bezeichnet FINK (2011) die Integration 
von Wahrscheinlichkeiten als „vollständig kontraproduktiv“. Seiner Meinung nach verleiten sie den Planer zu einem „Tunnelblick“ auf die Zukunft und hemmen das Denken in alternativen Zukünften - und dies widerspricht dem Ansatz der „multiplen Zukunft“. Nach Aussage von FINK (2011) verzichten in der Praxis 90\% der Unternehmen bei der Anwendung der Szenario-Technik auf die Integration von Wahrscheinlichkeiten. Zu einem ähnlichen Ergebnis kommt auch die Studie von HERZHOFF (2004: 175), die die Anwendung der Szenario-Technik in der chemischen Industrie untersucht hat.

Im Folgenden wird die Konsistenzanalyse betrachtet, um zu ermitteln, ob das Instrument fähig ist, Kernpunkt (7) und (8) der Grundstruktur zu unterstützen. Ziel des Instruments ist es, aus den in Kernpunkt (6) gebildeten Zukunftsprojektionen konsistente (widerspruchsfreie) Szenarien zu entwickeln. Durch die Konsistenz wird entscheidend die Qualität und die Glaubwürdigkeit der Zukunftsbilder verbessert (GAUSEMEIER ET AL. 1995: 254; FINK ET AL. 2002: 87f). Basis der Konsistenzanalyse ist die Konsistenzmatrix, in der anhand einer definierten Skala alle denkbaren Projektionspaare nach ihrem Konsistenzmaß bewertet werden. Das Konsistenzmaß stellt das Ausmaß dar, in dem das Eintreffen der zwei Projektionen zusammenpasst (GÖTZE 1993: 155). Dabei ist es ausreichend, nur auf einer Seite der Konsistenzmatrix die Projektionspaare zu bewerten, weil es sich - im Gegensatz zur Einflussanalyse - nicht um eine gerichtete Beziehung handelt (GAUSEMEIER ET AL. 1995: 255; GÖTZE 1993: 255; REIBNITZ 1992: 51). Der Arbeits- und Zeitaufwand sowie die Komplexität der Konsistenzmatrix werden bestimmt durch die Anzahl der integrierten Zukunftsprojektionen. Während GAUSEMEIER ET AL. (1992: 258) und REIBNITZ (1992: 78ff.) in eine Beispielrechnung 38 Projektionen miteinbeziehen, berücksichtigen FINK ET AL. (2002: 278ff.) gar 65 Zukunftsprojektionen. In Abbildung 13 wird die Problematik der Komplexität der Einflussmatrix durch die Integration einer solch hohen Zahl an Zukunftsprojektionen hervorgehoben. Die zunehmende Komplexität und der ansteigende Arbeits- und Zeitaufwand äußern sich in der Anzahl der zu beurteilenden Projektionspaare. 


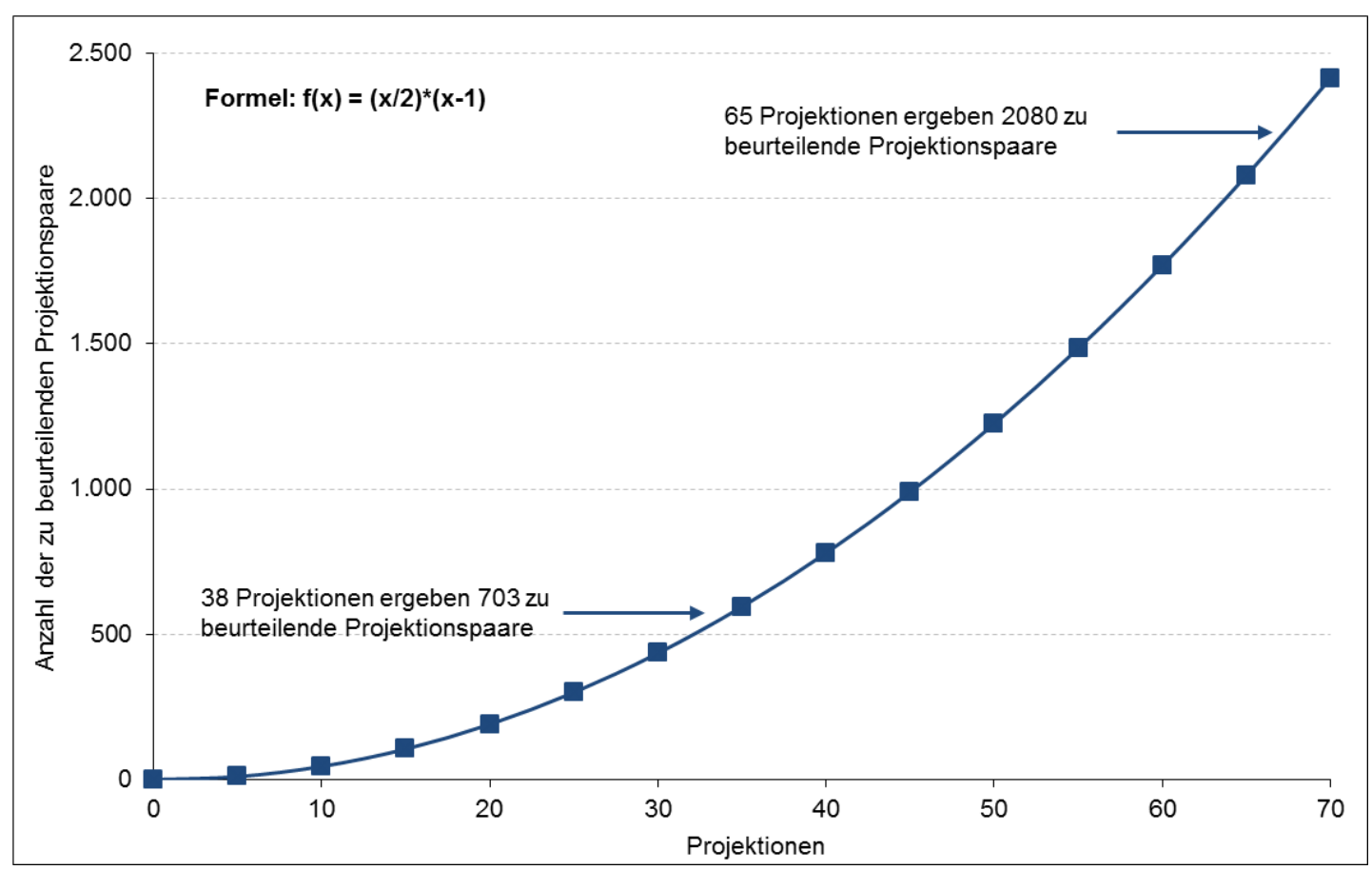

Abbildung 13: Anstieg der Komplexität der Konsistenzmatrix Quelle: EIGENE DARSTELLUNG

Der Anwender der Konsistenzmatrix muss bei 38 Projektionen 703 Projektionspaare und bei 65 Projektionen 2080 Projektionspaare beurteilen (Abbildung 13). Zur Unterstützung der nicht einfachen Bewertung (GÖTZE 1993: 161) eignet sich das Verfahren von REIBNITZ (1992: 50ff.), das mittels aufeinander aufbauender Fragen den Anwender bei der Beurteilung unterstützt. Trotz der Methodik von REIBNITZ führen die hohe Komplexität und der damit verbundene erhebliche Arbeits- und Zeitaufwand für die Bewertung der einzelnen Projektionspaare zu der Einsicht, dass aufgrund der großen Konsistenzmatrix die Konsistenzanalyse, wie sie GAUSEMEIER ET AL. (1995: 254ff.), GÖTZE (1993: 155ff.), FINK ET AL. (2006: 43ff.) und REIBNITZ (1992: 49ff.) beschreiben, nicht brauchbar ist, um die Prozessschritte (7) und (8) der Grundstruktur zu unterstützen.

Um eine Anwendbarkeit des Instruments zu erreichen, wäre eine drastische Verringerung der Zahl der Zukunftsprojektionen erforderlich, um den Umfang der Matrix zu senken. Der Reduzierung sind aber Grenzen gesetzt, um die Aussagekraft der Szenarien zu erhalten. Bei der Berücksichtigung von nur sechs ${ }^{31}$ bedeutenden Faktoren (Schlüsselfaktoren oder Deskriptoren), was in etwa zu $18^{32}$ Zukunftsprojektionen

${ }^{31}$ Die Berücksichtigung von sechs bedeutenden Faktoren stellt die Untergrenze dar. GAUSEMEIER ET AL. (1995: 258) betrachten 17 bedeutende Faktoren, REIBNITZ (1992: 88) und FINK ET AL. (2002: 291) sogar 18.

32 Unter der Annahme, dass im Durchschnitt drei Zukunftsprojektionen je Schlüsselfaktor oder Deskriptor gebildet werden. GAUSEMEIER ET AL. (1995: 258) bilden in der Beispielrechnung in ihrem Buch durchschnittlich 2,2 Zukunftsprojektionen je bedeutendem Faktor, FINK ET AL. (2002: 278ff.) gar 3,6. 
führt, müsste der Anwender der Konsistenzmatrix 153 Projektionspaare bewerten. Der damit verbundene Arbeits- und Zeitaufwand für die nicht unkomplizierte Beurteilung der Projektionspaare führt zu der Erkenntnis, dass trotz der bereits maximalen Reduktion die Komplexität zu groß ist, um das Instrument zur Durchführung der Kernpunkte (7) und (8) einzusetzen. Folglich wird von einem Gebrauch des Instruments abgesehen und dieses in der Arbeit nicht weiter betrachtet.

Aufgrund des beträchtlichen Zeitbedarfs zur Erstellung der Szenarien, verursacht durch die komplexen Instrumente Einflussanalyse, Konsistenzanalyse und CrossImpact-Analyse, können die etablierten Verfahren der induktiven Szenario-Technik nicht angewandt werden, um die Zielstellung der Arbeit zu erreichen. Selbst REIBNITZ (1992: 276), ehemalige Mitarbeiterin des BATTELLE-INSTITUTS, erkennt die Notwendigkeit einer Vereinfachung des Verfahrens. Infolgedessen wird eine optimierte Methodik entwickelt, basierend auf der Grundstruktur der induktiven Szenario-Technik, welche die Komplexität und den damit verbundenen Zeitbedarf senkt (Kapitel 4). Durch diese Komplexitätsreduzierung ist es möglich, das Verfahren der induktiven Szenario-Technik einzusetzen, um die Ziele der Studie zu erfüllen. 
Im Folgenden wird die formelle Ausrichtung (Kapitel 4.1) und die optimierte Methodik der induktiven Szenario-Technik erläutert, mit der die Szenarien des Ethanolmarktes in der EU27 und den USA für das Jahr 2023 gebildet werden. Die Methodik unterteilt sich in die drei Kernschritte: „Szenariofeld-Analyse“ (Kapitel 4.2), „Szenario-Prognostik“ (Kapitel 4.3) und "Szenario-Bildung und -Konsistenzprüfung“ (Kapitel 4.4). Abbildung 14 stellt das methodische Vorgehen des optimierten Verfahrens der Szenario-Technik grafisch dar.

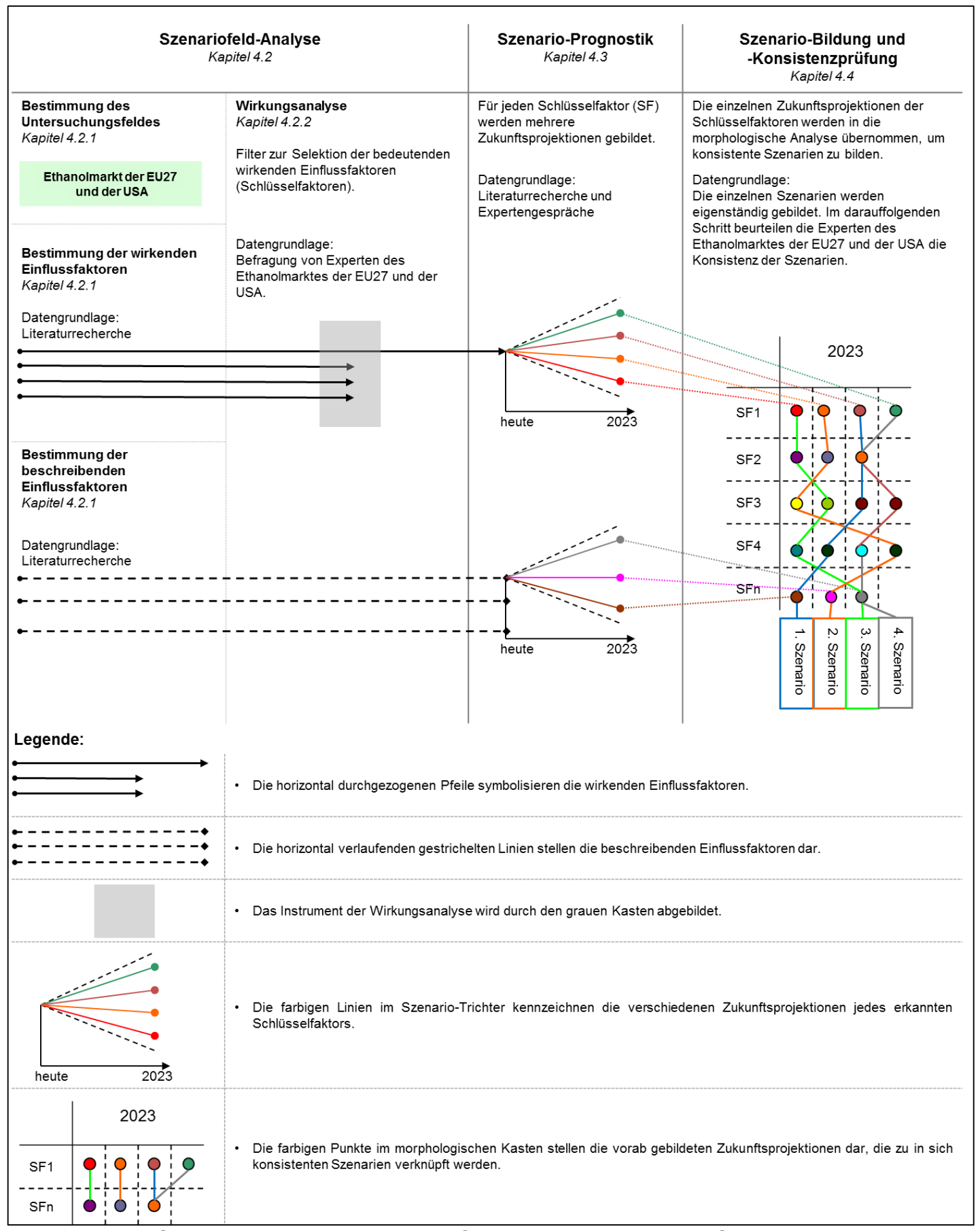

Abbildung 14: Schematische Darstellung - Optimierte Methodik der Szenario-Technik Quelle: EIGENE DARSTELLUNG 


\subsection{Formelle Ausrichtung der Szenario-Technik}

Die Methodik der Szenario-Technik kann ausschließlich zur Erstellung von „Marktentwicklungsszenarien“ genutzt werden (FINK ET AL. 2002: 138). Solchen „Marktentwicklungsszenarien“ sind zwei Merkmale inhärent: (1) Stehen die Märkte der Zukunft im Zentrum der Szenario-Erstellung - in dieser Arbeit der Ethanolmarkt der EU27 und der USA im Jahre 2023 - und (2) werden im Prozess der Szenarioerstellung nur nicht lenkbare, externe Einflussgrößen betrachtet (GAUSEMEIER ET AL. 1995: 106), also Faktoren, auf die die Entscheidungsträger der Szenarien keinen Einfluss nehmen können.

Darüber hinaus werden mithilfe der Szenario-Technik nur explorative „Was-wärewenn-Szenarien“ und keine antizipativen „Was-muss-geschehen-dassSzenarien“ erstellt (GAUSEMEIER ET AL. 1995: 111), da es das Ziel der Arbeit ist, den Ethanolmarkt der EU27 und der USA im Jahr 2023 durch unterschiedliche Szenarien zu beschreiben. Bei explorativen Szenarien wird der Ist-Zustand als Ausgangspunkt der Szenario-Erstellung analysiert und genutzt, um vorwärtsgewandt unterschiedliche Entwicklungen aufzuzeigen. Durch die Entwicklung explorativer Szenarien wird dem Ansatz der „multiplen Zukünfte“ (Abbildung 10) Rechnung getragen.

Ferner werden die Szenarien unabhängig von erwünschten Zielen der späteren Szenario-Anwender gebildet, um unbeeinflusst mögliche Entwicklungen des Ethanolmarktes aufzeigen zu können. Aufgrund dessen handelt es sich nicht um präskriptive, sondern um deskriptive Szenarien, die auf der Basis von Kausalitätsbeziehungen erstellt werden (GAUSEMEIER ET AL. 1995: 111; FINK ET AL. 2002: 62). Nur durch die Kombination eines explorativen Ausgangspunktes und einer deskriptiven Zielrichtung können Zukünfte des Ethanolmarktes unvoreingenommen erstellt werden.

Ein aus dem Forschungsbedarf abgeleitetes Ziel dieser Arbeit besteht darin, durch Szenarien eine breitere Abdeckung möglicher zukünftiger Entwicklungen des Ethanolmarktes der EU27 und der USA zu erreichen. Aus diesem Grund ist die Erstellung von Extrem- und Trendszenarien möglich und das Denken in alternativen Zukünften wird nicht durch einen systematischen Ausschluss möglicher Zukünfte eingeschränkt.

Bezüglich des Zeithorizonts handelt es sich um die Erstellung langfristiger Szenarien, da ein Planungszeitraum von über fünf Jahren betrachtet wird. Darüber hinaus wird, wie auch schon in Kapitel 3.4 genauer erläutert, auf die Inklusion von Wahrscheinlichkeiten verzichtet, um das besonders bei der Erstellung von Extremszenarien wichtige Denken in alternativen Zukünften nicht zu hemmen. 
Die Kriterien sowie die Möglichkeiten der formellen Ausrichtung der SzenarioTechnik sind in der folgenden Tabelle 6 grafisch zusammengefasst. Die formelle Ausrichtung, die für die optimierte Methodik der induktiven Szenario-Technik von Relevanz ist, ist mit der Farbe Grün hinterlegt. Die Spalte „Kommentar“ dient der Erläuterung der formellen Ausrichtung.

\begin{tabular}{|c|c|c|c|}
\hline Kriterium & \multicolumn{2}{|c|}{ Möglichkeit der formellen Ausrichtung } & Kommentar \\
\hline Lenkbarkeit der Einflussgrößen & $\begin{array}{l}\text { Lenkbare } \\
\text { Einflussgrößen }\end{array}$ & $\begin{array}{l}\text { Nicht lenkbare } \\
\text { Einflussgrößen }\end{array}$ & $\begin{array}{l}\text { Die Entscheidungsträger können die } \\
\text { Einflussgrößen nicht beeinflussen } \\
\text { (Umfeldszenarien). }\end{array}$ \\
\hline Ausgangspunkt der Szenario-Erstellung & $\begin{array}{l}\text { Antizipatives } \\
\text { Szenario }\end{array}$ & $\begin{array}{l}\text { Exploratives } \\
\text { Szenario }\end{array}$ & $\begin{array}{l}\text { Der Ist-Zustand ist Ausgangspunkt der } \\
\text { Szenario-Erstellung. }\end{array}$ \\
\hline Zielgerichtetheit der Szenario-Erstellung & $\begin{array}{l}\text { Präskriptives } \\
\text { Szenario }\end{array}$ & $\begin{array}{l}\text { Deskriptives } \\
\text { Szenario }\end{array}$ & $\begin{array}{l}\text { Die Szenarien werden unabhängig vom Ziel } \\
\text { der Szenario-Anwender erstellt. }\end{array}$ \\
\hline Inhaltliche Ausrichtung des Szenarios & Trendszenario & Extremszenario & $\begin{array}{l}\text { Es werden sowohl Trend- als auch } \\
\text { Extremszenarien berücksichtigt, um das } \\
\text { Denken in alternativen Zukünften nicht } \\
\text { einzuschränken. }\end{array}$ \\
\hline Zeithorizont des Szenarios & kurzfristig & langfristig & $\begin{array}{l}\text { Ab einem Betrachtungshorizont von mehr als } \\
5 \text { Jahren wird das Szenario als langfristig } \\
\text { bezeichnet. }\end{array}$ \\
\hline Berücksichtigung von Wahrscheinlichkeiten & ja & nein & $\begin{array}{l}\text { Das Denken in alternativen Zukünften soll } \\
\text { durch die Inklusion von Wahrscheinlichkeiten } \\
\text { nicht gestört werden. }\end{array}$ \\
\hline
\end{tabular}

Tabelle 6: Formelle Ausrichtung des optimierten Verfahrens der Szenario-Technik Quelle: EIGENE DARSTELLUNG

\subsection{Szenariofeld-Analyse}

Das zentrale Ziel der „Szenariofeld-Analyse“ - erster Kernschritt der optimierten induktiven Szenario-Technik - ist es, die Schlüsselfaktoren des Szenariofeldes zu bestimmen, um den „Blick in die Zukunft“ (GAUSEMEIER ET AL. 1995: 167) vorzubereiten. Die Schlüsselfaktoren sind kennzeichnende Faktoren des in diesem Kapitel zu analysierenden Szenariofeldes, welches sich aus dem Untersuchungsfeld und dem Einflussbereich zusammensetzt.

Zur Ermittlung der Schlüsselfaktoren bedarf es der folgenden beiden Schritte: (1) der Bestimmung des Untersuchungsfeldes sowie der wirkenden und der beschreibenden Einflussfaktoren in Kapitel 4.2.1 und (2) der Wirkungsanalyse in Kapitel 4.2.2. Diese beiden Schritte werden im Folgenden ausführlich vorgestellt. 


\subsubsection{Bestimmung des Untersuchungsfeldes sowie der wirkenden und der beschreibenden Einflussfaktoren}

Bestimmung des Untersuchungsfeldes ${ }^{33}$

Im ersten Schritt wird das Untersuchungsfeld bestimmt, welches mithilfe des optimierten Verfahrens der induktiven Szenario-Technik analysiert werden soll. Zum besseren Verständnis sei angemerkt, dass der Ethanolmarkt der EU27 (Kapitel 5.1.1.1) und der USA (Kapitel 6.1.1.1) das Untersuchungsfeld der vorliegenden Arbeit darstellt. Das Untersuchungsfeld ist Teil des Szenariofeldes und somit auch Bestandteil der zu entwickelnden Szenarien.

Für eine Beschreibung des Untersuchungsfeldes und um Missverständnisse hinsichtlich des Untersuchungsfeldes frühzeitig zu vermeiden, ist es erforderlich, die folgenden drei Punkte zu erläutern:

1. Ist-Situation des Untersuchungsfeldes

2. Historische Entwicklung des Untersuchungsfeldes

3. Abgrenzung des Untersuchungsfeldes zu anderen Märkten

\section{Bestimmung der wirkenden Einflussfaktoren}

Nachdem im ersten Schritt das Untersuchungsfeld definiert wurde, werden nun die auf das Untersuchungsfeld wirkenden Einflussfaktoren bestimmt. Wirkende Einflussfaktoren sind unabhängige, nicht lenkbare, externe Faktoren, die den Einflussbereich charakterisieren.

Zur Unterstützung der Ermittlung der wirkenden Einflussfaktoren wird der auf das Untersuchungsfeld einwirkende Einflussbereich nach der „SEPT formula“ (Social, Economical, Political, Technological) von WILSON (1998: 87) in die Bereiche „Einflussbereich Politik“, „Einflussbereich Technologie“, „Einflussbereich Ökonomie“ und „Einflussbereich Gesellschaft“ unterteilt ${ }^{34}$. Hintergrund dieser viergliedrigen Aufteilung des Einflussbereichs ist das Bemühen, zu verhindern, dass ausschließlich Einflussfaktoren aus einem Teilbereich genannt werden, um die "Gefahr schleichender Schwerpunktbildung“ (GAUSEMEIER ET AL. 1995: 167) zu minimieren. Ziel ist es, aus allen relevanten Bereichen des Szenariofeldes wirkende Einflussfaktoren zu ermitteln und zu beschreiben (FINK ET AL. 2002: 76).

\footnotetext{
${ }^{33}$ Wie in Abbildung 14 ersichtlich, ist dieses Kapitel in die drei Bereiche "Bestimmung des Untersuchungsfeldes“, „Bestimmung der wirkenden Einflussfaktoren“ und „Bestimmung der beschreibenden Einflussfaktoren" unterteilt.

${ }^{34}$ Die „SEPT formula“ entspricht der „PEST-Analyse“ von JOHNSON ET AL. (2011: 78), lediglich die Reihenfolge der genannten Einflussbereiche (Political, Economical, Social, Technological) unterscheidet sich.
} 
Es gibt eine Vielzahl von Ansätzen zur Bestimmung wirkender Einflussfaktoren, die in der Arbeit von GAUSEMEIER ET AL. (1995: 173ff.) beschrieben werden. Zu den Ansätzen zählen auch die in der vorliegenden Arbeit angewandten Methoden: Die Ermittlung der wirkenden Einflussfaktoren für den Ethanolmarkt der EU27 (Kapitel 5.1.1.2) und der USA (Kapitel 6.1.1.2) erfolgt anhand einer umfangreichen Literaturrecherche und eigens geführter Expertengespräche. Bei der Anzahl zu bestimmender Einflussfaktoren gibt es keine Beschränkung. Die Anzahl der Einflussfaktoren ist somit vollständig von der Komplexität des zu betrachtenden Marktes abhängig.

Es ist weiterhin von entscheidender Bedeutung, eine wertneutrale Beschreibung der Einflussfaktoren in der Arbeit vorzunehmen, um eine möglichst problemlose Bearbeitung der folgenden Schritte zu ermöglichen. Die Beschreibung soll Missdeutungen und abweichende Interpretationen der Einflussfaktoren verhindern, insbesondere bei der Anwendung des Instrumentes der Wirkungsanalyse, da bei diesem Instrument die Bewertung der Einflussfaktoren von mehreren unabhängig voneinander befragten Personen erfolgt (GAUSEMEIER ET AL. 1995: 186).

Abbildung 15 dient zur Unterstützung der folgenden Erläuterung, wie eine Beschreibung eines Einflussfaktors erfolgt.

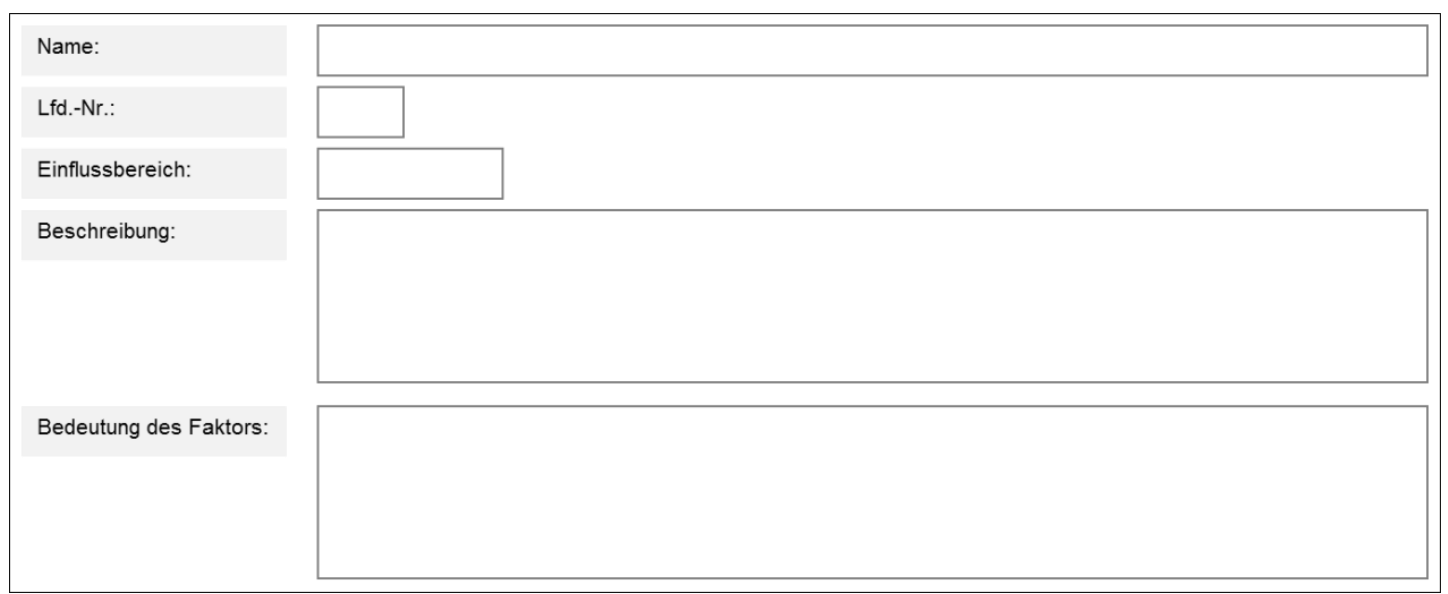

Abbildung 15: Struktur zur Beschreibung eines Einflussfaktors

Quelle: In Anlehnung an GAUSEMEIER ET AL. 1995: 183; FINK ET AL. 2002: 78

Ein auf das Untersuchungsfeld wirkender Einflussfaktor erhält in dem Feld „Name" eine präzise und leicht verständliche Bezeichnung (GAUSEMEIER ET AL. 1995: 184; FINK ET AL. 2002: 78). Im nächsten Textfeld wird eine „Laufende Nummer" (Lfd.-Nr.) zur eindeutigen Identifizierung des Einflussfaktors vergeben. Die Angabe, welchem Einflussbereich der jeweilige Einflussfaktor zuzuordnen ist, erfolgt im Feld „Einflussbereich“. Das Eingabefeld „Beschreibung“ wird dazu genutzt, den Einflussfaktor inhaltlich präzise zu beschreiben. Die Charakterisierung muss, darauf weist REIBNITZ (1992: 45) hin, wertneutral formuliert sein, da bei einer gerichteten Formulierung die Gefahr besteht, dass Zukunftsprojektionen in lediglich eine Rich- 
tung weitergedacht werden und nicht, wie von der induktiven Szenario-Technik gefordert, in alternativen Zukünften (Abbildung 10). In dem Textfeld „Bedeutung des Faktors" wird der Einfluss des Faktors auf das Untersuchungsfeld beschrieben. Die Bedeutung des Faktors begründet, warum der wirkende Einflussfaktor in dem weiteren Prozess der Szenario-Erstellung berücksichtigt werden muss ${ }^{35}$.

Im kommenden Unterpunkt werden die beschreibenden Einflussfaktoren genauer erläutert und der Unterschied zwischen wirkenden und beschreibenden Einflussfaktoren erklärt.

\section{Bestimmung der beschreibenden Einflussfaktoren}

Beschreibende Einflussfaktoren sind abhängige, nicht lenkbare, externe Faktoren. Sie stellen das Untersuchungsfeld als Teil des Szenariofeldes in den zu entwickelnden Szenarien dar, während die wirkenden Einflussfaktoren die Entwicklung des Einflussbereichs aufzeigen.

Die Unterteilung in wirkende und beschreibende Einflussfaktoren ist unerlässlich, um das im Kapitel 4.2.2 erläuterte Instrument der Wirkungsanalyse ${ }^{36}$ anwenden zu können. Nähme man keine Unterteilung der Einflussfaktoren vor, so hätte dies zur Folge, dass alle Einflussfaktoren (wirkende und beschreibende) auf die Stärke ihres Einflusses auf das Untersuchungsfeld überprüft werden müssten. Dies würde zu dem Ergebnis führen, dass alle Einflussfaktoren, die das Untersuchungsfeld repräsentieren (beschreibende Einflussfaktoren) herausgefiltert werden, da sie aufgrund ihrer Spezifität keine starke Wirkung auf das Untersuchungsfeld ausüben. Die das Untersuchungsfeld kennzeichnenden, beschreibenden Einflussfaktoren sind aber von unbedingter Notwendigkeit bei der Erstellung der Szenarien, damit die Entwicklungen des Untersuchungsfeldes in den Szenarien abgebildet werden kann.

Die in der vorliegenden Arbeit vorgenommene Unterteilung in wirkende und beschreibende Einflussfaktoren ist ein Novum in der Szenario-Technik, aber aus Sicht des Autors unbedingt erforderlich, um eine zielgerichtete Anwendbarkeit der Wirkungsanalyse zu erreichen.

\footnotetext{
${ }^{35}$ Durch das beschriebene Vorgehen können sowohl quantitative als auch qualitative Einflussfaktoren in den Prozess der Szenario-Erstellung integriert werden. Durch diese Flexibilität kommt es zu keinem systematischen Ausschluss möglicher bedeutender Faktoren, was einer der großen Vorteile der Methodik ist (Kapitel 3.1).

${ }^{36}$ In der Wirkungsanalyse werden die wirkenden Einflussfaktoren nach der Stärke ihrer Wirkung auf das Untersuchungsfeld beurteilt. Nur solche wirkenden Einflussfaktoren, die durch ihre starke Wirkung auf das Untersuchungsfeld als bedeutend erkannt werden, werden im zweiten Kernschritt „Szenario-Prognostik“ (Kapitel 4.3) weiter betrachtet.
} 
Die Bestimmung der beschreibenden Einflussfaktoren erfolgt auf Grundlage des bestehenden Forschungsstandes. Um eine Vergleichbarkeit der vorliegenden Studie mit den zitierten Studien des Kapitels 2.3 zu gewährleisten, ist es unabdingbar, die folgenden drei beschreibenden Einflussfaktoren in den Prozess zu integrieren: „Ethanolnachfrage“, „Ethanolproduktion“ und „Ethanolhandel“. Des Weiteren ist es möglich, noch weitere beschreibende Einflussfaktoren hinzuzufügen. Die beschreibenden Einflussfaktoren werden nach dem gleichen Muster wie die wirkenden Einflussfaktoren erläutert. Der einzige Unterschied ist, dass das Feld „Bedeutung des Faktors“" wegfällt, da die Faktoren das Untersuchungsfeld darstellen, es aber nicht beeinflussen.

Nach Abschluss der Bearbeitung der drei beschriebenen Bereiche - „Bestimmung des Untersuchungsfeldes“, „Bestimmung der wirkenden Einflussfaktoren“ und „Bestimmung der beschreibenden Einflussfaktoren“ - ist die Erstellung einer Darstellung, wie in Abbildung 16 beispielhaft abgebildet, möglich. Sowohl die Anzahl als auch die Verteilung der Einflussfaktoren in der Darstellung dient lediglich einem besseren Verständnis und hat keine bindende Wirkung. Dieses Beispiel wird in den folgenden Kapiteln fortgesetzt und auch dort nur zum besseren Verständnis der Methodik.

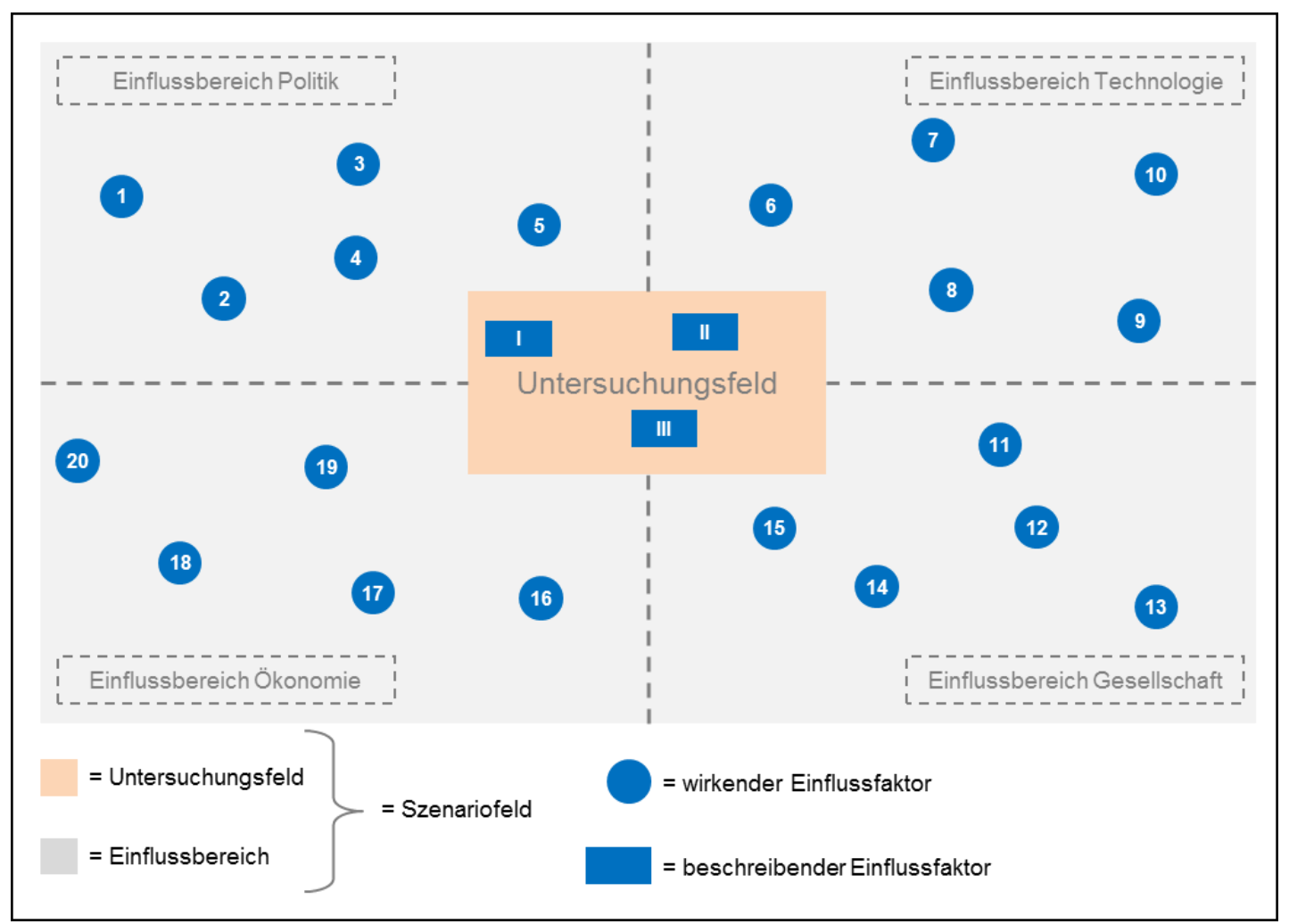

Abbildung 16: Darstellung des Szenariofeldes mit beschreibenden und wirkenden Einflussfaktoren

Quelle: EIGENE DARSTELLUNG 


\subsubsection{Wirkungsanalyse}

Das Ziel der Wirkungsanalyse ist es, die wirkenden Schlüsselfaktoren zu bestimmen; dies sind jene wirkenden Einflussfaktoren, die das Untersuchungsfeld am stärksten beeinflussen (GAUSEMEIER ET AL. 1995: 189).

Notwendig ist die Wirkungsanalyse, da nur durch eine starke Reduktion der bisher nicht begrenzten Anzahl an wirkenden Einflussfaktoren - es werden ausschließlich die wirkenden Einflussfaktoren betrachtet - der Prozess der Szenario-Technik beherrschbar und überschaubar bleibt (MILLETT/HONTON 1991: 71). Vielmehr besteht bei einer nicht erfolgten Reduktion die Gefahr, dass die zentralen Entwicklungen des Szenariofeldes aufgrund der zahlreichen Einflussfaktoren nicht deutlich zu erkennen sind (GAUSEMEIER ET AL. 1995: 187f.).

Bei dem Instrument der Wirkungsanalyse wird die Beziehung zwischen den wirkenden Einflussfaktoren und dem Untersuchungsfeld beurteilt (GÖTZE 1993: 107). Nur die wirkenden Einflussfaktoren, die durch ihre starke Wirkung auf das Untersuchungsfeld als bedeutend erkannt werden, werden als Schlüsselfaktoren im Prozess weiter betrachtet. Alle anderen wirkenden Einflussfaktoren werden in den folgenden Schritten des Prozesses der optimierten induktiven Szenario-Technik nicht weiter berücksichtigt. Das Instrument fungiert somit als Filter, der alle weniger relevanten wirkenden Einflussfaktoren herausfiltert (AYE 2009: 168). Ergänzend sei gesagt, dass sich in den etablierten Verfahren der Szenario-Technik kein einheitliches Vorgehen bei der Erstellung der Wirkungsanalyse finden lässt (AYE 2009: 169).

Die Durchführung des Instruments der Wirkungsanalyse erfolgt anhand eines Fragebogens; Abbildung 17 zeigt einen Ausschnitt dieses Fragebogens. 


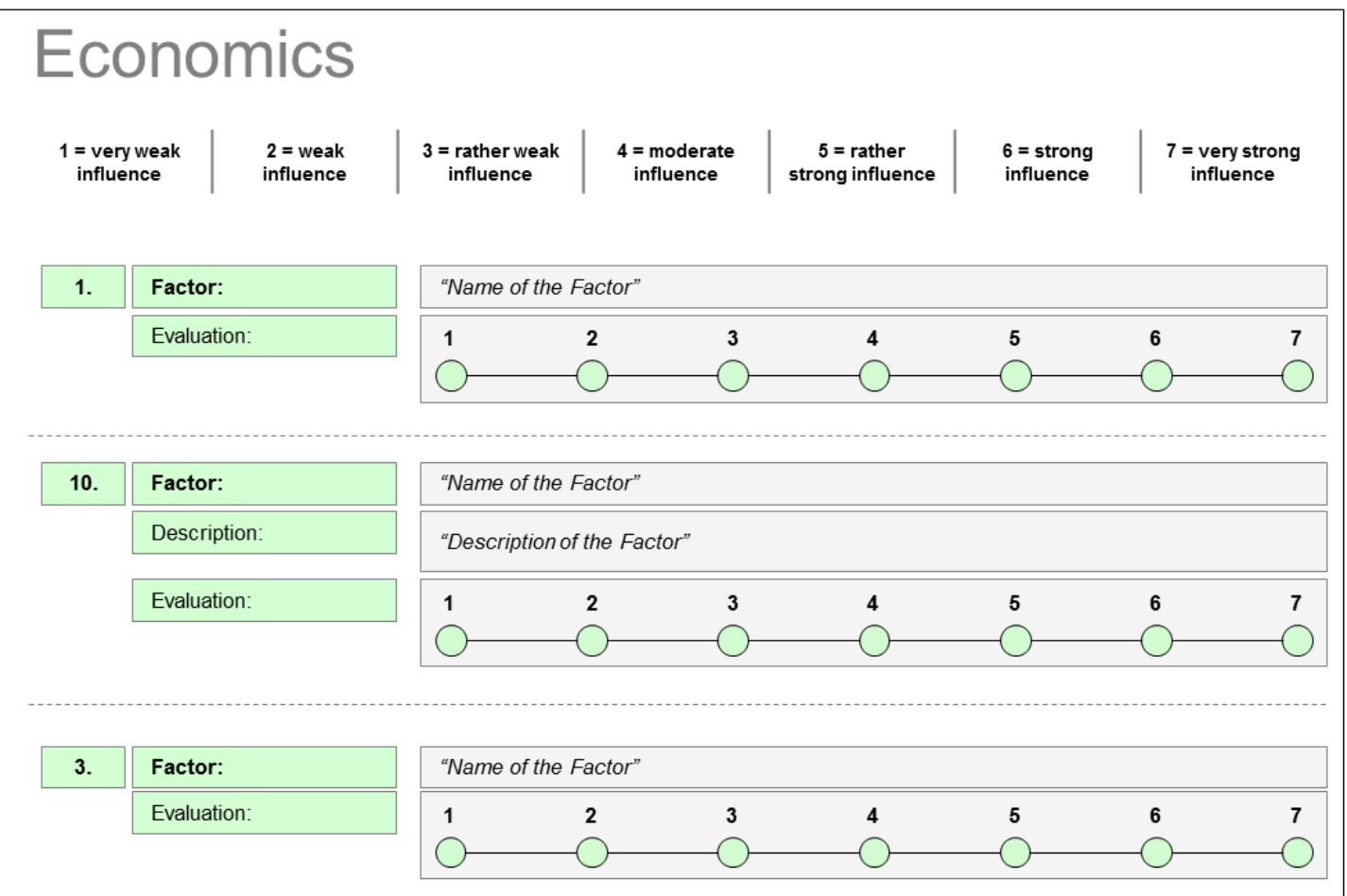

Abbildung 17: Ausschnitt des Fragebogens zur Durchführung der Wirkungsanalyse Quelle: EIGENE DARSTELLUNG

In dem Fragebogen werden die einzelnen wirkenden Einflussfaktoren entsprechend ihrem zugehörigen Einflussbereich untereinander geordnet. Jeder Einflussfaktor wird anhand der Felder „Factor" und wahlweise mittels des Bereichs „Description“ erläutert. Die Skalierung, um die Stärke des Einflusses des Faktors auf das Untersuchungsfeld zu messen, befindet sich darunter. Es wurde eine siebenstufige Likert-Skala gewählt, die es den Experten ermöglicht, Einschätzungen der Stärke der auf das Untersuchungsfeld wirkenden Einflussfaktoren vorzunehmen. Hierdurch wird die Selektion jener wirkenden Einflussfaktoren ermöglicht, die das Untersuchungsfeld entscheidend bis zum Jahr 2023 beeinflussen werden. Die Einschätzung erfolgt durch Experten des Ethanolmarktes in der EU27 und den USA, die unabhängig voneinander den Fragebogen ausfüllen und somit nicht die Möglichkeit haben, sich über ihre Bewertung auszutauschen.

Die Selektion der wirkenden Einflussfaktoren erfolgt auf Basis des arithmetischen Mittelwertes. Die sieben bis acht Faktoren mit dem höchsten Mittelwert, folglich mit dem nach Einschätzung der befragten Experten stärksten Einfluss auf das Untersuchungsfeld, werden als Schlüsselfaktoren in der "Szenario-Prognostik“ weiter betrachtet. Besitzen zwei Einflussfaktoren den identischen Mittelwert, so ist der Faktor mit der geringeren Standardabweichung zu bevorzugen. Die anderen wirkenden Einflussfaktoren, mit einem weniger starken Einfluss auf das Untersuchungsfeld, 
werden nicht weiter in der Arbeit berücksichtigt. Durch die Bestimmung der wirkenden Schlüsselfaktoren wird das erste Zie ${ }^{37}$ der Dissertation erreicht.

Die beschreibenden Einflussfaktoren, die das Untersuchungsfeld darstellen, werden ebenfalls in dem Kernschritt der "Szenario-Prognostik“ weiter betrachtet. Aufgrund dessen werden sie auch als beschreibende Schlüsselfaktoren bezeichnet.

Nach Durchführung und Auswertung der Wirkungsanalyse sind die Schlüsselfaktoren ermittelt, die im weiteren Prozess der Szenario-Technik betrachtet werden. Diese sind in der folgenden Abbildung 18 als grüne Kreise/Rechtecke dargestellt, während die grauen Kreise die in der Wirkungsanalyse aussortierten wirkenden Einflussfaktoren symbolisieren.

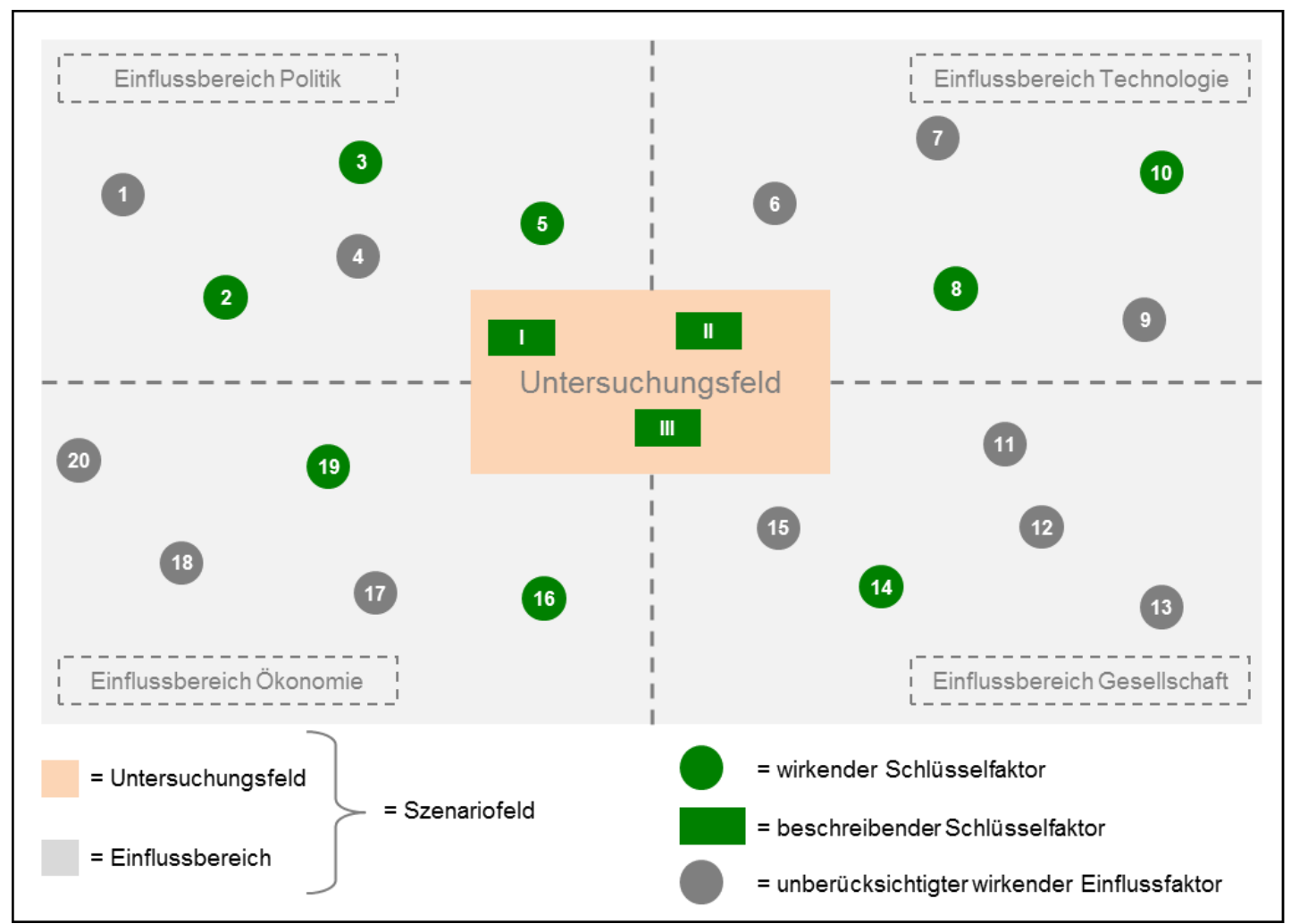

Abbildung 18: Darstellung des Szenariofeldes nach Durchführung der Wirkungsanalyse Quelle: EIGENE DARSTELLUNG

\subsection{Szenario-Prognostik}

Ziel der „Szenario-Prognostik“ - zweiter Kernschritt der Szenario-Technik - ist es, für jeden in Kapitel 4.2 erkannten Schlüsselfaktor mindestens eine Zukunftsprojektion zu bilden. Bei diesem Kernschritt der Szenario-Technik erfolgt der „Blick in die

${ }^{37}$ Das erste Ziel der Dissertation besteht darin, die Faktoren zu identifizieren die das Untersuchungsfeld (Ethanolmarkt der EU27 und der USA) entscheidend, also am stärksten beeinflussen werden (Kapitel 1.1). 
Zukunft“ (FINK ET AL. 2002: 82; GAUSEMEIER ET AL. 1995: 221), wodurch sich die „Szenario-Prognostik“ grundlegend von der „Szenariofeld-Analyse“38 unterscheidet.

Dem Kernschritt „Szenario-Prognostik“ kommt entscheidende Bedeutung für den Gesamtprozess der Szenario-Technik zu, da sich die im Kernschritt „SzenarioBildung und -Konsistenzprüfung" erstellten Szenarien aus den Zukunftsprojektionen, die im Zuge der Szenario-Prognostik gebildet werden, zusammensetzen. Damit entscheiden die Zukunftsprojektionen grundlegend über Qualität und Inhalt der späteren Szenarien (GAUSEMEIER ET AL. 1995: 221).

Die Anzahl der zu bildenden Zukunftsprojektionen wird in der Szenario-Prognostik nicht vorab festgelegt, sondern ist von der Unsicherheit hinsichtlich der langfristigen Entwicklung des jeweiligen Schlüsselfaktors abhängig. Dadurch unterscheidet sich das beschriebene Vorgehen der Szenario-Technik von den etablierten Verfahren, die die Anzahl an Zukunftsprojektionen auf maximal drei (GAUSEMEIER ET AL. 1995: 222) bzw. zwei (REIBNITZ 1992: 51) beschränken. Ausschlaggebend für die Entscheidung, die Anzahl an Zukunftsprojektionen nicht zu limitieren, ist das Bestreben, das Denken in alternativen Zukünften zu fördern. Dabei erfüllt das Erstellen von unterschiedlichen Zukunftsprojektionen den Grundsatz der „multiplen Zukunft" (GAUSEMEIER ET AL. 2009: 59f.), der in Abbildung 10 durch das Trichtermodell symbolisiert wird. Die Erfüllung dieses Grundsatzes ist eine entscheidende Stärke der Szenario-Technik (GAUSEMEIER ET AL. 1995: 222), denn dadurch kann die spezifische Unsicherheit der zukünttigen Entwicklung jedes Schlüsselfaktors in dem Prozess der „Szenario-Bildung“ berücksichtigt werden.

Die formelle Ausrichtung der zu erstellenden Zukunftsprojektionen stimmt mit der Gesamtausrichtung der Szenario-Technik, die in Kapitel 4.1 beschrieben wurde, überein: Die Zukunftsprojektionen sind somit explorativ, sie sind deskriptiv, sie betrachten einen langfristigen Zeithorizont und auf die Inklusion von Wahrscheinlichkeiten wird verzichtet. Ferner liegt der im Vergleich zu den etablierten Verfahren der Szenario-Technik grundlegende Unterschied darin, dass sowohl Trend- als auch Extremprojektionen entwickelt werden dürfen ${ }^{39}$. Der Autor sieht auch in dieser Entscheidung eine Stärkung des Grundsatzes des Denkens in alternativen Zukünften,

\footnotetext{
${ }^{38}$ Die Aufgabe der "Szenariofeld-Analyse“ ist es, durch die Bestimmung der Schlüsselfaktoren" den Blick in die Zukunft vorzubereiten (GAUSEMEIER ET AL. 1995: 167).

${ }^{39}$ Bei den etablierten Verfahren der Szenario-Technik von GAUSEMEIER ET AL. (1995: 224), FINK ET AL. (2002: 83) und REIBNITZ (1992: 51) ist zu entscheiden, ob entweder Trend- oder Extremprojektionen gebildet werden.
} 
da hierdurch eine unnötige und künstliche Beschränkung des möglichen Zukunftsraums verhindert wird.

Im kommenden Abschnitt wird erläutert, wie die Zukunftsprojektionen für den Ethanolmarkt der EU27 und der USA gebildet werden ${ }^{40}$. Ausgangspunkt bei der Bildung und Beschreibung der Zukunftsprojektionen ist die Analyse der historischen Entwicklung und der Ist-Situation. Die Ist-Analyse dient dazu, den Blick in die Zukunft vorzubereiten (GAUSEMEIER ET AL. 1995: 228), um ausgehend von der Gegenwart die Zukunftsprojektionen auf Grundlage von umfangreichen Literaturrecherchen und Expertengesprächen zu entwickeln.

Bei der Erstellung der Zukunftsprojektionen ist auf folgende vier Kriterien zu achten (GAUSEMEIER ET AL. 1995: 226f.):

1. Die Zukunftsprojektionen der Schlüsselfaktoren sollen nachvollziehbar und glaubwürdig sein.

2. Die erstellten Projektionen müssen sich eindeutig voneinander abgrenzen.

3. Für jeden Schlüsselfaktor muss mindestens eine Zukunftsprojektion gebildet werden.

4. Die Summe der Zukunftsprojektionen soll möglichst vollständig die Entwicklungsmöglichkeiten des Schlüsselfaktors abdecken.

Darüber hinaus müssen die Zukunftsprojektionen kurz und prägnant beschrieben werden, um eine Eintragung der Projektionen in den morphologischen Kasten zu ermöglichen und eine zielgerichtete Anwendbarkeit der morphologischen Analyse ${ }^{41}$ zu erreichen. Zur eindeutigen Identifizierung der Zukunftsprojektionen erhält jede Projektion eine Kennung, bestehend aus der bereits vergebenen laufenden Nummer und einem Buchstaben des lateinischen Alphabets.

Mit Abschluss der Szenario-Prognostik ist für jeden Schlüsselfaktor mindestens eine Zukunftsprojektion gebildet worden. In der folgenden Abbildung 19 ist das theoretische Ergebnis der Szenario-Prognostik grafisch dargestellt. Die Ist-Situation der

\footnotetext{
${ }^{40}$ Die etablierten Verfahrend der Szenario-Technik zeigen kein einheitliches Vorgehen zur Beschreibung der Projektionen:

REIBNITZ (1992: 78ff.) beschreibt die einzelnen Zukunftsprojektionen nur äußerst knapp, begründet diese aber ausführlicher, damit der Leser nachvollziehen kann, warum jede einzelne Projektion von Relevanz ist.

Demgegenüber erhält im Vorgehen von GAUSEMEIER ET AL. (1995: 242ff.) und FINK ET AL. (2002. 278ff.) jede Zukunftsprojektion eine kurze, prägnante Überschrift und wird im anschließenden Text genauer dargestellt. In der Erläuterung der Projektionen unterscheiden sich aber die Verfahren von GAUSEMEIER ET AL. und FINK ET AL.: Zwar beschreiben beide die Projektionen ausführlich, aber GAUSEMEIER ET AL. begründen zusätzlich, warum jede einzelne Projektion gebildet wurde.

${ }^{41}$ Die Durchführung der morphologischen Analyse wird in Kapitel 4.4 erklärt.
} 
wirkenden und der beschreibenden Schlüsselfaktoren ist grün gekennzeichnet, im Gegensatz zu den in verschiedenen Farbtönen dargestellten Zukunftsprojektionen. Die unterschiedlichen Farben der Zukunftsprojektionen symbolisieren die Eigenständigkeit und die Verschiedenheit der Projektion im Vergleich zur Ist-Situation und zu den anderen Zukunftsprojektionen dieses Schlüsselfaktors.

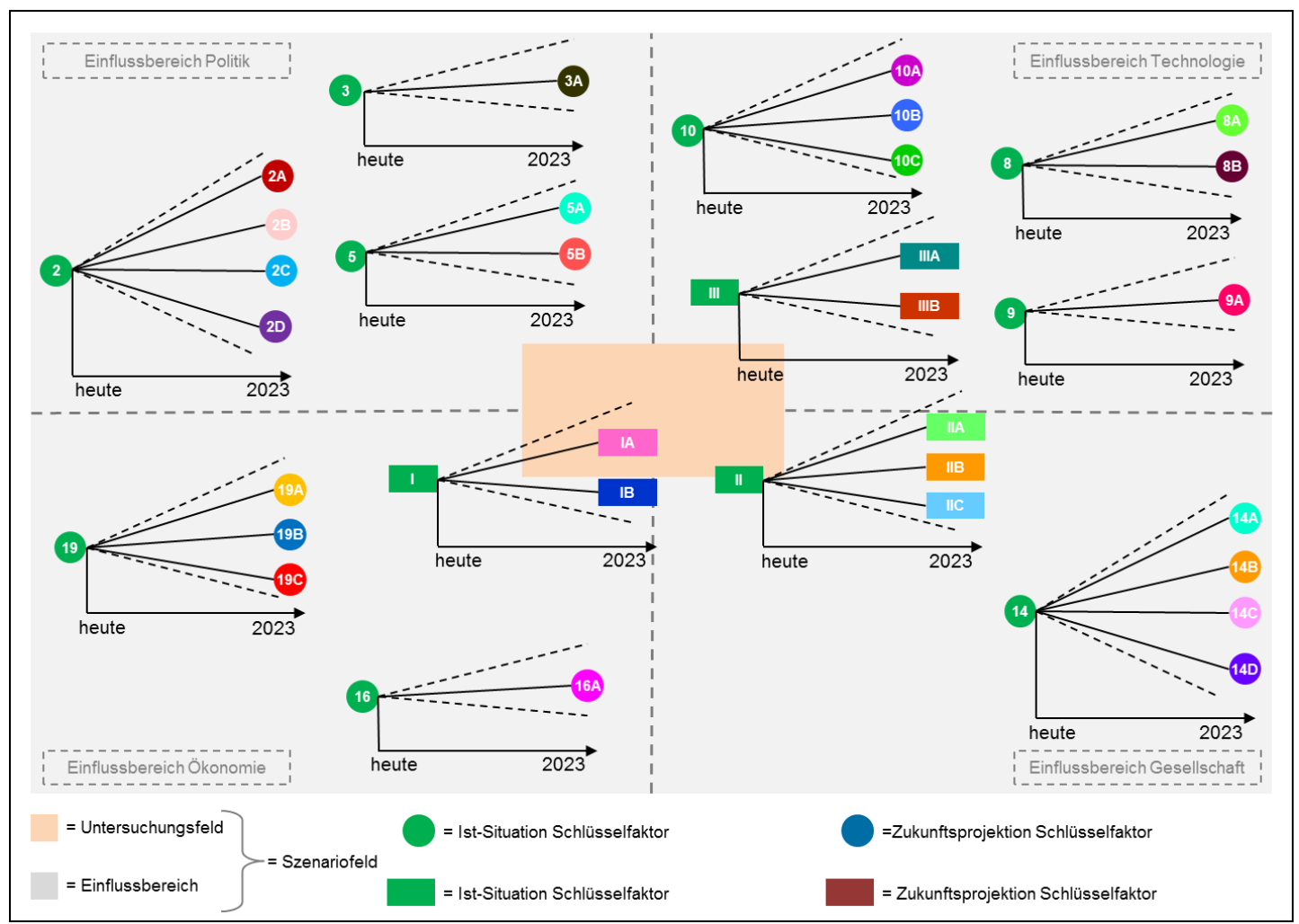

Abbildung 19: Darstellung des Szenariofeldes nach Durchführung der Szenario-Prognostik Quelle: EIGENE DARSTELLUNG

\subsection{Szenario-Bildung und -Konsistenzprüfung}

Der letzte Kernschritt der optimierten Methodik der induktiven Szenario-Technik unterteilt sich in die beiden Abschnitte (1) Szenario-Bildung und (2) -Konsistenzprüfung.

\section{Szenario-Bildung}

Ziel der Szenario-Bildung ist es, aus den in der Szenario-Prognostik entwickelten Zukunftsprojektionen konsistente Szenarien des Ethanolmarktes der EU27 und der USA zu bilden. Die Erstellung konsistenter Szenarien erfolgt im Gegensatz zu den etablierten Verfahren der Szenario-Technik ${ }^{42}$ nicht durch die Anwendung der Kon-

\footnotetext{
${ }^{42}$ Etablierte Verfahren der Szenario-Technik: GAUSEMEIER ET AL. 1995: 254ff.; GÖTZE 1993: 155ff.; FINK ET AL. 2006 : 43ff.; REIBNITZ 1992 : $49 \mathrm{ff}$.
} 
sistenzanalyse $^{43}$. Ersetzt wird das Instrument durch die morphologische Analyse, welche auf der Methode des morphologischen Kastens von Fritz Zwicky ${ }^{44}$ (KOSOW/GABNER 2008: 49) basiert.

Die Stärke der morphologischen Analyse ist die Fähigkeit, Informationen in komprimierter Form aufzunehmen und strukturiert darzustellen, um komplexe Probleme zu Iösen (SCHLICKSUPP 2004: 82; SCHLICKSUPP 1989: 84). Der Vorteil des Instruments ist seine Einfachheit in der Anwendung, durch die eine ausschließliche Konzentration auf das Problem möglich ist. Sowohl KOSOW und GABNER (2008: 49) als auch FINK und SIEBE (2006: 88) sind der Überzeugung, dass sich das Instrument der morphologischen Analyse dazu eignet, konsistente Szenarien zu erstellen. Ein weiterer Vorzug der morphologischen Analyse ist, dass sich das Instrument optimal in die bereits beschriebene optimierte Methodik der induktiven SzenarioTechnik integriert, indem es auf den Ergebnissen der Szenario-Prognostik aufbaut. Im Folgenden wird das Instrument der morphologischen Analyse erläutert, das in den Kapiteln 5.3.1 und 6.3.1 zur Anwendung kommen wird. Die Grundlage der morphologischen Analyse bildet die in Abbildung 20 dargestellte Matrix.

\footnotetext{
${ }^{43}$ Grund für die Entscheidung gegen die Konsistenzanalyse ist der hohe Zeitbedarf bei der Anwendung des Instruments. Erkannt wurde dieses Problem bei der Analyse des induktiven Ansatzes der Szenario-Technik, beschrieben in Kapitel 3.4.

${ }^{44}$ Der Schweizer Astrophysiker Fritz Zwicky ist der bedeutendste Morphologe der Gegenwart. Er entwickelte zahlreiche morphologische Methoden, die am weitesten verbreitete ist die "Methode des Morphologischen Kastens" (SCHLICKSUPP 2004: 77). Weitere Informationen zu den Arbeiten im Bereich der morphologischen Forschung sind der Veröffentlichung ZWICKY 1989 zu entnehmen.
} 


\begin{tabular}{|c|c|c|c|c|c|c|}
\hline & \multirow{2}{*}{\multicolumn{2}{|c|}{ Schlüsselfaktor }} & \multicolumn{4}{|c|}{ Zukunftsprojektionen 2023} \\
\hline & & & A & B & c & D \\
\hline \multirow{3}{*}{ Politik } & $\mathrm{SF}_{2}$ & Name & (2A & (28) & (2c) & (2D) \\
\hline & $\mathrm{SF}_{3}$ & Name & (3A) & & & \\
\hline & SF 5 & Name & (6A) & 68 & & \\
\hline \multirow{2}{*}{ Ökonomie } & SF 1 & Name & $16)^{-}$ & & & \\
\hline & $\mathrm{SF} 1$ & Name & (19A) & 198 & 190 & \\
\hline \multirow{3}{*}{ Technologie } & $\mathrm{SF} 8$ & Name & $8 A$ & $88^{-1}$ & & \\
\hline & $\mathrm{SF} 9$ & Name & $9 A^{-}$ & & & \\
\hline & $\mathrm{SF} 1$ & Name & (10A & 108 & 100 & \\
\hline Gesellschaft & SF 1 & Name & (14A) & -748 & (140) & (140 \\
\hline \multirow{4}{*}{ Untersuchungsfeld } & $\mathrm{SFI}$ & Name & IA & B & & \\
\hline & SFI & Name & IIA & 118 & Nic & \\
\hline & SF II & Name & IIIA & $\mathrm{MB}$ & & \\
\hline & & & & 1. Szenari & & \\
\hline
\end{tabular}

Abbildung 20: Matrix zur Durchführung der morphologischen Analyse

Quelle: In Anlehnung an SCHLICKSUPP 1989: 86; HENTZE ET AL. 1989: 108

In der Spalte „Schlüsselfaktor“ (Abbildung 20) befinden sich die im Kernschritt „Szenariofeld-Analyse“ (Kapitel 4.2) identifizierten beschreibenden und wirkenden Schlüsselfaktoren. Sie werden mittels ihrer laufenden Nummer (Lfd.-Nr.) und ihres Namens beschrieben. Die Reihenfolge der Anordnung der Schlüsselfaktoren erfolgt entsprechend ihrer Zugehörigkeit zum jeweiligen Einflussbereich ${ }^{45} /$ Untersuchungs- $^{-}$ feld. Im oberen Abschnitt der morphologischen Analyse befinden sich die wirkenden Schlüsselfaktoren der vier Einflussbereiche, erst zum Ende werden die beschreibenden Schlüsselfaktoren des Untersuchungsfeldes in die morphologische Analyse aufgenommen. Diese Anordnung ist vorteilhaft für die Durchführung der morphologischen Analyse, denn die Bestimmung der Zukunftsprojektionen der beschreibenden Schlüsselfaktoren des Untersuchungsfeldes ist erst nach der Auswahl aller Zukunftsprojektionen der wirkenden Schlüsselfaktoren möglich.

In die Felder der Matrix unter der Überschrift „Zukunftsprojektionen 2023“ werden alle Projektionen des zugehörigen Schlüsselfaktors eingetragen. Diese wurden im vorangegangenen Kernschritt „Szenario-Prognostik“ (Kapitel 4.3) gebildet. Die Summe aller eingetragenen Zukunftsprojektionen bildet das sogenannte „morphologische Feld“ (KOSOW/GABNER 2008: 49).

\footnotetext{
${ }^{45}$ Der Einflussbereich unterteilt sich, wie in Kernschritt "Szenariofeld-Analyse" beschrieben, in die Bereiche: Politik, Ökonomie, Technologie und Gesellschaft.
} 
Im Folgenden wird erläutert, wie aus der Kombination von Zukunftsprojektionen in sich konsistente Szenarien erstellt werden. Die Konsistenz eines Szenarios ist entscheidend für seine Glaubwürdigkeit und wird nur erreicht, wenn sich die einzelnen Zukunftsprojektionen des Szenarios nicht wechselseitig ausschließen bzw. wenn das gemeinsame Vorkommen der Zukunftsprojektionen in einem Szenario realistisch ist (GAUSEMEIER ET AL. 1995: 254; FINK ET AL. 2002: 88; AYE 2009: 243). Zur Erstellung der konsistenten Szenarien kommt die intuitive, nicht die systematische Vorgehensweise zur Anwendung ${ }^{46}$. Bei der Erstellung der Szenarien durch die intuitive Vorgehensweise wird für jeden Schlüsselfaktor eine Zukunftsprojektion aus dem „morphologischen Feld“ ausgewählt. Diese ausgewählten Schlüsselfaktoren werden über einen Linienzug miteinander verbunden (KOSOW/GAßNER 2008: 49), wie es beispielhaft in Abbildung 20 dargestellt ist. Bei der Auswahl der einzelnen Zukunftsprojektionen ist auf Widerspruchsfreiheit zwischen den einzelnen Projektionen zu achten, um am Ende konsistente und damit glaubwürdige Szenarien zu erhalten. Bei der Bildung der Szenarien ist deren Anzahl einzig und allein von der zu behandelnden Thematik abhängig. Hervorzuheben ist aber, dass sich alle Szenarien eindeutig voneinander abgrenzen müssen (REIBNITZ 1992: 52; GAUSEMEIER ET AL. 1995: 252ff.), einzelne Zukunftsprojektionen aber Bestandteil mehrerer Szenarien sein dürfen. Wichtig ist, dass alle erstellten Szenarien gleich gewichtet zu betrachten sind. Dies bedeutet, dass jedes Szenario mit gleicher Wahrscheinlichkeit eintreten kann.

In der folgenden Abbildung 21 ist beispielhaft das theoretische Ergebnis des Prozesses der Szenario-Bildung des „1. Szenarios“ dargestellt.

\footnotetext{
${ }^{46}$ Ausschlaggebend für die Nutzung der intuitiven Vorgehensweise ist, dass bei der systematischen Vorgehensweise alle denkbaren Zukunftsprojektionspaare auf ihr Konsistenzmaß bewertet werden müssen (RITCHEY 2011: 6). Diese Analyse erfolgt durch eine Matrix, die in Aufbau, Durchführung und Komplexität identisch mit der Konsistenzmatrix ist, welche in der Konsistenzanalyse zur Anwendung kommt (RITCHEY 2011: 7). Die Anwendung einer solchen komplexen und zeitaufwendigen Matrix gilt es aber schließlich durch das Verfahren der morphologischen Analyse zu verhindern, da eine Matrix in dieser Größe bereits in Kapitel 3.4 als nicht geeignet erkannt wurde.
} 


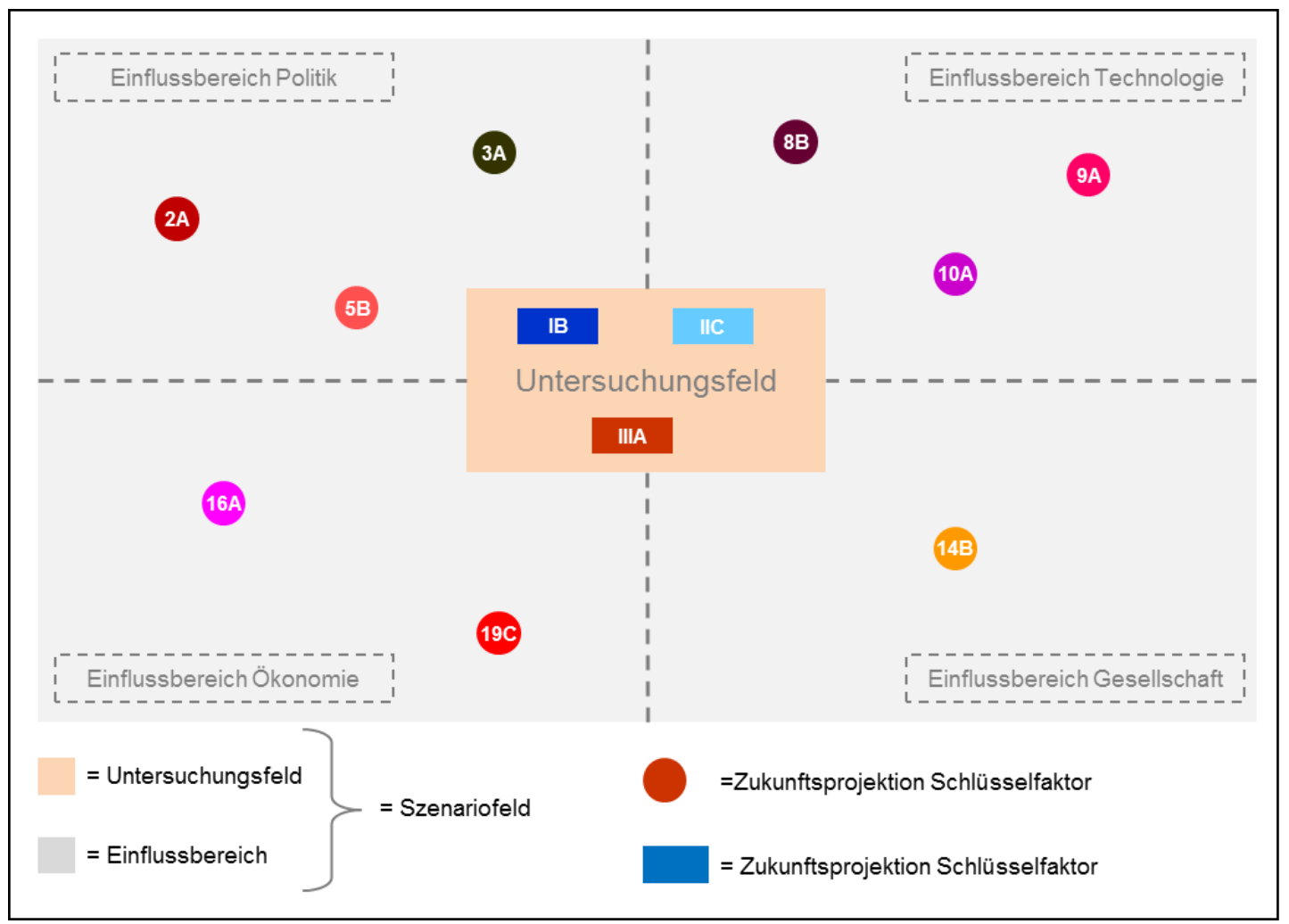

Abbildung 21: Das 1. Szenario, dargestellt als Szenariofeld Quelle: EIGENE DARSTELLUNG

Im Folgenden wird die Beschreibung der Szenarien erläutert. Ziel ist es, aus den Zukunftsprojektionen des Szenarios ein verständliches Zukunftsbild zu erzeugen, das die Experten des Marktes verstehen (GAUSEMEIER ET AL. 1995: 307ff.).

Bei der Beschreibung der Szenarien werden ausschließlich die Ergebnisse der Szenario-Bildung erläutert ${ }^{47}$. Zur Beschreibung der Szenarien schildern FINK ET AL. (2002: 88ff.) eine Vielzahl unterschiedlicher Verfahren. Diese Verfahren, wie beispielsweise das Storytelling, das Zeichnen einer Landkarte der Zukunft oder das Vorführen einer szenischen Darstellung, werden aufgrund des hohen Zeitbedarfs zur Durchführung und der fehlenden Genauigkeit in der Darstellung der Ergebnisse abgelehnt. Die Methode, die in den Kapiteln 5.3 und 6.3 zur Anwendung kommen wird, ist die formelle Beschreibung. Bei dieser Form gilt es, für die unterschiedlichen Szenarien als erstes eine prägnante Überschrift zu formulieren, die das Interesse an dem Inhalt der Szenarien weckt und die Richtung der Entwicklung des Ethanolmarktes aufzeigt (GAUSEMEIER ET AL. 1995: 312; FINK ET AL 2002: 94). Die genaue Beschreibung eines Szenarios erfolgt anhand der zugehörigen Zukunftsprojektionen, die es in einem Text zu verknüpfen gilt, um ein Zukunftsbild des Szenariofeldes im Kopf des Lesers zu erzeugen (GAUSEMEIER ET AL. 1995: 312).

\footnotetext{
${ }^{47}$ Auf keinen Fall werden die Ergebnisse kreativ erweitert, wie dies GAUSEMEIER ET AL. (1995: 312) empfehlen, damit sich die Leser besser in das Szenario hineinversetzen können.
} 


\section{Konsistenzprüfung}

Ziel der Konsistenzprüfung ist die Sicherstellung, dass alle Szenarien in sich widerspruchsfrei sind. Da die Anwendung der morphologischen Analyse zur SzenarioBildung für den Ethanolmarkt der EU27 und der USA durch den Autor der vorliegenden Studie erfolgt, ist ein solcher zusätzlicher Schritt wissenschaftlich notwendig. Zur Konsistenzprüfung wird ein Fragebogen entwickelt. In diesem wird den Experten der beiden Ethanolmärkte jedes Szenario einzeln erläutert und die Experten beurteilen mittels einer fünfstufigen Likert-Skala ${ }^{48}$ die Widerspruchsfreiheit jedes Szenarios. Darüber hinaus werden die befragten Experten gebeten, in einem Kommentarfeld mögliche Konsistenzprobleme innerhalb des Szenarios zu erläutern.

Szenarien werden als konsistent erachtet, wenn die Experten dem Szenario eine „sehr gute“ oder „gute“ Konsistenz attestieren und nicht mehr als zwei Experten ein identisches Konsistenzproblem in einem Szenario erkannt haben. Wird allerdings bei einem Szenario mit einer nach Auffassung der Experten „sehr guten“ oder „guten“ Konsistenz ein mögliches Konsistenzproblem von mehr als zwei Experten benannt, so wird dieses mögliche Problem in der Arbeit diskutiert, um daraufhin zu entscheiden, ob eine Veränderung des Szenarios notwendig ist. Ziel der Änderung ist es, ein widerspruchsfreies Szenario zu erhalten. Falls ein Szenario nach Einschätzung der befragten Experten lediglich eine „moderate“, „schlechte“ oder „sehr schlechte" Konsistenz besitzt, so wird dieses Szenario nicht weiter betrachtet oder wird mittels der abgegebenen Kommentare der befragten Experten analysiert, mit dem Ziel, die einzelnen Konsistenzprobleme zu beheben.

Mit Abschluss der Konsistenzprüfung ist sichergestellt, dass alle im weiteren Verlauf der Studie betrachteten Szenarien in sich widerspruchsfrei sind.

Im folgenden Kapitel 5 wird die optimierte induktive Szenario-Technik zur Erstellung von Szenarien für den Ethanolmarkt der EU27 im Jahr 2023 genutzt.

\footnotetext{
${ }^{48}$ Die fünfstufige Likert-Skala unterteilt sich in die Bereiche: „sehr gut“, „gut“, „moderat“,
} "schlecht" und "sehr schlecht". 
Ziel dieses Kapitels ist es, sowohl die Faktoren zu identifizieren, die den Ethanolmarkt bis zum Jahr 2023 entscheidend beeinflussen werden, als auch die Szenarien ${ }^{49}$ zu bilden, die den Ethanolmarkt im Jahr 2023 beschreiben. Die Erreichung beider Ziele erfolgt mittels der drei Kernschritte „EU27 Szenariofeld-Analyse“ (Kapitel 5.1), „EU27 Szenario-Prognostik“ (Kapitel 5.2) sowie „EU27 Szenario-Bildung und -Konsistenzprüfung" (Kapitel 5.3). Anschließend werden die Ergebnisse der Untersuchung in Kapitel 5.4 betrachtet. Die einzelnen Teilschritte der Untersuchung sind in Abbildung 22 grafisch dargestellt.

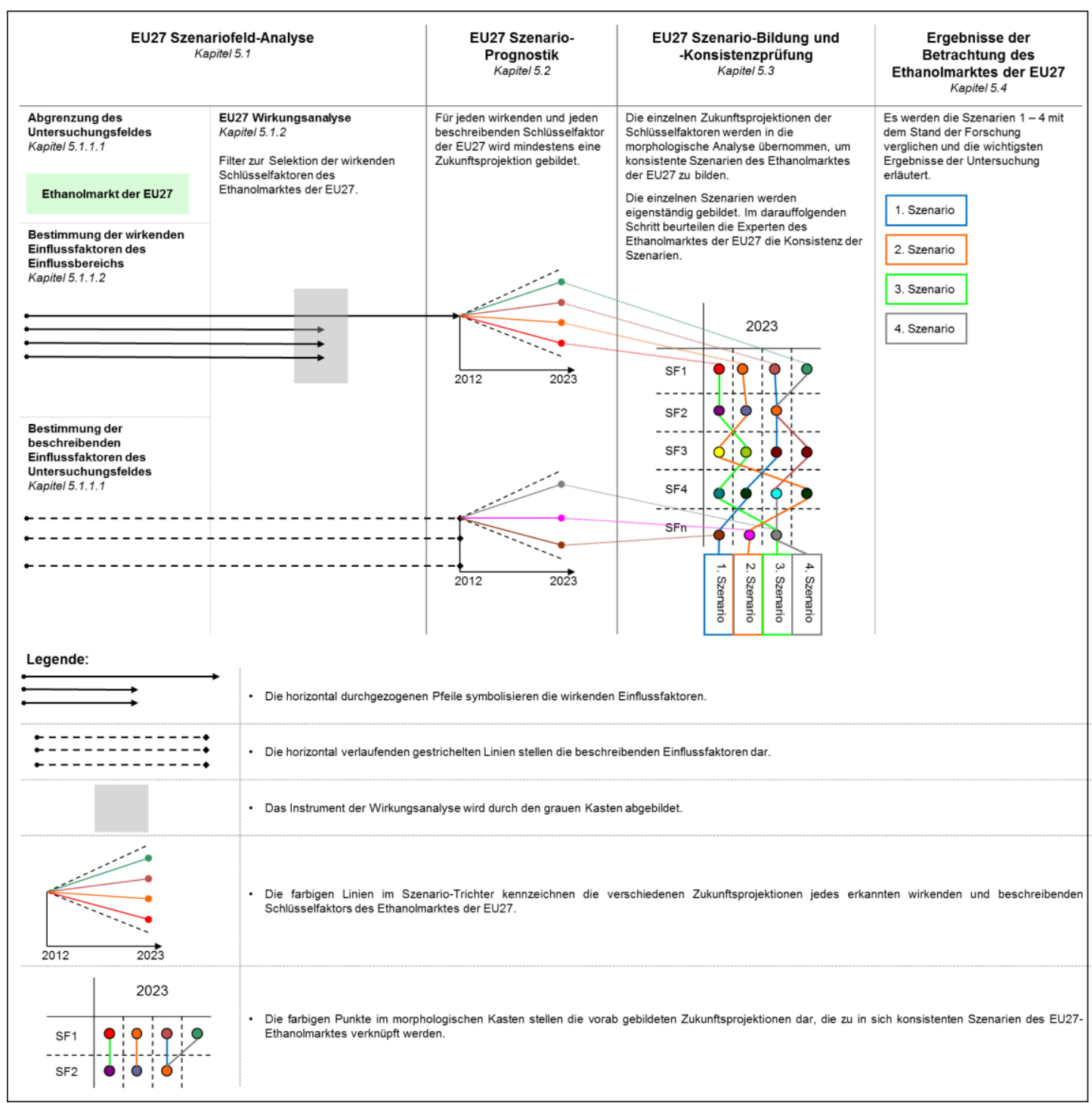

Abbildung 22: Schematische Darstellung - Vorgehen zur Bildung der Szenarien des Ethanolmarktes der EU27 im Jahr 2023

Quelle: EIGENE DARSTELLUNG

${ }^{49}$ Die formelle Ausrichtung der Szenarien entspricht den Vorgaben für Marktentwicklungsszenarien, beschrieben in Kapitel 4.1. 


\subsection{EU27 Szenariofeld-Analyse}

In der „EU27 Szenariofeld-Analyse“ gilt es, die Faktoren zu identifizieren, die den Ethanolmarkt der EU27 bis zum Jahr 2023 entscheidend beeinflussen werden. Damit erfült dieser Kernschritt das erste Ziel der Dissertation ${ }^{50}$ und ermöglicht durch die Bestimmung der wirkenden und beschreibenden Schlüsselfaktoren die Durchführung der „EU27 Szenario-Prognostik“ in Kapitel 5.2. Um dies zu erreichen, bedarf es der folgenden beiden Schritte: (1) der Beschreibung des EU27-Szenariofeldes (Kapitel 5.1.1) und (2) der EU27-Wirkungsanalyse (Kapitel 5.1.2).

\subsubsection{Beschreibung des EU27-Szenariofeldes}

Das EU27-Szenariofeld besteht aus dem Untersuchungsfeld und dem Einflussbereich. Nach inhaltlicher und geographischer Abgrenzung des Untersuchungsfelds wird dieses anhand beschreibender Einflussfaktoren dargestellt (Kapitel 5.1.1.1). Die Beschreibung des Einflussbereichs erfolgt mittels wirkender Einflussfaktoren (Kapitel 5.1.1.2).

\subsubsection{Abgrenzung des Untersuchungsfeldes und Bestimmung der be- schreibenden Einflussfaktoren}

Das Untersuchungsfeld befasst sich ausschließlich mit dem Ethanol-Kraftstoffmarkt in der EU27. Dies impliziert, dass die Verwendung von Ethanol in der chemischen Industrie und in der Nahrungsmittelindustrie nicht betrachtet wird. Ebenso ist auch die zukünftige Entwicklung von anderen Biokraftstoffen als Ethanol, wie beispielsweise Biodiesel, kein Bestandteil des Untersuchungsfeldes. Des Weiteren ist im Untersuchungsfeld der Ethanolmarkt außerhalb der EU27 nicht mit inbegriffen. Die historische Entwicklung und der Ist-Zustand des Untersuchungsfeldes „Ethanolmarkt der EU27“ sind ausführlich im Kapitel 2.1 erläutert.

Zur Darstellung der Entwicklung des Untersuchungsfeldes in den unterschiedlichen Szenarien werden die folgenden vier beschreibenden Einflussfaktoren genutzt: Ethanolnachfrage, Ethanolproduktion, Ethanolhandel und Ethanolpreis ${ }^{51}$. Die Berücksichtigung dieser Faktoren ermöglicht einen Vergleich mit dem referierten Stand

\footnotetext{
${ }^{50}$ Das erste Ziel der Dissertation ist es, die Faktoren zu identifizieren, die den Ethanolmarkt der EU27 bis zum Jahr 2023 entscheidend beeinflussen werden.

${ }^{51}$ Die Auswahl der Faktoren wurde persönlich mit den folgenden fünf Experten diskutiert: Dr. Christoph Berg - F.O. Licht, Dr. Marco Veselka - CropEnergies AG, Prof. Dr.-Ing. Achim Loewen - Hochschule für angewandte Wissenschaft und Kunst in Göttingen, Dr. Hinrich Harling und Dr. Andreas von Felde - KWS SAAT AG.
} 
der Forschung in Kapitel 2.3. Jeder beschreibende Einflussfaktor wird mittels der drei Punkte „Name“, „Lfd. Nr.“ und „Beschreibung“ dargestellt. Der Punkt „Beschreibung" umfasst eine Erläuterung des Einflussfaktors und gibt an, wie die zukünftige Entwicklung des Einflussfaktors gemessen wird.

\begin{tabular}{|c|c|c|}
\hline Name: & Ethanolnachfrage & \\
\hline Lfd. Nr.: & 1 & \\
\hline Beschreibung: & \multicolumn{2}{|c|}{$\begin{array}{l}\text { Der Einflussfaktor misst die Ethanolnachfrage für den Kraftstoffbereich in der } \\
\text { EU27. Die nachgefragte Menge wird gemessen in Milliarden Liter pro Jahr. }\end{array}$} \\
\hline Name: & Ethanolproduktion & \\
\hline Lfd. Nr.: & II & \\
\hline Beschreibung: & \multicolumn{2}{|c|}{$\begin{array}{l}\text { Der Einflussfaktor erfasst die Ethanolproduktion für den Kraftstoffbereich in der } \\
\text { EU27. Die Produktionsmenge wird gemessen in Milliarden Liter pro Jahr. }\end{array}$} \\
\hline Name: & Ethanolhandel & \\
\hline Lfd. Nr.: & III & \\
\hline Beschreibung: & \multicolumn{2}{|c|}{$\begin{array}{l}\text { Der Einflussfaktor betrachtet den Ethanolhandel der EU27 mit dem Rest der } \\
\text { Welt. Die Werte werden in der Einheit Milliarden Liter pro Jahr angegeben. }\end{array}$} \\
\hline Name: & Ethanolpreis & \\
\hline Lfd. Nr.: & IV & \\
\hline Beschreibung: & \multicolumn{2}{|c|}{$\begin{array}{l}\text { Der Einflussfaktor erfasst die Entwicklung des Ethanolpreises. Der Preis basiert } \\
\text { auf dem Ethanolweltmarktpreis; dies ist der Preis für wasserfreies Ethanol in } \\
\text { São Paulo, Brasilien. Die Preisentwicklung wird real (Basisjahr 2005) in der } \\
\text { Einheit US-\$ je Hektoliter beschrieben. }\end{array}$} \\
\hline
\end{tabular}

\subsubsection{Bestimmung der wirkenden Einflussfaktoren}

Kennzeichnend für die wirkenden Einflussfaktoren ist ihr Einfluss auf das Untersuchungsfeld „Ethanolmarkt der EU27“. Die wirkenden Einflussfaktoren beschreiben die vier Bereiche des Einflussbereichs, nämlich Ökonomie, Technologie, Politik und Gesellschaft. Entsprechend dieser Unterteilung sind auch die wirkenden Einflussfaktoren im Folgenden gegliedert. Die Auswahl der Faktoren basiert auf intensiver Literaturrecherche unter Beachtung der genannten Einflussfaktoren, die in den Studien 
zum Stand der Forschung referiert wurden (Anhang: 1) und fünf persönlich geführten Expertengesprächen ${ }^{52}$. Jeder wirkende Einflussfaktor wird anhand der folgenden fünf Punkte beschrieben „Name“, „Lfd. Nr.“, „Einflussbereich“, „Beschreibung“ und „Bedeutung des Einflussfaktors“. Der letzte Punkt „Bedeutung des Faktors“ wird genutzt, um zu erläutern, wie sich der jeweilig wirkende Einflussfaktor auf das Untersuchungsfeld auswirkt, wodurch sich die Notwendigkeit der Betrachtung dieses Einflussfaktors erklärt.

\section{Ökonomie}

Name:

Lfd. Nr.:

Einflussbereich:

Beschreibung:

Bedeutung des

Einflussfaktors:
Benzinnachfrage in der EU27

1

Ökonomie

Die Benzinnachfrage in der EU27 wird mittels der Einheit Milliarden Liter pro Jahr beschrieben.

Über 95\% des Ethanolabsatzes in der EU27 erfolgt als Beimischung zu Benzin (WOOD MACKENZIE 2010: 64). Dementsprechend entscheiden die Benzinnachfrage in der EU27 und die erlaubte Beimischungshöhe von Ethanol zu Benzin maßgeblich über die Marktgröße des europäischen Ethanolmarktes.

Eine Abnahme der Benzinnachfrage, wie sie von mehreren Forschungseinrichtungen - EUROPEAN COMMISSION JOINT RESEARCH CENTRE, INSTITUT FOR ENERGY ET AL. (2011: 24), OPEC (2011: 169) und WOOD MACKENZIE (2010: 40) - prognostiziert wird, senkt folglich die zukünftige potenzielle Ethanolnachfrage in der EU27.

52 Es wurden diesbezüglich persönliche Gespräche mit den folgenden Experten geführt : Dr. Christoph Berg - F.O. Licht; Dr. Marco Veselka - CropEnergies AG; Prof. Dr.-Ing. Achim Loewen - Hochschule für angewandte Wissenschaft und Kunst; Dr. Hinrich Harling und Dr. Andreas von Felde - KWS SAAT AG. 


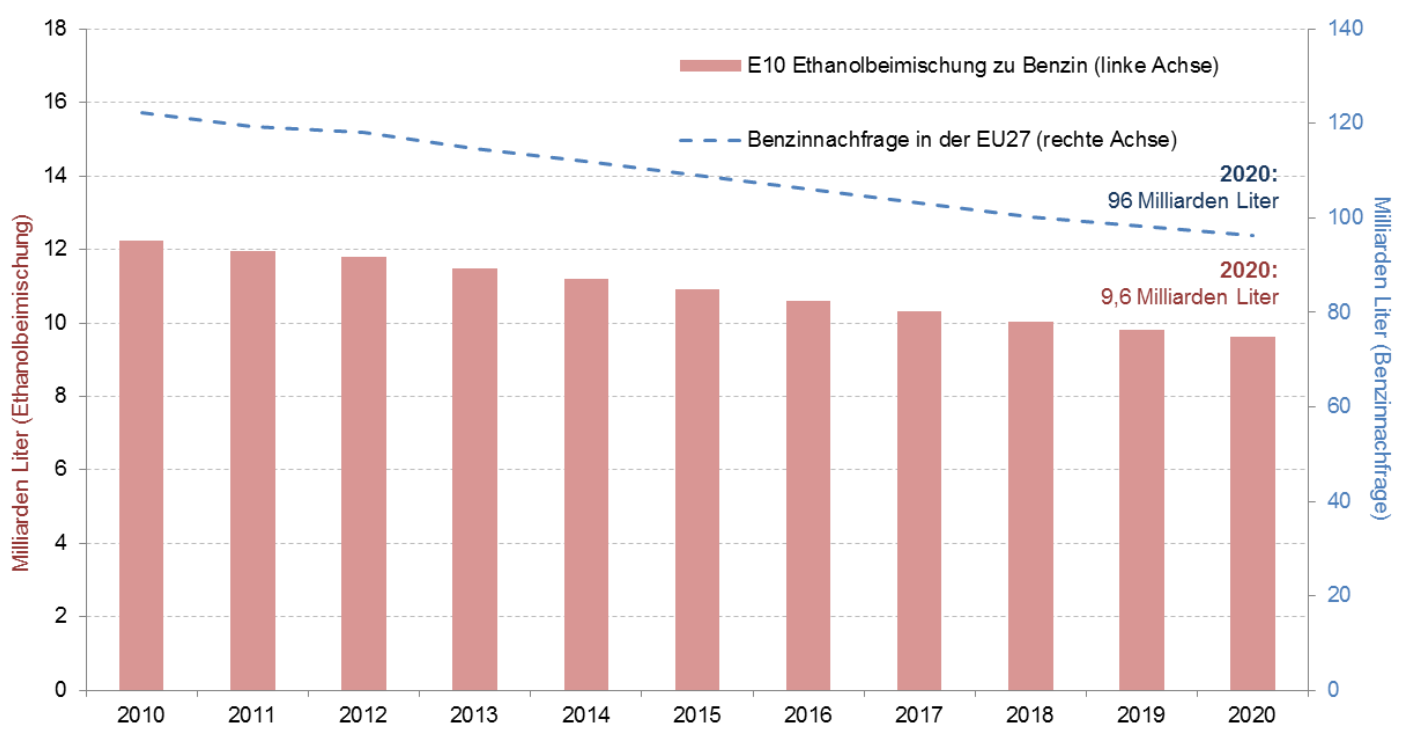

Abbildung 23: Einfluss der Benzinnachfrage auf die Ethanolnachfrage in der EU27

Quelle: In Anlehnung an EUROPEAN COMMISSION JOINT RESEARCH CENTRE, INSTITUT FOR ENERGY ET AL. 2011: 24; RICHTLINIE 2009/30/EG ANHANG I; EIGENE BERECHNUNG

In Abbildung 23 sind die prognostizierte Entwicklung der Benzinnachfrage und die daraus abgeleitete Gesamtgröße des Ethanolmarktes dargestellt. Basierend auf der Annahme, dass die erlaubte Beimischung von Ethanol zu Benzin weiterhin $10 \%$ vol beträgt (RICHTLINIE 2009/30/EG ANHANG I), ergibt sich für das Jahr 2020 eine maximale Ethanolnachfrage von rund 9,6 Milliarden Litern. Ein weiterer Rückgang der Benzinnachfrage bis zum Jahr 2023 würde den europäischen Ethanolmarkt weiter schrumpfen lassen.

Name:

Lfd. Nr.:

Einflussbereich:

Beschreibung:

Bedeutung des Einflussfaktors:
Rohölpreis

2

Ökonomie

Die Preisentwicklung von Rohöl wird in realen Werten (Basisjahr 2010) in der Einheit US-\$ je Barrel (158,987 Liter) angegeben und anhand der Rohölsorte „West Texas Intermediate“ (WTI) dargestellt.

Derzeit ist der Rohölpreis für die Entwicklung des Ethanolmarktes der EU27 von sekundärer Bedeutung, da Ethanol von der Mineralölindustrie nur eingesetzt wird, um die politische Vorgabe der Beimischung von „erneuerbaren Energien" zum Gesamtenergieverbrauch im Verkehrssektor zu erfüllen (RICHTLINIE 2009/28/EG ARTIKEL 3 (4)) ${ }^{53}$. Trotz der politischen Vorgaben sind es oft die Gesetze des Marktes, die am Ende entscheiden, da diese unab-

${ }^{53}$ In Text zu Einflussfaktor 18a wird ausführlich auf die Bedeutung der politischen Vorgabe der Beimischung von erneuerbaren Energien zum Gesamtenergieverbrauch im Verkehrsbereich eingegangen. 
hängig von Wahlausgängen und politischen Zielen dauerhaft von Bestand sind (HENNIGES 2007: 243). Ohne den Einfluss der Politik ist Ethanol ein Substitut für fossiles Benzin und konkurriert mit diesem auf dem Kraftstoffmarkt der EU27 (EUROPEAN COMMISSION JOINT RESEARCH CENTRE, INSTITUTE FOR PROSPECTIVE TECHNOLOGICAL STUDIES 2010a: 87; F.O. LICHT/AGRA INFORMA 2009: 21; HENNIGES 2007: 36). Die Höhe des Benzinpreises korreliert stark mit dem Rohölpreis (IER ET AL. 2010: 54). Folglich entscheidet der Rohölpreis über die Höhe des Benzinpreises und damit über die Konkurrenzfähigkeit von Ethanol zu Benzin.

Der Rohölpreis ist des Weiteren von Bedeutung, da er bei den Bürgern einen Einfluss auf die Akzeptanz der politischen Förderung von „erneuerbaren Energien“ im Verkehrssektor hat. So rechtfertigt ein ansteigender Rohölpreis die politische Förderung von Biokraftstoffen, um die Abhängigkeit der EU27 von Importen fossiler Kraftstoffe zu senken ${ }^{54}$. Auf der anderen Seite könnte ein fallender Rohölpreis bei den Bürgern für Unmut sorgen und die Politik in Bedrängnis bringen, da sich durch die politisch verordnete Beimischung von biogenen Treibstoffen zu fossilen Kraftstoffen der Preis für Benzin und Diesel an der Zapfsäule erhöht (HENNIGES 2007: 241).

\begin{tabular}{|c|c|}
\hline Name: & Ethanolimporte aus Brasilien in die EU27 \\
\hline Lfd. Nr.: & 3 \\
\hline Einflussbereich: & Ökonomie \\
\hline Beschreibung: & $\begin{array}{l}\text { Die Ethanolimporte aus Brasilien in die EU27 werden in der Einheit Milliarden } \\
\text { Liter pro Jahr erfasst }^{55} \text {. }\end{array}$ \\
\hline $\begin{array}{l}\text { Bedeutung des } \\
\text { Einflussfaktors: }\end{array}$ & $\begin{array}{l}\text { Im Jahr } 2011 \text { ist Brasilien nach den USA mit einer Ethanolproduktion von } 21 \\
\text { Milliarden Litern (F.O. LICHT 2012f: 74) und einem Ethanolexport von } 2 \text { Milliar- } \\
\text { den Litern der zweitgrößte Ethanolmarkt der Welt (F.O. LICHT 2012a: 304). Im } \\
\text { Jahr } 2011 \text { wurden } 0,3 \text { Milliarden Liter Ethanol in die EU27 exportiert } \\
\text { (THOMASSON 2011: } 11 \text { f.). Die Entwicklung des brasilianischen Ethanolexports } \\
\text { für den Zeitraum von } 2007 \text { bis } 2011 \text { ist in der nachfolgenden Abbildung } 24 \text { dar- } \\
\text { gestellt. Beachtlich ist das hohe Potenzial Brasiliens, das sich darin zeigt, dass } \\
\text { bereits im Jahr } 2008 \text { über } 5 \text { Milliarden Liter Ethanol auf den Weltmarkt expor- } \\
\text { tiert wurden. }\end{array}$ \\
\hline
\end{tabular}

${ }^{54}$ Die Stärkung der Energieunabhängigkeit der EU27 ist eines der wichtigsten politischen Argumente für die Förderung von erneuerbaren Energien im Verkehrsbereich (RICHTLINIE 2009/28/EG ERW (1)).

${ }^{55}$ Die Datenerfassung zum weltweiten Ethanolhandel hat sich in den letzten Jahren deutlich erschwert, da Ethanol in den meisten Fällen nicht mehr in Reinform, sondern in unterschiedlicher Beimischung zu Benzin und als Ethyl-Tertiär-Butylether (ETBE) gehandelt wird (F.O. LICHT 2012a: 300). 


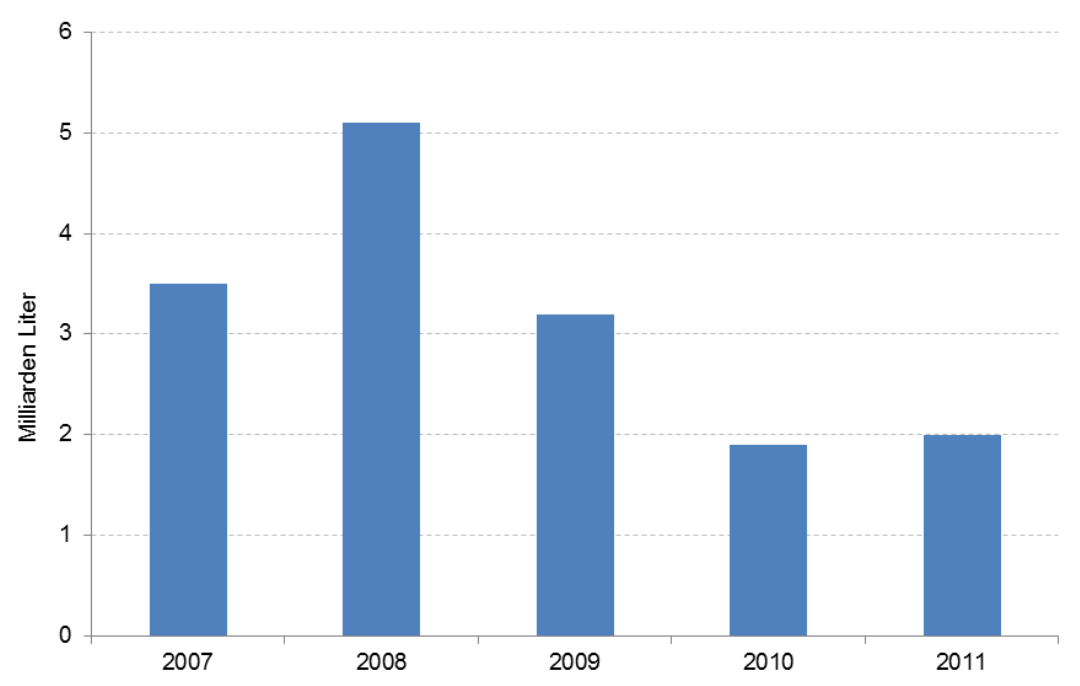

Abbildung 24: Entwicklung der brasilianischen Ethanolexporte Quelle: In Anlehnung an F.O. LICHT 2012a: 304

Der brasilianische Exportmarkt profitiert momentan und voraussichtlich auch in Zukunft von seinen im Verhältnis zum Ethanolmarkt der EU27 geringeren Produktionskosten. Im Durchschnitt der Jahre 2010 bis 2012 betrugen diese in Brasilien um die 55 US-\$ je Hektoliter, wohingegen die Herstellungskosten in der EU27 um durchschnittlich 20 US-\$ je Hektoliter höher lagen (BERG 2012d: 2). Von großer Bedeutung, insbesondere hinsichtlich des Kernziels der Kohlenstoffdioxidreduktion in der EU27, sind die hohen $\mathrm{CO}_{2}$-Einsparungen von Zuckerrohr-Ethanol aus Brasilien im Vergleich zu fossilem Kraftstoff (IFEU 2004: 23; F.O. LICHT/AGRA INFORMA 2009:56). Entsprechend den offiziellen Zahlen der EU27 in der RICHTLINIE 2009/28/EC (ANHANG V) kommt Ethanol aus Zuckerrohr auf eine $\mathrm{CO}_{2}$-Einsparung von $71 \% \mathrm{im}$ Vergleich $\mathrm{zu}$ fossilem Kraftstoff, womit Zuckerrohr-Ethanol die höchste $\mathrm{CO}_{2}$-Einsparung aller biogenen Kraftstoffe der ersten Generation erreicht und alle derzeitigen und zukünftigen politischen Vorgaben der $\mathrm{CO}_{2}$-Reduktion, festgelegt in der RICHTLINIE 2009/28/EC ARTIKEL 17 (2) ${ }^{56}$, erfüllt.

Darüber hinaus besitzt Brasilien ein enormes Flächenpotenzial, um die Produktion und den Export von Zuckerrohr-Ethanol weiter auszubauen (F.O. LICHT/AGRA INFORMA 2009: 168; INTERNATIONAL ENERGY AGENCY BIOENERGY 2007: 17). Dieser Ausbau wird durch politische Investitionsprogramme gefördert (INDUSTRY WEEK 2012:1), erfolgte aber in

${ }^{56}$ In der RICHTLINIE 2009/28/EC ARTIKEL 17 (2) sind die politisch geforderten $\mathrm{CO}_{2}$ Reduktionswerte für Biokraftstoffe im Vergleich zu fossilem Kraftstoff festgelegt: Bis zum Jahr 2017 müssen alle Biokraftstoffe eine Kohlenstoffdioxid-Einsparung von 35\% erreichen. Ab dem 1. Januar 2017 erhöht sich die geforderte Reduktion auf $50 \%$ und ab dem 1. Januar 2018 müssen alle Produktionsanlagen, die am oder nach dem 1. Januar 2017 die Produktion aufgenommen haben, eine $\mathrm{CO}_{2}$-Minderung von $60 \%$ erreichen. 
den letzten Jahren trotz des Einstiegs global agierender Mineralölkonzerne wie z.B. Royal Dutch Shell plc. in den brasilianischen Ethanolmarkt ${ }^{57}$ deutlich langsamer als ursprünglich gedacht (F.O. LICHT 2013d: 294f.).

Obwohl der Ausbau der Produktionskapazitäten derzeit eher langsam erfolgt, ist die OECD/FAO (2012: 99) der Auffassung, dass in Zukunft Brasilien den Weltmarkt mit Zuckerrohr-Ethanol beliefern wird, da es die ökonomischen und landwirtschaftlichen Voraussetzungen dafür besitzt.

Name:

Lfd. Nr.:

Einflussbereich:

Beschreibung:

Bedeutung des Einflussfaktors:
Ethanolimporte aus den USA in die EU27

4

Ökonomie

Die Ethanolimporte aus den USA in die EU27 werden in der Einheit Milliarden Liter pro Jahr erfasst.

Die USA sind nicht nur der größte Ethanolproduzent der Welt (F.O. LICHT 2012f: 73f.), sondern auch der weltgrößte Ethanolexporteur mit 4,5 Milliarden Litern im Jahr 2011 (F.O. LICHT 2012a: 304). In der folgenden Abbildung 25 ist die Entwicklung der US-amerikanischen Ethanolexporte in den Jahren von 2007 bis 2011 dargestellt.

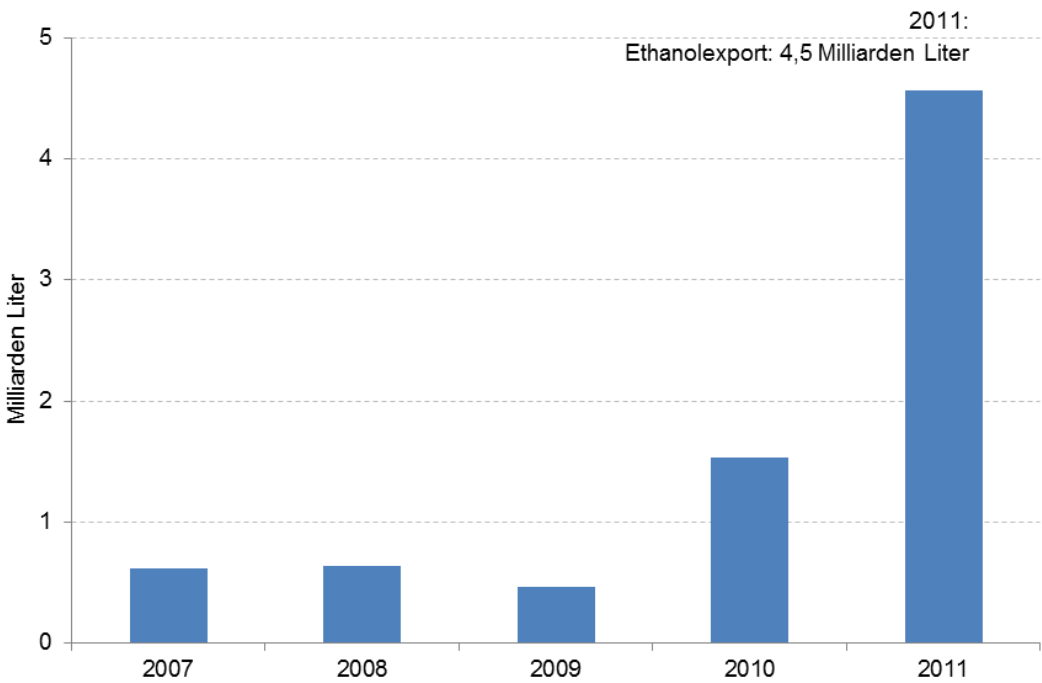

Abbildung 25: Entwicklung der US-amerikanischen Ethanolexporte Quelle: In Anlehnung an F.O. LICHT 2012a: 304

Einer der wichtigsten Exportmärkte für die USA ist die EU27. Im Jahr 2011 haben die USA eine Milliarde Liter in die EU27 exportiert; den größten Anteil davon als E90 (90\% Ethanol und 10\% Benzin), um einen geringeren Importzoll

${ }^{57}$ Die Unternehmen Royal Dutch Shell plc. und der brasilianische Zucker- und Ethanolproduzent Cosan Ltd. gründeten 2011 das Joint Venture Raízen, das mit einer Ethanolproduktion von jährlich 2 Milliarden Litern der größte Ethanolproduzent in Brasilien ist (BEVILL 2011a; SHELL 2013). 
zu entrichten. Darüber hinaus sind die US-amerikanischen Produktionskosten für Ethanol im Vergleich zu den Herstellungskosten in der EU27 deutlich geringer. Die somit günstigen Importe setzten den europäischen Ethanolpreis stark unter Druck und zwangen etliche Ethanolproduzenten, die Produktion aufgrund fehlender Profitabilität auszusetzen (F.O. LICHT 2011b: 66; USDA FOREIGN AGRICULTURAL SERVICE 2011: 19).

Der Exportmarkt ist für die USA auch in Zukunft von Interesse, da der heimische Markt aufgrund der „Blend Wall“-Problematik gesättigt ist. Solange die „Blend Wall“ besteht, ist der Exportmarkt für die USA eine der wenigen Möglichkeiten, ein zusätzliches Nachfragewachstum zu generieren, um die Überproduktion auf dem Weltmarkt abzusetzen und einen Wertverfall des Ethanols in den USA zu verhindern (Kapitel 2.2) (F.O. LICHT 2012a: 302; JESSEN 2012: 34). Darüber hinaus besitzen die USA die notwendige Infrastruktur im Inland und an den Häfen, um noch größere Ethanolmengen in die EU27 zu exportieren (THOMASSON 2011: 16).

Die USA betonen zwar, dass sie Ethanol für den US-amerikanischen Markt und nicht für den Weltmarkt produzieren wollen (F.O. LICHT 2012a: 301), dennoch hat das Jahr 2011 beispielhaft gezeigt, wie stark die negativen Auswirkungen für die europäischen Ethanolproduzenten sind, wenn sich die USA entschließen, eine für ihre Verhältnisse nur geringe Ethanolmenge in die EU27 zu exportieren.

Name:

Ethanolimporte in die EU27 aus Ländern mit zollfreiem Marktzugang

Lfd. Nr.: 5

Einflussbereich:

Ökonomie

Beschreibung:

Die Ethanolimporte aus Ländern mit zollfreiem Marktzugang in die EU27 werden in der Einheit Milliarden Liter pro Jahr erfasst.

Bedeutung des Ethanolexportierende Länder mit freiem Marktzugang zum Ethanolmarkt der

Einflussfaktors: EU27 müssen keinen Einfuhrzoll in Höhe von 19,2 €/hl (KN-Code 2207 10) für unvergälltes Ethanol oder 10,2 €/hl (KN-Code 220720$)$ für vergälltes Ethanol entrichten (EUROPÄISCHE KOMMISSION 2011: 182).

Freien Marktzugang in die EU27 besitzen die Staaten, die unter eines der drei folgenden Abkommen fallen (USDA FOREIGN AGRICULTURAL SERVICE 2012: 16; F.O. LICHT/AGRA INFORMA 2009: 150ff.):

1. Das „Cotonou Agreement“ für Staaten aus Afrika, der Karibik und dem pazifischen Raum mit Ausnahme von Südafrika

2. Die „Everything But Arms (EBA)“-Initiative, dem die am niedrigsten entwickel- 


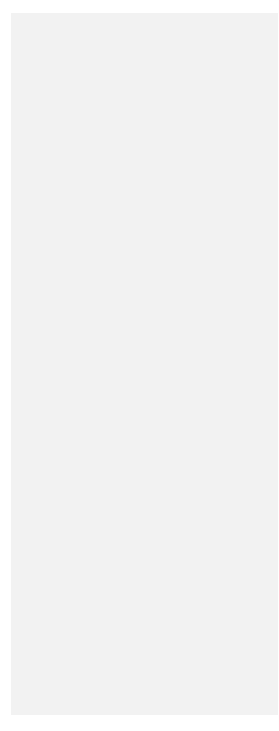

ten Staaten angehören

3. Das „Generalized System of Preferences Plus“ (GSP)

Die Staaten, die unter eines der drei genannten Abkommen fallen, sind von Bedeutung für die EU27, da sich deren Wettbewerbsposition durch den zollfreien Zutritt zum Ethanolmarkt der EU27 sowohl gegenüber den Ethanolproduzenten der EU27 als auch gegenüber den Ethanolexporteuren aus den USA und Brasilien, die den vollen Importzollsatz zu entrichten haben, verbessert. Trotz des genannten Vorteils ist der momentane Ethanolexport dieser Staaten in die EU27 gering (F.O. LICHT 2012a: 301ff.). Im Vergleich zu den anderen Ländern wird den Staaten Mittel- und Südamerikas zukünftig das größte Potenzial eingeräumt, vom freien Marktzugang in die EU27 durch die Produktion von Zuckerrohr-Ethanol zu profitieren (F.O. LICHT/AGRA INFORMA 2009: 188).

Name:

Lfd. Nr.:

Einflussbereich:

Beschreibung:

Bedeutung des Einflussfaktors:
Der Markt für Nebenprodukte der Ethanolherstellung

6

Ökonomie

Bei der Ethanolproduktion fallen für die Tierernährung verwendbare Nebenprodukte an, wobei die Art der Produkte abhängig vom verwendeten Rohstoff ist. Wird Weizen als Rohstoff genutzt, entsteht getrocknete Getreideschlempe als Nebenprodukt, welches auch als DDGS (Distillers‘ Dried Grains with Solubles) bezeichnet wird. Bei der Verwertung der Zuckerrübe fallen die Nebenprodukte Zuckerrübenschnitzel und Vinasse an (HENNIGES 2007: 53f.; IFEU 2008: 11; KASTENHUBER 2007: 67).

Die Entwicklung des Faktors wird mittels der Marktpreise der Nebenprodukte in der Einheit Euro je Tonne beschrieben.

Der Verkauf von Nebenprodukten als Eiweißfutter für die Tierernährung ist für die Ethanolproduzenten der EU27 von Bedeutung, da sie hierdurch einen wesentlichen Teil ihres Umsatzes generieren (HENNIGES 2007: 56; KASTENHUBER 2007: 67). So erzielten die Ethanolproduzenten in den USA im Zeitraum von Oktober 2011 bis Juni 2013 durchschnittlich 23\% ihres Umsatzes durch den Verkauf von DDGS (AGRICULTURAL MARKETING RESOURCE CENTER 2013). Da DDGS ein international gehandeltes Produkt ist, ist ein ähnlicher Wert für die EU27 zu erwarten.

Durch den Verkauf der Nebenprodukte sinkt die Abhängigkeit der Ethanolproduzenten vom Ethanolpreis; dies bietet innen die Möglichkeit, auch in Zeiten niedriger Ethanolpreise wirtschaftlich zu produzieren. Insbesondere die

${ }^{58}$ Die folgenden Staaten gehören dem GSP an: Bolivien, Kolumbien, Costa Rica, Ecuador, Guatemala, Honduras, Panama, Peru, El Salvador, Venezuela, Georgien, Sri Lanka, Mongolei und Moldawien (USDA FOREIGN AGRICULTURAL SERVICE 2012: 16). 
hohe Korrelation zwischen den Rohstoffkosten und dem Preis für Nebenprodukte mildert das Risiko hoher Rohstoffkosten ${ }^{59}$ für die Ethanolproduzenten (CROPENERGIES AG 2013a: 30).

Die Bedeutung der Nebenprodukte ist beispielsweise an der CropEnergies AG dem zweitgrößten Ethanolproduzenten der EU27 ${ }^{60}$ - zu erkennen. Das Unternehmen hat gezielt die Marken ProtiGrain und ProtiWanze aufgebaut, um die Nebenprodukte der Ethanolproduktion zu vermarkten (CROPENERGIES AG 2013a: 30).

Name:

Lfd. Nr.:

Einflussbereich:

Beschreibung:

Bedeutung des

Einflussfaktors:
Nachfrage nach ETBE

7

Ökonomie

ETBE (Ethyl-Tertiär-Butyl-Ether) ist ein Ether, der Ottokraftstoffen beigemischt wird und der Erhöhung der Oktanzahl und damit der Verbesserung der Klopffestigkeit des Kraftstoffs dient. ETBE besteht zu 45\% aus Ethanol und zu 55\% aus Iso-Buten (F.O. LICHT 2011: 148f.; EFOA 2013; HENNIGES 2007: 190f.). Die Entwicklung der Nachfrage nach ETBE wird in der Einheit Milliarden Liter pro Jahr beschrieben.

Durch den hohen Anteil von Ethanol in ETBE steigert die Nachfrage nach ETBE den Absatz von Ethanol in der EU27 (HENNIGES 2007: 190f.). ETBE kann Benzin bis zu $15 \%$ vol beigemischt werden, was einem Ethanolanteil von $6,7 \%$ vol entspricht (F.O. LICHT 2011: 148). Die folgende Abbildung 26 zeigt die durch die Beimischung von ETBE zu Benzin in der EU27 abgesetzte Menge Ethanol.

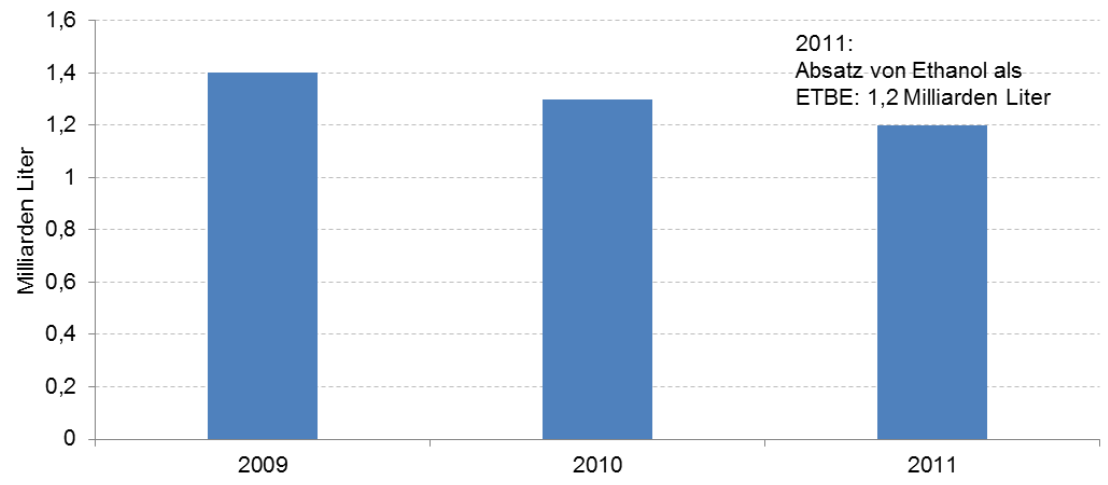

Abbildung 26: Absatz von Ethanol in Form von ETBE in der EU27 Quelle: In Anlehnung an F.O. LICHT 2011c: 149.

\footnotetext{
${ }^{59}$ Rohstoffkosten sind von hoher Relevanz, da sie mit über 50\% der Gesamtproduktionskosten der mit Abstand wichtigste Kostenfaktor bei der Ethanolproduktion sind (HENNIGES 2007: 55ff.; F.O. LICHT 2010c: 453).

${ }^{60}$ Mit einer Gesamtkapazität von derzeit 1,2 Milliarden Litern (der Kauf von Ensus ist berücksichtigt) ist die CropEnergies AG nach Abengoa Bioenergy der zweitgrößte Ethanolproduzent in der EU27 (CROPENERGIES AG 2013a: 4; EUROOBSERV'ER 2013: 7; CROPENERGIES AG 2013b).
} 
Wie in Abbildung 26 zu erkennen ist, wurden im Jahr 2011 1,2 Milliarden Liter Ethanol in Form von ETBE in der EU27 abgesetzt; dies entspricht 20\% der Gesamtnachfrage nach Ethanol in der EU27. Damit ist der Absatz von ETBE nach der Beimischung von Ethanol zu Benzin als E5 oder E10 der zweitwichtigste Absatzweg. Allerdings ist die Nachfrage nach ETBE seit 2009 zurückgegangen (F.O. LICHT 2011c: 148). Verantwortlich hierfür ist die Substitution von ETBE durch die Direktbeimischung von Ethanol zu Benzin. Durch die Nutzung von ETBE verbessert sich die Klopffestigkeit des Ottokraftstoffs zwar mehr als durch die Beimischung von Ethanol, doch ist die Nutzung von Ethanol günstiger als die von ETBE (F.O. LICHT 2011c: 149).

Name:

Lfd. Nr.:

Einflussbereich:

Beschreibung:

Bedeutung des

Einflussfaktors:
Vertikale strategische Kooperationen im Ethanolmarkt

\section{8}

Ökonomie

Es wird die Entwicklung von vertikalen strategischen Kooperationen in der EU27 betrachtet. Zur Messung des Einflussfaktors wird die Ist-Situation als Referenzsituation beschrieben, um davon ausgehend unterschiedliche Projektionen zu bilden.

Der Einflussfaktor ist von Bedeutung, da es durch die vertikalen Kooperationen von Unternehmen zu einer schnelleren Entwicklung des Ethanolmarktes kommen könnte. Beispielhaft hierfür ist das Gemeinschaftsprojekt „Project LIBERTY“, in dem POET - zweitgrößter Ethanolproduzent der USA - und DSM - ein niederländisches Unternehmen, das sich in dem Projekt auf die Entwicklung von Enyzmen zur Aufspaltung von Zellulose spezialisiert hat - zusammenarbeiten. Zweck der Kooperation ist die Erforschung der Produktion von Zellulose-Ethanol und der Bau einer kommerziellen 95-Millionen-Liter-Zellulose-Ethanolanlage in Emmetsburg (lowa) mit dem Ziel, die Technologie zur Produktion von ZelluloseEthanol an andere Unternehmen auszulizenzieren (POET-DSM ADVANCED BIOFUELS 2012; ETHANOL PRODUCER MAGAZINE 2013a).

Ebenso beispielhaft und zukunftsweisend ist die Kooperation zwischen dem Mineralölkonzern Shell und dem brasilianischen Unternehmen Cosan, die das Joint Venture Raízen gebildet haben und damit größter Ethanolproduzent in Brasilien sind (RAÍZEN 2013). Der Einstieg der großen Mineralölkonzerne in die Produktion von Ethanol ist von großem Interesse, da diese Unternehmen das Kapital besitzen, die Produktion auszuweiten, und Zugang zu der Infrastruktur haben, um Ethanol weltweit zu vermarkten. 
Name:

Lfd. Nr.:

Einflussbereich:

Einflussfaktor 9a:

Beschreibung 9a:

Einflussfaktor 9b:

Beschreibung 9b:

Bedeutung der

Faktoren 9a/9b:
Produktionskosten von Ethanol der ersten Generation ${ }^{61}$

$9 a / 9 b$

Ökonomie

Produktionskosten von Ethanol der ersten Generation - ohne Rohstoffkosten

Es werden die Produktionskosten für Ethanol der ersten Generation in der EU27 ohne Berücksichtigung der Rohstoffkosten betrachtet, da diese separat in Einflussfaktor 9b erfasst werden. Die Entwicklung der Produktionskosten wird in der Einheit Euro je Hektoliter beschrieben.

Rohstoffkosten der Ethanolproduktion der ersten Generation

Der Einflussfaktor betrachtet im Gegensatz zu Einflussfaktor 9a ausschließlich die Entwicklung der Rohstoffkosten für die Ethanolproduktion der ersten Generation in der EU27. Dargestellt werden die Rohstoffkosten in der Einheit US-\$ je Tonne.

Die Produktionskosten von Ethanol der ersten Generation sind von Bedeutung, da sich in Abhängigkeit vom Marktpreis entscheidet, ob eine profitable Produktion von Ethanol in der EU27 möglich ist.

Im Folgenden werden drei Beispiele genannt, in denen die Produktionskosten der europäischen Ethanolproduzenten von besonderer Relevanz sind:

1. Für die Wettbewerbsfähigkeit von Ethanol im Vergleich zu den Biokraftstoffen Biobutanol (Einflussfaktor 17) und BtL (Einflussfaktor 16) sind die Herstellungskosten entscheidend. Dies kann damit begründet werden, dass diese biogenen Treibstoffe genauso politisch gefördert werden wie Ethanol und ebenfalls Benzin beigemischt werden. Folglich können sie auch Ethanol-Kraftstoff am Markt substituieren.

2. Die Ethanolproduzenten der EU27 stehen in Konkurrenz zu Ethanolimporteuren, insbesondere aus Brasilien und den USA. Diese beiden Staaten produzieren Ethanol zu deutlich geringeren Kosten als die europäischen Produzenten (BERG 2012d: 2; HENNIGES 2007: 55ff.), können derzeit aber nicht von diesem Vorteil profitieren, da die Politik der EU27 den europäischen Markt durch hohe Importzölle schützt (Einflussfaktor 22). Sollten die Zölle sinken oder gar fallen, werden die Produktionskosten für Ethanol entscheiden, ob die Produzenten der EU27 weiter am Markt bestehen können.

${ }^{61}$ Der Einflussfaktor „Produktionskosten von Ethanol der ersten Generation“ unterteilt sich in die beiden Einflussfaktoren: „Produktionskosten von Ethanol der ersten Generation - ohne Rohstoffkosten“ (9a) und „Rohstoffkosten der Ethanolproduktion der ersten Generation“ (9b). Diese gesonderte Betrachtung der Rohstoffe ist sinnvoll, da deren Kosten über $50 \%$ der gesamten Herstellungskosten ausmachen und folglich von besonderer Relevanz für die $\mathrm{Zu}$ kunft des Ethanolmarktes der EU27 sind (HENNIGES 2007: 55ff.; F.O. LICHT 2010c: 453). Die Bedeutung der Einflussfaktoren 9a/9b wird gemeinsam erläutert, um Redundanzen zu vermeiden. 
3. Da Ethanol ein Substitut für Benzin ist, bemisst sich die Wettbewerbsfähigkeit von Ethanol gegenüber Benzin im Falle keiner politischen Einflussnahme an der Höhe der Herstellungskosten von Ethanol im Verhältnis zu Benzin $^{62}$. Es ist zu beachten, dass aufgrund des geringeren Energiegehalts von Ethanol (21,2 MJ/L) im Vergleich zu Benzin (32,1 MJ/L) ein Liter Ethanol 34\% günstiger als ein Liter Benzin sein muss, damit beide Kraftstoffe je Energieeinheit den gleichen Wert besitzen (MASSACHUSETTS INSTITUTE OF TECHNOLOGY 2007: 1).

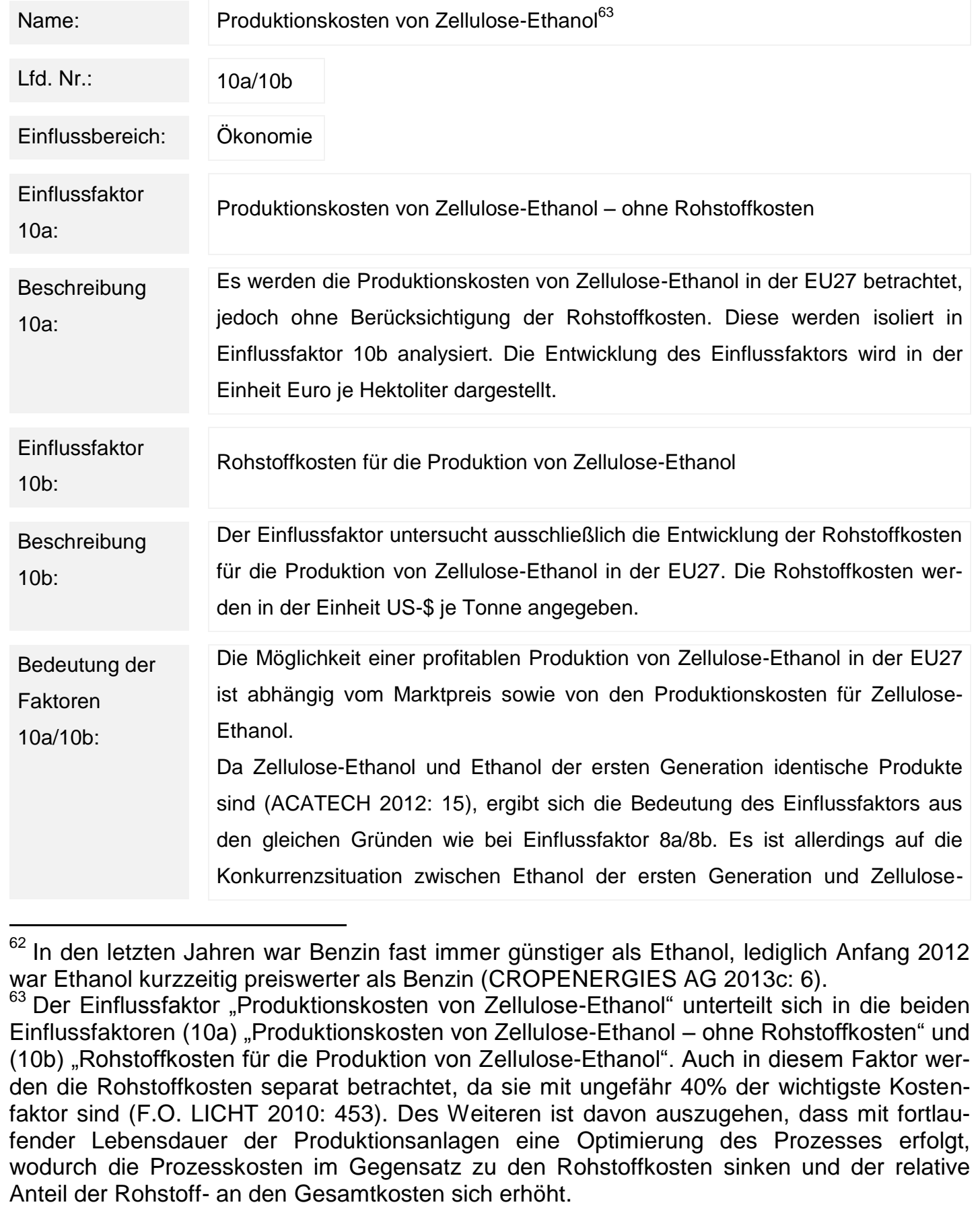

Es werden die Produktionskosten von Zellulose-Ethanol in der EU27 betrachtet, jedoch ohne Berücksichtigung der Rohstoffkosten. Diese werden isoliert in Einflussfaktor 10b analysiert. Die Entwicklung des Einflussfaktors wird in der Einheit Euro je Hektoliter dargestellt.

Rohstoffkosten für die Produktion von Zellulose-Ethanol

Der Einflussfaktor untersucht ausschließlich die Entwicklung der Rohstoffkosten für die Produktion von Zellulose-Ethanol in der EU27. Die Rohstoffkosten werden in der Einheit US-\$ je Tonne angegeben.

Die Möglichkeit einer profitablen Produktion von Zellulose-Ethanol in der EU27 ist abhängig vom Marktpreis sowie von den Produktionskosten für ZelluloseEthanol.

Da Zellulose-Ethanol und Ethanol der ersten Generation identische Produkte sind (ACATECH 2012: 15), ergibt sich die Bedeutung des Einflussfaktors aus den gleichen Gründen wie bei Einflussfaktor $8 a / 8 b$. Es ist allerdings auf die Konkurrenzsituation zwischen Ethanol der ersten Generation und Zellulose-

\footnotetext{
${ }^{62}$ In den letzten Jahren war Benzin fast immer günstiger als Ethanol, lediglich Anfang 2012 war Ethanol kurzzeitig preiswerter als Benzin (CROPENERGIES AG 2013c: 6).

${ }^{63}$ Der Einflussfaktor „Produktionskosten von Zellulose-Ethanol“ unterteilt sich in die beiden Einflussfaktoren (10a) „Produktionskosten von Zellulose-Ethanol - ohne Rohstoffkosten“ und (10b) „Rohstoffkosten für die Produktion von Zellulose-Ethanol“. Auch in diesem Faktor werden die Rohstoffkosten separat betrachtet, da sie mit ungefähr $40 \%$ der wichtigste Kostenfaktor sind (F.O. LICHT 2010: 453). Des Weiteren ist davon auszugehen, dass mit fortlaufender Lebensdauer der Produktionsanlagen eine Optimierung des Prozesses erfolgt, wodurch die Prozesskosten im Gegensatz zu den Rohstoffkosten sinken und der relative Anteil der Rohstoff- an den Gesamtkosten sich erhöht.
} 
Ethanol hinzuweisen. Denn wenn die Politik keinen Einfluss nimmt, entscheiden die Produktionskosten, welcher der beiden Produktionswege vorteilhafter ist.

\section{Technologie}

Name:

Lfd. Nr.:

Einflussbereich:

Beschreibung:

Bedeutung des

Einflussfaktors:
Anzahl an Flexible Fuel Vehicles (FFV) in der EU27

11

Technologie

FFV sind Kraftfahrzeuge, die einen Verbrennungsmotor besitzen, der fähig ist, jegliches Ethanol-Benzin-Gemisch zwischen E0 und E85 als Kraftstoff zu nutzen (U.S. ENERGY INFORMATION ADMINISTRATION 2007:1).

Gemessen wird die Entwicklung des Faktors anhand der Anzahl der zugelassenen FFV in der EU27.

FFV nutzen höhere Ethanolbeimischungen zu Benzin, folglich würde ein Anstieg der Zahl der zugelassenen FFV in der EU27 die europäische Ethanolnachfrage steigern.

Der brasilianische Markt könnte in dieser Hinsicht ein Vorbild für die EU27 sein. Im Jahr 2003 hat Volkswagen das erste FFV mit der Bezeichnung "Gol Power 1.6“ erfolgreich auf dem brasilianischen Markt eingeführt. Seitdem ist der Marktanteil der FFV am gesamten brasilianischen Automobilmarkt rasant angestiegen (F.O.LICHT/AGRA INFORMA 2009: 35f.). So waren im Jahr 2009 90\% aller neu zugelassenen Personenkraftwagen in Brasilien FFV (F.O. LICHT 2009: 70). Dies zeigt, dass die Technologie funktioniert und ausgereift ist. Nach Aussage von HENNIGES (2007: 241) könnte die brasilianische Entwicklung beispielhaft für die EU27 sein. So sind in Schweden bereits 130.000 FFV zugelassen (EUROPEAN BEST PROJECT 2010: 61).

Des Weiteren ist es von Vorteil, dass die FFV-Technologie vergleichsweise günstig ist, die zusätzlichen Kosten pro Pkw betragen circa 300 Euro. Darüber hinaus bieten bereits zahlreiche Automobilkonzerne in der EU27 FFV ${ }^{64}$ an. Ein großer Vorteil der FFV-Technologie im Gegensatz zu anderen neuen Technologien wie beispielsweise Erdgas ist, dass es kein „Chicken or the EggDilemma“ gibt, da die FFV immer die Möglichkeit haben, in der Zeit des Ausbaus der flächendeckenden Infrastruktur des E85 Tankstellennetzes bei bereits bestehenden konventionellen Tankstellen zu tanken.

Trotz des großen Potenzials ist es wichtig zu erwähnen, dass im Jahr 2010 bei einer Gesamtzahl von 147 Millionen benzinbetriebenen Autos (EUROPÄISCHE KOMMISSION EUROSTAT 2012) lediglich 0,17 Millionen FFV in der EU27 zugelassen waren (EUROPEAN BEST PROJECT 2010: 60). Demzufolge liegt

${ }^{64}$ Folgende Automobilkonzerne in der EU27 haben FFV im Portfolio: Volkswagen, Ford, Renault, Volvo, Citroen, Peugeot, Cadillac, Chrysler, Mitsubishi, Audi, Seat, Skoda, Nissan, Fiat, Saab, Dodge, Dacia (EUROPEAN BEST PROJECT 2010: 61). 
der Marktanteil der FFV in der EU27 bei lediglich 0,1\%. Die geringe Bedeutung zeigt sich auch an den Zulassungszahlen aus dem Jahr 2011: Nur 0,2\% aller neu zugelassenen Autos in der EU27 besitzen die FFV-Technologie (ICCT 2012: 81).

Name Anzahl benzinbetriebener Pkw, die mit mittleren Ethanolbeimischungen fahren können (E15 - E40)

Lfd. Nr.:

Einflussbereich:

Beschreibung:

Bedeutung des

Einflussfaktors:
12

Technologie

Es wird die Anzahl benzinbetriebener Pkw in der EU27 betrachtet, die mit mittleren Ethanolbeimischungen zu Benzin (E15 - E40) fahren können. Die Beschreibung der Entwicklung des Faktors erfolgt anhand der Anzahl an Pkw in der EU27, unterteilt nach deren technischer Fähigkeit, unterschiedlich hohe Ethanolbeimischungen zu Benzin als Kraftstoff zu nutzen.

Dieser wirkende Einflussfaktor ist von Relevanz, denn je mehr Personenkraftwagen Benzin mit mittleren Ethanolbeimischungen (E15 - E40) einsetzen können, desto stärker könnte die zukünftige Ethanolnachfrage in der EU27 ansteigen.

Im Folgenden wird der Einfluss des Faktors auf die Ethanolnachfrage anhand des Anstiegs der möglichen Beimischung von E10 auf E15 beispielhaft dargestellt ${ }^{65}$ : Bleibt die maximal mögliche technische Ethanolbeimischung zu Benzin bei $10 \%$ vol (E10), so ist die Nachfrage nach Ethanol auf 9,6 Milliarden Liter im Jahr 2020 begrenzt, da die Benzinnachfrage voraussichtlich 96 Milliarden Liter betragen wird $^{66}$. Aber durch einen Anstieg der Beimischung auf 15\% vol (E15) könnte die Ethanolnachfrage in der EU27 um weitere 4,8 Milliarden Liter auf 14,4 Milliarden Liter ansteigen (Abbildung 27) ${ }^{67}$.

\footnotetext{
${ }^{65}$ In dem Beispiel wird auf die Einbeziehung von politischen Faktoren wie der erlaubten Höhe der Beimischung von Ethanol zu Benzin, beschrieben in Einflussfaktor 19b, bewusst verzichtet, um nur die mögliche Wirkung des technischen Einflussfaktors auf den Ethanolmarkt darzustellen.

${ }^{66}$ Die Prognose der Entwicklung der Benzinnachfrage in der EU27 basiert auf einer Studie der EUROPEAN COMMISSION JOINT RESEARCH CENTRE; INSTITUT FOR ENERGY ET AL. (2011: 24).

${ }^{67}$ Zur Vereinfachung des Beispiels wird davon ausgegangen, dass alle Pkw in der EU27 E15 tanken können und dies auch tun. Eine genaue Betrachtung der Entwicklung dieses Faktors befindet sich in Kapitel 5.2.4.
} 


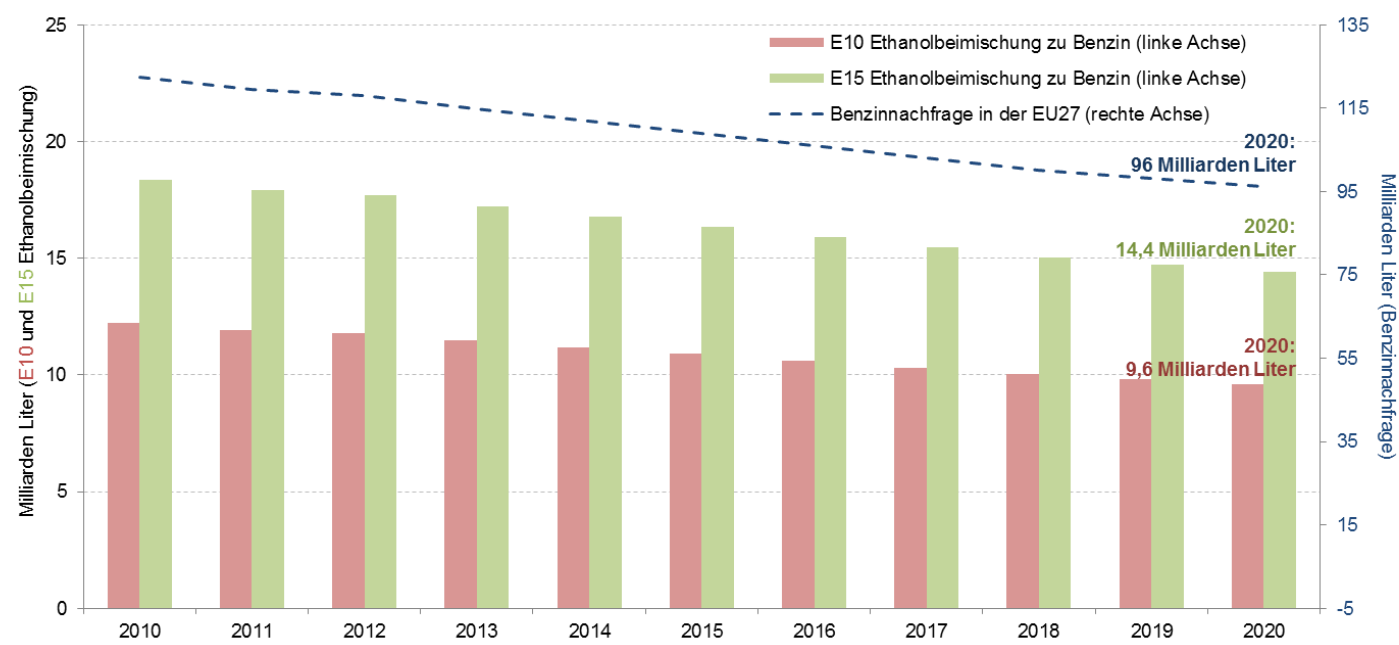

Abbildung 27: Höhere Ethanolnachfrage in der EU27 durch die Nutzung von E15 Quelle: In Anlehnung an EUROPEAN COMMISSION JOINT RESEARCH CENTRE; INSTITUT FOR ENERGY ET AL. 2011: 24; EIGENE BERECHNUNG

Dass die Personenkraftfahrzeuge in der EU27 in Zukunft fähig sein werden, Benzin mit mittleren Ethanolgehalten als Kraftstoff zu nutzen, ist realistisch. In den USA können bereits alle Kraftfahrzeuge ab Baujahr 2001 E15 als Kraftstoff verwenden (EPA 2012a; U.S. ENERGY INFORMATION ADMINISTRATION 2011a: 84). In Europa sind sogar alle verkauften Personenkraftwagen von VW ab Baujahr 2010 in der Lage, E20 als Kraftstoff zu verbrennen (SEYFRIED 2012).

Name:

Lfd. Nr.:

Einflussbereich:

Beschreibung:

Bedeutung des

Einflussfaktors:

\section{Anzahl der Elektrofahrzeuge in der EU27}

13

Technologie

Unter dem Begriff Elektrofahrzeuge werden Batterie- und Hybridfahrzeuge zusammengefasst. Batteriefahrzeuge sind Automobile mit einem Elektromotor, die lediglich gespeicherten Strom als Energiequelle nutzen (VDA 2011: 8). Hybridfahrzeuge besitzen dagegen sowohl einen Verbrennungs- als auch einen Elektromotor und können sowohl Kraftstoffe als auch gespeicherte Elektrizität als Energiequelle nutzen (CHAU/WONG 2002: 1954).

Die Entwicklung dieses Faktors wird anhand der Anzahl an zugelassenen Elektrofahrzeugen in der EU27 gemessen.

Elektrofahrzeuge haben keinen oder einen geringeren Benzinverbrauch im Vergleich $z u$ konventionellen Kraftfahrzeugen mit Ottomotor (ENVIRONMENTAL PROTECTION AGENCY 2012). Folglich sinkt die Benzinnachfrage und damit auch die Ethanolnachfrage in der EU27, wenn Elektrofahrzeuge zukünftig Fahrzeuge mit Benzinmotor substituieren.

Darüber hinaus ist es wichtig, die Entwicklung dieses Faktors zu betrachten, denn in RICHTLINIE 2009/28/EC Artikel 3 (4c) ist festgelegt, dass die Elektrizitätsmenge, gewonnen aus erneuerbaren Energiequellen und verbraucht 
im Straßenverkehr, dem 10\%-Ziel 68 2,5-fach angerechnet wird. Dementsprechend senkt ein Anstieg der Nutzung von erneuerbarer Elektrizität im Straßenverkehr den Bedarf an Ethanol-Kraftstoff, um das Ziel der Nutzung von $10 \%$ erneuerbarer Energie im Verkehrssektor zu erfüllen (OECD/FAO 2011: 87).

Alle führenden Automobilkonzerne arbeiten an der Entwicklung von Elektrofahrzeugen oder bieten diese bereits auf dem europäischen Markt an (ICF INTERNATIONAL/ECOLOGIC INSTITUTE 2011: 79ff.). Trotzdem wird nach Prognosen des EUROPEAN COMMISSION JOINT RESEARCH CENTRE, INSTITUT FOR PROSPECTIVE TECHNOLOGICAL STUDIES (2010b: 25f.) der Marktanteil für Elektroautos zwar zukünftig steigen, der Automobilmarkt im Jahr 2023 aber weiterhin von traditionellen Pkw bestimmt sein. Der Faktor wird dennoch berücksichtigt, da aufgrund der 2,5-fachen Anrechnung auf das 10\%-Ziel ein schon geringer Marktanteil den europäischen Ethanolmarkt beeinflussen könnte.

Name:

Lfd. Nr.:

Einflussbereich:

Beschreibung:

Bedeutung des Einflussfaktors:
Enzym-Technologie zur Produktion von Zellulose-Ethanol

14

Technologie

Der Faktor betrachtet die Entwicklung der Enzym-Technologie zur Produktion von Zellulose-Ethanol. In diesem Prozess werden Enzyme eingesetzt, um Zellulose in C5 und C6 Zucker aufzuspalten, die im darauf folgenden Prozessschritt zu Ethanol fermentiert werden (NATIONAL RENEWABLE ENERGY LABORATORY 2007: 4; SUN/CHENG 2002: 6).

Der Einflussfaktor wird dargestellt, indem die Ist-Situation der Enzym-Technologie als Referenzsituation beschrieben wird, um davon ausgehend unterschiedliche Entwicklungen des Einflussfaktors aufzuzeigen.

Durch einen Fortschritt der Enzym-Technologie kann sowohl die Ausbeute an Zucker (C5 und C6) je eingesetzter Menge Zellulose als auch die Umwandlungsgeschwindigkeit von Zellulose zu Zucker gesteigert werden, wodurch sich sowohl der Ethanolertrag je Tonne des eingesetzten Rohstoffs als auch die Produktivität der gesamten Produktionsanlage erhöht (NATIONAL RENEWABLE ENERGY LABORATORY 2007: 4). Ferner ist die Entwicklung der Enzym-Technologie von Relevanz, da der regelmäßige Kauf von Enzymen mit zu den bedeutendsten Kostenfaktoren bei der Produktion von Zellulose-Ethanol gehört (F.O. LICHT 2010c: 453).

Entscheidend wäre eine technologische Weiterentwicklung, die die Flexibilität hinsichtlich der Rohstoffnutzung verbessert, sodass die gleichzeitige Verwendung unterschiedlicher Rohstoffe wie beispielsweise Weizenstroh, Roggenstroh,

${ }^{68}$ Das $10 \%$-Ziel ist in Kapitel 2.1 und Einflussfaktor 18a beschrieben. 
Maisstroh und Miscanthus möglich ist. Dies würde die Versorgung der Anlage mit Rohstoffen deutlich vereinfachen und die Produktionskosten senken, da die Anlage dann nicht von der Preisentwicklung eines einzelnen Rohstoffs abhängig wäre, sondern die jeweils günstigsten Rohstoffe zur Produktion von ZelluloseEthanol einkaufen könnte.

\begin{tabular}{|c|c|}
\hline Name: & Kohlenstoffdioxidausstoß von Ethanol-Kraftstoff ${ }^{69}$ \\
\hline Lfd. Nr.: & $15 a / 15 b$ \\
\hline Einflussbereich: & Technologie \\
\hline $\begin{array}{l}\text { Einflussfaktor } \\
15 \mathrm{a}:\end{array}$ & Kohlenstoffdioxidausstoß von Ethanol-Kraftstoff der ersten Generation \\
\hline $\begin{array}{l}\text { Beschreibung } \\
15 \mathrm{a}:\end{array}$ & $\begin{array}{l}\text { Der Einflussfaktor betrachtet die Entwicklung der } \mathrm{CO}_{2} \text {-Gesamtemissionen bei } \\
\text { der Produktion von Ethanol-Kraftstoff der ersten Generation in der EU27. Ge- } \\
\text { messen wird die Entwicklung in der Einheit } \mathrm{gCO}_{2 e q} / \mathrm{MJ} \text {. }\end{array}$ \\
\hline $\begin{array}{l}\text { Einflussfaktor } \\
15 \mathrm{~b}:\end{array}$ & Kohlenstoffdioxidausstoß von Ethanol-Kraftstoff aus Zellulose \\
\hline $\begin{array}{l}\text { Beschreibung } \\
\text { 15b: }\end{array}$ & $\begin{array}{l}\text { Es werden die } \mathrm{CO}_{2} \text {-Gesamtemissionen bei der Herstellung von Ethanol- } \\
\text { Kraftstoff aus Zellulose in der EU27 analysiert. Der zukünftige Verlauf des Ein- } \\
\text { flussfaktors wird in der Einheit } \mathrm{gCO}_{2 e q} / \mathrm{MJ} \text { angegeben. }\end{array}$ \\
\hline $\begin{array}{l}\text { Bedeutung der } \\
\text { Faktoren } \\
15 \mathrm{a} / 15 \mathrm{~b} \text { : }\end{array}$ & $\begin{array}{l}\text { Der Einflussfaktor ist von Bedeutung, da die } \mathrm{CO}_{2} \text {-Gesamtemissionen von Etha- } \\
\text { nol je geringer sind, desto höher die } \mathrm{CO}_{2} \text {-Einsparung im Vergleich zu fossilen } \\
\text { Kraftstoffen und damit der Nutzen für die Umwelt ist. Dies ist wichtig, denn das } \\
\text { Kernziel der politischen Förderung von erneuerbaren Energien ist die Reduktion } \\
\text { des Kohlenstoffdioxidausstoßes im Verkehrssektor (RICHTLINIE 2009/28/EG } \\
\text { ERW 1). } \\
\text { Zur Erreichung der } \mathrm{CO}_{2} \text {-Reduktion hat die Politik Ziele hinsichtlich der prozen- } \\
\text { tualen } \mathrm{CO}_{2} \text {-Einsparung von Biokraftstoffen im Verhältnis zu fossilen Kraftstoffen } \\
\text { formuliert }{ }^{70} \text {. Verfehlen die Biokraftstoffe diese Ziele, werden sie nicht den „er- }\end{array}$ \\
\hline
\end{tabular}

69 Der Einflussfaktor „Kohlenstoffdioxidausstoß von Ethanol-Kraftstoff“ gliedert sich in die beiden Faktoren 15a: „Kohlenstoffdioxidausstoß von Ethanol-Kraftstoff der ersten Generation“ und 15b: „Kohlenstoffdioxidausstoß von Ethanol-Kraftstoff aus Zellulose“. Die beiden Faktoren werden einzeln betrachtet, da sich der Produktionsprozess und die $\mathrm{CO}_{2}$ Emissionen zur Herstellung von Zellulose-Ethanol und Ethanol der ersten Generation deutlich unterscheiden. Die Bedeutung der beiden Faktoren wird jedoch im Folgenden gemeinsam betrachtet, denn Ethanol der ersten und zweiten Generation muss die identischen politischen Vorgaben zur Treibhausgasreduktion im Vergleich zu Fossilbrennstoff erfüllen, um politisch gefördert zu werden.

${ }^{70}$ In der RICHTLINIE 2009/28/EC ARTIKEL 17 (2) sind die politisch geforderten $\mathrm{CO}_{2}$ Reduktionsziele für Biokraftstoffe im Vergleich zu fossilem Kraftstoff festgelegt: Bis zum Jahr 2017 müssen alle Biokraftstoffe eine Kohlenstoffdioxid-Einsparung von 35\% erreichen. Ab dem 1. Januar 2017 erhöht sich die geforderte Reduktion auf 50\% und ab dem 1. Januar 
neuerbaren Energien“, beschrieben in Einflussfaktor 18a, angerechnet (RICHTLINIE 2009/28/EG ARTIKEL17 (2)). Dies hätte zur Folge, dass diese Biokraftstoffe nicht politisch gefördert werden und in direkter Konkurrenz zu fossilen Kraftstoffen stehen.

Die Berechnung der prozentualen Kohlenstoffdioxideinsparung von Biokraftstoffen im Vergleich zu fossilem Kraftstoff erfolgt über die folgende Formel (RICHTLINIE 2009/28/EG ANHANG V 4.):

Einsparung $=\left(E_{F}-E_{B}\right) / E_{F}$

$E_{B}=$ Gesamtemissionen bei der Verwendung des Biokraftstoffs

$E_{F}=$ Gesamtemissionen des Komparators für Fossilbrennstoff

Im Folgenden werden die maximalen $\mathrm{CO}_{2}$-Gesamtemissionen von Biokraftstoffen in Abhängigkeit von der politisch geforderten prozentualen $\mathrm{CO}_{2}$ Einsparung ${ }^{71}$ berechnet. Zur Ermittlung dessen wird die Formel wie folgt umgestellt: $E_{B}=E_{F}-\left(\right.$ Einsparung $\left.{ }^{*} E_{F}\right)$

\section{$\mathrm{CO}_{2}$-Einsparung von $35 \%$ im Verhältnis zu fossilem Kraftstoff}

$\mathrm{E}_{\mathrm{F}}=$ Gesamtemissionen des Komparators für Fossilbrennstoff $83,8 \mathrm{gCO}_{2 \mathrm{eq}} / \mathrm{MJ}$

Einsparung $=0,35$

$\mathrm{E}_{\mathrm{B}}=\mathrm{E}_{\mathrm{F}}-\left(\right.$ Einsparung $\left.{ }^{*} \mathrm{E}_{\mathrm{F}}\right)=83,8-(0,35 * 83,8)=54,5 \mathrm{gCO}_{2 \mathrm{eq}} / \mathrm{MJ}$

\section{$\mathrm{CO}_{2}$-Einsparung von $50 \%$ im Verhältnis zu fossilem Kraftstoff}

$\mathrm{E}_{\mathrm{F}}=$ Gesamtemissionen des Komparators für Fossilbrennstoff 83,8 $\mathrm{gCO}_{2 \mathrm{eq}} / \mathrm{MJ}$

Einsparung $=0,5$

$\mathrm{E}_{\mathrm{B}}=\mathrm{E}_{\mathrm{F}}-\left(\right.$ Einsparung $\left.{ }^{*} \mathrm{E}_{\mathrm{F}}\right)=83,8-(0,5 * 83,8)=41,9 \mathrm{gCO}_{2 \mathrm{eq}} / \mathrm{MJ}$

Damit Biokraftstoffe eine 35-prozentige und 50-prozentige $\mathrm{CO}_{2}$-Reduktion im Vergleich $\mathrm{zu}$ fossilen Kraftstoffen erfüllen, dürfen sie eine maximale $\mathrm{CO}_{2-}$ Gesamtemission von 54,5 $\mathrm{gCO}_{2 \mathrm{eq}} / \mathrm{MJ}$ beziehungsweise 41,9 $\mathrm{gCO}_{2 \mathrm{eq}} / \mathrm{MJ}$ nicht überschreiten. In der folgenden Tabelle 7 werden diese Werte in die Spalte „maximale $\mathrm{E}_{\mathrm{B}}\left(\mathrm{gCO}_{2 e q} / \mathrm{MJ}\right)$ “ eingetragen und mit den aktuellen Werten der $\mathrm{CO}_{2}$ Gesamtemissionen für Ethanol, unterteilt in die Rohstoffe Weizen, Zuckerrübe und Zellulose (Weizenstroh) sowie mit und ohne Berücksichtigung des ILUCFaktors $^{72}$, verglichen.

Ist bei dem Vergleich die Zahl der $\mathrm{CO}_{2}$-Gesamtemission des Ethanol-Kraftstoffs grün gefärbt, so liegt der $\mathrm{CO}_{2}$-Ausstoß des biogenen Kraftstoffs unter der maximal politisch geforderten $\mathrm{CO}_{2}$-Emission und wird politisch gefördert. Rot bedeutet, dass der Ethanol-Kraftstoff die politischen Vorgaben der Kohlenstoffdioxidreduktion nicht erfüllt und damit auch nicht von der Politik gefördert wird.

2018 müssen alle Produktionsanlagen, die am oder nach dem 1. Januar 2017 die Produktion aufgenommen haben, eine $\mathrm{CO}_{2}$-Minderung von $60 \%$ erreichen.

${ }^{71}$ Die politische geforderte prozentuale $\mathrm{CO}_{2}$-Einsparung von Biokraftstoffen im Verhältnis zu fossilen Kraftstoffen wird in Einflussfaktor 19b betrachtet.

72 Die Bedeutung des ILUC-Faktor auf den Ethanolmarkt der EU27 wird in Einflussfaktor 21 erläutert. 


\begin{tabular}{|c|c|c|c|}
\hline & $\begin{array}{c}\text { maximale } E_{B} \\
\left(\mathrm{gCO}_{2 e q} / \mathrm{MJ}\right)\end{array}$ & $\begin{array}{c}\mathrm{E}_{\mathrm{B}} \text { (ohne ILUC) } \\
\left(\mathrm{gCO}_{2 \mathrm{eq}} / \mathrm{MJ}\right)\end{array}$ & $\begin{array}{c}\mathrm{E}_{\mathrm{B}} \text { (mit ILUC) } \\
\left(\mathrm{gCO}_{2 \mathrm{eq}} / \mathrm{MJ}\right)\end{array}$ \\
\hline Weizen (35\%) & 54,5 & $>46$ & $<58$ \\
\hline Weizen $(50 \%)$ & 41,9 & $<46$ & $<58$ \\
\hline Zuckerrübe (35\%) & 54,5 & $>33$ & $>46$ \\
\hline Zuckerrübe (50\%) & 41,9 & $>33$ & $<46$ \\
\hline Zellulose $^{73}(35 \%)$ & 54,5 & $>11$ & $>11$ \\
\hline Zellulose (50\%) & 41,9 & $>11$ & $>11$ \\
\hline
\end{tabular}

Tabelle 7: Maximale $\mathrm{CO}_{2}$-Gesamtemissionen von Biokraftstoffen in Abhängigkeit von der politisch geforderten prozentualen $\mathrm{CO}_{2}$-Reduktion im Verhältnis $\mathrm{zu}$ fossilen Kraftstoffen

Quelle: RICHTLINIE 2009/28/EG ANHANG V; EUROPÄISCHE KOMMISSION 2012:2; EIGENE BERECHNUNG

In der Tabelle 7 ist zu erkennen, dass Zellulose-Ethanol alle derzeitigen und zukünftigen Vorgaben der $\mathrm{CO}_{2}$-Reduktion im Vergleich zu fossilem Kraftstoff erfüllt. Bei Ethanol aus Zuckerrüben ist dies ähnlich, lediglich bei einer Berücksichtigung des ILUC-Wertes und einem Anstieg der geforderten $\mathrm{CO}_{2-}$ Einsparung auf $50 \%$ erreicht die Zuckerrübe nicht mehr die politisch geforderte $\mathrm{CO}_{2}$-Reduktion.

Von besonderer Relevanz ist die Betrachtung dieses Einflussfaktors für Weizen-Ethanol, dem wichtigsten Rohstoff zur Ethanolproduktion in der EU27 (EUROPEAN COMMISSION JOINT RESEARCH CENTRE, INSTITUT FOR ENERGY ET AL. 2010: 74). Sollte es zu keiner Optimierung des Prozesses der Ethanolproduktion aus Weizen kommen, um einen geringeren $\mathrm{CO}_{2}$-Ausstoß zu erreichen, werden die politisch geforderten $\mathrm{CO}_{2}$-Einsparungen im Vergleich zu fossilem Kraftstoff ab dem Jahr 2017, unabhängig von der Berücksichtigung eines ILUC-Faktors, nicht erreicht, wodurch Weizen-Ethanol nicht weiter politisch gefördert würde und in direkter Konkurrenz zu fossilem Benzin stünde.

Name: Nachfrage nach BtL in der EU27

Lfd. Nr.: 16

Einflussbereich: Technologie

Beschreibung:

BtL (Biomass to Liquids) ist ein synthetischer Kraftstoff, der aus Zucker, Stärke, Zellulose und Lignin produziert werden kann. Der Biokraftstoff kann problemlos Benzin und Diesel beigemischt werden und die Motoren der Kraftfahrzeuge sind ohne technische Modifikationen fähig, den Kraftstoff zu nutzen (EUROPEAN BIOFUELS 2013b; U.S. DEPARTMENT OF ENERGY 2013d; FNR 2009: 83ff.).

\footnotetext{
${ }^{73}$ Zur Ethanolproduktion aus Zellulose wird in diesem Beispiel der Rohstoff Weizenstroh genutzt. Da es ein Nebenprodukt der Weizenproduktion ist, wird diesem kein ILUC-Wert angerechnet.
} 
Bedeutung des

Einflussfaktors:

Die zukünftige Entwicklung der Nachfrage nach BTL in der EU27 wird in der Einheit Milliarden Liter pro Jahr beschrieben.

Der synthetische Biokraftstoff BtL ist für die Entwicklung des Ethanolmarktes der EU27 relevant, da BtL Benzin beigemischt werden kann, wodurch eine Substitution von Ethanol möglich ist. Darüber hinaus wird BtL wie auch Ethanol, unter der Voraussetzung einer ausreichenden $\mathrm{CO}_{2}$-Reduktion im Vergleich $\mathrm{zu}$ fossilen Kraftstoffen, den „erneuerbaren Energien“ im Verkehrssektor angerechnet und politisch gefördert (EUROPEAN BIOFUELS 2013b; U.S. DEPARTMENT OF ENERGY 2013d).

BtL besitzt gegenüber Ethanol den Vorteil, dass der Kraftstoff durch Veränderungen des Prozesses an die Bedürfnisse der Motoren und der Infrastruktur angepasst werden kann und weder hygroskopisch noch korrodierend wirkt, womit der Gebrauch von BtL in Ottomotoren und Pipelines auch in hohen Beimischungen $\mathrm{zu}$ Benzin problemlos möglich ist (EUROPEAN BIOFUELS 2013b; U.S. DEPARTMENT OF ENERGY 2013d; FNR 2009: 83ff.).

Es gibt mehrere Projekte in der EU27 zur Erprobung der Produktion von BtL, aber bis zum Jahr 2012 noch keine kommerzielle Produktion ${ }^{74}$, da die Produktionskosten von BtL, trotz der besseren Qualität und des höheren Heizwertes $^{75}$ (33,45 MJ/L), im Vergleich zu anderen Biokraftstoffen noch zu hoch sind (FNR 2009: 23; MASSACHUSETTS INSTITUTE OF TECHNOLOGY 2007).

Name: Nachfrage nach Biobutanol in der EU27

Lfd. Nr.: 17

Einflussbereich: Technologie

Beschreibung:

Bedeutung des

Einflussfaktors:

Biobutanol ist ein Alkohol, der durch die Fermentation von Zucker, Stärke und Zellulose produziert wird. Er kann Benzin beigemischt werden und wird von Ottomotoren problemlos als Kraftstoff genutzt (EUROPEAN BIOFUELS 2013a; FNR 2009: 98f.; U.S. DEPARTMENT OF ENERGY 2013c).

Der Einflussfaktor betrachtet die Entwicklung der Biobutanolnachfrage in der EU27, beschrieben wird der Faktor in der Einheit Milliarden Liter pro Jahr.

Der biogene Kraftstoff Biobutanol hat für den Ethanolmarkt der EU27 eine Bedeutung, da er Ethanol-Kraftstoff substituieren kann. Biobutanol wird wie Ethanol aus den Rohstoffen Weizen, Zuckerrübe, Mais, Zuckerrohr und Zellulose produziert und kann Benzin beigemischt werden. Unter der Voraussetzung einer ausreichenden $\mathrm{CO}_{2}$-Reduktion wird Biobutaol den „erneuerbaren Energien“ im

\footnotetext{
${ }^{74}$ Die beiden finnischen Unternehmen UPM und Vapo planen den Bau von kommerziellen Anlagen zur Produktion von BtL. Beide Vorhaben werden im Rahmen des „NER300“ Programms der Europäischen Kommission mit 170 Millionen Euro und 88,5 Millionen Euro gefördert (EUROPEAN COMMISSION 2012b; EUROPEAN BIOFUELS 2013b).

${ }^{75}$ Heizwert von Benzin $(32,1 \mathrm{MJ} / \mathrm{L})$; Heizwert von Ethanol $(21,2 \mathrm{MJ} / \mathrm{L})$ (MASSACHUSETTS INSTITUTE OF TECHNOLOGY 2007).
} 
Verkehrssektor angerechnet und damit wie Ethanol politisch gefördert (FNR 2009: 98f; EUROPEAN BIOFUELS 2013a).

Biobutanol hat im Vergleich zu Ethanol die folgenden Vorteile (EUROPEAN BIOFUELS 2013a; FNR 2009: 98f.; U.S. DEPARTMENT OF ENERGY 2013c; TECHNOLOGY REVIEW 2007):

- Biobutanol hat mit 27,2 MJ/L eine höhere Energiedichte als Ethanol mit 21,2 $\mathrm{MJ} / \mathrm{L}$.

- Ottomotoren können Benzin sowohl mit niedrigen als auch mit hohen Biobutanolbeimischungen ( $5 \% \mathrm{vol}-85 \% \mathrm{vol}$ ) ohne technische Modifikationen nutzen.

- Biobutanol kann durch die bestehenden Pipelines transportiert werden, da der Kraftstoff im Gegensatz zu Ethanol nicht hygroskopisch wirkt.

- Biobutanol kann auch in höheren Beimischungen zu Benzin das bestehende Tankstellennetz nutzen, da der Kraftstoff nicht korrodierend wirkt und die Aluund Polymerkomponenten in den Zapfsäulen nicht angreift.

Trotz der zahlreichen Vorteile von Biobutanol und obwohl Unternehmen wie Butamax Advanced Biofuels $\mathrm{LLC}^{76}$ und $\mathrm{Gevo}^{77}$ intensiv an der Entwicklung von Biobutanol forschen, gibt es im Jahr 2012 lediglich eine Demonstrationsanlage in Hull, England, zur Herstellung von Biobutanol in der EU27 (BUTAMAX ADVANCED BIOFUELS 2013). Grund dafür sind die hohen Produktionskosten von Biobutanol, wodurch die Konkurrenzfähigkeit gegenüber anderen biogenen Treibstoffen noch nicht gegeben ist (FNR 2009: 98f).

\section{Politik}

Name:

Lfd. Nr.:

Einflussbereich:

Einflussfaktor

18a:
EU27-Richtlinien für Kraftstoff-Ethanol ${ }^{78}$ (Erneuerbare-Energien-Richtlinie)

$18 a / 18 b$

Politik

Der politisch geforderte prozentuale Anteil von erneuerbaren Energien am Endenergieverbrauch im Verkehrsbereich

\footnotetext{
${ }^{76}$ Butamax Advanced Biofuels LLC ist ein im Jahr 2009 gegründetes Joint Venture zwischen dem britischen Mineralölkonzern BP und dem US-amerikanischen Chemieunternehmen DuPont. Ziel des Unternehmens ist es, den Produktionsprozess zur Herstellung von Biobutanol zu verbessern und Biobutanol großtechnisch zu produzieren (BUTAMAX ADVANCED BIOFUELS 2013).

77 Gevo ist ein US-amerikanisches Unternehmen, das an der Optimierung des Prozesses zur Herstellung von Biobutanol arbeitet und seine Technologie zur Produktion von Biobutanol an Hersteller auslizenziert (GEVO 2013).

${ }^{78}$ Der Einflussfaktor „Richtlinien für Kraftstoff-Ethanol in der EU27“ unterteilt sich in die zwei Einflussfaktoren 18a und 18b. Die Faktoren werden nicht einzeln betrachtet, da sie beide in der RICHTLINIE 2009/28/EG (Erneuerbare-Energien-Richtlinie) beschrieben sind und sich ergänzen.
} 
Beschreibung

18a:

Bedeutung des

Faktors 18a:
Der Einflussfaktor ist derzeit in der RICHTLINIE 2009/28/EG (ARTIKEL 3 (4)) als politische Vorgabe für das Jahr 2020 formuliert $^{79}$.

Die Berechnung des prozentualen Anteils der erneuerbaren Energien im Verkehrssektor erfolgt über die folgende Formel ${ }^{80}$ :

Anteil erneuerbare Energie $=\left(E_{E} \times 100\right) / E_{G}$

$E_{E}=$ Zusammenrechnung aller Arten von Energie aus erneuerbaren Quellen, die bei allen Verkehrsträgern verbraucht werden (RICHTLINIE 2009/28/EG ARTIKEL 3 (4b)).

$E_{G}=$ Addition von Ottokraftstoff, Dieselkraftstoff sowie verbrauchten Biokraftstoffen und Elektrizität im Straßen- und Schienenverkehr (RICHTLINIE 2009/28/EG ARTIKEL 3 (4a)).

Die Entwicklung des Einflussfaktors wird mittels des von der EU27 geforderten prozentualen Anteils von erneuerbaren Energien am Gesamtenergieverbrauch im Verkehrsbereich beschrieben.

Der Einflussfaktor ist von zentraler Bedeutung für den Ethanolmarkt der EU27, da er die Nutzung von erneuerbaren Energien im Verkehrssektor fördert. Ethanol wird zwar durch den Faktor nicht direkt gefördert, doch sind sich alle führenden Experten einig, dass die Biokraftstoffe - Ethanol und Biodiesel - den größten Beitrag zur Erfüllung der politisch geforderten Verwendung von erneuerbaren Energien im Verkehrsbereich leisten werden (EUROPÄISCHEN KOMMISSION 2012: 7; EUROPEAN COMMISSION JOINT RESEARCH CENTRE, INSTITUTE FOR ENERGY ET AL. 2011: 46 ff.; EUROPEAN COMMISSION JOINT RESEARCH CENTRE, INSTITUTE FOR PROSPECTIVE TECHNOLOGICAL STUDIES 2010a: 33).

Sollte der Einflussfaktor wegfallen, würde die Ethanolnachfrage in der EU27 stark sinken, da es für die europäische Mineralölindustrie keinen Grund mehr gäbe, die hohen Beimischungen von Ethanol zu Benzin aufrechtzuerhalten ${ }^{81}$. Dass der Absatz der in der EU27 frei werdenden Ethanolmengen auf dem Weltmarkt erfolgt, ist als unwahrscheinlich einzustufen, da die Herstellungskosten in der EU27 zu hoch sind, um mit den USA oder Brasilien konkurrieren zu können (BERG 2012d: 2). Folglich bedeutet ein Rückgang der europäischen Ethanolnachfrage auch eine Minderung der Ethanolproduktion in der EU27.

Die politisch geforderte Mindesteinsparung von Kohlenstoffdioxid bei Biokraftstoffen im Verhältnis zu fossilen Kraftstoffen

Der Einflussfaktor ist in der RICHTLINIE 2009/28/EG (ARTIKEL 17 (2)) be-
Einflussfaktor

18b:

Beschreibung

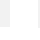

\footnotetext{
${ }^{79}$ In der RICHTLINIE 2009/28/EG (ARTIKEL 3 (4)) ist das Ziel formuliert, dass im Jahr 2020 $10 \%$ der eingesetzten Energie im Verkehrssektor aus erneuerbaren Energiequellen bezogen werden soll.

${ }^{80}$ Eine ausführlichere Beschreibung der Berechnung des Einflussfaktors befindet sich in Kapitel 5.2.6.

81 Die EU27 besitzt eine hohe Überproduktion an Benzin. Die Mineralölkonzerne werden daher ein Ende der Beimischungspflicht von Biokraftstoffen zu fossilen Kraftstoffen begrüBen, um den Absatz von Benzin in der EU27 zu erhöhen (WOOD MACKENZIE 2013: 8).
} 
18b:

Bedeutung des

Faktors 18b: schrieben. Die Berechnung der prozentualen $\mathrm{CO}_{2}$-Einsparung erfolgt über die Formel (RICHTLINIE 2009/28/EG ANHANG V 4.):

Einsparung $=\left(E_{F}-E_{B}\right) / E_{F}$

$E_{B}=$ Gesamtemissionen bei der Verwendung des Biokraftstoffs

$E_{F}=$ Gesamtemissionen des Komparators für Fossilbrennstoffe

Die Entwicklung des Einflussfaktors wird anhand der politisch geforderten prozentualen $\mathrm{CO}_{2}$-Einsparung von Biokraftstoffen im Verhältnis zu fossilen Kraftstoffen beschrieben.

Das Kernziel der EU27 ist es, durch die Nutzung von Ethanol den Kohlenstoffdioxidausstoß im Verkehrsbereich zu senken (RICHTLINIE 2009/28/EG ERW 1). Um dieses Ziel zu erfüllen, müssen die verwendeten Biokraftstoffe in der EU27 festgelegte prozentuale $\mathrm{CO}_{2}$-Einsparungen im Vergleich zu fossilem Kraftstoff erreichen ${ }^{82}$. Verfehlen die Kraftstoffe diese Einsparungen, dürfen sie nicht den in Einflussfaktor 18a beschriebenen „erneuerbaren Energien“ angerechnet werden und dienen somit nicht der Erreichung des politisch geforderten Anteils an erneuerbaren Energien im Verkehrsbereich (RICHTLINIE 2009/28/EG ARTIKEL 17 (2)). Dadurch werden diese Biokraftstoffe nicht politisch gefördert und stehen auf dem Kraftstoffmarkt in direkter Konkurrenz zu fossilem Benzin.

EU27-Richtlinien zur Kraftstoffqualität ${ }^{83}$ (Kraftstoffqualitätsrichtlinie)

$19 a / 19 b$

Lfd. Nr.:

Einflussbereich:

Politik

Einflussfaktor

19a:

Beschreibung

19a:

Bedeutung des
Vorgabe der EU27 zur Kohlenstoffdioxideinsparung von Kraftstoffen im Verkehrsbereich

Der Einflussfaktor ist in der Richtlinie 2009/30/EG beschrieben. Diese legt fest, dass die im Verkehrsbereich der EU27 eingesetzten Kraftstoffe im Jahr 2020 mindestens $6 \%$ weniger Lebenszyklustreibhausgase je Energieeinheit freisetzen als die verwendeten fossilen Kraftstoffe im Jahr 2010.

Die Entwicklung des Faktors wird anhand der politisch bestimmten prozentualen Kohlenstoffdioxideinsparung je Energieeinheit dargestellt.

Der Einflussfaktor dient der Unterstützung der Reduktion des $\mathrm{CO}_{2}$-Ausstoßes

${ }^{82}$ In der RICHTLINIE 2009/28/EC (ARTIKEL 17 (2)) sind die politisch geforderten $\mathrm{CO}_{2^{-}}$ Reduktionswerte für Biokraftstoffe im Vergleich zu fossilem Kraftstoff festgelegt: Bis zum Jahr 2017 müssen alle Biokraftstoffe eine Kohlenstoffdioxid-Einsparung von 35\% erreichen. Ab dem 1. Januar 2017 erhöht sich die geforderte Reduktion auf $50 \%$ und vom 1. Januar 2018 an müssen alle Produktionsanlagen, die am oder nach dem 1. Januar 2017 die Produktion aufgenommen haben, eine $\mathrm{CO}_{2}$-Minderung von $60 \%$ erreichen.

83 Der Einflussfaktor „EU27-Richtlinien zur Kraftstoffqualität (Kraftstoffqualitätsrichtlinie)" unterteilt sich in die zwei Einflussfaktoren 19a und 19b. Beide Faktoren sind Bestandteil der RICHTLINIE 2009/30/EG und werden aus diesem Grund gemeinsam betrachtet. 
im Verkehrsbereich - Kernziel der EU27 zur Förderung von Biokraftstoffen.

Die prozentuale Einsparung des Kohlenstoffdioxidausstoßes im Kraftstoffbereich kann im Vergleich zum verwendeten fossilen Kraftstoff im Jahr 2010 durch zwei Ansätze erfolgen: (1) bei der Mineralölförderung (Upstream), beispielsweise durch die Verringerung sowohl des Entweichens als auch des gezielten Verbrennens von Gasen an den Förderstätten, (2) im „Downstream“-Bereich, nämlich durch die Nutzung von erneuerbaren Energien wie Biokraftstoffen oder dem Gebrauch von Elektrizität im Straßenverkehr (RICHTLINIE 2009/30/EG ARTIKEL 7a).

Durch die Möglichkeit der Verwendung von Ethanol-Kraftstoff wird die Ethanolnachfrage in der EU27 gefördert, insbesondere die nach Ethanol mit einer hohen $\mathrm{CO}_{2}$-Einsparung im Vergleich zu fossilen Kraftstoffen, wie beispielsweise Zuckerrohrethanol aus Brasilien oder Ethanol der zweiten Generation (RICHTLINIE 2009/30/EG ANHANG V). Dies ist dadurch zu erklären, dass infolge der hohen $\mathrm{CO}_{2}$-Einsparungen dieses Ethanols geringere Mengen eingesetzt werden müssen, um die politische Vorgabe zu erfüllen.

Des Weiteren ist der Einflussfaktor von Relevanz, da die beiden wichtigen politischen Richtlinien 2009/30/EG (Kraftstoffqualitätsrichtlinie) und 2009/28/EG (Erneuerbare-Energien-Richtlinie) in Beziehung zueinander stehen. Um im Jahr 2020 im Vergleich zu 2010 6\% weniger Lebenszyklustreibhausgase je Energieeinheit Kraftstoff freizusetzen ${ }^{84}$ (RICHTLINIE 2009/30/EG ARTIKEL 7a), müssen, wie in RICHTLINIE 2009/28/EG (ARTIKEL 3 (4)) beschrieben, im Jahr 2020 10\% der eingesetzten Energie im Verkehrssektor aus erneuerbaren Energiequellen stammen und im Durchschnitt eine im Vergleich zu fossilem Kraftstoff 60-prozentige $\mathrm{CO}_{2}$-Reduktion erreichen.

Einflussfaktor

19b:

Beschreibung

19b:
Die maximal erlaubte prozentuale Beimischung von Ethanol zu Benzin

Der Einflussfaktor beschreibt die in Volumen ausgedrückte maximal erlaubte Beimischung von Ethanol zu Benzin in der EU27, die derzeitig 10\% vol beträgt (RICHTLINIE 2009/30/EG ANHANG I).

Der zukünftige Verlauf des Faktors wird in Volumenprozent angegeben.

${ }^{84}$ Basierend auf der Annahme, dass die politisch festgelegte prozentuale $\mathrm{CO}_{2}$-Reduktion im Kraftstoffbereich ausschließlich aus der Nutzung von erneuerbaren Energien erfolgt. 
Bedeutung des

Faktors 19b:
Name:

Lfd. Nr.:

Einflussbereich:

Beschreibung:

Bedeutung des Einflussfaktors:
Die Ethanolbeimischung zu Benzin ist für den Ethanolmarkt von großer Bedeutung, da die Beimischungsmenge annähernd der Gesamtnachfrage nach Ethanol entspricht.

Die maximal erlaubte Beimischung von derzeitig 10\% vol Ethanol zu Benzin (RICHTLINIE 2009/30/EG ANHANG I) begrenzt die Ethanolnachfrage in der EU27 in Abhängigkeit von der Höhe der Benzinnachfrage. Der Einflussfaktor wird zukünftig weiter an Bedeutung gewinnen, da die maximal mögliche Beimischung von Ethanol zu Benzin infolge des prognostizierten Rückgangs der Benzinnachfrage in der EU27 sinken wird (EUROPEAN COMMISSION 2012a: 95; EUROPEAN COMMISSION JOINT RESEARCH CENTRE, INSTITUT FOR ENERGY ET AL. 2011: 24). Die Abbildung 27 zeigt die maximale Beimischungsmenge von Ethanol zu Benzin in den Jahren von 2010 bis 2020 unter der Annahme der maximal erlaubten Beimischung von $10 \%$ vol Ethanol zu Benzin. Aus der Abbildung ist zu erkennen, dass die politische Vorgabe die Ethanolnachfrage in der EU27 im Jahr 2020 auf rund 10 Milliarden Liter begrenzt und dadurch ein zukünftiges Marktwachstum der Ethanolindustrie verhindert ${ }^{85}$. Eine Anhebung der erlaubten Beimischung auf $15 \%$ vol würde die Situation entspannen und ein weiteres potentielles Marktwachstum von 4,8 Milliarden Litern im Jahr 2020 ermöglichen.

Bestimmungen der Mitgliedsstaaten der EU27 zur Förderung von EthanolKraftstoff

20

Politik

Um in jedem Mitgliedsland der EU27 den in der RICHTLINIE 2009/28/EG (ARTIKEL 3 (4)) festgelegten prozentualen Anteil von erneuerbaren Energien am Gesamtenergieverbrauch des Verkehrsbereiches zu erfüllen ${ }^{86}$, fördern die Staaten über die Instrumente der Beimischungspflicht von Biokraftstoffen zu Fossilbrennstoff und/oder der Verbrauchssteuerbefreiung sowie -senkung die Nutzung von Ethanol in der EU27 (F.O. LICHT 2012d: 420f.).

Die zukünftige Entwicklung des Einflussfaktors wird mittels der von den Mitgliedsstaaten zur Förderung des Absatzes von Ethanol-Kraftstoff eingesetzten politischen Instrumente dargestellt.

Wie bereits in dem Feld „Beschreibung“ erläutert, fördert die Politik der EU27 Ethanol nicht direkt, sondern bestimmt in der RICHTLINIE 2009/EG/28 (ARTIKEL 3 (4)) lediglich, dass ein bestimmter Anteil der verbrauchten Gesamtener-

\footnotetext{
${ }^{85}$ Auch BLOOMBERG NEW ENERGY FINANCE (2010: 8) hat die Bedeutung des Einflussfaktors erkannt und ist der Auffassung, dass die erlaubte Beimischung erhöht werden muss, um ein zukünftiges starkes Wachstum des Ethanolmarktes zu ermöglichen.

${ }^{86}$ Genauere Informationen zu der RICHTLINIE 2009/EG/28 (ARTIKEL 3 (4)) befinden sich im Text zu Einflussfaktor 18a.
} 
gie im Verkehrsbereich aus erneuerbaren Ernergiequellen stammen muss derzeitig liegt die Vorgabe bei 10\% für das Jahr 2020. Diese Verordnung gilt für jedes Mitgliedsland und die Politik eines jeden Staates ist verpflichtet, in einem nationalen Aktionsplan darzulegen, wie sie diese Verordnung erfüllen will (RICHTLINIE 2009/28EG ARTIKEL 4 (1)).

Die Politik der einzelnen Mitgliedsstaaten ist von Relevanz, da sie entscheidet, welche erneuerbaren Energien durch politische Eingriffe gefördert werden. Somit erfolgt die derzeitige europaweite Förderung von Ethanol durch Quotensysteme und/oder die Verbrauchssteuerbefreiung sowie -senkung, da sich die Mitgliedsstaaten entschlossen haben, in dieser Form die politische Vorgabe der Nutzung von erneuerbaren Energien im Verkehrssektor zu erfüllen (F.O. LICHT 2012d: 420f.). Folglich können sich die Staaten der EU27 auch entscheiden, auf die politische Förderung von Ethanol-Kraftstoff $z u$ verzichten und stattdessen andere erneuerbare Energien wie z.B. die Nutzung von Elektrizität im Zug- und Automobilverkehr oder die Verwendung biogener Treibstoffe im Flugverkehr zu unterstützen. Dies hätte starke Auswirkungen auf den Ethanolmarkt der EU27, denn ohne die politische Förderung würde Ethanol auf dem europäischen Kraftstoffmarkt in direkter Konkurrenz zu fossilem Benzin stehen ${ }^{87}$.

Name:

Lfd. Nr.:

Einflussbereich:

Beschreibung:

Bedeutung des Einflussfaktors:
Bestimmungen der EU27 betreffend ILUC (Indirect Land Use Change)

21

Politik

Der Fachausdruck ILUC (Indirect Land Use Change) bezeichnet den Anbau von Pflanzen für die Produktion von Biokraftstoffen auf Ackerland, welches für die Produktion von Nahrungs- und Futtermitteln geeignet ist. Dadurch wird die Nahrungs- und Futtermittelproduktion auf Flächen verdrängt, die zuvor nicht zur landwirtschaftlichen Erzeugung genutzt wurden. Der Umbruch dieser Flächen zur Bewirtschaftung führt zu hohen Kohlenstoffdioxidemissionen, die den Biokraftstoffen angerechnet werden.

Die Höhe des ILUC-Faktors wird mittels der Einheit $\mathrm{gCO}_{2 \mathrm{eq}} / \mathrm{MJ}$ beschrieben.

Kernziel der EU27 ist es, durch die Verwendung von Kraftstoff-Ethanol den $\mathrm{CO}_{2}$-Ausstoß im Verkehrsbereich zu senken. Allerdings verschlechtert sich die $\mathrm{CO}_{2}$-Bilanz von Ethanol durch die Berücksichtigung eines ILUC-Faktors.

Die Höhe des ILUC-Faktors variiert, abhängig von den verwendeten Rohstoffen, die zur Ethanolproduktion genutzt werden. Die folgenden kulturspezifischen ILUC-Werte wurden von der EUROPÄISCHEN

\footnotetext{
${ }^{87}$ Beispielhaft für den Aufstieg und Fall eines Biokraftstoffs infolge der sich ändernden politischen Bestimmungen ist der B100-Markt in Deutschland. Der Markt ist im Jahr 1995 aufgrund der Steuerbegünstigungen von B100 im Vergleich zu fossilem Diesel entstanden und nach der Aufhebung dieser Begünstigungen im Jahr 2007 wieder beinahe vollständig verschwunden (UFOP 2011: 9).
} 
KOMMISSION (2012: 22) veröffentlicht und basieren auf einer Studie des IFPRI (2011: 13) (Tabelle 8).

\begin{tabular}{l|c}
\multicolumn{1}{c|}{ Rohstoffe } & \multicolumn{2}{c}{$\mathbf{g C O}_{\mathbf{2 e q}} / \mathbf{M J}$} \\
\hline Getreide und sonstige stärkehaltige Pflanzen & 12 \\
\hline Zuckerpflanzen & 13 \\
\hline Ölpflanzen & 55
\end{tabular}

Tabelle 8: Kulturspezifische ILUC-Werte

Quelle: EUROPÄISCHE KOMMISSION 2012: 2

Die Bedeutung des ILUC-Faktors zeigt sich beispielsweise an dem Rohstoff Weizen - dem wichtigsten Rohstoff zur Ethanolproduktion in der EU27 (EUROPEAN COMMISSION JOINT RESEARCH CENTRE, INSTITUT FOR ENERGY ET AL. 2010:74). Der ILUC-Faktor für Weizen beträgt $12 \mathrm{gCO}_{2 \mathrm{eq}} / \mathrm{MJ}$ (Tabelle 8); dies bedeutet, dass bei der Produktion von Ethanol aus Weizen unter Berücksichtigung des ILUC-Faktors $12 \mathrm{gCO}_{2 \mathrm{eq}} / \mathrm{MJ}$ mehr Kohlenstoffdioxid ausgestoBen werden als ohne ILUC-Faktor. Durch die Berücksichtigung des ILUCFaktors verschlechtert sich die $\mathrm{CO}_{2}$-Bilanz so stark, dass Weizen-Ethanol nicht mehr die derzeitige politische Vorgabe einer $35 \% \mathrm{CO}_{2}$-Einsparung ${ }^{88} \mathrm{im}$ Vergleich zu fossilem Kraftstoff erfüllt ${ }^{89}$. Folglich darf es nicht der Gruppe „erneuerbare Energien“ angerechnet werden und erhält somit keine politische Unterstützung mehr.

Ethanol aus der Zuckerrübe erreicht trotz ILUC-Faktor noch eine 35\% $\mathrm{CO}_{2}$ Reduktion, aber nicht mehr die ab 2017 geforderte $\mathrm{CO}_{2}$-Einsparung von 50\% ${ }^{90}$

${ }^{88}$ Die Vorgaben für eine politische Förderung von Biokraftstoffen hinsichtlich der $\mathrm{CO}_{2}$ Einsparung im Vergleich zu fossilem Kraftstoff sind im Text zu Einflussfaktor 18b erläutert.

${ }^{89}$ Folgendes Beispiel zeigt die Bedeutung des ILUC-Faktors für die Ethanolproduktion aus Weizen. Weizen-Rechnung 1 erfolgt ohne ILUC-Faktor und Weizen-Rechnung 2 mit ILUCFaktor (RICHTLINIE 2009/28/EG ANHANG V (4); RICHTLINIE 2009/28/EG ANHANG V (19); EUROPÄISCHE KOMMISSION 2012: 22):

\section{Weizen-Rechnung 1:}

$\mathrm{E}_{\mathrm{B} 1}$ : Ethanol aus Weizen (Erdgas als Prozessbrennstoff in konventioneller Anlage) 46 $\mathrm{gCO}_{2 \mathrm{eq}} / \mathrm{MJ}$

$\mathrm{E}_{\mathrm{F}}$ : Gesamtemissionen des Komparators für Fossilbrennstoffe $83,8 \mathrm{gCO}_{2 \mathrm{eq}} / \mathrm{MJ}$

Einsparung $=\left(E_{F}-E_{B 1}\right) / E_{F}=(83,8-46) / 83,8=45 \%>35 \% \rightarrow$ Auflage der RICHTLINIE 2009/28/EG ARTIKEL 17 (2) erfüllt.

\section{Weizen-Rechnung 2:}

$\mathrm{E}_{\mathrm{B} 2}$ : Ethanol aus Weizen (Erdgas als Prozessbrennstoff in konventioneller Anlage) 46 $\mathrm{gCO}_{2 \mathrm{eq}} / \mathrm{MJ}+$ ILUC-Faktor $12 \mathrm{gCO}_{2 \mathrm{eq}} / \mathrm{MJ}=58 \mathrm{gCO}_{2 \mathrm{eq}} / \mathrm{MJ}$

$\mathrm{E}_{\mathrm{F}}$ : Gesamtemissionen des Komparators für Fossilbrennstoffe $83,8 \mathrm{gCO}_{2 \mathrm{eq}} / \mathrm{MJ}$

Einsparung $=\left(E_{\mathrm{F}}-\mathrm{E}_{\mathrm{B} 2}\right) / \mathrm{E}_{\mathrm{F}}=(83,8-58) / 83,8=31 \%<35 \% \rightarrow$ Auflage der RICHTLINIE 2009/28/EG ARTIKEL 17 (2) nicht erfüllt.

${ }^{90}$ Folgende Berechnung zeigt die Bedeutung des ILUC-Faktors für die Ethanolproduktion aus Zuckerrüben. Zuckerrüben-Rechnung 1 erfolgt ohne ILUC-Faktor und ZuckerrübenRechnung 2 mit ILUC Faktor (RICHTLINIE 2009/28/EG ANHANG V (4); RICHTLINIE 2009/28/EG ANHANG V (19); EUROPÄISCHE KOMMISSION 2012: 22): 
(RICHTLINIE 2009/28/EC ARTIKEL 17 (2)). Dementsprechend würde auch Zuckerrüben-Ethanol ab 2017 nicht mehr politisch gefördert werden.

Zusammenfassend ist festzuhalten, dass durch die Einführung eines ILUCFaktors Ethanol-Kraftstoffe der ersten Generation langfristig nicht mehr die politisch geforderten $\mathrm{CO}_{2}$-Einsparungen im Vergleich $\mathrm{zu}$ fossilem Kraftstoff erreichen. Dementsprechend werden sie nicht mehr politisch gefördert und stehen auf dem Kraftstoffmarkt in direkter Konkurrenz zu fossilem Benzin, wodurch die Nachfrage nach Ethanol stark sinken wird.

Name:

Lfd. Nr.:

Einflussbereich:

Beschreibung:

Bedeutung des Einflussfaktors:
Zölle auf Ethanolimporte in die EU27

22

Politik

Es wird die Höhe der Zölle für Ethanolimporte in die EU27 betrachtet und mittels der Einheit Euro je Hektoliter beschrieben. Die Importzölle werden derzeit per Verordnung von der Europäischen Kommission festgelegt und sind im KNCode 2207 beschrieben (EUROPÄISCHE KOMMISSION 2011: 182).

Die EU27 erhebt im Gegensatz zu den USA und Brasilien hohe Importzölle auf Ethanol (F.O. LICHT 2012b: 169). Diese schützen die europäische Produktion, da die Herstellungskosten von Ethanol in den USA und Brasilien im Vergleich zur EU27 deutlich geringer sind (BERG 2012d: 2f.).

Das Jahr 2011 hat beispielhaft die Notwendigkeit der Importzölle für die europäischen Ethanolproduzenten gezeigt: Die USA exportierten 2011 eine Milliarde Liter Ethanol in die EU27, den Hauptteil als E90 ${ }^{91}$ (90\% Ethanol und $10 \%$ Benzin), um einen geringeren Importzoll an die EU27 zu entrichten. Infolge dessen sank der europäische Ethanolpreis und zwang mehrere Ethanolproduzenten der EU27 die Produktion auszusetzen, da die Produktionsanlagen nicht mehr kostendeckend betrieben werden konnten (F.O.

\section{Zuckerrüben-Rechnung 1:}

$\mathrm{E}_{\mathrm{B} 1}$ : Ethanol aus Zuckerrüben $33 \mathrm{gCO}_{2 \mathrm{eq}} / \mathrm{MJ}$

$\mathrm{E}_{\mathrm{F}}$ : Gesamtemissionen des Komparators für Fossilbrennstoffe $83,8 \mathrm{gCO}_{2 \mathrm{eg}} / \mathrm{MJ}$

Einsparung $=\left(E_{F}-E_{B 1}\right) / E_{F}=(83,8-33) / 83,8=61 \%>50 \% \rightarrow$ Auflage der RICHTLINIE 2009/28/EG ARTIKEL 17 (2) für das Jahr 2017 erfüllt.

\section{Zuckerrüben-Rechnung 2:}

$\mathrm{E}_{\mathrm{B} 2}$ : Ethanol aus Zuckerrüben $33 \mathrm{gCO}_{2 \mathrm{eq}} / \mathrm{MJ}+\mathrm{ILUC}$-Faktor $13 \mathrm{gCO}_{2 \mathrm{eq}} / \mathrm{MJ}=46 \mathrm{gCO}_{2 \mathrm{eq}} / \mathrm{MJ}$ $\mathrm{E}_{\mathrm{F}}$ : Gesamtemissionen des Komparators für Fossilbrennstoffe $83,8 \mathrm{gCO}_{2 e \mathrm{e}} / \mathrm{MJ}$

Einsparung $=\left(\mathrm{E}_{\mathrm{F}}-\mathrm{E}_{\mathrm{B} 2}\right) / \mathrm{E}_{\mathrm{F}}=(83,8-46) / 83,8=45 \%<50 \% \rightarrow$ Auflage der RICHTLINIE 2009/28/EG ARTIKEL 17 (2) für das Jahr 2017 nicht erfüllt.

${ }^{91}$ Die Ethanolimporte als E90 wurden 2011 lediglich unter KN-Code 38249097 mit einem Wertzollsatz von 6,5\% verzollt (F.O. LICHT 2012a: 303; USDA FOREIGN AGRICULTURAL SERVICE 2012: 16). Dieser liegt deutlich unter den regulären Zollsätzen von $19,2 € / h$ für unvergälltes Ethanol und 10,2 $€ / h$ lür vergälltes Ethanol (EUROPÄISCHE KOMMISSION 2011: 182). Die EU27 hat 2012 reagiert und die Zollbestimmungen angepasst, um einen weiteren Import von E90 zu geringeren Zollsätzen zu verhindern (F.O. LICHT 2012a: 303). 
LICHT 2011b: 66; USDA FOREIGN AGRICULTURAL SERVICE 2011: 19). Das Beispiel zeigt, dass eine Senkung oder Aufhebung der Importzölle die europäische Ethanolproduktion unter starken Kostendruck setzen würde.

\section{Gesellschaft}

Name:

Tank-oder-Teller-Diskussion in der EU27

Lfd. Nr.:

23

Einflussbereich:

Gesellschaft

Beschreibung:

In der Tank-oder-Teller-Diskussion geht es um die Nutzung landwirtschaftlicher Rohstoffe zur Produktion von Biokraftstoffen anstatt der Verwendung als Nahrungs- oder Futtermittel (INTERNATIONAL ENERGY AGENCY 2011b: 40). Bei der Betrachtung des Faktors wird ausschließlich die Intensität der Diskussion beschrieben und nicht, ob die Diskussion wissenschaftlich gerechtfertigt ist.

Zur Messung der Entwicklung des Einflussfaktors wird die Ist-Situation als Referenzsituation beschrieben und davon ausgehend begründet, warum in Zukunft die Stärke der Diskussion zunimmt, abnimmt oder konstant bleibt.

Bedeutung des Die Tank-oder-Teller-Diskussion ist von Relevanz, da sie die politische Förderung von Biokraftstoffen beeinflusst. Die Debatte verläuft kontrovers und Ethanol als bedeutendster Biokraftstoff der Welt steht in ihrem Zentrum (INTERNATIONAL ENERGY AGENCY 2011b: 40; OECD/FAO 2012: 89) ${ }^{92}$. Die Tank-oder-Teller-Diskussion wurde bereits in den Jahren 2007/2008 und 2012 intensiv geführt. Im Jahr 2007/2008 sorgten die stark ansteigenden Agrarpreise nach einer Periode niedriger Rohstoffkosten für eine Tank-oder-TellerDiskussion; verstärkt unter anderem durch eine Veröffentlichung der WORLD BANK DEVELOPMENT PROSPECTS GROUP (2008), die den Preisanstieg der Agrarrohstoffe im Wesentlichen auf das Wachstum der Produktion von Biokraftstoffen zurückführte ${ }^{93}$. Infolge der Diskussion wurden zahlreiche Nachhaltigkeitsvorschriften in die europäischen Richtlinien zur Förderung von Biokraftstoffen aufgenommen (F.O. LICHT/AGRA INFORMA 2009: 159). Im Jahr 2012 kam es in der EU27 erneut zu einer starken Tank-oder-TellerDiskussion, denn nach der Trockenheit im Sommer 2012 waren in den USA die Agrarpreise angestiegen. Die geführte Debatte ist - neben der ILUC-Diskussion - ein Grund, warum die Kommissare Günther Oettinger und Connie Hedegard im Oktober 2012 vorschlugen, die Beimischung von Biokraftstoffen der ersten

\footnotetext{
${ }^{92}$ Beispielhaft für die kontroverse Diskussion ist der Titel der „Bild“-Zeitung vom 1. April 2011 „Neuer E10-Irrsinn - Öko-Sprit macht Lebensmittel teurer!“" gefolgt von einer ganzen Seite, auf der unter der Überschrift „Wahnsinn E10! So macht die Öko-Plörre unsere Lebensmittel teurer" dem Leser erklärt wird, dass Ethanol die Preise für Lebensmittel erhöht (BILD 2011). ${ }^{93} \mathrm{Im}$ Jahr 2010 korrigierte die WORLD BANK DEVELOPMENT PROSPECTS GROUP (2010: 2) ihre Aussage und erkannte, dass sie den Einfluss der Biokraftstoffe auf den Preisanstieg der Agrarrohstoffe zu stark bewertet hatte.
} 
Generation auf 5\% zu begrenzen (EUROPÄISCHE KOMMISSION 2012: 16) und ab 2021 lediglich Biokraftstoffe der zweiten Generation politisch zu fördern (EUROPÄISCHE KOMMISSION 2012: 3). Werden die Vorschläge in dieser Form umgesetzt, werden sie die Zukunft des Marktes weitreichend beeinflussen.

Schon im Jahr 2011 warnte die OECD/FAO (2011: 87) davor, dass bei einem weiteren Wachstum des Biokraftstoffmarktes die Tank-oder-Teller-Diskussion zunehmen würde und die Politik zu Kürzungen der Förderung von Biokraftstoffen bewegen könnte.

Name:

Lfd. Nr.:

Einflussbereich:

Beschreibung:

Bedeutung des

Einflussfaktors:
Diskussion in der EU27 über die Auswirkungen von Ethanol auf die Umwelt

24

Gesellschaft

Bei diesem Einflussfaktor werden die Richtung ${ }^{94}$ und die Intensität der Diskussion in der EU27 über die Auswirkungen von Ethanol auf die Umwelt betrachtet. Ob die Debatte wissenschaftlich korrekt ist, wird nicht begutachtet.

Um die zukünftige Diskussion darzustellen, wird die Ist-Situation als Referenzsituation beschrieben. Davon ausgehend werden unterschiedliche Projektionen gebildet, die aufzeigen, wie sich die Diskussion in Richtung und Intensität im Vergleich zur Ist-Situation verändert hat.

Die gesellschaftliche Diskussion über die Auswirkungen von Ethanol auf die Umwelt ist von Bedeutung, denn das Kernziel der politischen Förderung von erneuerbaren Energien im Verkehrsbereich und damit auch von Ethanol ist die $\mathrm{CO}_{2}$-Reduktion und damit der Umweltschutz (RICHTLINIE 2009/28/EG ERW 1). Folglich könnte eine positive Debatte - „Ethanol schützt die Umwelt“ - wie auch eine negative Diskussion - „Ethanol schadet dem Klima“ - die politische Einstellung zu Ethanol-Kraftstoff beeinflussen und Folgen für die zukünftige politische Förderung von Biokraftstoffen haben. Schon die Tank-oder-TellerDiskussion im Jahr 2007/2008 und im Sommer 2012 hat gezeigt, welch starken Einfluss eine gesellschaftliche Diskussion auf die politische Ausgestaltung der Förderung von Biokraftstoffen in der EU27 haben kann (Einflussfaktor 23).

Darüber hinaus beeinflussen Richtung und Intensität der Diskussion auch die Einstellung der Verbraucher zu Ethanol. So könnte eine positive Diskussion den Ethanolabsatz fördern, da sich mehr Autofahrer entscheiden würden, Benzin mit höheren Ethanolbeimischungen zu tanken, um die Umwelt zu schützen. Dementsprechend könnte aber auch eine negative Diskussion den Ethanolabsatz hemmen.

${ }^{94}$ Durch „Richtung“ wird ausgedrückt, ob die gesellschaftliche Diskussion die Wirkung von Ethanol-Kraftstoff auf die Umwelt positiv, negativ oder neutral betrachtet. 
Name:

Lfd. Nr.:

Einflussbereich:

Beschreibung:

Bedeutung des

Einflussfaktors:
Diskussion in der EU27 über den Nutzen von Ethanol zur Förderung der Energieunabhängigkeit der EU27

25

\section{Gesellschaft}

Bei diesem Einflussfaktor werden die Richtung und die Intensität der Diskussion in der EU27 über den Nutzen von Ethanol-Kraftstoff zur Förderung der Energieunabhängigkeit der EU27 begutachtet. Die wissenschaftliche Korrektheit der Debatte wird nicht betrachtet.

Die Entwicklung der Debatte wird durch eine Beschreibung der Ist-Situation dargestellt, um davon ausgehend Zukunftsprojektionen des Einflussfaktors zu bilden.

Der Einflussfaktor ist von Relevanz, da sowohl eine positive - „Ethanol fördert die Energieunabhängigkeit“ - als auch eine negative - „Ethanol fördert nicht die Energieunabhängigkeit“ - gesellschaftliche Diskussion die Einstellung der Politiker und damit auch die politische Förderung von Ethanol beeinflussen könnte. Die Reduzierung der Abhängigkeit von Energieimporten ist einer der Gründe, warum erneuerbare Energien im Verkehrsbereich in der EU27 politisch gefördert werden (RICHTLINIE 2009/28/EG ERW 1). Der Faktor ist des Weiteren bedeutend, da die Debatte auch die Einstellung der Verbraucher gegenüber Ethanol und damit ihre Entscheidung, Benzin mit einer höheren oder geringeren Ethanolbeimischung an der Zapfsäule zu tanken, beeinflussen könnte.

Das Thema Energieunabhängigkeit ist für die EU27 von hoher Relevanz, da im Jahr 2012 85\% (3,8 Milliarden Barrel) des verbrauchten Rohöls aus dem Ausland in die EU27 importiert wurden (EUROPEAN COMMISSION 2013a: 67); dies entspricht einem monetären Wert von 421 Milliarden US-\$ (EUROPÄISCHE KOMMISSION 2013). In Abbildung 28 ist die Herkunft des importierten Rohöls der EU27 aus dem Jahr 2012 grafisch dargestellt. Dabei ist anzumerken, dass manche Exportstaaten aufgrund ihrer politisch instabilen Lage oder diktatorischen Regimes hinsichtlich ihrer langfristigen Liefersicherheit als kritisch zu betrachten sind, was die Notwendigkeit der Förderung von heimischen erneuerbaren Energien verstärkt 


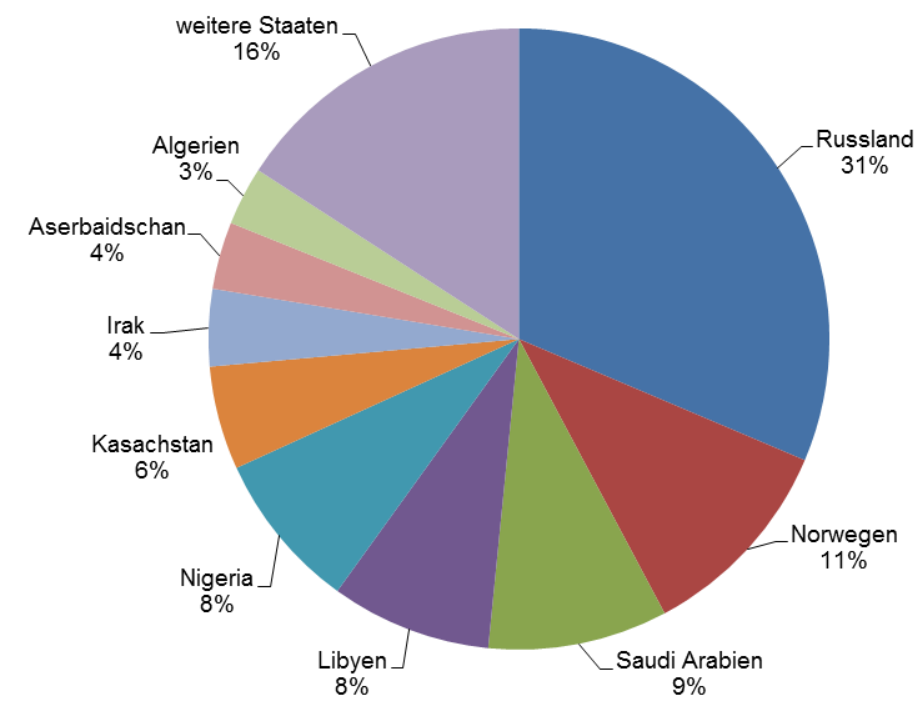

Abbildung 28: Herkunft der Rohölimporte in die EU27

Quelle: In Anlehnung an EUROPÄISCHE KOMMISSION 2013

In der folgenden Abbildung 29 sind nochmals alle beschreibenden und wirkenden Einflussfaktoren des Ethanolmarktes der EU27 zusammenfassend dargestellt. 


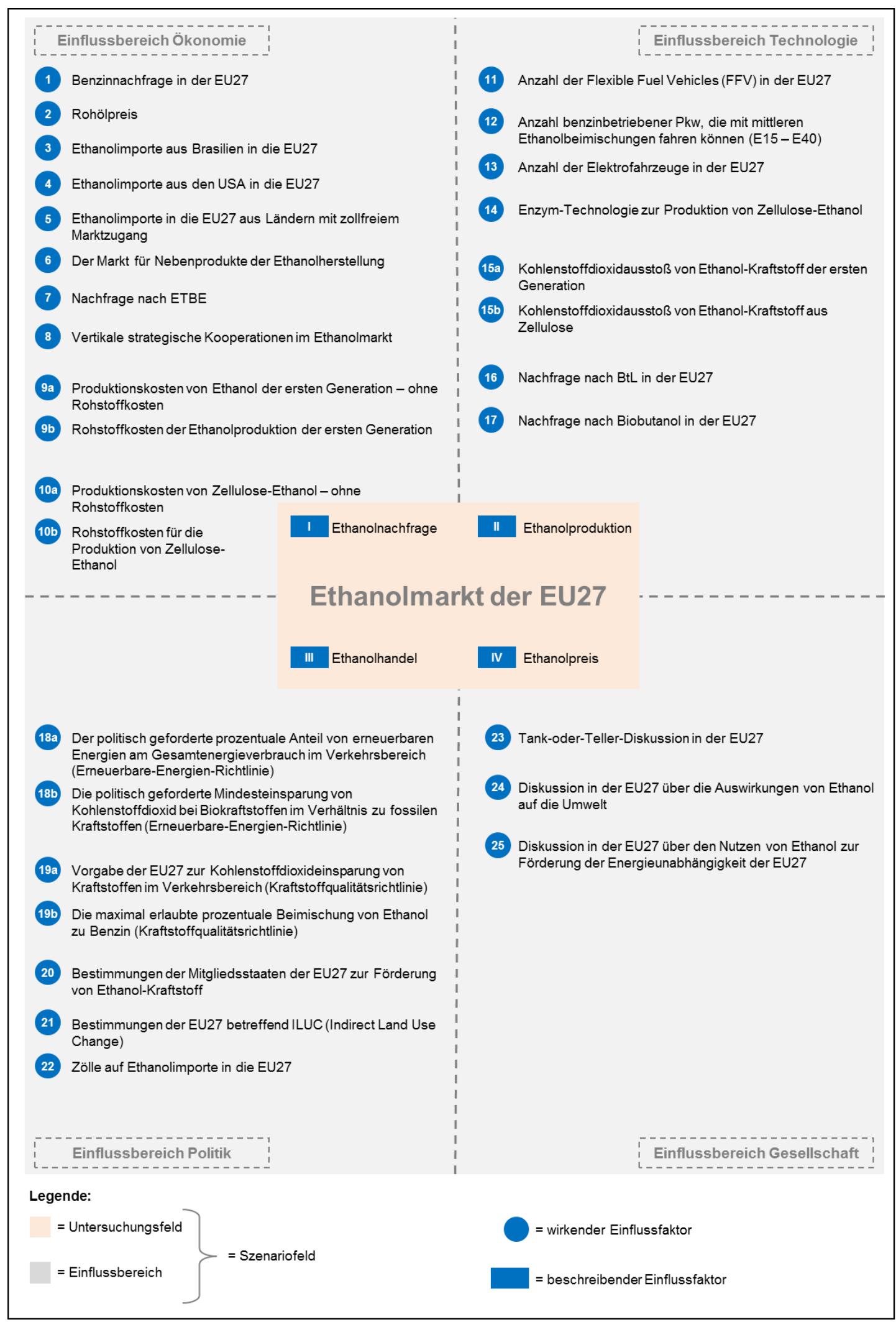

Abbildung 29: Zusammenfassung der beschreibenden und wirkenden Einflussfaktoren des EU27-Szenariofeldes

Quelle: EIGENE DARSTELLUNG 


\subsubsection{EU27-Wirkungsanalyse}

Im zweiten Schritt der EU27-Szenariofeld-Analyse werden mittels der Wirkungsanalyse $^{95}$ - Durchführung der Wirkungsanalyse (Kapitel 5.1.2.1) und Auswertung der Wirkungsanalyse (Kapitel 5.1.2.2) - die wirkenden Schlüsselfaktoren identifiziert, die den Ethanolmarkt der EU27 bis zum Jahr 2023 am stärksten beeinflussen werden.

\subsubsection{Durchführung der Wirkungsanalyse}

Zur Messung der Stärke der Beeinflussung der einzelnen wirkenden Einflussfaktoren auf den Ethanolmarkt der EU27 wurde ein standardisierter schriftlicher Fragebogen entwickelt. Dieser befindet sich in Anhang 2 und gliedert sich in drei Abschnitte:

1. Einführung in die Thematik

2. Beurteilung der wirkenden Einflussfaktoren

3. Erhebung der persönlichen Daten der Probanden

Im Anschluss an eine Erklärung, dass es sich um eine wissenschaftliche Studie handelt, wurde den Experten im ersten Abschnitt das Ziel des Fragebogens sowie der Dissertation erläutert. Danach wurde ihnen dargelegt, wie sie den Fragebogen zu beantworten haben, und ausdrücklich darauf hingewiesen, dass die Antworten anonymisiert ausgewertet werden.

Der zweite Abschnitt stellte den Kernteil der Befragung dar. Hier erfolgte durch die Probanden die Beurteilung der Wirkungsstärke der einzelnen Faktoren, die den Ethanolmarkt der EU27 beeinflussen. Die wirkenden Einflussfaktoren waren nach Einflussbereichen, zu denen sie gehören, in der Reihenfolge Ökonomie, Technologie, Politik und Gesellschaft geordnet. Jeder Einflussfaktor wurde benannt und, falls notwendig, durch eine kurze Beschreibung charakterisiert. Unterhalb jedes Einflussfaktors befand sich eine siebenstufige Likert-Skala ${ }^{96}$, anhand derer die Befragten die Wirkungsstärke des Faktors auf den Ethanolmarkt der EU27 bewerteten. Zum Abschluss des Fragebogens wurden im dritten Abschnitt die Experten nach dem Herkunftsland und der Branche ihres Unternehmens bzw. ihrer Organisation befragt sowie gebeten, ihre Kontaktdaten anzugeben, damit innen die Ergebnisse der Befragung zugeschickt werden konnten.

\footnotetext{
${ }^{95}$ Eine ausführliche methodische Beschreibung der Wirkungsanalyse befindet sich in Kapitel 4.2.2.

${ }^{96}$ Es wird mit einer siebenstufigen Likert-Skala mit der Abstufung „sehr starker Einfluss“; „,starker Einfluss“; „eher starker Einfluss“; „moderater Einfluss“; „eher schwacher Einfluss“; „schwacher Einfluss“ und „sehr schwacher Einfluss“ gearbeitet, um eine einheitliche Datengewinnung und eine Vergleichbarkeit der Ergebnisse zu ermöglichen.
} 
Vor dem Start der Erhebung wurde der Fragebogen durch Pretests mit Experten ${ }^{97}$ des Ethanolmarktes überprüft. Durch die Tests konnten missverständliche Beschreibungen einzelner Einflussfaktoren identifiziert werden sowie die Fragestellung auf Eindeutigkeit und die Likert-Skale auf ihre Eignung als Messinstrument überprüft werden. Darüber hinaus gaben die Pretests Aufschluss über die für den Fragebogen benötigte Bearbeitungszeit. Die erkannten Mängel wurden vor der Datenerhebung behoben.

Die Probanden waren ausschließlich Experten, die sich hauptberuflich mit dem Ethanolmarkt der EU27 beschäftigten und zum Teil maßgeblich die Zukunft des europäischen Ethanolmarktes beeinflussen werden. Um Experten aus allen Bereichen des Ethanolmarktes in die Erhebung zu integrieren, erfolgte die Datenerhebung sowohl auf der „World Biofuels 2012“98-Konferenz vom 9. bis zum 10. Mai 2012 in Sevilla (Spanien) als auch durch eine Onlinebefragung im Juni 2012. Zur Gewährleistung der Teilnahme möglichst vieler Experten an der Befragung, unabhängig von ihrem Herkunftsland, war der Fragebogen in englischer Sprache verfasst. Die Befragung wurde auf der „World Biofuels 2012“-Konferenz durchgeführt, da an dieser Konferenz Fachleute des europäischen Ethanolmarktes teilnahmen, die aus verschiedenen Staaten Europas und den USA stammten sowie für unterschiedliche Industrien, Politik und Forschungseinrichtungen arbeiteten. Um die Wertigkeit der Fragebögen zu erhöhen, wurde jeder Fragebogen in eine Mappe geheftet. Auf der Konferenz wurde diese im Anschluss an eine kurze gegenseitige Vorstellung an den jeweiligen Experten übergeben. Die Beantwortung des Fragebogens erfolgte eigenständig und die Mappen wurden zu einem späteren Zeitpunkt wieder zurückgegeben. Jenen Experten, die bis kurz vor Ende der Konferenz den Fragebogen noch nicht zurückgegeben hatten, wurde ein beschrifteter Briefumschlag übergeben, um sowohl die Rücksendung zu vereinfachen als auch - und viel wichtiger - um sie höflich daran zu erinnern, den Fragebogen noch vor Ende der Konferenz auszufül$\operatorname{len}^{99}$. Da die Experten gerne als Probanden fungierten, bestand keine Notwendig-

\footnotetext{
${ }^{97}$ Pretests zur Überprüfung des Fragebogens wurden durchgeführt mit: Dr. Christoph Berg F.O. Licht, Dr. Marco Veselka - CropEnergies AG, Dr. Hinrich Harling - KWS SAAT AG und Dr. Andreas von Felde - KWS SAAT AG.

${ }^{98}$ Besonderer Dank gilt Herrn Dr. Christoph Berg von F.O. Licht, der es ermöglicht hat, die Befragung während der Konferenz durchzuführen. Die Konferenz wurde organisiert von F.O. Licht und Abengoa Bioenergy.

${ }_{99}$ Das Vorgehen, zu Beginn der Befragung die wichtigsten und einflussreichsten Experten der Konferenz zu bitten, den Fragebogen auszufüllen, damit die anderen Fachleute sähen, wie diese an der Befragung teilnahmen, um so deren Bereitschaft zur Teilnahme zu steigern, hat sich als erfolgreich herausgestellt.
} 
keit, ein Incentive zu verteilen. Es wurde lediglich angeboten, ihnen die Ergebnisse der Befragung zukommen zu lassen.

Um noch weitere Experten zu befragen, insbesondere solche, die sich während der „F.O. Licht World Ethanol \& Biofuels“-Konferenz ${ }^{100}$ und des Fachkongresses „Kraftstoffe der Zukunft 2012“"101 bereit erklärt hatten, an der Befragung teilzunehmen, wurde der bereits bestehende Fragebogen mittels der Software des Anbieters Onlineumfragen.com in, was Inhalt und Struktur betrifft, identischer Form als Onlinefragebogen erstellt. Die befragten Experten erhielten per E-Mail ein kurzes persönliches Anschreiben, in dem an das Treffen auf der Konferenz erinnert wurde, sowie einen Link, um den Fragebogen zu starten. Da es sich um den identischen Fragebogen handelte, der beide Male selbstständig von den Experten ausgefüllt wurde, wurden die Ergebnisse aus der schriftlichen Befragung und der Onlinebefragung gemeinsam ausgewertet.

\subsubsection{Auswertung der Wirkungsanalyse}

Im Rahmen der Konferenz „World Biofuels 2012“ in Sevilla (Spanien) wurden 47 der insgesamt 79 Experten des Ethanolmarktes der EU27 befragt. Die Datenerhebung 32 weiterer Experten erfolgte mittels des Onlinefragebogens. 61 der Probanden arbeiteten für Unternehmen/Organisationen aus der EU27, die im Wesentlichen aus Deutschland, Großbritannien, Spanien und Belgien stammten. Weitere 11 Probanden waren bei Unternehmen/Organisationen aus dem außereuropäischen Ausland beschäftigt (Abbildung 30).

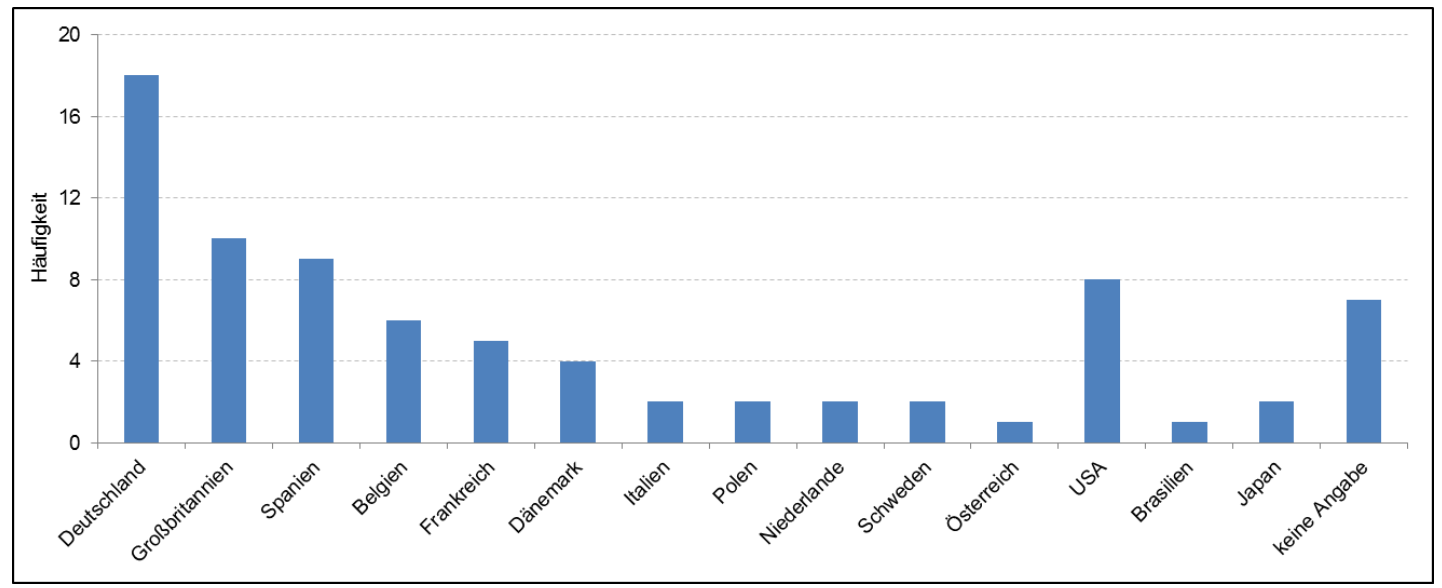

Abbildung 30: Wirkungsanalyse EU27 - Herkunftsland der Unternehmen/Organisationen der befragten Experten

Quelle: EIGENE DARSTELLUNG

${ }^{100}$ Die Konferenz fand vom 7. bis 10. November 2011 in Barcelona, Spanien statt.

${ }^{101}$ Der Fachkongress fand am 23. und 24. Januar 2012 in Berlin statt. 
Die befragten Experten kamen sowohl aus allen wichtigen Industriezweigen des Ethanolmarktes, wie beispielsweise der Ethanolindustrie, der Mineralölindustrie und der Enzymtechnologie, als auch aus politischen Institutionen der Mitgliedsstaaten und den Generaldirektionen der Europäischen Kommission (Abbildung 31).

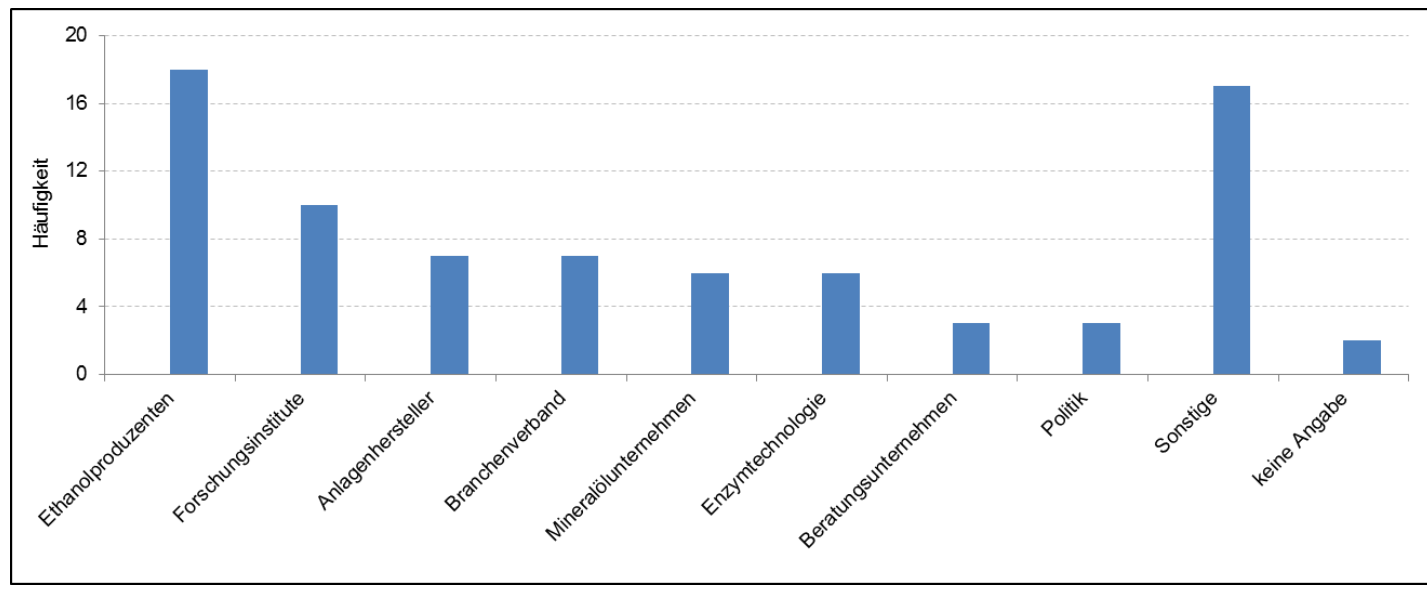

Abbildung 31: Wirkungsanalyse EU27 - Herkunftsbranche der Unternehmen/Organisationen der befragten Experten

Quelle: EIGENE DARSTELLUNG

In der folgenden Tabelle 9 finden sich alle wirkenden Einflussfaktoren, abfallend geordnet nach der Stärke ihres Einflusses auf den Ethanolmarkt der EU27 bis zum Jahr 2023. Die Stärke der Wirkung jedes Einflussfaktors ist anhand des Mittelwertes $^{102}$ zu erkennen: Je höher der Mittelwert, desto stärker wirkt der Einflussfaktor nach Ansicht der Befragten auf den Ethanolmarkt der EU27.

\begin{tabular}{|c|c|c|c|c|c|}
\hline $\begin{array}{l}\text { Einfluss- } \\
\text { faktor }\end{array}$ & & $\mathbf{n}$ & $\begin{array}{l}\text { Mittel- } \\
\text { wert }\end{array}$ & $\begin{array}{l}\text { Standard- } \\
\text { abweichung }\end{array}$ & $\begin{array}{l}\text { Einfluss- } \\
\text { bereich }\end{array}$ \\
\hline $18 \mathrm{a}$ & $\begin{array}{l}\text { Der politisch geforderte prozentuale Anteil } \\
\text { von erneuerbaren Energien am Gesamt- } \\
\text { energieverbrauch im Verkehrsbereich }\end{array}$ & 62 & 6,11 & 1,026 & Politik \\
\hline $19 b$ & $\begin{array}{l}\text { Die maximal erlaubte prozentuale Beimi- } \\
\text { schung von Ethanol zu Benzin }\end{array}$ & 62 & 5,94 & 1,143 & Politik \\
\hline 20 & $\begin{array}{l}\text { Bestimmungen der Mitgliedsstaaten der } \\
\text { EU27 zur Förderung von Ethanol-Kraftstoff }\end{array}$ & 68 & 5,84 & 1,128 & Politik \\
\hline $9 b$ & $\begin{array}{l}\text { Rohstoffkosten der Ethanolproduktion der } \\
\text { ersten Generation }\end{array}$ & 70 & 5,81 & 1,171 & Ökonomie \\
\hline $19 a$ & $\begin{array}{l}\text { Vorgabe der EU27 zur Kohlenstoffdioxid- } \\
\text { einsparung von Kraftstoffen im Verkehrs- } \\
\text { bereich }\end{array}$ & 64 & 5,61 & 1,002 & Politik \\
\hline 22 & Zölle auf Ethanolimporte in die EU27 & 67 & 5,36 & 1,334 & Politik \\
\hline $18 b$ & $\begin{array}{l}\text { Die politisch geforderte Mindesteinsparung } \\
\text { von Kohlenstoffdioxid bei Biokraftstoffen im } \\
\text { Verhältnis zu fossilen Kraftstoffen }\end{array}$ & 64 & 5,33 & 1,169 & Politik \\
\hline 21 & $\begin{array}{l}\text { Bestimmungen der EU27 betreffend ILUC } \\
\text { (Indirect Land Use Change) }\end{array}$ & 68 & 5,1 & 1,384 & Politik \\
\hline
\end{tabular}

\footnotetext{
${ }^{102}$ Wirkungsstärke der Mittelwerte auf den Ethanolmarkt der EU27: $7=$ „sehr starker Einfluss“; 6 = „starker Einfluss“; 5 = „eher starker Einfluss“; 4 = „moderater Einfluss“; 3 = „,eher schwacher Einfluss“; 2 = „schwacher Einfluss“ und 1 = „sehr schwacher Einfluss“.
} 


\begin{tabular}{|c|c|c|c|c|c|}
\hline 2 & Rohölpreis & 79 & 5,05 & 1,319 & Ökonomie \\
\hline 12 & $\begin{array}{l}\text { Anzahl benzinbetriebener Pkw, die mit } \\
\text { mittleren Ethanolbeimischungen fahren } \\
\text { können (E15-E40) }\end{array}$ & 76 & 5,01 & 1,553 & Technologie \\
\hline 1 & Benzinnachfrage in der EU27 & 79 & 4,97 & 1,664 & Ökonomie \\
\hline $10 \mathrm{~b}$ & $\begin{array}{l}\text { Rohstoffkosten für die Produktion von } \\
\text { Zellulose-Ethanol }\end{array}$ & 70 & 4,94 & 1,735 & Ökonomie \\
\hline $15 a$ & $\begin{array}{l}\text { Kohlenstoffdioxidausstoß von Ethanol- } \\
\text { Kraftstoff der ersten Generation }\end{array}$ & 77 & 4,88 & 1,298 & Technologie \\
\hline 6 & $\begin{array}{l}\text { Der Markt für Nebenprodukte der Ethanol- } \\
\text { herstellung }\end{array}$ & 76 & 4,67 & 1,482 & Ökonomie \\
\hline 4 & Ethanolimporte aus den USA in die EU27 & 79 & 4,53 & 1,299 & Ökonomie \\
\hline $10 \mathrm{a}$ & $\begin{array}{l}\text { Produktionskosten von Zellulose-Ethanol - } \\
\text { ohne Rohstoffkosten }\end{array}$ & 70 & 4,51 & 1,767 & Ökonomie \\
\hline $9 a$ & $\begin{array}{l}\text { Produktionskosten von Ethanol der ersten } \\
\text { Generation - ohne Rohstoffkosten }\end{array}$ & 69 & 4,43 & 1,334 & Ökonomie \\
\hline 14 & $\begin{array}{l}\text { Enzym-Technologie zur Produktion von } \\
\text { Zellulose-Ethanol }\end{array}$ & 76 & 4,42 & 1,659 & Technologie \\
\hline 3 & Ethanolimporte aus Brasilien in die EU27 & 79 & 4,34 & 1,3 & Ökonomie \\
\hline $15 b$ & $\begin{array}{l}\text { Kohlenstoffdioxidausstoß von Ethanol- } \\
\text { Kraftstoff aus Zellulose }\end{array}$ & 75 & 4,31 & 1,627 & Technologie \\
\hline 11 & $\begin{array}{l}\text { Anzahl der Flexible Fuel Vehicles (FFV) in } \\
\text { der EU27 }\end{array}$ & 77 & 4,26 & 1,72 & Technologie \\
\hline $23^{103}$ & Tank-oder-Teller-Diskussion in der EU27 & 77 & 4,22 & 1,527 & Gesellschaft \\
\hline 8 & $\begin{array}{l}\text { Vertikale strategische Kooperationen im } \\
\text { Ethanolmarkt }\end{array}$ & 67 & 4,1 & 1,257 & Ökonomie \\
\hline 24 & $\begin{array}{l}\text { Diskussion in der EU27 über die Auswir- } \\
\text { kungen von Ethanol auf die Umwelt }\end{array}$ & 75 & 4,08 & 1,468 & Gesellschaft \\
\hline 25 & $\begin{array}{l}\text { Diskussion in der EU27 über den Nutzen } \\
\text { von Ethanol zur Förderung der Energieun- } \\
\text { abhängigkeit der EU27 }\end{array}$ & 75 & 4 & 1,48 & Gesellschaft \\
\hline 5 & $\begin{array}{l}\text { Ethanolimporte in die EU27 aus Ländern } \\
\text { mit zollfreiem Marktzugang }\end{array}$ & 76 & 3,93 & 1,279 & Ökonomie \\
\hline 7 & Nachfrage nach ETBE & 75 & 3,52 & 1,528 & Ökonomie \\
\hline 17 & Nachfrage nach Biobutanol in der EU27 & 72 & 3,49 & 1,565 & Technologie \\
\hline 13 & Anzahl der Elektrofahrzeuge in der EU27 & 77 & 3,27 & 1,536 & Technologie \\
\hline 16 & Nachfrage nach BtL in der EU27 & 72 & 3,24 & 1,716 & Technologie \\
\hline
\end{tabular}

Tabelle 9: Wirkungsanalyse EU27 - Anordnung der wirkenden Einflussfaktoren hinsichtlich der Stärke des Einflusses auf den Ethanolmarkt der EU27

Quelle: EIGENE DARSTELLUNG

Um die Übersichtlichkeit der in Tabelle 9 dargestellten Ergebnisse zu verbessern, sind in der folgenden Abbildung 32 die Mittelwerte der einzelnen wirkenden Einflussfaktoren dargestellt. Die jeweilige Farbe der einzelnen Einflussfaktoren symbolisiert die Zugehörigkeit zu einem der vier Einflussbereiche Ökonomie, Technologie, Politik und Gesellschaft.

\footnotetext{
${ }^{103}$ Der gesellschaftliche Einflussfaktor 23: „Tank-oder-Teller-Diskussion in der EU27“ besitzt mit einem Mittelwert von 4,22 lediglich einen „moderaten Einfluss“ bis „eher starken Einfluss" auf den Ethanolmarkt der EU27. Damit ist dieser Einflussfaktor zwar der wichtigste gesellschaftliche Faktor, aber im Vergleich zu anderen Einflussfaktoren von eher geringer Relevanz. Dieses Ergebnis ist darauf zurückzuführen, dass die Befragung im Mai und Juni des Jahres 2012 erfolgte und somit vor dem Aufkommen der Tank-oder-Teller-Diskussion im Sommer 2012 und der Entscheidung der Generaldirektionen Energie und Klimaschutz, die Nutzung von Biokraftstoffen der ersten Generation aufgrund der ILUC- und der Tank-oderTeller-Diskussion zu beschränken (EUROPÄISCHE KOMMISSION 2012: 16).
} 


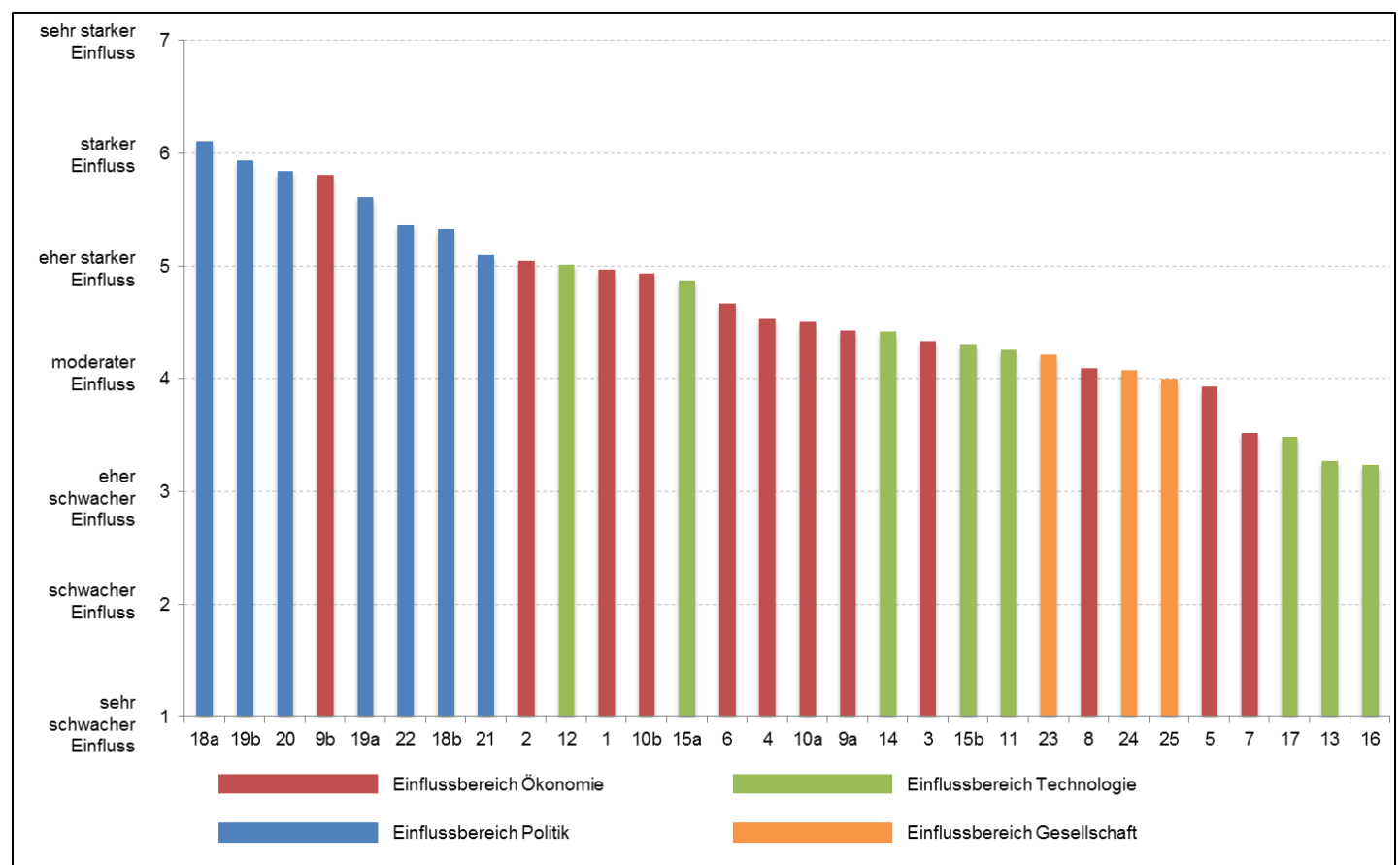

Abbildung 32: Wirkungsanalyse EU27 - Vergleich der Mittelwerte der wirkenden Einflussfaktoren

Quelle: EIGENE DARSTELLUNG

Der Vergleich der Mittelwerte der einzelnen wirkenden Einflussfaktoren ermöglicht es, das erste Ziel der Dissertation zu erreichen: Die Faktoren zu identifizieren, die den Ethanolmarkt der EU27 bis zum Jahr 2023 entscheidend beeinflussen werden. Das sind die wirkenden Einflussfaktoren des Einflussbereichs Politik ${ }^{104}$ mit den Nummern 18a, 19b, 20, 19a, 22, 18b und 21 sowie der ökonomische Einflussfaktor 9b. Da von den ersten acht bedeutendsten Einflussfaktoren sieben dem Einflussbereich Politik entstammen, ist davon auszugehen, dass der zukünftige Ethanolmarkt der EU27, wie bereits in der Vergangenheit, ein weiterhin hoch politisch beeinflusster Markt sein wird und die langfristige Entwicklung des Marktes stark von politischen Entscheidungen abhängig ist.

Zur genaueren Analyse des entscheidenden politischen Einflussbereichs wurde eine Faktoranalyse durchgeführt. Ihr Ziel ist es, darzustellen, wie die wirkenden Faktoren des Einflussbereichs Politik thematisch zusammenhängen, um dessen innere Struktur zu verdeutlichen. In der Faktoranalyse werden sowohl alle politischen Einfluss-

\footnotetext{
${ }^{104}$ Die folgenden wirkenden Einflussfaktoren des Einflussbereichs Politik sind für die Entwicklung des Ethanolmarktes bis zum Jahr 2023 von hoher Bedeutung: 18a: „Der politisch geforderte prozentuale Anteil von erneuerbaren Energien am Gesamtenergieverbrauch im Verkehrsbereich“; 19b: „Die maximal erlaubte prozentuale Beimischung von Ethanol zu Benzin“; 20: „Bestimmungen der Mitgliedsstaaten der EU27 zur Förderung von EthanolKraftstoff“; 19a: „Vorgabe der EU27 zur Kohlenstoffdioxideinsparung von Kraftstoffen im Verkehrsbereich“; 22: „Z̈̈lle auf Ethanolimporte in die EU27“; 18b: „Die politisch geforderte Mindesteinsparung von Kohlenstoffdioxid bei Biokraftstoffen im Verhältnis zu fossilen Kraftstoffen“ und 21: „Bestimmungen der EU27 betreffend ILUC (Indirect Land Use Change)“.
} 
faktoren als auch die beiden ökonomischen Faktoren 3: „Ethanolimporte aus Brasilien in die EU27“ und 4: „Ethanolimporte aus den USA in die EU27“ berücksichtigt. Die ausgewählten Daten besitzen mit einem Wert von 0,632 im Kaiser-Meyer-OlkinKriterium $^{105}$ und einer Signifikanz nach Barlett von 0,000 die Güte, die Faktoranalyse durchzuführen (BACKHAUS ET AL. 2006: 274ff.). In der folgenden Tabelle 10 sind die Ergebnisse der Analyse dargestellt, welche mittels der Hauptkomponentenanalyse als Extraktionsmethode und der orthogonalen VarimaxRotationsmethode ermittelt wurden ${ }^{106}$.

\begin{tabular}{|c|c|c|c|c|}
\hline \multicolumn{2}{|r|}{ Einflussfaktoren } & $\begin{array}{l}\text { Thema 1: } \\
\text { Politische Förde- } \\
\text { rung von Ethanol }\end{array}$ & $\begin{array}{l}\text { Thema 2: } \\
\text { Politische Vorga- } \\
\text { ben zur Treibhaus- } \\
\text { gasreduktion für } \\
\text { Ethanol }\end{array}$ & $\begin{array}{l}\text { Thema 3: } \\
\text { Ethanolimporte in } \\
\text { die EU27 }\end{array}$ \\
\hline $19 b$ & $\begin{array}{l}\text { Die maximal erlaubte pro- } \\
\text { zentuale Beimischung von } \\
\text { Ethanol zu Benzin }\end{array}$ & 0,888 & & \\
\hline $18 a$ & $\begin{array}{l}\text { Der politisch geforderte } \\
\text { prozentuale Anteil von er- } \\
\text { neuerbaren Energien am } \\
\text { Gesamtenergieverbrauch im } \\
\text { Verkehrsbereich }\end{array}$ & 0,764 & & \\
\hline 20 & $\begin{array}{l}\text { Bestimmungen der Mit- } \\
\text { gliedsstaaten der EU27 zur } \\
\text { Förderung von Ethanol- } \\
\text { Kraftstoff }\end{array}$ & 0,761 & & \\
\hline $18 b$ & $\begin{array}{l}\text { Die politisch geforderte Min- } \\
\text { desteinsparung von Kohlen- } \\
\text { stoffdioxid bei Biokraftstoffen } \\
\text { im Verhältnis zu fossilen } \\
\text { Kraftstoffen }\end{array}$ & & 0,794 & \\
\hline 21 & $\begin{array}{l}\text { Bestimmungen der EU27 } \\
\text { betreffend ILUC (Indirect } \\
\text { Land Use Change) }\end{array}$ & & 0,753 & \\
\hline $19 a$ & $\begin{array}{l}\text { Vorgabe der EU27 zur Koh- } \\
\text { lenstoffdioxideinsparung von } \\
\text { Kraftstoffen im Verkehrsbe- } \\
\text { reich }\end{array}$ & & 0,717 & \\
\hline 4 & $\begin{array}{l}\text { Ethanolimporte aus den USA } \\
\text { in die EU27 }\end{array}$ & & & 0,832 \\
\hline 3 & $\begin{array}{l}\text { Ethanolimporte aus Brasilien } \\
\text { in die EU27 }\end{array}$ & & & 0,698 \\
\hline 22 & $\begin{array}{l}\text { Zölle auf Ethanolimporte in } \\
\text { die EU27 }\end{array}$ & & & 0,592 \\
\hline & $\begin{array}{l}\text { Cronbachs Alpha }{ }^{10 /} \\
\% \text { der Varianz }\end{array}$ & $\begin{array}{l}0,794 \\
35 \%\end{array}$ & $\begin{array}{l}0,686 \\
19 \%\end{array}$ & $\begin{array}{l}0,615 \\
15 \%\end{array}$ \\
\hline
\end{tabular}

Tabelle 10: Wirkungsanalyse EU27 - Ergebnisse der Faktoranalyse Quelle: EIGENE DARSTELLUNG

\footnotetext{
${ }^{105} \mathrm{Im}$ Kaiser-Meyer-Olkin-Kriterium („Measure of Sampling Adequacy (MSA)“) sind die Daten mit einem Wert von 0,632 als "mittelmäßig“ (MSA $\geq 0,6)$ bis „ziemlich gut" (MSA $\geq 0,7)$ einzuordnen, was eine Durchführung der Faktoranalyse ermöglicht (BACKHAUS ET AL. 2006: 276).

${ }_{106}^{106}$ In der Faktoranalyse werden Faktorladungen von unter 0,5 nicht angezeigt.

107 Die Werte des Cronbachs Alpha zur Reliabilitätsanalyse (Thema 1: 0,794; Thema 2: 0,686 und Thema 3: 0,615) sind mindestens als akzeptabel zu beurteilen (GARSON 2012: $14)$.
} 
Das erste Thema ${ }^{108}$ „Politische Förderung von Ethanol“ vereint die drei wirkenden Einflussfaktoren (19b/18a/20) mit dem stärksten Einfluss auf den Ethanolmarkt der EU27 (Tabelle 10). Dies zeigt die enorme Bedeutung des Themas für die Zukunft des Marktes, was die Betrachtung der politischen Förderung von Ethanol unabdingbar macht, um eine frühzeitige Entwicklung des Marktes zu erkennen. Die Einflussfaktoren mit den Nummern 18b, 21 und 19a lassen sich mittels des zweiten Themas „Politische Vorgaben zur Treibhausgasreduktion für Ethanol“ zusammenfassen. Dieses zweite Thema besitzt zwar mit einem Mittelwert von 5,3 ${ }^{109}$ eine geringere Wirkungsstärke als das erste mit einem Mittelwert von 6,0. Es gehört aber im Vergleich zu den anderen wirkenden Einflussfaktoren eindeutig zu den entscheidenden Größen, die die Zukunft des europäischen Ethanolmarktes beeinflussen werden. Im Gegensatz zu den ersten beiden ist das dritte Thema „Ethanolimporte in die EU27“, bestehend aus den Einflussfaktoren 4, 3 und 22, nicht politisch. Es wurde aber dennoch in die Faktoranalyse aufgenommen, um zu zeigen, dass sich der wirkende politische Faktor 22: „Zölle auf Ethanolimporte in die EU27“ von den anderen politischen Faktoren abgrenzt und nicht von den beiden politischen Themen abgedeckt wird. Dies ist bei der Bestimmung der wirkenden Schlüsselfaktoren am Ende dieses Kapitels von Relevanz.

Neben den bereits genannten Faktoren sind des Weiteren die beiden wirkenden Einflussfaktoren 2: „Rohölpreis“ und 1: „Benzinnachfrage in der EU27“ aus dem Einflussbereich Ökonomie sowie der wirkende technische Einflussfaktor 12: „Anzahl benzinbetriebener Pkw, die mit mittleren Ethanolbeimischungen fahren können (E15 - E40)“von Bedeutung. Diese haben einen „eher starken Einfluss“ auf die zukünftige Entwicklung des Ethanolmarktes ${ }^{110}$.

108 Um Unklarheiten zu vermeiden, wird der Begriff „Themen“ in dieser Studie stellvertretend
für den üblicherweise in der Faktoranalyse benutzten Begriff „Faktor“ verwendet, da der Be-
griff „Faktor“ bereits als Beschreibung eines wirkenden Einflussfaktors in der Arbeit genutzt
wird.
109 Der Mittelwert und damit die Wirkungsstärke der Themen „Politische Förderung von
Ethanol“ und „Politische Vorgaben der Treibhausgasreduktion für Ethanol“ errechnet sich
aus dem Mittelwert der Wirkungsstärken der einzelnen zugehörigen wirkenden Einflussfakto-
ren.
110 Durch die Befragung lassen sich nicht nur die Faktoren mit einem starken Einfluss auf die
Zukunft des Marktes bestimmen, sondern auch jene, die nur von geringer Bedeutung sind
(Tabelle 9). Auffallend ist, dass die drei Einflussfaktoren aus dem Einflussbereich Technolo-
gie 17: „Nachfrage nach Biobutanol“; 13: „Anzahl der Elektrofahrzeuge in der EU27“ und 16:
"Nachfrage nach BtL in der EU27“, die jeweils eine Zukunftstechnologie beschreiben, den
Ethanolmarkt der EU27 von allen betrachteten Faktoren am schwächsten beeinflussen wer-
den. Daraus wird erstens ersichtlich, dass die befragten Experten in den nächsten 10 Jahren
erwarten, dass Ethanol der wichtigste Biokraftstoff für die Beimischung zu Benzin bleibt und
weder von Biobutanol noch von BtL substituiert wird. Zweitens lässt dies erkennen, dass es
keinen starken Anstieg der Anzahl der Elektrofahrzeuge in der EU27 geben wird, welche die
Beimischung von Ethanol zu Benzin ersetzt, um das politische Ziel des prozentual geforder- 
Die wirkenden Einflussfaktoren, die im nächsten Kernschritt „EU27 SzenarioPrognostik" als wirkende Schlüsselfaktoren weiter betrachtet werden, sind jene wirkenden Einflussfaktoren mit dem stärksten Einfluss auf den Ethanolmarkt der EU27. Wie bereits ausführlich erläutert und in Tabelle 9 ersichtlich, sind dies Einflussfaktoren des Einflussbereichs Politik sowie der ökonomische Einflussfaktor 9b. Um in der Szenario-Prognostik jedoch nicht nur politische und einen ökonomischen Einflussfaktor weiter zu betrachten, werden die beiden durch die Faktoranalyse gebildeten politischen Themen „Politische Förderung von Ethanol“ und „Politische Vorgaben zur Treibhausgasreduktion für Ethanol“ - abgekürzt durch T1 und T2 - stellvertretend für die darin enthaltenen Faktoren weiter betrachtet. Ferner gehen die nächsten fünf Einflussfaktoren (9b/22/2/12/1) mit der höchsten Wirkungsstärke als Schlüsselfaktoren in die Szenario-Prognostik ein. Es werden ausschließlich diese Einflussfaktoren weiter betrachtet, um sowohl die zentralen Entwicklungen des Marktes in den Szenarien aufzuzeigen als auch um den Prozess der „SzenarioBildung und -Konsistenzprüfung" beherrschbar und überschaubar zu halten (MILLETT/HONTON 1991: 71; GAUSEMEIER ET AL. 1995: 187f.). ${ }^{111}$

In der folgenden Abbildung 33 sind sowohl die beiden politischen Themen (T1/T2), die im weiteren Vorgehen genauso wie alle anderen wirkenden Einflussfaktoren als Schlüsselfaktoren bezeichnet und identisch behandelt werden, wie auch die weiteren wirkenden Schlüsselfaktoren des Ethanolmarktes der EU27 zusammengefasst abgebildet.

ten Anteils von erneuerbaren Energien am Gesamtenergieverbrauch im Verkehrsbereich zu erreichen.

${ }_{111}$ Auf die Berücksichtigung des nächstwichtigsten Einflussfaktors 10b: „Rohstoffkosten für die Produktion von Zellulose-Ethanol" als wirkender Schlüsselfaktor wurde bewusst verzichtet, da die vier wirkenden Einflussfaktoren 10a, 10b, 14 und 15, die den Einfluss von Zellulose-Ethanol auf die Entwicklung des europäischen Ethanolmarktes beschreiben, einen nur durchschnittlich starken Einfluss auf den Ethanolmarkt aufweisen (Tabelle 9). Dies weist darauf hin, dass die meisten Experten davon ausgehen, dass in Zukunft weiterhin Ethanol der ersten Generation der wichtigere Produktionsweg bleiben wird. 


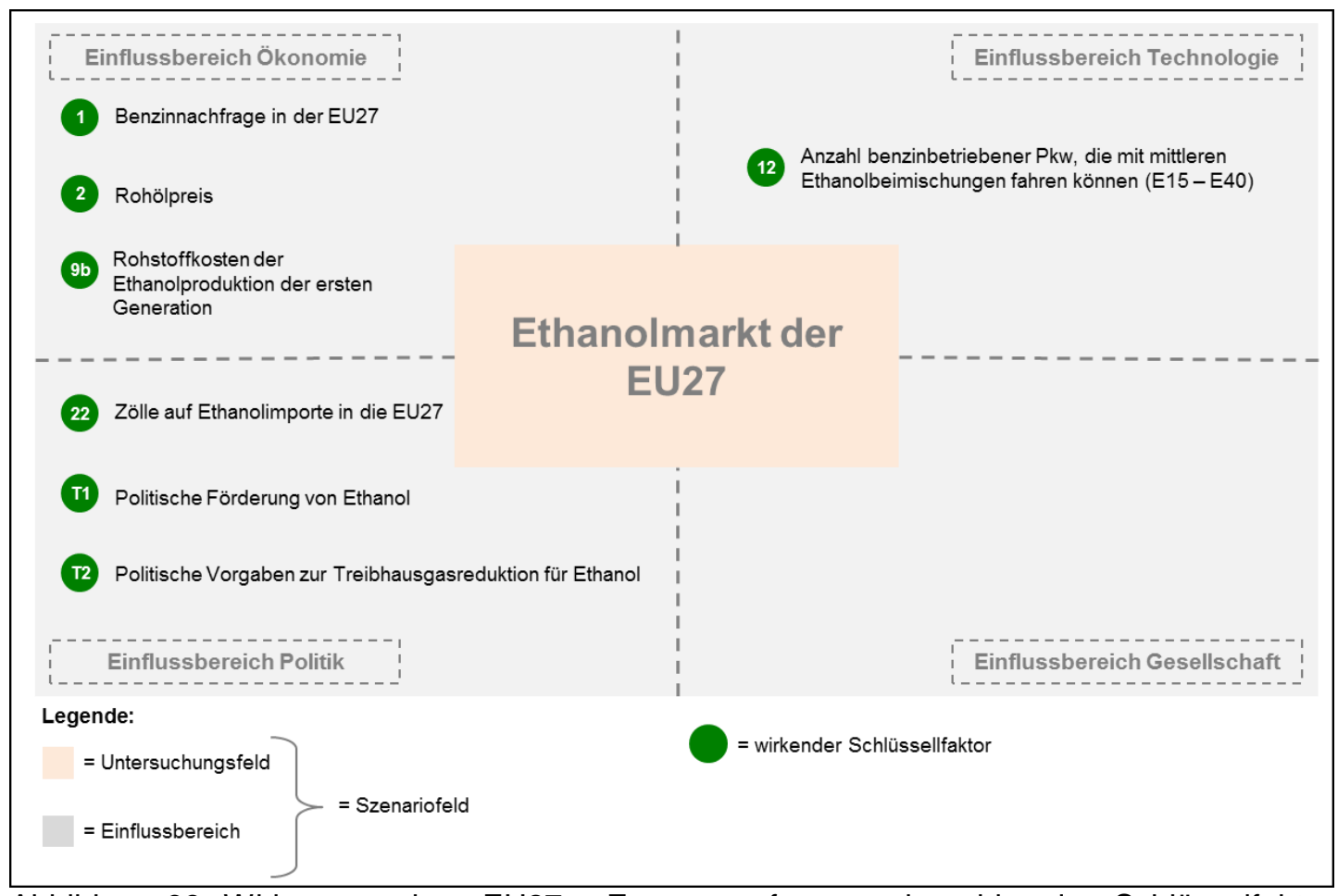

Abbildung 33: Wirkungsanalyse EU27 - Zusammenfassung der wirkenden Schlüsselfaktoren

Quelle: EIGENE DARSTELLUNG

5.2

EU27 Szenario-Prognostik

Ziel des zweiten Kernschrittes „EU27 Szenario-Prognostik“ ist es, für jeden Schlüsselfaktor ${ }^{112}$ mindestens eine Zukunftsprojektion zu bilden, die den Faktor im Jahr 2023 beschreibt ${ }^{113}$. Die Schlüsselfaktoren werden in folgender Reihenfolge betrachtet (Tabelle 11):

\section{Schlüsselfaktor}

\begin{tabular}{l|l}
\hline 1 & Benzinnachfrage in der EU27 \\
\hline 2 & Rohölpreis \\
\hline $9 b$ & Rohstoffkosten der Ethanolproduktion der ersten Generation \\
\hline 12 & $\begin{array}{l}\text { Anzahl benzinbetriebener Pkw, die mit mittleren Ethanolbeimischungen fahren können (E15 - } \\
\text { E40) }\end{array}$ \\
\hline 22 & Zölle auf Ethanolimporte in die EU27 \\
\hline T1 & Politische Förderung von Ethanol \\
\hline T2 & Politische Vorgaben zur Treibhausgasreduktion für Ethanol \\
\hline I & Ethanolnachfrage \\
\hline
\end{tabular}

${ }^{112}$ Die in Kapitel 5.1.1.1 bestimmten beschreibenden Einflussfaktoren - Ethanolnachfrage, Ethanolproduktion, Ethanolhandel und Ethanolpreis - werden in der Szenario-Prognostik als beschreibende Schlüsselfaktoren weiter betrachtet.

${ }_{113}$ Das methodische Vorgehen zur Erstellung der einzelnen Zukunftsprojektionen ist in Kapitel 4.3 beschrieben. 


\begin{tabular}{l|l} 
II & Ethanolproduktion \\
III & Ethanolhandel \\
IV & Ethanolpreis
\end{tabular}

Tabelle 11: EU27 Szenario-Prognostik - Reihenfolge der Betrachtung der Schlüsselfaktoren Quelle: EIGENE DARSTELLUNG

\subsubsection{Benzinnachfrage in der EU27}

Die Zukunftsprojektion des Schlüsselfaktors „Benzinnachfrage in der EU27“ wird in der Einheit Milliarden Liter pro Jahr dargestellt. Abbildung 34 zeigt die Entwicklung der Benzin- und Dieselnachfrage für den Transportbereich in der EU27 im Zeitraum von 1990 bis 2010 .

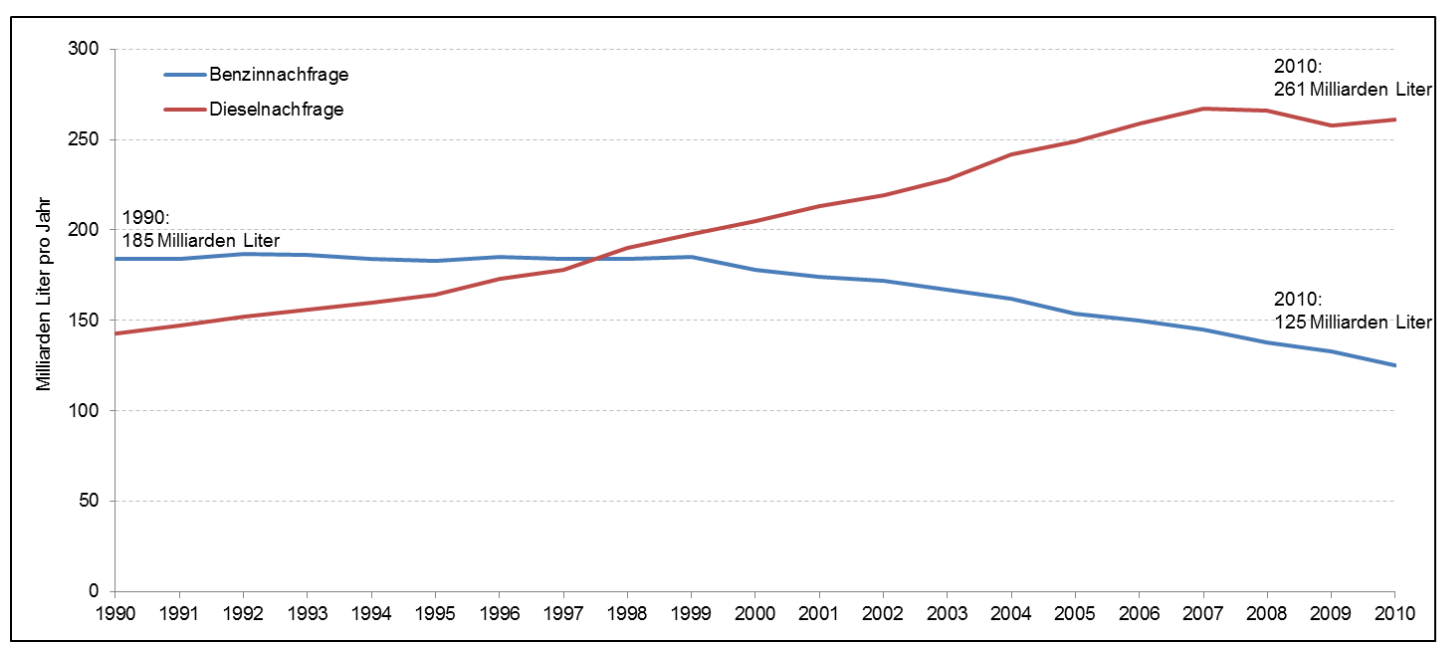

Abbildung 34: Entwicklung der Nachfrage nach Benzin und Diesel im Transportbereich in der EU27 in den Jahren von 1990 bis 2010

Quelle: In Anlehnung an EUROPEAN COMMISSION 2012a: 95

In Abbildung 34 ist zu erkennen, dass die Nachfrage nach Benzin um durchschnittlich 3,5\% pro Jahr von 185 Milliarden Litern im Jahr 1999 auf 125 Milliarden Liter im Jahr 2010 gesunken ist. Im Gegensatz dazu ist ein, abgesehen von den letzten Jahren, anhaltender Anstieg der Nachfrage nach Diesel bis auf 261 Milliarden Liter im Jahr 2010 feststellbar. Im Vergleich zum Jahr 1990 ist die EU27 im Jahr 2010 ein Dieselmarkt, mit einem Diesel-Benzin-Verhältnis in Höhe von 2,1 zu $1^{114}$. Die beständige Abnahme der Nachfrage nach Benzin in der EU27 ist auf folgende drei Einflüsse zurückzuführen: (1) Immer mehr Dieselfahrzeuge werden in der EU27 zugelassen und substituieren benzinbetriebene Kraftfahrzeuge, wodurch die Anzahl an benzinbetriebenen Pkw, trotz eines leicht wachsenden Pkw-Marktes gesunken

\footnotetext{
${ }^{114}$ Aufgrund der Entwicklung von einem Benzinmarkt zu einem Dieselmarkt entsteht in den europäischen Raffinerien eine Überproduktion an Benzin und der Kraftstoff wird in die USA, nach Afrika und in den Mittleren Osten exportiert (WOOD MACKENZIE 2013: 8; WOOD MACKENZIE 2010: 60).
} 
ist $^{115}$ (ACEA 2011b: 13; FRAUNHOFER ISI ET AL. 2010: 39). (2) Aufgrund des geringeren Benzinverbrauchs infolge der gestiegenen Effizienz der neueren Kraftfahrzeuge im Vergleich zu den älteren Modellen sowie (3) durch die Beimischung von Ethanol zu Benzin, wodurch Benzin substituiert wird (ACEA 2011a: 54; SHELL 2009: 36).

Die Zukunftsprojektion für den Schlüsselfaktor, die in die Szenario-Bildung eingeht, wird auf Grundlage der in Abbildung 35 dargestellten Projektionen gebildet. Die Projektionen stammen vom JEC, einer Forschungsgemeinschaft, bestehend aus den Organisationen JRC (European Commission Joint Research Centre), dem EUCAR (European Council for Automotive Research \& Development) und dem CONCAWE (Oil Companies' European Association for Environment, Health and Safety in Refining and Distribution).

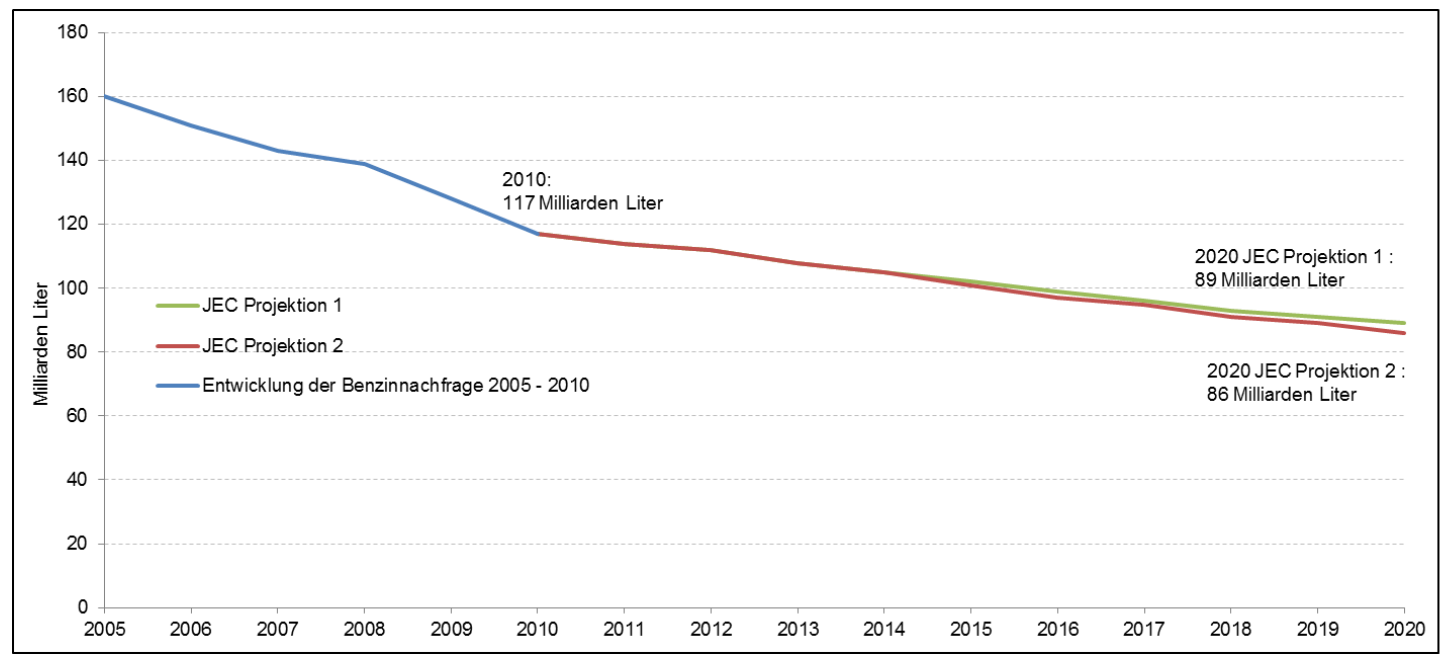

Abbildung 35: Zukünftige Entwicklung der Nachfrage nach Benzin im Straßenverkehr der EU27

Quelle: In Anlehnung an EUROPEAN COMMISSION JOINT RESEARCH CENTRE; INSTITUT FOR ENERGY ET AL. 2011:24

Es werden die Projektionen des JEC weiter betrachtet, da sie die Entwicklung der Nachfrage nach Benzin ausschließlich für den Bereich Straßenverkehr aufzeigen und damit genau dort, wo Ethanol als Beimischung eingesetzt wird ${ }^{116}$ (WOOD MACKENZIE 2010: 64). Darüber hinaus werden die Projektionen als qualitativ hoch-

\footnotetext{
${ }^{115}$ Im Jahr 1990 waren nur 13\% der in der EU15 neu zugelassenen Autos Dieselfahrzeuge, im Jahr 2010 waren es bereits 55\%. Insbesondere auf solch wichtigen Märkten wie Frankreich, Belgien und Spanien liegt der Verkauf von Kraftfahrzeugen mit Dieselmotor bei über 70\% (ACEA 2011b: 20). Angaben für die EU15 zeigen eine eindeutige Tendenz für die EU27, da 83\% der Pkw-Flotte der EU27 in den Staaten der EU15 zugelassen sind (ACEA 2011b: 36).

${ }_{116}$ In Abbildung 35 ist die Entwicklung der Nachfrage nach Benzin im Straßenverkehr dargestellt und nicht, wie in Abbildung 34, für den Transportbereich; darum sind die Werte im Zeitraum von 2005 bis 2010 nicht identisch.
} 
wertig eingeschätzt, da sie in Zusammenarbeit von Einrichtungen erstellt wurden, die sich in ihrem Wissen ergänzen und somit eine umfassendere Sicht auf die Entwicklung des Schlüsselfaktors besitzen ${ }^{117}$.

Die Projektionen (JEC Projektion 1 und JEC Projektion 2), die sich insbesondere für die Betrachtung eines langfristigen Zeithorizonts nur unerheblich unterscheiden, zeigen beide eine konstante Reduktion der Benzinnachfrage in der EU27. Ausgehend von 117 Milliarden Litern im Jahr 2010 reduziert sich die nachgefragte Menge an Benzin im Straßenverkehr auf 89 Milliarden Liter (JEC Projektion 1) und auf 86 Milliarden Liter (JEC Projektion 2) bis zum Jahr 2020; dies entspricht einer jährlichen prozentualen Reduktion von 2,46\% (JEC Projektion 1) und 2,76\% (JEC Projektion 2). Die Projektionen bestätigen sich bei einem Vergleich mit den Prognosen der Organisationen OPEC (2011: 168) und WOOD MACKENZIE (2010: 40), die ebenfalls eine Reduktion der Benzinnachfrage in der EU27 prognostizieren ${ }^{118}$. Da sich die beiden Projektionen des JEC nur unerheblich unterscheiden, wird im Folgenden ausschließlich die JEC Projektion 1 weiter betrachtet.

Für die zukünftig sinkende Nachfrage nach Benzin im Straßenverkehr der EU27 sind dieselben Gründe zu nennen, die schon zur Abnahme der Benzinnachfrage in der Zeit von 1990 - 2010 geführt haben: Die Verdrängung von Kraftfahrzeugen mit Benzinmotor durch Diesel-Pkw, der geringere Benzinverbrauch durch effizientere Kraftfahrzeuge und die Substitution von Benzin durch Ethanol (WOOD MACKENZIE 2010: 41; U.S. ENERGY INFORMATION ADMINISTRATION 2011b: 122, SHELL 2009: 36)

Die Zukunftsprojektion, die in den dritten Kernschritt „EU27 Szenario-Bildung und -Konsistenzprüfung“ eingeht, basiert auf der „JEC Projektion 1“ (Abbildung 35). Aus der Projektion wird die angenommene Höhe der Substitution von Benzin durch Ethanol herausgerechnet, wodurch die Entwicklung der Benzinnachfrage in der EU27 unabhängig von der zukünftigen Ethanolnutzung ersichtlich wird ${ }^{119}$. Da die JEC Projektion 1 die Entwicklung des Schlüsselfaktors lediglich bis zum Jahr 2020

\footnotetext{
${ }^{117}$ Das JRC besitzt große Erfahrung bei der Entwicklung von Zukunftsprojektionen durch die Unterabteilung „Institute for Prospective Technological Studies“, das EUCAR ist spezialisiert auf die Forschung in der Automobilindustrie und das CONCAWE deckt den Bereich der Entwicklung der Treibstoffe ab.

${ }_{118}$ Die Daten von der OPEC (2011: 168) und WOOD MACKENZIE (2010: 40) wurden nicht in die Abbildung 35 mit aufgenommen, da sie die Entwicklung für den gesamten Transportbereich und nicht explizit für den Straßenverkehr angeben.

${ }_{119}$ Die Nichtbeachtung der Ethanolnutzung ist notwendig, um bei der Szenario-Bildung (Kapitel 5.3.1) unabhängig von der Auswahl der Zukunftsprojektion des Schlüsselfaktors „Ethanolnachfrage" konsistente Szenarien erstellen zu können.
} 
angibt, werden die Daten für die Jahre 2021, 2022 und 2023 mittels einer linearen Trendberechnung dargestellt (Abbildung 36).

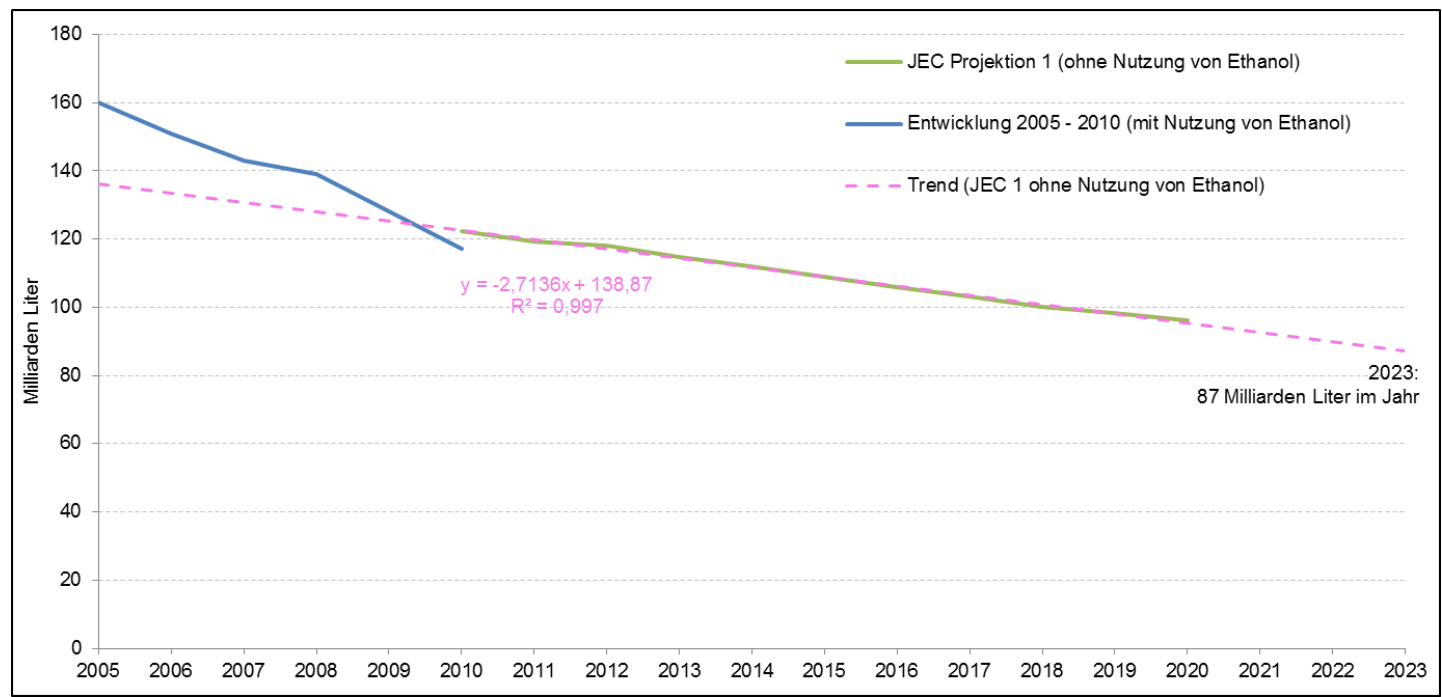

Abbildung 36: Entwicklung der Nachfrage nach Benzin im Straßenverkehr in der EU27 bis zum Jahr 2023

Quelle: In Anlehnung an EUROPEAN COMMISSION JOINT RESEARCH CENTRE; INSTITUT FOR ENERGY ET AL. 2011: 24, EIGENE BERECHNUNG

Die Trendberechnung ist aufgrund des sehr guten Bestimmtheitsmaßes von 0,997 aussagekräftig. Auf Grundlage der Trendberechnung ergibt sich für das Jahr 2023 eine Benzinnachfrage für den Straßenverkehr in der EU27 in Höhe von 87 Milliarden Litern. Die Zukunftsprojektion 1A, die in die „EU27 Szenario-Bildung und -Konsistenzprüfung" eingeht, ist wie folgt beschrieben:

1A | Rückgang der Benzinnachfrage in der EU27 auf 87 Milliarden Liter pro Jahr

\subsubsection{Rohölpreis}

Die Zukunftsprojektionen des zweiten Schlüsselfaktors „Rohölpreis“ werden in der Einheit US-\$ je Barrel (158,987 Liter) angegeben. Die langfristige Entwicklung des Rohölpreises wird anhand der Rohölsorte „West Texas Intermediate“ (WTI) ${ }^{120}$ beschrieben. WTI wird an der „New York Mercantile Exchange“ gehandelt und als Referenzsorte zur Preisbildung auf den weltweiten Rohölmärkten genutzt (U.S. ENERGY INFORMATION ADMINISTRATION 2012a; OECD 2012: 142). Aufgrund ihrer Funktion als Referenzsorte eignet sich WTI dazu, die Projektionen des Schlüsselfaktors aufzuzeigen.

\footnotetext{
${ }^{120}$ West Texas Intermediate ist eine leichte, süßliche Rohölsorte mit geringem Schwefelgehalt (U.S. ENERGY INFORMATION ADMINISTRATION 2012b: 219).
} 
In Abbildung 37 werden sowohl die reale Preisentwicklung zum Basisjahr 2010 für WTI in US-\$ je Barrel seit dem Jahr 1980 dargestellt als auch geschichtliche Ereignisse genannt, die den Rohölpreis beeinflusst haben.

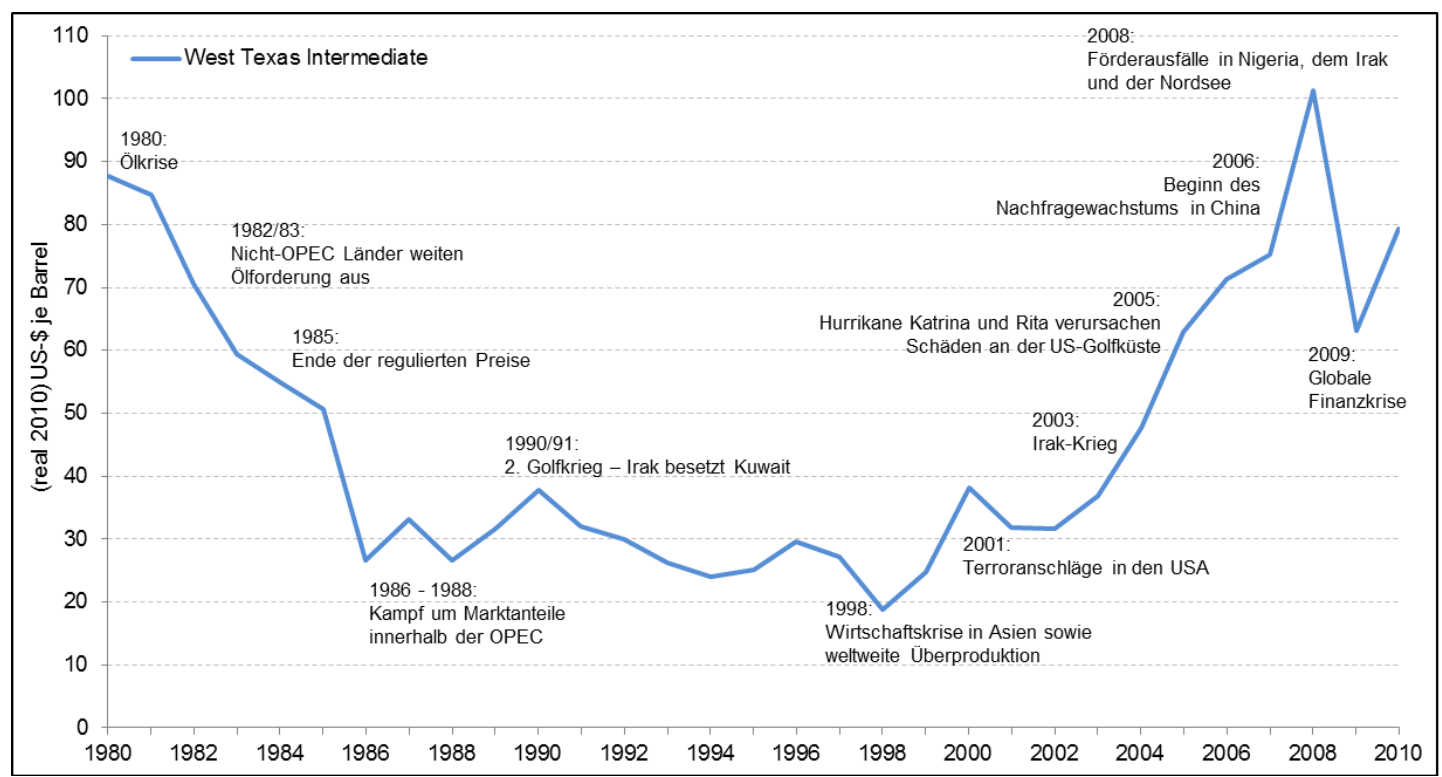

Abbildung 37: Reale Entwicklung des Rohölpreises WTI 1980 - 2010

Quelle: In Anlehnung an OECD 2012: 143; U.S. ENERGY INFORMATION ADMINISTRATION 2012b: 24; U.S. ENERGY INFORMATION ADMINISTRATION 2012c: 2

Die folgenden Ausführungen beschreiben die Entwicklung des Rohölpreises von 1980 bis 2010, wie in Abbildung 37 dargestellt: Nach den hohen Rohölpreisen im Jahr 1980 (87,68 US-\$), die durch die Ölkrise und den ersten Golfkrieg zwischen dem Iran und dem Irak verursacht wurden, sank der Preis je Barrel Rohöl bis zum Jahr 1986 schrittweise auf 26,69 US-\$. Dieser Rückgang kann maßgeblich auf die Überproduktion der OPEC zurückgeführt werden, die durch Saudi-Arabiens Streben nach höheren Marktanteilen verursacht wurde. Die 1990er Jahre waren hingegen durch niedrige Ölpreise gekennzeichnet - nur der zweite Golfkrieg 1990/91 verursachte einen kurzfristigen Anstieg des Rohölpreises auf 37,76 US-\$. Nach Überwindung der Wirtschaftskrise in Asien und einer Reduktion der Förderziele der OPEC kam es zu einem kontinuierlichen Preisanstieg bis auf 101,24 US-\$ im Jahre 2008. Der Preisanstieg wurde durch den dritten Golfkrieg im Irak (2003), die Hurrikane Katrina und Rita (2005), die starke Schäden an der US-Golfküste verursachten, sowie insbesondere durch den Beginn der wachsenden Rohölnachfrage aufstrebender Volkswirtschaften, speziell China, hervorgerufen. Bedingt durch die weltweite Finanzkrise ist die Nachfrage nach Rohöl zwischenzeitlich drastisch gesunken; im Jahr 2009 fielen die Preise für Rohöl in kürzester Zeit auf 63,04 US-\$. Mit Einsetzen der wirtschaftlichen Erholung stiegen die Rohölpreise wieder auf 79,4 US-\$ im Jahr 2010 (OECD 2012: 142). 
Aus der Abbildung 37 wird ebenso ersichtlich, dass die Zeit des günstigen Rohöls wie in den Jahren von 1986 bis 2003 vorbei ist und der Rohölpreis seit 2007 stark volatil ist (INTERNATIONAL ENERGY AGENCY 2011a: 61). Die Erstellung einer langfristig richtigen Prognose des Ölpreises ist fast unmöglich, da unvorhersehbare, oft kurzfristig wirkende Ereignisse wie beispielsweise Naturkatastrophen oder Förderausfälle in wichtigen Produktionsregionen die Entwicklung des Rohölpreises maßgeblich beeinflussen können. Aufgrund dessen gilt es, sich insbesondere auf die essentiell langfristig global wirkenden Faktoren zu konzentrieren. Die U.S. ENERGY INFORMATION ADMINISTRATION (2012b:23) nennt die folgenden vier:

1. Das Angebot an Rohöl und anderen Treibstoffen ${ }^{121}$ von Nicht-OPEC-Staaten.

2. Investitionen der OPEC in die zukünftige Rohölförderung und Entscheidungen der OPEC über Rohölfördermengen.

3. Die wirtschaftliche Konkurrenzfähigkeit anderer Treibstoffe im Vergleich zu Rohöl.

4. Die weltweite Nachfrage nach Rohöl und anderen Treibstoffen.

Die folgende Abbildung 38 zeigt einen Vergleich der Einschätzung der langfristigen Entwicklung des Rohölpreises bis zum Jahr 2025. Die Werte sind in (real 2010) US$\$$ je Barrel WTI angegeben ${ }^{122}$.

\footnotetext{
${ }^{121}$ Unter der Bezeichnung „andere Treibstoffe“ fasst die U.S. Energy Information Administration die folgenden Produkte zusammen: Biokraftstoffe, Bitumen (Ölsand), Coal-to-Liquid (CtL), Biomass-to-Liquid (BtL), Gas-to-Liquid (GtL), Extra-Schweröl und Ölschiefer.

122 Die International Energy Agency nutzt für ihre Projektionen den „Average IEA Crude Oil Import Price" und die INFORUM gibt die Preisentwicklung mittels des durchschnittlichen USAmerikanischen RAC Preises (Refiner Acquisition Cost) an - trotz dieses Unterschieds sind die Preise problemlos miteinander vergleichbar (INTERNATIONAL ENERGY AGENCY 2011a: 62).
} 


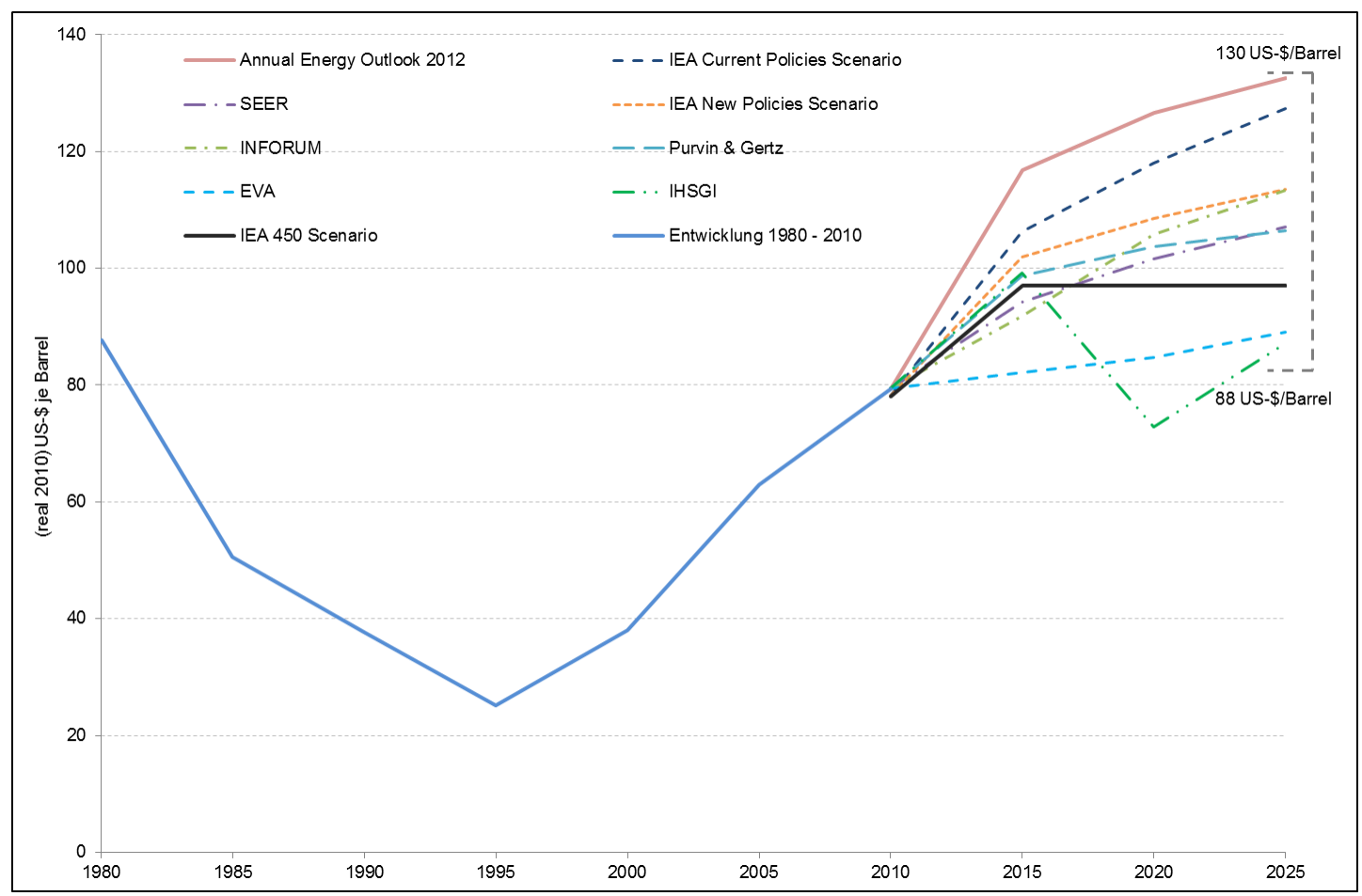

Abbildung 38: Entwicklung des Rohölpreises (real 2010) 1980 - 2025

Quelle: In Anlehnung an U.S. ENERGY INFORMATION ADMINISTRATION 2012b: 23ff.; INTERNATIONAL ENERGY AGENCY 2011a: 61f.

Alle Organisationen in Abbildung 38 erwarten einen Anstieg des Rohölpreises bis zum Jahr 2023. Aus der Betrachtung der einzelnen Projektionen ergibt sich ein Zukunftsraum der zwischen (real 2010) 88 und 130 US-\$ je Barrel liegt. Um dieser Ungewissheit der zukünftigen Entwicklung des Rohölpreises gerecht zu werden, werden zwei Projektionen in der weiteren Arbeit berücksichtigt. Als erstes die Projektion „Annual Energy Outlook 2012“123, gebildet von der U.S. ENERGY INFORMATION ADMINISTRATION (2012b: 23ff.), die einen Anstieg des Rohölpreises auf 130 US-\$/Barrel bis zum Jahr 2025 prognostiziert. Sowie, um den unteren Bereich

${ }^{123}$ Die Projektion „Annual Energy Outlook 2012“, die einen Anstieg auf (real 2010) 132 US\$/Barrel bis zum Jahr 2025 prognostiziert, basiert auf folgenden Annahmen (U.S. ENERGY INFORMATION ADMINISTRATION 2012b: 24f.):

- Weiterhin robustes Wirtschaftswachstum in Nicht-OECD-Staaten, eingeschlossen China und Indien.

- Nur geringes Wirtschaftswachstum in zahlreichen OECD-Staaten.

- Steigende Nachfrage nach Rohöl und anderen Treibstoffen in Nicht-OECD-Staaten (im Vergleich zu 2010: Anstieg der Nachfrage um 21 Millionen Barrel pro Tag bis zum Jahr 2035).

- Konstante bis leichte Steigerung der Nachfrage nach Rohöl und anderen Treibstoffen in der OECD.

- OPEC hält ihren Marktanteil an der weltweiten Rohölproduktion in Höhe von $42 \%$.

- Anstieg der Rohölproduktion von Nicht-OPEC-Staaten. Die Förderung erfolgt aus schwer zugänglichen Reserven zu hohen Förderkosten (high-cost reserves). Die Erschließung der Reserven ist aufgrund des hohen technischen Fortschritts möglich.

- Anstieg der Bitumen-Produktion in Kanada. 
des Zukunftsraums abzubilden, die Prognose „IEA 450 Scenario“124 entwickelt von der INTERNATIONAL ENERGY AGENCY (2011a: 61f.), die eine moderate Steigerung des Rohölpreises auf 97 US-\$/Barrel darstellt.

Basierend auf den vorangegangenen Erläuterungen werden die beiden Zukunftsprojektionen 2A und 2B im dem dritten Kernschritt „EU27 Szenario-Bildung und -Konsistenzprüfung" weiter betrachtet:

2A | Anstieg des Rohölpreises (WTI) auf (real 2010) 130 US-\$ je Barrel

2B | Moderater Anstieg des Rohölpreises (WTI) auf (real 2010) 97 US-\$ je Barrel

\subsubsection{Rohstoffkosten der Ethanolproduktion der ersten Generation}

Im Gegensatz zu den USA, in denen Mais der dominierende Rohstoff zur Produktion von Ethanol ist (U.S. DEPARTMENT OF ENERGY 2012a), werden in der EU27 verschiedene landwirtschaftliche Kulturen zur Ethanolproduktion genutzt. In der folgenden Abbildung 39 ist die Verteilung der Rohstoffe dargestellt, die zur Produktion von 3 Milliarden Litern Ethanol-Kraftstoff in der EU27 im Jahr 2008 eingesetzt wurden (F.O. LICHT 2012f: 74).

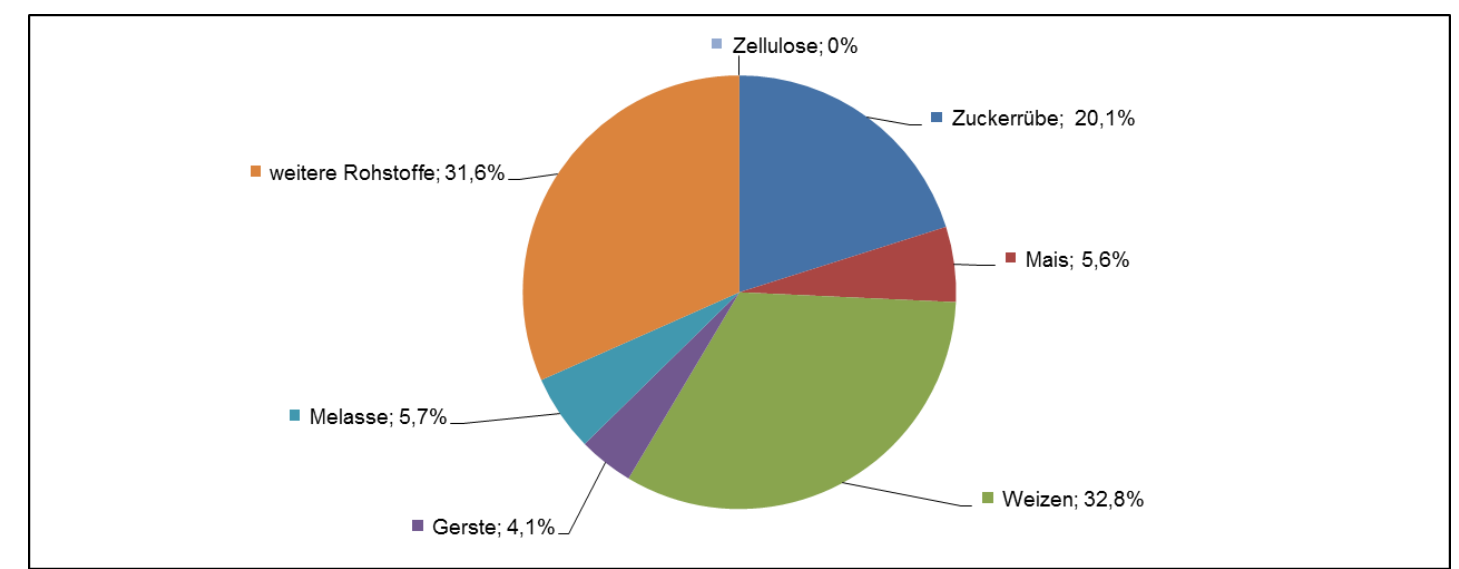

Abbildung 39: Rohstoffe zur Produktion von Ethanol in der EU27

Quelle: In Anlehnung an EUROPEAN COMMISSION JOINT RESEARCH CENTRE, INSTITUT FOR ENERGY ET AL. 2010: 74

Die Einrichtungen WOOD MACKENZIE (2010: 66) und ECOFYS ET AL. (2011: 30) zeigen für das Jahr 2008 eine ähnliche Verteilung der Rohstoffnutzung ${ }^{125}$. Der wichtigste Rohstoff zur Ethanolproduktion ist Weizen, gefolgt von Zuckerrübe, Melasse und Mais. In der Gruppe „weitere Rohstoffe" befinden sich unter anderem Grund-

\footnotetext{
${ }^{124}$ Die Prognose der INTERNATIONAL ENERGY AGENCY (2011a: 62) basiert auf der Annahme, dass es in Zukunft zu keinem Anstieg der Nachfrage nach Rohöl und infolgedessen auch zu keiner teuren Rohölförderung in Nicht-OPEC Staaten kommen wird.

${ }_{125}$ Neuere Daten zur Rohstoffnutzung konnten leider nicht gefunden werden. Da aber die Anlagen, die 2008 in Betrieb waren, aktuell auch noch Ethanol herstellen, ist die grundlegende Nutzung der Rohstoffe auf das Jahr 2013 übertragbar.
} 
stoffe wie Roggen, Triticale, Weinalkohol, Molke und Papierpulpe. Darüber hinaus ist zu erkennen, dass Zellulose als Rohstoff zur Ethanolherstellung noch keine Bedeutung besitzt. Bei der Betrachtung der Rohstoffe, unterteilt in die beiden Gruppen Stärke und Zucker, ist zu erkennen, dass Stärke (Weizen, Mais und Gerste) der wesentliche Rohstoff zur Ethanolproduktion in der EU27 ist. Da Weizen der wichtigste Rohstoff zur Ethanolherstellung ist und da das EUROPEAN COMMISSION JOINT RESEARCH CENTRE, INSTITUT FOR ENERGY ET AL. (2010: 74), die OECD/FAO (2012: 125) und das USDA (2012: 20) prognostizieren, dass Weizen auch zukünftig der wichtigste Rohstoff zur Ethanolproduktion in der EU27 sein wird $^{126}$, steht in diesem Kapitel die zukünftige Entwicklung des Weizenpreises im Zentrum der Beobachtung.

Im Folgenden werden die Ist-Situation sowie die Preisentwicklung bis zum Jahr 2023 anhand der Sorte Hard Red Winter Wheat - FOB (Free on Board) an der USamerikanischen Golfküste - betrachtet. Abbildung 40 zeigt die Entwicklung des (real 2005) Weizenpreises von 1989 bis 2010.

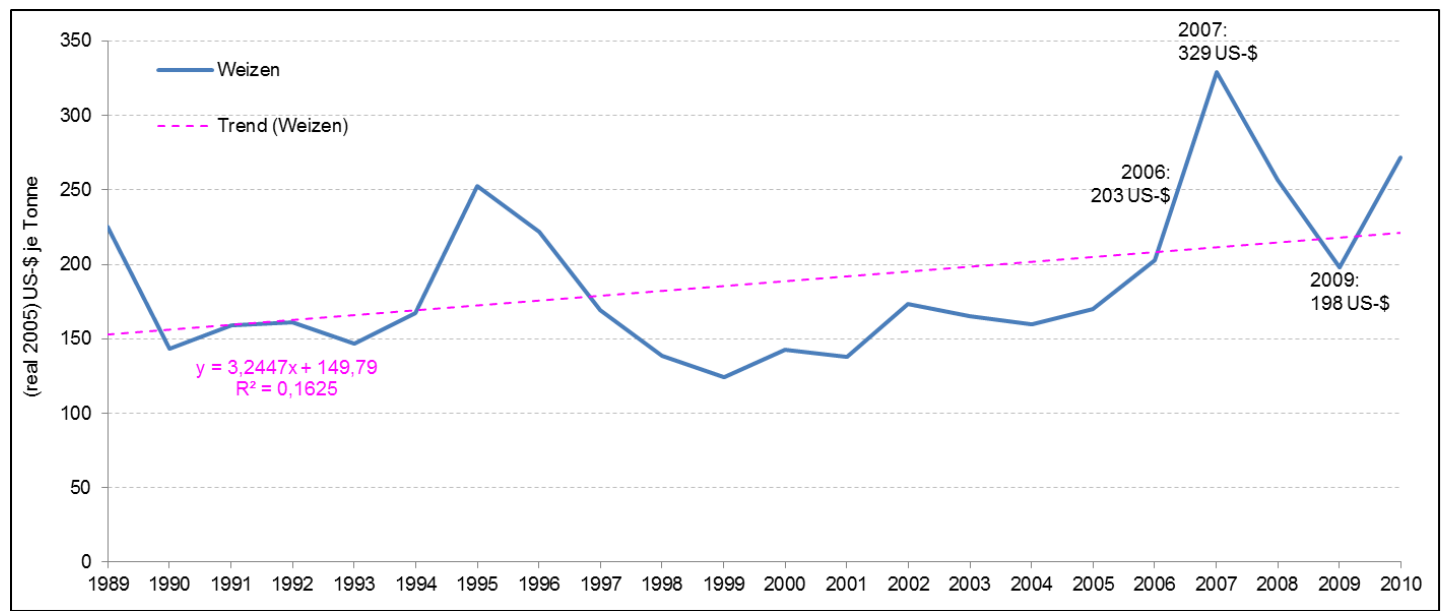

Abbildung 40: Entwicklung des (real 2005) Weizenpreises $1989-2010$

Quelle: In Anlehnung an OECD/FAO 2012: 121, EIGENE BERECHNUNG

Mittels der eingefügten linearen Trendlinie ist ein realer Preisanstieg des Weizenpreises im Zeitraum von 1989 bis 2010 zu erkennen. In der Zeit von 1989 bis 1996 hat sich der Weizenpreis volatil verhalten; insbesondere im Jahr 1995 kam es zu einem starken Preisanstieg. Die Jahre von 1997 bis 2006 waren hingegen geprägt durch konstant niedrige Weizenpreise von unter (real 2005) 200 US-\$ je Tonne. Diese Phase endete 2006, seitdem schwankt der Weizenpreis stark. Im Zeitraum

\footnotetext{
${ }^{126}$ Das EUROPEAN COMMISSION JOINT RESEARCH CENTRE, INSTITUT FOR ENERGY ET AL. (2011: 38) prognostiziert, dass im Jahr 2020 60\% der Ethanolgewinnung in der EU27 aus dem Rohstoff Weizen erfolgt.
} 
von 2006 bis 2007 stieg der Weizenpreis um 126 US-\$ auf 329 US-\$ je Tonne und fiel wieder auf 198 US-\$ im Jahr 2009.

Die folgende Abbildung 41 zeigt die prognostizierte Entwicklung des Weizenpreises bis zum Jahr 2021, dargestellt anhand der Projektionen der Organisationen OECD/FAO, USDA und World Bank ${ }^{127}$.

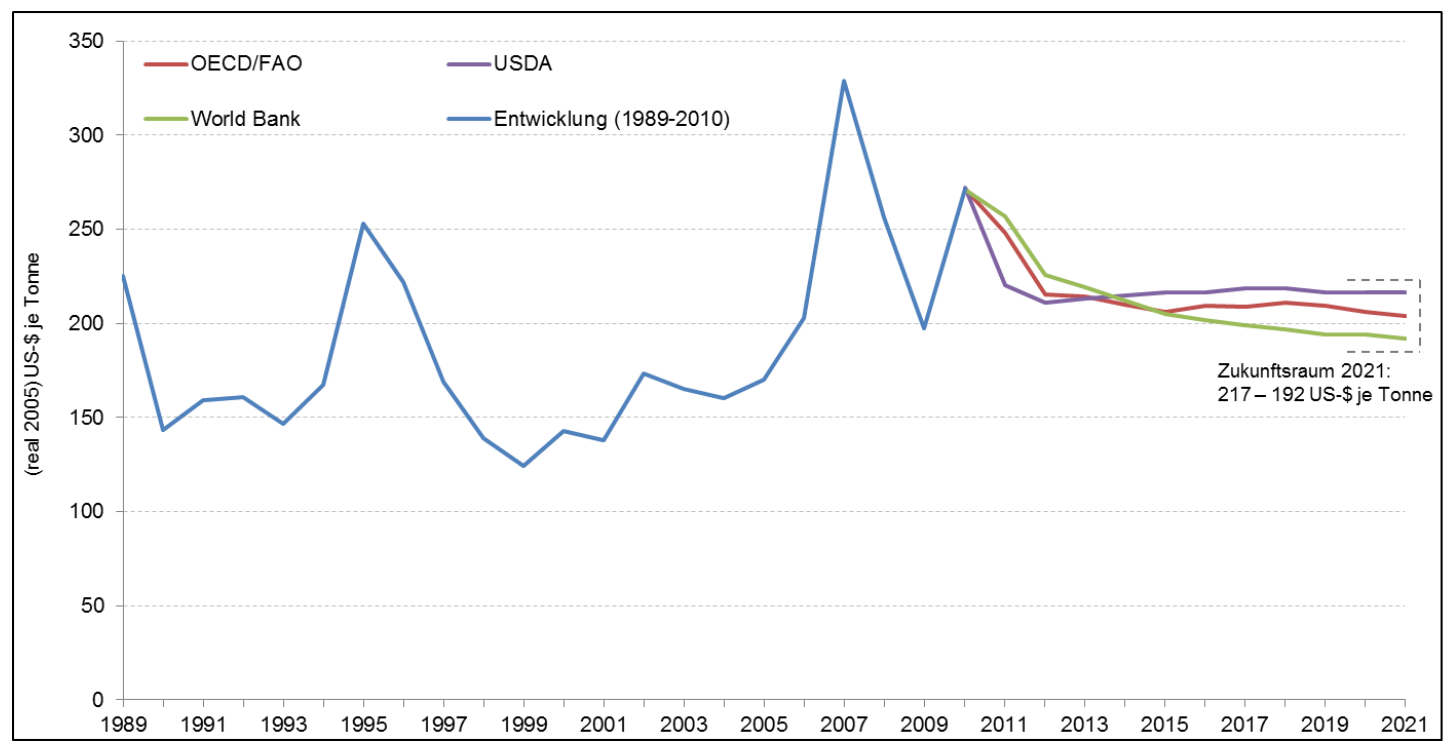

Abbildung 41: Prognostizierte Entwicklung des (real 2005) Weizenpreises bis 2021 Quelle: In Anlehnung an OECD/FAO 2012: 121; USDA 2012: 70; WORLD BANK DEVELOPMENT PROSPECTS GROUP 2012: 1

Alle drei Einrichtungen sowie das FAPRI (2011a) ${ }^{128}$ gehen von einer sehr ähnlichen Entwicklung des Weizenpreises bis zum Jahr 2021 aus. Es wird prognostiziert, dass der Weizenpreis (real 2005) bis zum Jahr 2014 auf ungefähr 210 US-\$ je Tonne sinkt und bis zum Jahr 2021 auf diesem Preisniveau verharrt. Im Jahr 2021 ergibt sich aus den drei Projektionen ein Zukunftsraum von 217 bis 192 US-\$ je Tonne. Bei einem Vergleich der Projektionen mit der Vergangenheit ist zu erkennen, dass der zukünftige Weizenpreis über den Weizenpreisen der Jahre 1997 bis 2006 liegt und langfristig gesehen einen geringen Preisanstieg aufweist.

Um den Zukunftsraum (217 - 192 US-\$ je Tonne) in dem dritten Kernschritt darzustellen, wird die Projektion der OECD/FAO genutzt, da sie für das Jahr 2021 einen Weizenpreis (real 2005) von 204 US-\$ je Tonne prognostiziert, der in der Mitte des durch die verschiedenen Projektionen gebildeten Zukunftsraums liegt. Aufgrund der geringen Größe des Zukunftsraums reicht die Projektion der OECD/FAO aus, um

\footnotetext{
${ }^{127}$ Die Organisationen OECD/FAO und World Bank stellen die Entwicklung des Weizenpreises anhand der Sorte „Hard Red Winter Wheat" dar. Das USDA nutzt den „Farm Price“, um die Entwicklung des Weizenpreises aufzuzeigen.

${ }^{128}$ Die prognostizierten Daten vom FAPRI sind nicht in der Abbildung 41 enthalten, da sie die Entwicklung des Weizenpreises nur nominal angeben.
} 
ihn abzubilden. Da diese Projektion nur einen Weizenpreis bis zum Jahr 2021 prognostiziert, wird auf Grundlage der Daten der Jahre 2014 bis 2021 die reale Preisentwicklung mithilfe einer linearen Trendberechnung bis zum Jahr 2023 berechnet (Abbildung 42). Für das Jahr 2023 geht ein Weizenpreis (real 2005) von 206 US-\$ je Tonne in die „EU27 Szenario-Bildung und -Konsistenzprüfung“ ein.

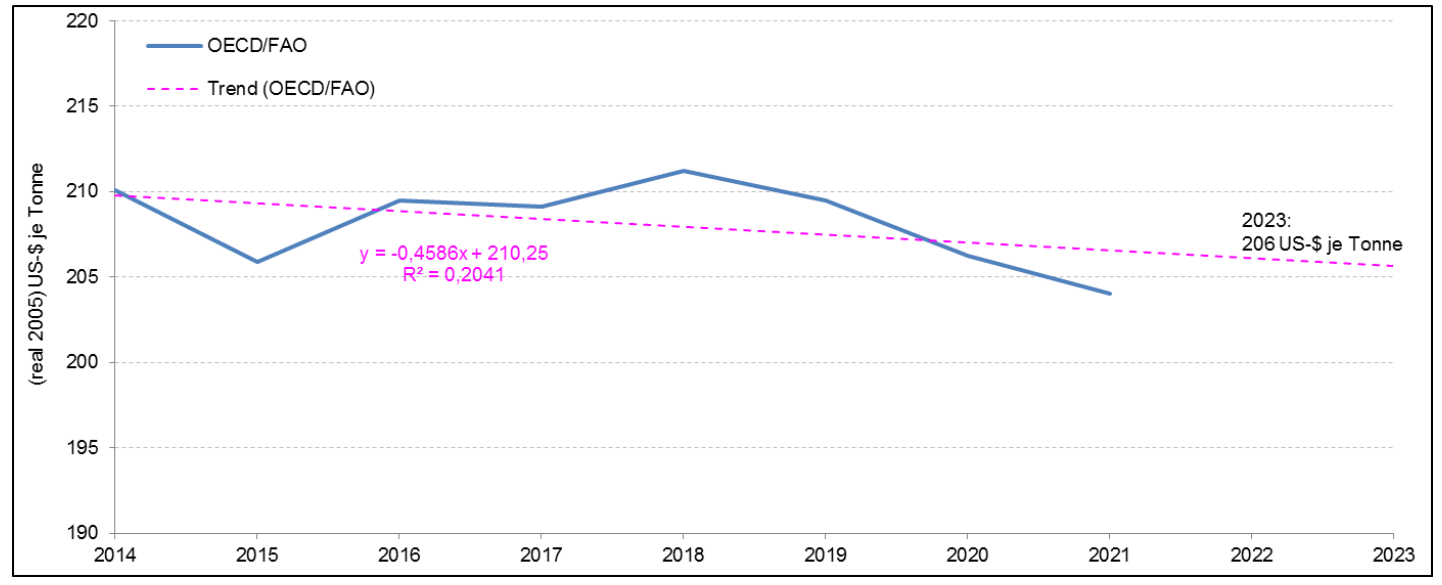

Abbildung 42: Prognostizierte Entwicklung des (real 2005) Weizenpreises bis 2023

Quelle: In Anlehnung an OECD/FAO 2012: 121; USDA 2012: 70; WORLD BANK DEVELOPMENT PROSPECTS GROUP 2012: 1; EIGENE BERECHNUNG

Auch wenn die Projektionen der Einrichtungen OECD/FAO, USDA und World Bank einen relativ konstanten Verlauf des Weizenpreises im Zeitraum von 2014 bis 2021 prognostizieren (Abbildung 42), müssen sich die Ethanolproduzenten auf volatile Märkte einrichten. Infolge der prognostizierten geringen Lagerbestände im Verhältnis zum Verbrauch von Weizen ${ }^{129}$ werden unerwartete Produktionsausfälle von bedeutenden Weizen produzierenden Staaten ${ }^{130} \mathrm{zu}$ starken Preisanstiegen führen (OECD/FAO 2012: 128). Diese Preisschwankungen werden durch keine Zukunftsprojektion abgebildet, da sie infolge nicht vorhersehbarer Ereignisse wie beispielsweise einer Dürre auftreten und nur kurzfristig auf den Weizenpreis einwirken, sodass sich der Preis langfristig ${ }^{131}$ wieder normalisiert.

Basierend auf den vorangegangenen Erläuterungen wird folgende Zukunftsprojektion in der Arbeit weiter berücksichtigt:

9bA | Weizenpreis (real 2005) 206 US-\$ je Tonne

\footnotetext{
129 In den Jahren 2014 bis 2021 beträgt die "Stock-to-use ratio“ nach Prognosen der OECD/FAO (2012: 123) nur knapp über 30\%.

${ }^{130}$ Als wichtige Weizen produzierende Staaten sind die USA, Russland, China, Indien und die Europäische Union zu nennen (OECD/FAO 2012: 123).

${ }^{131}$ Das USDA (2012: 60) und die OECD/FAO (2012: 121) nennen die folgenden Faktoren, die den Weizenpreis langfristig beeinflussen: Bevölkerungswachstum, Wachstum des ProKopf-Einkommens, ansteigende Energiekosten und geringere Produktivitätssteigerungen in der Landwirtschaft.
} 


\subsubsection{Anzahl benzinbetriebener Pkw, die mit mittleren Ethanolbeimi- schungen fahren können (E15 - E40)}

Die Entwicklung des technischen Schlüsselfaktors wird in der Einheit Millionen Pkw in der EU27, gegliedert nach der Fähigkeit der Pkw, unterschiedlich hohe Ethanolbeimischungen zu Benzin als Kraftstoff zu nutzen, angegeben.

Wie in Abbildung 43 ersichtlich, ist die Anzahl benzinbetriebener Pkw seit Jahren rückläufig. Die Anzahl sank von 154,3 Millionen Pkw im Jahr 2000 auf 147,1 Millionen Pkw im Jahr 2010. Zwar wächst der Pkw-Markt in der EU27 geringfügig, aber benzinbetriebene Pkw werden im stärkeren Maße durch Dieselfahrzeuge ersetzt (ACEA 2011b: 13; FRAUNHOFER ISI ET AL. 2010: 39). Benzinbetriebene Pkw, die im Jahr 2005 oder später in der EU27 zugelassen wurden, können Benzin mit einer 10-prozentigen Ethanolbeimischung (E10) als Kraftstoff verwenden; Kraftfahrzeuge, die vor 2005 die Zulassung bekamen, sind mindestens fähig, Beimischungen von 5\% Ethanol zu Benzin (E5) als Treibstoff zu verbrennen (EUROPEAN COMMISSION JOINT RESEARCH CENTRE, INSTITUT FOR ENERGY ET AL. 2011: 42; WOOD MACKENZIE 2010: 144f.; ACEA 2012a).

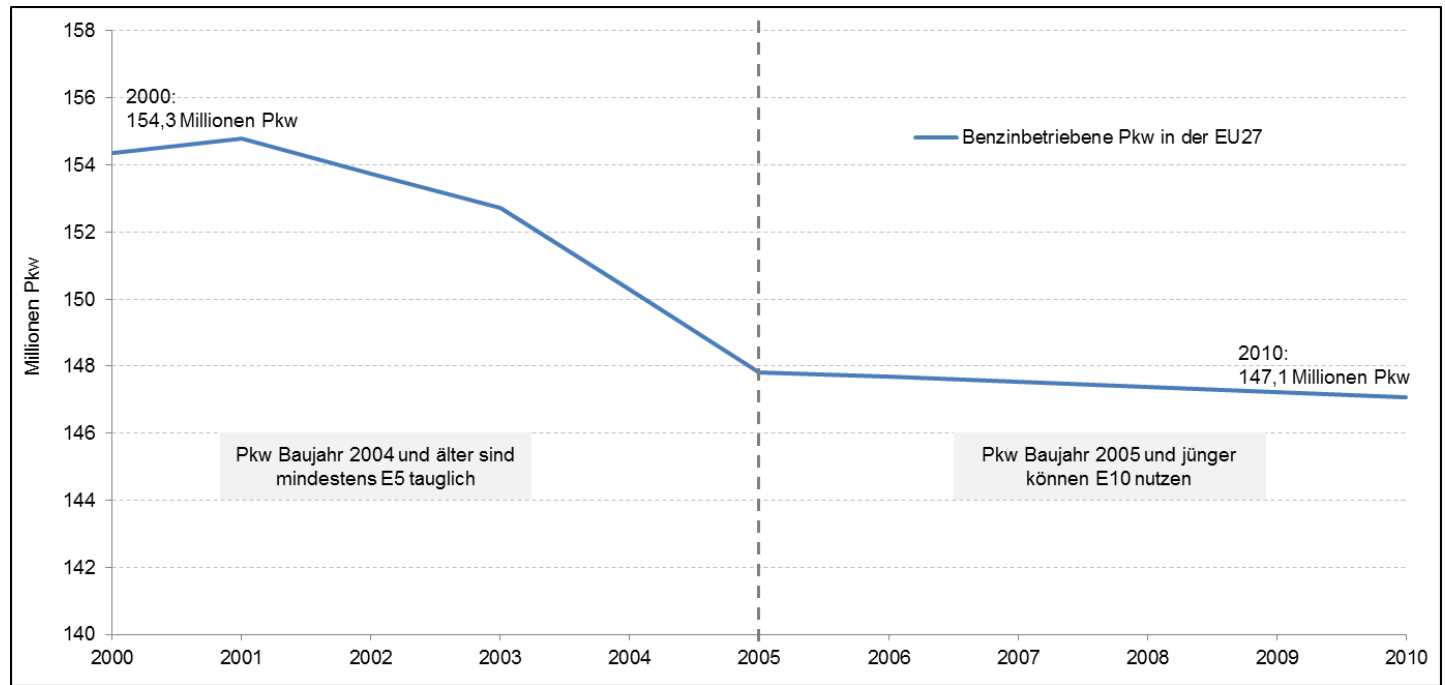

Abbildung 43: Entwicklung der Anzahl benzinbetriebener Pkw in der EU27 und deren Ethanolverträglichkeit

Quelle: In Anlehnung an EUROPÄISCHE KOMMISSION EUROSTAT 2012; FRAUNHOFER ISI ET AL. 2010: 39; EUROPEAN COMMISSION JOINT RESEARCH CENTRE, INSTITUT FOR ENERGY ET AL. 2011: 42

Die Ermittlung der Zukunftsprojektionen für das Jahr 2023 erfolgt in vier aufeinander aufbauenden Schritten:

1. Ermittlung der Anzahl benzinbetriebener Pkw in der EU27 im Jahr 2023.

2. Aufteilung der Pkw in unterschiedliche Altersklassen.

3. Bestimmung von zwei Projektionen, ab welchem Baujahr die Pkw eine bestimmte prozentuale Ethanolbeimischung zu Benzin als Kraftstoff verwenden können. 
4. Ermittlung der Anzahl an Pkw in der EU27 im Jahr 2023, unterteilt nach deren Fähigkeit, unterschiedlich hohe Ethanolbeimischungen zu Benzin als Kraftstoff zu nutzen.

Im ersten Schritt wird ausgehend von 147,1 Millionen Pkw im Jahr 2010 ein weiterer Rückgang der benzinbetriebenen Pkw bis zum Jahr 2023 in der EU27 erwartet. Die Institute FRAUNHOFER ISI ET AL. (2010: 39) prognostizieren für das Jahr 2023 eine benzinbetriebene Pkw-Flotte von 145,2 Millionen ${ }^{132}$. Der durchschnittliche jährliche Rückgang um $0,1 \%$ ist sowohl auf die Substitution benzinbetriebener Fahrzeuge durch dieselbetriebene Pkw als auch auf das geringe Wachstum $(1,1 \%$ pro Jahr) der Automobilflotte in der EU27 zurückzuführen (FRAUNHOFER ISI ET AL. 2010: 39, WOOD MACKENZIE 2010: 41; U.S. ENERGY INFORMATION ADMINISTRATION 2011b: 122).

Basierend auf den prognostizierten 145,2 Millionen Pkw im Jahr 2023 gilt es, im zweiten Schritt festzustellen, wann diese Kraftfahrzeuge gebaut werden. Zur Ermittlung dessen werden die Angaben von WOOD MACKENZIE (2010: 144) Tabelle 12 erste und zweite Zeile - genutzt ${ }^{133}$. Durch die Multiplikation des prozentualen Anteils an der Gesamtflotte im Jahr 2023 mit den prognostizierten 145,2 Millionen benzinbetriebenen Pkw ergeben sich die folgenden Werte (Tabelle 12): Von den 145,2 Millionen benzinbetriebenen Pkw im Jahr 2023 sind 10,2 Millionen Baujahr 2023, 39,2 Millionen Baujahr 2022 bis 2018, 47,9 Millionen Baujahr 2017 bis 2013 und 47,9 Millionen älter als Baujahr 2013.

\footnotetext{
${ }^{132}$ Die Projektion wurde unter Leitung des Fraunhofer-Instituts für System- und Innovationsforschung und in Zusammenarbeit mit den folgenden weiteren europäischen Instituten erstellt: NEA Transport Research and Training, Trasporti e Territorio SRL, Transport \& Mobility Leuven, Institute for Economic Policy Research University of Karlsruhe, Institute for Prospective Technological Studies European Commission und The Netherlands Organisation for Applied Scientific Research. Diese sind im Bereich der Mobilität und der Erstellung von Zukunftsprojektionen führend.

133 Die prognostizierten Daten von WOOD MACKENZIE (2010: 144) sind bei einem Vergleich mit dem durchschnittlichen Alter der Pkw von 8,2 Jahren in der EU27 im Jahr 2009 als realistisch zu betrachten (ACEA 2011b: 35).
} 


\begin{tabular}{|c|c|c|}
\hline Alter der Pkw im Jahr 2023 & $\begin{array}{l}\text { Prozentualer Anteil an der } \\
\text { Gesamtflotte im Jahr } 2023\end{array}$ & $\begin{array}{c}\text { Anzahl benzinbetriebener } \\
\text { Pkw }\end{array}$ \\
\hline Neu (Baujahr: 2023) & $7 \%$ & 10,2 Millionen \\
\hline 1 - 4 Jahre (Baujahr: $2022-2018)$ & $27 \%$ & 39,2 Millionen \\
\hline $\begin{array}{c}5 \text { - } 10 \text { Jahre (Baujahr: } 2017 \text { - } \\
\text { 2013) }\end{array}$ & $33 \%$ & 47,9 Millionen \\
\hline $\begin{array}{c}\text { >10 Jahre (Baujahr: Älter als Bau- } \\
\text { jahr 2013) }\end{array}$ & $33 \%$ & 47,9 Millionen \\
\hline Total & $100 \%$ & 145,2 Millionen \\
\hline
\end{tabular}

Tabelle 12: Anzahl der benzinbetriebenen Pkw in der EU27 im Jahr 2023

Quelle: In Anlehnung an WOOD MACKENZIE 2010: 144; EIGENE BERECHNUNG

Im dritten Schritt werden hinsichtlich der Fähigkeit der benzinbetriebenen Pkw, mittlere Ethanolbeimischungen zu Benzin als Kraftstoff zu verbrennen, für das Jahr 2023 zwei Projektionen gebildet. Die Erstellung von zwei unterschiedlichen Projektionen ist notwendig, da zum heutigen Zeitpunkt keine eindeutige Prognose möglich ist. Die Annahmen zur Bildung der zwei Projektionen stammen vom EUROPEAN COMMISSION JOINT RESEARCH CENTRE, INSTITUT FOR ENERGY ET AL. (2011: 26ff.).

Die zwei Annahmen gehen von folgenden unterschiedlichen Situationen im Jahr 2023 aus:

Annahme 1: „Hohe Ethanolverträglichkeit der Pkw“

- Alle zugelassenen Pkw in der EU27 im Jahr 2023 vertragen E10 als Kraftstoff.

- Alle zugelassenen Pkw in der EU27 ab dem Jahr 2015 können E20 als Kraftstoff nutzen.

Annahme 2: "Geringe Ethanolverträglichkeit der Pkw“

- Alle zugelassenen Pkw in der EU27 im Jahr 2023 vertragen E10 als Kraftstoff.

- Zugelassene Pkw bis zum Ende des Zukunftshorizonts vertragen keine höheren Ethanolbeimischungen als E10.

Die erste Projektion „Hohe Ethanolverträglichkeit der Pkw“ basiert auf der Einschätzung, dass die Automobilindustrie in die Weiterentwicklung der Technologie zur Nutzung von mittleren Ethanolbeimischungen investiert, um den $\mathrm{CO}_{2}$-Ausstoß ihrer Pkw-Flotte zu senken (OSEC 2009: 54; ACEA 2012c). Infolgedessen werden technische Probleme am Verbrennungsmotor und an den Dichtungen gelöst, die eine Nutzung von E20 bisher verhindern (WOOD MACKENZIE 2010: 102ff.). Beispielhaft für diese Entwicklung ist der Automobilkonzern Volkswagen, bei dem alle in Europa 
verkauften Autos Baujahr 2010 und jünger bereits E20 nutzen können (SEYFRIED 2012).

Im Gegensatz zur ersten Projektion basiert die zweite Prognose „Geringe Ethanolverträglichkeit der Pkw" auf der Annahme, dass die technischen Probleme am Verbrennungsmotor zur Nutzung mittlerer Ethanolbeimischungen von den meisten Automobilkonzernen bis zum Jahr 2023 nicht gelöst werden, wodurch die Verwendung von E20 im Jahr 2023 nicht möglich sein wird (WOOD MACKENZIE 2010: 102ff.). Außerdem liegt der Projektion die Annahme zugrunde, dass viele Automobilkonzerne Bauteile verwenden, die E10, aber nicht E20 vertragen, um Kosten zu senken. In beiden Projektionen sind alle Kraftfahrzeuge E10-tauglich. Auf die Betrachtung benzinbetriebener Pkw, die vor 2005 zugelassen wurden und nur E5 vertragen, wird aufgrund ihrer geringen Anzahl im Jahr 2023 in der weiteren Arbeit verzichtet ${ }^{134}$.

Für die Projektion „Hohe Ethanolverträglichkeit der Pkw“ ergeben sich im vierten Schritt aufgrund der Berechnungen zu Anzahl und Alter der benzinbetriebenen Pkw in der EU27 im Jahr 2023 (Schritt: 2) und aufgrund der Annahmen hinsichtlich der Verträglichkeit mittlerer Ethanolbeimischungen zu Benzin (Schritt: 3) folgende Ergebnisse (Abbildung 44): 78 Millionen Pkw in der EU27 können E20 als Kraftstoff nutzen, dies sind 54\% der gesamten benzinbetriebenen Pkw im Jahr 2023. Die restlichen 67 Millionen Pkw - 46\% der gesamten benzinbetriebenen Pkw-Flotte - sind E10-tauglich.

\footnotetext{
${ }^{134}$ Bei vergleichbaren Berechnungen, durchgeführt durch das JEC (European Commission Joint Research Centre, EUCAR und CONCAWE) für das Jahr 2020, mithilfe des Fleet and Fuels (F\&F) Model, haben benzinbetriebene Pkw Baujahr 2005 und älter einen Anteil an der Pkw-Flotte von lediglich 3\% (EUROPEAN COMMISSION JOINT RESEARCH CENTRE, INSTITUT FOR ENERGY ET AL. 2011: 20). Folglich kann diese Altersgruppe aufgrund ihrer geringen Bedeutung in der weiteren Studie vernachlässigt werden.
} 


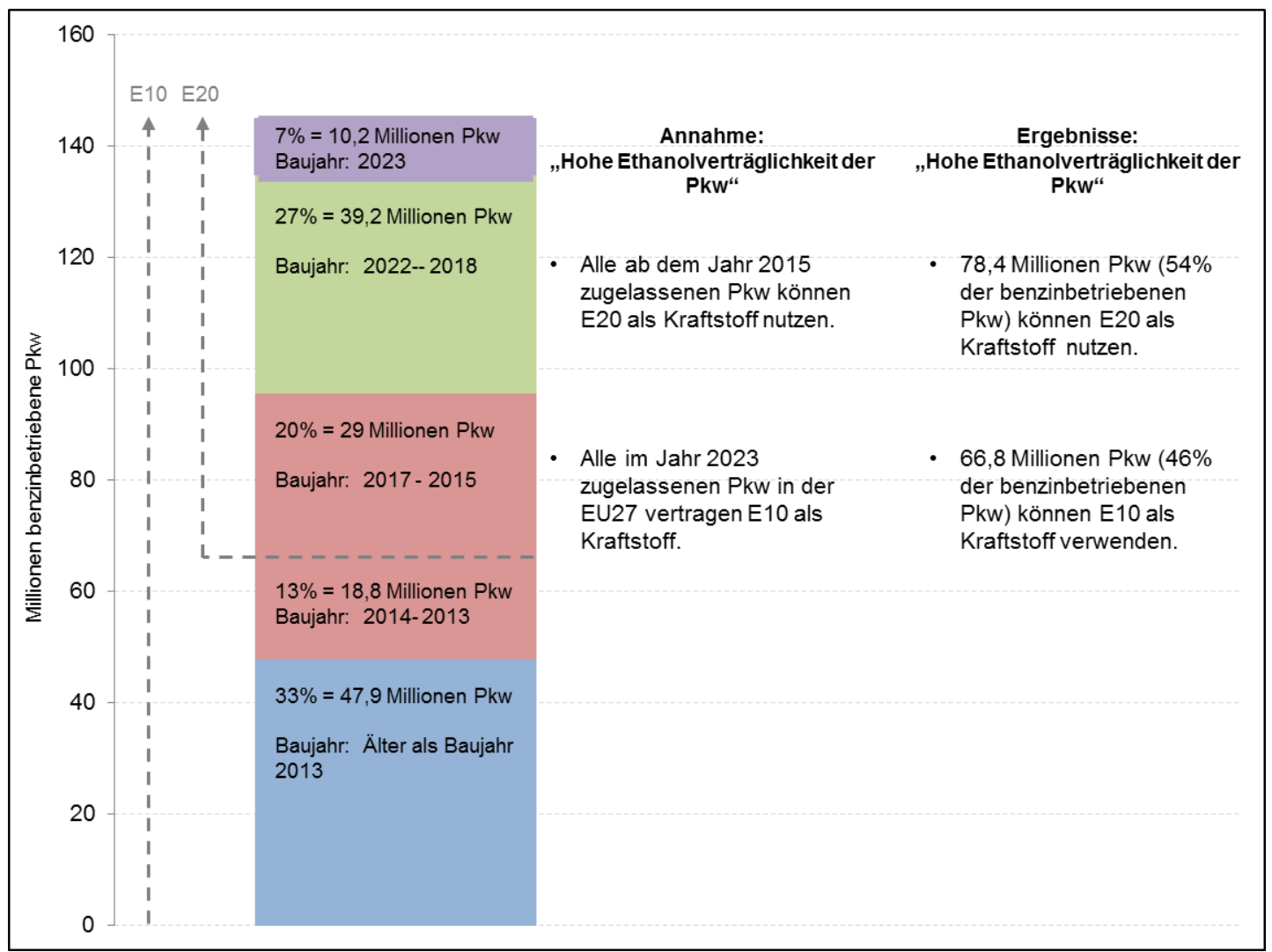

Abbildung 44: Darstellung der Ergebnisse der Projektion „Hohe Ethanolverträglichkeit der Pkw"

Quelle: EIGENE BERECHNUNG

Die zweite Projektion („Geringe Ethanolverträglichkeit der Pkw“), die ebenso wie die erste Projektion auf den Schritten zwei und drei basiert, zeigt im vierten Schritt folgendes Ergebnis (Abbildung 45): Alle benzinbetriebenen Pkw in der EU27 im Jahr 2023 (145,2 Millionen) können E10, aber nicht E20 als Kraftstoff nutzen. 


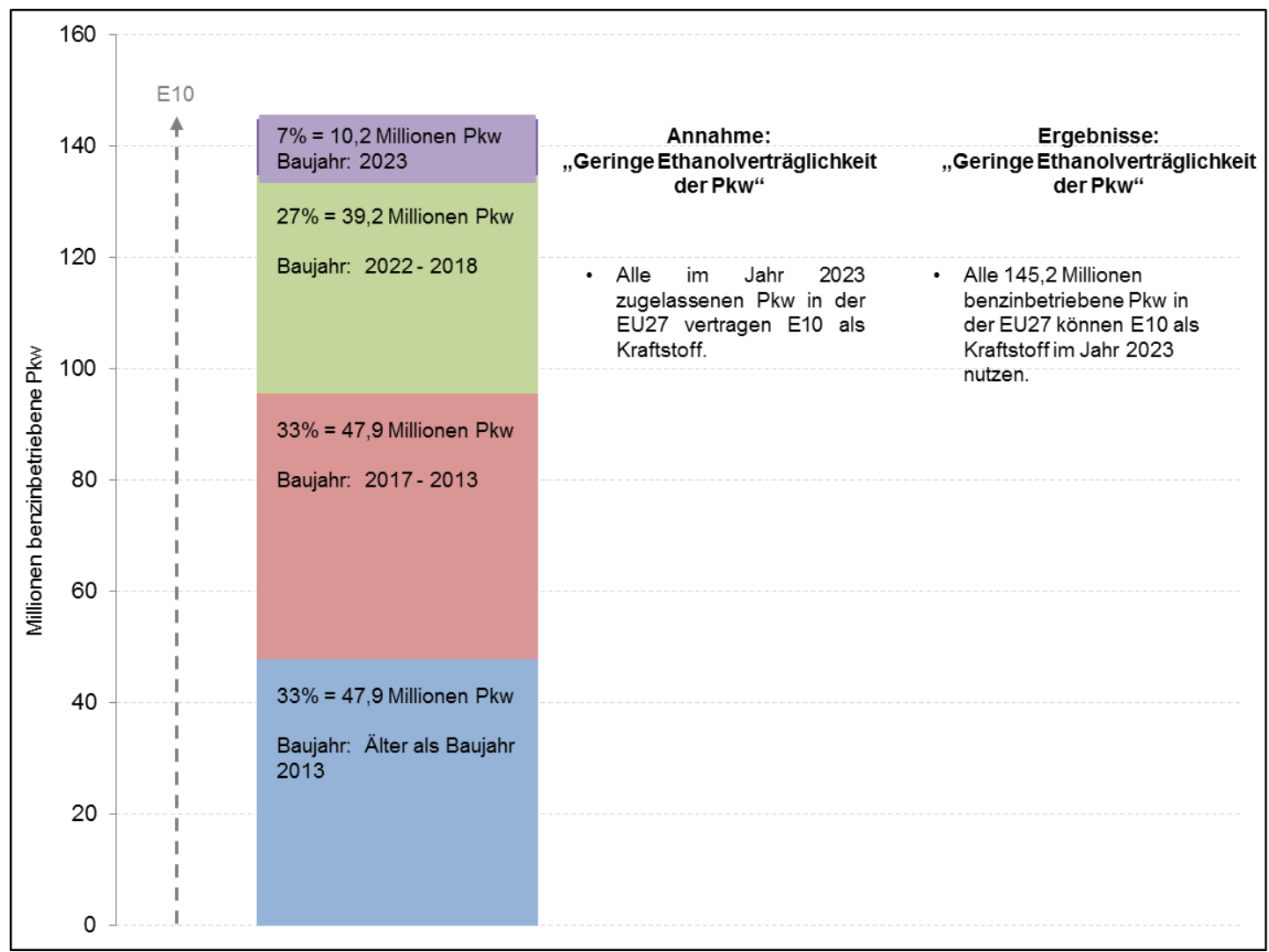

Abbildung 45: Darstellung der Ergebnisse der Projektion „Geringe Ethanolverträglichkeit der Pkw"

Quelle: EIGENE BERECHNUNG

Die Ergebnisse der zwei Projektionen bilden die Grundlage der Zukunftsprojektionen, die mitsamt ihrer Beschreibungen in den dritten Kernschritt eingehen:

$12 \mathrm{~A}$

Hohe Ethanolverträglichkeit konventioneller Pkw

Beschreibung:

54\% (78 Millionen) der benzinbetriebenen Pkw können E20, die verbleibenden 46\% (67

Millionen) E10 als Kraftstoff nutzen.

Geringe Ethanolverträglichkeit konventioneller Pkw

12B Beschreibung:

Alle der 145 Millionen benzinbetriebene Pkw in der EU27 können E10, aber nicht E20 als

Kraftstoff verwenden.

\subsubsection{Zölle auf Ethanolimporte in die EU27}

Die EU27 erhebt hohe Zölle auf den Import von Ethanol; trotzdem ist die EU27 der größte Ethanolimporteur der Welt. Im Jahr 2011 wurden 1,3 Milliarden Liter Ethanol, hauptsächlich aus den USA und Brasilien, in die EU27 importiert (F.O. LICHT 2012a: 300f.; F.O. LICHT 2011b: 87; THOMASSON 2011: 11). 
Die derzeitigen Importzölle für Ethanol sind per Verordnung von der europäischen Kommission unter dem KN-Code $2207^{135}$ festgelegt. Der KN-Code 220710 bestimmt den Zollsatz für „Ethylalkohol mit einem Alkoholgehalt von $80 \%$ vol oder mehr, unvergällt ${ }^{136 ،, ~ e r ~ b e t r a ̈ g t ~ 19,2 ~ € / h l . ~ D e r ~ Z o l l s a t z ~ f u ̈ r ~ „ E t h y l a l k o h o l ~ u n d ~ B r a n n t-~}$ wein mit beliebigem Alkoholgehalt, vergällt" liegt bei 10,2 €/hl, er ist festgelegt unter KN-Code $220720^{137}$ (EUROPÄISCHE KOMMISSION 2011: 182). Darüber hinaus werden seit März 2012 auch Ethanol-Benzin-Mischungen mit einem Ethanolgehalt von 70\% oder mehr durch den KN-Code 220720 verzollt (F.O. LICHT 2012a: 303). Zuvor wurden diese Mischungen unter KN-Code 38249097 mit einem deutlich geringeren Wertzollsatz von 6,5\% verzollt ${ }^{138}$ (F.O. LICHT 2012a: 303; USDA FOREIGN AGRICULTURAL SERVICE 2012: 16). Durch diese Veränderung der Zollbestimmungen erhofft sich die EU27, die hohen Einfuhren von günstigem E90 insbesondere aus den USA zu stoppen. Im Jahr 2011 wurden eine Milliarde Liter E90, bei einem Gesamtimport von 1,3 Milliarden Litern, in die EU27 importiert (F.O. LICHT 2012a: 300) ${ }^{139}$.

Ausgenommen von den soeben beschriebenen Zollbestimmungen sind Staaten, die unter eines der folgenden Programme fallen: Die „Everything But Arms (EBA) Initiative“, das "Cotonou Agreement" (Staaten in Afrika, der Karibik und dem Pazifischen Raum mit Ausnahme von Südafrika), die „Euro-Med Agreements“ und das „Generalised System of Preferences Plus“ (GSP) (USDA FOREIGN AGRICULTURAL SERVICE 2012: 16.; F.O. LICHT/AGRA INFORMA 2009: 150ff.). Sie haben somit freien Marktzugang zu dem Ethanolmarkt der EU27. Der Einfluss dieser Staaten auf den Ethanolmarkt der EU27 ist gering, da die größten Importmengen aus den USA und Brasilien stammen (F.O. LICHT 2012a: 300). Aufgrund dessen wird der Schwer-

\footnotetext{
${ }^{135}$ Der KN-Code gibt die „Kombinierte Nomenklatur“ zur Einordnung des Produktes in den Zolltarif der Europäischen Union an (BUNDESANSTALT FÜR LANDWIRTSCHAFT UND ERNÄHRUNG 2013).

${ }^{136}$ Das Vergällen bewirkt, dass Ethanol für den menschlichen Verzehr ungenießbar wird. Die Vergällung erfolgt durch die Zugabe von Substanzen entsprechend der Verordnung der Kommission 3199/93 (USDA FOREIGN AGRICULTURAL SERVICE 2012: 16).

${ }^{137}$ Die meisten Mitgliedsstaaten der EU27 erlauben nur die Einfuhr von unvergälltem Ethanol, mit Ausnahme der Staaten Großbritannien, Finnland, Dänemark, Niederlande, Tschechien und der Slowakei, die auch den Import von vergälltem Ethanol genehmigen (EUROPÄISCHE KOMMISSION 2011: 182).

${ }^{138}$ Der Zollsatz unter KN-Code 38249097 ist deutlich geringer, denn erst ab einem Ethanolpreis von $157 € /$ hl liegt der Wertzollsatz von $6,5 \%$ über dem Zollsatz, festgelegt im KNCode 220720 (F.O. LICHT 2011b: 86).

${ }_{139}$ Infolge der günstigen E90-Importe sank der Ethanolpreis in der EU27 und zwang etliche Ethanolproduzenten, die Produktion aufgrund fehlender Profitabilität auszusetzen. (F.O. LICHT 2012a: 300; USDA FOREIGN AGRICULTURAL SERVICE 2012: 16f., F.O. LICHT 2011a: 66). Die Importzölle sind für die Ethanolproduzenten der EU27 von hoher Relevanz, da die Ethanolherstellungskosten in den USA und Brasilien im Vergleich zur EU27 deutlich geringer sind (BERG 2012d: 2f.).
} 
punkt auf die Entwicklung der Zollbestimmungen für Staaten wie Brasilien und die USA gelegt, die keinen freien Marktzugang zur EU27 besitzen ${ }^{140}$.

Aufgrund der Unsicherheit hinsichtlich der Entwicklung der Zollbestimmungen für in die EU27 importiertes Ethanol bis zum Jahr 2023 werden zwei Zukunftsprojektionen entwickelt. Die erste Prognose „Hohe Zölle auf Ethanolimporte in die EU27“ geht davon aus, dass die heutigen Zollsätze noch im Jahr 2023 bestehen. Im Gegensatz dazu prognostiziert die zweite Projektion eine zollfreie Einfuhr von Ethanol in die EU27.

Die Projektion „Hohe Zölle auf Ethanolimporte in die EU27“ basiert auf der Annahme, dass die Verordnungen der Kommission unter KN-Code 220710 und KN-Code 220720 sowie die Verordnung, dass Ethanol-Benzin-Mischungen mit einem Ethanolgehalt von $70 \%$ oder mehr durch den KN-Code 220720 verzollt werden, noch im Jahr 2023 bestehen. Darüber hinaus werden bei der Projektion zukünftige, heute noch unbekannte Wege, die es den Händlern ermöglichen, den Importzoll zu umgehen oder zu deutlich geringeren Zollsätzen Ethanol in die EU27 zu exportieren, durch Verordnungen der Europäischen Kommission versperrt ${ }^{141}$. Ein aktuelles Beispiel hierfür ist die im Jahr 2011 erlassene Verordnung zur Verzollung von E90Importen unter KN-Code 220720.

Die Beibehaltung der jetzigen Zollbestimmungen mit dem Ziel, die EU27 vor günstigen Ethanolimporten zu schützen, ist realistisch. Denn ein Ziel der politischen Unterstützung von Bioenergie und damit auch von Ethanol ist die Schaffung von Beschäftigung und die Förderung der regionalen Entwicklung insbesondere in ländlichen und entlegenen Gebieten (RICHTLINIE 2009/28/EG ERW 1). Die Erreichung dieses Ziels ist aber nur möglich, wenn das Ethanol auch in der EU27 produziert und nicht importiert wird. Des Weiteren sehen F.O. LICHT/AGRA INFORMA (2009: 141), EUROPEAN COMMISSION JOINT RESARCH CENTRE, INSTITUT FOR PROSPECTIVE TECHNOLOGICAL STUDIES (2010a: 15) sowie HENNIGES/ZEDDIES (2006: 2) die politische Förderung von Ethanol auch als ein Mittel zur Unterstützung der europäischen Landwirtschaft. Diese bleibt nur erhalten, solange das Ethanol in der EU27 produziert wird.

\footnotetext{
${ }^{140}$ Auch in der Wirkungsanalyse (Kapitel 5.1.2) wurde der Einfluss der USA und Brasiliens auf den Ethanolmarkt der EU27 deutlich höher bewertet als der von Staaten mit freiem Marktzugang in die EU27.

${ }^{141}$ Durch die hohen Zollsätze lohnt es sich für Händler, nach Schlupflöchern zu suchen, um die hohen Importzölle zu umgehen (F.O. LICHT 2012a: 300). Aufgrund dessen ist auch zukünftig zu erwarten, dass neue Wege gefunden werden, die es ermöglichen, die Zollbestimmungen der EU27 zu umgehen (F.O. Licht 2011b: 88).
} 
Die zweite Projektion „Zollfreie Einfuhr von Ethanol in die EU 27“ sagt voraus, dass bis zum Jahr 2023 alle Importzölle aufgehoben wurden und Ethanol zollfrei in die EU27 importiert werden kann. Diese Projektion wird als realistisch betrachtet, da in den USA zum Jahreswechsel (2011 - 2012) der Importzoll in Höhe 0,54 US-\$ je Gallone ausgelaufen ist (F.O. LICHT 2012b: 169). Damit haben die beiden größten Ethanolmärkte der Welt - USA und Brasilien - geringere Zollbeschränkungen im Vergleich zur EU27, wodurch sich der Druck auf die europäische Gesetzgebung erhöht, dem Beispiel der USA zu folgen (F.O. LICHT 2012c: 222). Auch die INTERNATIONAL ENERGY AGENCY (2011a: 29) fordert einen weltweiten Abbau der Zollbeschränkungen für Ethanol, damit der Markt besser auf Angebots- und Nachfrageschwankungen in den einzelnen Regionen reagieren kann. Darüber hinaus ist es das politische Kernziel der Förderung von Ethanol-Kraftstoff in der EU27, die Treibhausgasemission im Verkehrsbereich zu verringern (RICHTLINIE 2009/28/EG ERW 1). Folglich wäre es nur sinnvoll, unabhängig von der Herkunft das Ethanol mit der besten $\mathrm{CO}_{2}$-Bilanz im Verkehrssektor einzusetzen. Die Zollbeschränkungen erschweren aber die Einfuhr von Ethanol mit einer guten $\mathrm{CO}_{2}$-Bilanz wie beispielsweise Zuckerrohr-Ethanol aus Brasilien ${ }^{142}$ und sind damit der Erreichung des Kernziels hinderlich.

Auf die Entwicklung einer dritten Projektion, die einen Anstieg der Zollsätze darstellt, wird verzichtet, da die Zölle auf Ethanol insbesondere im Vergleich zu den USA und Brasilien bereits hoch sind (F.O. LICHT 2012a: 300) und die EU27 auf Importe angewiesen ist, um die Beimischungspflichten in den einzelnen Mitgliedsstaaten zu erfüllen (ECOFYS ET AL. 2011: 46).

Die zuvor beschriebenen zwei Zukunftsprojektionen werden in dem Kernschritt der „EU27 Szenario-Bildung und -Konsistenzprüfung“ weiter betrachtet:

Hohe Zölle auf Ethanolimporte in die EU27

Beschreibung:

22A Die derzeitigen Importzölle, festgelegt per Verordnung unter KN-Code 2207, bleiben bestehen. Ethanol-Benzin-Mischungen mit einem Ethanolgehalt von $70 \%$ oder mehr werden ebenfalls unter KN-Code 2207 verzollt.

22B $\mid$ Zollfreie Einfuhr von Ethanol in die EU27

\subsubsection{Politische Förderung von Ethanol}

Der Schlüsselfaktor T1 hat den stärksten Einfluss auf den Ethanolmarkt der EU27 bis zum Jahr 2023. Die politische Förderung von Ethanol-Kraftstoff erfolgt durch die RICHTLINIE 2009/28/EG (INTERNATIONAL ENERGY AGENCY 2011b: 19; EU-

142 Entsprechend der RICHTLINIE 2009/28/EG (Anhang V) hat Ethanol aus Zuckerrohr die beste $\mathrm{CO}_{2}$-Bilanz von Biokraftstoffen der ersten Generation. 
ROPEAN COMMISSION JOINT RESEARCH CENTRE, INSTITUT FOR ENERGY ET AL. 2011: 15; BLOOMBERG NEW ENERGY FINANCE 2010: 8). Kernpunkt der RICHTLINIE ist der ARTIKEL 3 (4), in dem das Ziel formuliert ist, dass jedes Mitgliedsland der EU27 im Jahr 2020 mindestens 10\% des Endenergieverbrauchs im Verkehrssektor aus erneuerbaren Energiequellen beziehen muss. Die Berechnung des prozentualen Energieverbrauchs im Verkehrssektor aus erneuerbaren Energiequellen erfolgt durch die folgende Formel:

Anteil erneuerbare Energie $=\left(E_{E} \times 100\right) / E_{G}$

$E_{E}=$ Zusammenfassung aller Arten von Energie aus erneuerbaren Quellen, die bei allen Verkehrsträgern verbraucht werden (RICHTLINIE 2009/28/EG ARTIKEL $3(4 b))$.

$E_{G}=$ Addition von Ottokraftstoff, Dieselkraftstoff sowie verbrauchten Biokraftstoffen und Elektrizität im Straßen- und Schienenverkehr (RICHTLINIE 2009/28/EG ARTIKEL 3 (4a)).

Bei der Berechnung von $E_{E}$ wird der Energiegehalt von Biokraftstoffen, verbraucht im Verkehrsbereich und hergestellt aus zellulosehaltigem Non-Food-Material, Abfällen und lignozellulosehaltigem Material, doppelt angerechnet (RICHTLINIE 2009/28/EG ARTIKEL 21 (2)), die aus erneuerbaren Energiequellen erzeugte und von Verkehrsträgern genutzte Elektrizitätsmenge sogar mit dem 2,5-fachen Energiegehalt (RICHTLINIE 2009/28/EG ARTIKEL 3 (4)). Darüber hinaus gibt es keine Begrenzung der Anrechnung von Biokraftstoffen der ersten Generation zur Erreichung des $10 \%$-Ziels.

Das von der EU27 bestimmte 10\%-Ziel fördert Biokraftstoffe und somit auch Ethanol nicht direkt ${ }^{143}$. Die Experten sind sich aber einig (EUROPÄISCHEN KOMMISSION 2012: 7; EUROPEAN COMMISSION JOINT RESEARCH CENTRE, INSTITUTE FOR ENERGY ET AL. 2011: 46 ff.; EUROPEAN COMMISSION JOINT RESEARCH CENTRE, INSTITUTE FOR PROSPECTIVE TECHNOLOGICAL STUDIES 2010a: 33), dass Biokraftstoffe den mit Abstand größten Anteil dazu beitragen werden, das Ziel im Jahr 2020 zu erreichen; laut Branchenangaben werden voraussichtlich acht

\footnotetext{
${ }^{143}$ Biokraftstoffe werden nicht durch die EU27, sondern durch die Regierungen der einzelnen Mitgliedsstaaten direkt gefördert. Die RICHTLINIE 2009/28/EG (ARTIKLE 4 (1)) verpflichtet die Mitgliedsstaaten der EU27, einen „Nationalen Aktionsplan für erneuerbare Energien" auszuarbeiten, in dem jedes Mitgliedsland darlegt, wie sie das vorgegebene Ziel der EU27 erreichen will. Von den Mitgliedsstaaten werden die Instrumente der Beimischungspflicht von Ethanol und Biodiesel zu Fossilbrennstoff und/oder die Befreiung von Steuern auf Biokraftstoffe genutzt, um deren Anteil am Kraftstoffverbrauch zu erhöhen (F.O. LICHT 2012d: 420f.). Weitere Informationen zu diesem Thema befinden sich in Kapitel 5.1.1.2/Einflussfaktor 20.
} 
Prozent der gesamten zehn Prozent durch den Verbrauch von Biokraftstoffen der ersten Generation erfüllt (AGRA-EUROPE 2012: EU-NACHRICHTEN 9).

In Zukunft sind Veränderungen der politischen Förderung von Ethanol zu erwarten. Energiekommissar Günther Oettinger und die Klimakommissarin Connie Hedegard haben diesbezüglich am 17. Oktober 2012 einen Vorschlag unterbreitet (EUROPÄlSCHE KOMMISSION 2012). Die Punkte dieses Vorschlags, die die politische Förderung von Ethanol-Kraftstoff betreffen, sind im Folgenden genannt:

- Das Ziel, dass 10\% der eingesetzten Energie im Verkehrssektor im Jahr 2020 aus erneuerbaren Energiequellen stammen sollen, bleibt bestehen (EUROPÄISCHE KOMMISSION 2012: 9).

- Auf $5 \%$ begrenzte Anrechnung von Biokraftstoffen, produziert aus Getreide, sonstigen stärkehaltigen Pflanzen, Zuckerpflanzen und Ölpflanzen auf das 10\%Ziel (EUROPÄISCHE KOMMISSION 2012: 16).

- Vierfache Anrechnung des Energiegehalts von Biokraftstoffen, beispielsweise produziert aus Stroh und Algen, auf das 10\%-Ziel (EUROPÄISCHE KOMMISSION 2012: 16) ${ }^{144}$.

- Ab dem Jahr 2021 sollen Biokraftstoffe der ersten Generation nicht mehr politisch gefördert werden (EUROPÄISCHE KOMMISSION 2012: 3).

Aufgrund der zu erwartenden politischen Veränderungen und der damit verbundenen Unsicherheit kann eine einzige Zukunftsprojektion die möglichen Entwicklungen des Schlüsselfaktors nicht abdecken. Um dieser Situation gerecht zu werden, werden im Folgenden drei Projektionen gebildet, die jeweils eine Situation des Schlüsselfaktors im Jahr 2023 beschreiben.

Die erste Zukunftsprojektion „Politische Förderung von Biokraftstoffen der ersten und zweiten Generation“ geht davon aus, dass die derzeitigen (Jahr 2012) politischen Vorgaben noch im Jahr 2023 bestehen werden; folgende Punkte umfasst die Projektion:

- Ziel, $10 \%$ der verwendeten Energie im Verkehrsbereich aus erneuerbaren Energiequellen zu produzieren.

\footnotetext{
${ }^{144}$ Folgende weitere Rohstoffe werden ebenfalls mit dem Vierfachen ihres Energiegehalts dem 10\%-Ziel angerechnet (EUROPÄISCHE KOMMISSION 2012: 24f.): Biomasse-Anteil gemischter Siedlungsabfälle, Biomasse-Anteil von Industrieabfällen, Stroh, Tierdung und Klärschlamm, freie Fettsäuren aus der Herstellung von Palmöl (POME) und leere Palmfruchtbündel, Tallölpech, Rohglyzerin, Bagasse, Traubentrester und Weintrub, Nussschalen, Hülsen, Maiskolben und Rinde, Zweige, Blätter, Sägemehl und Sägespäne.
} 
- Keine Begrenzung der Anrechnung von Biokraftstoffen der ersten Generation auf das $10 \%$-Ziel.

- Biokraftstoffe der zweiten Generation werden gefördert, indem ihr Energiegehalt auf das 10\%-Ziel doppelt angerechnet wird.

Die Projektion ist realistisch, da sie auf der Ist-Situation basiert, die in dieser Form bis zum Jahr 2020 bestehen soll. Ferner handelt es sich bei den Äußerungen von Energiekommissar Günther Oettinger und Klimakommissarin Connie Hedegard zur Änderung der genannten politischen Rahmenbedingungen für Biokraftstoffe lediglich um einen Vorschlag (EUROPÄISCHE KOMMISSION 2012), der sowohl noch im EU-Parlament als auch mit den Mitgliedsstaaten der EU27 verhandelt werden muss (F.O. LICHT 2012e: 28).

Die zweite Zukunftsprojektion „Begrenzung der Nutzung von Biokraftstoffen der ersten Generation auf 5\% und starke Förderung von Biokraftstoffen der zweiten Generation“ basiert auf dem Vorschlag der Kommissare Günther Oettinger und Connie Hedegard (EUROPÄISCHE KOMMISSION 2012: 9ff.). Die Kernpunkte der Projektion sind folgende:

- Ziel, $10 \%$ der verwendeten Energie im Verkehrsbereich aus erneuerbaren Energiequellen zu produzieren.

- Auf $5 \%$ begrenzte Anrechnung von Biokraftstoffen der ersten Generation auf das 10\%-Ziel.

- Vierfache Anrechnung des Energiegehalts von Biokraftstoffen der zweiten Generation auf das $10 \%$-Ziel.

Die Projektion wird als realistisch betrachtet, da sie auf den Vorschlägen der Kommissare basiert (EUROPÄISCHE KOMMISSION 2012: 9ff.). Darüber hinaus gilt es, die Projektion zu betrachten, da sie den Argumenten der „Tank-oder-TellerDiskussion" - welche in den Sommermonaten des Jahres 2012 intensiv geführt wurde (FRANKFURTER ALLGEMEINE ZEITUNG 2012) - durch eine Begrenzung der Nutzung von Biokraftstoffen der ersten Generation und durch eine stärkere Förderung von Biokraftstoffen der zweiten Generation folgt. Somit erfüllt diese Zukunftsprojektion die gesellschaftlichen Ansprüche an Biokraftstoffe in der EU27. Des Weiteren ist die stärkere Förderung von Biokraftstoffen der zweiten Generation realistisch, da diese Biokraftstoffe im Vergleich zu biogenen Treibstoffen der ersten 
Generation höhere $\mathrm{CO}_{2}$-Einsparungen erreichen ${ }^{145}$ und damit dem Kernziel der Senkung der $\mathrm{CO}_{2}$-Emissionen in der EU27 entsprechen (RICHTLINIE 2009/28/EG ERW 1).

Die dritte Zukunftsprojektion „Ausschließliche Förderung von Biokraftstoffen der 2. Generation" geht noch einen Schritt weiter im Vergleich zur zweiten Projektion. Sie prognostiziert eine alleinige politische Förderung von Biokraftstoffen der zweiten Generation im Jahr 2023; folgende Punkte enthält die Projektion:

- Ziel, 10\% der verwendeten Energie im Verkehrsbereich aus erneuerbaren Energiequellen zu produzieren.

- Keine Anrechnung von Biokraftstoffen der ersten Generation auf das 10\%-Ziel.

- Mehrfache Anrechnung des Energiegehalts von Biokraftstoffen der zweiten Generation auf das $10 \%$-Ziel.

Die Projektion ist glaubwürdig aus denselben Gründen wie bei der zweiten Zukunftsprojektion, zum einen in Bezug auf die „Tank-oder-Teller-Diskussion“ und zum anderen auf die höhere $\mathrm{CO}_{2}$-Einsparung durch die Nutzung von Biokraftstoffen der zweiten Generation. Darüber hinaus ist die Projektion als realistisch zu betrachten, da die Kommissare Günther Oettinger und Connie Hedegard ab dem Jahr 2021 ausschließlich Biokraftstoffe der zweiten Generation fördern wollen (EUROPÄlSCHE KOMMISSION 2012: 3).

Die drei Zukunftsprojektionen werden in dem dritten Kernschritt „EU27 SzenarioBildung und -Konsistenzprüfung" weiter betrachtet:

Politische Förderung von Biokraftstoffen der ersten und zweiten Generation Beschreibung:

T1A Es ist das Ziel, dass 10\% der Energie im Verkehrssektor aus erneuerbaren Energiequellen stammen. Biokraftstoffe der ersten Generation können unbegrenzt dem $10 \%$-Ziel angerechnet werden. Biokraftstoffe der zweiten Generation werden besonders gefördert, indem ihr Energiegehalt dem 10\%-Ziel doppelt angerechnet wird.

T1B Auf $5 \%$ begrenzte Nutzung von Biokraftstoffen der ersten Generation und starke politische Förderung von Biokraftstoffen der zweiten Generation Beschreibung:

Es ist das Ziel, dass 10\% der Energie im Verkehrssektor aus erneuerbaren Energiequellen stammen. Auf $5 \%$ begrenzte Anrechnung von Biokraftstoffen der ersten Generation auf das 10\%-Ziel. Biokraftstoffe der zweiten Generation werden stark gefördert, indem ihr Energiegehalt dem 10\%-Ziel vierfach angerechnet wird.

\footnotetext{
${ }^{145}$ Noch deutlicher wird der Unterschied zwischen erster und zweiter Generation bei Berücksichtigung eines kulturspezifischen ILUC-Faktors (RICHTLINIE 2009/28/EG ANHANG V; EUROPÄISCHE KOMMISSION 2012: 24).
} 
Ausschließliche politische Förderung von Biokraftstoffen der zweiten Generation

$\mathrm{T} 1 \mathrm{C}$ Beschreibung:

Es ist das Ziel, dass 10\% der Energie im Verkehrssektor aus erneuerbaren Energiequellen stammen. Biokraftstoffe der ersten Generation werden nicht mehr politisch unterstützt. Biokraftstoffe der zweiten Generation werden stark gefördert, indem ihr Energiegehalt dem $10 \%$-Ziel mehrfach angerechnet wird.

\subsubsection{Politische Vorgaben der Treibhausgasreduktion für Ethanol}

In den RICHTLINIEN 2009/28/EG und 2009/30/EG sind die gesetzlichen Vorgaben bezüglich der Anforderungen der Treibhausgasreduktion für Ethanol im Verhältnis zu Fossilbrennstoff formuliert (USDA FOREIGN AGRICULTURAL SERVICE 2012: 5; EUROPEAN COMMISSION JOINT RESEARCH CENTRE, INSTITUT FOR ENERGY ET AL. 2011: 14). Ethanol-Kraftstoff muss die im Folgenden beschriebenen $\mathrm{CO}_{2}$-Reduktionswerte im Verhältnis zu Fossilbrennstoff erreichen ${ }^{146}$, um politisch gefördert zu werden und auf das Ziel, im Jahr 2020 10\% des Endenergieverbrauchs im Verkehrssektor aus erneuerbaren Energiequellen zu beziehen, angerechnet zu werden (RICHTLINIE 2009/28/EG ARTIKEL 17 (1); RICHTLINIE 2009/30/EG ARTIKEL 7b). Derzeit muss Ethanol-Kraftstoff eine 35-prozentige Minderung der Treibhausgasemissionen im Verhältnis zu Fossilbrennstoffen erreichen. Ab dem 1. Januar 2017 wird die geforderte $\mathrm{CO}_{2}$-Einsparung auf 50\% angehoben. Anlagen, die am 1 . Januar 2017 oder später die Ethanolproduktion aufnehmen, müssen ab dem 1. Januar 2018 eine Reduktion der Treibhausgasemissionen von mindestens 60\% erreichen. Diese Vorgaben bestehen sowohl für produziertes als auch für in die EU27 importiertes Ethanol (RICHTLINIE 2009/28/EG ARTIKEL 17 (1)).

Das Thema ILUC ${ }^{147}$ (Indirect Land Use Change) wird seit einigen Jahren intensiv diskutiert (USDA FOREIGN AGRICULTURAL SERVICE 2012: 8), ist aber in den derzeit geltenden Richtlinien 2009/28/EG und 2009/30/EG nicht berücksichtigt. Zur Ermittlung des Einflusses des ILUC-Faktors auf die $\mathrm{CO}_{2}$-Bilanz von Biokraftstoffen beauftragte die Europäische Kommission verschiedene Forschungseinrichtungen ${ }^{148}$.

\footnotetext{
${ }^{146}$ Die Berechnung der prozentualen $\mathrm{CO}_{2}$-Einsparung von Ethanol im Verhältnis zu Fossilbrennstoff erfolgt über die Formel (RICHTLINIE 2009/28/EG ANHANG V):

Einsparung $=\left(E_{F}-E_{B}\right) / E_{F}$

$E_{B}=$ Gesamtemissionen bei der Verwendung von Biokraftstoff oder flüssigem Biobrennstoff

$E_{F}=$ Gesamtemissionen des Komparators für Fossilbrennstoffe

Für die fossile Vergleichsgröße $\mathrm{E}_{\mathrm{F}}$ wird der Wert $83 \mathrm{gCO}_{2 e q} / \mathrm{MJ}$ verwendet.

${ }^{147}$ Eine genaue Beschreibung des ILUC-Faktors befindet sich in Kapitel 5.1.1.2 unter Einflussfaktor 21.

${ }^{148}$ Die Veröffentlichung „Indirect Land Use Change from increased biofuels demand“ (EUROPEAN COMMISSION JOINT RESEARCH CENTRE, INSTITUT FOR ENERGY 2010) vergleicht die Ergebnisse unterschiedlicher Studien zum Thema ILUC.
} 
Um das Thema genauer zu untersuchen wurde das International Food Policy Research Institute (IFPRI) ausgewählt. Dieses Institut erarbeitete und veröffentlichte daraufhin die Studie „Assessing the Land Use Change Consequences of European Biofuel Policies“ (IFPRI 2011), die mithilfe des Modells MIRAGE-Biof zu dem Ergebnis kommt, dass sich die Treibhausgasreduktionswerte von Ethanol durch den ILUC-Faktor, abhängig vom zur Produktion genutzten Rohstoff, verschlechtern (IFPRI 2011: 13). Des Weiteren kommt auch das JOINT RESEARCH CENTRE, INSTITUTE FOR ENERGY (2011: 16) ausgehend von den Ergebnissen der IFPRIStudie zu ähnlichen Resultaten. In der folgenden Abbildung 46 sind die ILUC-Werte der einzelnen Rohstoffe in der Einheit $\mathrm{gCO}_{2 \mathrm{eq}} / \mathrm{MJ}$ angegeben.

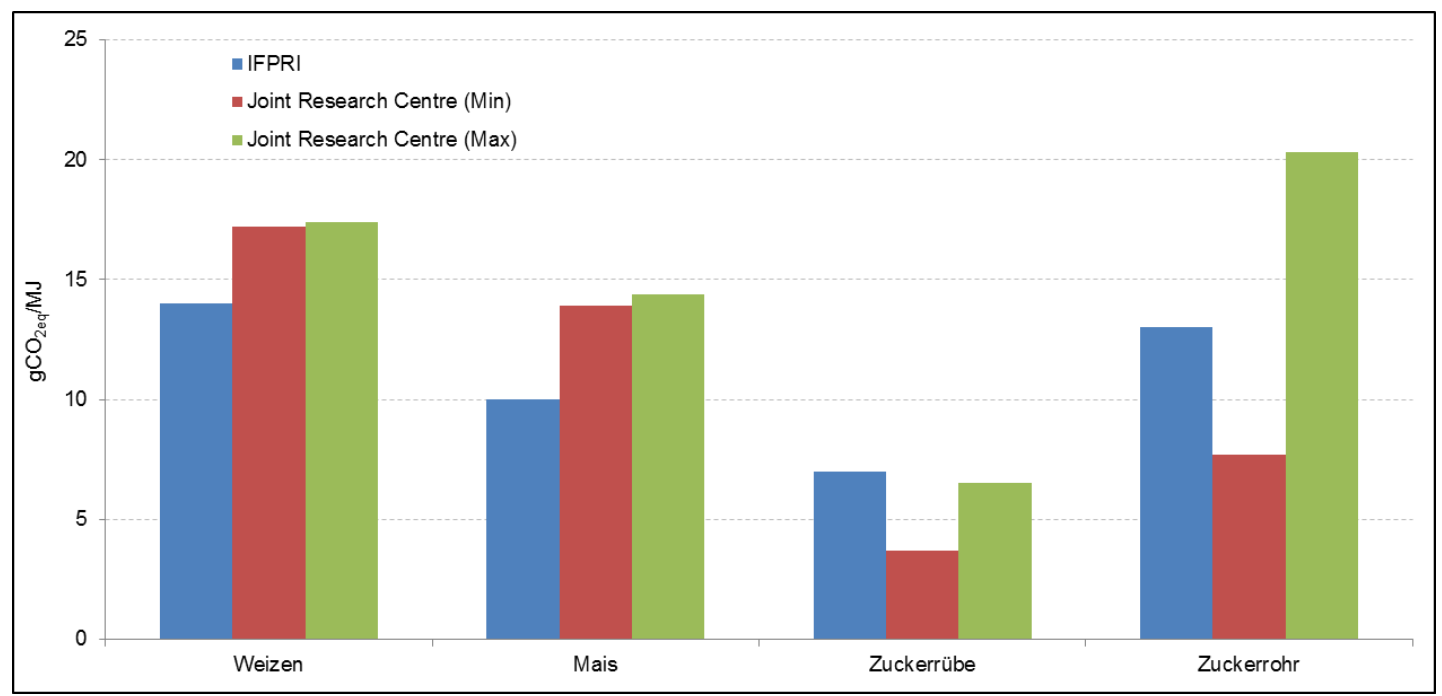

Abbildung 46: ILUC-Werte für Ethanol aus Weizen, Mais, Zuckerrübe und Zuckerrohr Quelle: In Anlehnung an IFPRI 2011: 13; JOINT RESEARCH CENTRE, INSTITUTE FOR ENERGY 2011: 16

Zum besseren Verständnis der hohen Relevanz des ILUC-Faktors wird die $\mathrm{CO}_{2}$ Bilanz der Ethanolproduktion aus Weizen betrachtet. Der ILUC-Wert für Weizen beträgt basierend auf den Berechnungen des IFPRI $14 \mathrm{gCO}_{2 \mathrm{eq}} / \mathrm{MJ}$. Dies bedeutet, dass bei der Berücksichtigung des ILUC-Faktors die Produktion von Ethanol aus Weizen $14 \mathrm{gCO}_{2 \mathrm{eq}} / \mathrm{MJ}$ mehr freisetzt als ohne ILUC-Faktor. Dadurch verschlechtert sich die $\mathrm{CO}_{2}$-Bilanz von Ethanol so stark, dass Weizen-Ethanol nicht mehr die politische Vorgabe einer 35-prozentigen $\mathrm{CO}_{2}$-Reduzierung im Vergleich zu Fossilbrennstoff erfüllt, welche ohne Beachtung eines ILUC-Faktors problemlos erreicht wur$\mathrm{de}^{149}$.

\footnotetext{
${ }^{149}$ In Kapitel 5.1.1.2/Einflussfaktor 21 wird die Wirkung des ILUC-Faktors auf die $\mathrm{CO}_{2}$-Bilanz von Ethanol ausführlich dargestellt.
} 
Die Kommissare Günther Oettinger (Generaldirektion Energie) und Connie Hedegard (Generaldirektion Klimaschutz) haben am 17. Oktober 2012 einen Vorschlag ${ }^{150}$ zur Änderung der gesetzlichen Vorgaben für Biokraftstoffe präsentiert (EUROPÄlSCHE KOMMISSION 2012). Vorgeschlagene Veränderungen, die diesen Schlüsselfaktor betreffen, sind im Folgenden genannt:

- Ethanolanlagen, die ihre Produktion nach dem 1. Juli 2014 aufnehmen, müssen eine Treibhausgasemissionseinsparung von 60\% im Vergleich zu Fossilbrennstoff erreichen (EUROPÄISCHE KOMMISSION 2012: 12).

- Ethanolanlagen, die vor dem 1. Juli 2014 in Betrieb sind, müssen bis zum 31. Dezember 2017 eine $\mathrm{CO}_{2}$-Reduktion von mindestens 35\% und ab dem 1. Januar 2018 eine $\mathrm{CO}_{2}$-Einsparung von mindestens $50 \%$ im Vergleich zu Fossilbrennstoff erreichen (EUROPÄISCHE KOMMISSION 2012: 12).

- Ein ILUC-Faktor wird bei der Bilanzierung der $\mathrm{CO}_{2}$-Einsparungen von Ethanol bis zum Jahr 2020 nicht berücksichtigt. Es muss aber an die Kommission gemeldet werden, wie hoch die geschätzten Treibhausgasemissionen infolge indirekter Landnutzungsänderungen sind; folgende kulturspezifische ILUC-Werte dienen zur Berechnung dieser Daten (Tabelle 13) (EUROPÄISCHE KOMMISSION 2012: $12)^{151}$ :

\begin{tabular}{l|r} 
Rohstoff & gCO2eq/ MJ \\
\hline Getreide und sonstige stärkehaltige Pflanzen & 12 \\
\hline Zuckerpflanzen & 13 \\
\hline Ölpflanzen & 55
\end{tabular}

Tabelle 13: Kulturspezifische ILUC-Werte bei der Produktion von Biokraftstoffen

Quelle: In Anlehnung an EUROPÄISCHE KOMMISSION 2012: 22.

- Ab dem Jahr 2021 soll ein ILUC-Faktor in der Berechnung der $\mathrm{CO}_{2}$-Emissionen bei der Herstellung von Ethanol berücksichtigt werden (EUROPÄISCHE KOMMISSION 2012: 3).

Basierend auf den genannten Informationen werden im Folgenden die zwei Zukunftsprojektionen „Strengere Vorgaben der Treibhausgasreduktion für Ethanol“ und „Strengere Vorgaben der Treibhausgasreduktion für Ethanol und Berücksichtigung des ILUC-Faktors“ erläutert.

${ }^{150}$ Der Titel des Vorschlags lautet: „Vorschlag für eine RICHTLINIE DES EUROPÄISCHEN PARLAMENTS UND DES RATES“" zur Änderung der Richtlinie 98/70/EG über die Qualität von Otto- und Dieselkraftstoffen und zur Änderung der Richtlinie 2009/28/EG zur Förderung der Nutzung von Energie aus erneuerbaren Quellen" (EUROPÄISCHE KOMMISSION 2012). ${ }^{151}$ Bei der Betrachtung der ILUC-Werte ist zu beachten, dass die guten Werte der Zuckerrübe (Abbildung 46) nicht in dem Vorschlag der Kommissionen Energie und Klimaschutz berücksichtigt wurden. Die ILUC-Werte der anderen Rohstoffe orientieren sich an den Werten des IFPRI. 
Die erste Projektion „Strengere Vorgaben der Treibhausgasreduktion für Ethanol" basiert auf der Annahme, dass die aktuellen Regelungen in den RICHTLINIEN 2009/28/EG (ARTIKEL 17 (2)) und 2009/30/EG (ARTIKEL 7b (2)) noch im Jahr 2023 gültig sind. Dies bedeutet, dass Ethanolproduzenten im Jahr 2023 eine $\mathrm{CO}_{2}$ Reduktion im Verhältnis zu Fossilbrennstoffen von 50\% erreichen müssen in Anlagen, die vor dem 1. Januar 2017 erbaut wurden, und von 60\% in Produktionsstätten, die ab dem 1. Januar 2017 die Ethanolproduktion aufgenommen haben. Die Projektion ist realistisch, denn $\mathrm{CO}_{2}$-Einsparungen von $50 \%$ bzw. $60 \%$ im Verhältnis zu Fossilbrennstoff sind anspruchsvoll und fordern von einigen Produktionsprozessen zur Ethanolherstellung Verbesserungen ihrer $\mathrm{CO}_{2}$-Bilanz ${ }^{152}$, womit die Projektion im Einklang mit dem politischen Kernziel der Verringerung der Treibhausgasemissionen steht (RICHTLINIE 2009/28/EG ERW 1). Auch die Nicht-Berücksichtigung eines ILUC-Faktors ist realistisch, da der Faktor von Interessenverbänden energisch kritisiert wird und die Richtigkeit der Berechnungen des IFPRI sowohl von der Industrie als auch der Wissenschaft bezweifelt wird (EPURE 2013; FINKBEINER 2013).

Die zweite Zukunftsprojektion „Strengere Vorgaben der Treibhausgasreduktion für Ethanol und Berücksichtigung des ILUC-Faktors“ basiert auf dem Vorschlag der Kommissionen Energie und Klimaschutz vom 17. Oktober 2012 (EUROPÄISCHE KOMMISSION 2012). Demzufolge müssen die Ethanolproduzenten im Jahr 2023 in Anlagen, die vor dem 1. Juli 2014 die Produktion aufgenommen haben, eine $\mathrm{CO}_{2}$ Reduktion von 50\% im Vergleich zu fossilem Kraftstoff erreichen. Produktionsanlagen, die ab dem 1. Juli 2014 in Betrieb gehen werden, sogar eine 60-prozentige $\mathrm{CO}_{2}$-Einsparung. Diese Zukunftsprojektion unterscheidet sich grundlegend von der ersten Projektion durch die Berücksichtigung eines rohstoffabhängigen ILUCFaktors bei der Berechnung der Treibhausgasemissionen zur Herstellung von Ethanol. Die Höhe der ILUC-Werte wird den veröffentlichten Werten aus dem Vorschlag der Kommissionen Energie und Klimaschutz entsprechen (Tabelle 13) (EUROPÄlSCHE KOMMISSION 2012: 22). Die höheren Anforderungen bezüglich der $\mathrm{CO}_{2}{ }^{-}$ Reduzierung sind realistisch, da sie dem politischen Kernziel der Reduktion von Treibhausgasen entsprechen (RICHTLINIE 2009/28/EG ERW 1). Die Berücksichti-

152 Produktionsprozesse zur Ethanolherstellung, die derzeit nicht die 60-prozentige $\mathrm{CO}_{2}$ Einsparung im Verhältnis zu Fossilbrennstoff erfüllen (RICHTLINIE 2009/28/EG ANHANG V):

- Ethanol aus Weizen (Prozessbrennstoff nicht spezifiziert)

- Ethanol aus Weizen (Braunkohle als Prozessbrennstoff in KWK-Anlage)

- Ethanol aus Weizen (Erdgas als Prozessbrennstoff in konventioneller Anlage)

- Ethanol aus Weizen (Erdgas als Prozessbrennstoff in KWK-Anlage)

- Ethanol aus Mais, in der Gemeinschaft erzeugt (Erdgas als Prozessbrennstoff in KWKAnlage) 
gung eines ILUC-Faktors im Jahr 2023 ist ebenfalls sinnvoll aufgrund der eindeutigen Aussage der Kommissare Günther Oettinger und Connie Hedegard, ab dem Jahr 2021 einen ILUC-Faktor bei der Herstellung von Ethanol in die Berechnungen der $\mathrm{CO}_{2}$-Emissionen einzubeziehen (EUROPÄISCHE KOMMISSION 2012: 3).

Auf Grundlage der Erläuterungen werden die zwei Zukunftsprojektionen samt Beschreibung weiter betrachtet:

Strengere Vorgaben der Treibhausgasreduktion für Ethanol

Beschreibung:

Um politisch gefördert zu werden, muss Ethanol, welches in Anlagen hergestellt wird, die die

T2A Produktion vor dem 1. Januar 2017 aufgenommen haben, eine 50-prozentige $\mathrm{CO}_{2}$-Reduktion im Vergleich zu Fossilbrennstoff erreichen. Ethanol, hergestellt in Produktionsstätten, die die Herstellung nach dem 1. Januar 2017 beginnen werden, müssen eine 60-prozentige Treibhausgasreduktion erfüllen. Ein ILUC-Faktor wird in der Zukunftsprojektion nicht berücksichtigt.

Strengere Vorgaben der Treibhausgasreduktion für Ethanol und Berücksichtigung des ILUC-Faktors

Beschreibung:

T2B Damit Ethanol politisch gefördert wird, muss es, wenn es in Anlagen hergestellt wird, die vor dem 1. Januar 2014 die Produktion begonnen haben, eine 50-prozentige $\mathrm{CO}_{2}$-Reduktion im Vergleich zu Fossilbrennstoff erfüllen. Ethanol, produziert in Anlagen, die nach dem 1. Januar 2014 ihre Produktion starten werden, müssen eine Treibhausgasreduktion von 60\% erreichen. Ein ILUC-Faktor wird in der Projektion berücksichtigt.

\subsubsection{Ethanolnachfrage}

Im Gegensatz zu den vorherigen Kapiteln, in denen wirkende Schlüsselfaktoren Gegenstand der Untersuchung waren, wird im Folgenden der beschreibende Schlüsselfaktor „Ethanolnachfrage“ betrachtet.

Die Ethanolnachfrage lag zwischen 1992 und 2002 bei unter 0,5 Milliarden Litern (Abbildung 47). Erst ab dem Jahr 2003 kam es aufgrund von politischen Maßnahmen zur Förderung des Ethanolabsatzes ${ }^{153}$ zu einem starken Nachfragewachstum. In den darauf folgenden neun Jahren (2003 - 2012) ist die Nachfrage auf 5,6 Milliarden Liter angestiegen, was einem durchschnittlichen jährlichen Wachstum von $31,4 \%$ entspricht.

\footnotetext{
${ }^{153}$ Die wichtigsten politischen Maßnahmen zur Förderung der Ethanolnachfrage in der EU27 im Jahr 2003 sind in den RICHTLINIEN 2003/30/EG und 2003/96/EG festgehalten. Eine ausführliche Beschreibung der beiden RICHTLINIEN befindet sich in Kapitel 2.1.
} 


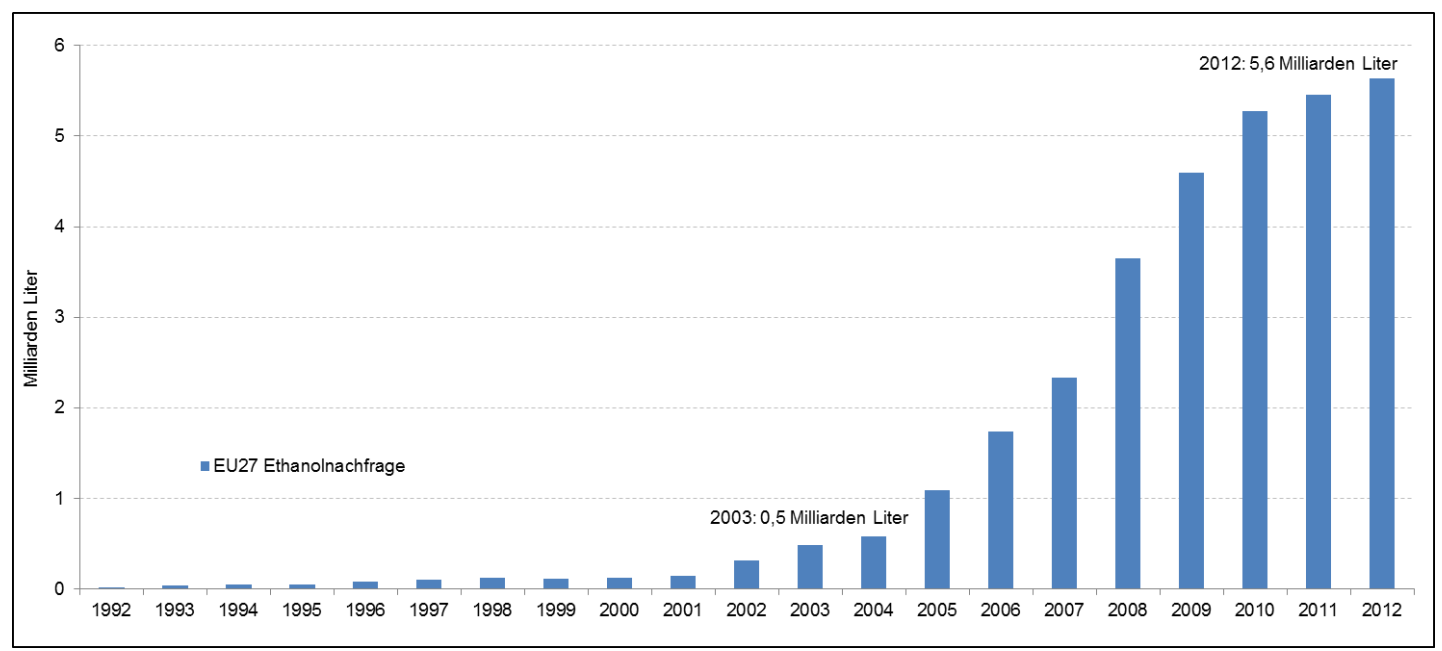

Abbildung 47: Entwicklung der Ethanolnachfrage in der EU27 im Zeitraum von 1992 bis 2012

Quelle: In Anlehnung an EUROSTAT 2012; F.O. LICHT 2012f: 74

Gefolgt von Frankreich (0,5 Milliarden Liter), Großbritannien (0,4 Milliarden Liter) und Spanien (0,3 Milliarden Liter) war Deutschland im Jahr 2011 mit 1 Milliarde Litern der wichtigste Markt für Ethanol-Kraftstoff in der EU27 (EUROOBSERV'ER 2013: 2). Der Absatz von Ethanol-Kraftstoff erfolgt als 5- oder 10-prozentige Beimischung zu Benzin und in Form von ETBE (WOOD MACKENZIE 2010: 64). Der Verkauf von Ethanol als E85 für FFV ist in der EU27 von geringer Bedeutung. Der Marktanteil der FFV an dem gesamten Pkw-Markt der EU27 beträgt lediglich 0,1\% (EUROPEAN BEST PROJECT 2010: 60).

Im Folgenden wird die Entwicklung der Ethanolnachfrage bis zum Jahr 2023 betrachtet. Abbildung 48 zeigt die Einschätzung der langfristigen Entwicklung der Ethanolnachfrage von unterschiedlichen internationalen Forschungsinstituten sowie die drei Zukunftsprojektionen - IA, IB und IC - die im Kernschritt „EU27 SzenarioBildung und -Konsistenzprüfung“ weiter betrachtet werden. 


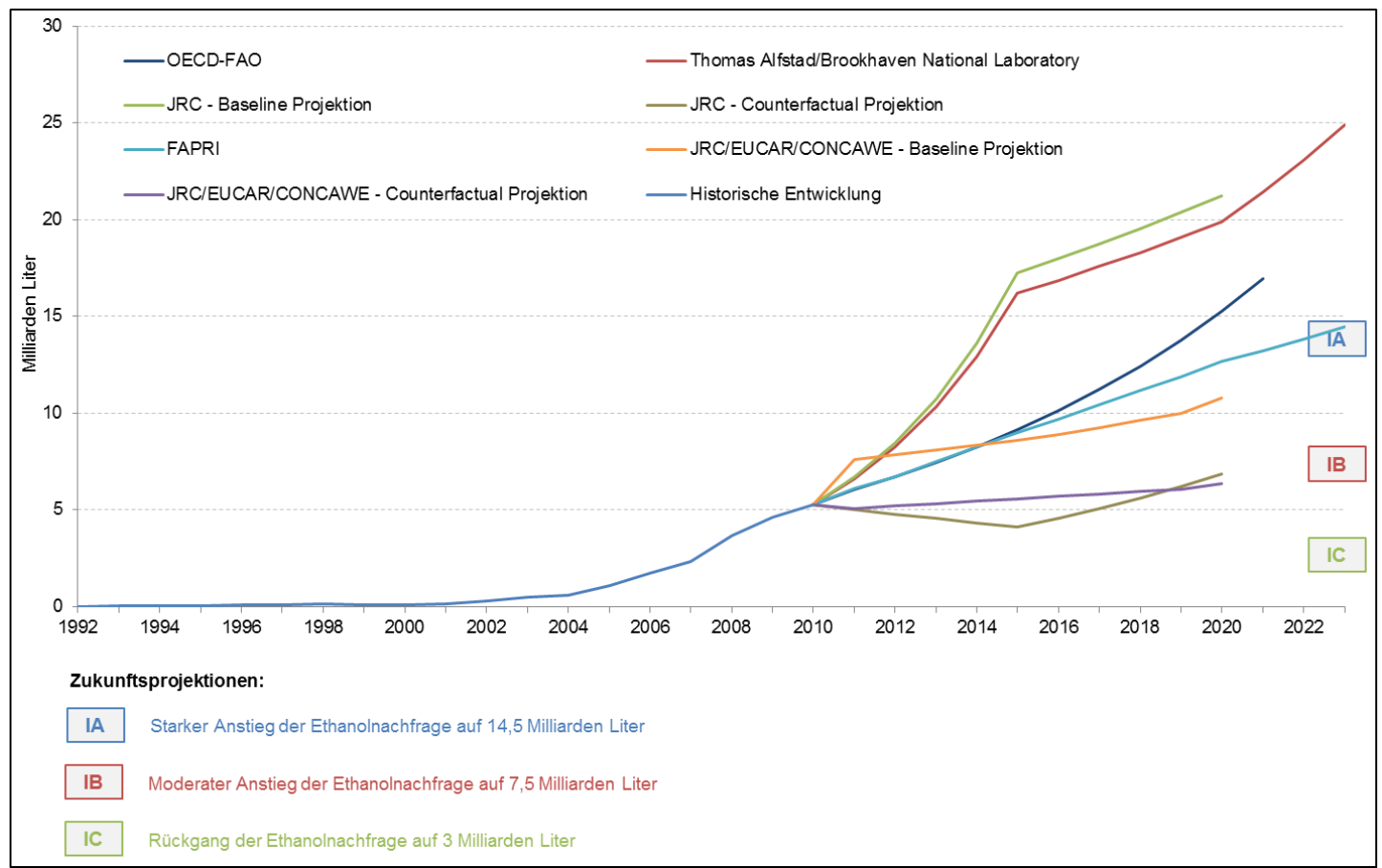

Abbildung 48: Zukünftige Entwicklung der Ethanolnachfrage in der EU27

Quelle: In Anlehnung an OECD/FAO 2012: 224; ALFSTAD 2008: A2f.; EUROPEAN COMMISSION JOINT RESEARCH CENTRE, INSTITUTE FOR PROSPECTIVE TECHNOLOGICAL STUDIES 2010a: 36; FAPRI 2011c: 117; EUROPEAN COMMISSION JOINT RESEARCH CENTRE, INSTITUT FOR ENERGY ET AL. 2011: 41; EUROSTAT 2012; EIGENE BERECHNUNG

Die Prognosen der internationalen Forschungsinstitute zur langfristigen Entwicklung der Ethanolnachfrage beschreiben einen Zukunftsraum mit einer oberen Grenze von 25 Milliarden Litern (Thomas Alfstad/Brookhaven National Laboratory) und einer Untergrenze von 6,3 Milliarden Litern (Abbildung 48) (JRC/EUCAR/CONCAWE Counterfactual Projektion). Dieser Zukunftsraum, in dem jede Prognose einen Anstieg der Ethanolnachfrage erwartet, wird in der weiteren Arbeit nur teilweise beachtet. Die drei Zukunftsprojektionen IA, IB und IC bilden den Zukunftsraum, der in der weiteren Studie betrachtet wird; dieser beschreibt eine Ethanolnachfrage zwischen 14,5 Milliarden Litern und 3 Milliarden Litern im Jahr 2023. Im Folgenden wird jede Zukunftsprojektion - IA, IB und IC - einzeln beschrieben und in diesem Zusammenhang erläutert, warum ein Anstieg der Ethanolnachfrage auf über 14,5 Milliarden Liter unrealistisch, aber ein Rückgang der Nachfrage auf 3 Milliarden Liter durchaus möglich ist.

In der Zukunftsprojektion IA wird ein starker Anstieg der Ethanolnachfrage in der EU27 auf 14,5 Milliarden Liter beschrieben. Diese Ethanolnachfrage entspricht der Summe der Ethanolmengen, die die einzelnen Staaten der EU27 ihren Angaben zufolge einsetzen werden, um das politische Ziel der Nutzung von 10\% erneuerbarer Energie im Verkehrssektor im Jahr 2020 zu erfüllen. Die jeweiligen Werte der 
Ethanolnachfrage sind den „Nationalen Aktionsplänen für erneuerbare Energien“, die jedes Mitgliedsland veröffentlichen muss, entnommen. Voraussetzung dieser Zukunftsprojektion ist die Annahme, dass die derzeitigen politischen Vorgaben, wie in RICHTLINIE 2009/28/EG beschrieben, noch im Jahr 2023 von Bedeutung sind und es keine politische Begrenzung der Nutzung von Ethanol der ersten Generation gibt $^{154}$. In der folgenden Abbildung 49 ist die von den Mitgliedsstaaten der EU27 geplante Entwicklung der Ethanolnachfrage dargestellt.



Abbildung 49: Zielvorgaben der Mitgliedsstaaten zur Nutzung von Ethanol-Kraftstoff

Quelle: In Anlehnung an BUNDESMINISTERIUM FÜR WIRTSCHAFT; FAMILIE UND JUGEND 2010: 79; BELGIUM NATIONAL RENEWABLE ENERGY ACTION PLAN 2010: 94; REPUBLIC OF BULGARIA MINISTRY OF ECONOMY, ENERGY AND TOURISM 2010: 168; REPUBLIC OF CYPRUS MINISTRY OF COMMERCE, INDUSTRY AND TOURISM 2010: 9; CZECH REPUBLIK MINISTRY OF INDUSTRY AND TRADE 2012: 83; KLIMA- OG ENERGIMINISTERIET 2010: 109; MAJANDAS- JA KOMMUNIKATSIOONIMINISTEERIUM 2010: 61; FINLAND MINISTRY OF EMPLOYMENT AND THE ECONOMY ENERGY DEPARTMENT 2010: 19; MINISTÉRE DE L'ĖCOLOGIE, DE L'ĖNERGIE, DU DĖVELOPPEMENT DURABLE ET DE LA MER 2009: 100; BUNDESREPUBLIK DEUTSCHLAND 2009: 119; GREECE MINISTRY OF ENVIRONMENT ENERGY \& CLIMATE CHANGE 2010: 107; HUNGARY 2010: 204; IRELAND 2010: 142; ITALIAN NATIONAL RENEWABLE ENERGY ACTION PLAN 2010: 169; REPUBLIC OF LATVIA 2010: 95; LITHUANIA 2010: 130; LE GOUVERNMENT DU GRAND-DUCHE DE LUXEMBOURG MINISTĖRE DE L'ÉCONOMIE ET DU COMMERCE EXTÉRIEUR 2010: 110; MALTA 2011: 80; NETHERLANDS 2010: 113; POLAND MINISTER OF ECONOMY 2010: 137; PORTUGAL 2010: 130; ROMANIA 2010: Table 5.7d; MINISTRY OF ECONOMY AND CONSTRUCTION OF THE SLOVAK REPUBLIC 2010: 74; SLOVENIA 2010: 130; GOBIERNO DE ESPANA MINISTERIO DE INDUSTRIA, TURISMO Y COMERCIO 2010: 156; SWEDEN REGERINGSKANSLIET 2010: 116; UNITED KINGDOM 2010: 156

Ein Anstieg der Ethanolnachfrage von derzeit 5,6 Milliarden Litern auf 14,5 Milliarden Liter setzt ein prozentuales Wachstum von $160 \%$ in den nächsten elf Jahren voraus. Eine noch stärkere Steigerung der Ethanolnachfrage, wie etwa von den Organisationen „JRC - Baseline“, „Thomas Alfstad/Brookhaven National Laborato-

${ }^{154}$ Die beschriebene Entwicklung der politischen Förderung von Ethanol wird in der Zukunftsprojektion T1A/Kapitel 5.2.6 dargestellt. 
ry" und der "OECD-FAO" prognostiziert (Abbildung 48), wird als nicht realistisch betrachtet. Denn bereits bei einer Ethanolnachfrage von 14,5 Milliarden Litern und einer Benzinnachfrage von 87 Milliarden Litern ${ }^{155}$ im Jahr 2023 muss jedes Kraftfahrzeug seine technisch maximal mögliche Ethanolbeimischung zu Benzin tanken, um den genannten Ethanolverbrauch zu erreichen ${ }^{156}$. Hierbei ist zu beachten, dass es möglich ist, dass nicht alle Kraftfahrzeugbesitzer bereit sein werden, die technisch höchstmögliche Ethanolbeimischung zu Benzin zu tanken. So hat beispielsweise E10 in Deutschland - dem wichtigsten Ethanolmarkt der EU27 - lediglich einen Marktanteil von 14,8\% am gesamten Benzinmarkt im Jahr 2012, da die Verbraucher weiterhin Ottokraftstoff mit einem Volumenanteil von nur fünf Prozent bevorzugen (F.O. LICHT 20133: 500). Darüber hinaus hat sich das Wachstum der Ethanolnachfrage in der EU27 seit 2010 deutlich verlangsamt (Abbildung 47) und der Ist-Wert der Ethanolnachfrage in Höhe von 5,6 Milliarden Litern (F.O. LICHT 2012f: 74) liegt bereits 2,5 Milliarden Liter hinter dem Plan-Wert der Mitgliedsstaaten in Höhe von 8,1 Milliarden Litern (Abbildung 49).

Die zweite Zukunftsprojektion IB prognostisiert einen moderaten Anstieg der Ethanolnachfrage auf 7,5 Milliarden Liter bis zum Jahr 2023. Die Prognose basiert auf der Annahme einer 5-prozentigen Begrenzung der Anrechnung von Biokraftstoffen der ersten Generation auf das 10\%-Ziel, wie von Energiekommissar Günther Oettinger und Klimakommissarin Connie Hedegard am 17. Oktober 2012 vorgeschlagen (EUROPÄISCHE KOMMISSION 2012: 16) ${ }^{157}$. Infolge dieser politischen Entscheidung steigt die Nachfrage nach Ethanol der ersten Generation nur moderat auf 7,5 Milliarden Liter, was in etwa der Hälfte der von den Mitgliedsstaaten geplanten Nutzung von 14,5 Milliarden Litern Ethanol-Kraftstoff entspricht. Eine stärkere Nachfrage nach Ethanol der zweiten Generation, um die Beschränkung der Nutzung von Ethanol der ersten Generation zu substituieren, wird aufgrund der fehlenden Verfügbarkeit von Zellulose-Ethanol nicht erwartet. Dies gleicht der Prognose der

\footnotetext{
${ }^{155}$ Die Beschreibung der Zukunftsprojektion zur Entwicklung der Benzinnachfrage bis zum Jahr 2023 befindet sich in Kapitel 5.2.1.

${ }_{156}$ Die folgende Berechnung basiert auf den Zukunftsprojektionen: $1 \mathrm{~A}$ „Rückgang der Benzinnachfrage in der EU27 auf 87 Milliarden Liter" (Kapitel 5.2.1) und 12A „Hohe Ethanolverträglichkeit der Pkw im Jahr 2023“" (Kapitel 5.2.4). Durch die Multiplikation der Benzinnachfrage von 87 Milliarden Litern mit der technisch möglichen Ethanolbeimischung zu Kraftfahrzeugen - 54\% der Kraftfahrzeuge vertragen E20 und 46\% der Kraftfahrzeuge vertragen lediglich E10 - ergibt sich eine Ethanolnachfrage von 13,4 Milliarden Litern, die restlichen 1,1 Milliarden Liter werden von FFV in der EU27 nachgefragt.

${ }^{157}$ Diese politische Entwicklung wird in der Zukunftsprojektion T1B/Kapitel 5.2.6 beschrieben.
} 
OECD/FAO (2012: 224), die von einer Zellulose-Ethanolproduktion von lediglich 0,4 Milliarden Litern in der EU27 für das Jahr 2021 ausgeht ${ }^{158}$.

Ein Rückgang der Ethanolproduktion auf drei Milliarden Liter wird in der dritten Zukunftsprojektion IC vorhergesagt. Die Prognose basiert auf der Ankündigung von Energiekommissar Günther Oettinger und Connie Hedegard, ab dem Jahr 2021 ausschließlich Biokraftstoffe der zweiten Generation politisch zu fördern (EUROPÄISCHE KOMMISSION 2012: 3). Infolge der fehlenden politischen Unterstützung bricht die Nachfrage nach Ethanol der ersten Generation in der EU27 ein. Um das politische Ziel der Nutzung von 10\% erneuerbarer Energie im Verkehrssektor trotzdem zu erfüllen, schlägt die Politik vor, den Energiegehalt von Zellulose-Ethanol auf das $10 \%$-Ziel mehrfach anzurechnen ${ }^{159}$. Basierend auf diesem Vorschlag steigt die Nachfrage nach Zellulose-Ethanol auf 3 Milliarden Liter an. Eine stärkere Nachfrage nach Zellulose-Ethanol wird sowohl aufgrund der zu geringen Produktion von Zellulose-Ethanol in der EU27 als auch durch die mehrfache Anrechnung des Energiegehalts nicht erwartet, denn dadurch sind deutlich geringere Mengen ZelluloseEthanol ausreichend, um das 10\%-Ziel zu erreichen. Im Folgenden sind nochmals die drei Zukunftsprojektionen des beschreibenden Schlüsselfaktors dargestellt, die in der weiteren Studie betrachtet werden:
IA | Starker Anstieg der Ethanolnachfrage auf 14,5 Milliarden Liter
IB | Moderater Anstieg der Ethanolnachfrage auf 7,5 Milliarden Liter
IC | Rückgang der Ethanolnachfrage auf 3 Milliarden Liter

\subsubsection{Ethanolproduktion}

Entsprechend der Ethanolnachfrage in der EU27 lag die Ethanolproduktion in den Jahren von 1992 bis 2003 bei unter 0,5 Milliarden Litern. Erst im Jahr 2003 kam es aufgrund von politischen Maßnahmen zur Förderung der Ethanolnachfrage (Kapitel 2.1) auch zu einem Anstieg der Ethanolproduktion in der EU27. In den folgenden sieben Jahren stieg die Produktion von 0,4 Milliarden Litern im Jahr 2003 auf 4,3 Milliarden Liter im Jahr 2012 (Abbildung 50); dies entspricht einem durchschnittlichen jährlichen Produktionswachstum von 30,2\%. Der Rückgang der Ethanolpro-

\footnotetext{
${ }^{158}$ Die Prognose der OECD/FAO (2012: 224) ist als realistisch zu betrachten, denn die derzeitige Produktion von Zellulose-Ethanol in der EU27 beträgt lediglich 0,03 Milliarden Liter. Darüber hinaus bestehen noch immer verfahrenstechnische und logistische Probleme bei der kommerziellen Produktion von Zellulose-Ethanol und der Bau von Produktionsanlagen ist mit hohen Kapitalkosten verbunden.

${ }_{159}$ Die politischen Rahmenbedingungen dieser Projektion sind in der Zukunftsprojektion T1C/Kapitel 5.2.6 dargestellt.
} 
duktion im Jahr 2012 ist auf die günstigen Ethanolimporte aus den USA und Brasilien zurückzuführen, die den europäischen Ethanolpreis senkten und zahlreiche Ethanol-produzenten zwangen, die Produktion zu unterbrechen, da eine kostendeckende Herstellung nicht mehr möglich war (F.O. LICHT 2012a: 300; USDA FOREIGN AGRICULTURAL SERVICE 2012: 16f.; F.O. LICHT 2011a: 66). Der größte Teil der Ethanolproduktion erfolgt aus den landwirtschaftlichen Rohstoffen Weizen und Zuckerrübe (EUROPEAN COMMISSION JOINT RESEARCH CENTRE, INSTITUT FOR ENERGY ET AL. 2010: 74); die Herstellung von Zellulose-Ethanol besitzt nur eine untergeordnete Rolle: Im Jahr 2010 wurden lediglich 0,029 Milliarden Liter produziert (OECD/FAO 2012: 224).

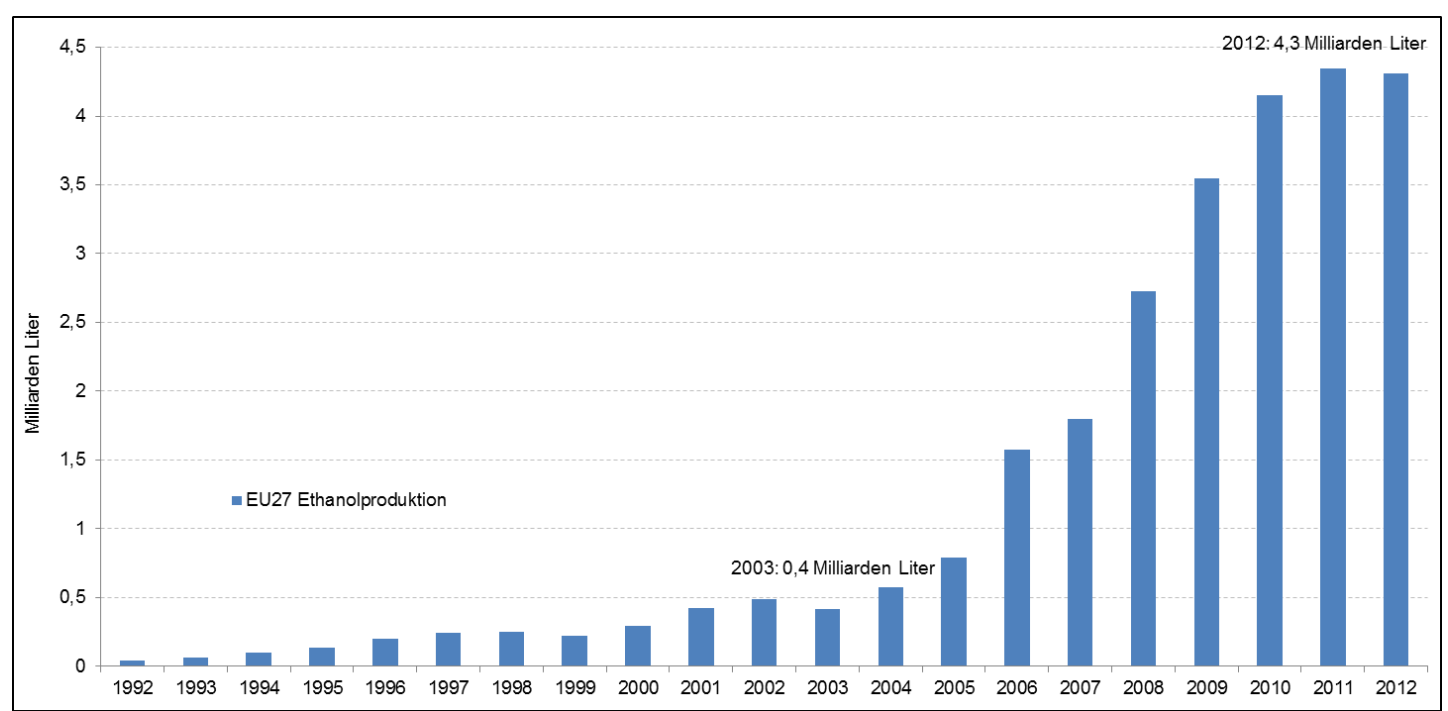

Abbildung 50: Entwicklung der Ethanolproduktion in der EU27

Quelle: In Anlehnung an F.O. LICHT 2012c: 323; BIOFUELS PLATFORM 2012: 2

Im folgenden Abschnitt werden die drei Zukunftsprojektionen IIA, IIB und IIC beschrieben, die die Ethanolproduktion in der EU27 im Jahr 2023 darstellen.

Basierend auf einer Ethanolnachfrage von 14,5 Milliarden Litern (Zukunftsprojektion la/Kapitel 5.2.8) und einem Ethanolimport von 1,5 Milliarden Litern (Zukunftsprojektion IIIA/Kapitel 5.2.10) beschreibt die Zukunftsprojektion IIA einen starken Anstieg der Ethanolproduktion auf 13 Milliarden Liter, davon sind 12,6 Milliarden Liter Ethanol der ersten Generation und nur 0,4 Milliarden Liter Ethanol der zweiten Generation aus Zellulose ${ }^{160}$. Grundlage der Projektion sind die zukünftige politische Förderung von Ethanol der ersten Generation (Zukunftsprojektion T1A/Kapitel 5.2.6) sowie die Annahme, dass die Verunsicherung der Ethanolindustrie hinsichtlich der zukünftigen politischen Förderung von Ethanol der ersten Generation gewichen ist und die Industrie wieder in den Bau von Anlagen zur Ethanolproduktion investiert -

160 Eine Produktion von 0,4 Milliarden Litern Zellulose-Ethanol entspricht der Prognose der OECD/FAO (2012: 224). 
die Produktionskapazitäten steigen von derzeit 8 Milliarden Litern auf über 13 Milliarden Liter an (USDA FORGEIGN AGRICULTURAL SERVICE 2012: 11).

Die zweite Zukunftsprojektion IIB beschreibt eine Ethanolproduktion von 6 Milliarden Litern. Dieser Projektion liegen eine Ethanolnachfrage von 7,5 Millarden Litern (Zukunftsprojektion IB/Kapitel 5.2.8) und ein Ethanolimport von 1,5 Milliarden Litern (Zukunftsprojektion IIIA/ Kapitel 5.2.10) zugrunde. Basis der Projektion ist die politische Entscheidung, die Anrechnung von Biokraftstoffen der ersten Generation auf das 10\%-Ziel auf fünf Prozent zu begrenzen (Zukunftsprojektion T1B/Kapitel 5.2.6). Die Ethanolproduktion der zweiten Generation beträgt trotz starker politischer Förderung lediglich 0,4 Milliarden Liter, wie von der OECD/FAO (2012: 224) vorhergesagt. Die Produktion von 5,6 Milliarden Litern Ethanol der ersten Generation ist problemlos möglich, da bereits Produktionskapazitäten von 8 Milliarden Litern bestehen (USDA FORGEIGN AGRICULTURAL SERVICE 2012: 11).

Die Zukunftsprojektion IIC beschreibt eine Produktion von 3 Milliarden Litern Ethanol, ausschließlich gewonnen aus Zellulose, im Jahr 2023. Voraussetzung der Prognose ist eine Zellulose-Ethanolnachfrage von 3 Milliarden Litern in der EU27 (Zukunftsprojektion IC/Kapitel 5.2.8). Der Anstieg der Nachfrage nach Zellulose-Ethanol basiert auf der politischen Entscheidung, im Jahr 2023 ausschließlich Biokraftstoffe der zweiten Generation zu fördern (Zukunftsprojektion T1C/Kapitel 5.2.6). Eine Ethanolproduktion von 3 Milliarden Litern Zellulose-Ethanol in der EU27 ist als realistisch zu betrachten: Die momentane Produktion beträgt zwar lediglich 0,029 Milliarden Liter (OECD/FAO 2012: 224), allerdings gibt es in der EU27 bereits zahlreiche Produktionsanlagen, die der Erprobung der kommerziellen Produktion von Zellulose-Ethanol dienen (Tabelle 14). 


\begin{tabular}{|c|c|c|c|}
\hline Land & Investor & Status & $\begin{array}{c}\text { Jährliche Produktions- } \\
\text { kapazität (Milliarden } \\
\text { Liter) }\end{array}$ \\
\hline Dänemark & Inbicon (DONG Energy) & in Betrieb & 0,0054 \\
\hline \multirow{4}{*}{ Frankreich } & Futurol (Total, Teros) & Projekt & Keine Angabe \\
\hline & LED (Abengoa Bioenergia) & Projekt & 0,063 \\
\hline & $\begin{array}{l}\text { KL Energy Europe (KL Energy } \\
\text { Corp. (US)) }\end{array}$ & Projekt & Keine Angabe \\
\hline & Tereos, Deinove & Projekt & Keine Angabe \\
\hline Deutschland & Clariant & in Betrieb & 0,0025 \\
\hline Finnland & Fibre EtOH & Projekt & 0,025 \\
\hline Spanien & $\begin{array}{l}\text { Biocarburantes de Castilla y } \\
\text { Leon (Abengoa Bioenergia) }\end{array}$ & in Betrieb & 0,005 \\
\hline \multirow{4}{*}{ Schweden } & SEKAB & Projekt & 0,0063 \\
\hline & $\begin{array}{l}\text { Härjedalens Miljöbränsle AB, } \\
\text { National Bio Energy Co. Ltd., } \\
\text { Dragon Power Co. Ltd. }\end{array}$ & Projekt & 0,0038 \\
\hline & Sala Heby Energi & Projekt & 0,005 \\
\hline & Domsjö Fabriker & Projekt & 0,011 \\
\hline \multirow{2}{*}{ Großbritannien } & TMO Renewables Ltd. & in Betrieb & 0,005 \\
\hline & Ineos Bio & Projekt & 0,03 \\
\hline
\end{tabular}

Tabelle 14: Anlagen und Projekte zur Produktion von Zellulose-Ethanol in der EU27

Quelle: In Anlehnung an F.O. LICHT 2010c: 451; GRUPPO MOSSI \& GHISOLFI 2012; CLARIANT 2012

Darüber hinaus wurde im Oktober 2013 die erste kommerzielle Anlage zur Produktion von Zellulose-Ethanol in der EU27 durch das Unternehmen Gruppo Mossi \& Ghisolfi in Crescentino, Italien, eröffnet (BETA RENEWABLES 2013). Auch in den USA bauen die Unternehmen POET \& DSM, ABENGOA BIOENERGY und DuPont $^{161}$ (POET-DSM ADVANCED BIOFUELS 2012; ABENGOA BIOENERGY 2012; DUPONT 2012) die ersten kommerziellen Anlagen zur Herstellung von ZelluloseEthanol. Die Inbetriebnahme dieser Anlagen kann zeigen, dass die Technologie zur Produktion von Zellulose-Ethanol in kommerziellem Maßstab Marktreife besitzt. Sollte dies der Fall sein, könnte die Produktion von Zellulose-Ethanol in der EU27 stark steigen, da andere Unternehmen die Technologie zur Herstellung von ZelluloseEthanol von den genannten Unternehmen einlizensieren und in der EU27 Produktionsanlagen errichten würden, um ebenfalls kommerziell zu produzieren.

Ein stärkeres Wachstum der Produktion von Zellulose-Ethanol als 3 Milliarden Liter bis zum Jahr 2023 wird in der EU27 aufgrund des erforderlichen hohen Investions-

${ }^{161}$ Eine genauere Beschreibung der drei US-amerikanischen Anlagen befindet sich in Kapitel 2.2. 
volumens nicht erwartet. Zur Herstellung von 3 Milliarden Litern Zellulose-Ethanol werden in der EU27 31 Produktionsanlagen mit einer durchschnittlichen Jahreskapazität von 0,095 Milliarden Litern ${ }^{162}$ errichtet. Der Bau einer Anlage kostet nach Berechnungen von BLOOMBERG NEW ENERGY FINANCE (2013: 2) 0,23 Milliarden Euro ${ }^{163}$, woduch sich ein Gesamtinvestionsbedarf bis zum Jahr 2023 von 7,2 Milliarden Euro ergibt. Zum Vergleich: Der Aufbau der identischen Produktionskapazitäten durch Ethanolanlagen der ersten Generation würde nur 0,9 ${ }^{164}$ Milliarden Euro kosten.

In der „EU27 Szenario-Bildung und -Konsistenzprüfung“ werden demnach drei Zukunftsprojektionen weiter betrachtet:

IIA

Starker Anstieg der Ethanolproduktion der ersten Generation auf 13 Milliarden Liter und keine bedeutende Produktion von Zellulose-Ethanol

IIB

Moderater Anstieg der Ethanolproduktion der ersten Generation auf 6 Milliarden Liter und keine bedeutende Produktion von Zellulose-Ethanol

IIC Keine Produktion von Ethanol der ersten Generation, aber starker Anstieg der Produktion von Zellulose-Ethanol auf 3 Milliarden Liter

\subsubsection{Ethanolhandel}

Die EU27 ist im Zeitraum von 2003 bis 2012 ein Ethanolimporteur, lediglich im Jahr 2004 hatte die EU27 eine ausgeglichene Handelsbilanz. Die Ethanolimporte sind seit 2003 von 0,07 Milliarden Litern auf 1,3 Milliarden Liter im Jahr 2012 angestiegen (Abbildung 51). Mit einem Import von 1,3 Milliarden Litern ist die EU27 im Jahr 2012 der weltgrößte Ethanolimporteur gewesen. Die Importe stammen fast ausschließlich aus den beiden wichtigsten ethanolproduzierenden Staaten USA und Brasilien (F.O. LICHT 2012a: 301f.).

\footnotetext{
${ }^{162}$ Es wird mit einer durchschnittlichen Jahreskapazität von 0,095 Milliarden Litern gerechnet, dies entspricht der Kapazität der ersten kommerziellen Anlagen, die von Abengoa Bioenergy und dem Gemeinschaftprojekt "Project LIBERTY“ (POET und DSM) in den USA errichtet werden.

${ }^{163}$ Es wird ein Wechselkurs von 1,25 US-\$/Euro für das Jahr 2023 zugrunde gelegt, dieser beruht auf Berechnungen des EUROPEAN COMMISSION DIRECTORATE - GENERAL FOR ENERGY (2009: 16ff.).

${ }^{164}$ Der Bau einer Produktionsanlage mit einer Jahreskapazität von 0,380 Milliarden Litern kostet 0,112 Milliarden Euro, somit ergibt sich ein Investionsbedarf von 0,9 Milliarden Euro, um eine Produktionskapazität von 3 Milliarden Litern zu errichten - es wurde mit einem Wechselkurs von 1,25 US-\$/Euro gerechnet (UNIVERSITY OF NEBRASKA 2007: 6).
} 


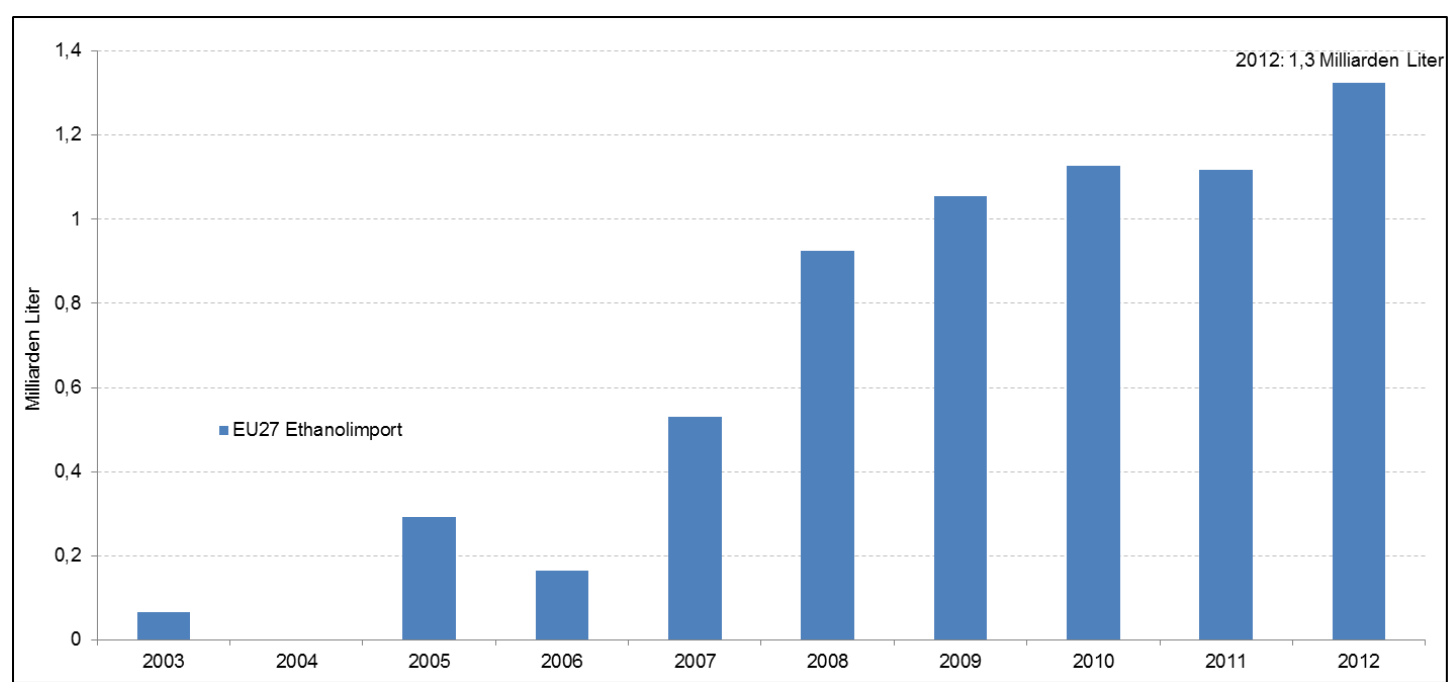

Abbildung 51: Entwicklung der Ethanolimporte in die EU27 im Zeitraum von 2003 bis 2012 Quelle: F.O. LICHT 2012f: 74; BERG 2012c: 19; EUROSTAT 2012

Der Zukunftsraum des Schlüsselfaktors im Jahr 2023 wird mittels der zwei Zukunftsprojektionen IIIA und IIIB dargestellt. Beide Zukunftsprojektionen beschreiben, ebenso wie zahlreiche etablierte Forschungsinstitute ${ }^{165}$, die EU27 als Nettoimporteur von Ethanol-Kraftstoff.

Die erste Zukunftsprojektion IIIA prognostiziert für das Jahr 2023 einen Import von Ethanol der ersten Generation entsprechend dem Niveau des Jahres 2012 in Höhe von 1,5 Milliarden Litern. Diese Projektion basiert auf der Annahme, dass die derzeitig hohen Importzölle für Ethanol, festgelegt unter KN-Code $2207^{166}$, noch im Jahr 2023 bestehen werden (EUROPÄISCHE KOMMISSION 2011: 182) ${ }^{167}$.

Die zweite Zukunftprojektion IIIB erwartet für das Jahr 2023 einen deutlich stärkeren Ethanolimport der ersten Generation in Höhe von 4,5 Milliarden Litern in die EU27. Grundlage dessen ist die politische Entscheidung, keine Einfuhrzölle auf Ethanolimporte mehr zu erheben, sodass die Ethanolproduzenten aus den USA und Brasilien zollfrei in die EU27 exportieren können. Aufgrund der in beiden Ländern im Vergleich zur EU27 geringeren Produktionskosten (BERG 2012d: 2; HENNIGES 2007: $55 \mathrm{ff}$.) wird ein starker Anstieg der Ethanolimporte erwartet.

\footnotetext{
${ }^{165}$ Folgende internationale Forschungsinstitute sind sich einig, dass die EU27 langfristig als Nettoimporteur auf dem Ethanolmarkt auftreten wird: OECD/FAO 2012: 224; ALFSTAD 2008: A2f.; EUROPEAN COMMISSION JOINT RESEARCH CENTRE, INSTITUTE FOR PROSPECTIVE TECHNOLOGICAL STUDIES 2010a: 36; EUROPEAN COMMISSION JOINT RESEARCH CENTRE, INSTITUT FOR ENERGY ET AL. 2011: 41.

${ }^{166}$ Eine genaue Beschreibung der derzeitigen Zollsätze für Ethanol-Kraftstoff erfolgt in Kapitel 5.2.5.

${ }_{167}$ Die Zollbestimmungen sind in der Zukunftsprojektion 22A/Kapitel 5.2 .5 beschrieben und werden ebenfalls in der „EU27 Szenario-Bildung und -Konsistenzprüfung“ weiter betrachtet.
} 
Basierend auf den vorangegangenen Ausführungen werden die folgenden zwei Zukunftsprojektionen für den beschreibenden Schlüsselfaktor weiter betrachtet:

IIIA | Konstante Ethanolimporte in Höhe von 1,5 Milliarden Litern

IIIB | Starker Anstieg der Ethanolimporte auf 4,5 Milliarden Liter

\subsubsection{Ethanolpreis}

Die Entwicklung des Schlüsselfaktors Ethanolpreis IV wird mithilfe des EthanolWeltmarktpreises dargestellt; dies ist der Ethanolpreis für wasserfreies Ethanol der ersten Generation, produziert in Brasilien, Sao Paulo (FAPRI 2011c: 117). Der Ethanol-Weltmarktpreis lässt Rückschlüsse auf den Ethanolpreis in der EU27 zu, da diese beiden stark miteinander korrelieren (INTERNATIONAL SUGAR ORGANIZATION 2011: 34). Der Einfluss des Weltmarktpreises auf den Ethanolpreis der EU27 ist hoch, da die EU27 der weltgrößte Ethanolimporteur ist (USDA 2012: 4; F.O. LICHT 2012a: 300). Außerdem ist davon auszugehen, dass die Preisbeeinflussung noch im Jahr 2023 besteht, da Brasilien nach Studien der Organisationen FAPRI (2011c: 117), OECD/FAO (2012: 95) und EUROPEAN COMMISSION JOINT RESEARCH CENTRE ET AL. (2010: 78) der mit Abstand weltgrößte Ethanolexporteur im Jahr 2023 sein und die EU27 mit Ethanol beliefern wird.

Die folgende Abbildung 52 zeigt die reale (2005) Preisentwicklung für Ethanol der ersten Generation in den Jahren von 2001 bis 2011.

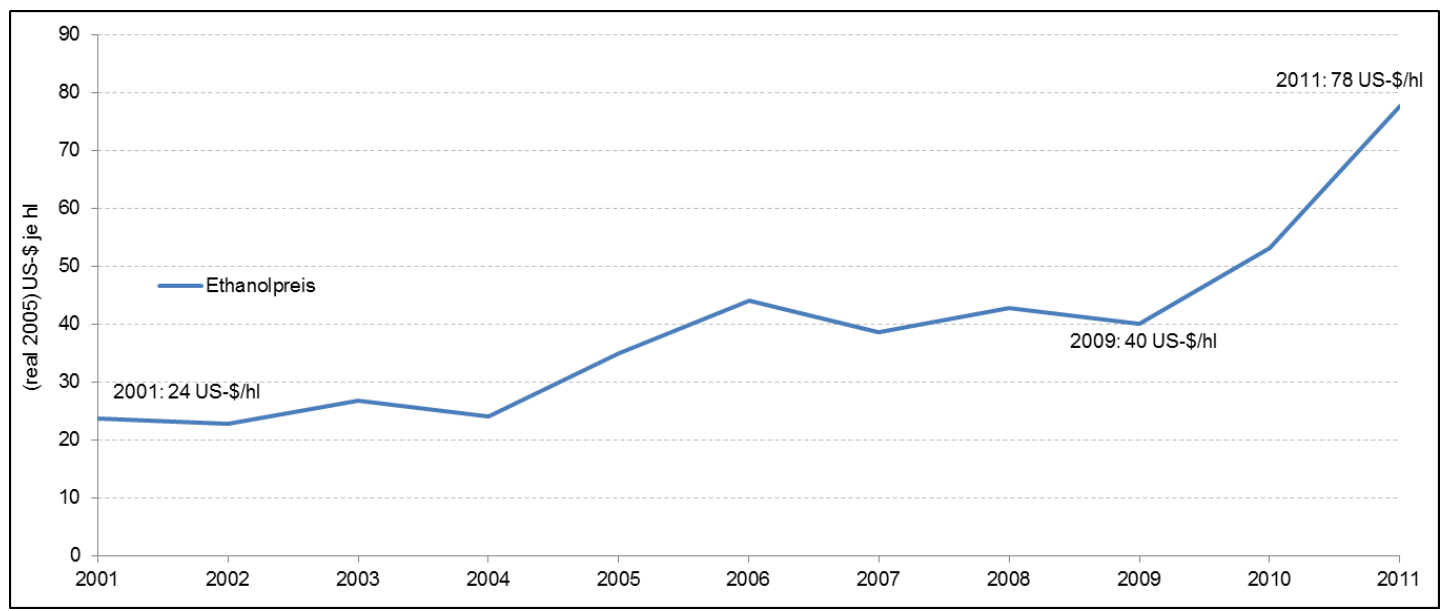

Abbildung 52: Entwicklung des (real 2005) Ethanol-Weltmarktpreises in den Jahren von 2001 bis 2011

Quelle: In Anlehnung an OECD/FAO 2012: 89

In der Zeit von 2001 bis 2009 ist der Preis für Ethanol von 24 US-\$/hl auf 40 US-\$/hl angestiegen. Einen noch stärkeren Preisanstieg gab es in den Jahren von 2009 bis 2011, in denen sich der Ethanolpreis von 40 US- $\$ /$ hl auf 78 US- $\$ / h l$ beinahe verdoppelte. Der Preisanstieg in den Jahren 2009 bis 2011 ist zurückzuführen auf die 
abnehmende Ethanolproduktion in Brasilien ${ }^{168}$, das geringere Wachstum der Ethanolproduktion in den $U_{S A}{ }^{169}$ sowie zu einem geringeren Anteil auf die allgemein hohen Energiepreise (OECD/FAO 2012: 88).

In der folgenden Abbildung 53 ist die von der OECD/FAO (2012: 89) prognostizierte Entwicklung des (real 2005) Ethanol-Weltmarktpreises bis zum Jahr 2023 dargestellt.

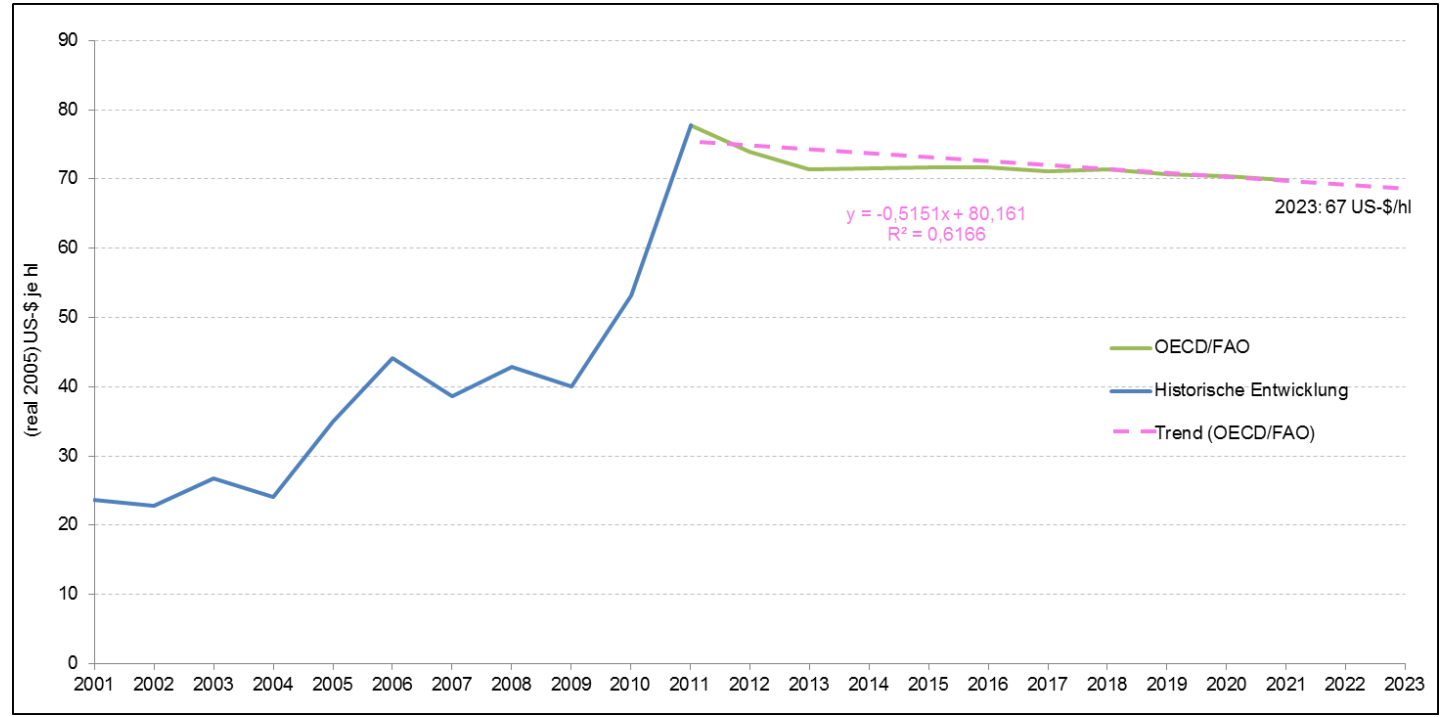

Abbildung 53: Zukünftige Entwicklung des (real 2005) Ethanol-Weltmarktpreises bis zum Jahr 2023

Quelle: In Anlehnung an OECD/FAO 2012: 89; EIGENE BERECHNUNG

Nach dem starken Preisanstieg für Ethanol im Jahr 2011 auf 78 US-\$/hl prognostiziert die OECD/FAO (2012: 89) einen Rückgang des Ethanolpreises im Jahr 2012 sowie in den folgenden Jahren einen relativ konstanten Preis zwischen 69 US-\$/hl und 71 US-\$/hl. Auf Grundlage der erwarteten Preise der Jahre 2011 bis 2021 wurden mithilfe einer linearen Trendberechnung die Ethanolpreise für die Jahre 2022 und 2023 berechnet; darauf basierend ergibt sich für das Jahr 2023 ein Preis von 67 US- $\$ / h^{170}$.

Die konstante langfristige Entwicklung des Ethanol-Weltmarktpreises begründet die OECD/FAO (2012: 89) mit der ebenfalls konstanten Entwicklung der Rohstoffpreise für Weizen und Mais (Kapitel 5.2.3 und 6.2.3). Darüber hinaus erwartet die

\footnotetext{
${ }^{168}$ In Brasilien ist die Ethanolproduktion um 5,4 Milliarden Liter zurückgegangen von ursprünglich 28 Milliarden Litern im Jahr 2010 auf 22,6 Milliarden Liter im Jahr 2011 (F.O. LICHT 2011a: 67).

${ }_{169}$ Die USA konnten das Wachstum der vorherigen Jahre nicht erreichen. Während das Produktionswachstum in den Jahren 2007 bis 2010 bei durchschnittlich 8,5 Milliarden Litern lag, erreichten die USA im Zeitraum von 2010 bis 2011 lediglich einen Anstieg der Ethanolproduktion um 2 Milliarden Liter (F.O. LICHT 2011a: 67).

170 Das Forschungsinstitut FAPRI (2011c: 117) erwartet einen fast identischen EthanolWeltmarktpreis von 68 US-\$/hl für das Jahr 2023.
} 
OECD/FAO (2012: 89) nicht, dass der Ethanol-Weltmarktpreis einem möglichen starken Anstieg des Rohölpreises folgen wird. Bedeutenden Einfluss auf den Ethanolpreis haben der US-amerikanische Renewable Fuel Standard 2 (RFS 2) und die steigende Nachfrage nach Ethanol in Brasilen aufgrund der wachsenden Anzahl an FFV (OECD/FAO 2012: 114f.). Die Ethanolnachfrage in der EU27 hat nur einen geringen Einfluss auf den Ethanol-Weltmarktpreis, da die EU27 im Gegensatz zu den USA und Brasilien ein deutlich kleinerer Ethanolmarkt ist (EUROPEAN COMMISSION JOINT RESEARCH CENTRE, INSTITUTE FOR PROSPECTIVE TECHNOLOGICAL STUDIES 2010a: 44f.).

Da die EU27 momentan und möglicherweise auch im Jahr 2023 Zölle auf Ethanolimporte erheben wird (Kapitel 5.2.5), ist es notwendig, den Importzoll dem EthanolWeltmarktpreis anzurechnen. Die EU27 erhebt einen Importzoll von 19,2 €/hl auf unvergälltes Ethanol und einen Zollsatz von 10,2 €/hl auf vergälltes Ethanol (EUROPÄISCHE KOMMISSION 2011: 182). Da die meisten Mitgliedsstaaten der EU27 nur die Einfuhr von unvergälltem Ethanol erlauben ${ }^{171}$ (EUROPÄISCHE KOMMISSION 2011: 182), wird in der weiteren Betrachtung mit einem Zollsatz von 19,2 €/hl gerechnet. Damit der Zollsatz dem prognostizierten realen (2005) EthanolWeltmarktpreis angerechnet werden kann, wird der Zollsatz auf US-amerikanische Dollar umgerechnet und vom Jahr 2023 auf das Jahr 2005 abgezinst. Es wird mit einem prognostizierten Wechselkurs von 1,25 US-\$/Euro und einem Abzinsungsfaktor von $1,9835249 \%{ }^{172}$ gerechnet. Aufgrund der genannten Annahmen ergibt sich ein realer Importzollsatz für das Jahr 2005 für unvergälltes Ethanol in Höhe von 10 US- $\$ / h$ l. Auf Grundlage des prognostizierten Ethanol-Weltmarktpreises von (real 2005) 65 US- $\$ / \mathrm{hl}^{173}$ und der Berücksichtigung bzw. Nichtberücksichtigung des Importzollsatzes in Höhe von (real 2005) 10 US-\$/hl werden die folgenden beiden Zukunftsprojektionen in der weiteren Arbeit betrachtet: (1) „Ethanolpreis (real 2005) 65 US-\$/hl“ und (2) „Ethanolpreis (real 2005) 75 US-\$/hl“174.

Des Weiteren geht noch eine dritte Zukunftsprojektion für den Marktpreis für Zellulose-Ethanol in der EU27 in die „Szenario-Bildung und -Konsistenzprüfung“ ein. Der

\footnotetext{
${ }^{171}$ Lediglich die Staaten Großbritannien, Finnland, Dänemark, Niederlande, Tschechien und die Slowakei erlauben auch die Einfuhr von vergälltem Ethanol (EUROPÄISCHE KOMMISSION 2011: 182)

${ }_{172}$ Der Abzinsungsfaktor von 1,9835249\% beruht auf Berechnungen der OECD/FAO (2012: 89).

${ }^{173}$ Basierend auf einer Diskussion mit Dr. Christoph Berg (BERG 2012b) wird ein Ethanolpreis von 65 US-\$/hl weiter betrachtet anstatt der errechneten 67 US-\$/hl.

174 Das Beratungsunternehmen LMC INTERNATIONAL (2011: 110) prognostiziert ebenfalls einen Ethanolpreis in der EU27 von ungefähr 75 US-\$/hl.
} 
Preis für Zellulose-Ethanol orientiert sich am Weltmarktpreis für Ethanol der ersten Generation, wird aber in der EU27 preislich höher liegen, da aufgrund der mehrfachen Anrechnung des Energiegehalts des biogenen Kraftstoffs auf das politische Ziel der prozentualen Nutzung von erneuerbaren Energien im Verkehrssektor Zellulose-Ethanol in Europa einen höheren Wert für die Mineralölindustrie besitzt ${ }^{175}$.

Die zuvor beschriebenen drei Zukunftsprojektionen werden im dritten Kernschritt weiter betrachtet:

IVA

Ethanolpreis (real 2005) 65 US-\$ je Hektoliter

Beschreibung:

Keine Berücksichtigung eines Importzolls für Ethanol in der Zukunftsprojektion.

Ethanolpreis (real 2005) 75 US-\$ je Hektoliter

IVB Beschreibung:

Die Zukunftsprojektion berücksichtigt den Importzoll für Ethanol in Höhe von (real 2005) 10 US-\$ je Hektoliter.

Der Preis für Zellulose-Ethanol ist höher als der Preis für Ethanol der ersten Generation

IVC Beschreibung:

Da der Energiegehalt von Zellulose-Ethanol dem 10\%-Ziel mehrfach angerechnet wird, ist der Preis für Zellulose-Ethanol höher als der Preis für Ethanol der ersten Generation.

Zum Abschluss der „EU27 Szenario-Prognostik“ sind in der folgenden Abbildung 54 alle Zukunftsprojektionen der sieben wirkenden und der vier beschreibenden Schlüsselfaktoren zusammengefasst dargestellt.

\footnotetext{
${ }^{175}$ Der höhere Preis für Biokraftstoffe, deren Energiegehalt dem 10\%-Ziel mehrfach angerechnet wird, ist bereits am Preis für Biodiesel, hergestellt aus Used Cooking Oil (UCO), zu erkennen. Dessen Energiegehalt wird dem 10\%-Ziel doppelt angerechnet und erzielt deshalb einen höheren Marktpreis im Vergleich zu Biodiesel, der aus landwirtschaftlichen Rohstoffen wie beispielsweise Raps produziert wird, und deren Energiegehalt dem 10\%-Ziel nur einfach angerechnet wird (CHATHAM HOUSE 2013: 14).
} 


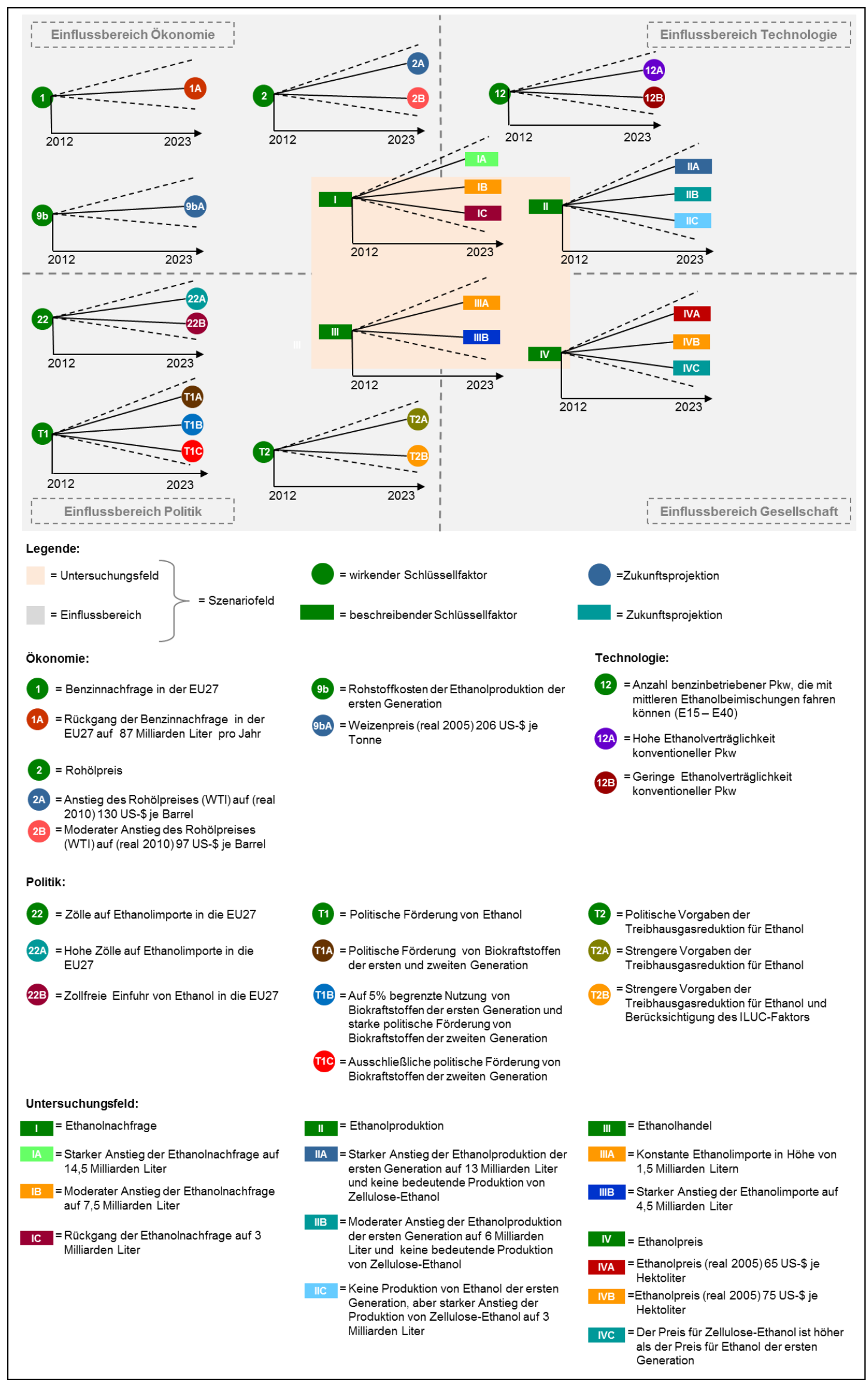

Abbildung 54: Zusammenfassung der Zukunftsprojektionen der wirkenden und beschreibenden Schlüsselfaktoren des Ethanolmarktes der EU27

Quelle: EIGENE DARSTELLUNG 


\subsection{EU27 Szenario-Bildung und -Konsistenzprüfung}

Ziel des dritten und letzten Kernschritts „EU27 Szenario-Bildung und -Konsistenzprüfung“ ist es, aus den einzelnen Zukunftsprojektionen konsistente Szenarien zu bilden, die den Ethanolmarkt der EU27 im Jahr 2023 beschreiben. In Kapitel 5.3.1 werden die Zukunftsprojektionen mithilfe des morphologischen Kastens zu in sich konsistenten Szenarien verknüpft. Im Anschluss wird die Konsistenz jedes Szenarios durch eine Expertenbefragung überprüft; Kapitel 5.3.2 beschreibt die Durchführung und Kapitel 5.3.3 die Auswertung dieser Prüfung.

\subsubsection{Szenario-Bildung}

Die Erstellung der Szenarien erfolgt durch den morphologischen Kasten, dargestellt in Abbildung 55. Im oberen Abschnitt des Kastens befinden sich die Zukunftsprojektionen der wirkenden Schlüsselfaktoren, jeweils zugeordnet zu ihrem Einflussbereich. Die Reihenfolge der Anordnung der Bereiche - beginnend mit der Politik, gefolgt von der Ökonomie und der Technologie - entspricht der Stärke ihres Einflusses auf den Ethanolmarkt der EU27 bis zum Jahr $2023^{176}$. Die Zukunftsprojektionen, die das Untersuchungsfeld beschreiben, werden erst im unteren Abschnitt des morphologischen Kastens betrachtet, da ihre Bestimmung von der Auswahl der Zukunftsprojektionen der wirkenden Schlüsselfaktoren abhängig ist. Jede Zukunftsprojektion ist mittels ihrer Nummer und ihres Buchstaben eindeutig zu identifizieren. Die Verknüpfung der einzelnen Zukunftsprojektionen zu Szenarien erfolgt durch den Verfasser ${ }^{177}$ dieser Studie. Im Folgenden sind die vier Szenarien „Strong Growth of First Generation Ethanol“, „5\% Cap for First Generation Ethanol“, „Growth of Cellulosic Ethanol“ und „No Import Tariff“ einzeln beschrieben ${ }^{178}$.

\footnotetext{
${ }^{176}$ Einflussbereich „Politik“ - Schlüsselfaktor: 22, T1 und T2 - besitzt mit einem Mittelwert von 5,55 den stärksten Einfluss auf den Ethanolmarkt der EU27. Gefolgt vom Einflussbereich "Ökonomie" - Schlüsselfaktor: 1,2 und 9 - mit einer durchschnittlichen Wirkungsstärke von 5,27 und dem Bereich „Technologie“, bestehend aus dem Schlüsselfaktor 12, mit einer Wirkungsstärke von 5,01.

${ }_{177}$ Die erstellten Szenarien wurden mit den folgenden Experten besprochen: Dr. Helmut Born - Deutscher Bauernverband, Dr. Christoph Berg - F.O. Licht, Dr. Clemens Heikaus und Dr. Paolo Corvo - Clariant, Dr. Hinrich Harling, Dr. Beate Rother und Dr. Andreas von Felde - KWS SAAT AG.

${ }_{178}$ Die Szenarien sind in englischer Sprache verfasst, damit alle Experten des europäischen Ethanolmarktes unabhängig von ihrer Herkunft das Szenario verstehen und an der Konsistenzprüfung (Kapitel 5.3.2) teilnehmen können.
} 


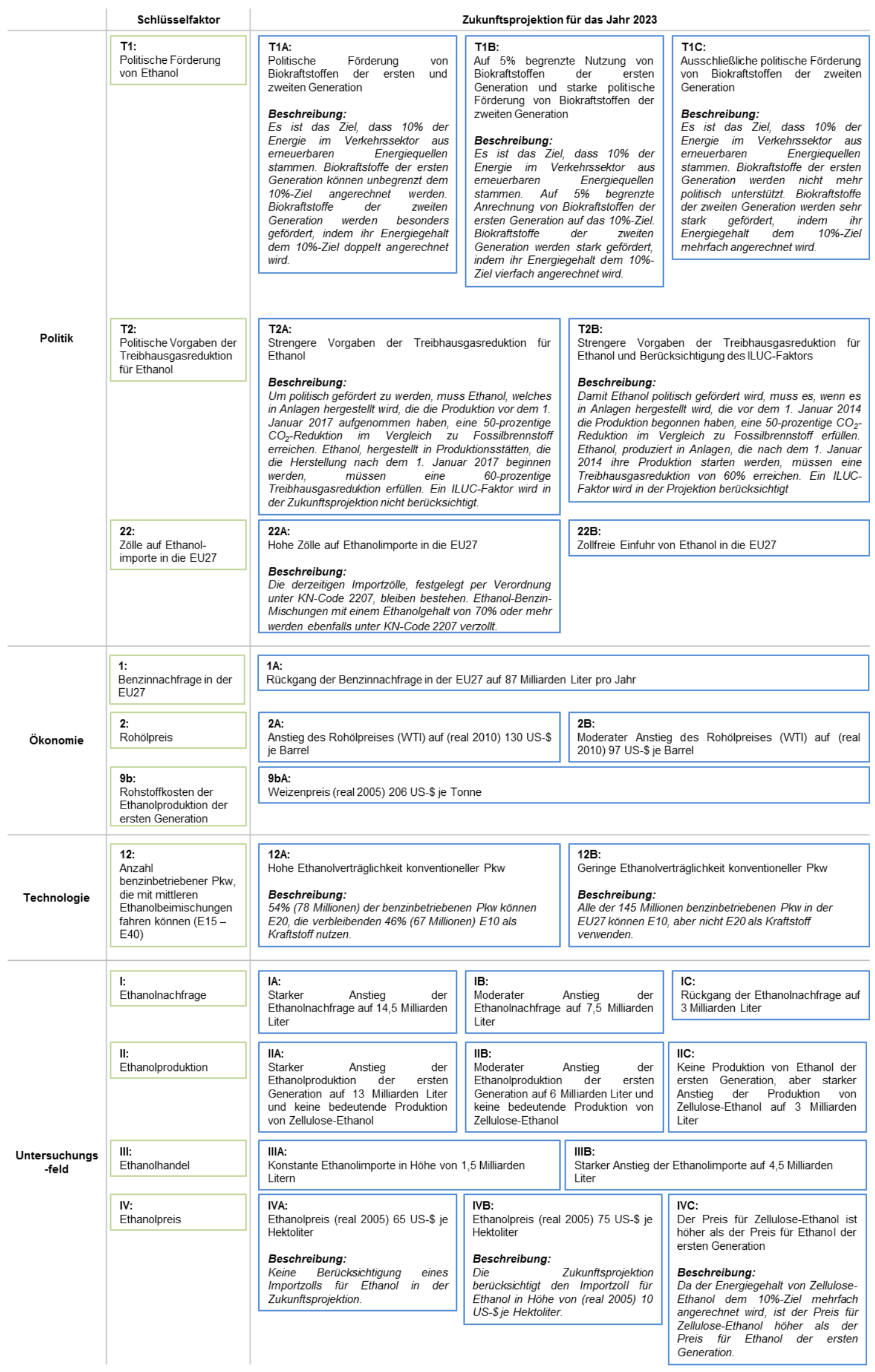

Abbildung 55: Morphologischer Kasten zur Bildung konsistenter Szenarien des Ethanolmarktes der EU27 im Jahr 2023

Quelle: EIGENE DARSTELLUNG 


\section{Scenario 1: Strong Growth of First Generation Ethanol}

Zukunftsprojektionen: T1A, T2A, 22A, 1A, 2A, 9bA, 12A, IA, IIA, IIIA, IVA

In the year 2023, after years of constant growth, the demand for fuel ethanol is around 14.5 billion liters in the EU27. The main part of the demanded ethanol - 13 billion liters - is produced in the EU27 as first generation ethanol out of wheat, corn and sugar beets. The political support for biofuels can be considered as the key driver for the constantly growing fuel ethanol market. The political goal is that $10 \%$ of the energy used in the transport sector derive from renewable sources and biofuels play the key role in achieving this goal. There is no cap for the counting of first generation biofuels towards the $10 \%$ goal; also, second generation biofuels get supported by having their energy content double counted towards the $10 \%$ goal. Due to high production costs and missing production capacity, there is no significant production of cellulosic ethanol in the EU27 in the year 2023. Ethanol has to achieve the following mandatory political targets concerning GHG savings in order to get counted towards the $10 \%$ goal:

- Ethanol produced in plants built until January 1st, 2017 has to achieve a GHG reduction of $50 \%$ compared to fossil fuels.

- Ethanol produced in plants that have started production after January 1st, 2017 has to reach a GHG reduction of $60 \%$ compared to fossil fuels.

- An ILUC-Factor is not included in the calculation of the $\mathrm{CO}_{2}$ balance of ethanol.

Plants in the EU27 built before and after January 1st, 2017 are able to meet these requirements. In order to satisfy the demand, around 1.5 billion liters of ethanol, which have to pay high import tariffs, are imported into the EU27. All conventional gasoline vehicles in the EU27 are able to use E10 as a gasoline fuel and 54\% of all conventional gasoline vehicles can run on ethanol blends up to $20 \%$. The WTI crude oil price constantly rises to (real 2010) 130 US-\$ per barrel in the year 2023 while the gasoline demand continually decreases. The ethanol producers are able to profitably produce ethanol for a price of around (real 2005) 75 US-\$/hl and for costs for wheat of approximately (real 2005) 206 US-\$/ton.

\section{Scenario 2: 5\% Cap for First Generation Ethanol}

Zukunftsprojektionen: T1B, T2A, 22A, 1A, 2B, 9bA, 12A, IB, IIB, IIIB, IVB

In the year 2023, the demand for fuel ethanol is around 7.5 billion liters in the EU27. The main part of the demanded ethanol -6 billion liters - is produced in the EU27 
as first generation ethanol out of wheat, corn and sugar beets. The modest growth of the ethanol market is due to the political goal that $10 \%$ of the energy used in the transport sector derive from renewable sources. The ethanol market has not grown further because of the political cap to count no more than $5 \%$ of first generation biofuel towards the $10 \%$ goal. Cellulosic ethanol is highly politically supported by quadruple counting the energy content towards the $10 \%$ goal. But due to high production costs and missing production capacity, there is no significant production of cellulosic ethanol in the year 2023. Ethanol has to achieve the following mandatory political targets concerning GHG savings in order to get counted towards the $10 \%$ goal:

- Ethanol produced in plants built until January 1st, 2017 has to achieve a GHG reduction of $50 \%$ compared to fossil fuels.

- Ethanol produced in plants that have started production after January 1st, 2017 has to reach a GHG reduction of $60 \%$ compared to fossil fuels.

- An ILUC-Factor is not included in the calculation of the $\mathrm{CO}_{2}$ balance of ethanol. Plants in the EU27 built before and after January 1st, 2017 are able to meet these requirements. In the year 2023, the EU27 protects their domestic ethanol market and charges high import tariffs, but imports of about 1.5 billion liters still reach the EU27. All conventional gasoline vehicles in the EU27 are able to run on E10, but the vehicles are not capable of using higher ethanol blends. The price for WTI crude oil rises moderately up to (real 2010) 97 US-\$ per barrel in the year 2023 while the gasoline demand decreases continually in the EU27. The ethanol producers are able to profitably produce first generation ethanol for a price of around (real 2005) 75 US-\$/ hl and for costs for wheat of approximately (real 2005) 206 US-\$/ ton.

\section{Scenario 3: Growth of Cellulosic Ethanol}

Zukunftsprojektionen: T1C, T2B, 22A, 1A, 2A, 9bA, 12B, IC, IIC, IVC

In the year 2023, politics exclusively support second generation biofuels. It is the political goal that $10 \%$ of the energy used in the transport sector derive from renewable sources. However, first generation biofuels are not counted towards the $10 \%$ goal which pushes wheat, corn and sugar beet ethanol out of the fuel market. In contrast, cellulosic ethanol receives strong political support by multiple counting their energy content towards the $10 \%$ goal and substitutes first generation ethanol. Moreover, unlike cellulosic ethanol producers, most first generation ethanol producers are not capable of achieving the required GHG savings in order to get counted towards 
the $10 \%$ goal. The Directorate-General (DG) Energy and Climate Action require the following mandatory GHG reduction targets:

- Ethanol produced in plants built until January 1st, 2014 has to achieve a GHG reduction of $50 \%$ compared to fossil fuels.

- Ethanol produced in plants that have started production after January 1st, 2014 has to reach a GHG reduction of $60 \%$ compared to fossil fuels.

- A raw material dependent and scientifically proved ILUC-Factor is integrated into the calculation of the $\mathrm{CO}_{2}$ balance of ethanol.

Due to the ILUC-Factor, most European first generation ethanol producers are not capable of achieving the required GHG savings. In the year 2023, the demand for cellulosic ethanol is about 3 billion liters and gets completely supplied by the domestic production in the EU27. The demand of around 3 billion liters cellulosic ethanol is sufficient for the fuel market, since the energy content of cellulosic ethanol is counted multiple times towards the $10 \%$ goal. All conventional gasoline vehicles in the EU27 are able to run on E10. The WTI crude oil price constantly rises up to (real 2010) 130 US-\$ per barrel in the year 2023 while the gasoline demand continually decreases. The ethanol producers are able to profitably produce cellulosic ethanol, since the market accepts a higher price compared to first generation ethanol due to the political decision to count the energy content multiple times towards the $10 \%$ goal.

\section{Scenario 4: No Import Tariff}

Zukunftsprojektionen: T1A, T2B, 22B, 1A, 2A, 9bA, 12B, IC, IIB, IIIB, IVC In the year 2023 , it is the political goal that $10 \%$ of the energy used in the transport sector derives from renewable sources, and biofuels play the key role in achieving this objective. Furthermore, after the political decision to abolish import tariffs for fuel ethanol, all countries can export ethanol duty free into the EU27. Brazil uses the free trade situation and exports around 4.5 billion liters cane ethanol to the EU27. Brazilian ethanol has got a very good $\mathrm{CO}_{2}$ balance and fulfills the following mandatory political targets concerning GHG savings in order to get counted towards the $10 \%$ goal:

- Ethanol produced in plants built until January 1st, 2014 has to achieve a GHG reduction of $50 \%$ compared to fossil fuels.

- Ethanol produced in plants that have started production after January 1st, 2014 has to reach a GHG reduction of $60 \%$ compared to fossil fuels. 
- A raw material dependent and scientifically proved ILUC-Factor is integrated into the calculation of the $\mathrm{CO}_{2}$ balance of ethanol.

Most European first generation ethanol producers cannot compete with the Brazilian imports, since they are not capable of producing ethanol that fulfills the mandatory political GHG reduction targets. Unlike first generation ethanol, cellulosic ethanol produced in the EU27 is able to fulfill these targets. Moreover, it is strongly politically supported by multiple counting the energy content of cellulosic ethanol towards the $10 \%$ goal. The production of cellulosic ethanol is around 3 billion liters in the EU27 in the year 2023. The increase in the cellulosic ethanol production is needed to meet the demand for fuel ethanol of around 7.5 billion liters. The WTI crude oil price constantly rises up to (real 2010) 130 US-\$ per barrel in the year 2023 while the gasoline demand continually decreases. Moreover, all conventional gasoline vehicles are capable of running on E10 in the year 2023. The European producers are able to economically produce cellulosic ethanol - in contrast to first generation ethanol because of the higher market price for cellulosic ethanol compared to cane ethanol. The market value of cellulosic ethanol is higher due to the political decision to count the energy content of cellulosic ethanol multiple times towards the $10 \%$ goal.

\subsubsection{Durchführung der Konsistenzprüfung}

Durch die Konsistenzprüfung wird sichergestellt, dass alle vier Szenarien in sich widerspruchsfrei sind. Um dies zu überprüfen, wurde ein standardisierter Onlinefragebogen ${ }^{179}$ entwickelt, der sich in drei Abschnitte gliedert.

1. Einführung in die Thematik

2. Konsistenzbeurteilung der Szenarien

3. Erhebung der persönlichen Daten der Probanden

Im ersten Abschnitt wurde den Probanden das Ziel der Dissertation und des Fragebogens beschrieben. Anschließend wurde erläutert, wie der Fragebogen zu beantworten ist, und explizit darauf hingewiesen, dass ausschließlich die Konsistenz der Szenarien zu bewerten ist.

Der Hauptteil der Befragung befand sich in Abschnitt 2. Dort beurteilten die Teilnehmer die Konsistenz der Szenarien. Jedes Szenario wurde den Probanden einzeln mittels einer Zusammenfassung vorgestellt sowie mit einem Link versehen, um zu dem vollständig ausformulierten Szenario zu gelangen. Des Weiteren wurden die

${ }^{179}$ Der Onlinefragebogen ist in Anhang 3 abgebildet. 
Kernpukte des Szenarios genannt. Zur Beurteilung der Konsistenz des Szenarios wurden eine fünfstufige Likert-Skala ${ }^{180}$ sowie ein Kommentarfeld verwendet. In diesem konnten die Probanden mögliche Inkonsistenzen im Szenario beschreiben.

Zum Abschluss der Befragung wurden die Probanden gebeten, sowohl das Herkunftsland und die Branche des Unternehmens/der Organisation anzugeben, für welche/s sie arbeiteten, als auch ihre persönlichen Kontaktdaten zu nennen, um innen die Ergebnisse der Erhebung zuschicken zu können.

Die Struktur des Fragebogens - insbesondere die Nennung der Kernpunkte der Szenarien - hat sich in den Pretests ${ }^{181}$ als geeignet erwiesen. Darüber hinaus konnten durch die Tests die Beschreibungen der Szenarien verbessert, die Likert-Skala auf ihre Eignung als Messinstrument und die Fragestellung auf Eindeutigkeit überprüft sowie die Bearbeitungsdauer des Fragebogens festgestellt werden.

An der Befragung haben ausschließlich Experten des Ethanolmarktes der EU27 teilgenommen, da nur diese die Expertise besaßen, die Konsistenz der Szenarien zu beurteilen. Die Experten beschäftigten sich hauptberuflich mit dem Ethanolmarkt und besetzten zum Teil Positionen in der Politik und in Unternehmen, in denen sie die Entwicklung des Marktes bis zum Jahr 2023 beeinflussen konnten. Um möglichst viele Experten des europäischen Ethanolmarktes zu befragen, war der Fragebogen in englischer Sprache verfasst. Der Großteil der befragten Experten wurde bereits in der Wirkungsanalyse befragt oder erklärte sich während der „F.O. Licht World Ethanol \& Biofuels“-Konferenz $2012^{182}$ bereit, an der Erhebung teilzunehmen. Die Erhebung wurde im Zeitraum von Mitte Januar 2013 bis Mitte Februar 2013 durchgeführt.

\subsubsection{Auswertung der Konsistenzprüfung}

Mittels der Auswertung der Konsistenzprüfung ergibt sich, ob die vier gebildeten Szenarien in sich konsistent sind oder auf Grundlage der Anmerkungen der befragten Experten überarbeitet werden müssen. Die Ergebnisse der Befragung sind valide, da jedes Szenario von mindestens 46 der befragten 53 Experten beurteilt wurde. Die befragten Experten stammten sowohl aus der Politik als auch aus allen ent-

\footnotetext{
${ }^{180}$ Die fünfstufige Likert-Skala besitzt folgende Abstufung: „sehr gute Konsistenz“, „gute Konsistenz", „moderate Konsistenz“, „schlechte Konsistenz" und „sehr schlechte Konsistenz“. ${ }^{181}$ Die folgenden Experten führten den Pretest durch: Dr. Helmut Born - Deutscher Bauernverband, Dr. Christoph Berg - F.O. Licht, Dr. Clemens Heikaus und Dr. Paolo Corvo - Clariant, Dr. Hinrich Harling, Dr. Beate Rother und Dr. Andreas von Felde - KWS SAAT AG.

182 Die Konferenz fand vom 5. bis 8. November 2012 in München statt.
} 
scheidenden Branchen des europäischen Ethanolmarktes (Abbildung 56) sowie aus ganz Europa, insbesondere Deutschland, Großbritannien und Frankreich (Abbildung $57)$.

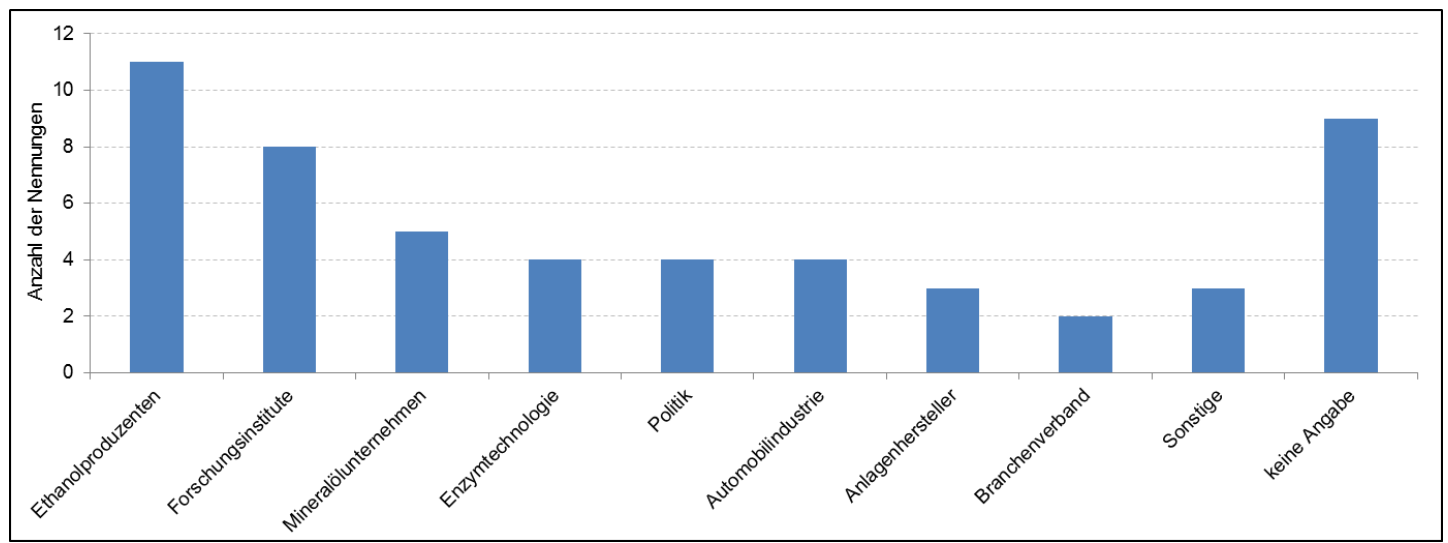

Abbildung 56: Konsistenzanalyse EU27 - Herkunftsbranche der Unternehmen/Organisationen der befragten Experten

Quelle: EIGENE DARSTELLUNG

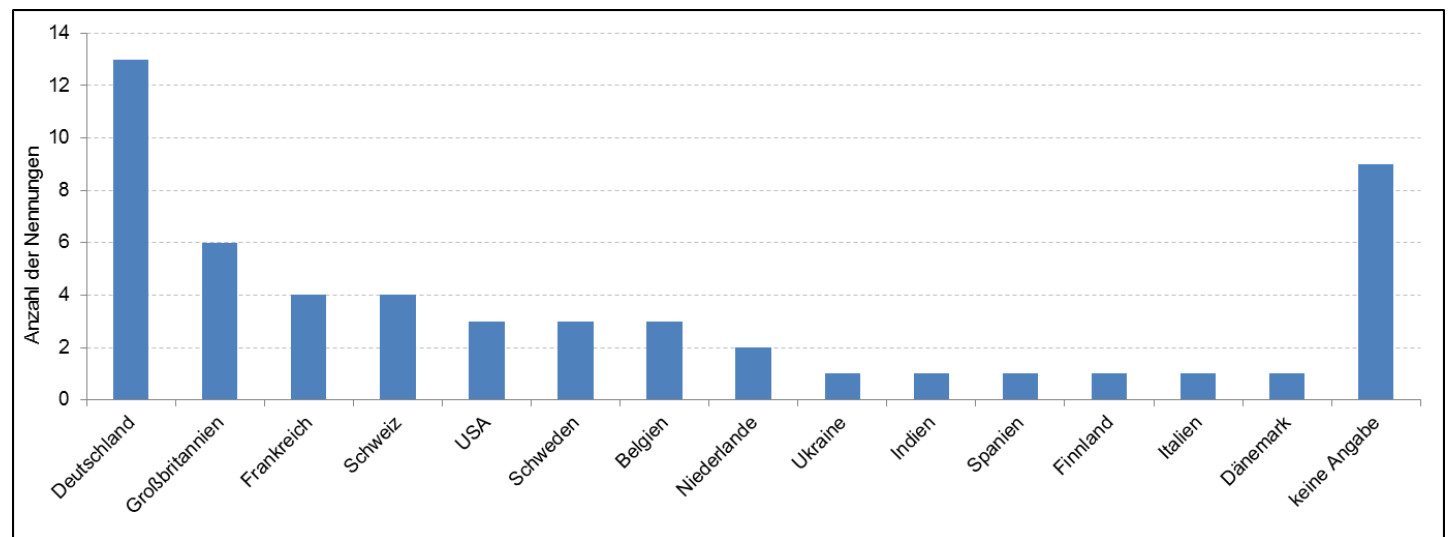

Abbildung 57: Konsistenzanalyse EU27 - Herkunftsland der Unternehmen/Organisationen der befragten Experten

Quelle: EIGENE DARSTELLUNG

Jedes Szenario wird anhand der Beantwortung der folgenden beiden Fragen einzeln analysiert:

1. Wie schätzen die Experten die Konsistenz des Szenarios ein?

2. Befindet sich im Szenario ein mögliches Konsistenzproblem, das von mehr als zwei Befragten genannt wurde ${ }^{183}$ ?

Ein Szenario, dem eine „sehr gute Konsistenz" oder "gute Konsistenz" bescheinigt wurde und bei dem nicht mehr als zwei Probanden auf ein identisches Konsistenzproblem hingewiesen haben, bleibt unverändert. In Szenarien, in denen mindestens drei Experten in der zweiten Frage eine mögliche Inkonsistenz beschreiben, unab-

\footnotetext{
${ }^{183}$ Anmerkungen zur Konsistenz, die lediglich ein- oder zweimal genannt wurden, werden aufgrund ihrer geringen Relevanz bei mindestens 46 befragten Experten je Szenario nicht weiter betrachtet.
} 
hängig vom Konsistenzwert der ersten Frage, gilt es, die mögliche Inkonsistenz in der Studie zu erörtern, um daraufhin zu entscheiden, ob das Szenario verändert werden sollte. Ferner werden Szenarien mit lediglich einer moderaten, schlechten oder sehr schlechten Konsistenz nicht weiter betrachtet oder es wird das gesamte Szenario mittels der Kommentare der Experten analysiert, um die einzelnen Konsistenzprobleme zu lösen.

\section{Szenario 1: Strong Growth of First Generation Ethanol}

Obwohl das erste Szenario mit einem Mittelwert von 2,1 $1^{184}$ eine gute Konsistenz besitzt, werden im Folgenden zwei mögliche Konsistenzprobleme diskutiert: Als erstes die Konsistenz zwischen den beiden Kernpunkten [1.4] „Stricter GHG regulations for ethanol“ und [4.1] „Strong increase of the production of first generation ethanol up to around 13 billion liters" und anschließend die Konsistenz der Kernpunkte [1.3] „Political support for second generation biofuels“ und [4.2] „No significant production of cellulosic ethanol“.

Anlass für die Diskussion der ersten möglichen Inkonsistenz sind die im Folgenden genannten drei Kommentare der befragten Experten ${ }^{185}$; in diesen wird angemerkt, dass die Ethanolproduzenten der ersten Generation Schwierigkeiten haben werden, die strengeren Vorgaben der Treibhausgasreduktion zu erfüllen, wodurch es zu keinem starken Anstieg der Ethanolproduktion der ersten Generation kommen wird.

- "One point I would say, is that stricter GHG emissions enforced by the government may mean that domestic production would struggle to meet the necessary standards."

- "My only concern would be that stricter GHG regulations may mean that domestically they will be unable to support 13 billion liters of production."

- "Can first generation producers achieve the GHG reduction targets that have been set?"

Ein starkes Produktionswachstum für Ethanol der ersten Generation auf 13 Milliarden Liter und strengere $\mathrm{CO}_{2}$-Reduktionswerte für Ethanol können entgegen den vorgebrachten Einwänden durchaus als konsistent betrachtet werden. Die Ethanolproduzenten werden in der Lage sein, die im Szenario genannten $\mathrm{CO}_{2}{ }^{-}$

\footnotetext{
${ }^{184} 53$ Experten beurteilten die Konsistenz des ersten Szenarios, das einen Mittelwert von 2,1 und eine Standardabweichung von 0,64 besitzt.

${ }_{185}$ Zur besseren Lesbarkeit wurden die Kommentare der Experten sprachlich geringfügig korrigiert, inhaltlich blieben sie unverändert.
} 
Reduktionswerte ${ }^{186}$ für das Jahr 2023 zu erfüllen, da diese bereits in der RICHTLINIE 2009/28/EG (ARTIKEL 17 (2)) des Europäischen Parlaments und des Rates vom 23. April 2009 festgesetzt und aufgrund dessen beim Bau neuerer Anlagen berücksichtigt wurden. Ältere Anlagen können zur Verbesserung ihrer $\mathrm{CO}_{2}$-Bilanz nachgerüstet werden, beispielsweise durch den Anbau einer Biogasanlage, durch die effizientere Nutzung der Getreideschlempe als Nebenprodukt oder durch das Auffangen, das Reinigen und anschließende Verflüssigen von $\mathrm{CO}_{2}$, um es an die Getränkeindustrie zu verkaufen ${ }^{187}$.

Problematisch wäre ein starker Ausbau der Ethanolproduktion der ersten Generation, wenn ein ILUC-Faktor in der Berechnung der $\mathrm{CO}_{2}$-Bilanz bei der Herstellung von Ethanol berücksichtigt werden müsste. Das ist in diesem Zukunftsbild - im Gegensatz zu den Szenarien 3 und 4 - aber nicht der Fall.

Basierend auf den folgenden Kommentaren wird die Konsistenz zwischen den Kernpunkten [1.3] und [4.2] analysiert. Einige Experten erkennen eine mögliche Inkonsistenz darin, dass es trotz politischer Förderung für Biokraftstoffe der zweiten Generation keine bedeutende Produktion dieser Biokraftstoffe in der EU27 gibt.

- "How can political support for second generation biofuels result in no home production of second generation biofuels?"

- "If there is a strong political support for $2 \mathrm{G}$ ethanol which is translated in adequate measures, there will be a considerable production of cellulosic ethanol."

- "Are you sure that $1.3 \& 4.2$ do not contradict?"

Zwar werden Biokraftstoffe der zweiten Generation im Szenario politisch gefördert, indem ihr Energiegehalt dem 10\%-Ziel zweifach angerechnet wird, doch erfolgt dies bereits seit dem Jahr 2009 und bis jetzt gibt es in der EU27 nur eine sehr geringe Zellulose-Ethanolproduktion in Höhe von 0,029 Milliarden Litern (RICHTLINIE 2009/28/EG ARTIKEL 21 (2); OECD/FAO 2012: 224). Auch zukünftig wird in dem Szenario keine bedeutende Produktion von Zellulose-Ethanol erwartet, da die Produktionskosten zu hoch sind, um mit Biokraftstoffen der ersten Generation konkurrieren zu können. Eine politische Unterstützung für Biokraftstoffe der zweiten Generation muss nicht zu deren erhöhter Produktion führen. Auch in den USA besteht

\footnotetext{
${ }^{186}$ Ethanolproduzenten müssen im Jahr 2023 eine Reduzierung des $\mathrm{CO}_{2}$-Ausstoßes im Verhältnis zu Fossilbrennstoff von 50\% erlangen in Anlagen, die vor dem 1. Januar 2017 in Betrieb genommen wurden, sowie von $60 \%$ in Anlagen, die ab dem 1. Januar 2017 die Ethanolproduktion aufnehmen werden.

${ }_{187}$ In Kooperation mit dem Unternehmen Tyczka Energie GmbH veredelt die Crop Energies $A G$ an ihrem Standort in Zeitz Kohlenstoffdioxid, welches beispielsweise als Kohlensäure in der Getränkeindustrie oder als Kühlmittel verwendet wird (CROPENERGIES AG 2012: 2).
} 
seit 2007 eine starke politische Förderung der Produktion von Zellulose-Ethanol durch festgesetzte Verbrauchsmandate im RFS 2, doch auch diese haben noch zu keiner relevanten Produktion von Zellulose-Ethanol geführt (U.S. GOVERNMENT INFORMATION 2007: 121 STAT. 1519ff.) ${ }^{188}$.

Zusammenfassend ist daher festzuhalten, dass keine Inkonsistenz zwischen den beiden Kernpunkten besteht und das Szenario unverändert weiter betrachtet werden kann.

\section{Szenario 2: 5\% Cap for First Generation Ethanol}

Das zweite Szenario hat eine gute Konsistenz und besitzt mit einem Mittelwert von 2,0 $0^{189}$ nach Meinung der befragten Experten die beste Konsistenz der vier Szenarien. Da auch keine möglichen Inkonsistenzen von mehr als zwei Experten genannt wurden, bleibt das Szenario in dieser Form bestehen.

\section{Szenario 3: Growth of Cellulosic Ethanol}

Nach Einschätzung der 46 Probanden besitzt das dritte Szenario mit einem Mittelwert von 2,2 und einer Standardabweichung von 0,81 eine gute Konsistenz.

Im Gegensatz zum ersten Szenario, in dem zwei mögliche Konsistenzprobleme analysiert wurden, wird im Folgenden ausschließlich der Kernpunkt [4.3]: „No fuel ethanol imports" betrachtet, der von vier Experten kommentiert wurde. In den Kommentaren wird von den Experten angemerkt, dass ein Import von Zellulose-Ethanol, beispielsweise aus den USA oder Brasilien in die EU27, berücksichtigt werden sollte.

- "I think that we still will see imports for fuel ethanol from the USA or Brazil as they will probably also develop cellulosic ethanol."

- “... and why no parallel imports?"

- "Why are there no imports of ethanol if the tariff is kept unchanged and the price of oil rises significantly, encouraging blends of fuel ethanol with conventional fossil fuels?"

- "In this scenario, you would have more exports from Brazil."

Falls es im Jahr 2023 eine größere Produktion von Zellulose-Ethanol geben sollte, so findet diese infolge der starken politischen Förderung von „Advanced: Cellulosic

\footnotetext{
188 Die US-amerikanische Zellulose-Ethanolproduktion betrug im Durchschnitt der Jahre 2009 bis 2011 lediglich 0,027 Milliarden Liter (OECD/FAO 2012: 224).

${ }_{189}$ Das zweite Szenario wurde von 48 Experten des europäischen Ethanolmarktes beurteilt und besitzt einen Mittelwert von 2,0 und eine Standardabweichung von 0,79.
} 
Biofuel" durch den RFS $2^{190}$ in den USA statt (U.S. GOVERNMENT INFORMATION 2007: 121 STAT. 1521ff.). Dort werden derzeitig auch die weltweit ersten kommerziellen Anlagen zur Zellulose-Ethanolproduktion errichtet ${ }^{191}$. Es ist aber nicht zu erwarten, dass die USA zukünttig Zellulose-Ethanol in die EU27 exportieren werden, da sie selbst große Mengen davon benötigen, um die jährlich ansteigenden Verbrauchsmandate für „Advanced: Cellulosic Biofuel“ erfüllen zu können.

Ein Anstieg der Zellulose-Ethanolproduktion aus dem Rohstoff Bagasse ist in Brasilien möglich. Allerdings erfolgte bereits der Ausbau der Produktionskapazitäten zur Herstellung von Zuckerrohr-Ethanol in den letzten Jahren deutlich langsamer als ursprünglich gedacht (F.O. LICHT 2013d: 294f.). Kommt es doch zu einer Produktion, wird das Ethanol für den heimischen Bedarf genutzt, da dieser infolge der steigenden Zulassungen von FFV wachsen wird (F.O. LICHT 2009: 70). Darüber hinaus besteht noch der Einfluss der USA, die Zellulose-Ethanol aus Brasilien importieren werden, um ihr Verbrauchsmandat für „Advanced: Cellulosic Biofuel“ zu erfüllen. Diese Wirkung ist vergleichbar mit dem derzeitigen Import von Zuckerrohr-Ethanol, um das Verbrauchsmandat „Advanced: Unspecified Biofuel“ zu erfüllen (F.O. LICHT 2012h: 193).

Zusammenfassend ist festzuhalten, dass ein Import von geringen Mengen Zellulose-Ethanol aus Brasilien - nicht aus den USA - in die EU27 denkbar ist. Allerdings ist es ebenso realistisch, gar keinen Import von Zellulose-Ethanol in die EU27 in Betracht zu ziehen. Da dieses Szenario den Wandel des europäischen Ethanolmarktes von der ersten Generation zu einer erfolgreichen zweiten Generation mit starker heimischer Produktion darstellt, wurde auf die Annahme eines Imports von Zellulose-Ethanol bewusst verzichtet.

\section{Szenario 4: No Import Tariff}

46 Probanden haben das vierte Szenario beurteilt und es besitzt mit einem Mittelwert von 2,2 und einer Standardabweichung von 1 eine gute Konsistenz. Aufgrund dessen und da keine Inkonsistenzen innerhalb des Szenarios mehr als zwei Mal genannt wurden, bleibt das Szenario unverändert.

\footnotetext{
${ }^{190}$ Der RFS 2 gibt jährliche Mandate für Biokraftstoffe vor, die im US-amerikanischen Transportbereich verbraucht werden müssen. Das Verbrauchsmandat für „Advanced: Cellulosic Biofuel" steigt seit dem Jahr 2010 kontinuierlich an und wird nach derzeitiger politischer Gesetzgebung bis auf 61 Milliarden Liter im Jahr 2022 ansteigen (U.S. GOVERNMENT INFORMATION 2007: 121 STAT. 1522). Eine genauere Beschreibung des RFS 2 befindet sich in Kapitel 6.2.5.

${ }^{191}$ In Kapitel 2.2 sind die Anlagen beschrieben.
} 


\subsection{Ergebnisse der Betrachtung des Ethanolmarktes der EU27}

Die zwei in der Einleitung (Kapitel 1.1) definierten Ziele der Studie wurden durch die optimierte Methodik der induktiven Szenario-Technik ${ }^{192}$ erfüllt.

Wie bereits in der Vergangenheit werden auch bis zum Jahr 2023 die Faktoren des Einflussbereichs Politik den Ethanolmarkt der EU27 entscheidend beeinflussen ${ }^{193}$. Die politischen Faktoren lassen sich mit Ausnahme des Faktors 22: „Zölle auf Ethanolimporte in die EU27“ zu den beiden Themen „Politische Förderung von Ethanol“ 194 und „Politische Vorgaben der Treibhausgasreduktion für Ethanol“ ${ }^{195}$ zusammenfassen. Insbesondere das Thema „Politische Förderung von Ethanol“, das alle drei Faktoren mit der stärksten Wirkung auf den europäischen Ethanolmarkt vereint, gilt es zu betrachten, um frühzeitig zu erkennen, zu welchem der folgenden Szenarien hin sich der Ethanolmarkt der EU27 entwickeln wird. Der einzige nicht politisch wirkende Einflussfaktor von vergleichbarer Relevanz ist der ökonomische Faktor 9b, der die Zukunft der „Rohstoffkosten der Ethanolproduktion der ersten Generation" betrachtet. Im Folgenden werden die vier Szenarien ${ }^{196}$, die den Ethanolmarkt der EU27 im Jahr 2023 darstellen, anhand ihrer Kernpunkte beschrieben und das spezifische der einzelnen Szenarien herausgestellt, um sie eindeutig voneinander abzugrenzen.

\section{Szenario 1: Strong Growth of First Generation Ethanol \\ Beschreibung:}

In diesem Szenario wird ein weiterhin starker Anstieg der Ethanolnachfrage (14,5 Milliarden Liter) und der Ethanolproduktion (13 Milliarden Liter) sowie ein konstanter Ethanolimport von 1,5 Milliarden Litern erwartet. Der Treiber dieses Wachstums ist das politische Ziel, dass 10\% der eingesetzten Energie im Verkehrssektor aus erneuerbaren Energiequellen bezogen werden sollen. Es kommt zu keiner Begrenzung der Anrechnung von Biokraftstoffen der ersten Generation auf das 10\%-Ziel.

\footnotetext{
${ }^{192}$ Die optimierte Methodik der induktiven Szenario-Technik ist ausführlich in Kapitel 4 beschrieben.

${ }^{193}$ Eine genaue Beschreibung der Stärke der Wirkung der einzelnen Faktoren auf den Ethanolmarkt der EU27 bis zum Jahr 2023 befindet sich in Kapitel 5.1.2.2.

${ }^{194}$ Das Thema „Politische Förderung von Ethanol“ besteht aus den wirkenden Einflussfaktoren 19b: „Die maximal erlaubte prozentuale Beimischung von Ethanol zu Benzin“, 18a: „Der politisch geforderte prozentuale Anteil von erneuerbaren Energien am Gesamtenergieverbrauch im Verkehrsbereich“ und 20: „Bestimmungen der Mitgliedsstaaten der EU27 zur Förderung von Ethanol-Kraftstoff".

${ }^{195}$ Die einzelnen wirkenden Einflussfaktoren 18b: „Die politisch geforderte Mindesteinsparung von Kohlenstoffdioxid bei Biokraftstoffen im Verhältnis zu fossilen Kraftstoffen“, 21: „Bestimmungen der EU27 betreffend ILUC (Indirect Land Use Change)“ und 19a: „Vorgabe der EU27 zur Kohlenstoffdioxideinsparung von Kraftstoffen im Verkehrsbereich“ werden zu dem Thema „Politische Vorgaben der Treibhausgasreduktion für Ethanol“ zusammengefasst. ${ }^{196}$ In Kapitel 5.3.1 sind die vier Szenarien ausführlich beschrieben.
} 
Darüber hinaus wird auch auf die Berücksichtigung eines ILUC-Faktors in der Berechnung der $\mathrm{CO}_{2}$-Emissionen zur Herstellung von Ethanol verzichtet, wodurch die Ethanolproduzenten die politischen Vorgaben hinsichtlich der Treibhausgasreduktion im Verhältnis zu Fossilbrennstoff erfüllen. Die hohe Nachfrage nach Ethanol ist trotz des Rückgangs der Benzinnachfrage möglich, da auch die Automobilindustrie Kraftfahrzeuge verkauft, die E20 als Ottokraftstoff nutzen können.

\section{Abgrenzung:}

In diesem Szenario wird ein zukünftig starkes Wachstum des europäischen Ethanolmarktes der ersten Generation beschrieben. Dieses Wachstum ist vergleichbar mit jenem des Zeitraums von 2003 bis 2010, wodurch die Jahre 2011 und 2012, in denen der Ethanolmarkt deutlich langsamer gewachsen ist, eine Ausnahme darstellen. Ein Wandel des Biokraftstoffmarktes von der ersten Generation zur zweiten Generation, wie es derzeitig von Energiekommissar Günther Oettinger und Klimakommissarin Connie Hedegard gefordert wird, findet nicht statt. Stattdessen kommt es zu einem politischen Umdenken und der Bereitschaft, weiterhin Biokraftstoffe der ersten Generation zu fördern. Die politische Unterstützung überzeugt die Ethanolindustrie, wieder in neue Anlagen zu investieren, um die Produktionskapazitäten in der EU27 von derzeitig 8 Milliarden Litern (USDA FOREIGN AGRICULTURAL SERVICE 2012: 11) auf über 13 Milliarden Liter auszubauen.

\section{Szenario 2: 5\% Cap for First Generation Ethanol \\ Beschreibung:}

Im zweiten Szenario ist die europäische Ethanolnachfrage auf 7,5 Milliarden Liter und die Ethanolproduktion auf 6 Milliarden Liter angestiegen, bei einem konstanten Ethanolimport von 1,5 Milliarden Litern. Trotz des politischen Ziels der Nutzung von $10 \%$ erneuerbarer Energie im Verkehrssektor ist die europäische Ethanolindustrie deutlich langsamer gewachsen als im Zeitraum von 2003 bis 2010. Grund dafür ist die politische Entscheidung der 5-prozentigen Begrenzung der Anrechnung von Biokraftstoffen der ersten Generation auf das 10\%-Ziel. Die politischen Vorgaben bezüglich der $\mathrm{CO}_{2}$-Einsparungen im Vergleich zu Fossilbrennstoff werden von den Ethanolproduzenten der ersten und zweiten Generation erfüllt, da kein ILUC-Faktor berücksichtigt wird. Trotz der starken politischen Förderung von Zellulose-Ethanol durch die vierfache Anrechnung des Energiegehalts auf das 10\%-Ziel ist die Produktion von Zellulose-Ethanol aufgrund zu hoher Herstellungskosten und fehlender Produktionskapazitäten gering. 


\section{Abgrenzung:}

Zwar wird ein Wachstum des europäischen Ethanolmarktes der ersten Generation bis zum Jahr 2023 prognostiziert, doch fällt die Höhe dieses Wachstums deutlich geringer aus als in der Vergangenheit. Grund dafür ist das politische Ziel, einen Wandel des europäischen Biokraftstoffmarktes von der ersten Generation zur zweiten Generation zu erreichen. Dies ist an den politischen Entscheidungen zu erkennen, sowohl die Anrechnung von Biokraftstoffen der ersten Generation auf das 10\%Ziel zu begrenzen als auch Biokraftstoffe der zweiten Generation durch die vierfache Anrechnung ihres Energiegehalts auf das 10\%-Ziel stark zu fördern. Obwohl die politischen Voraussetzungen zum Wandel damit gesetzt sind, kommt es trotzdem nicht zu der erwünschten Veränderung des Ethanolmarktes, sondern zum Stillstand. Dies wird zum einen durch die politische Entscheidung der Beschränkung der Ethanolnachfrage der ersten Generation und zum anderen durch die hohen Herstellungskosten und fehlenden Produktionskapazitäten, die ein Wachstum der Ethanolproduktion der zweiten Generation in der EU27 verhindern, bewirkt.

\section{Szenario 3: Growth of Cellulosic Ethanol}

\section{Beschreibung:}

Es werden ausschließlich Biokraftstoffe der zweiten Generation politisch gefördert, indem ihr Energiegehalt dem politischen Ziel der Nutzung von 10\% erneuerbarer Energie im Verkehrssektor mehrfach angerechnet wird. Infolgedessen kommt es zu einem Einbruch des Ethanolmarktes der ersten Generation, aber zu einem starken Anstieg der Zellulose-Ethanolnachfrage auf drei Milliarden Liter, welche vollständig durch die europäische Produktion gedeckt wird. Aufgrund der mehrfachen Anrechnung des Energiegehalts von Zellulose-Ethanol auf das 10\%-Ziel ist eine Ethanolnachfrage von 3 Milliarden Litern ausreichend, um das Ziel zu erreichen. Darüber hinaus erfüllt Zellulose-Ethanol, trotz der Berücksichtigung eines ILUC-Faktors bei der Ermittlung der $\mathrm{CO}_{2}$-Emissionen bei der Produktion von Ethanol, die strengeren politischen Anforderungen hinsichtlich der $\mathrm{CO}_{2}$-Reduktion von Biokraftstoffen im Vergleich zu Fossilbrennstoff.

\section{Abgrenzung:}

In diesem Szenario hat ein erfolgreicher Wandel des europäischen Ethanolmarktes von der ersten zur zweiten Generation stattgefunden. Dies ist auf die starke politische Förderung von Biokraftstoffen der zweiten Generation zurückzuführen, indem der Energiegehalt von Zellulose-Ethanol dem 10\%-Ziel mehrfach angerechnet wird und Ethanol der ersten Generation keine politische Förderung mehr erfährt. Zu er- 
klären ist dies außerdem durch den technischen Fortschritt bei der Produktion von Zellulose-Ethanol. Im Vergleich zu den ersten beiden beschreibt dieses Szenario den technisch am weitesten entwickelten Ethanolmarkt, da es eine erfolgreiche kommerzielle Produktion von Zellulose-Ethanol in der EU27 gibt.

\section{Szenario 4: No Import Tariff}

Beschreibung:

Basierend auf der politischen Forderung, dass 10\% der eingesetzten Energie im Verkehrssektor aus erneuerbaren Energiequellen produziert werden sollen, steigt die Ethanolnachfrage auf 7,5 Milliarden Liter an. Die Importzölle für Ethanol werden aufgehoben und Brasilien nutzt diese Situation und exportiert 4,5 Milliarden Liter Zuckerrohr-Ethanol in die EU27. Brasilianisches Zuckerrohr-Ethanol besitzt eine sehr gute $\mathrm{CO}_{2}$-Bilanz und erfüllt die strengeren politischen Vorgaben der Treibhausgasreduktion, die auch eine Berücksichtigung des ILUC-Faktors bei der Berechnung der $\mathrm{CO}_{2}$-Emissionen bei der Herstellung von Ethanol-Kraftstoff fordern. Ethanol der ersten Generation, welches in der EU27 produziert wird, ist nicht in der Lage, mit den brasilianischen Ethanolimporten zu konkurrieren, da es die politischen Vorgaben der $\mathrm{CO}_{2}$-Reduktion im Gegensatz zur europäischen ZelluloseEthanolproduktion nicht erfült. Diese wiederum steigt infolge der politischen Förderung durch die mehrfache Anrechnung ihres Energiegehalts auf das 10\%-Ziel auf 3 Milliarden Liter an. Die Produktion von Zellulose-Ethanol in der EU27 ist konkurrenzfähig zu den Ethanolimporten aus Brasilien, da für Zellulose-Ethanol aufgrund der mehrfachen Anrechnung des Energiegehalts des Biokraftstoffs auf das 10\%-Ziel ein höherer Marktpreis bezahlt wird.

\section{Abgrenzung:}

Das Szenario unterscheidet sich von den ersten drei Szenarien, da sich die politische Förderung von Biokraftstoffen ausschließlich am Ziel der maximalen Treibhausgasreduktion orientiert. Aufgrund dessen wird (1) der Außenschutz der EU27 aufgehoben, um den Ethanol-Kraftstoff mit der höchsten $\mathrm{CO}_{2}$-Reduktion unabhängig von seiner Herkunft in der EU27 nutzen zu können. (2) Wird ein ILUC-Faktor in der Berechnung der $\mathrm{CO}_{2}$-Emissionen zur Herstellung von Ethanol berücksichtigt und (3) die politisch geforderte prozentuale Treibhausgasreduktion im Vergleich zu fossilem Kraftstoff erhöht. Außerdem werden (4) Biokraftstoffe der zweiten Generation durch die mehrfache Anrechnung des Energiegehalts auf das 10\%-Ziel gefördert. Infolgedessen kommt es zu einem hohen Import von Zuckerrohr-Ethanol aus Brasilien sowie zu einem Wandel der europäischen Ethanolproduktion der ersten Generation 
hin zur kommerziellen Produktion von Zellulose-Ethanol. Von allen vier Szenarien beschreibt dieses den politisch fortschrittlichsten europäischen Ethanolmarkt, da die EU27 den internationalen Handel nicht durch Importzölle beschränkt.

Die vier Szenarien bilden einen breiten Zukunftsraum ab, in dem sowohl ein starkes Wachstum („Strong Growth of First Generation Ethanol“) als auch ein Schrumpfen („Growth of Celulosic Ethanol“) des Ethanolmarktes in der EU27 prognostiziert wird. Darüber hinaus stellen die Szenarien: „Strong Growth of First Generation Ethanol“, „5\% Cap for First Generation Ethanol“ und „No Import Tariff“ die EU27 als Ethanolimporteur dar, wohingegen das Szenario "Growth of Cellulosic Ethanol“ weder Importe noch Exporte von Ethanol annimmt (Abbildung 58).

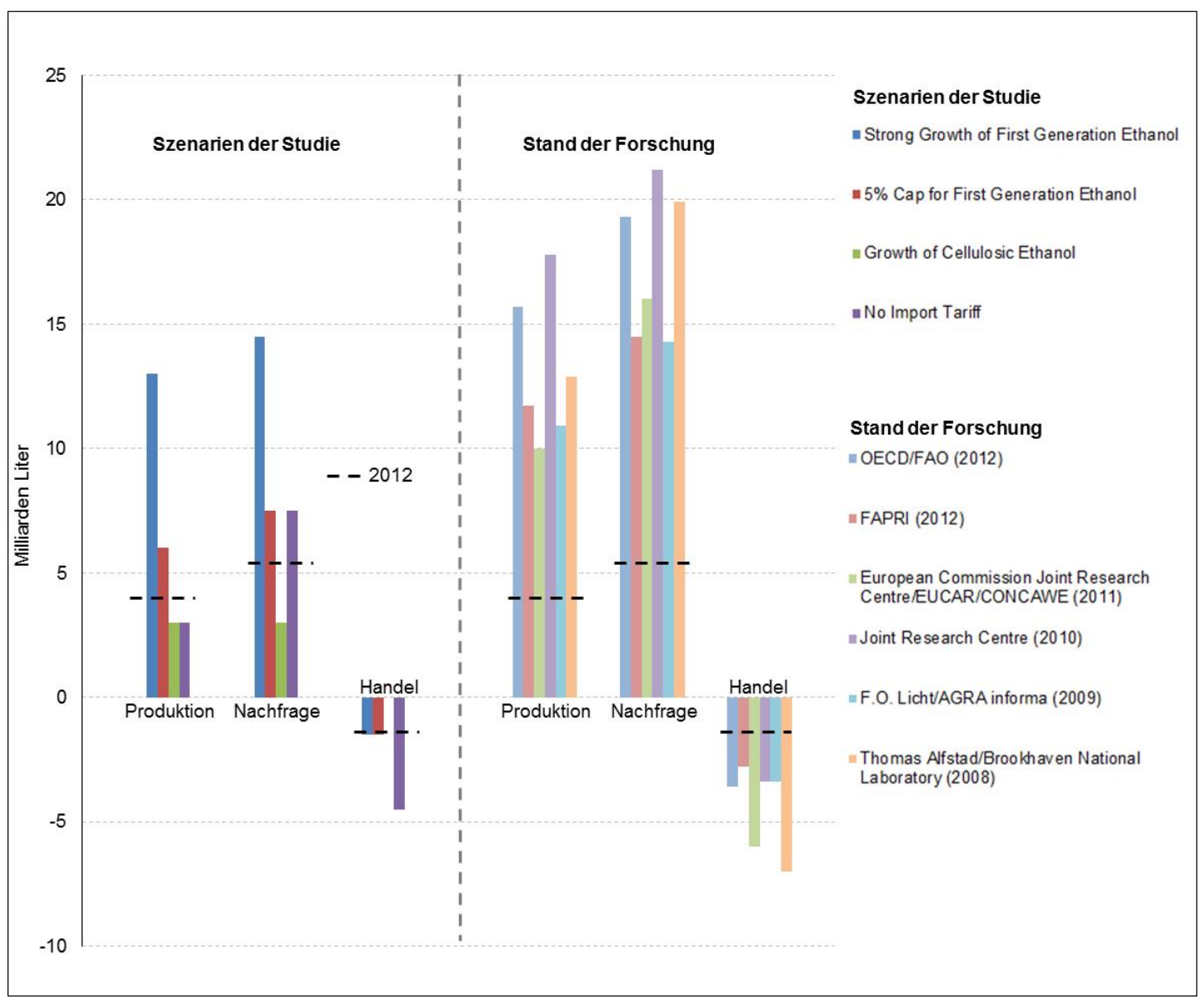

Abbildung 58: EU27: Vergleich der Szenarien der Studie mit dem Stand der Forschung Quelle: In Anlehnung an OECD/FAO 2012: 87ff.; FAPRI 2012: 1ff.; EUROPEAN COMMISSION JOINT RESEARCH CENTRE, INSTITUT FOR ENERGY ET AL. 2011: 5ff.; EUROPEAN COMMISSION JOINT RESEARCH CENTRE, INSTITUT FOR PROSPECTIVE TECHNOLOGICAL STUDIES ET AL. 2010a: 9ff.; F.O. LICHT/AGRA INFORMA 2009: 133ff.; ALFSTAD 2008: $1 \mathrm{ff}$.

Im Vergleich zum Stand der Forschung wird bei der Betrachtung des Zukunftsraums dieser Studie (Abbildung 58) ersichtlich, dass die Prognosen der Forschungsinstitute, die allesamt einen starken Anstieg des Ethanolmarktes der EU27 voraussagen, 
nicht ausreichend sind, um die unterschiedlichen Entwicklungsmöglichkeiten des Ethanolmarktes der EU27 bis zum Jahr 2023 darzustellen. Das erste Szenario „Strong Growth of First Generation Ethanol“ (Ethanolnachfrage: 14,5 Milliarden Liter; Ethanolproduktion: 13 Milliarden Liter; Ethanolhandel: 1,5 Milliarden Liter) entspricht in etwa den Prognosen, die im Stand der Forschung beschrieben wurden. Die anderen drei Szenarien „5\% Cap for First Generation Ethanol“, „Growth of Cellulosic Ethanol” und „No Import Tariff” beschreiben alternative Entwicklungen des Ethanolmarktes, die bisher noch nicht betrachtet wurden. Damit Unternehmen sich auf alle realistischen Entwicklungsmöglichkeiten ausreichend vorbereiten können, ist es unabdingbar, auch diese drei Szenarien in dem Prozess der strategischen Planung zu berücksichtigen. Dies ist besonders deshalb von Relevanz, da sich die Abgeordneten des Europaparlaments im September 2013 entschieden haben, der Neuausrichtung zur Förderung von Biokraftstoffen in der EU27, wie sie von den Kommissaren Günther Oettinger und Connie Hedegard vorgeschlagen wurde, in den Kernpunkten zu folgen (F.O. LICHT 2013h: 83; F.O. LICHT 2013g: 62; AGRA-EUROPE 2013a: EU-NACHRICHTEN 1). 
Bei der Betrachtung des US-amerikanischen Ethanolmarktes werden (1) die Faktoren identifiziert, die den Ethanolmarkt bis zum Jahr 2023 essentiell beeinflussen, und (2) wird der Markt im Jahr 2023 mittels unterschiedlicher Szenarien ${ }^{197}$ beschrieben. Um die beiden Ziele zu erreichen, kommt die in Kapitel 4 beschriebene Methodik der induktiven Szenario-Technik ${ }^{198}$ zur Anwendung, die sich in die drei Kernschritte „USA Szenariofeld-Analyse“ (Kapitel 6.1), „USA Szenario-Prognostik“ (Kapitel 6.2) und „USA Szenario-Bildung und -Konsistenzprüfung“ (Kapitel 6.3) unterteilt. Anschließend werden die Ergebnisse der Untersuchung in Kapitel 6.4 betrachtet. Die folgende Abbildung 59 stellt die einzelnen Schritte der Untersuchung dar.

\footnotetext{
${ }^{197}$ Wie in Kapitel 4.1 dargestellt, entspricht die formelle Ausrichtung der Szenarien den Vorgaben für Marktentwicklungsszenarien.

${ }_{198} \mathrm{Da}$ die in Kapitel 4 dargestellte induktive Szenario-Technik sowohl in Kapitel 5 „Ethanolmarkt der EU27 im Jahr 2023“ als auch in Kapitel 6 "Ethanolmarkt der USA im Jahr 2023“ zur Anwendung kommt, sind die beiden Kapitel vom Aufbau her identisch.
} 


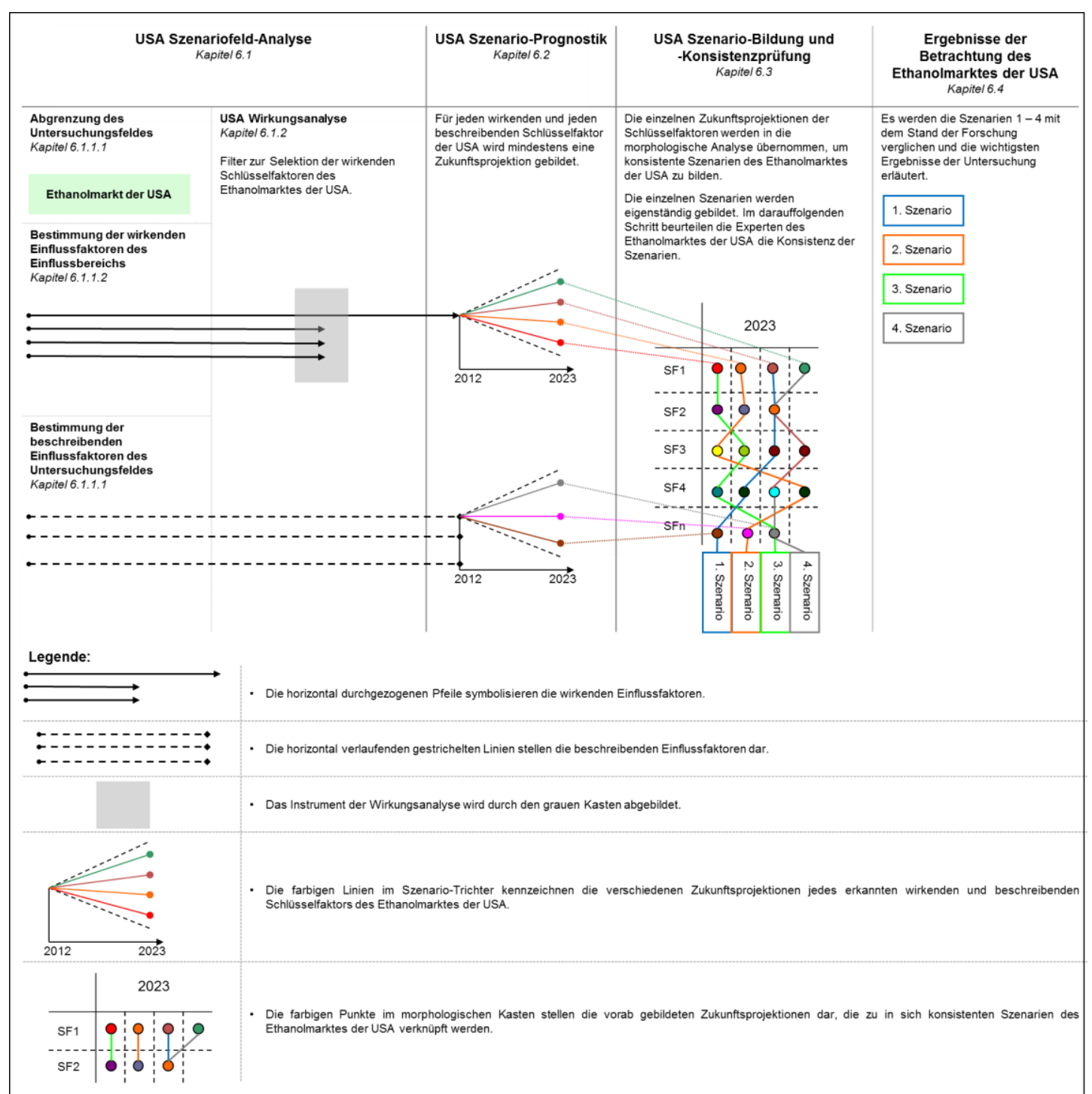

Abbildung 59: Schematische Darstellung - Vorgehen zur Bildung der Szenarien des Ethanolmarktes der USA im Jahr 2023

Quelle: EIGENE DARSTELLUNG

\subsection{USA Szenariofeld-Analyse}

Der erste Kernschritt der Szenario-Technik dient der Bestimmung der Faktoren, die den US-amerikanischen Ethanolmarkt bis zum Jahr 2023 maßgeblich beeinflussen werden. Somit erfüllt die Szenariofeld-Analyse das erste Ziel der Dissertation ${ }^{199}$ und schafft durch die Bestimmung der wirkenden und beschreibenden Schlüsselfaktoren die Voraussetzungen zur Durchführung der „USA Szenario-Prognostik“ (Kapitel 6.2). Die Szenariofeld-Analyse unterteilt sich in die beiden Schritte: (1) Beschreibung des USA-Szenariofeldes in Kapitel 6.1.1 und (2) USA Wirkungsanalyse in Kapitel 6.1.2.

199 Das erste Ziel der Studie besteht darin, die Faktoren zu identifizieren, die den Ethanolmarkt der USA bis zum Jahr 2023 entscheidend beeinflussen werden (Kapitel 1.1). 


\subsubsection{Beschreibung des USA-Szenariofeldes}

Das USA-Szenariofeld setzt sich zusammen aus dem Untersuchungsfeld „Ethanolmarkt der USA“ - und den vier Einflussbereichen Ökonomie, Technologie, Politik und Gesellschaft. Im folgenden Kapitel 6.1.1.1 erfolgt sowohl die inhaltliche/geografische Abgrenzung als auch die Darstellung des Untersuchungsfeldes mittels beschreibender Einflussfaktoren. Anschließend werden in Kapitel 6.1.1.2 die vier Einflussbereiche anhand wirkender Einflussfaktoren beschrieben.

\subsubsection{Abgrenzung des Untersuchungsfeldes und Bestimmung der be- schreibenden Einflussfaktoren}

Im Untersuchungsfeld wird ausschließlich der US-amerikanische EthanolKraftstoffmarkt betrachtet. Folglich sind weder die Märkte anderer Biokraftstoffe, wie beispielsweise Biodiesel, noch die Produktion, die Verwendung und der Handel mit Ethanol in der chemischen Industrie sowie in der Nahrungsmittelindustrie Bestandteile des Untersuchungsfeldes. Ethanolmärkte außerhalb der USA gehören dem Untersuchungsfeld ebenfalls nicht an. Eine ausführliche Beschreibung der historischen Entwicklung und der Ist-Situation des Untersuchungsfeldes „Ethanolmarkt der USA“ befindet sich in Kapitel 2.2.

Um die Entwicklung des Untersuchungsfeldes in den unterschiedlichen Szenarien aufzuzeigen und mit dem Stand der Forschung (Kapitel 2.3) zu vergleichen, werden die folgenden vier beschreibenden Einflussfaktoren „Ethanolnachfrage“, „Ethanolproduktion“, „Ethanolhandel“ und „Ethanolpreis“200 definiert. Die Darstellung jedes Faktors erfolgt mittels der drei Punkte: „Name“, „Lfd. Nr“ und „Beschreibung“. Der Punkt "Beschreibung“ ist von besonderer Bedeutung, da dieser eine Erklärung des Einflussfaktors umfasst und angibt, mittels welcher Messgröße die Entwicklung des Faktors dargestellt wird.

\begin{tabular}{l|l|}
\hline Name: & Ethanolnachfrage \\
\hline Lfd. Nr:: & I \\
\hline Beschreibung: & $\begin{array}{l}\text { Der Einflussfaktor misst die Ethanolnachfrage für den Kraftstoffbereich in den } \\
\text { USA. Die nachgefragte Menge wird in Milliarden Litern pro Jahr angegeben. }\end{array}$ \\
\hline
\end{tabular}

${ }^{200}$ Die Auswahl der beschreibenden Einflussfaktoren wurde mit den folgenden Experten diskutiert: Dr. Christoph Berg - F.O. Licht; Dr. Marco Veselka - CropEnergies AG; Prof. Dr.Ing. Achim Loewen - Hochschule für angewandte Wissenschaft und Kunst in Göttingen, Dr. Hinrich Harling und Dr. Andreas von Felde - KWS SAAT AG. 


\begin{tabular}{|c|c|c|}
\hline Name: & Ethanolproduktion & \\
\hline Lfd. Nr.: & II & \\
\hline Beschreibung: & \multicolumn{2}{|c|}{$\begin{array}{l}\text { Der Einflussfaktor erfasst die Ethanolproduktion für den Kraftstoffbereich in den } \\
\text { USA. Die Produktionsmenge wird in der Einheit Milliarden Liter pro Jahr ge- } \\
\text { messen. }\end{array}$} \\
\hline Name: & Ethanolhandel & \\
\hline Lfd. Nr.: & III & \\
\hline Beschreibung: & \multicolumn{2}{|c|}{$\begin{array}{l}\text { Der Einflussfaktor betrachtet den Ethanolhandel der USA mit dem Rest der } \\
\text { Welt. Die Werte werden in der Einheit Milliarden Liter pro Jahr angegeben. }\end{array}$} \\
\hline Name: & Ethanolpreis & \\
\hline Lfd. Nr.: & IV & \\
\hline Beschreibung: & \multicolumn{2}{|c|}{$\begin{array}{l}\text { Der Einflussfaktor beschreibt die Entwicklung des US-amerikanischen Ethanol- } \\
\text { preises in der Einheit US-\$ je Liter. }\end{array}$} \\
\hline
\end{tabular}

\subsubsection{Bestimmung der wirkenden Einflussfaktoren}

Jeder wirkende Einflussfaktor ist durch seinen Einfluss auf die Entwicklung des Untersuchungsfeldes „Ethanolmarkt der USA“ gekennzeichnet. Die wirkenden Einflussfaktoren beschreiben die vier Einflussbereiche Ökonomie, Technologie, Politik und Gesellschaft und werden anhand der fünf Punkte „Name“, „Lfd. Nr.“, „Einflussbereich“, „Beschreibung“ und „Bedeutung des Einflussfaktors“ einzeln erläutert. Von besonderer Relevanz ist der Punkt „Bedeutung des Einflussfaktors“, da er die Notwendigkeit der Betrachtung des Faktors erklärt, indem beschrieben wird, wie der wirkende Einflussfaktor das Untersuchungsfeld beeinflusst.

Die Bestimmung der 29 wirkenden Einflussfaktoren stützt sich auf fünf persönliche Expertengespräche ${ }^{201}$ sowie insbesondere auf eine intensive Literaturrecherche unter Berücksichtigung der genannten Einflussfaktoren, die in den Studien zum Stand der Forschung referiert wurden (Anhang: 1). Die wirkenden Einflussfaktoren sind im Folgenden entsprechend ihrer Zugehörigkeit zu einem Einflussbereich aufgelistet.

${ }^{201}$ Die Auswahl der wirkenden Einflussfaktoren konnte mit den folgenden Experten des USamerikanischen Ethanolmarktes besprochen werden: Dr. Christoph Berg - F.O. Licht; Dr. Marco Veselka - CropEnergies AG; Prof. Dr.-Ing. Achim Loewen - Hochschule für angewandte Wissenschaft und Kunst in Göttingen, Dr. Hinrich Harling und Dr. Andreas von Felde - KWS SAAT AG. 


\section{Ökonomie}

Name:

Lfd. Nr.:

Einflussbereich:

Beschreibung:

Bedeutung des

Einflussfaktors:
Benzinnachfrage in den USA

1

\section{Ökonomie}

Die Benzinnachfrage in den USA wird in der Einheit Milliarden Liter pro Jahr beschrieben.

Über 99\% des US-amerikanischen Ethanolabsatzes erfolgt als 10-prozentige Beimischung zu Benzin (U.S. ENERGY INFORMATION ADMINISTRATION 2012j: 29). Demzufolge entscheiden die US-amerikanische Benzinnachfrage und die Höhe der erlaubten Beimischung von Ethanol zu Benzin über die potenzielle Größe des Ethanolmarktes.

Der Faktor ist von besonderer Relevanz, da sich die USA seit dem Jahr 2011 an der E10-„Blend Wall“202 befinden (F.O. LICHT 2013b: 213). Aufgrund der fehlenden Infrastruktur an den Tankstellen (Einflussfaktor 8) ist eine prozentuale Erhöhung der Ethanolbeimischung auf $15 \%$ vol momentan nicht möglich (F.O. LICHT 2013c: 341), wodurch ein weiteres Wachstum des Ethanolmarktes nur durch einen Anstieg der Benzinnachfrage zu erreichen ist. Dies ist allerdings nicht zu erwarten. So prognostiziert die U.S. ENERGY INFORMATION ADMINISTRATION (2013a: 84) einen langfristigen Rückgang der USamerikanischen Benzinnachfrage (Abbildung 60).

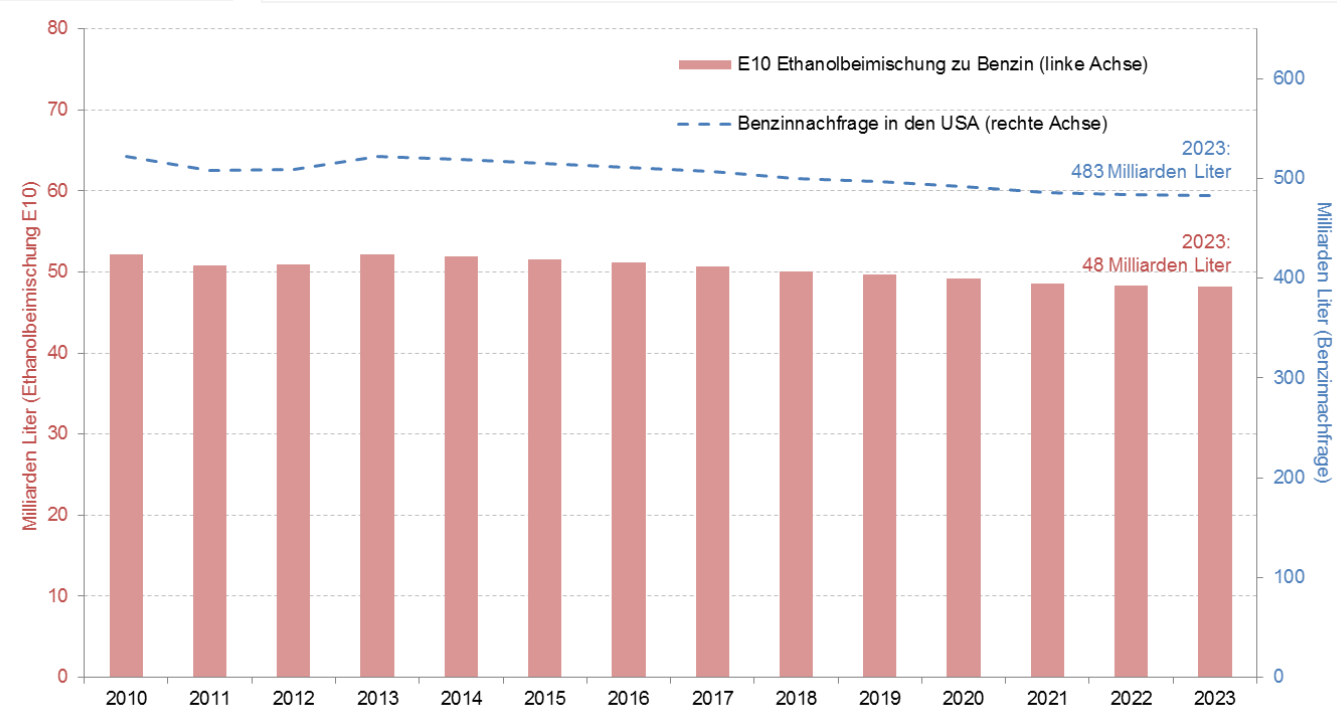

Abbildung 60: Einfluss der Benzinnachfrage auf die Ethanolnachfrage in den USA Quelle: In Anlehnung an U.S. ENERGY INFORMATION ADMINISTRATION 2012b: 98; EIGENE BERECHNUNG

Durch den erwarteten Rückgang der Benzinnachfrage sinkt auch die Höhe der

${ }^{202}$ Die „Blend Wall“ ist die maximale Menge Ethanol, die dem US-amerikanischen Benzin beigemischt werden kann (CONGRESSIONAL RESEARCH SERVICE 2010a: 5; OECD/FAO 2012: 110). Eine genauere Beschreibung der „Blend Wall“-Problematik befindet sich in Kapitel 2.2. 
möglichen Ethanolnachfrage von 51 Milliarden Litern im Jahr 2012 auf 48 Milliarden Liter im Jahr $2023^{203}$ (Abbildung 60). Diese Entwicklung widerspricht den Zielen des Renewable Fuel Standard (RFS 2) (Einflussfaktor 29), in dem ein starkes Wachstum der Verbrauchsmandate für Biokraftstoffe bis zum Jahr 2022 vorgegeben ist (U.S. GOVERNMENT INFORMATION 2007: 121 STAT. 1522).

Name:

Lfd. Nr.:

Einflussbereich:

Beschreibung:

Bedeutung des Einflussfaktors:
Rohölpreis in den USA

2

\section{Ökonomie}

Der Rohölpreis wird in realen Werten (Basisjahr 2010) in der Einheit US-\$ je Barrel angegeben. Die Entwicklung des Rohölpreises wird anhand der Rohölsorte „West Texas Intermediate“ (WTI) dargestellt.

Für die derzeitige Entwicklung des US-amerikanischen Ethanolmarktes ist die Höhe des Rohölpreises von zweitrangiger Bedeutung. Dies liegt darin begründet, dass die US-amerikanische Mineralölindustrie unabhängig von der Höhe des Rohölpreises dem Benzin Ethanol beimischt, um die im RFS 2 (Einflussfaktor 29) festgelegten Verbrauchsmandate für Biokraftstoffe zu erfüllen.

Politische Vorgaben wie der RFS 2 sind jedoch abhängig von dem Ausgang von Wahlen und den sich ändernden Einstellungen von Politikern und besitzen damit nicht die dauerhafte Gültigkeit wie die Gesetze des Marktes (HENNIGES 2007: 243). Ohne den Einfluss der Politik ist Ethanol ein Substitut von Benzin und konkurriert mit diesem auf dem US-amerikanischen Kraftstoffmarkt ${ }^{204}$ (F.O. LICHT/AGRA INFORMA 2009: 21; HENNIGES 2007: 36). Da die Höhe des Benzinpreises stark mit dem Rohölpreis korreliert (IER ET AL. 2010: 54), entscheidet der Rohölpreis maßgeblich über die Konkurrenzfähigkeit von Ethanol gegenüber Benzin. Hierbei ist allerdings zu beachten, dass aufgrund der im Vergleich zu Benzin (32,1 MJ/L) geringeren Energiedichte von Ethanol (21,2 $\mathrm{MJ} / \mathrm{L}$ ) der Preis für Ethanol 34\% günstiger sein muss, damit die Kosten je Energieeinheit identisch sind (MASSACHUSETTS INSTITUTE OF TECHNOLOGY 2007: 1).

Des Weiteren ist der Einflussfaktor relevant, da ein hoher Rohölpreis die Akzeptanz der politischen Förderung von Biokraftstoffen durch die US-amerikanische Bevölkerung erhöht, um die Abhängigkeit von Rohölimporten zu verringern (USDA 2008: 31). So kam es auch in der Vergangenheit immer dann zu einer

${ }^{203}$ Die Berechnung basiert auf der Annahme, dass dem US-amerikanischen Benzin weiterhin ausschließlich $10 \%$ vol Ethanol beigemischt werden kann.

${ }^{204} \mathrm{Da}$ Ethanol-Kraftstoff dem Benzin beigemischt wird, ist die Substitution von Benzin nur bis zu einer bestimmten Menge möglich. Sie ist abhängig von der Entwicklung der Ethanolverträglichkeit der Kraftfahrzeuge (Einflussfaktor 15), der von der EPA genehmigten prozentualen Höhe der Beimischung von Ethanol zu Benzin (Einflussfaktor 24) und der Fähigkeit der Tankstellen, Benzin mit mittleren Ethanolbeimischungen zu verkaufen (Einflussfaktor 8). 
starken politischen Unterstützung von Biokraftstoffen und zum Bau von Anlagen zur Produktion von Biokraftstoffen, wenn die Rohölpreise stark angestiegen sind, wie beispielsweise in den 1970er Jahren und im Jahr 2008 (INTERNATIONAL ENERGY AGENCY 2010a: 358; HENNIGES 2007: 70).

Auf der anderen Seite könnte ein niedriger Rohölpreis die politische Förderung von Biokraftstoffen unter Druck setzen. Da sich die Bürger der USA darüber beschweren könnten, dass sie infolge des politisch geforderten Verbrauchs von Bio-kraftstoffen höhere Preise an den Zapfsäulen bezahlen müssen.

Name: Ethanolimporte aus Brasilien in die USA

Lfd. Nr.: 3

Einflussbereich:

Ökonomie

Beschreibung:

Der Faktor betrachtet die Ethanolimporte aus Brasilien in die USA und die Entwicklung des Faktors wird in der Einheit Milliarden Liter pro Jahr dargestellt.

Bedeutung des Der brasilianische Ethanolmarkt ist im Jahr 2012 mit einer Ethanolproduktion Einflussfaktors: von 20,5 Milliarden Litern und einem Exportvolumen von 2,3 Milliarden Litern hinter den USA - der zweitgrößte Ethanolmarkt der Welt (F.O. LICHT 2013f: 387; F.O. LICHT 2012f: 72; BERG 2012C: 179). In der folgenden Abbildung $61^{205}$ ist die Entwicklung der brasilianischen Ethanolimporte in die USA dargestellt.

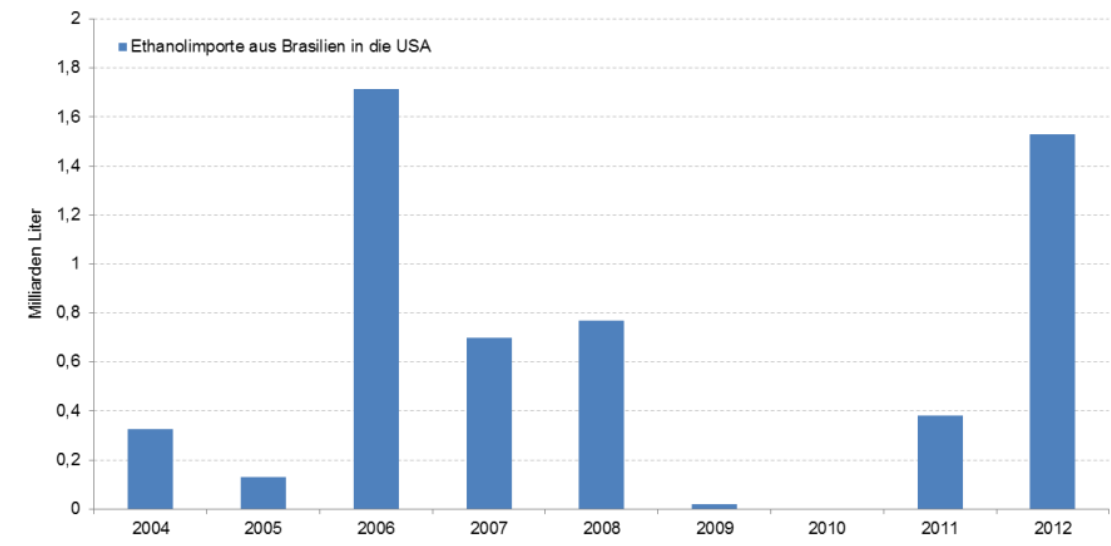

Abbildung 61: Entwicklung der Ethanolimporte aus Brasilien in die USA Quelle: U.S. ENERGY INFORMATION ADMINISTRATION 2013c

Die Ethanolimporte von brasilianischem Zuckerrohr-Ethanol in die USA sind im Zeitraum von 2009 bis 2012 auf 1,5 Milliarden Liter angestiegen (Abbildung 61). Es werden von den USA jährlich ansteigende Mengen brasilianischen Zuckerrohr-Ethanols nachgefragt, da der Kraftstoff eine 50-prozentige Treibhausgasreduktion im Vergleich zu Benzin besitzt und somit der Erfüllung des Ver-

${ }^{205}$ Für das Jahr 2010 wurde von der U.S. ENERGY INFORMATION ADMINISTRATION kein Importwert angegeben. 
brauchsmandats „Advanced: Unspecified Biofuel“ dient (U.S. GOVERNMENT INFORMATION 2007: 121 STAT. 1519; OECD/FAO 2012: 94; F.O. LICHT 2012a: 301). Dadurch steht brasilianisches Zuckerrohr-Ethanol in direkter Konkurrenz zur US-amerikanischen Produktion von Ethanol aus Sorghum und Zuckerrohr, welches ebenfalls dem Verbrauchsmandat „Advanced: Unspecified Biofuel" angerechnet werden kann (U.S. GOVERNMENT INFORMATION 2007: 121 STAT. 1519ff.), wobei zu beachten ist, dass Zuckerrohr-Ethanol aus Brasilien eine hohe Konkurrenzfähigkeit besitzt, da die Produktionskosten vergleichbar mit denen von Mais-Ethanol in den USA sind (BERG 2012c: 11).

Darüber hinaus sind die Importe von Zuckerrohr-Ethanol aus Brasilien von Relevanz, da sie im Gegensatz zur US-amerikanischen Ethanolproduktion aus Mais im Corn Belt den Anforderungen des "Low Carbon Fuel Standard“ in Kalifornien genügen und von der ansässigen Mineralölindustrie importiert werden, um die Verbrauchsmandate des RFS 2 zu erfüllen (Einflussfaktor 22) (F.O. LICHT 2012h: 193; F.O. LICHT 2011a: 65). Des Weiteren hat sich die Konkurrenzfähigkeit brasilianischer Importe im Vergleich zur US-amerikanischen Produktion durch die Aufhebung des US-amerikanischen Importzollsatzes für Ethanol in Höhe von 0,54 US-\$/Gallone im Jahr 2011 verbessert (F.O. LICHT 2011a: 65). Brasilien besitzt ferner ein hohes Flächenpotenzial, um die Produktion sowie den Export von Zuckerrohr-Ethanol zukünftig zu steigern (F.O. LICHT/AGRA INFORMA 2009: 168; INTERNATIONAL ENERGY AGENCY BIOENERGY 2007: 17). Allerdings ist anzumerken, dass der Ausbau der Produktion trotz politischer Unterstützung in den Jahren 2008 bis 2012 deutlich langsamer erfolgte als ursprünglich geplant (F.O. LICHT 2013d: 294f.; INDUSTRY WEEK 2012: 1).

Name:

Lfd. Nr.:

Einflussbereich:

Beschreibung:

Bedeutung des Einflussfaktors:
Ethanolexporte aus den USA nach Brasilien

4

Ökonomie

Die Ethanolexporte aus den USA nach Brasilien werden in der Einheit Milliarden Liter pro Jahr angegeben.

Die USA importieren nicht nur Zuckerrohr-Ethanol aus Brasilien (Einflussfaktor 3), sondern exportieren auch Mais-Ethanol nach Brasilien ${ }^{206}$. Die USA haben sich seit 2006 von einem Ethanolimporteur zu einem Ethanolexporteur gewandelt und waren im Jahr 2011 der weltgrößte Exporteur (F.O. LICHT 2012a: 301). Dabei gehört Brasilien neben der EU27 und Kanada zu den wichtigsten

${ }^{206}$ Diese außergewöhnliche Situation des zweiseitigen Handels zwischen den USA und Brasilien mit dem chemisch identischen Produkt besteht schon seit mehreren Jahren und ist das Ergebnis der US-amerikanischen Einstufung von Zuckerrohr-Ethanol als „Advanced: Unspecified Biofuel“", wodurch es in den USA eine höhere Wertigkeit als in Brasilien besitzt (U.S. GOVERNMENT INFORMATION 2007: 121 STAT. 1519ff.). 
Exportmärkten der USA (F.O. LICHT 2012a: 302). In der folgenden Abbildung 62 ist die Entwicklung der US-amerikanischen Ethanolexporte nach Brasilien dargestellt.

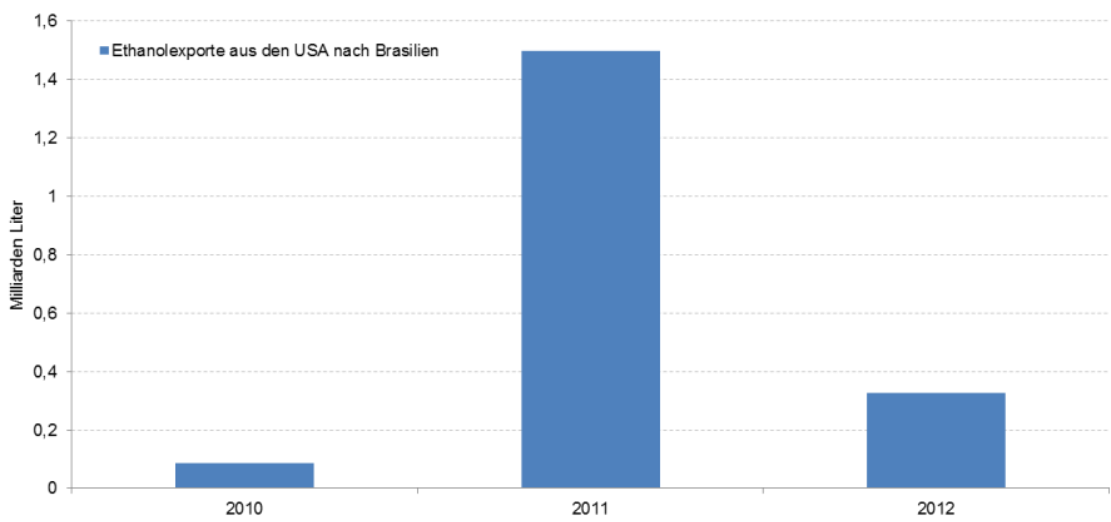

Abbildung 62: Entwicklung der US-amerikanischen Ethanolexporte nach Brasilien

Quelle: U.S. ENERGY INFORMATION ADMINISTRATION 2013d

Die USA exportierten im Jahr 2011 mit 1,5 Milliarden Litern deutlich höhere Ethanolmengen nach Brasilien als in den Jahren 2010 (0,08 Milliarden Liter) und 2012 (0,3 Milliarden Liter) (Abbildung 62). Dies ist auf den starken Rückgang der brasilianischen Ethanolproduktion um 4,5 Milliarden Liter im Jahr 2011 und auf die US-amerikanische Ethanolüberproduktion infolge der "Blend Wall“Problematik zurückzuführen (F. O. LICHT 2012f: 74). Somit stellte der brasilianische Markt im Jahr 2011 eine Möglichkeit dar, die Überproduktion abzusetzen und einen Preisverfall des Ethanols in den USA zu verhindern (F.O. LICHT 2012a: 302).

Der brasilianische Ethanolmarkt ist von Bedeutung, da er ein zusätzlicher Absatzmarkt für US-amerikanischens Ethanol ist und zukünftig wie bereits im Jahr 2011 dazu dienen kann, eine US-amerikanische Überproduktion abzubauen. Darüber hinaus könnte zukünftig der zweiseite Handel zwischen den USA und Brasilien zunehmen. Dies wäre dann der Fall, wenn Brasilien höhere Mengen Mais-Ethanol aus den USA bezöge, um den heimischen Markt mit Ethanol zu versorgen, und im Gegenzug größere Mengen Zuckerrohr-Ethanol in die USA exportierte, damit das ansteigende Verbrauchsmandat für „Advanced: Unspecified Biofuel“ erfüllt werden kann.

Allerdings ist zu beachten, dass die betrachteten Exportmengen um ein Vielfaches geringer sind im Vergleich zur US-amerikanischen Binnennachfrage von über 50 Milliarden Litern (F.O. LICHT 2012f: 74).

Name:

Ethanolexporte aus den USA in die EU27

Lfd. Nr.:

5

Einflussbereich: Ökonomie 
Beschreibung:

Bedeutung des Einflussfaktors:
Die Ethanolexporte aus den USA in die EU27 werden in der Einheit Milliarden Liter pro Jahr dargestellt.

Der Ethanolmarkt der EU27 nimmt Einfluss auf den US-amerikanischen Ethanolmarkt, da er einen zusätzlichen Markt für Ethanol darstellt. So wurden bereits im Jahr 2011 1,1 Milliarden Liter in die EU27 exportiert. Der Ethanolexport im Jahr 2011 erfolgte als E90 (90\% Ethanol und 10\% Benzin), um einen geringeren Zollsatz zu entrichten und dadurch die Konkurrenzfähigkeit des Biokraftstoffs gegenüber dem in Europa produzierten Ethanol zu verbessern. Im Jahr 2012 sind allerdings die Exporte auf 0,38 Milliarden Liter zurückgegangen, da seit März 2012 E90 von der EU27 mit dem vollen Zollsatz belegt wird ${ }^{207}$ und die US-amerikanische Ethanolproduktion im Jahr 2012 gesunken ist (Abbildung 63) (F.O. LICHT 2012a: 302).

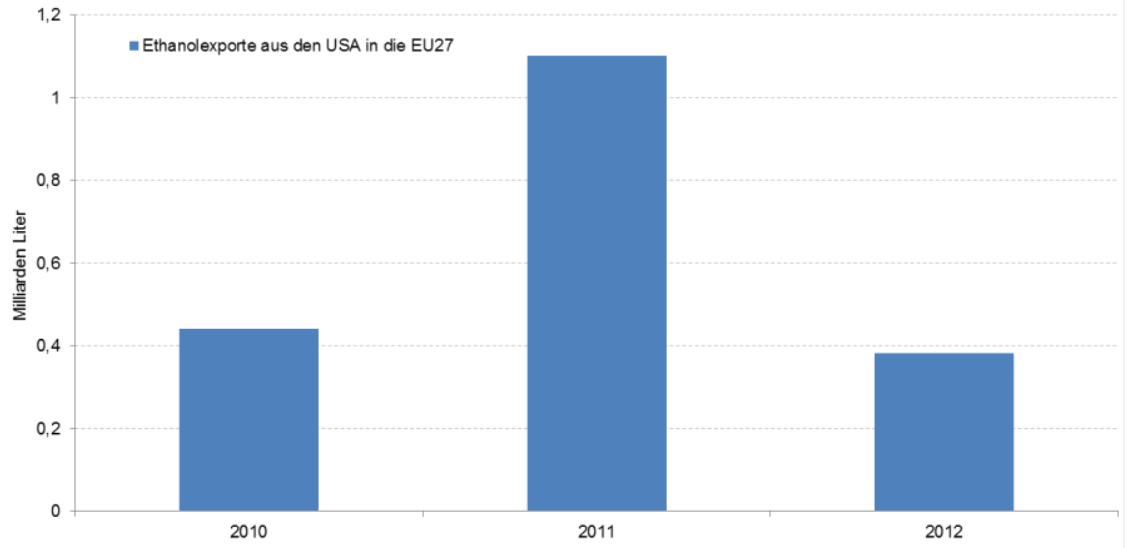

Abbildung 63: Entwicklung der US-amerikanischen Ethanolexporte in die EU27 Quelle: U.S. ENERGY INFORMATION ADMINISTRATION 2013e

Trotz der Neuregelung der Verzollung von E90 ist der europäische Ethanolmarkt als Absatzmarkt für US-amerikanisches Ethanol auch zukünftig bedeutsam, da die Produktionskosten für Ethanol in den USA geringer sind als in der EU27 $7^{208}$ und die EU27 auf Ethanolimporte angewiesen ist, um die politischen Ziele der Biokraftstoffnutzung zu erfüllen (ECOFYS ET AL. 2011: 46). Jedoch ist anzumerken, dass die Ethanolnachfrage in der EU27 seit dem Jahr 2010 nur noch moderat angestiegen ist (Abbildung 2).

Ethanolexporte aus den USA ins Ausland (Exporte nach Brasilien und die EU27 werden nicht berücksichtigt)

6

${ }^{207}$ Eine genaue Beschreibung der Zollbestimmungen in der EU27 befindet sich in Kapitel 5.2 .5 .

${ }^{208}$ Im Zeitraum von 2010 bis Anfang 2012 waren die US-amerikanischen Produktionskosten für Ethanol im Vergleich zu denen in der EU27 um durchschnittlich 20 US-\$/hl geringer (BERG 2012d: 2). 
Einflussbereich:

Beschreibung:

Bedeutung des

Einflussfaktors:

\section{Ökonomie}

Es werden die Ethanolexporte aus den USA ins Ausland betrachtet. Dabei werden die Exporte nach Brasilien und in die EU27 nicht berücksichtigt, da diese separat in Einflussfaktor 4 und 5 dargestellt sind. Die Entwicklung der Exporte wird in der Einheit Milliarden Liter pro Jahr angegeben.

Die ausländischen Ethanolmärkte stellen zusätzliche Absatzmärkte für USamerikanisches Ethanol dar und müssen aus diesem Grund näher betrachtet werden.

Bereits im Jahr 2011 wurden 0,25 Milliarden Liter Ethanol in die Vereinigten Arabischen Emirate exportiert, die Ethanol-Kraftstoff verwenden, um die Oktanzahl von Benzin anzuheben und damit die Klopffestigkeit des Kraftstoffs zu verbessern (Abbildung 64) (F.O. LICHT 2013f: 390). Die Philippinen, Südkorea und Japan importieren derzeit nur geringe Mengen Ethanol aus den USA (Abbildung 64), besitzen allerdings ein hohes Marktpotenzial. F.O. LICHT (2013f: 390) prognostiziert für den philippinischen Markt ein zukünftiges Absatzpotenzial von 0,45 Milliarden Litern, da die Regierung im November 2012 ein Programm zur Beimischung von 10\% Ethanol zu Benzin verabschiedet hat, die Philippinen selbst jedoch nur sehr geringe Produktionskapazitäten zur Ethanolherstellung besitzen. Südkorea (2012: 0,3 Milliarden Liter) und Japan (2012: 0,58 Milliarden Liter) importieren bereits seit Jahren größere Mengen Ethano ${ }^{209}$ (F.O. LICHT 2013f: 388), bisher allerdings nicht aus den USA (Abbildung 64). Folglich sind die Märkte für die USA von Interesse, um den Ethanolexport zu erhöhen.

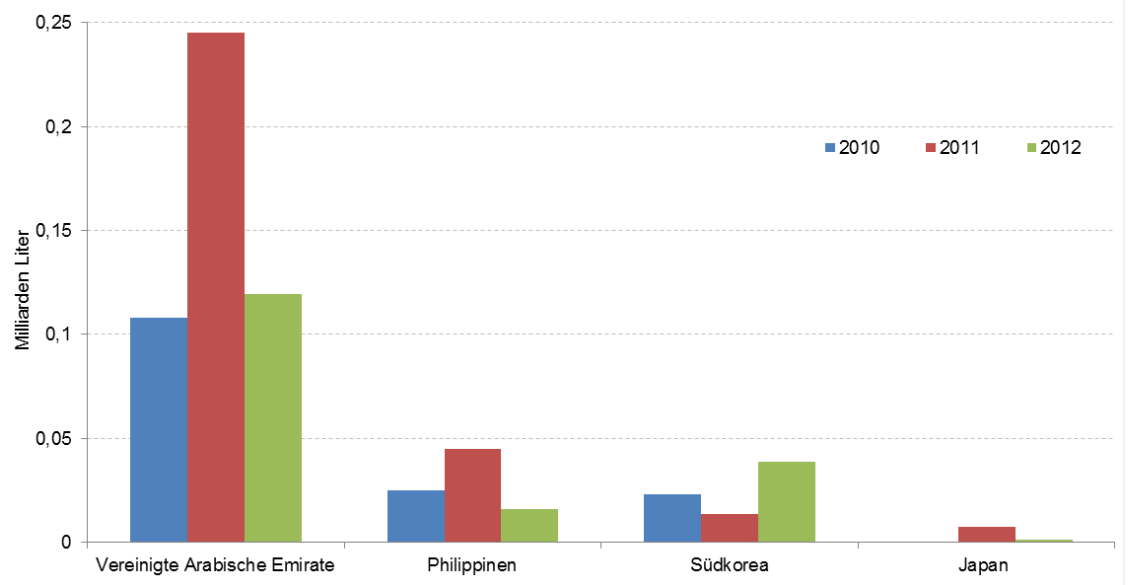
Abbildung 64: Entwicklung der US-amerikanischen Ethanolexporte ins Ausland $^{210}$

Quelle: In Anlehnung an U.S. ENERGY INFORMATION ADMINISTRATION 2013 e

Wie bereits erwähnt, ist die Betrachtung der ausländischen Märkte relevant, da

${ }^{209}$ Südkorea und Japan importieren seit 2009 jährlich über 0,23 Milliarden Liter bzw. 0,5 Milliarden Liter Ethanol aus dem Ausland (F.O. LICHT 2013f: 388).

${ }^{210}$ Die Daten für die US-amerikanischen Ethanolexporte nach Japan für die Jahre 2010 und 2012 sind in der Abbildung dargestellt. Allerdings sind sie so gering, dass sie nur schwer zu erkennen sind (2010: 0,159 Millionen Liter; 2012: 1,431 Millionen Liter). 
diese die Nachfrage nach US-amerikanischem Ethanol steigern und somit neue Wachstumsimpulse für den US-amerikanischen Markt erzeugen könnten. Sie könnten vor allem dann in den Fokus der Aufmerksamkeit rücken, wenn in Zukunft die US-amerikanische Binnennachfrage beispielsweise aufgrund einer andauernden „Blend Wall“-Problematik nicht weiter ansteigen sollte.

Name:

US-amerikanische Infrastruktur, die benötigt wird, um das Ethanol von den Produktionsanlagen zu den Märkten an die Ost- und Westküste der USA zu transportieren

Lfd. Nr.: 7

\section{Einflussbereich:}

\section{Ökonomie}

Beschreibung:

Der Faktor betrachtet die Infrastruktur, mit deren Hilfe das Ethanol von den Produktionsanlagen im Mittleren Westen zu den Ethanolmärkten an der Ostund Westküste der USA transportiert werden kann. Die Entwicklung des Faktors wird beschrieben, indem die Ist-Situation als Referenzsituation zugrunde gelegt wird, um davon ausgehend unterschiedliche Projektionen zu bilden, die den Faktor im Jahr 2023 beschreiben.

Bedeutung des Die Ethanolproduktion erfolgt im Mittleren Westen der USA, insbesondere in Einflussfaktors: den Staaten lowa, Minnesota, South Dakota, Nebraska, Illinois und Indiana (U.S. DEPARTMENT OF ENERGY 2013e). In der folgenden Abbildung 65 sind die Ethanolanlagen als grüne Punkte eingezeichnet. Hierbei ist zu beachten: Je größer der Durchmesser des Punktes, desto höher ist die Produktionskapazität der Anlage.

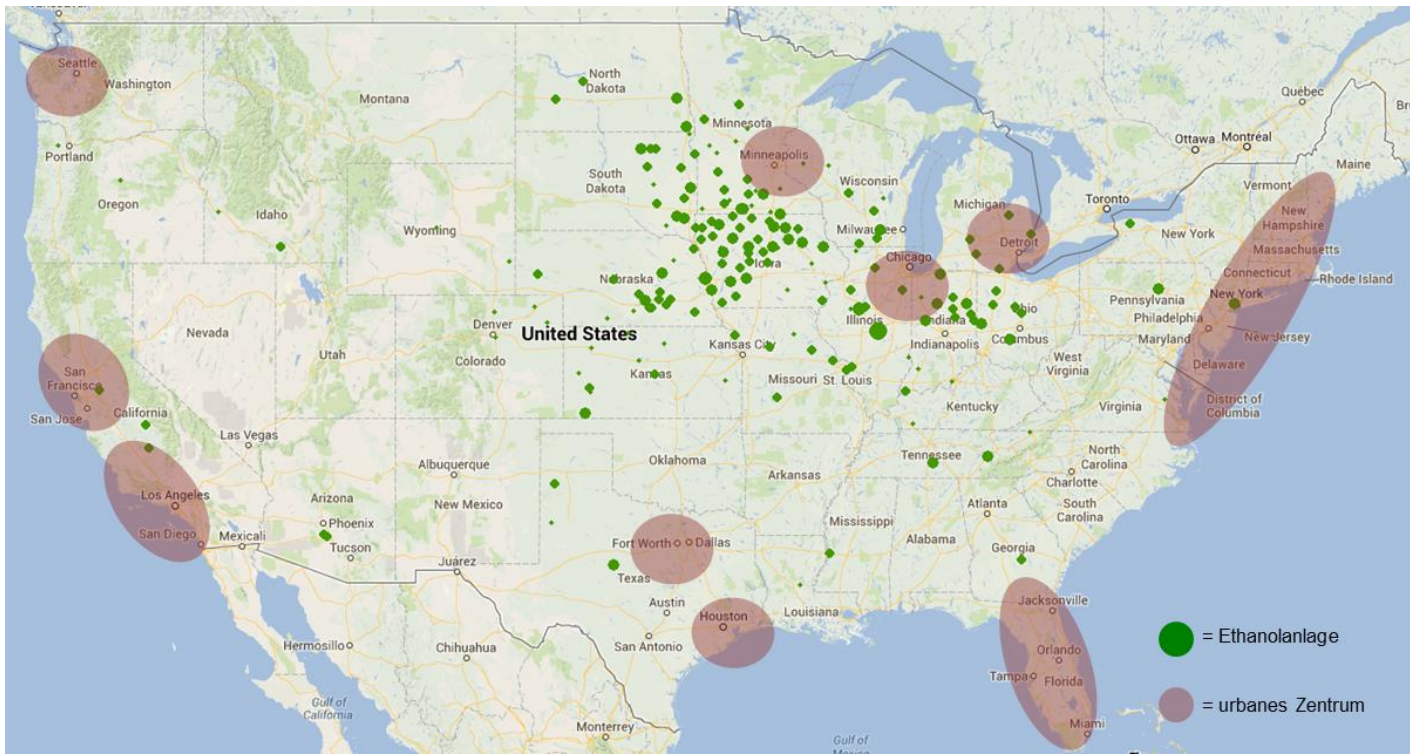

Abbildung 65: Standort der US-amerikanischen Ethanolanlagen

Quelle: In Anlehnung an NATIONAL RENEWABLE ENERGY LABORATORY 2013; UNITED STATES CENSUS BUREAU 2013 
Der Faktor ist deshalb bedeutend, da sich die Nachfrage nach Benzin und damit der Ethanolmarkt nicht im Mittleren Westen, sondern in den urbanen Zentren an der Ost- und Westküste (in Abbildung 65 rot markiert) befindet. Folglich muss das Ethanol an die Küsten transportiert werden. Doch ein kostengünstiger Transport mittels einer bereits bestehenden Pipeline ${ }^{211}$ ist nicht möglich, da Ethanol im Gegensatz zu Benzin sowohl hygroskopisch als auch korrodierend wirkt (U.S. DEPARTMENT OF ENERGY 2013e). Infolgedessen muss Ethanol zu hohen Kosten per Zug und Lastwagen zu den Märkten transportiert werden, wodurch sich die Konkurrenzfähigkeit gegenüber brasilianischem Ethanol verschlechtert, welches per Schiff direkt zu den Häfen der Ballungsgebiete transportiert wird (U.S. DEPARTMENT OF ENERGY 2013e).

Name: US-amerikanische Tankstelleninfrastruktur für den Verkauf von Benzin mit mittlerem Ethanolgehalt (E15 - E40)

Lfd. Nr.:

Einflussbereich:

Beschreibung:

Bedeutung des

Einflussfaktors:
8

\section{Ökonomie}

Um die Entwicklung der US-amerikanischen Tankstelleninfrastruktur für den Verkauf von Benzin mit mittlerem Ethanolgehalt (E15 - E40) aufzuzeigen, wird die Ist-Stituation als Referenzsituation beschrieben, um davon ausgehend die Entwicklung des Faktors durch unterschiedliche Zukunftsprojektionen darzustellen.

Seit dem Jahr 2011 übt der Einflussfaktor entscheidenden Einfluss auf den USamerikanischen Ethanolmarkt aus, da die US-amerikanischen Tankstellen aus technischen Gründen ${ }^{212}$ nicht in der Lage sind, den von der EPA zugelassenen Kraftstoff E15 (15\% Ethanol und 85\% Benzin) zu verkaufen. Infolgedessen ist die Überwindung der derzeitigen E10-„Blend Wall“213 nicht möglich, wodurch die US-amerikanische Ethanolnachfrage nicht weiter ansteigen kann, was die Erfüllung der Verbrauchsmandate des RFS 2 (Einflussfaktor 22) unmöglich macht (F.O. LICHT 2013c: 341).

Dieser Faktor wird auch in Zukunft entscheidend sein, denn um die ansteigenden Mandate des RFS 2 erfüllen zu können, ist auch der Verkauf von E15 nicht

${ }^{211}$ Über den Bau einer neuen Pipeline für den Transport von Ethanol wird seit längerem
nachgedacht und die Unternehmen POET - zweitgrößter US-amerikanischer Ethanolprodu-
zent - und Magellan Midstream Partners gründeten zur Förderung des Projektes 2009 ein
Joint Venture. Die geplante Pipeline soll das Ethanol aus lowa, Illinois, Indiana und Ohio bis
nach New Jersey transportieren. Jedoch sind seitdem keine weiteren Projektfortschritte zu
erkennen, was angesichts der prognostizierten Baukosten von 3,4 Milliarden US-\$ und ei-
nem Rückgang der US-amerikanischen Ethanolproduktion im Jahr 2012 wenig verwunder-
lich ist (U.S. DEPARTMENT OF ENERGY 2013e; POET 2009; F.O. LICHT 2012f: 74).
212 Die Zapfsäulen an den US-amerikanischen Tankstellen vertragen kein E15, weil dadurch
die Dichtungen erodieren und undicht werden (NACS 2012b: 30; U.S. ENERGY INFORMA-
TION ADMINISTRATION 2011c).
${ }_{213}$ Eine ausführliche Beschreibung der „Blend Wall“-Problematik befindet sich in Kapitel 2.2. 
ausreichend. Die US-amerikanischen Tankstellen müssen fähig sein, E30 (30\% Ethanol und $70 \%$ Benzin) flächendeckend in den USA anzubieten (Abbildung 73), was den Einbau eines weiteren Lagertanks zum Verkauf von E30 oder die Umrüstung der Tankstelle auf „Blender Pumps“214 unumgänglich macht (U.S. ENERGY INFORMATION ADMINISTRATION 2012h).

Name:

US-amerikanische Tankstelleninfrastruktur für den Verkauf von E85

Lfd. Nr.: 9

Einflussbereich:

Ökonomie

Beschreibung:

Die Entwicklung der Tankstelleninfrastruktur für den Verkauf von E85 (85\% Ethanol und 15\% Benzin) wird anhand der Anzahl an Tankstellen, die E85 in den USA verkaufen, beschrieben.

Bedeutung des

Die Betrachtung der Entwicklung der Tankstelleninfrastruktur für den Verkauf Einflussfaktors: von E85 ist wichtig, da die Verfügbarkeit von E85 Voraussetzung ist, damit mehr Flexible Fuel Vehicles (FFV) in den USA gekauft werden. Für den USamerikanischen Kraftfahrzeugfahrer lohnt sich der Kauf eines FFV erst, wenn er in der Lage ist, den Kraftstoff E85 möglichst flächendeckend zu tanken ${ }^{215}$. In den USA gibt es zwar schon zahlreiche Tankstellen, die E85 anbieten (in Abbildung 66 sind diese als türkise Punkte gekennzeichnet), doch befinden sich diese zum größten Teil im Mittleren Westen und nicht in den urbanen Zentren (in Abbildung 66 rot markiet) an der Ost- und Westküste, wo der Großteil des Benzins nachgefragt wird (U.S. DEPARTMENT OF ENERGY 2013e).

${ }^{214}$ Eine „Blender Pump“ ist eine Zapfanlage, die dem Autofahrer unterschiedliche EthanolBenzin-Mischungen anbieten kann. Typische Mischungen sind E10, E15, E20, E30, E40 sowie E85. Eine „Blender Pump“ kann unterschiedliche Mischungen verkaufen, da sie den Kraftstoff nicht nur einem, sondern zwei unabhängigen Tanks entnimmt, wobei der eine mit Benzin und der andere mit Ethanol gefüllt ist. Entsprechend der gewünschten EthanolBenzin-Mischung pumpt die „Blender Pump“ die entsprechenden Mengen Kraftstoff aus dem jeweiligen Tank und vermischt sie, bevor sie in das Kraftfahrzeug des Kunden fließen (U.S. DEPARTMENT OF ENERGY 2012b).

${ }^{215}$ Vorteil der FFV-Technologie ist es, dass es kein "Chicken or the Egg - Dilemma“ gibt, da die Autofahrer in der Zeit des Ausbaus der flächendeckenden Infrastruktur des E85Tankstellennetzes auch E10 tanken können. 


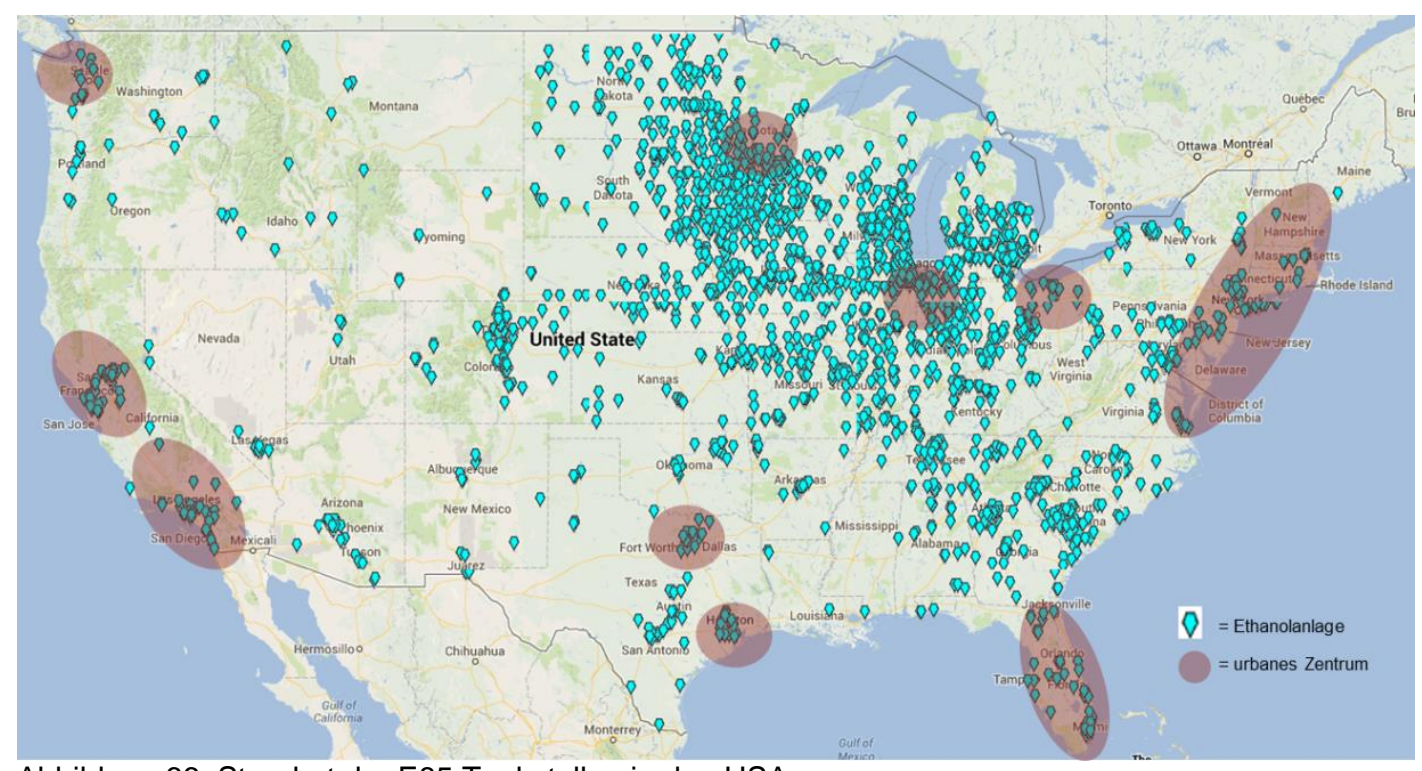

Abbildung 66: Standort der E85 Tankstellen in den USA

Quelle: In Anlehnung an NATIONAL RENEWABLE ENERGY LABORATORY 2013; UNITED STATES CENSUS BUREAU 2013

Die Entwicklung der FFV-Technologie ist zudem relevant, da sich die USA an der E10-,Blend Wall“ befinden, die ein weiteres Wachstum der USamerikanischen Ethanolnachfrage derzeit verhindert (F.O. LICHT 2013b: 213). Durch den Verkauf von E85 wäre ein weiterer Anstieg der Ethanolnachfrage möglich, da aufgrund des hohen Ethanolanteils schon verhältnismäßig geringe Absatzmengen von E85 eine bedeutende Steigerung der Ethanolnachfrage bewirken würden.

Die U.S. ENERGY INFORMATION ADMINISTRATION (2013a: 154) prognostiziert eine E85-Nachfrage von 5,3 Milliarden Litern für das Jahr 2025, was einer Ethanolnachfrage von 4,5 Milliarden Litern entspricht. Um diese erwartete, starke Steigerung des E85-Absatzvolumens von derzeit 0,146 Milliarden Litern $z u$ erreichen, muss es zu einem Ausbau der Tankstelleninfrastruktur kommen (U.S. ENERGY INFORMATIOM ADMINISTRATION 2012j: 29). Allerdings sind die Kosten für den Um- bzw. Einbau einer neuen Zapfanlage hoch (zwischen 22.000 und 80.000 US-\$), was viele Tankstellenbetreiber davon abhalten könnte, in den Bau zu investieren (U.S. ENERGY INFORMATION ADMINISTRATION 2007: 4). Politische Programme wie das „Renewable Energy Assistance Program" des USDA, das den Bau von Zapfanlagen, die dem Autofahrer mittlere und hohe Ethanolbeimischungen zu Benzin verkaufen können, finanziell unterstützt, sind daher weiterhin dringend notwendig (ETHANOL PRODUCER MAGAZINE 2013b).

Name: Preis für DDGS in den USA

Lfd. Nr.: 
Einflussbereich:

Beschreibung:

Bedeutung des

Einflussfaktors:

\section{Ökonomie}

Bei der Ethanolproduktion aus Mais durch das Trockenmahlverfahren entsteht DDGS (Distillers' Dried Grains with Solubles) als Nebenprodukt, welches als Proteinfutter in der Tierernährung verwendet wird (IOWA STATE UNIVERSITY 2007: 175). Die Entwicklung des Faktors wird anhand des Marktpreises in der Einheit US-\$ je Tonne beschrieben.

Der Verkauf von DDGS ist für die US-amerikanischen Ethanolproduzenten von großer Bedeutung, da sie dadurch einen wesentlichen Anteil ihres Umsatzes generieren (AGRICULTURAL MARKETING RESOURCE CENTER 2013). Der prozentuale Umsatzanteil ist im Zeitraum von 2005 bis 2013 entscheidend gestiegen. Die US-amerikanischen Ethanolproduzenten erzielen seit Oktober 2011 durchschnittlich 23\% ihres Umsatzes durch den Verkauf von DDGS (Abbildung 67).



Abbildung 67: Umsatzanteil durch den Verkauf von DDGS

Quelle: AGRICULTURAL MARKETING RESOURCE CENTER 2013

Durch die gewachsene Bedeutung des Verkaufs von DDGS sinkt die starke Abhängigkeit der Ethanolproduzenten vom Ethanolpreis und gibt innen die Möglichkeit, auch in Zeiten niedriger Ethanolpreise noch profitabel zu produzieren (AGRICULTURAL MARKETING RESOURCE CENTER 2012). Insbesondere durch die Korrelation zwischen dem Maispreis und dem Preis für DDGS wird das Risiko der hohen Rohstoffkosten gemildert, was ein entscheidender Vorteil ist, da die Rohstoffkosten rund $80 \%$ der Produktionskosten ausmachen (AGRICULTURAL MARKETING RESOURCE CENTER 2012).

11

Lfd. Nr.:

Einflussbereich: Ökonomie 
Beschreibung:
Bedeutung des
Einflussfaktors:

Der Einflussfaktor betrachtet die Entwicklung von vertikalen strategischen Kooperationen im Ethanolmarkt der USA. Dargestellt wird die Entwicklung des Faktors, indem die Ist-Situation als Referenzwert beschrieben wird, um davon ausgehend unterschiedliche Zukunftsprojektionen für das Jahr 2023 zu bilden.

Vertikale strategische Kooperationen werden betrachtet, da es durch diese zu einer schnelleren Weiterentwicklung des US-amerikanischen Ethanolmarktes kommen könnte. Beispielhaft für eine solche Zusammenarbeit ist das Projekt „Project LIBERTY“ oder das Gemeinschaftsunternehmen „Butamax Advanced Biofuels LLC“.

Die Unternehmen POET - zweitgrößter Ethanolproduzent der USA ${ }^{216}$ - und DSM - ein niederländisches Unternehmen, das sich in diesem Projekt auf die Entwicklung von Enzymen zur Aufspaltung von Zellulose spezialisiert hat arbeiten gemeinsam am „Project LIBERTY“. Ziel dieses Gemeinschaftsprojekts ist die Weiterentwicklung der kommerziellen Produktion von Zellulose-Ethanol, um im ersten Schritt eine kommerzielle 95-Millionen-Liter-ZelluloseEthanolanlage in Emmetsburg (lowa) zu errichten und im zweiten Schritt die erprobte Technologie an andere Unternehmen auszulizenzieren (POET-DSM ADVANCED BIOFUELS 2012; POET 2013).

Das Unternehmen „Butamax Advanced Biofuels LLC“ ist ein im Jahr 2009 gegründetes Joint Venture zwischen dem US-amerikanischen Chemieunternehmen DuPont und dem britischen Mineralölkonzern BP. Das Joint Venture arbeitet an der Entwicklung der kommerziellen Produktion von Biobutanol ${ }^{217}$. Eine erste Demonstrationsanlage wurde bereits in Hull, England errrichtet (BUTAMAX ADVANCED BIOFUELS 2013). Wie im Beispiel von Butamax ist eine vertikale Kooperation mit einem führenden Mineralölkonzern von besonderer Bedeutung für den Biokraftstoffmarkt, da diese Unternehmen sowohl die finanziellen Mittel besitzen, um langfristig an der Entwicklung von Biokraftstoffen zu forschen, als auch den Zugang zur Infrastruktur haben, um Biokraftstoffe weltweit zu vermarkten.

\begin{tabular}{l|l} 
Name: & Produktionskosten von Mais-Ethanol \\
\hline Lfd. Nr.: & $12 \mathrm{a} / 12 \mathrm{~b}$ \\
\hline
\end{tabular}

${ }^{216}$ Nach ADM ist POET mit einer Ethanolproduktion von über 5 Milliarden Litern der zweitgrößte Ethanolproduzent der Welt (F.O. LICHT 2011d: 353).

217 In Einflussfaktor 21 wird der Biokraftstoff Biobutanol genauer betrachtet und die Bedeutung für den US-amerikanischen Ethanolmarkt erläutert.

218 Der Einflussfaktor „Produktionskosten von Mais-Ethanol“ unterteilt sich in die beiden Einflussfaktoren (12a) „Rohstoffkosten für die Produktion von Mais-Ethanol“ und (12b) „Produktionskosten von Mais-Ethanol - ohne Rohstoffkosten“. Diese Aufteilung wurde gewählt, da die Rohstoffkosten für Mais $80 \%$ der gesamten Produktionskosten ausmachen und deshalb von solch hoher Bedeutung für die zukünftige Entwicklung des US-amerikansichen Ethanolmarktes sind, dass sie isoliert betrachtet werden müssen (AGRICULTURAL MARKETING RESOURCE CENTER 2012). Die Bedeutung der Faktoren $12 \mathrm{a}$ und $12 \mathrm{~b}$ wird gemeinsam erläutert, um unnötige Wiederholungen zu vermeiden. 


\begin{tabular}{|c|c|}
\hline Einflussbereich: & Ökonomie \\
\hline $\begin{array}{l}\text { Einflussfaktor } \\
\text { 12a: }\end{array}$ & Rohstoffkosten bei der Produktion von Mais-Ethanol \\
\hline $\begin{array}{l}\text { Beschreibung } \\
\text { 12a: }\end{array}$ & $\begin{array}{l}\text { Der Einflussfaktor betrachtet die Rohstoffkosten zur Produktion von Mais- } \\
\text { Ethanol. Die Entwicklung des Maispreises wird in der Einheit US-\$ je Tonne } \\
\text { angegeben. }\end{array}$ \\
\hline $\begin{array}{l}\text { Einflussfaktor } \\
\text { 12b: }\end{array}$ & Produktionskosten von Mais-Ethanol - ohne Rohstoffkosten \\
\hline $\begin{array}{l}\text { Beschreibung } \\
\text { 12b: }\end{array}$ & $\begin{array}{l}\text { Der Faktor beschreibt die Produktionskosten zur Herstellung von Mais-Ethanol } \\
\text { in den USA. Die Rohstoffkosten werden nicht berücksichtigt, da diese in Ein- } \\
\text { flussfaktor } 12 \text { a separat betrachtet werden. Dargestellt werden die Produktions- } \\
\text { kosten in US- } \$ \text { je Hektoliter. }\end{array}$ \\
\hline $\begin{array}{l}\text { Bedeutung der } \\
\text { Faktoren } \\
\text { 12a/12b: }\end{array}$ & 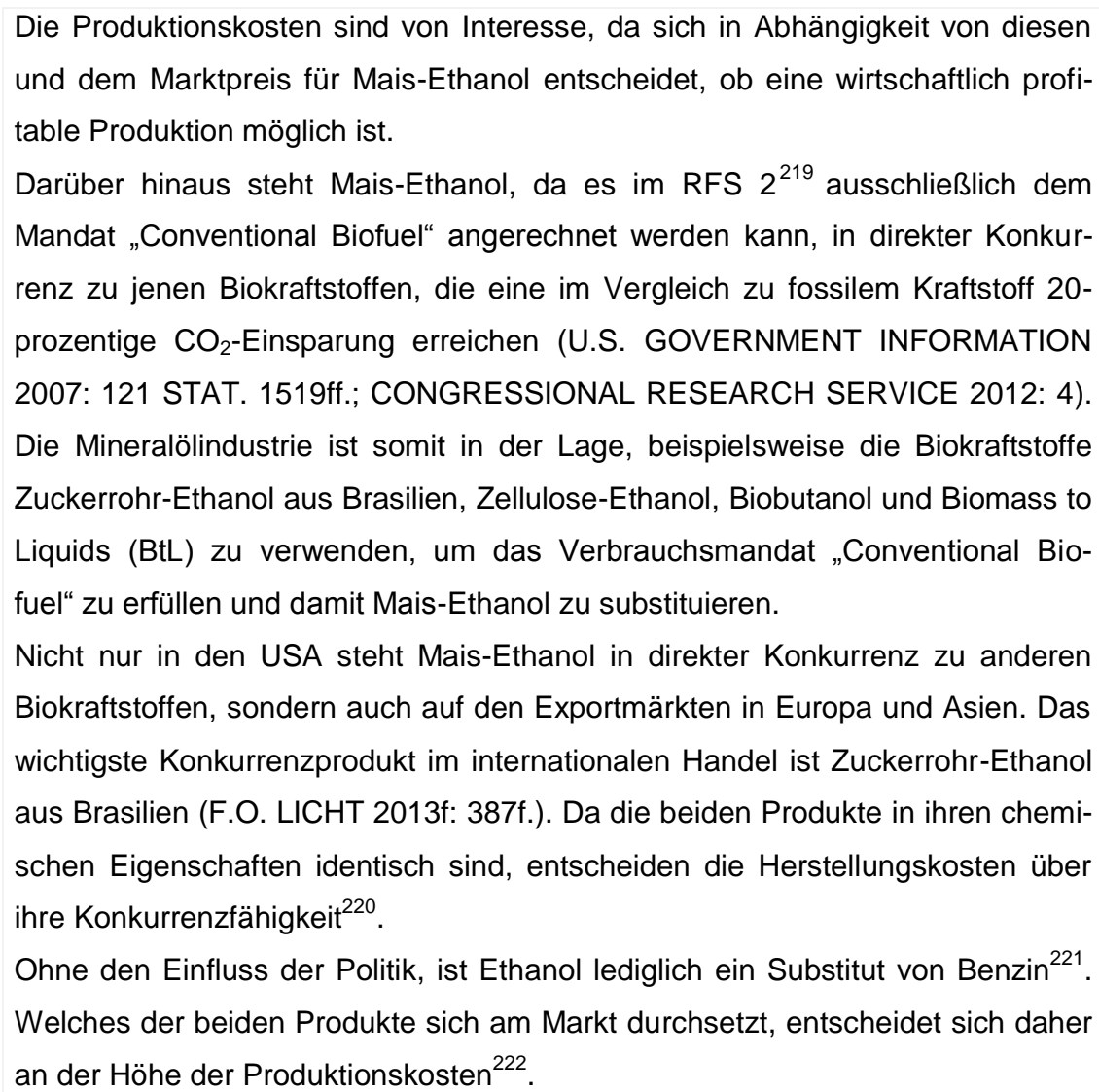 \\
\hline
\end{tabular}

${ }^{219}$ Die Struktur des RFS 2 ist ausführlich in Einflussfaktor 22 beschrieben.

${ }^{220} \mathrm{Im}$ Vergleich zu Fossilbrennstoff unterscheiden sich Mais-Ethanol und ZuckerrohrEthanol in der Höhe der Treibhausgasreduktion. Dies könnte für Zuckerrohr-Ethanol ein entscheidender Vorteil in Bezug auf den zukünftigen Export in die EU27 sein, denn Zuckerrohr-Ethanol erfüllt im Gegensatz zu Mais-Ethanol die in Zukunft steigenden politischen Anforderungen an die $\mathrm{CO}_{2}$-Reduktion (Kapitel 5.2.7) (RICHTLINIE 2009/28/EG ANHANG V). ${ }^{221}$ Allerdings ist Ethanol kein vollkommenes Substitut von Benzin, da die Tankstellen derzeitig lediglich in der Lage sind, eine maximale Ethanolbeimischung zu Benzin von 10\% zu verkaufen, und die Kraftfahrzeuge nur Benzin mit einem Ethanolanteil von 10\% oder $15 \%$ als Kraftstoff verwenden können (U.S. GOVERNMENT INFORMATION 2011: 4662; F.O. LICHT 2013c: 341). 
Name:

Lfd. Nr.:

Einflussbereich:

Einflussfaktor

13a:

Beschreibung

13a:

Einflussfaktor

13b:

Beschreibung

13b:

Bedeutung der

Faktoren

13a/13b:
Produktionskosten von Zellulose-Ethanol ${ }^{223}$

$13 a / 13 b$

Ökonomie

Rohstoffkosten bei der Produktion von Zellulose-Ethanol

Die Rohstoffkosten zur Produktion von Zellulose-Ethanol in den USA werden in US-\$ je Tonne angegeben.

Produktionskosten von Zellulose-Ethanol - ohne Rohstoffkosten

Der Einflussfaktor betrachtet die Produktionskosten von Zellulose-Ethanol in den USA. Hierbei werden die Rohstoffkosten nicht berücksichtigt, da diese in Einflussfaktor $13 \mathrm{a}$ isoliert dargestellt werden. Beschrieben werden die Herstellungskosten in US-\$ je Hektoliter.

Der Faktor ist deshalb bedeutend, da sich in Abhängigkeit von den Produktionskosten und dem Preis für Zellulose-Ethanol entscheidet, ob die Herstellung rentabel ist.

Darüber hinaus sind die Produktionskosten entscheidend, da das Mandat für „Advanced: Cellulosic Biofuel“ auch durch weitere Biokraftstoffe, wie beispielsweise Biobutanol und BtL, erfüllt werden kann. Voraussetzung für die Substitution von Zellulose-Ethanol ist, dass die biogenen Treibstoffe aus den Rohstoffen Zellulose, Hemizellulose oder Lignin produziert werden und eine 60-prozentige Treibhausgasreduktion im Vergleich zu den fossilen Kraftstoffen, welche sie ersetzen, erreichen (U.S. GOVERNMENT INFORMATION 2007: 121 STAT. 1519ff.; CONGRESSIONAL RESEARCH SERVICE 2012: 4). Darüber hinaus muss der Einflussfaktor betrachtet werden, da die US-amerikanische ZelluloseEthanolproduktion zukünftig in Konkurrenz zu importiertem Zellulose-Ethanol aus Brasilien, produziert aus Bagasse ${ }^{224}$, stehen könnte.

Sollte es zukünftig keine politische Unterstützung für Biokraftstoffe in den USA

${ }^{222}$ Bei dem Vergleich der Herstellungskosten ist allerdings darauf zu achten, dass der Ethanolpreis $(21,2 \mathrm{MJ} / \mathrm{L}) 34 \%$ geringer sein muss als der von Benzin $(32,1 \mathrm{MJ} / \mathrm{L})$, damit die Kosten je Energieeinheit identisch sind (MASSACHUSETTS INSTITUTE OF TECHNOLOGY 2007: 1).

${ }^{223}$ Der wirkende Einflussfaktor „Produktionskosten von Zellulose-Ethanol“ gliedert sich in die beiden Einflussfaktoren (13a) "Rohstoffkosten zur Produktion von Zellulose-Ethanol“ und (13b) „Produktionskosten von Zellulose-Ethanol - ohne Rohstoffkosten“. Die gesonderte Betrachtung der Rohstoffkosten ist sinnvoll, da diese der mit Abstand wichtigste Kostenfaktor bei der Herstellung von Zellulose-Ethanol ist und dadurch entscheidend die zukünftige Entwicklung des US-amerikanischen Zellulose-Ethanolmarktes beeinflusst (F.O. LICHT 2010: 453). Die Bedeutung der Faktoren 13a und 13b wird gemeinsam beschrieben, um redundante Erklärungen zu vermeiden.

${ }^{224}$ Das Unternehmen Raízen - ein Joint Venture des Mineralölkonzerns Royal Dutch Shell plc. und des brasilianischen Zucker- und Ethanolproduzenten Cosan Ltd. - investiert in den Bau einer 40 Millionen Liter Zellulose-Ethanolanlage in Brasilien. Darüber hinaus ist der Bau weiterer acht Zellulose-Ethanolanlagen bis zum Jahr 2024 geplant (BLOOMBERG 2013). 
mehr geben, steht Zellulose-Ethanol in direkter Konkurrenz zu Mais-Ethanol. Da die beiden Biokraftstoffe chemisch identisch sind, entscheidet ausschließlich die Höhe der Produktionskosten über die Vorteilhaftigkeit eines der beiden Produkte (ACATECH 2012: 15). Des Weiteren besteht ohne politische Unterstützung, wie auch schon in Einflussfaktor 12 beschrieben, eine Konkurrenzsituation zwischen Ethanol und fossilem Benzin.

\section{Technologie}

Name:

Lfd. Nr.:

Einflussbereich:

Beschreibung:

Bedeutung des

Einflussfaktors:
Anzahl der Flexible Fuel Vehicles in den USA

14

Technologie

Flexible Fuel Vehicles (FFV) sind Kraftfahrzeuge mit einem Verbrennungsmotor, der fähig, ist ein Benzin-Ethanol-Gemisch mit bis zu 85\% vol Ethanol (E85) als Kraftstoff zu verwenden (U.S. ENERGY INFORMATION ADMINISTRATION 2007: 1)

Beschrieben wird der Faktor mittles der Anzahl an zugelassenen FFV in den USA.

Durch einen Anstieg der zugelassenen FFV in den USA wird auch die Ethanolnachfrage steigen, da FFV Benzin mit hohen Ethanolgehalten als Kraftstoff nutzen können. In der Regel tanken FFV den Kraftstoff E85, der aus 85\% vol Ethanol und 15\% vol Benzin besteht. Aufgrund des im Vergleich zu regulärem Benzin (E10) hohen Ethanolanteils von E85 können schon geringe Absatzsmengen eine erkennbare Nachfragesteigerung nach Ethanol bewirken.

Die Entwicklung des FFV-Marktes ist derzeit von besonderer Bedeutung für den US-amerikanischen Ethanolmarkt, da sich dieser an der E10-,Blend Wall“ befindet, infolgedessen die Binnennachfrage nach Ethanol seit 2011 stagniert (F.O. LICHT 2013b: 213). Durch den Verkauf von E85 an FFV wäre jedoch eine Steigerung der US-amerikanischen Ethanolnachfrage wieder möglich; allerdings erfordert dies ein Wachstum des FFV-Marktes. Beispielhaft für eine solche Entwicklung ist der brasilianische Markt, in dem seit der Einführung des „Gol Power 1.6“ von Volkswagen im Jahr 2003 der Marktanteil der FFV am Gesamtautomobilmarkt stark gestiegen ist - so waren im Jahr 2009 90\% aller neu zugelassenen Kraftfahrzeuge FFV (F.O LICHT/AGRA INFORMA 2009: 35f.; F.O. LICHT 2009: 70). Die FFV-Technologie besitzt den großen Vorteil gegenüber anderen neuen Antriebstechnologien wie beispielsweise der Elektromobilität, dass es kein „Chicken or the Egg-Dilemma“ gibt, da die FFV in der Zeit des Ausbaus einer flächendeckenden Infrastruktur (Einflussfaktor 9) kon- 
ventionellen Kraftstoff tanken können.

In den USA werden FFV bereits von allen bedeutenden US-amerikanischen Automobilmarken sowie einer Reihe von Importeuren angeboten ${ }^{225}$ und die U.S. ENERGY INFORMATION ADMINISTRATION (2013f) prognostiziert einen starken Anstieg der Anzahl an zugelassenen FFV auf 18,2 Millionen bis zum Jahr 2023 (Abbildung 68).

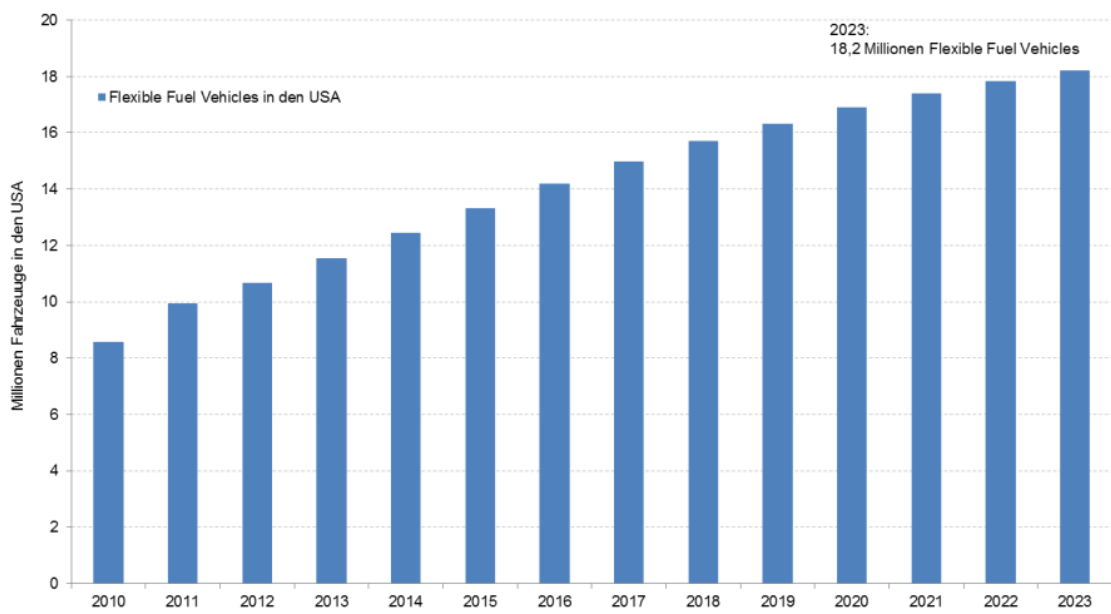

Abbildung 68: Entwicklung der Anzahl an Flexible Fuel Vehicles in den USA Quelle: U.S. ENERGY INFORMATION ADMINISTRATION $2013 f$

Allerdings ist der derzeitige Verbrauch von Ethanol als E85 minimal, lediglich $0,28 \%(0,146$ Milliarden Liter) des US-amerikanischen Ethanolabsatzes erfolgt über FFV (U.S. ENERGY INFORMATION ADMINISTRATION 2012j: 29). Auch zukünftig ist trotz des erwarteten starken Anstiegs der Anzahl an FFV zu beachten, dass der Großteil der prognostizierten 278 Millionen ${ }^{226}$ zugelassenen Kraftfahrzeuge im US-amerikanischen Straßenverkehr im Jahr 2023 weiterhin konventionellen fossilen Kraftstoff tanken wird ${ }^{227}$.

Name: Anzahl der Kraftfahrzeuge, die mit mittleren Ethanolbeimischungen zu Benzin (E15 - E40) fahren können

Lfd. Nr.: 15

Einflussbereich: Technologie

${ }^{225}$ Folgende Marken bieten FFV auf dem US-amerikansichen Markt an: Audi, Bentley Motors, Buick, Cadillac, Chevrolet, Chrysler, Dodge, Ford, GMC, Jaguar, Jeep, Land Rover, Lincoln, Mazda, Mercedes-Benz, Mercury, Nissan, Ram, Toyota, Volkswagen (U.S. DEPARTMENT OF ENERGY 2013g).

${ }^{226}$ Die Prognose, dass es im Jahr 2023 in den USA 278 Millionen zugelassene Kraftfahrzeuge gibt, ist ausführlich in Kapitel 6.2.4 beschrieben.

${ }^{227}$ Basierend auf den genannten Prognosen für das Jahr 2023 - 18,2 Millionen Flexible Fuel Vehicles/278 Millionen zugelassene Kraftfahrzeuge - besitzen die Flexible Fuel Vehicles einen Marktanteil von nur 6,5\% am gesamten US-amerikanischen Automobilmarkt. 
Beschreibung:

Bedeutung des

Einflussfaktors:
Der Faktor dient der Darstellung der Anzahl an Kraftfahrzeugen in den USA, die mit mittleren Ethanolbeimischungen zu Benzin (E15 - E40) fahren können. Beschrieben wird die Entwicklung des Faktors anhand der Anzahl an USamerikanischen Kraftfahrzeugen, unterteilt nach den unterschiedlich hohen Ethanolbeimischungen zu Benzin, die die Fahrzeuge als Kraftstoff nutzen können.

Der Faktor ist von entscheidender Relevanz, da die 10-prozentige Ethanolbeimischung zu Benzin der mit großem Abstand wichtigste Absatzweg für USamerikanisches Ethanol ist ${ }^{228}$. Derzeit vertragen alle zugelassenen Kraftfahrzeuge in den USA den Kraftstoff E10, die neueren Fahrzeuge ab Baujahr 2001 auch schon E15 (U.S. ENERGY INFORMATION ADMINISTRATION 2007; EPA 2012a; U.S. ENERGY INFORMATION ADMINISTRATION 2011a: 84).

Basierend auf dem prognostizierten Rückgang der US-amerikanischen Benzinnachfrage auf 484 Milliarden Liter bis zum Jahr 2022 (Einflussfaktor 1) sowie auf der Annahme, dass die Kraftfahrzeuge auch in Zukunft höchstens E15 als Kraftstoff nutzen können, ergibt sich ein maximaler US-amerikanischer Ethanolabsatz von 77 Milliarden Litern im Jahr 2022 (Abbildung 73) (U.S. ENERGY INFORMATION ADMINISTRATION 2012b: 98). Zur Erfüllung der Verbrauchsmandate des RFS 2 im Jahr 2022 (Einflussfaktor 22) bedarf es jedoch eines Ethanolabsatzes von mindestens 132 Milliarden Litern (U.S. GOVERNMENT INFORMATION 2007: 121 STAT. 1519ff.). Folglich muss es in den nächsten Jahren zu einer technischen Weiterentwicklung der Kraftfahrzeuge kommen. Aus Abbildung 73 ist ersichtlich, dass alle US-amerikanischen Fahrzeuge technisch fähig sein müssen, E30 (30\% vol Ethanol und 70\% vol Benzin) als Kraftstoff zu verwenden, um die politische Vorgabe im Jahr 2022 zu erfüllen ${ }^{229}$.

An der Weiterentwicklung der Kraftfahrzeuge wird bereits von der Automobilindustrie gearbeitet; so sind alle verkauften Kraftfahrzeuge von VW in Europa ab dem Baujahr 2010 in der Lage, E20 als Kraftstoff zu nutzen (SEYFRIED 2012).

Anzahl der Elektrofahrzeuge in den USA

16

Lfd. Nr.:

Einflussbereich:

Technologie

Beschreibung:
Der Faktor vereint unter dem Begriff Elektrofahrzeuge Batterie- und Hybridfahrzeuge. Batteriefahrzeuge besitzen einen Elektromotor und nutzen gespeicherte Elektrizität als Energiequelle (VDA 2011: 8). Hybridfahrzeuge hingegen besitzen einen Elektro- sowie einen Verbrennungsmotor, wodurch sie fähig sind

${ }^{228}$ Im Jahr 2012 wurden über 99\% des Ethanols auf dem US-amerikanischen Markt als 10prozentige Beimischung zu Benzin verkauft (U.S. ENERGY INFORMATION ADMINISTRATION 2012j: 29).

${ }^{229}$ Der Ethanolabsatz durch FFV wird in dieser Berechnung nicht berücksichtigt, da der USamerikanische FFV-Markt isoliert in Einflussfaktor 14 betrachtet wird. 
Bedeutung des

Einflussfaktors: sowohl gespeicherte Elektrizität als auch Flüssigkraftstoff als Antriebsenergie zu nutzen (CHAU/WONG 2002: 1954).

Dargestellt wird die Entwicklung des Faktors anhand der Anzahl der zugelassenen Elektrofahrzeuge in den USA.

Da Elektrofahrzeuge keinen oder nur einen geringeren Benzinverbrauch im Vergleich zu konventionellen Kraftfahrzeugen haben (ENVIRONMENTAL PROTECTION AGENCY 2012), sinkt mit steigender Anzahl an Elektrofahrzeugen die US-amerikanische Benzinnachfrage. Dies verschärft die aktuelle „Blend Wall“-Problematik und senkt die Gesamtmenge an Ethanol, die Benzin beigemischt werden kann, was zu einem Rückgang der Ethanolnachfrage führt.

Darüber hinaus könnte durch eine steigende Anzahl an Elektrofahrzeugen die Bedeutung des gesellschaftlichen Themas „Energieunabhängigkeit der USA“230 und damit einhergehend die politische Unterstützung für Biokraftstoffe sinken. Dies ist damit zu begründen, dass sich die US-amerikanischen Bürger nicht mehr durch die rohölexportierenden Staaten erpressbar fühlen, weil sie durch die Elektromobilität eine Technologie besitzen, um Rohöl im Transportbereich zu substituieren.

Alle führenden Automobilkonzerne haben bereits erste Elektrofahrzeuge auf dem US-amerikanischen Markt und forschen intensiv an der weiteren Entwicklung $^{231}$. In der folgenden Abbildung 69 ist die von der U.S. ENERGY INFORMATION ADMINISTRATION (2013f) prognostizierte Entwicklung der Anzahl an Elektrofahrzeugen in den USA im Zeitraum von 2010 bis 2023 dargestellt.

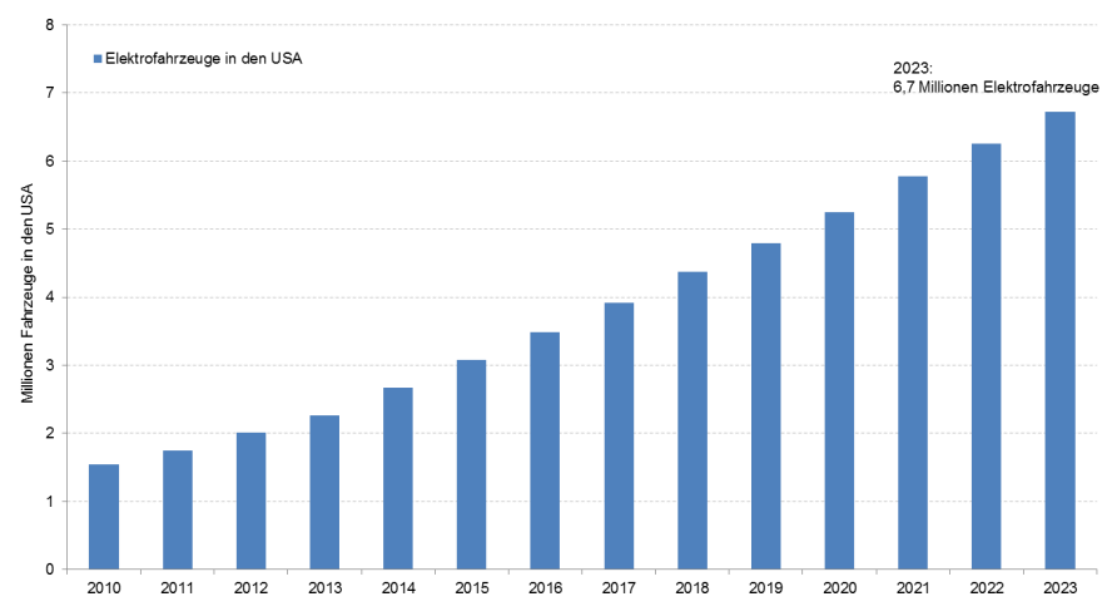

Abbildung 69: Entwicklung der Anzahl an Elektrofahrzeugen in den USA ${ }^{232}$ Quelle: U.S. ENERGY INFORMATION ADMINISTRATION $2013 f$

Aus Abbildung 69 wird ersichtlich, dass die Anzahl der Elektrofahrzeuge nach

${ }^{230}$ Das Thema „Energieunabhängigkeit der USA“ wird ausführlich in Kapitel 6.2.7 diskutiert. ${ }^{231}$ Folgende Automobilmarken bieten Elektrofahrzeuge auf dem US-amerikanischen Markt an: Acura, Audi, BMW, Buick, Cadillac, Chevrolet, Coda Automotive, Ford, Fiat, GMC, Honda, Hyundai, Infiniti, Kia, Lexus, Lincoln, Mercedes-Benz, Mercury, Nissan. Porsche, Scion, Smart, Subaru, Tesla, Toyota, Volkswagen, Wheego Electric Cars (U.S. DEPARTMENT OF ENERGY 2013g).

232 Der starke Anstieg der Anzahl an Elektrofahrzeugen ist auf das Wachstum der Hybridfahrzeuge und nicht auf das der Batteriefahrzeuge zurückzuführen. 
Prognosen der U.S. ENERGY INFORMATION ADMINISTRATION bis zum Jahr 2023 stetig auf 6,7 Millionen Fahrzeuge ansteigen wird; dies entspricht im Zeitraum von 2010 bis 2023 einem durchschnittlichen jährlichen Wachstum von $12,2 \%$

Trotz des erwarteten starken Wachstums der Zahl an Hybridfahrzeugen ist jedoch anzumerken, dass weiterhin konventionelle Kraftfahrzeuge ${ }^{233}$ den mit Abstand größten Anteil der prognostizierten 278 Millionen US-amerikanischen Kraftfahrzeuge im Jahr 2023 stellen werden (Kapitel 6.2.4).

Name:

Lfd. Nr.:

Einflussbereich:

Beschreibung:

Bedeutung des Einflussfaktors:
Enzym-Technologie zur Produktion von Zellulose-Ethanol

17

Technologie

Der Faktor dient der Darstellung der Enzym-Technologie zur Produktion von Zellulose-Ethanol in den USA. Enzyme werden im Produktionsprozess eingesetzt, um den Rohstoff Zellulose in C5- und C6-Zucker aufzuspalten, die im folgenden Schritt zu Ethanol fermentiert werden (NATIONAL RENEWABLE ENERGY LABORATORY 2007: 4; SUN/CHENG 2002: 6).

Zur Darstellung des Faktors wird die Ist-Situation der Enzym-Technologie als Referenzsituation beschrieben, um darauf basierend Zukunftsprojektionen zu bilden, die die Enzymtechnologie im Jahr 2023 beschreiben.

Eine weiterentwickelte Enzym-Technologie kann die Ausbeute an Zucker je eingesetzter Rohstoffmenge optimieren sowie die Freisetzung des Zuckers (C5 und C6) beschleunigen. Dadurch verbessern sich sowohl der Ethanolertrag im Verhältnis zur eingesetzten Rohstoffmenge als auch die Produktionsleistung der gesamten Anlage (NATIONAL RENEWABLE ENERGY LABORATORY 2007: 4).

Bedeutend wäre darüber hinaus eine Optimierung der Enzym-Technologie dahingehend, dass die gleichzeitige Verwendung unterschiedlicher Rohstoffe möglich ist. Dies würde die Rohstoffversorgung vereinfachen, insbesondere für Anlagen, die sich in Gebieten befinden, in denen sowohl Maisstroh als auch Weizenstroh als Rohstoff zur Verfügung stehen, wie beispielsweise im Norden von Kansas oder im Süden von Nebraska (U.S. DEPARTMENT OF ENERGY/OAK RIDGE NATIONAL LABORATORY 2011: 29ff.). Dies gäbe den Anlagenbetreibern die Möglichkeit, den jeweils günstigeren Rohstoff einzukaufen und dadurch die Produktionskosten zu senken ${ }^{234}$, und würde die Abhängigkeit von den rohstoffliefernden Landwirten mindern.

Die Entwicklung der Enzym-Technologie könnte auch dazu führen, dass die

${ }^{233}$ Konventionelle Kraftfahrzeuge sind Fahrzeuge, die fossilen Kraftstoff als Antriebsenergie nutzen (U.S. ENERGY INFORMATION ADMINISTRATION 2013f).

${ }_{234}$ Die Rohstoffkosten sind der wichtigste Kostenfaktor bei der Produktion von ZelluloseEthanol in den USA (F.O. LICHT 2010c: 453). 
Kosten für den regelmäßigen Kauf der Enzyme sänken. Dies ist von Interesse, da die Enzyme zu den wichtigsten Kostenfaktoren bei der Produktion von Zellulose-Ethanol gehören (F.O. LICHT 2010c: 453).

Name:

Lfd. Nr.:

Einflussbereich:

Beschreibung:

Bedeutung des

Einflussfaktors:
Treibhausgasbilanz von Mais-Ethanol

18

Technologie

Der Faktor betrachtet die Lebenszyklustreibhausgasbilanz von Mais-Ethanol, produziert und verbraucht in den USA. In der Berechnung der Treibhausgasbilanz werden direkte und indirekte $\mathrm{CO}_{2}$-Emissionen ${ }^{235}$ von Ethanol-Kraftstoff berücksichtigt. Beschrieben wird der Faktor anhand der prozentualen Treibhausgasreduktion im Vergleich zu fossilem Benzin ${ }^{236}$.

Da Mais-Ethanol eine 20-prozentige $\mathrm{CO}_{2}$-Reduktion im Vergleich zu fossilem Benzin erreichen muss, um dem Verbrauchsmandat „Conventional Biofuel“ des RFS 2 angerechnet zu werden, ist die Betrachtung des Faktors wichtig (Abbildung 70) (U.S. GOVERNMENT INFORMATION 2007: 121 STAT. 1522; CONGRESSIONAL RESEARCH SERVICE 2012: 6; CONGRESSIONAL RESEARCH SERVICE 2010c: 6). Verfehlt Mais-Ethanol die politisch geforderte prozentuale Treibhausgasreduktion, so wird der Kraftstoff nicht politisch gefördert und steht dadurch in direkter Konkurrenz zu fossilem Benzin. Aufgrund des zu erwartenden Rückgangs der US-amerikanischen Benzinnachfrage (Einflussfaktor 1) (U.S. ENERGY INFORMATION ADMINISTRATION 2012b: 98) ist davon auszugehen, dass die US-amerikanische Mineralölindustrie auf eine freiwillige Beimischung von Ethanol zu Benzin verzichten wird, um den abnehmenden Benzinabsatz nicht noch stärker zu senken.

Des Weiteren ist infolge einer zu geringen $\mathrm{CO}_{2}$-Reduktion auch ein Ethanolexport in die EU27 nicht möglich, da dort eine Treibhausgasreduktion von mindestens $35 \%$ im Vergleich zu Fossilbrennstoff politisch gefordert ist ${ }^{237}$. Möglich wäre allerdings ein Export nach Brasilien, Asien oder in den mittleren Osten (Einflussfaktor 4 und 6).

\footnotetext{
${ }^{235}$ In der Betrachtung der indirekten $\mathrm{CO}_{2}$-Emissionen wird auch der Treibhausgasausstoß infolge indirekter Landnutzungsänderungen (Indirect Land Use Change) berücksichtigt (U.S. GOVERNMENT INFORMATION 2007: 121 STAT. 1520; CONGRESSIONAL RESEARCH SERVICE 2012: 6; CONGRESSIONAL RESEARCH SERVICE 2010c: 7).

${ }^{236}$ Der Vergleichsmaßstab bezieht sich auf die Lebenszyklustreibhausgasbilanz von fossilem Benzin aus dem Jahr 2005 (CONGRESSIONAL RESEARCH SERVICE 2012: 6).

${ }_{237}$ In der RICHTLINIE 2009/28/EC ARTIKEL 17 (2) sind die politisch geforderten $\mathrm{CO}_{2}$ Reduktionswerte für Biokraftstoffe im Vergleich zu fossilem Kraftstoff festgelegt: Bis zum Jahr 2017 müssen alle Biokraftstoffe eine Kohlenstoffdioxid-Einsparung von 35\% erreichen. Ab dem 1. Januar 2017 erhöht sich die geforderte Reduktion auf 50\% und vom 1. Januar 2018 an müssen alle Produktionsanlagen, die am oder nach dem 1. Januar 2017 die Produktion aufgenommen haben, eine $\mathrm{CO}_{2}$-Minderung von $60 \%$ erreichen.
} 
Name:

Lfd. Nr.:

Einflussbereich:

Beschreibung:

Bedeutung des

Einflussfaktors:
Treibhausgasbilanz von Zellulose-Ethanol

19

Technologie

Es wird die Lebenszyklustreibhausgasbilanz von Zellulose-Ethanol betrachtet, welches in den USA produziert und verbraucht wird. In der Ermittlung der Treibhausgasbilanz werden sowohl die direkten als auch die indirekten Treibhausgasemissionen berücksichtigt, was auch die Anrechnung von $\mathrm{CO}_{2-}$ Emissionen infolge indirekter Landnutzungsänderungen (ILUC) beinhaltet. Dargestellt wird der Faktor mittels der prozentualen $\mathrm{CO}_{2}$-Reduktion im Vergleich zu fossilem Benzin ${ }^{238}$.

Die Höhe der prozentualen $\mathrm{CO}_{2}$-Reduktion im Vergleich zu Benzin ist für Zellulose-Ethanol entscheidend, da die Politik im RFS 2 entsprechende Anforderungen an Biokraftstoffe formuliert hat ${ }^{239}$.

Damit Zellulose-Ethanol dem Verbrauchsmandat „Advanced: Cellulosic Biofuel“ - mit 60,5 Milliarden Litern das größte Mandat (Einflussfaktor 23) - angerechnet werden kann, muss es mindestens eine 60-prozentige Treibhausgasreduktion im Vergleich zu fossilem Benzin erreichen (U.S. GOVERNMENT INFORMATION 2007: 121 STAT. 1520ff.). Erfüllt Zellulose-Ethanol jedoch lediglich eine $\mathrm{CO}_{2}$-Einsparung zwischen $59 \%$ und $50 \%$, so kann der Biokraftstoff nur der Untergruppe „Advanced: Unspecified Biofuel“ angerechnet werden, die mit 15 Milliarden Litern deutlich kleiner ist, und steht in direkter Konkurrenz zu beispielsweise Zuckerrohr-Ethanol aus Brasilien oder US-amerikanischem Ethanol, produziert aus Sorghum (U.S. GOVERNMENT INFORMATION 2007: 121 STAT. 1519ff.). Falls auch keine 50-prozentige, sondern nur eine 20prozentige Treibhausgaseinsparung erreicht wird, so dient Zellulose-Ethanol lediglich der Erfüllung des Verbrauchsmandats „Conventional Biofuel“ und steht damit in Konkurrenz zu Mais-Ethanol (U.S. GOVERNMENT INFORMATION 2007: 121 STAT. 1520ff.).

Verfehlt Zellulose-Ethanol auch eine 20-prozentige $\mathrm{CO}_{2}$-Einsparung, so kann der Kraftstoff keinem Verbrauchsmandat des RFS 2 angerechnet werden. Damit besäße es für die Mineralölindustrie keinen politischen Wert und stünde in direkter Konkurrenz zu fossilem Benzin (U.S. GOVERNMENT INFORMATION 2007: 121 STAT. 1520ff.).

${ }^{238}$ Der Vergleichswert bezieht sich wie bei Einflussfaktor 18 auf die Lebenszyklustreibhausgasbilanz von fossilem Benzin, basierend auf den Werten des Jahres 2005 (CONGRESSIONAL RESEARCH SERVICE 2012: 6).

${ }_{239}$ In Einflussfaktor 23 sind die im RFS 2 beschriebenen politischen Anforderungen an die $\mathrm{CO}_{2}$-Reduktion von Biokraftstoffen im Verhältnis zu fossilen Kraftstoffen dargestellt. 
Name:

Lfd. Nr.:

Einflussbereich:

Beschreibung:

Bedeutung des

Einflussfaktors:
Nachfrage nach BtL in den USA

20

Technologie

BtL (Biomass-to-Liquid) ist ein synthetischer Kraftstoff, der aus Zucker, Stärke, Zellulose und Lignin produziert werden kann. Er kann problemlos Benzin beigemischt werden und die Motoren der Kraftfahrzeuge sind in der Lage, den Biokraftstoff in hohen Beimischungsmengen zu Benzin als Kraftstoff zu verwenden (U.S. DEPARTMENT OF ENERGY 2013d; EUROPEAN BIOFUELS 2013b; FNR 2009: 83ff.). Beschrieben wird die Entwicklung der US-amerikanischen BtL-Nachfrage in Milliarden Litern pro Jahr.

Der Biokraftstoff BtL kann abhängig vom verwendeten Rohstoff und der erzielten $\mathrm{CO}_{2}$-Reduktion im Vergleich zu fossilem Benzin den unterschiedlichen Verbrauchsmandaten - „Advanced: Cellulosic Biofuel“, „Advanced: Unspecified Biofuel“ und "Conventional Biofuel“ - des RFS 2 angerechnet werden (Abbildung 70). Aus diesem Grund und da BtL Benzin beigemischt werden kann, ist der Biokraftstoff in der Lage, Ethanol-Kraftstoff zu substituieren (U.S. GOVERNMENT INFORMATION 2007: 121 STAT. 1519ff.; U.S. DEPARTMENT OF ENERGY 2013d).

Darüber hinaus besitzt BtL gegenüber Ethanol den Vorteil, dass er weder hygroskopisch noch korrodierend wirkt. So ist zum einen ein günstiger Transport des Kraftstoffs durch bereits bestehende Pipelines von den Produktionsstätten zu den urbanen Zentren an der Ost- und Westküste möglich; zum anderen und dies ist noch wichtiger - können die Zapfsäulen an den Tankstellen und die Ottomotoren der Kraftfahrzeuge Benzin mit hohen BtL-Beimischungen nutzen (U.S. DEPARTMENT OF ENERGY 2013d; EUROPEAN BIOFUELS 2013b; FNR 2009: 83ff.). Infolgedessen besteht bei der Verwendung von BtL keine „Blend Wall“-Problematik, die derzeit ein weiteres Wachstum des USamerikanischen Ethanolmarktes verhindert (F.O. LICHT 2013b: 213).

Trotz der hohen Kraftstoffqualität und des hohen Heizwertes von $\mathrm{BtL}^{240}$ gibt es bisher aber nur eine sehr geringe kommerzielle Produktion in den USA ${ }^{241}$, da die Produktionskosten im Vergleich zu Ethanol zu hoch sind (U.S. DEPARTMENT OF ENERGY 2013d; FNR 2009: 23).

Nachfrage nach Biobutanol in den USA

21

${ }^{240}$ Der Heizwert von BtL liegt bei 33,45 MJ/L und damit über dem von Benzin mit 32,1 MJ/L und Ethanol mit 21,2 MJ/L (MASSACHUSETTS INSTITUTE OF TECHNOLOGY 2007).

${ }^{241}$ Das Unternehmen KiOR produziert seit 2012 in Columbus (Mississippi) in einer ersten kleineren kommerziellen Anlage BtL aus Zellulose (KIOR 2013). 


Einflussbereich:

Bedeutung des

Einflussfaktors:
Technologie

Biobutanol $\left(\mathrm{C}_{4} \mathrm{H}_{10} \mathrm{O}\right)$ ist wie Ethanol $\left(\mathrm{C}_{2} \mathrm{H}_{6} \mathrm{O}\right)$ ein Alkohol, der durch die Fermentation von landwirtschaftlichen Rohstoffen gewonnen wird. Biobutanol kann in hohen prozentualen Mengen Benzin beigemischt und dennoch problemlos von Ottomotoren als Kraftstoff verwendet werden (U.S. DEPARTMENT OF ENERGY 2013c; EUROPEAN BIOFUELS 2013a; FNR 2009: 98f.). Dargestellt wird die jährliche US-amerikanische Biobutanolnachfrage in Milliarden Litern.

Es gilt diesen Faktor zu betrachten, da Ethanol-Kraftstoff durch Biobutanol substituiert werden kann, denn Biobutanol ist den unterschiedlichen Verbrauchsmandaten des RFS 2 - „Advanced: Cellulosic Biofuel“, „Advanced: Unspecified Biofuel“ und "Conventional Biofuel“ - anrechenbar und kann Benzin beigemischt werden ${ }^{242}$ (U.S. GOVERNMENT INFORMATION 2007: 121 STAT. 1519ff.; U.S. DEPARTMENT OF ENERGY 2013c).

Biobutanol besitzt darüber hinaus den Vorteil, dass die bereits zugelassenen US-amerikanischen Kraftfahrzeuge ohne technische Veränderungen Benzin mit Biobutanolbeimischungen bis $85 \%$ vol problemlos als Kraftstoff verwenden können. Auch der Verkauf dieses Kraftstoffs durch das bestehende Tankstellennetz ist möglich, da Biobutanol im Gegensatz zu Ethanol nicht korrodierend wirkt. Infolgedessen besteht bei der Verwendung von Biobutanol keine „Blend Wall“-Problematik, die derzeit ein weiteres Wachstum des US-amerikanischen Ethanolmarktes verhindert. Darüber hinaus ist ein kostengünstiger Transport durch das bereits bestehende US-amerikanische Pipelinenetz möglich, da Biobutanol auch nicht hygroskopisch wirkt (U.S. DEPARTMENT OF ENERGY 2013c; F.O. LICHT 2013b: 213).

Trotz der genannten Vorteile sowie des höheren Energiewertes von Biobutanol (27,2 MJ/L) im Vergleich zu Ethanol $(21,1 \mathrm{MJ} / \mathrm{L})$ und Unternehmen wie Butamax Advanced Biofuels LLC ${ }^{243}$ und Gevo ${ }^{244}$, die intensiv an der Weiterentwicklung der kommerziellen Produktion des Kraftstoffes arbeiten, gibt es bisher keine kommerzielle Biobutanolproduktion in den USA ${ }^{245}$. Grund dafür sind die hohen Produktionskosten, derentwegen Biobutanol im Vergleich zu Ethanol

242 Die Anrechnung auf die unterschiedlichen Verbrauchsmandate des RFS 2 erfolgt in Abhängigkeit vom zur Herstellung verwendeten Rohstoff und von der erzielten Treibhausgasreduktion im Vergleich zu fossilem Benzin (U.S. GOVERNMENT INFORMATION 2007: 121 STAT. 1519ff.).

${ }^{243}$ Butamax Advanced Biofuels LLC ist ein Joint Venture zwischen dem Mineralölkonzern BP und dem Chemieunternehmen DuPont. Ziel der Zusammenarbeit ist die wirtschaftlich rentable kommerzielle Produktion von Biobutanol, um sowohl den Biokraftstoff selbst zu produzieren als auch die Technologie an andere Unternehmen auszulizenzieren (BUTAMAX ADVANCED BIOFUELS 2013).

${ }^{244}$ Gevo ist ein US-amerikanisches Unternehmen, das an der Optimierung des Prozesses zur kommerziellen Produktion von Biobutanol arbeitet, um seine Technologie an andere Hersteller auszulizenzieren (GEVO 2013).

${ }^{245}$ Zwar gibt es in den USA noch keine kommerzielle Biobutanolproduktion, allerdings haben die beiden Ethanolproduzenten Highwater Ethanol LLC und Lincolnway Energy LLC eine Vereinbarung mit Butamax Advanced Biofuels LLC geschlossen, um die Ethanolanlage bis 2014 in eine Biobutanolanlage umzubauen (ETHANOL PRODUCER MAGAZINE 2012g; BLOOMBERG 2012b). 
noch nicht wettbewerbsfähig ist (FNR 2009: 98f.; MASSACHUSETTS INSTITUTE OF TECHNOLOGY 2007).

\section{Politik}

Name:

Biokraftstoff-Verbrauchsmandate - Renewable Fuel Standard 2 (RFS 2)

Lfd. Nr.:

22

Einflussbereich:

Politik

Beschreibung:

Der Renewable Fuel Standard 2 ist Bestandteil des „ENERGY INDEPENDENCE AND SECURITY ACT“ (EISA) aus dem Jahr 2007 (U.S. GOVERNMENT INFORMATION 2007: 121 STAT. 1519). In diesem sind bis zum Jahr 2022 jährlich ansteigende Mandate für Biokraftstoffe festgelegt, die im USamerikanischen Transportbereich verbraucht werden müssen ${ }^{246}$ (U.S. GOVERNMENT INFORMATION 2007: 121 STAT. 1522).

Die Biokraftstoffe werden im RFS 2 in die beiden Gruppen „Conventional Biofuels“ und „Advanced Biofuels“ unterteilt. Unterscheidungskriterium ist sowohl der Rohstoff, der zur Herstellung genutzt wird, als auch die prozentuale Höhe der Treibhausgasreduktion ${ }^{247}$ im Vergleich zu fossilem Kraftstoff. Wird der Rohstoff Mais zur Ethanolproduktion genutzt, so gehört der biogene Treibstoff der Gruppe der „Conventional Biofuels“ an. Wird ein anderer Rohstoff zur Herstellung verwendet, so kann dieser Biokraftstoff sowohl der Gruppe „Advanced“ als auch der Gruppe „Conventional“ angerechnet werden. Die Gruppe „Advanced Biofuels“ unterteilt sich erneut in verschiedene Unterkategorien - „Advanced: Cellulosic Biofuel“, „Advanced: Biomass-Based Diesel“ und „Advanced: Unspecified Biofuel“ - entsprechend des verwendeten Rohstoffs (Abbildung 70) (U.S GOVERNMENT INFORMATION 2007: 121 STAT. 1520) ${ }^{248}$.

${ }^{246}$ Die Überwachung der Erfüllung der Verbrauchsmandate erfolgt durch die U.S. Environmental Protection Agency (EPA) (CONGRESSIONAL RESEARCH SERVICE 2012: 2).

${ }^{247}$ In Einflussfaktor 23 wird umfassend auf das Kriterium der Treibhausgasreduktion eingegangen.

248 Die einzelnen Mandate im RFS 2 besitzen eine hierarchische Struktur: In der Gruppe der „Advanced Biofuels“ können Biokraftstoffe, die den Verbrauchsmandaten „Advanced: Cellulosic Biofuel“ und „Advanced: Biomass-Based Diesel“ angerechnet werden auch das Mandat „Advanced: Unspecified Biofuel“ erfüllen. Darüber hinaus können die „Advanced Biofuels" auch das Mandat der "Conventional Biofuels" erfüllen (U.S. GOVERNMENT INFORMATION 2007: 121 STAT. 1521ff.; CONGRESSIONAL RESEARCH SERVICE 2012: 4) 


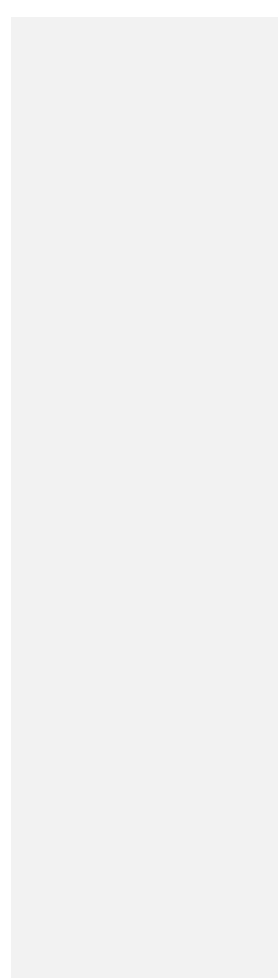

Bedeutung des Einflussfaktors:

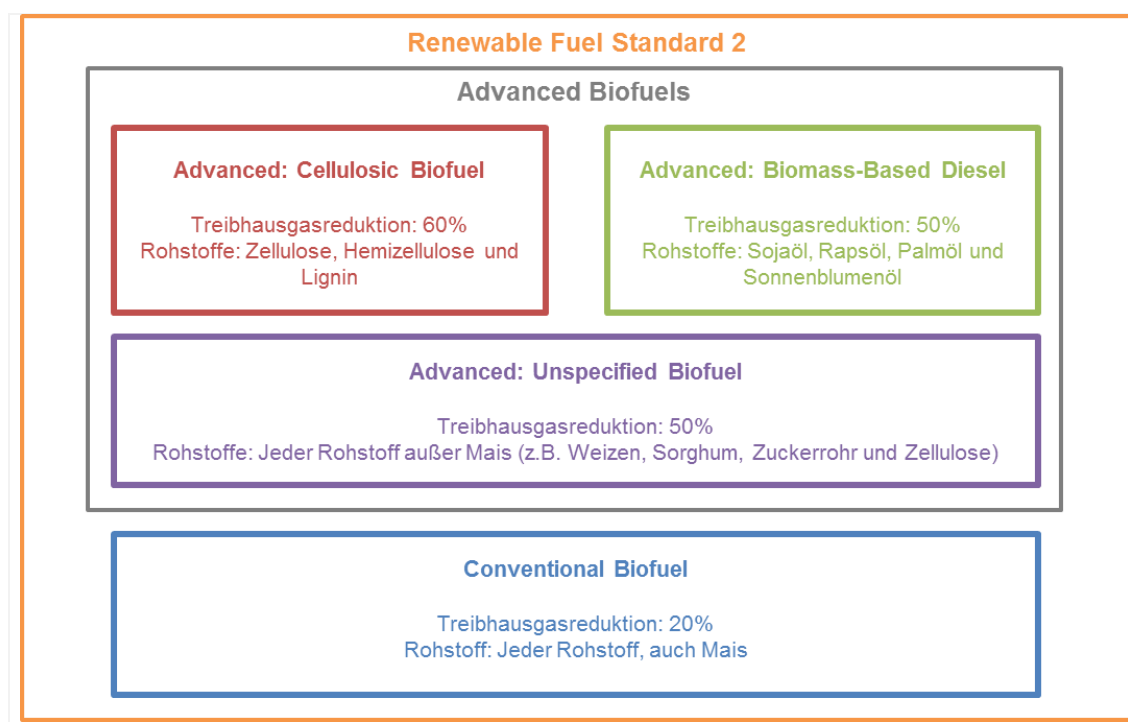

Abbildung 70: Struktur des RFS 2

Quelle: U.S. GOVERNMENT INFORMATION 2007: 121 STAT. 1519ff.; CONGRESSIONAL RESEARCH SERVICE 2012: 4

Die zukünftige Entwicklung des RFS 2 wird dargestellt, indem die Mandate jeder Gruppe und Unterkategorie in Milliarden Litern angegeben werden.

Die hohe Relevanz des wirkenden Einflussfaktors ist an der Entwicklung der US-amerikanischen Ethanolnachfrage zu erkennen (Abbildung 71). So hat sich diese im Zeitraum von 2008 bis 2010 entsprechend den Verbrauchsmandaten des RFS 2 entwickelt. Auf die Jahre 2011 und 2012 ist dies nur bedingt übertragbar, da die „Blend Wall“-Problematik ${ }^{249}$ einen weiteren Anstieg der Ethanolnachfrage verhinderte.

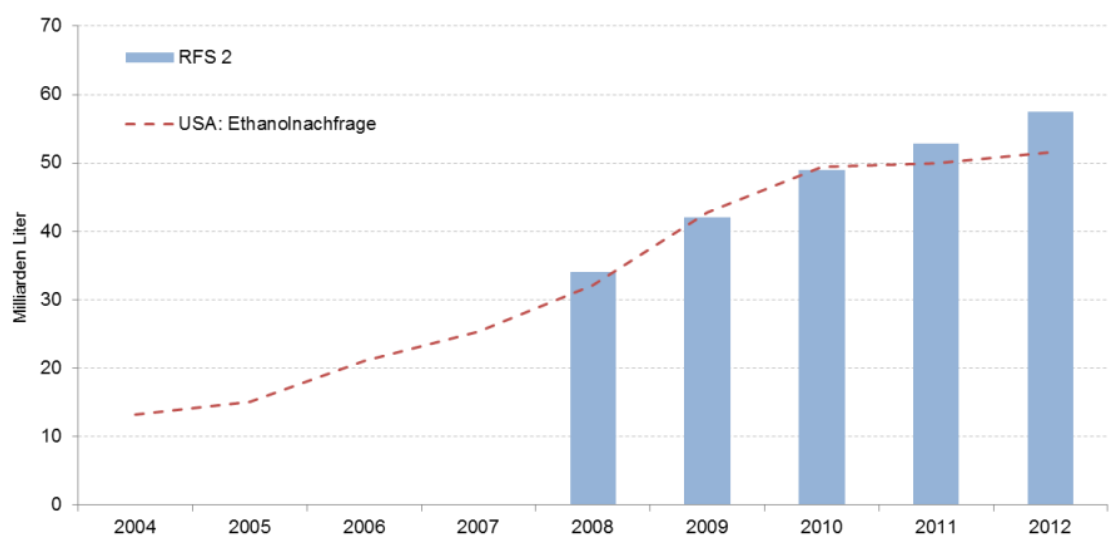

Abbildung 71: Einfluss des RFS 2 auf die Ethanolnachfrage Quelle: F.O. LICHT 2012f: 73f.; U.S. GOVERNMENT INFORMATION 2007: 121 STAT. 1519

Auch zukünftig wird der RFS 2 den US-amerikanischen Ethanolmarkt maßgeblich beeinflussen, da er die Verbrauchsmandate bis zum Jahr 2022 festlegt. Nach den Vorgaben des RFS 2 müsste es bis zum Jahr 2022 zu einem starken Anstieg der US-amerikanischen Biokraftstoffnachfrage auf 136

${ }^{249}$ Die „Blend Wall“ ist die maximale Menge Ethanol, die US-amerikanischem Benzin aus technischen Gründen beigemischt werden kann (CONGRESSIONAL RESEARCH SERVICE 2010a: 5; OECD/FAO 2012: 110). 


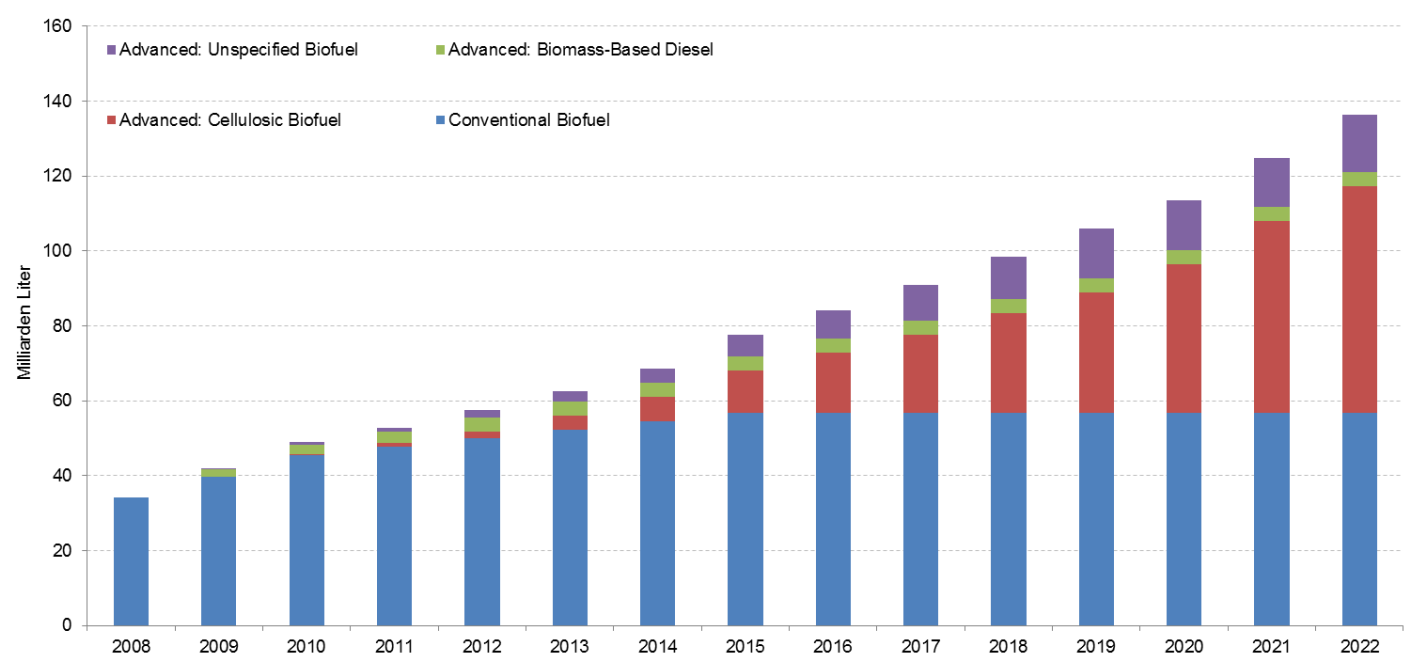

Abbildung 72: Entwicklung der Biokraftstoff-Verbrauchsmandate des RFS 2 Quelle: U.S. GOVERNMENT INFORMATION 2007: 121 STAT. 1522

Im RFS 2 bleibt zwar das Verbrauchsmandat für "Conventional Biofuel“ ab dem Jahr 2015 in Höhe von 57 Milliarden Litern konstant (Abbildung 72). Durch die jährliche Steigerung des Mandats „Advanced: Cellulosic Biofuel“ auf bis zu 61

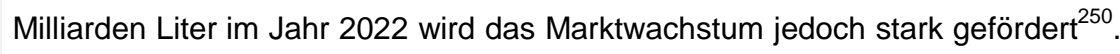
Des Weiteren ist für das Jahr 2022 ein Verbrauchsmandat in Höhe von 15 Milliarden Litern für die Untergruppe „Advanced: Unspecified Biofuel“ festgelegt.

Zusammenfassend kann festgehalten werden, dass der RFS 2 der entscheidende politische Treiber für die zukünftige Entwicklung des US-amerikanischen Ethanolmarktes sein wird. Allerdings ist anzumerken, dass die derzeitige „Blend Wall“-Problematik (F.O. LICHT 2013b: 213), der langsame Ausbau der Produktionskapazitäten zur Herstellung von Zellulose-Ethanol und die schwindende Unterstützung der US-amerikanischen Politik für Biokraftstoffe zu einer Absenkung des RFS 2 führen könnten (F.O. LICHT 2012c: 319).

Name:

Treibhausgasreduktionswerte für Biokraftstoffe - Renewable Fuel Standard 2 (RFS 2)

Lfd. Nr.:

Einflussbereich:

Beschreibung:
23

Politik

Wie bereits in Einflussfaktor 22 beschrieben, unterteilt der RFS 2 die Biokraftstoffe in unterschiedliche Gruppen. Die Unterteilung erfolgt entsprechend dem

${ }^{250}$ Der Markt für „Advanced: Cellulosic Biofuel“ im Jahr 2022 wird für sich alleine größer sein als der derzeitige US-amerikanische und europäische Ethanolmarkt zusammen. 
Bedeutung des

Einflussfaktors: zur Herstellung genutzten Rohstoff und der prozentualen Treibhausgasreduktion im Vergleich zu jenem fossilen Kraftstoff, den der Biokraftstoff substituiert ${ }^{251}$ (CONGRESSIONAL RESEARCH SERVICE 2012: 6). In Abbildung 70 ist unter anderem die geforderte prozentuale Kohlenstoffdioxidreduktion der einzelnen Biokraftstoffgruppen dargestellt.

Die Entwicklung des Faktors wird mittels der politisch geforderten prozentualen $\mathrm{CO}_{2}$-Reduktion der einzelnen Biokraftstoffgruppen im Vergleich zum ersetzten fossilen Kraftstoff angegeben ${ }^{252}$.

Nur, wenn die Biokraftstoffe die politisch geforderte prozentuale $\mathrm{CO}_{2}$-Reduktion im Vergleich zu fossilem Kraftstoff erreichen, dürfen sie dem jeweiligen Verbrauchsmandat angerechnet werden ${ }^{253}$. Dies begründet die Bedeutung des wirkenden Einflussfaktors.

Somit darf ein Biokraftstoff, der aus Zellulose, Hemizellulose oder Lignin produziert wird, nur dem Verbrauchsmandat „Advanced: Cellulosic Biofuel“ angerechnet werden, wenn er eine Treibhausgasreduktion von mindestens 60\% erreicht (U.S. GOVERNMENT INFORMATION 2007: 121 STAT. 1520). Erfüllt ein Biokraftstoff eine 50-prozentige $\mathrm{CO}_{2}$-Einsparung und ist er nicht aus dem Rohstoff Mais produziert worden, so ist er der Untergruppe „Advanced: Unspecified Biofuel“ anzurechnen. Biogene Treibstoffe, die ebenfalls eine 50prozentige Treibhausgasreduktion erreichen und aus den Rohstoffen Sojaöl, Rapsöl, Palmöl oder Sonnenblumenöl produziert wurden, können darüber hinaus noch der Untergruppe „Advanced: Biomass-Based Diesel“ angerechnet werden (U.S. GOVERNMENT INFORMATION 2007: 121 STAT. 1519).

Biogene Kraftstoffe, welche unabhängig vom verwendeten Rohstoff nur eine 20-prozentige Treibhausreduktion erfüllen, dienen noch der Erfüllung des Verbrauchsmandats „Conventional Biofuels“ (U.S. GOVERNMENT INFORMATION 2007: 121 STAT. 1522). Biokraftstoffe, die nicht einmal eine 20-prozentige Treibhausgasreduktion erreichen, dürfen keinem Verbrauchsmandat mehr angerechnet werden. Sie besitzen daher für die Mineralölindustrie keinen politischen Wert und stehen somit in direkter Konkurrenz zu fossilem Kraftstoff.

Höhe der genehmigten prozentualen Beimischung von Ethanol zu Benzin

24

Einflussbereich: Politik

${ }^{251}$ Die $\mathrm{CO}_{2}$-Emissionen durch „Indirect Land Use Changes“ (ILUC) werden in den USA seit dem Jahr 2010 berücksichtigt (CONGRESSIONAL RESEARCH SERVICE 2012: 6).

${ }^{252}$ Als Vergleichsmaßstab dient die Lebenszyklustreibhausgasbilanz von fossilem Kraftstoff aus dem Jahr 2005 (CONGRESSIONAL RESEARCH SERVICE 2012: 6).

${ }^{253}$ Die Voraussetzungen des verwendeten Rohstoffs für die Anrechnung auf das jeweilige Verbrauchsmandat (Abbildung 70) werden in Einflussfaktor 22 betrachtet. 
Beschreibung:

Bedeutung des

Einflussfaktors:

Der Faktor betrachtet die von der United States Environmental Protection Agency (EPA) genehmigte Höhe der prozentualen Beimischung von Ethanol zu Benzin. Die Entwicklung des wirkenden Einflussfaktors wird in Volumenprozent angegeben.

Da der US-amerikanische Ethanolabsatz ${ }^{254}$ als Beimischung zu Benzin erfolgt (U.S. ENERGY INFORMATION ADMINISTRATION 2012j: 29), beeinflussen die Benzinnachfrage (Einflussfaktor 1) und die von der EPA erlaubte Höhe der Ethanolbeimischung zu Benzin entscheidend die Größe des USamerikanischen Ethanolmarktes. Derzeit erlaubt die EPA für Kraftfahrzeuge ab dem Baujahr 2001 eine 15-prozentige (E15) Ethanolbeimischung zu Benzin, ältere Kraftfahrzeuge dürfen jedoch weiterhin nur E10 tanken (U.S. GOVERNMENT INFORMATION 2011: 4662; EPA 2012b).

In Abhängigkeit von der prognostizierten Entwicklung der US-amerikanischen Benzinnachfrage ist in Abbildung 73 dargestellt, welche Beimischungshöhe von Ethanol zu Benzin von der EPA erlaubt sein müsste, damit die im RFS 2 beschriebenen Verbrauchsmandate ${ }^{255}$ (Einflussfaktor 22) erfüllt werden könnten. Der Begriff „Blend Wall“ in Abbildung 73 beschreibt dabei die in Abhängigkeit von der erlaubten Beimischungshöhe maximal mögliche Ethanolbeimischung zu Benzin $^{256}$.

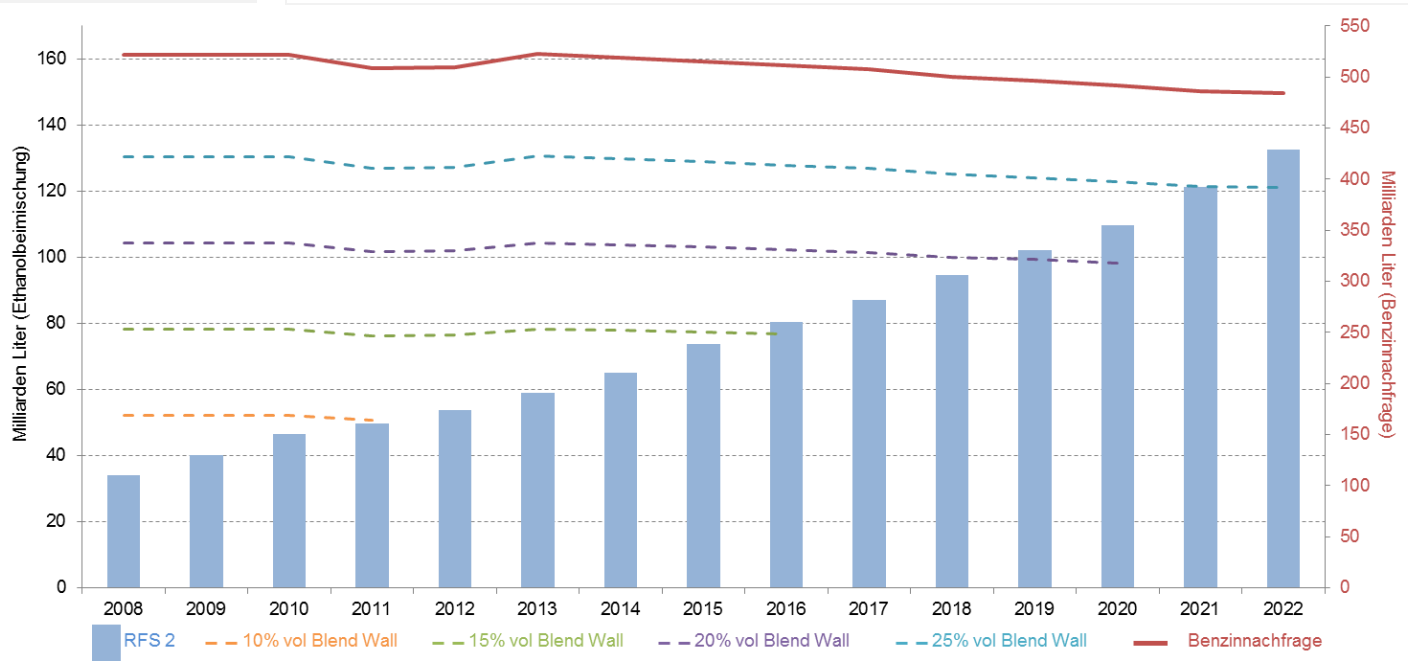

Abbildung 73: Bedeutung der erlaubten Höhe der Beimischung von Ethanol zu Benzin, um die Verbrauchsmandate des RFS 2 zu erfüllen

Quelle: U.S. GOVERNMENT INFORMATION 2007: 121 STAT. 1519ff.; U.S. ENERGY INFORMATION ADMINISTRATION 2012b: 98; EIGENE BERECHNUNG

Aus Abbildung 73 ist ersichtlich, dass die derzeit erlaubte Beimischungshöhe von $15 \%$ vol nicht ausreichend ist, um einen Ethanolabsatz von 132 Milliarden Litern im Jahr 2022 zu erreichen. Basierend auf der prognostizierten Benzin-

${ }^{254}$ Im Jahr 2012 wurden 99\% des Ethanolabsatzes als E10 verkauft (U.S. ENERGY INFORMATION ADMINISTRATION 2012j: 29).

${ }^{255}$ Das Verbrauchsmandat für „Advanced: Biomass-Based Diesel“ ist in Abbildung 73 nicht berücksichtigt worden, da ausschließlich die Beimischung von Ethanol zu Benzin betrachtet wird.

${ }^{256}$ Bei der Berechnung der „Blend Wall“ wird der mögliche Ethanolabsatz als E85 (85\% Ethanol und $15 \%$ Benzin) an FFV nicht berücksichtigt. 
nachfrage und einer weiterhin erlaubten Beimischung von $15 \%$ vol Ethanol zu Benzin würde die „Blend Wall“ im Jahr 2016 erreicht werden. Grund dafür ist eine maximal mögliche Ethanolbeimischung von 77 Milliarden Litern, aber ein Verbrauchsmandat von bereits 80 Milliarden Litern. Des Weiteren ist ersichtlich, dass auch eine erlaubte Beimischungshöhe von $20 \%$ vol oder $25 \%$ vol nicht ausreichend wären, um im Jahr 2022 eine Ethanolbeimischung von 132 Milliarden Litern zu erreichen. Erst eine Beimischungshöhe von $30 \%$ vol würde dies zulassen $^{257}$.

Aus der Betrachtung wird deutlich, dass die EPA die erlaubte Beimischungshöhe stark anheben muss, damit die Verbrauchsmandate des RFS 2 erfüllt werden können.

\begin{tabular}{l|l} 
Name: & Low Carbon Fuel Standard (LCFS) \\
\hline Lfd. Nr.: & 25 \\
\hline Einflussbereich: & Politik
\end{tabular}

Beschreibung:

Bedeutung des Der LCFS wurde im Jahr 2007 von Gouverneur Arnold Schwarzenegger eingeführt, um den $\mathrm{CO}_{2}$-Ausstoß im kalifornischen Verkehrssektor zu senken. Um dies zu erreichen, dürfen Kraftstoffe jährlich sinkende Standardwerte hinsichtlich des $\mathrm{CO}_{2}$-Ausstoßes je Megajoule $\left(\mathrm{gCO}_{2 e q} / \mathrm{MJ}\right)$ nicht überschreiten (CONGRESSIONAL RESEARCH SERVICE 2010b: 30; CALIFORNIA ENERGY COMMISSION 2013). Die Entwicklung des Faktors wird anhand des politischen Standardwertes in der Einheit $\mathrm{gCO}_{2 \mathrm{eq}} / \mathrm{MJ}$ angegeben.

Der Faktor ist bedeutend, da die US-amerikanischen Ethanolproduzenten im Corn Belt, die Mais als Rohstoff verwenden, nicht die vom Staate Kalifornien geforderten $\mathrm{CO}_{2}$-Standardwerte erfüllen ${ }^{258}$ (CONGRESSIONAL RESEARCH SERVICE 2010b: 30; CALIFORNIA ENERGY COMMISSION 2007: 1ff.; CALIFORNIA AIR RESOURCES BOARD 2007: 19). Dabei ist zu bedenken, dass die US-amerikanische Ethanolproduktion fast ausschließlich im Corn Belt und fast nur aus dem Rohstoff Mais erfolgt (U.S. ENERGY INFORMATION ADMINISTRATION 2013b: 1; U.S. ENERGY INFORMATION ADMINISTRATION 2012j: 6ff.)

Im Jahr 2012 lag der kalifornische Standardwert bei 95,37 $\mathrm{gCO}_{2 \mathrm{eq}} / \mathrm{MJ}$, während Berechnungen der CALIFORNIA ENERGY COMMISSION (2007: 1ff.) zufolge

${ }^{257}$ Weitere Voraussetzungen, damit eine Beimischung von 132 Milliarden Litern Ethanol realistisch ist, ist eine flächendeckende Tankstelleninfrastruktur, um diesen Kraftstoff in den USA zu verkaufen (Einflussfaktor 8). Außerdem müssen die Kraftfahrzeuge die hohe Beimischung von Ethanol zu Benzin vertragen können (Einflussfaktor 15) und schließlich müssen die Verbraucher gewillt sein, den Kraftstoff zu tanken.

${ }^{258}$ Die Ethanolanlagen in Kalifornien, die ebenfalls Mais als Rohstoff verwenden, erfüllen allerdings die Anforderungen des LCFS (CALIFORNIA ENERGY COMMISSION 2007: 1). 
die $\mathrm{CO}_{2}$-Emissionen der Ethanolproduktion im Corn Belt $99,4 \mathrm{gCO}_{2 e q} / \mathrm{MJ}$ betragen ${ }^{259}$. Folglich besteht für die kalifonische Mineralölindustrie kein Anreiz, das Ethanol aus dem Corn Belt Benzin beizumischen, da es die Erfüllung des LCFS verhindert. Stattdessen wird Zuckerrohr-Ethanol aus Brasilien importiert, da dieses einen $\mathrm{CO}_{2}$-Ausstoß von lediglich $73,4 \mathrm{~g} \mathrm{CO}_{2 \text { eq }}$ je Megajoule hat ${ }^{260}$ (CALIFORNIA ENERGY COMMISSION 2007: 5). Auch zukünftig wird sich an der Situation nichts ändern, da die Vorgaben des Staates Kalifornien mit jedem Jahr ansteigen: So ist ab dem Jahr 2020 ein Standardwert von 89,09 $\mathrm{gCO}_{2 e q} / \mathrm{MJ}$ zu erfüllen (CALIFORNIA AIR RESOURCES BOARD 2007: 19). Damit entgeht der Ethanolindustrie im Corn Belt der wichtigste USamerikanische Ethanolmarkt, denn Kalifornien besitzt den größten Benzinmarkt in den USA und eine jährliche Ethanolnachfrage von 5,6 Milliarden Litern (CONGRESSIONAL RESEARCH SERVICE 2010b: 30).

Darüber hinaus ist der wirkende Einflussfaktor bedeutend, da Bundesstaaten an der nördlichen Ostküste, wie beispielsweise New York, Massachusetts oder Connecticut, und die Staaten Oregon und Washington an der Westküste an der Einführung eines Low Carbon Fuel Standard nach dem Vorbild von Kalifornien arbeiten (CENTER FOR CLIMATE AND ENERGY SOLUTIONS 2013).

\section{Gesellschaft}

Name:

Tank-oder-Teller-Diskussion in den USA

Lfd. Nr.:

26

Einflussbereich:

Gesellschaft

Beschreibung:

Der Begriff „Tank-oder-Teller-Diskussion“ steht für die Debatte um die Verwendung landwirtschaftlicher Rohstoffe zur Produktion von Biokraftstoffen anstatt zur Nutzung als Nahrungsmittel (INTERNATIONAL ENERGY AGENCY 2011b: 40). In der Betrachtung dieses Faktors geht es nicht um die Klärung, ob die „Tank-oder-Teller-Diskussion“ wissenschaftlich korrekt ist, sondern ausschließlich um die Darstellung der Intensität der Debatte in den USA.

Dargestellt wird der Faktor, indem die Ist-Situation analysiert und als Referenzsituation beschrieben wird, um darauf basierend Projektionen zu bilden, die aufzeigen, ob die Debatte zunimmt, abnimmt oder konstant bleibt.

${ }^{259}$ Bei der Ermittlung der $\mathrm{CO}_{2}$-Emissionen der Biokraftstoffe werden die Treibhausgasemissionen infolge von "Indirect Land Use Change" berücksichtigt (CALIFORNIA ENERGY COMMISSION 2013).

${ }^{260}$ In Einflussfaktor 3 wird der Import von Zuckerrohr-Ethanol aus Brasilien in die USA betrachtet. 
Bedeutung des

Einflussfaktors:
Der US-amerikanische Ethanolmarkt steht als der mit Abstand größte Biokraftstoffmarkt der Welt im Zentrum der „Tank-oder-Teller-Diskussion“ ${ }^{261}$ (F.O. LICHT 2012f: 74).

Der Einflussfaktor ist von Interesse für die zukünftige Entwicklung des USamerikanischen Ethanolmarktes, da diese Diskussion die politische Unterstützung für Biokraftstoffe beeinflusst (F.O. LICHT 2012f: 68). Bereits in den Jahren 2007/2008 und 2012 gab es in den USA infolge ansteigender Agrarpreise eine starke „Tank-oder-Teller-Diskussion“. Während dieser Debatte forderten sogar der texanische Gouverneur Rick Perry im Jahr 2008 und Beverly Perdue, Gouverneurin von North Carolina im Jahr 2012, eine Aufhebung des RFS 2 (F.O. LICHT 2012g: 490; F.O. LICHT 2012f: 70). Zwar wurden beide Einwände von der EPA zurückgewiesen und auch die „Tank-oder-Teller-Diskussion“ schwächte sich nach einigen Monaten wieder ab, trotzdem blieb aufgrund der Debatte bei den Bürgern und den Politikern ein negativer Eindruck im Zusammenhang mit Biokraftstoffen zurück, der langfristig die politische Unterstützung für Ethanol schwächt (F.O. LICHT 2012f: 68).

Auch die OECD/FAO (2011: 87) weist darauf hin, dass es im Falle eines weiteren Wachstums des Biokraftstoffmarktes zu einer stärkeren „Tank-oder-TellerDiskussion“ kommen könnte. Die mögliche Folge daraus wäre eine Kürzung der Förderung von Biokraftstoffen durch die Politik.

Diskussion in den USA über die Auswirkungen von Ethanol auf die Umwelt

27

Lfd. Nr.:

Einflussbereich:

Gesellschaft

Beschreibung:

Bedeutung des

Einflussfaktors:
In diesem Faktor wird sowohl die Richtung als auch die Intensität der USamerikanischen Diskussion über die Auswirkungen von Ethanol auf die Umwelt begutachtet. Nicht betrachtet wird jedoch, ob die Disskussion wissenschaftlich korrekt ist.

Zur Darstellung des Faktors wird die Ist-Situation als Referenzwert beschrieben, um davon ausgehend unterschiedliche Projektionen für das Jahr 2023 zu bilden, die aufzeigen, wie sich die Diskussion in Richtung und Intensität verändert hat.

Die gesellschaftliche Diskussion über die Auswirkungen von Ethanol auf die Umwelt ist relevant, da die Reduktion des Treibhausgasausstoßes eines der Ziele der politischen Förderung von Biokraftstoffen durch den RFS 2 ist (U.S. GOVERNMENT INFORMATION 2007: 121 STAT. 1492). Auch die ObamaAdministration formuliert klar das Ziel der $\mathrm{CO}_{2}$-Reduktion im Kraftfahrzeugbe-

\footnotetext{
${ }^{261}$ Im Jahr 2011 wurden zur Herstellung von 52 Milliarden Litern Ethanol 124 Millionen Tonnen Mais verwendet. Dies entspricht $40 \%$ der US-amerikanischen Maisproduktion sowie einer Anbaufläche von 14 Millionen Hektar (F.O. LICHT 2012f: 74; U.S. ENERGY INFORMATION ADMINISTRATION 2012j: 8).
} 
reich (THE WHITE HOUSE 2013a; ETHANOL PRODUCER MAGAZINE 2013c). Folglich könnte sowohl eine Diskussion, in der Ethanol als umweltschützend verstanden wird, als auch eine Debatte, in der Ethanol als umweltschädlich gesehen wird, die zukünftige Ausgestaltung der politischen Förderung von Ethanol beeinflussen.

Des Weiteren kann die Richtung und die Intensität der Diskussion auch das Verhalten der Verbraucher ändern. Eine Interpretation von Ethanol als umweltschützend könnte Kraftfahrzeugbesitzer dazu bewegen, Benzin mit höheren Ethanolbeimischungen zu tanken. Eine Einschätzung von Ethanol als umweltschädlich wiederum könnte den Ethanolabsatz senken.

Name:

Lfd. Nr.:

Einflussbereich:

Beschreibung:

Bedeutung des

Einflussfaktors:
Diskussion über die Energieunabhängigkeit der USA

28

Gesellschaft

Der Faktor betrachtet die Diskussion und damit die Bedeutung des Themas „Energieunabhängigkeit der USA“ für die US-amerikanischen Bürger. Die Frage, ob die gesellschaftliche Bedeutung des Themas wissenschaftlich gerechtfertigt ist, wird nicht behandelt.

Dargestellt wird der Faktor, indem die Ist-Situation als Referenzsituation beschrieben wird, um davon ausgehend Projektionen zu bilden, die aufzeigen, ob die Bedeutung des Themas in Zukunft zunimmt, abnimmt oder konstant bleibt.

Der Faktor ist wichtig für die zukünftige Entwicklung des US-amerikanischen Ethanolmarktes, da das Kernziel der politischen Förderung von Biokraftstoffen die Verbesserung der Energieunabhängigkeit der USA ist. So ist es z.B. das primäre Ziel des „Energy Independence and Security Act“, in dem der RFS 2 beschrieben ist, „to move the United States toward greater energy independence and security" (U.S. GOVERNMENT INFORMATION 2007: 121 STAT. 1492). Ebenso verfolgt auch die Obama-Administration in ihrer Energiestrategie das Ziel einer höheren Energieunabhängigkeit (THE WHITE HOUSE 2013b). Folglich ist eine hohe gesellschaftliche Bedeutung des Themas „Energieunabhängigkeit der USA“ dem US-amerikanischen Ethanolmarkt dienlich, da es die derzeitige politische Förderung von Biokraftstoffen gesellschaftlich rechtfertigt und die zukünftige Ausgestaltung der politischen Förderung von EthanolKraftstoff positiv beeinflussen könnte. Andererseits könnte aber auch eine abnehmende gesellschaftliche Bedeutung des Themas die politischen Entscheidungsträger dazu bewegen, Biokraftstoffe in Zukunft weniger stark zu fördern. Ethanol-Kraftstoff ist mit dem Thema Energieunabhängigkeit stark verbunden, da Ethanol ein Substitut von Benzin ist und damit den Verbrauch sowie den Import von Rohöl in die USA reduzieren kann. Wichtig zu erwähnen ist dabei, dass das Thema „Energieunabhängigkeit der USA“ ausschließlich den Rohölimport betrifft, da die USA bei den restlichen Energieträgern - Erdgas, Kohle, 
erneuerbare Energien und Kernenergie - langfristige Selbstversorger sind ${ }^{262}$ (U.S. ENERGY INFORMATION ADMINISTRATION 2012e).

Name: Diskussion in den USA über den Nutzen von Ethanol für die US-amerikanische Wirtschaft

Lfd. Nr.:

Einflussbereich:

Beschreibung:

Bedeutung des

Einflussfaktors:
29

Gesellschaft

Bei diesem Faktor werden die Intensität und die Richtung der Diskussion über den Nutzen von Ethanol für die US-amerikanische Wirtschaft betrachtet. Jedoch wird der Aspekt, inwieweit die Debatte wissenschaftlich fundiert ist, nicht behandelt.

Dargestellt wird der Faktor, indem die Ist-Situation beschrieben wird, um darauf basierend Zukunftsprojektionen für das Jahr 2023 zu bilden.

Der Faktor ist von Bedeutung, da sowohl eine positive gesellschaftliche Diskussion „Ethanolproduktion fördert die US-amerikanische Wirtschaft" als auch eine negative Diskussion „Ethanolproduktion schadet der US-amerikanischen Wirtschaft" die Politiker in ihrer Einstellung zum Ethanol-Kraftstoff und damit auch die Ausgestaltung der zukünftigen politischen Förderung von Ethanol beeinflussen könnte.

Die Relevanz des Faktors zeigt sich des Weiteren an dem Verhalten der Interessenvertretungen der US-amerikanischen Ethanolindustrie - „Renewable Fuels Association“, „Growth Energy“ und „American Coalition for Ethanol“ -, die die Politik und die US-amerikanischen Bürger gezielt zu diesem Thema informieren. Sie weisen darauf hin, dass durch den Anstieg der US-amerikanischen Ethanolproduktion viele neue Beschäftigungsverhältnisse entstanden sind, insbesondere in den ruralen, ökonomisch schwach entwickelten Gebieten des Mittleren Westens ${ }^{263}$ (U.S. DEPARTMENT OF ENERGY 2013f).

Darüber hinaus könnte der Faktor auch die US-amerikanischen Kraftfahrzeugbesitzer beeinflussen, entsprechend der Richtung der Diskussion in Zukunft eine höhere oder geringere Ethanolbeimischung zu Benzin zu tanken.

Alle beschreibenden und wirkenden Einflussfaktoren des US-amerikanischen Ethanolmarktes sind in der folgenden Abbildung 74 zusammengefasst dargestellt.

${ }^{262}$ Angesichts der Tatsache, dass die USA in den anderen Energieträgern Selbstversorger sind, könnte man das Thema „Energieunabhängigkeit“ vereinfachend auch als „Rohölunabhängigkeit" bezeichnen.

${ }^{263}$ Nach Angaben der Renewable Fuels Association sind 365.000 neue Arbeitsplätze entstanden: 70.000 direkte Arbeitsplätze, 70.000 indirekte Arbeitsplätze (z.B. bei Anlagenherstellern) und 225.000 induzierte Arbeitsplätze in Branchen, die von den Ausgaben der Arbeitnehmer, die die direkten und indirekten Arbeitsplätze innehaben, profitieren (U.S. DEPARTMENT OF ENERGY 2013f). 


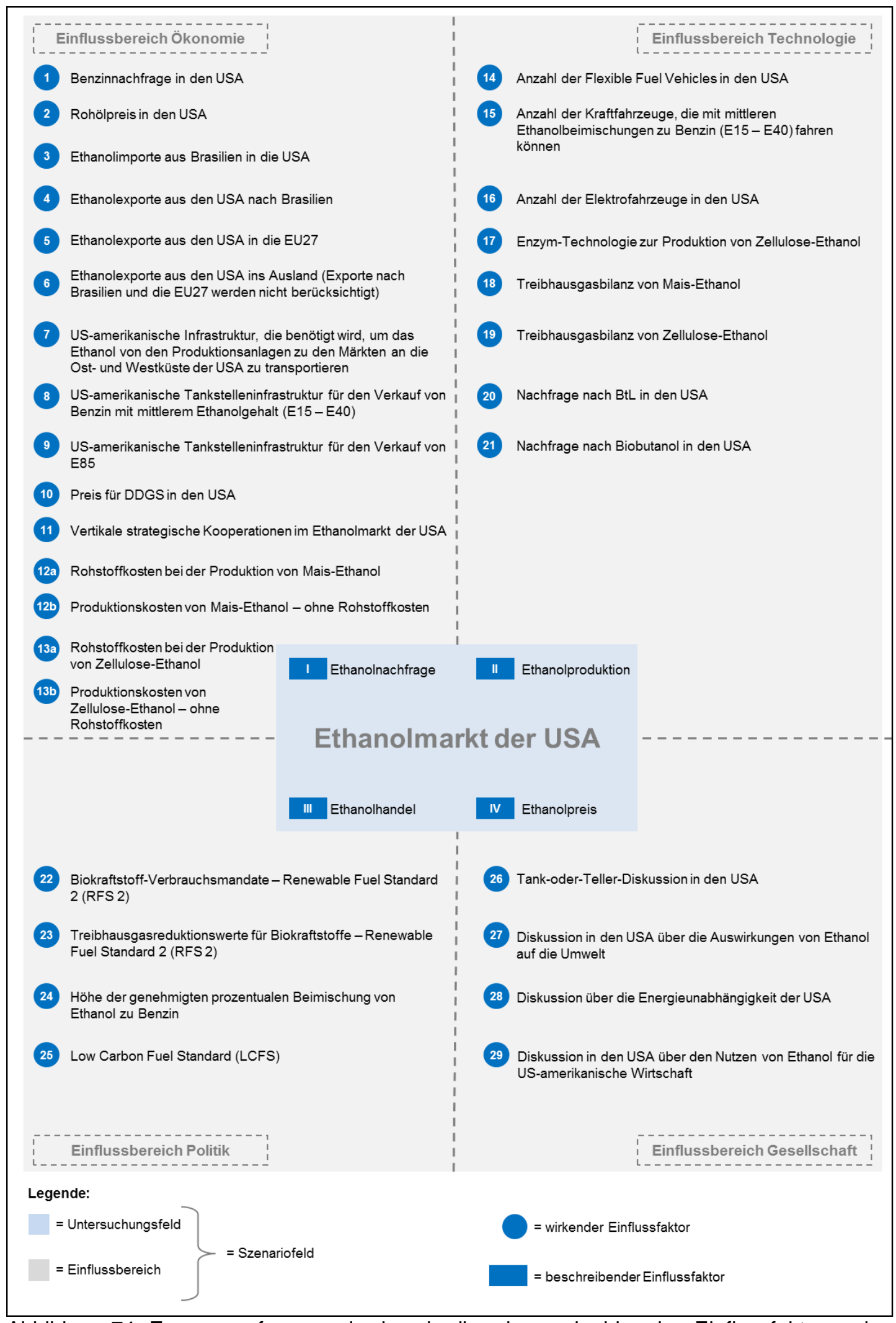

Abbildung 74: Zusammenfassung der beschreibenden und wirkenden Einflussfaktoren des USA-Szenariofeldes

Quelle: EIGENE DARSTELLUNG 


\subsubsection{USA Wirkungsanalyse}

Als Bestandteil der Szenariofeld-Analyse dient die Wirkungsanalyse ${ }^{264}$ der Bestimmung der wirkenden Schlüsselfaktoren, welche den US-amerikanischen Ethanolmarkt bis zum Jahr 2023 entscheidend beeinflussen werden.

\subsubsection{Durchführung der Wirkungsanalyse}

Mittels eines standardisierten schriftlichen Fragebogens wurde die Stärke der einzelnen auf den Ethanolmarkt der USA wirkenden Einflussfaktoren (Lfd. Nr. 1 bis 29) gemessen. Es ist zu beachten, dass der Fragebogen den identischen Aufbau wie der Fragebogen zur Durchführung der Wirkungsanalyse für den Ethanolmarkt der EU27 besitzt. Dies wurde bewusst so gehandhabt, da sich dieser als sehr geeignet erwiesen hat ${ }^{265}$. In Anhang 4 ist der Fragebogen für den US-amerikanischen Markt dargestellt. Er gliedert sich in drei Abschnitte:

1. Einführung in die Thematik

2. Beurteilung der wirkenden Einflussfaktoren

3. Erhebung der persönlichen Daten der Probanden

Im ersten Teil des Fragebogens ${ }^{266}$ wurde das Ziel des Fragebogens sowie der gesamten Studie erläutert. Des Weiteren wurde darauf hingewiesen, dass es sich um eine wissenschaftliche Arbeit handelt und die erhobenen Daten anonymisiert ausgewertet werden.

Der wichtigste Teil des Fragebogens befand sich im zweiten Abschnitt, in dem die einzelnen wirkenden Einflussfaktoren entsprechend ihrer Zugehörigkeit zu den Einflussbereichen aufgelistet waren. Jeder Einflussfaktor wurde benannt und, falls notwendig, mit einer kurzen Beschreibung versehen. Unterhalb jedes Faktors befand sich eine siebenstufige Likert-Skala ${ }^{267}$, mittels welcher die Probanden die Wirkungsstärke jedes Faktors auf die Entwicklung des US-amerikanischen Ethanolmarktes bis zum Jahr 2023 beurteilten.

Zum Abschluss der Befragung wurden die Experten nach dem Herkunftsland und der Branche des Unternehmens oder der Organisation, für welche sie arbeiteten,

${ }^{264}$ In Kapitel 4.2.2 befindet sich eine methodische Beschreibung der Wirkungsanalyse.

${ }^{265}$ Befragte Experten lobten den Aufbau und das Design des Fragebogens. Ferner gab es keine Rückfragen bezüglich der Fragestellung oder anderer Aspekte des Fragebogens.

${ }^{266}$ Da der Ethanolmarkt der USA im Mittelpunkt der Untersuchung steht und die Datenerhebung in den USA erfolgte, ist der Fragenbogen in englischer Sprache verfasst.

267 Die Likert-Skala besitzt die Abstufungen: „sehr starker Einfluss“; „starker Einfluss“; „eher starker Einfluss“; ,moderater Einfluss“; „eher schwacher Einfluss“; „,schwacher Einfluss“ und „sehr schwacher Einfluss“. Durch die Struktur der Likert-Skala sind eine einheitliche Datengewinnung und eine Vergleichbarkeit der Ergebnisse problemlos möglich. 
gefragt sowie gebeten, ihre persönlichen Kontaktdaten anzugeben, um ihnen die Ergebnisse der Erhebung zuschicken zu können.

Vor dem Start der Datenerhebung wurde der Fragebogen durch Pretests mit Experten des US-amerikanischen Ethanolmarktes ${ }^{268}$ überprüft. Da sich die Struktur des Fragebogens bei der Durchführung der Wirkungsanalyse für den Ethanolmarkt der EU27 bewährt hatte, lag der Schwerpunkt des Pretests auf der Prüfung der Eindeutigkeit der Fragestellung sowie der unmissverständlichen Beschreibung der einzelnen wirkenden Einflussfaktoren. Erkannte Schwächen am Fragebogen wurden vor dem Beginn der Erhebung ausgebessert.

Es wurden ausschließlich Experten des Ethanolmarktes der USA befragt, die sich hauptberuflich mit diesem Markt beschäftigten und zum Teil maßgeblich seine Entwicklung beeinflussen können. Um Experten aus allen Bereichen des USamerikanischen Ethanolmarktes befragen zu können, erfolgte die Datenerhebung im Rahmen des „International Fuel Ethanol Workshop“269 in Minneapolis (Minnesota) vom 4. bis zum 7. Juni 2012. Die jährlich stattfindende Veranstaltung wird von BBI International organisiert und ist zur Befragung sehr gut geeignet, da alle bedeutenden Unternehmen, Organisationen und Forschungseinrichtungen, die sich mit dem US-amerikanischen Ethanolmarkt beschäftigen, daran teilnehmen ${ }^{270}$.

Die Datenerhebung erfolgte, indem die Experten einzeln angesprochen und ihnen der Fragebogen in einer Mappe übergeben wurde. Die Experten beantworteten den Fragebogen selbstständig. Es bestand keine Notwendigkeit, innen ein Incentive anzubieten, da sie sehr gerne an der Befragung teilnahmen ${ }^{271}$.

\subsubsection{Auswertung der Wirkungsanalyse}

An der Wirkungsanalyse haben 85 Experten des US-amerikanischen Ethanolmarktes teilgenommen. Der Großteil der Experten arbeitete für US-amerikanische Unternehmen/Organisationen (Abbildung 75).

\footnotetext{
${ }^{268}$ Pretests wurden mit den folgenden Experten durchgeführt: Dr. Christoph Berg - F.O. Licht, Dr. Marco Veselka - CropEnergies AG, Dr. Hinrich Harling, Dr. Beate Rother und Dr. Andreas von Felde - KWS SAAT AG.

${ }^{269}$ Der "International Fuel Ethanol Workshop" und die "National Ethanol Conference" sind die beiden wichtigsten Konferenzen für den US-amerikanischen Ethanolmarkt, an dem alle wichtigen Entscheidungsträger teilnehmen.

${ }^{270}$ Im Jahr 2012 waren 87\% aller US-amerikanischen Ethanolproduzenten beim „International Fuel Ethanol Workshop“ vertreten (ETHANOL PRODUCER MAGAZINE 2012h).

${ }^{271}$ Einige Experten halfen mir sogar bei der Datenerhebung, indem sie mich ihren Geschäftspartnern vorstellten und diese baten, ebenfalls einen Fragebogen auszufüllen.
} 


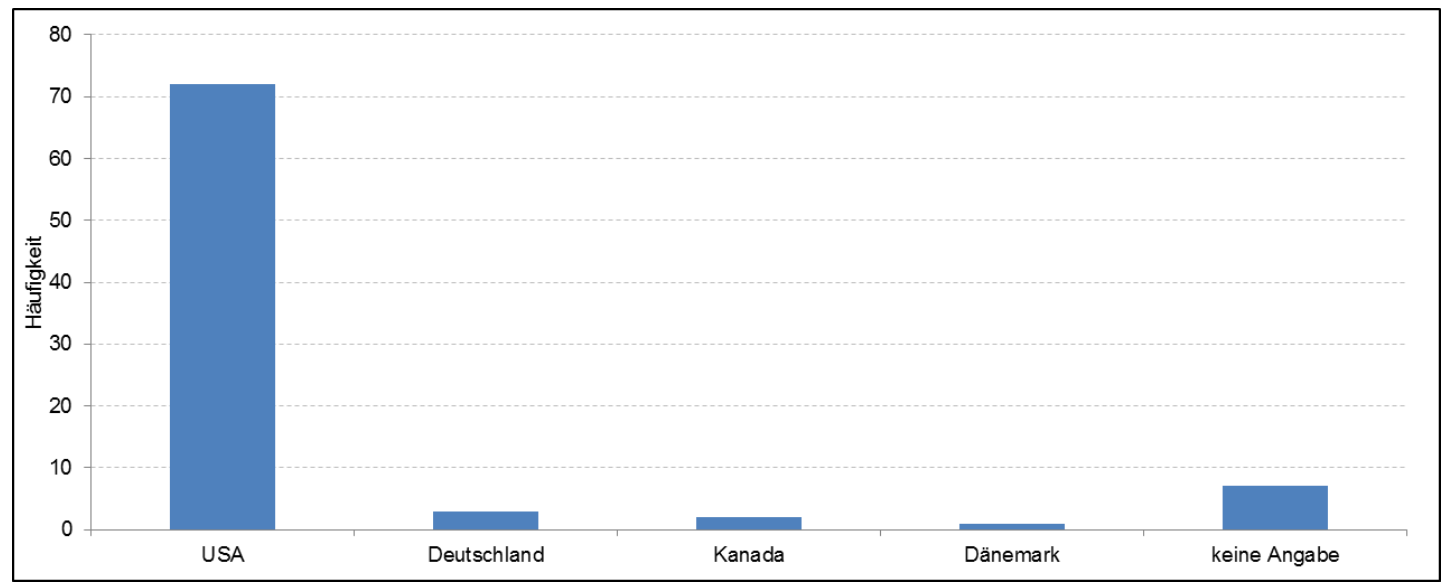

Abbildung 75: Wirkungsanalyse USA - Herkunftsland der Unternehmen/Organisationen der befragten Experten

Quelle: EIGENE DARSTELLUNG

Die meisten Probanden waren für Unternehmen/Organisationen beschäftigt, die in der Ethanolproduktion, dem Anlagenbau, der Unternehmensberatung sowie in der Enzymtechnologie tätig waren (Abbildung 76).

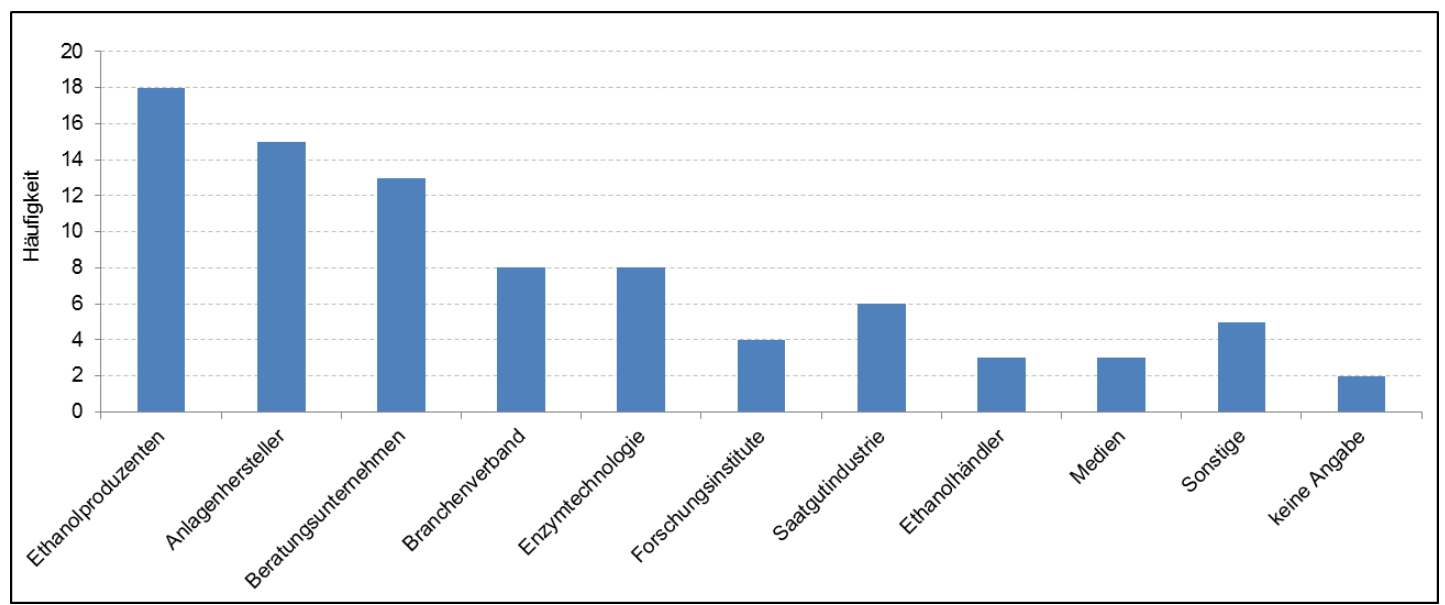

Abbildung 76: Wirkungsanalyse USA - Herkunftsbranche der Unternehmen/Organisationen der befragten Experten

Quelle: EIGENE DARSTELLUNG

In der folgenden Tabelle 15 sind die wirkenden Einflussfaktoren abfallend nach der Stärke ihrer Wirkung auf die Entwicklung des US-amerikanischen Ethanolmarktes bis zum Jahr 2023 angeordnet. Der Mittelwert jedes Einflussfaktors gibt die von den Experten erkannte Wirkungsstärke an. Je höher der Mittelwert ${ }^{272}$, desto stärker ist der Einfluss und damit die Bedeutung des Faktors für die Zukunft des Marktes.

${ }^{272}$ Bei der Interpretation der Mittelwerte gelten die Abstufungen der Likert-Skala: $7=$ „sehr starker Einfluss"; 6 = „starker Einfluss“; 5 = „eher starker Einfluss“; 4 = „moderater Einfluss“; 3 = „eher schwacher Einfluss“; 2 = „schwacher Einfluss“ und 1 = „sehr schwacher Einfluss“. 


\begin{tabular}{|c|c|c|c|c|c|}
\hline $\begin{array}{l}\text { Einfluss- } \\
\text { faktor }\end{array}$ & & $\mathbf{n}$ & $\begin{array}{l}\text { Mittel- } \\
\text { wert }\end{array}$ & $\begin{array}{l}\text { Standard- } \\
\text { abweichung }\end{array}$ & $\begin{array}{l}\text { Einfluss- } \\
\text { bereich }\end{array}$ \\
\hline 24 & $\begin{array}{l}\text { Höhe der genehmigten prozentualen } \\
\text { Beimischung von Ethanol zu Benzin }\end{array}$ & 76 & 6,11 & 1,053 & Politik \\
\hline 22 & $\begin{array}{l}\text { Biokraftstoff-Verbrauchsmandate - Re- } \\
\text { newable Fuel Standard } 2 \text { (RFS 2) }\end{array}$ & 78 & 6,08 & 1,078 & Politik \\
\hline 1 & Benzinnachfrage in den USA & 85 & 5,61 & 1,235 & Ökonomie \\
\hline $12 a$ & $\begin{array}{l}\text { Rohstoffkosten bei der Produktion von } \\
\text { Mais-Ethanol }\end{array}$ & 76 & 5,57 & 1,215 & Ökonomie \\
\hline 8 & $\begin{array}{l}\text { US-amerikanische Tankstelleninfrastruk- } \\
\text { tur für den Verkauf von Benzin mit mittle- } \\
\text { rem Ethanolgehalt (E15 - E40) }\end{array}$ & 84 & 5,54 & 1,197 & Ökonomie \\
\hline 2 & Rohölpreis in den USA & 85 & 5,44 & 1,017 & Ökonomie \\
\hline 15 & $\begin{array}{l}\text { Anzahl der Kraftfahrzeuge, die mit mittle- } \\
\text { ren Ethanolbeimischungen zu Benzin } \\
\text { (E15 - E40) fahren können }\end{array}$ & 84 & 5,37 & 1,278 & Technologie \\
\hline 28 & $\begin{array}{l}\text { Diskussion über die Energieunabhängig- } \\
\text { keit der USA }\end{array}$ & 84 & 5,15 & 1,135 & Gesellschaft \\
\hline 29 & $\begin{array}{l}\text { Diskussion in den USA über den Nutzen } \\
\text { von Ethanol für die US-amerikanische } \\
\text { Wirtschaft }\end{array}$ & 83 & 5,07 & 1,218 & Gesellschaft \\
\hline 9 & $\begin{array}{l}\text { US-amerikanische Tankstelleninfrastruk- } \\
\text { tur für den Verkauf von E85 }\end{array}$ & 77 & 5,06 & 1,341 & Ökonomie \\
\hline 23 & $\begin{array}{l}\text { Treibhausgasreduktionswerte für Bio- } \\
\text { kraftstoffe - Renewable Fuel Standard } 2 \\
\text { (RFS 2) }\end{array}$ & 72 & 5,03 & 1,289 & Politik \\
\hline 17 & $\begin{array}{l}\text { Enzym-Technologie zur Produktion von } \\
\text { Zellulose-Ethanol }\end{array}$ & 80 & 4,98 & 1,441 & Technologie \\
\hline $13 b$ & $\begin{array}{l}\text { Produktionskosten von Zellulose-Ethanol } \\
\text { - ohne Rohstoffkosten }\end{array}$ & 73 & 4,96 & 1,695 & Ökonomie \\
\hline $13 a$ & $\begin{array}{l}\text { Rohstoffkosten bei der Produktion von } \\
\text { Zellulose-Ethanol }\end{array}$ & 74 & 4,93 & 1,547 & Ökonomie \\
\hline 14 & $\begin{array}{l}\text { Anzahl der Flexible Fuel Vehicles in den } \\
\text { USA }\end{array}$ & 84 & 4,92 & 1,407 & Technologie \\
\hline $12 b$ & $\begin{array}{l}\text { Produktionskosten von Mais-Ethanol - } \\
\text { ohne Rohstoffkosten }\end{array}$ & 76 & 4,79 & 1,482 & Ökonomie \\
\hline 7 & $\begin{array}{l}\text { US-amerikanische Infrastruktur, die be- } \\
\text { nötigt wird, um das Ethanol von den } \\
\text { Produktionsanlagen zu den Märkten an } \\
\text { die Ost- und Westküste der USA zu } \\
\text { transportieren }\end{array}$ & 83 & 4,78 & 1,316 & Ökonomie \\
\hline 25 & Low Carbon Fuel Standard (LCFS) & 74 & 4,39 & 1,363 & Politik \\
\hline 11 & $\begin{array}{l}\text { Vertikale strategische Kooperationen im } \\
\text { Ethanolmarkt der USA }\end{array}$ & 76 & 4,37 & 1,198 & Ökonomie \\
\hline 21 & Nachfrage nach Biobutanol in den USA & 73 & 4,25 & 1,588 & Technologie \\
\hline 10 & Preis für DDGS in den USA & 74 & 4,24 & 1,412 & Ökonomie \\
\hline 18 & Treibhausgasbilanz von Mais-Ethanol & 76 & 4,21 & 1,379 & Technologie \\
\hline 4 & $\begin{array}{l}\text { Ethanolexporte aus den USA nach Brasi- } \\
\text { lien }\end{array}$ & 84 & 4,15 & 1,393 & Ökonomie \\
\hline 5 & Ethanolexporte aus den USA in die EU27 & 84 & 4,13 & 1,24 & Ökonomie \\
\hline 27 & $\begin{array}{l}\text { Diskussion in den USA über die Auswir- } \\
\text { kungen von Ethanol auf die Umwelt }\end{array}$ & 85 & 4,12 & 1,375 & Gesellschaft \\
\hline 3 & Ethanolimporte aus Brasilien in die USA & 84 & 4,08 & 1,328 & Ökonomie \\
\hline 19 & $\begin{array}{l}\text { Treibhausgasbilanz von Zellulose- } \\
\text { Ethanol }\end{array}$ & 74 & 4,05 & 1,423 & Technologie \\
\hline
\end{tabular}




\begin{tabular}{c|l|c|c|c|c}
\hline $26^{273}$ & Tank-oder-Teller-Diskussion in den USA & 85 & 4,02 & 1,309 & Gesellschaft \\
\hline 20 & Nachfrage nach BtL in den USA & 74 & 3,91 & 1,491 & Technologie \\
\hline 6 & $\begin{array}{l}\text { Ethanolexporte aus den USA ins Ausland } \\
- \text { die Ethanolexporte nach Brasilien und } \\
\text { in die EU27 werden nicht berücksichtigt }\end{array}$ & 83 & 3,66 & 1,417 & Ökonomie \\
\hline 16 & Anzahl der Elektrofahrzeuge in den USA & 84 & 2,82 & 1,364 & Technologie \\
\hline
\end{tabular}

Tabelle 15: Wirkungsanalyse USA - Anordnung der wirkenden Einflussfaktoren hinsichtlich der Stärke des Einflusses auf den Ethanolmarkt der USA

Quelle: EIGENE DARSTELLUNG

Aus Tabelle 15 sind die Faktoren zu identifizieren, die den Ethanolmarkt der USA bis zum Jahr 2023 entscheidend beeinflussen werden, womit das erste Ziel der Dissertation erfüllt werden kann. Die acht Faktoren mit dem stärksten Einfluss auf den Ethanolmarkt der USA sind die folgenden: 24: „Höhe der genehmigten prozentualen Beimischung von Ethanol zu Benzin“; 22: „Biokraftstoff-Verbrauchsmandate - Renewable Fuel Standard 2 (RFS 2)“; 1: „Benzinnachfrage in den USA“; 12a: „Rohstoffkosten bei der Produktion von Mais-Ethanol“; 8: „US-amerikanische Tankstelleninfrastruktur für den Verkauf von Benzin mit mittlerem Ethanolgehalt (E15 - E40)“; 2: „Rohölpreis in den USA“; 15: „Anzahl der Kraftfahrzeuge, die mit mittleren Ethanolbeimischungen zu Benzin (E15 - E40) fahren können“ und 28: „Diskussion über die Energieunabhängigkeit der USA“.

Zur Veranschaulichung der Ergebnisse sind die genannten Einflussfaktoren in Abbildung 77 dargestellt.

${ }^{273}$ Der wirkende Einflussfaktor 26: „Tank-oder-Teller-Diskussion in den USA“ besitzt nach Einschätzung der befragten Experten lediglich einen „moderaten Einfluss“ auf die Entwicklung des US-amerikanischen Ethanolmarktes und gehört damit zu den vier Faktoren mit dem geringsten Einfluss auf den US-amerikanischen Ethanolmarkt. Diese Beurteilung ist auf den Zeitpunkt der Erhebung zurückzuführen, die im Juni 2012 stattfand und somit kurz vor der intensiv geführten Tank-oder-Teller-Disskussion im Sommer und Herbst des Jahres 2012. Grund der damaligen Debatte waren die ansteigenden Agrarpreise aufgrund der Trockenheit im Mittleren Westen der USA (F.O. LICHT 2012f: 70). 


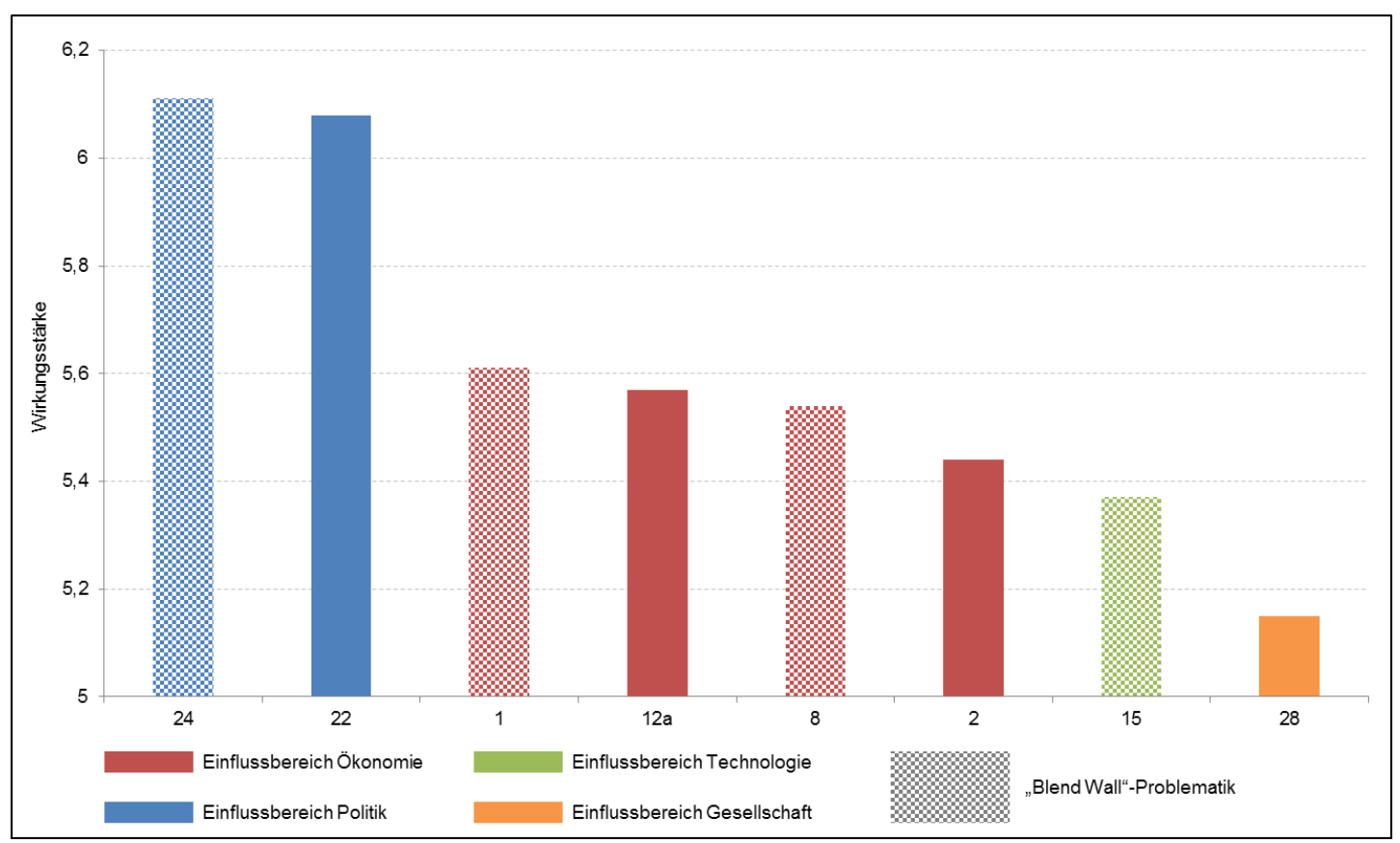

Abbildung 77: Wirkungsanalyse USA - Darstellung der acht bedeutendsten Einflussfaktoren für die langfristige Entwicklung des US-amerikanischen Ethanolmarktes ${ }^{274}$

Quelle: EIGENE DARSTELLUNG

Aus der Abbildung 77 ist ersichtlich, dass Einflussfaktoren aus allen vier Einflussbereichen zu den wirkungsstärksten Faktoren gehören. Die meisten Faktoren - 1, 12a, 8 und 2 - stammen aus dem Einflussbereich Ökonomie und bilden damit die größte Gruppe. Trotzdem sind die beiden mit Abstand wichtigsten Einflussfaktoren - 24: „Höhe der genehmigten prozentualen Beimischung von Ethanol zu Benzin“ und 22: „Biokraftstoff-Verbrauchsmandate - Renewable Fuel Standard 2 (RFS 2)“ -, die jeweils einen „starken Einfluss“ auf die zukünftige Entwicklung des Marktes besitzen, dem Bereich Politik zuzuordnen. Dies zeigt, dass der US-amerikanische Ethanolmarkt nach Auffassung der befragten Experten auch in Zukunft ${ }^{275}$ ein stark von politischen Entscheidungen beeinflusster Markt sein wird ${ }^{276}$.

Des Weiteren ist die entscheidende Bedeutung der „Blend Wall“-Problematik ${ }^{277} \mathrm{zu}$ erkennen, denn alle vier Faktore ${ }^{278}$ - in Abbildung 77 sind diese weiß gemustert -,

\footnotetext{
${ }^{274}$ Abstufung der Wirkungsstärke anhand der Likert-Skala: $6=$ „starker Einfluss“ und $5=$ "eher starker Einfluss".

${ }^{275}$ Den hohen Einfluss der Politik auf die historische Entwicklung sowie die gegenwärtige Situation des US-amerikanischen Ethanolmarktes ist ausführlich in Kapitel 2.2 dargestellt.

${ }_{276}$ Die hohe Relevanz der Politik bezieht sich nicht auf den Aspekt der Treibhausgasreduktion, da die beiden politischen Faktoren 23 und 25, die diese Thematik beschreiben, lediglich von durchschnittlicher Relevanz sind.

277 Die „Blend Wall“ beschreibt die Situation, dass nur eine begrenzte Beimischung von Ethanol zu Benzin möglich ist, wodurch die US-amerikanische Ethanolnachfrage beschränkt ist (CONGRESSIONAL RESEARCH SERVICE: 5). Seit dem Jahr 2011 ist die E10-„Blend Wall" erreicht und verhindert ein weiteres Wachstum der Ethanolnachfrage und damit die Erfüllung der Verbrauchsmandate des RFS 2 (F.O. LICHT 2013b: 213). Ein weiterer bereits prognostizierter Rückgang der US-amerikanischen Benzinnachfrage würde die Situation noch verschärfen, da dies die Ethanolbeimischung und damit auch die Ethanolnachfrage
} 
die in direktem Zusammenhang mit diesem Thema stehen, befinden sich unter den ersten acht Faktoren. Die vier Faktoren sind miteinander verknüpft, wobei eine besonders enge Verbindung zwischen den Faktoren 15, 24 und 8 besteht. Der Faktor 1 ist ein Teil des Themas, da dieser die zukünftige Entwicklung der USamerikanischen Benzinnachfrage beschreibt, während die anderen drei Faktoren (24, 8 und 15) die Ethanolmengen betrachten, die einem Liter Benzin beigemischt werden können. Dabei ist jedoch zu beachten, dass diese Faktoren (24, 8 und 15) voneinander abhängig sind. Denn erst, wenn die in Faktor 15 dargestellten USamerikanischen Kraftfahrzeuge eine mittlere Ethanolbeimischung zu Benzin als Kraftstoff verwenden können, ist die EPA (Faktor 24) auch bereit, diesen Kraftstoff zu genehmigen ${ }^{279}$. Darüber hinaus werden die Tankstellen erst dann ihre Zapfanlagen dem neuen Kraftstoff anpassen (Faktor 8), wenn sowohl die Kraftfahrzeuge den Kraftstoff vertragen als auch die EPA den Kraftstoff zugelassen hat. Folglich kann eine Steigerung der Ethanolbeimischung zu einem Liter Benzin nur erfolgen, wenn alle drei Faktoren diese höhere Ethanolbeimischung unterstützen ${ }^{280}$. Die hohe Bedeutung der Einflussfaktoren 8 und 15 zeigt darüber hinaus, dass nach Einschätzung der Experten auch zukünftig der wesentliche Ethanolabsatz als Beimischung zu Benzin an konventinelle Kraftfahrzeuge und nicht an FFV erfolgt ${ }^{281}$.

Von geringerer Bedeutung als die politischen Faktoren, aber trotzdem hoch relevant sind die beiden ökonomischen Faktoren 2: „Rohölpreis in den USA“ und 12a: „Rohstoffkosten bei der Produktion von Mais-Ethanol“, die die Konkurrenzfähigkeit von Ethanol im Vergleich zu Benzin beschreiben ${ }^{282}$. So beeinflusst der Rohölpreis stark

reduzieren würde. Eine ausführliche Beschreibung der derzeitigen „Blend Wall“-Problematik befindet sich in Kapitel 2.2.

${ }^{278}$ Die folgenden vier Faktoren bilden die „Blend Wall“-Problematik: 24: „Höhe der genehmigten prozentualen Beimischung von Ethanol zu Benzin“, 1: „Benzinnachfrage in den USA“, 8: „US-amerikanische Tankstelleninfrastruktur für den Verkauf von Benzin mit mittlerem Ethanolgehalt (E15 - E40)“ und 15: „Anzahl der Kraftfahrzeuge, die mit mittleren Ethanolbeimischungen zu Benzin (E15 - E40) fahren können“.

${ }^{279}$ Durch den Einfluss von Faktor 24 auf die „Blend Wall“-Problematik zeigt sich erneut die hohe Bedeutung der Politik für die Entwicklung des US-amerikanischen Ethanolmarktes.

${ }_{280}$ Der US-amerikanische Ethanolmarkt befindet sich derzeit an der E10-,Blend Wall“, da die Zapfsäulen an den US-amerikanischen Tankstellen E15 nicht verkaufen können. Die Zapfsäulen sind derzeit der limitierende Faktor, weil alle Kraftfahrzeuge ab Baujahr 2001 E15 nutzen können - das sind 62\% aller Fahrzeuge - und die EPA den Kraftstoff E15 im Jahr 2011 für alle Fahrzeuge Baujahr 2001 und jünger zugelassen hat (U.S. GOVERNMENT INFORMATION 2011: 4662; F.O. LICHT 2013c: 341; RFA 2012b).

${ }^{281}$ Die beiden Faktoren 9: „US-amerikanische Tankstelleninfrastruktur für den Verkauf von E85“ und 14: „Anzahl der Flexible Fuel Vehicles in den USA“, die die zukünftige Entwicklung des FFV-Marktes in den USA beschreiben, besitzen lediglich einen „eher starken Einfluss" und befinden sich somit im Vergleich zu den anderen Faktoren nur im oberen Mittelfeld.

${ }^{282}$ Die Konkurrenzsituation zwischen dem Benzinpreis und dem Ethanolpreis ist von Bedeutung, denn Ethanol ist in der Lage, Benzin auf dem Kraftstoffmarkt zu substituieren (U.S. ENERGY INFORMATION ADMINISTRATION 2012b: 98). 
den Benzinpreis, und die Rohstoffkosten für Mais sind entscheidend für die Produktionskosten von Ethanol (IER ET AL. 2010: 54; AGRICULTURAL MARKETING RESOURCE CENTER 2012).

Der wirkende Einflussfaktor 28: „Diskussion über die Energieunabhängigkeit der USA“ veranschaulicht, dass nach Auffassung der Experten die seit Jahren intensiv geführte Debatte auch in Zukunft von hoher Bedeutung sein wird. Die Relevanz des gesellschaftlichen Faktors ist nicht überraschend, da das Thema Energieunabhängigkeit das primäre politische Ziel ist, warum Biokraftstoffe in den USA gefördert werden (U.S. GOVERNMENT INFORMATION 2007: 121 STAT. 1492) ${ }^{283}$.

Im nächsten Kernschritt „USA Szenario-Prognostik“ werden ausschließlich die acht bereits beschriebenen Faktoren mit dem stärksten Einfluss auf die Entwicklung des US-amerikanischen Ethanolmarktes betrachtet (Abbildung 78). Diese werden im weiteren Prozess als wirkende Schlüsselfaktoren bezeichnet. Es werden bewusst nicht mehr Faktoren ausgewählt, einerseits damit die zentralen Entwicklungen des Marktes in den Szenarien klar darstellbar sind, andererseits damit der Kernschritt „USA Szenario-Bildung und -Konsistenzprüfung“ (Kapitel 6.3) beherrschbar und überschaubar bleibt (MILLETT/HONTON 1991: 71; GAUSEMEIER ET AL. 1995: $187 f.)^{284}$.

In der folgenden Abbildung 78 sind die wirkenden Schlüsselfaktoren des Szenariofeldes eingezeichnet.

\footnotetext{
${ }^{283}$ Mittels der Ergebnisse der Befragung lassen sich nicht nur die Faktoren bestimmen, die den US-amerikanischen Ethanolmarkt maßgeblich beeinflussen werden, sondern auch jene, die nach Auffassung der Probanden für die weitere Entwicklung des Marktes eine nur geringe Bedeutung haben.

Auffallend in diesem Zusammenhang ist, dass die Faktoren 4: „Ethanolexporte aus den USA nach Brasilien“, 5: „Ethanolexporte aus den USA in die EU27“" und 6: „Ethanolexporte aus den USA ins Ausland - die Ethanolexporte nach Brasilien und in die EU27 werden nicht berücksichtigt", welche allesamt den US-amerikanischen Exportexport betrachten, von nur geringer Relevanz für die zukünftige Entwicklung des Marktes sind (Tabelle 15). Dies wiederum zeigt die hohe Bedeutung der US-amerikanischen Binnennachfrage und betont die Wichtigkeit der „Blend Wall“-Problematik.

Darüber hinaus sind die drei technischen Faktoren 21: „Nachfrage nach Biobutanol in den USA“, 20: „Nachfrage nach BtL in den USA“ sowie 16: „Anzahl der Elektrofahrzeuge in den USA“, die jeweils eine Zukunftstechnologie beschreiben, nach Ansicht der befragten Experten von nur geringer Bedeutung. Für den Biokraftstoffmarkt zeigt dies, dass Ethanol auch in Zukunft der wesentliche Biokraftstoff sein und weder von Biobutanol noch von BtL substituiert werden wird. Ferner wird auch die Elektromobilität keine große Konkurrenz für den Ethanolmarkt darstellen.

${ }^{284}$ Die Berücksichtigung des nächstwichtigsten Faktors 29: „Diskussion in den USA über den Nutzen von Ethanol für die US-amerikanische Wirtschaft" im weiteren Prozess wäre nicht falsch. Es wird aber darauf verzichtet, da es einer starken Reduktion von Faktoren bedarf, um eine gute Durchführbarkeit der Methodik zu erreichen. Darüber hinaus wird der Einflussbereich Gesellschaft durch den Faktor 28 bereits repräsentiert, der die hohe gesellschaftliche Bedeutung des Themas Energieunabhängigkeit in den USA betrachtet.
} 


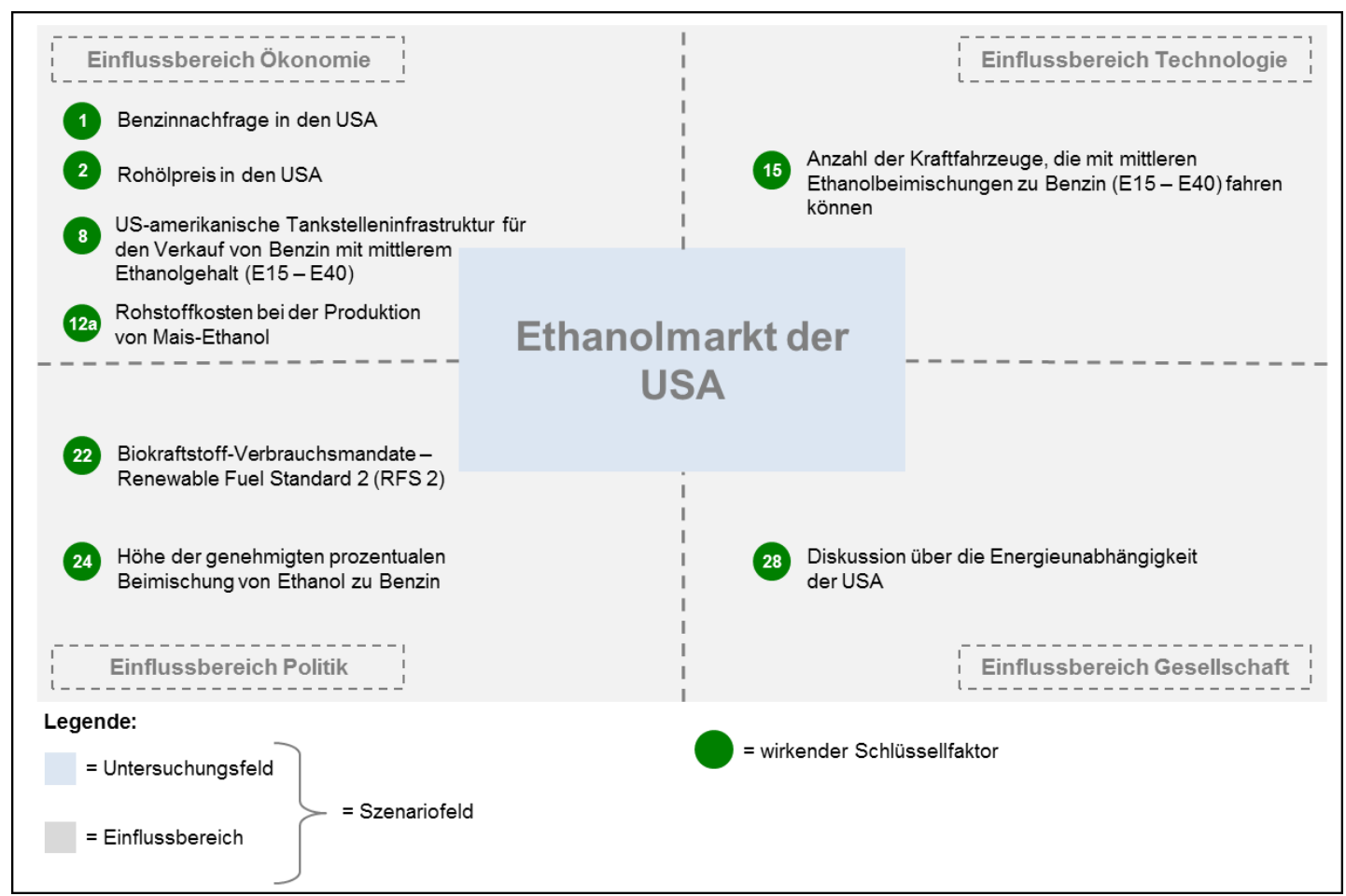

Abbildung 78: Wirkungsanalyse USA - Zusammenfassung der wirkenden Schlüsselfaktoren Quelle: EIGENE DARSTELLUNG

\subsection{USA Szenario-Prognostik}

Zweck des zweiten Kenschritts ist es, für jeden beschreibenden ${ }^{285}$ und wirkenden Schlüsselfaktor mindestens eine Zukunftsprojektion zu bilden, die den Faktor im Jahr 2023 beschreibt. Die Schlüsselfaktoren werden mit Ausnahme des Faktors 2: „Rohölpreis in den USA“ entsprechend der in Tabelle 16 dargestellten Reihenfolge bearbeitet. Für Faktor 2 werden die bereits für den Ethanolmarkt der EU27 gebildeten Zukunftsprojektionen (Kapitel 5.2.2) für den US-amerikanischen Markt übernommen ${ }^{286}$.

Schlüsselfaktor

\begin{tabular}{l|l}
\hline 1 & Benzinnachfrage in den USA \\
\hline 2 & Rohölpreis in den USA \\
\hline 8 & $\begin{array}{l}\text { US-amerikanische Tankstelleninfrastruktur für den Verkauf von Benzin mit mittlerem Ethanolge- } \\
\text { halt (E15 - E40) }\end{array}$ \\
\hline $12 a$ & Rohstoffkosten bei der Produktion von Mais-Ethanol \\
\hline
\end{tabular}

285 Die in Kapitel 6.1.1.1 gebildeten beschreibenden Einflussfaktoren des USamerikanischen Ethanolmarktes werden in der Szenario-Prognostik als beschreibende Schlüsselfaktoren weiter betrachtet.

${ }^{286}$ Die Folgenden beiden Zukunftsprojektionen 2A: „Moderater Anstieg des Rohölpreises (WTI) auf (real 2010) 97 US-\$ je Barrel“ und 2b: „Anstieg des Rohölpreises (WTI) auf (real 2010) 130 US-\$ je Barrel“ werden im dritten Kernschritt „USA Szenario-Bildung und -Konsistenzprüfung" weiter betrachtet. 


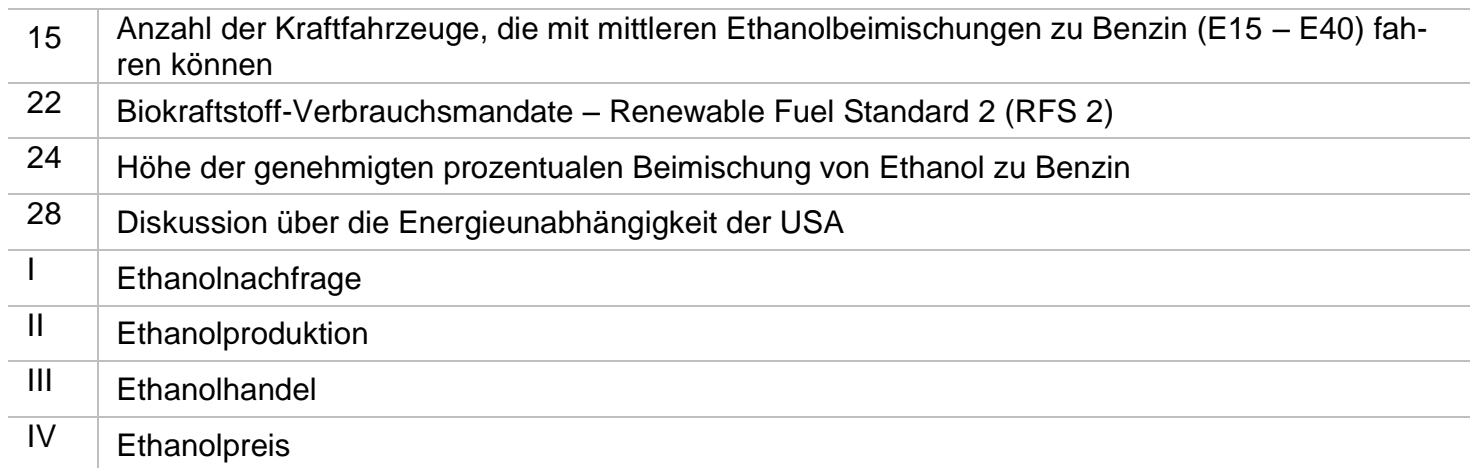

Tabelle 16: USA Szenario-Prognostik - Reihenfolge der Betrachtung der Schlüsselfaktoren Quelle: EIGENE DARSTELLUNG

\subsubsection{Benzinnachfrage in den USA}

Im Gegensatz zur EU27 sind die USA ein Benzinmarkt ${ }^{287}$ und die Benzinnachfrage ist im US-amerikanischen Transportbereich von 383 Milliarden Litern im Jahr 1980 auf 522 Milliarden Liter im Jahr 2010 angestiegen; dies entspricht einem durchschnittlichen jährlichen Wachstum von 1,04\% (Abbildung 79) (U.S. ENERGY INFORMATION ADMINISTRATION 2012b: 98$)^{288}$.

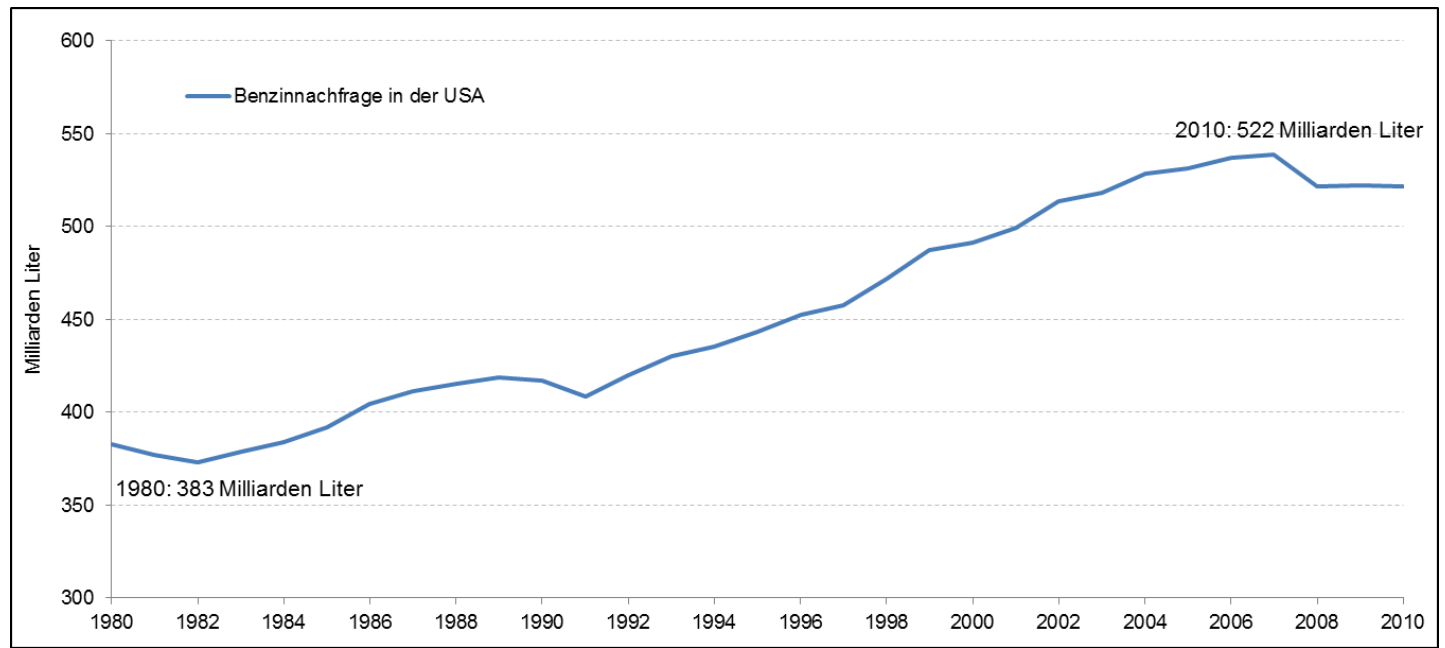

Abbildung 79: Historische Entwicklung der Benzinnachfrage in den USA

Quelle: In Anlehnung an U.S. ENERGY INFORMATION ADMINISTRATION 2012b: 98; OAK RIDGE NATIONAL LABORATORY 2012: 2-13; RITA 2012

${ }^{287}$ In den Daten zur Benzinnachfrage ist der dem Benzin beigemischte Ethanolanteil enthalten. Dementsprechend sind die Auswirkungen der Substitution von Benzin durch Ethanol in den Daten zur historischen und zur zukünftigen Entwicklung der Benzinnachfrage nicht ersichtlich. Die Nicht-Betrachtung der Ethanolnutzung ist notwendig, um im dritten Kernschritt „USA Szenario-Bildung und -Konsistenzprüfung“ unabhängig von der Auswahl der Zukunftsprojektion des Faktors: I "Ethanolnachfrage“ konsistente Szenarien erstellen zu können.

${ }^{288}$ Der Bedarf an Dieselkraftstoff lag in den USA im Jahr 2010 bei lediglich 192 Milliarden Litern (U.S. ENERGY INFORMATION ADMINISTRATION 2012b: 98). 
Der konstante Anstieg der Benzinnachfrage ist sowohl auf die Zunahme der Zahl an zugelassenen Kraftfahrzeugen ${ }^{289}$ von 149 Millionen im Jahr 1980 auf 230 Millionen im Jahr 2010 (U.S. Department of Transportation 2012a: 254f.) als auch auf den Anstieg der durchschnittlich gefahrenen Kilometer je Fahrzeug pro Jahr von 15.691 im Jahr 1980 auf 20.761 Kilometer im Jahr 2010 zurückzuführen (U.S. DEPARTMENT OF TRANSPORTATION 2012a: 254f.).

Im Folgenden wird die zukünftige Entwicklung der Benzinnachfrage bis zum Jahr 2023 betrachtet. Die U.S. ENERGY INFORMATION ADMINISTRATION (2012b: 98) prognostiziert eine Abnahme der Benzinnachfrage auf 483 Milliarden Liter bis zum Jahr 2023 (Abbildung 80), was einem durchschnittlichen jährlichen Nachfragerückgang von $0,6 \%$ entspricht.

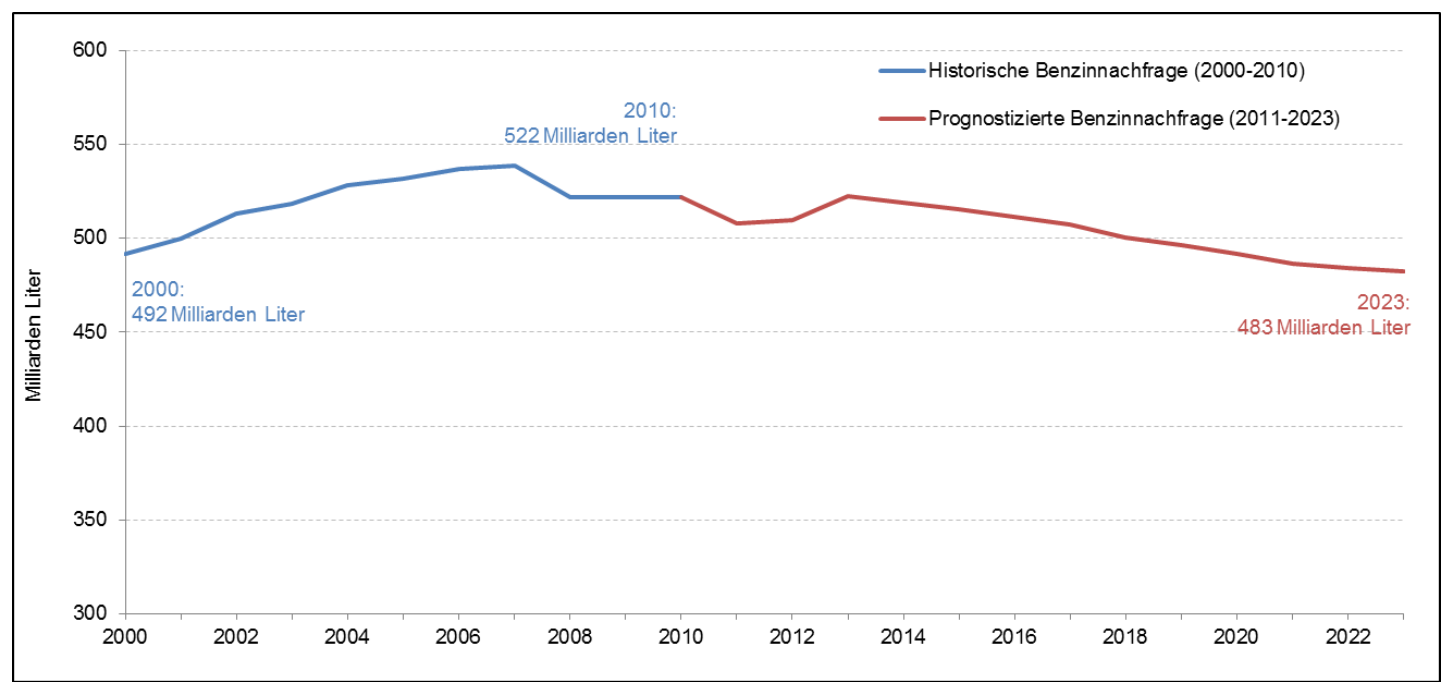

Abbildung 80: Zukünftige Entwicklung der Benzinnachfrage in den USA bis zum Jahr 2023 Quelle: In Anlehnung an U.S. ENERGY INFORMATION ADMINISTRATION 2012b: 98; OAK RIDGE NATIONAL LABORATORY 2012: 2-13, RITA 2012

Auch die Organisationen INTERNATIONAL ENERGY AGENCY (2011a: 556), OPEC (2011: 91) und die TRANSPORTATION RESEARCH BOARD (2011: 29) erwarten eine Abnahme der Benzinnachfrage in den USA bis zum Jahr 2023. Trotz des erwarteten Anstiegs der Zahl an zugelassenen Kraftfahrzeugen bis zum Jahr 2023 (Kapitel 5.2.4) (TRANSPORTATION RESEARCH BOARD 2011: 28) sowie bei Konstanz der durchschnittlich gefahrenen Kilometer je zugelassenem Fahrzeug pro $\mathrm{Jahr}^{290}$ (U.S. ENERGY INFORMATION ADMINISTRATION 2012b: 85) ist die Zukunftsprojektion der U.S. ENERGY INFORMATION ADMINISTRATION (2012b: 98)

${ }^{289}$ Eine Beschreibung der Entwicklung der Zahl an zugelassenen Kraftfahrzeugen in den USA im Zeitraum von 1990 bis 2010 befindet sich in Kapitel 5.2.4.

${ }^{290}$ Die U.S. ENERGY INFORMATION ADMINISTRATION (2012b: 85) prognostiziert eine durchschnittlich gefahrene Strecke von 20.165 Kilometern je zugelassenes Kraftfahrzeug im Jahr 2023; im Jahr 2010 lag dieser Wert bei 20.390 Kilometern. 
als realistisch zu betrachten. Denn verantwortlich für den Rückgang der Benzinnachfrage ist die gestiegene Effizienz der neueren und in Zukunft zugelassenen Kraftfahrzeuge in den USA (U.S. ENERGY INFORMATION ADMINISTRATION 2012f: 6). Die folgende Abbildung 81 zeigt die prognostizierte Treibstoffeffizienzsteigerung von Fahrzeugen in den USA.

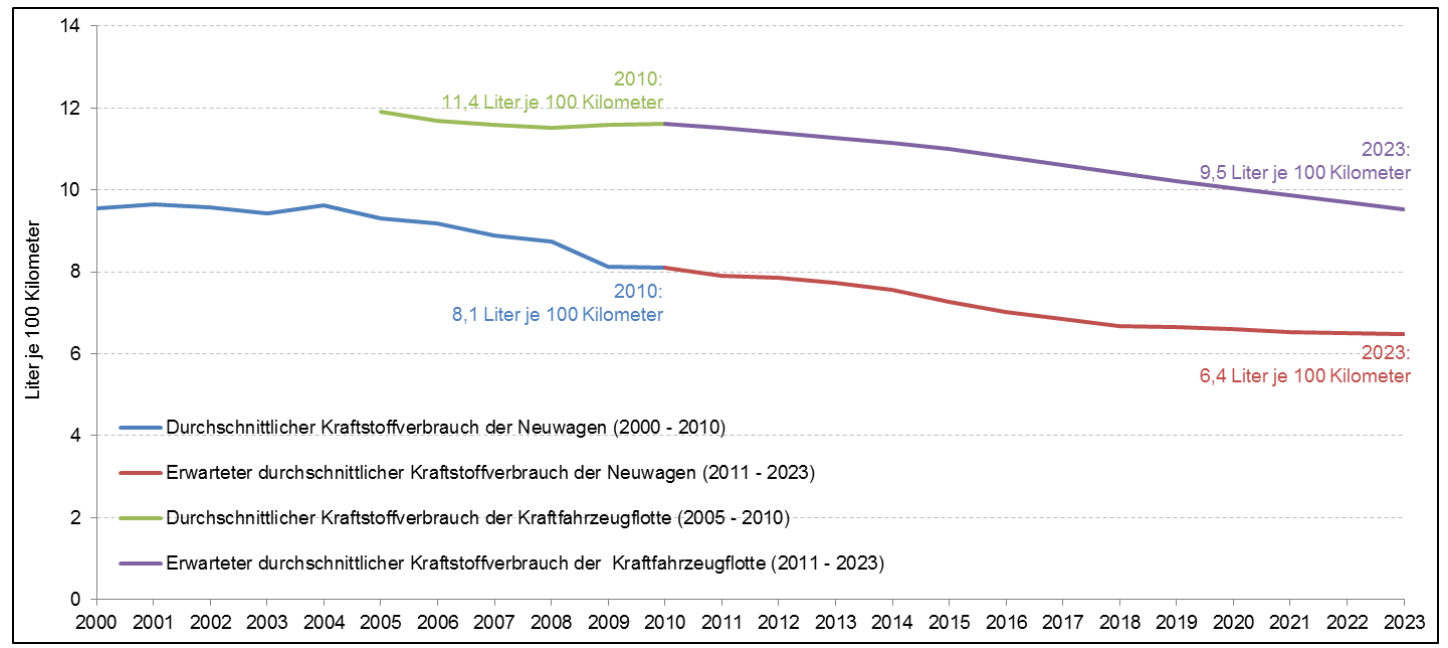

Abbildung 81: Effizienzsteigerung von Kraftfahrzeugen in den USA bis zum Jahr 2023 Quelle: In Anlehnung an U.S. ENERGY INFORMATION ADMINISTRATION 2012b: 30; U.S. ENERGY INFORMATION ADMINISTRATION 2012g; EIGENE BERECHNUNG

Die U.S. ENERGY INFORMATION ADMINISTRATION (2012g) erwartet, dass der durchschnittliche Kraftstoffverbrauch von Neuwagen von 8,1 Litern je 100 Kilometer im Jahr 2010 auf nur noch 6,4 Liter je 100 Kilometer im Jahr 2023 sinkt. Durch den Kauf von effizienteren Autos wird zeitversetzt auch der durchschnittliche Kraftstoffverbrauch der Automobilflotte auf den erwarteten Wert von 9,5 Litern je 100 Kilometer im Jahr 2023 sinken (U.S. ENERGY INFORMATION ADMINISTRATION 2012b: 30). Maßgeblich für diese Entwicklung sind die politischen Vorgaben zur verbesserten Treibstoffeffizienz von Kraftfahrzeugen, welche im „Energy Independence and Security Act“ formuliert sind (U.S. GOVERNMENT INFORMATION 2007: 121 STAT. 1499). So muss im Jahr 2020 ein "Corporate Average Fuel Economy Standard“Wert (CAFE) über die Gesamtflotte jedes Autobauers ${ }^{291}$ von mindestens 35 Meilen pro Gallone (höchstens 6,76 Liter je 100 Kilometer) erreicht werden (U.S. GOVERNMENT INFORMATION 2007: 121 STAT. 1499).

Die Zukunftsprojektion $1 \mathrm{~A}$, die in der weiteren Arbeit betrachtet wird, ist wie folgt beschrieben:

1A | Rückgang der Benzinnachfrage in den USA auf 483 Milliarden Liter pro Jahr

${ }^{291}$ Der CAFE-Wert bezieht sich auf die Neuwagen des Autobauers. 


\subsubsection{US-amerikanische Tankstelleninfrastruktur für den Verkauf von Benzin mit mittlerem Ethanolgehalt (E15 - E40)}

Mit einem Marktanteil von über $90 \%$ ist Benzin mit einer Ethanolbeimischung von 10\% derzeit das mit Abstand meistverkaufte Benzin in den USA. Weiterhin ist festzustellen, dass Tankstellen in den gesamten USA in der Lage sind, den Kraftstoff zu verkaufen (U.S. ENERGY INFORMATION ADMINISTRATION 2011c; NACS 2012b: 25). Seit Januar 2011 ist auf Grundlage einer Entscheidung der U.S. Environmental Protection Agency (EPA) auch der Vertrieb von Benzin mit einem Ethanolgehalt von 15\% (E15) für Kraftfahrzeuge ab dem Baujahr 2001 erlaubt (EPA 2012b; U.S. ENERGY INFORMATION ADMINISTRATION 2012h), was im Jahr 2012 immerhin $62 \%$ (140 Millionen) der in den USA zugelassenen Kraftfahrzeuge betrifft (U.S. ENERGY INFORMATION ADMINISTRATION 2012h; RFA 2012b). Trotz des groBen Marktes bieten nur wenige Tankstellen E15 an und jene Tankstellen, die bereits E15 verkaufen, befinden sich nicht in den urbanen Zentren, sondern in den ländlich geprägten Bundesstaaten Kansas, lowa und Nebraska (BLOOMBERG 2012a) ${ }^{292}$. Der Hauptgrund für das geringe Angebot von E15 ist, dass die meisten Tankstellen Zapfsäulen besitzen, die eine Beimischung von über 10\% Ethanol zu Benzin nicht vertragen, da sonst die Dichtungen erodieren und undicht werden (NACS 2012b: 30; U.S. ENERGY INFORMATION ADMINISTRATION 2011c). Darüber hinaus haben viele Tankstellen nur zwei Lagertanks für Benzin, in denen sich zum einen Benzin mit einem Ethanolgehalt von 10\% und zum anderen ein höherwertiges Benzin befinden. Folglich müssten Tankstellen, die E15 anbieten wollen, einen neuen Lagertank für E15 einbauen, die Tankstelle auf „Blender Pumps“293 umrüsten oder auf den Verkauf von E10 zugunsten von E15 verzichten ${ }^{294}$ (U.S. ENERGY INFORMATION ADMINISTRATION 2012h).

Die Tankstellen werden vorerst jedoch nicht auf den Verkauf von E10 verzichten, da dieses derzeit noch das Benzin mit dem bei weitem höchsten Marktanteil ist und 38\% der zugelassenen Fahrzeuge keine höheren Ethanolbeimischungen zu Benzin vertragen (U.S. ENERGY INFORMATION ADMINISTRATION 2012h; RFA 2012b). Die

\footnotetext{
292 Die fehlende Infrastruktur für den Verkauf von E15 ist momentan der entscheidende Grund für die „Blend Wall“-Problematik (F.O. LICHT 2013b: 213) (Kapitel 2.2).

${ }^{293}$ Eine "Blender Pump“ ist eine Zapfanlage, die dem Autofahrer unterschiedliche EthanolBenzin-Mischungen anbieten kann. Typische Mischungen sind E10, E15, E20, E30, E40 sowie E85. Eine „Blender Pump“ kann unterschiedliche Mischungen verkaufen, da sie den Kraftstoff nicht nur einem, sondern zwei unabhängigen Tanks entnimmt, wobei der eine mit Benzin und der andere mit Ethanol gefült ist. Entsprechend der gewünschten EthanolBenzin-Mischung pumpt die „Blender Pump“ die entsprechenden Mengen Kraftstoff aus dem jeweiligen Tank und vermischt sie, bevor sie in das Kraftfahrzeug des Kunden fließen (U.S. DEPARTMENT OF ENERGY 2012b).

${ }^{294}$ Voraussetzung der Substitution von E10 durch E15 ist, dass die Zapfsäule eine 15prozentige Beimischung von Ethanol zu Benzin verträgt.
} 
Erweiterung der Tankstelle um einen Lagertank für E15, insbesondere aber der Umbau auf „Blender Pumps“, sind mit hohen Kosten verbunden ${ }^{295}$. Tankstellenbetreiber zögern derzeit noch mit dem Umbau, wissen aber, dass sie mittel- bis langfristig Benzin mit mittleren Ethanolgehalten anbieten müssen, um auf dem Markt bestehen zu können (NACS 2012b: 32; U.S. ENERGY INFORMATION ADMINISTRATION 2012h; NACS 2011: 23), denn die Mineralölkonzerne werden in Zukunft gezwungen sein, dem Kraftstoff höhere Ethanolmengen beizumischen, um die politisch bestimmten Mandate des RFS 2 erfüllen zu können (NACS 2012b: 21; CONGRESSIONAL RESEARCH SERVICE 2010a: 3).

In der weiteren Studie wird die Prognose berücksichtigt, dass in den USA im Jahr 2023 eine flächendeckende Tankstelleninfrastruktur zum Verkauf von Benzin mit mittleren Ethanolbeimischungen besteht. Die Projektion ist aus folgenden Gründen als realistisch zu betrachten: Die Technologie, Benzin mit mittleren und hohen Ethanolgehalten zu verkaufen, ist durch den Einsatz von „Blender Pumps“ und Zapfanlagen, die bereits für den Verkauf von E85 in den USA eingesetzt werden, vorhanden (U.S. ENERGY INFORMATION ADMINISTRATION 2012i). Darüber hinaus wird die Anzahl an Fahrzeugen, die lediglich E10 vertragen, in Zukunft weiter abnehmen und die alten werden durch modernere Fahrzeuge ersetzt werden, die mittlere Ethanolbeimischungen zu Benzin als Kraftstoff nutzen können (Kapitel 6.2.4). Basierend auf dieser Entwicklung wird auch der Markt für Benzin mit mittleren Ethanolgehalten kontinuierlich ansteigen und die Tankstellenbetreiber davon überzeugen, in neue Zapfanlagen zu investieren, um von diesem wachsenden Markt zu profitieren. Der Verkauf von Benzin mit mittleren und hohen Ethanolgehalten ist des Weiteren für jene Tankstellenbetreiber von Vorteil, die zusätzlich zur Tankstelle noch ein Geschäft betreiben, in dem sie Süßwaren, Getränke, Zeitschriften und Tabakwaren verkaufen. $80 \%$ des verkauften Benzins in den USA werden von dieser Art von Tankstellen vertrieben (NACS 2012b: 9; NACS 2011: 6). Förderlich ist der Absatz von Benzin mit höheren Ethanolgehalten, da der Kraftstoff einen geringeren Energiegehalt im Vergleich zu E10 besitzt (U.S. DEPARTMENT OF ENERGY 2012b) ${ }^{296}$,

\footnotetext{
${ }^{295}$ Der Umbau einer durchschnittlichen Tankstelle mit vier Zapfsäulen kostet ungefähr 80.000 US-\$ (NACS 2011: 23). Da der Großteil der Tankstellen im Eigentum von privaten Besitzern ist, die zumeist nur eine oder wenige Tankstellen besitzen, sind Umbaukosten in dieser Höhe eine große Investition (NACS 2012a: 21; NACS 2011: 2). Die großen Mineralölkonzerne haben sich aus dem Einzelhandelsgeschäft vollkommen zurückgezogen. Einzig Exxon Mobil und Chevron besitzen noch einige wenige Tankstellen in den USA, doch liegt ihr Marktanteil bei unter einem Prozent (NACS 2011: 2).

${ }^{296}$ Im Vergleich zu Benzin ohne Ethanol (E0) verursacht E10 einen erhöhten Kraftstoffverbrauch von $3,7 \%$, E15 von 5,3\%, E20 von $7,7 \%$ und E85 von $27 \%$ (NATIONAL RENEWABLE ENERGY LABORATORY 2009: 3-1; OAK RIDGE NATIONAL LABORATORY 2007: 4; NACS 2012b: 29)
} 
wodurch die Autofahrer bei gleicher Kilometerleistung häufiger zur Tankstelle müssen und sich infolgedessen die Kundenzahl im Geschäft erhöht. Dies ist für die Tankstellenbesitzer von großer Relevanz, denn die Tankstellenshops erwirtschaften durchschnittlich 73\% des Gewinns der gesamten Tankstelle (NACS 2011: 6) ${ }^{297}$.

Basierend auf den vorangegangenen Ausführungen wird folgende Zukunftsprojektion weiter betrachtet:

8A In den USA besteht eine flächendeckende Tankstelleninfrastruktur für den Verkauf von Benzin mit mittleren Ethanolbeimischungen

\subsubsection{Rohstoffkosten bei der Produktion von Mais-Ethanol}

Mais ist in den USA der mit Abstand wichtigste Rohstoff zur Produktion von Ethanol (U.S. ENERGY INFORMATION ADMINISTRATION 2012j: 6) ${ }^{298}$. Im Jahr 2011 wurden 124 Millionen Tonnen Mais zur Ethanolproduktion verwendet - dies sind 40\% der gesamten US-amerikanischen Maisernte (315 Millionen Tonnen) (U.S. ENERGY INFORMATION ADMINISTRATION 2012j: 8). Abbildung 82 zeigt die reale (Basisjahr 2005) Entwicklung des US-amerikanischen Maispreises ${ }^{299}$ in den Jahren von 1989 bis 2010 .

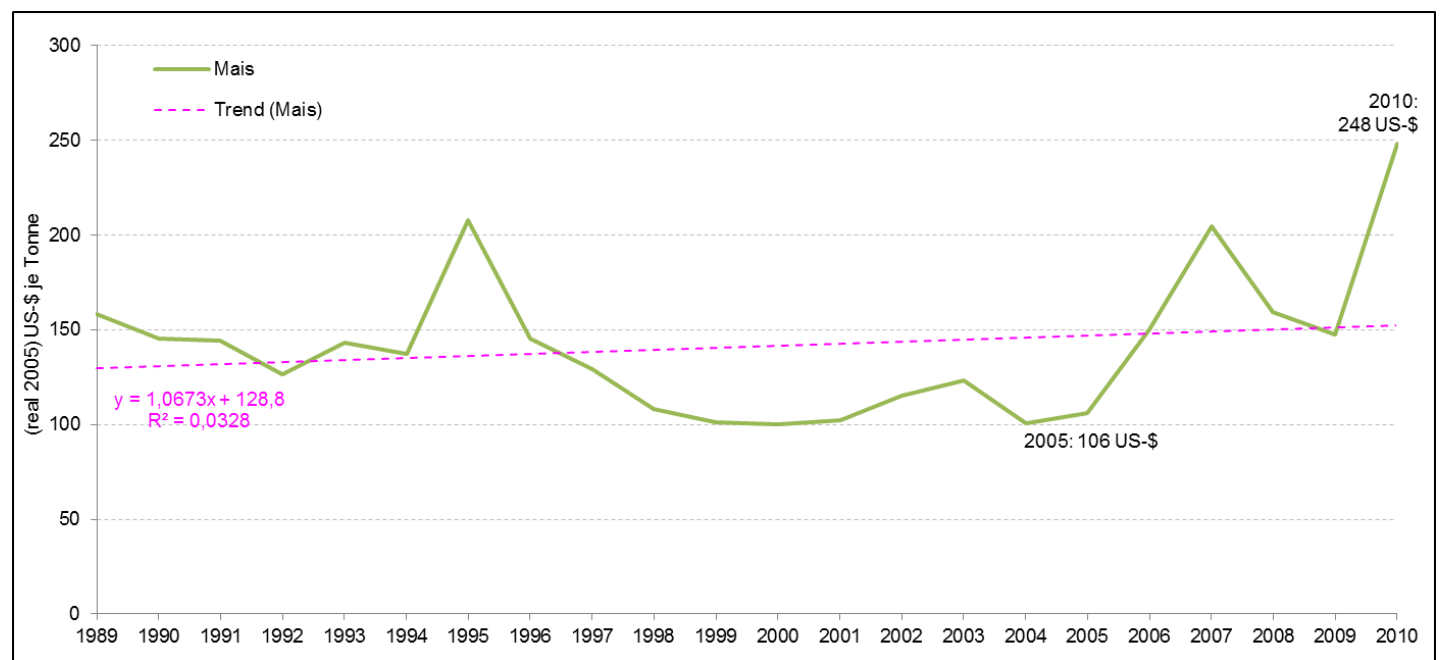

Abbildung 82: Entwicklung des (real 2005) Maispreises $1989-2010$

Quelle: In Anlehnung an OECD/FAO 2012: 121, EIGENE BERECHNUNG

In dem betrachteten Zeitraum (1989 - 2010) kam es erstmals 1995 zu einem kurzfristigen Preisanstieg, dem Jahre (1996 - 2006) niedriger Maispreise von unter (real

${ }^{297} 70 \%$ des gesamten Umsatzes einer durchschnittlichen Tankstelle wird durch den Verkauf von flüssigem Kraftstoff erwirtschaftet. Aufgrund von geringen Margen liegt der Gewinnbeitrag aber bei lediglich 27\% (NACS 2012b: 9; NACS 2011: 6).

${ }^{298} \mathrm{Im}$ Jahr 2012 gibt es in den USA 211 kommerzielle Ethanolanlagen mit einer Gesamtkapazität von 55,6 Milliarden Litern. Lediglich Anlagen mit einer Kapazität von insgesamt 0,53 Milliarden Litern nutzen einen anderen Rohstoff als Mais zur Produktion (RFA 2012c).

${ }^{299}$ Der Maispreis ist als Incoterm (International Commercial Term) F.O.B. (Free On Board) an der US-amerikanischen Golfküste angegeben. 
2005) 150 US-\$/Tonne folgten. Seit dem Jahr 2006 ist der Markt deutlich volatiler, so stiegen die Preise von 106 US-\$/Tonne auf 248 US-\$/Tonne in der Zeitspanne von 2005 bis 2010. Der langfristige Trend (1989 - 2010) zeigt einen Anstieg des US-amerikanischen Maispreises (Abbildung 82).

In Abbildung 83 wird die prognostizierte (real 2005) Entwicklung des Maispreises bis zum Jahr 2021 mittels der Projektionen der Organisationen OECD/FAO, USDA und World Bank dargestellt.

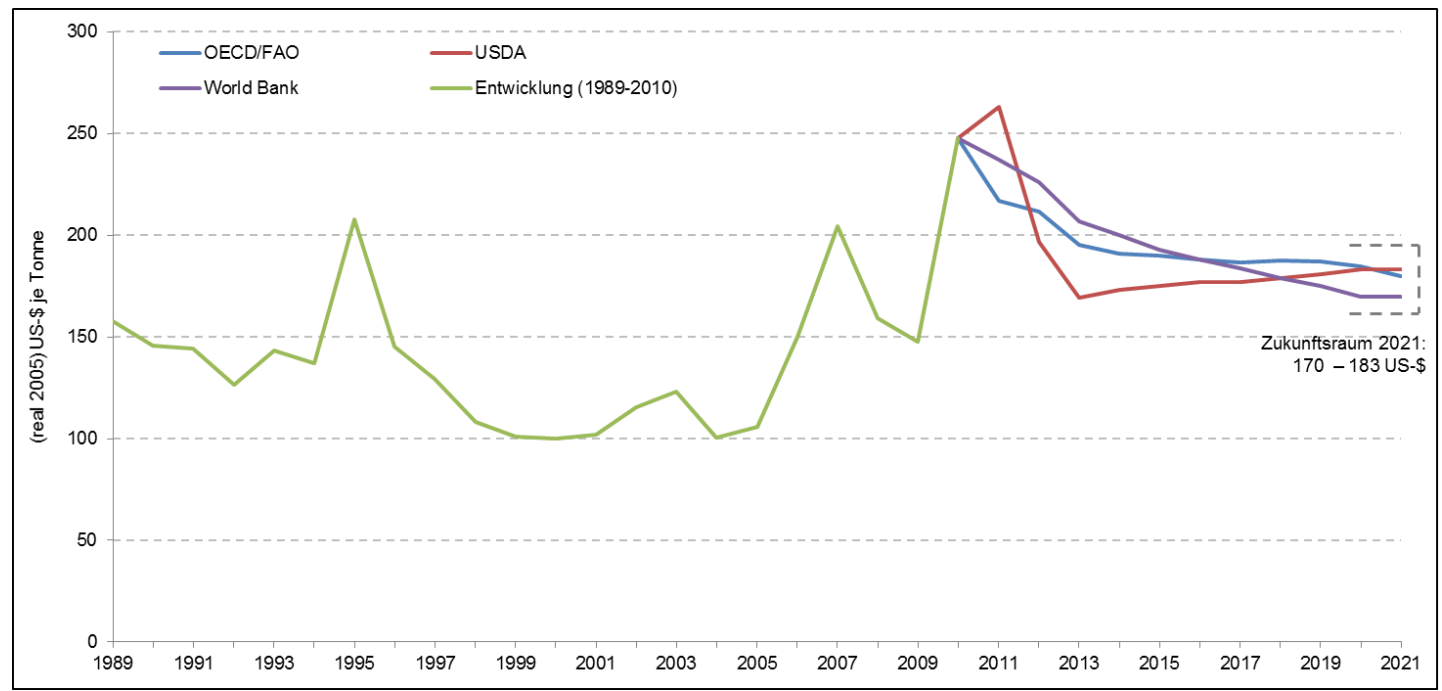

Abbildung 83: Prognostizierte Entwicklung des (real 2005) Maispreises bis 2021

Quelle: In Anlehnung an OECD/FAO 2012: 121; USDA 2012: 66; WORLD BANK DEVELOPMENT PROSPECTS GROUP 2012

In Abbildung 83 ist zu erkennen, dass die Organisationen OECD/FAO, USDA und World Bank ${ }^{300}$ unterschiedlich stark sinkende Maispreise vom Jahr 2010 bis zum Jahr 2014 prognostizieren. In den darauffolgenden Jahren (2015 - 2021) nähern sich die Projektionen jedoch stark an, sodass sich ein Zukunftsraum von (real 2005) 170 - 183 US-\$/Tonne für das Jahr 2021 ergibt $^{301}$. Aufgrund der geringen Unterschiede zwischen den einzelnen Zukunftsprojektionen ist eine Projektion ausreichend, um den Zukunftsraum im dritten Kernschritt „USA Szenario-Bildung und -Konsistenzprüfung" darzustellen. Es wird die Prognose der OECD/FAO gewählt, da diese zwischen den anderen beiden Projektionen liegt und den Zukunftsraum somit am ehesten beschreibt. Da die OECD/FAO die Entwicklung des Maispreises nur bis zum Jahr 2021 prognostiziert, wird mittels einer linearen Trendbe-

\footnotetext{
${ }^{300}$ Auch das FAPRI (2011b: 12) erwartet bis zum Jahr 2021 sinkende Maispreise. Die Daten sind in Abbildung 83 nicht enthalten, da diese nur nominal angegeben sind.

${ }^{301} \mathrm{Im}$ Gegensatz zur kurzfristigen Betrachtung ist bei einem Vergleich der Projektionen mit der langfristigen Vergangenheit (1989 - 2010) ein realer Preisanstieg zu erkennen, insbesondere im Vergleich zu den Maispreisen von unter 150 US-\$/Tonne in den Jahren von 1996 - 2006 (OECD/FAO 2012: 121).
} 
rechnung der Preis für das Jahr 2023 ermittelt (Abbildung 84). Ein Maispreis von (real 2005) 180 US-\$/Tonne wird als Zukunftsprojektion in der weiteren Studie berücksichtigt.

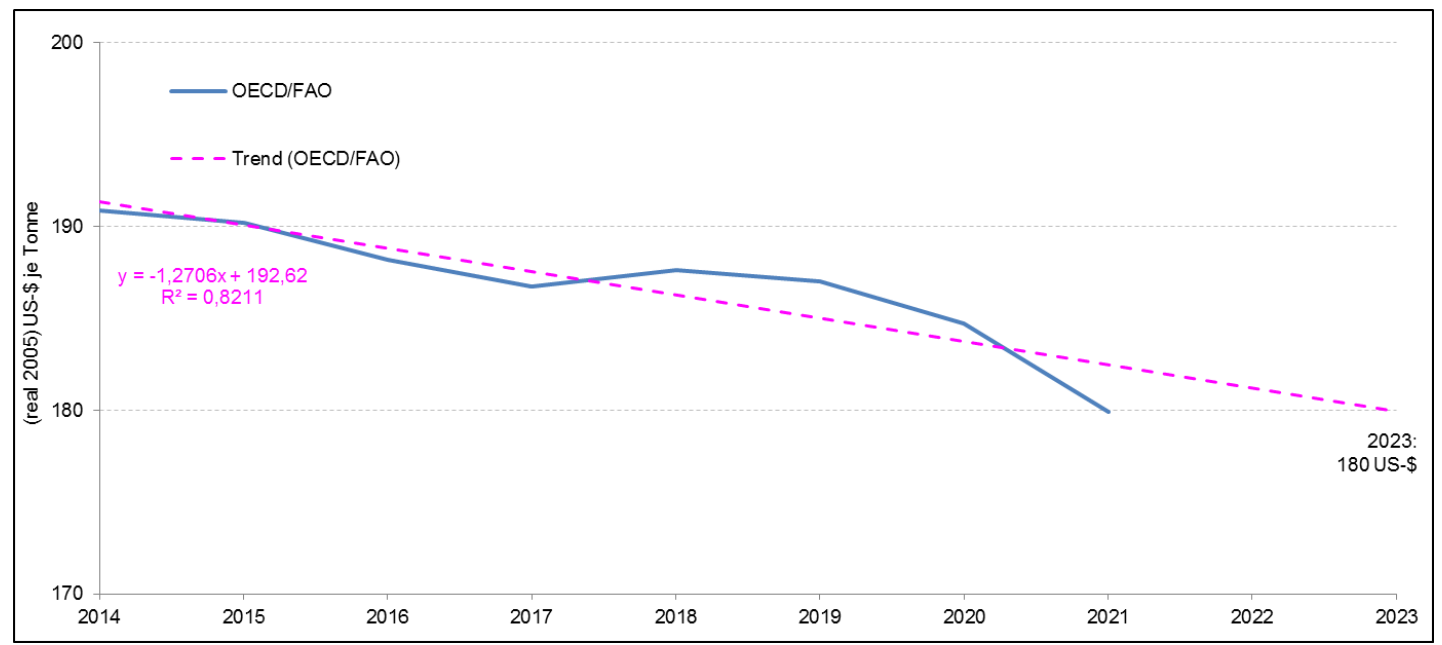

Abbildung 84: Prognostizierte Entwicklung des (real 2005) Maispreises bis 2023

Quelle: In Anlehnung an OECD/FAO 2012: 121; EIGENE BERECHNUNG

Durch die von den Organisationen OECD/FAO, USDA und World Bank prognostizierten relativ konstanten Entwicklungen des Maispreises im Zeitraum von 2014 bis 2021 dürfen sich die Ethanolproduzenten jedoch nicht täuschen lassen, denn der Maispreis wird auch in Zukunft infolge der historisch geringen Lagerbestände im Verhältnis zum Verbrauch (Stock-to-use ratio) ${ }^{302}$ volatil sein. So werden nicht erwartete Ernteausfälle von bedeutenden Mais produzierenden Staaten ${ }^{303} \mathrm{zu}$ starken kurzfristigen Preisschwankungen führen (OECD/FAO 2012: 128). Diese Preisveränderungen werden in den Prognosen nicht berücksichtigt, da sie durch Ereignisse verursacht werden wie z.B. Wetterextreme, die nicht langfristig vorhersehbar sind und nur kurzfristig wirken, sodass sich der Preis langfristig ${ }^{304}$ wieder normalisiert.

Die Projektionen der OECD/FAO, der USDA und der World Bank basieren auf der Annahme, dass die Verbrauchsmandate des RFS 2 für die Gruppe „Conventional Biofuel" bis zum Jahr 2022 unverändert bei einem Volumen von 57 Milliarden Litern bleiben. Ein Anstieg der Mandate für "Conventional Biofuel“ um 19 Milliarden Liter auf 76 Milliarden Liter, wie in der Zukunftsprojektion 22c (Kapitel 6.2.5) beschrieben,

302 Die OECD/FAO (2012: 123) erwartet in den Jahren von 2014 bis 2021 eine „Stock-to-use ratio" von nur knapp über $15 \%$.

${ }^{303}$ Als wichtige Mais produzierende Staaten sind die USA, Brasilien, China, Indien und die Europäische Union zu nennen (OECD/FAO 2012: 123).

${ }^{304}$ Einflüsse, die langfristig den Maispreis beeinflussen, sind beispielsweise Bevölkerungswachstum, Anstieg des Pro-Kopf-Einkommens, Wertverlust des US-\$, höhere Energiekosten, steigende Biokraftstoffproduktion und geringere Produktivitätssteigerungen in der Landwirtschaft. 
wird in den Prognosen nicht berücksichtigt. Zur Produktion von 19 Milliarden Litern Ethanol werden zusätzlich 40 Millionen Tonnen ${ }^{305}$ Mais benötigt. Dies entspricht $13 \%$ der US-amerikanischen Maisproduktion im Jahr 2011 und ist damit vom Umfang her vergleichbar mit den durchschnittlichen jährlichen US-amerikanischen Maisexporten der Jahre 2010, 2011 und 2012 in Höhe von jeweils etwa 45 Millionen Tonnen ${ }^{306}$ (USDA 2012: 66). Aufgrund der erhöhten Nachfrage nach Mais wird in Übereinstimmung mit der OECD/FAO (2012: 117) ein Preisanstieg von 16\% auf (real 2005) 209 US-\$/Tonne prognostiziert und als zweite Zukunftsprojektion in der weiteren Arbeit berücksichtigt.

Basierend auf den vorangegangenen Erläuterungen werden zwei Zukunftsprojektionen in der „USA Szenario-Bildung und -Konsistenzprüfung“ weiter betrachtet:

$12 \mathrm{aA}$ Maispreis (real 2005) 180 US-\$/Tonne

Maispreis (real 2005) 209 US-\$/Tonne

12aB Beschreibung:

Anstieg des Maispreises aufgrund der Erhöhung des Verbrauchsmandats „Conventional Biofuel" auf 76 Milliarden Liter.

\subsubsection{Anzahl der Kraftfahrzeuge, die mit mittleren Ethanolbeimischungen zu Benzin (E15 - E40) fahren können}

In den USA ist die Anzahl der Kraftfahrzeuge ${ }^{307}$ von 182 Millionen im Jahr 1990 auf 230 Millionen im Jahr 2010 gestiegen (Abbildung 85) ${ }^{308}$, was einem durchschnittlichen jährlichen Wachstum von 1,2\% entspricht. Ausschließlich in den Jahren 2009 und 2010 kam es aufgrund der Finanzkrise zu einem leichten Rückgang der Größe

\footnotetext{
${ }^{305}$ Es wird eine Tonne Mais zur Produktion von 471 Litern Ethanol benötigt (U.S. DEPARTMENT OF ENERGY 2013a).

${ }^{306}$ Die USA sind mit einem Exportvolumen von 45 Millionen Tonnen der mit Abstand wichtigste Maisexporteur der Welt (USDA 2013a).

${ }^{307}$ Die Anzahl der Kraftfahrzeuge errechnet sich aus der Addition von Light Duty Vehicle (Short Wheel Base) und Light Duty Vehicle (Long Wheel Base). Die beiden Gruppen - Short Wheel Base/Long Wheel Base - unterscheiden sich anhand ihres Radstands. Short Wheel Base sind Kraftfahrzeuge mit einem Radstand kleiner gleich 121 Zoll $(307 \mathrm{~cm})$, während Long Wheel Base Kraftfahrzeuge mit einem Radstand größer als 121 Zoll sind (U.S. DEPARTMENT OF TRANSPORTATION 2012a: 257). Die Begriffe Kraftfahrzeug und Automobil sind als Synonyme zu verstehen.

${ }^{308}$ Eine Unterteilung der Kraftfahrzeuge in Benzin- und Dieselfahrzeuge, wie sie für den europäischen Automobilmarkt vorgenommen wurde (Kapitel 5.2.4), ist für die Betrachtung des US-amerikanischen Marktes nicht notwendig, da Diesel nur einen sehr geringen Marktanteil besitzt (U.S. DEPARTMENT OF TRANSPORTATION 2012a: 256; U.S. ENERGY INFORMATION ADMINISTRATION 2012b: 85; WOOD MCKENZIE 2010: 108). Ferner wird erwartet, dass der Kraftstoff Diesel auch im Jahr 2023 von keiner großen Bedeutung sein wird (U.S. ENERGY INFORMATION ADMINISTRATION 2012b: 85; WOOD MCKENZIE 2010: 108).
} 
der Kraftfahrzeugflotte in den USA ${ }^{309}$ (CENTER FOR AUTOMOTIVE RESEARCH 2011: 2).

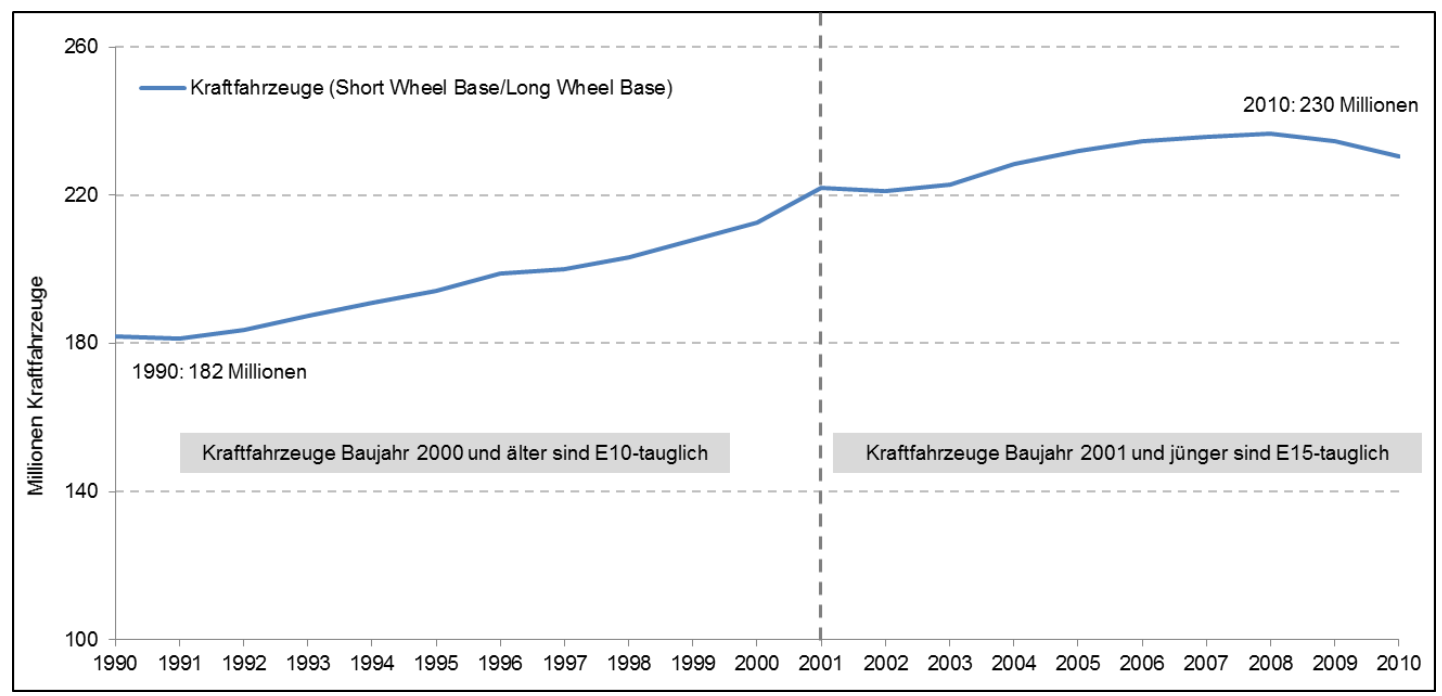

Abbildung 85: Entwicklung der Anzahl an Kraftfahrzeugen in den USA in den Jahren von 1990 bis 2010

Quelle: In Anlehnung U.S. DEPARTMENT OF TRANSPORTATION 2012a: 254f.; U.S. DEPARTMENT OF TRANSPORTATION 2012b; U.S. ENERGY INFORMATION ADMINISTRATION 2007; U.S. ENERGY INFORMATION ADMINISTRATION 2011a: 84; EIGENE BERECHNUNG

Alle ab dem Jahr 1970 verkauften Kraftfahrzeuge in den USA sind in der Lage, E10 als Kraftstoff zu nutzen (U.S. ENERGY INFORMATION ADMINISTRATION 2007). Darüber hinaus haben umfangreiche Tests der EPA ergeben, dass Fahrzeuge ab dem Baujahr 2001 auch den Kraftstoff E15 nutzen können (EPA 2012a; U.S. ENERGY INFORMATION ADMINISTRATION 2011a: 84). Somit vertragen alle Kraftfahrzeuge in den USA im Jahr 2010 den Kraftstoff E10 und 143 Millionen Fahrzeuge sogar E15 (RFA 2012b).

Basierend auf der Ist-Situation wird die Entwicklung des Einflussfaktors in vier aufeinander folgenden Schritten betrachtet. Während der erste Schritt der Ermittlung der Anzahl an zugelassenen Kraftfahrzeugen in den USA im Jahr 2023 dient, werden im zweiten Schritt die Fahrzeuge in vier Altersklassen unterteilt. Im dritten Schritt werden zwei unterschiedliche Annahmen gebildet, die darstellen, ab welchem Baujahr die Fahrzeuge eine bestimmte prozentuale Ethanolbeimischung zu Benzin als Kraftstoff nutzen können. Im letzten Schritt werden die Ergebnisse aus Schritt zwei und drei verknüpft, um die Anzahl an Kraftfahrzeugen im Jahr 2023 zu ermitteln, die eine festgelegte Ethanolbeimischung zu Benzin als Kraftstoff verwen-

\footnotetext{
${ }^{309}$ Mit 230 Millionen Kraftfahrzeugen besitzt die USA eine sehr hohe Automobildichte von über 700 Fahrzeugen je 1000 Einwohner (INTERNATIONAL ENERGY AGENCY 2011a: 114f.).
} 
den können. Die Ergebnisse aus Schritt vier werden als Zukunftsprojektionen in der Studie weiter betrachtet.

Im ersten Schritt wird die Größe der Automobilflotte in den USA im Jahr 2023 ermittelt. Die folgenden Ausführungen basieren auf einer Studie des CENTER FOR AUTOMOTIVE RESEARCH (2011: 2ff.), derzufolge die Entwicklung der Kraftfahrzeugflotte in den USA maßgeblich von der Anzahl an Haushalten sowie der durchschnittlichen Anzahl an Fahrzeugen je Haushalt abhängt. Bis zum Jahr 2023 wird ein Anstieg der Zahl der Haushalte von 117 Millionen im Jahr 2009 auf 134,5 Millionen im Jahr 2023 erwartet. Die Zahl der Kraftfahrzeuge pro Haushalt beträgt derzeit 2,05 und für das Jahr 2023 wird ein Wert von 2,07 prognostiziert. Aus der Multiplikation dieser beiden Zahlen errechnet sich für das Jahr 2023 eine Automobilflotte in den USA von 278 Millionen Kraftfahrzeugen. Die Ergebnisse des CENTER FOR AUTOMOTIVE RESEARCH (2011: 2ff.) werden durch die Organisationen U.S. ENERGY INFORMATION ADMINISTRATION (2013f) und INTERNATIONAL ENERGY AGENCY (2011a: 115) bestätigt, die ebenfalls einen langfristigen Anstieg der USamerikanischen Kraftfahrzeugflotte prognostizieren.

Im folgenden Schritt wird die Altersstruktur der 278 Millionen Kraftfahrzeuge im Jahr 2023 ermittelt, was analog zum Vorgehen von WOOD MACKENZIE (2010: 144) erfolgt, der dies für den Kraftfahrzeugmarkt der EU27 durchgeführt hat (Kapitel 5.2.4). Grundlage hierfür ist die Unterteilung der Kraftfahrzeuge nach ihrem Alter in die Klassen: „Neu“, „1-4 Jahre“, „5-10 Jahre“ und „>10 Jahre“ (Tabelle 17).

\begin{tabular}{|c|c|c|}
\hline $\begin{array}{l}\text { Alter der Kraftfahrzeuge im Jahr } \\
22023\end{array}$ & $\begin{array}{l}\text { Prozentualer Anteil an der } \\
\text { Gesamtflotte im Jahr } 2023\end{array}$ & Anzahl der Kraftfahrzeuge \\
\hline Neu (Baujahr: 2023) & $6,3 \%$ & 17,5 Millionen \\
\hline 1 - 4 Jahre (Baujahr: 2022 - 2018) & $19,4 \%$ & 53,9 Millionen \\
\hline $\begin{array}{c}5 \text { - } 10 \text { Jahre (Baujahr: } 2017 \text { - } \\
2013)\end{array}$ & $24,3 \%$ & 67,6 Millionen \\
\hline $\begin{array}{c}\text { >10 Jahre (Baujahr: Älter als Bau- } \\
\text { jahr 2013) }\end{array}$ & $50 \%$ & 139 Millionen \\
\hline Total & $100 \%$ & 278 Millionen \\
\hline
\end{tabular}

Tabelle 17: Alter und Anzahl der Kraftfahrzeuge in den USA im Jahr 2023

Quelle: In Anlehnung WOOD MACKENZIE 2010: 144; U.S. ENERGY INFORMATION ADMINISTRATION 2012b: 169; OAK RIDGE NATIONAL LABORATORY 2012: 3-14; EIGENE BERECHNUNG

Die prozentualen Werte in Tabelle 17 wurden wie folgt ermittelt: Die 6,3\% in der Altersklasse „Neu (Baujahr: 2023)“ basieren auf der Annahme der U.S. ENERGY 
INFORMATION ADMINISTRATION (2012b: 169), dass im Jahr 2023 17,5 Millionen Kraftfahrzeuge in den USA neu zugelassen werden ${ }^{310}$. Der Wert $50 \%$ in der Klasse „>10 Jahre (Baujahr: Älter als Baujahr 2013)“ beruht auf der Prognose, dass das heutige zehnjährige Durchschnittsalter der Fahrzeuge in den USA noch im Jahr 2023 bestehen wird (OAK RIDGE NATIONAL LABORATORY 2012: 3-14). Die Werte $19,4 \%$ und $24,3 \%$ für die Altersklassen „1 - 4 Jahre (Baujahr: 2022 - 2018)“ und „5 - 10 Jahre (Baujahr: 2017 - 2013)“ entstammen der Erwartung, dass die Kraftfahrzeuge in den beiden Altersklassen unabhängig vom Baujahr noch zu gleichen jährlichen Anteilen im Jahr 2023 genutzt werden ${ }^{311}$.

Im dritten Schritt werden zwei Annahmen bezüglich der Fähigkeit der Kraftfahrzeuge, im Jahr 2023 mittlere Ethanolbeimischungen zu Benzin als Kraftstoff verwenden zu können, gebildet. Die Bildung von zwei Annahmen ist notwendig, da zum heutigen Zeitpunkt ungewiss ist, inwieweit die zukünttig in den USA zugelassenen Fahrzeuge mittlere Ethanolgehalte im Benzin als Kraftstoff verwenden können ${ }^{312}$. Die zwei Annahmen „Hohe Ethanolverträglichkeit von Kraftfahrzeugen“ und „Geringe Ethanolverträglichkeit von Kraftfahrzeugen“ gehen von folgenden unterschiedlichen Situationen im Jahr 2023 aus:

Annahme 1: „Hohe Ethanolverträglichkeit von Kraftfahrzeugen“

- Alle zugelassenen Kraftfahrzeuge in den USA im Jahr 2023 vertragen E15 als Kraftstoff.

- Alle zugelassenen Kraftfahrzeuge in den USA ab dem Jahr 2015 vertragen E20 als Kraftstoff.

- Alle zugelassenen Kraftfahrzeuge in den USA ab dem Jahr 2020 vertragen E25 als Kraftstoff.

Annahme 2: „Geringe Ethanolverträglichkeit von Kraftfahrzeugen“

- Alle zugelassenen Kraftfahrzeuge in den USA im Jahr 2023 vertragen E15 als Kraftstoff.

${ }^{310}$ Der Wert von 6,3\% drückt das Verhältnis der Anzahl an neu zugelassenen Kraftfahrzeugen im Jahr 2023 (17,5 Millionen) zur gesamten US-amerikanischen Automobilflotte (278 Millionen) aus.

${ }^{311}$ Das Vorgehen zur Ermittlung der Prozentwerte für die Zeiträume „1 - 4 Jahre (Baujahr: 2022 - 2018)“ und „5 - 10 Jahre (Baujahr: 2017 - 2013)“ basiert auf dem Vorgehen von WOOD MACKENZIE (2010: 144), welches das Unternehmen zur Ermittlung der Daten für den Automobilmarkt der EU27 in Tabelle 12 angewandt hat.

${ }^{312}$ Die über mehrere Jahre andauernden Tests der EPA zur Zulassung von E15 zeigen die Schwierigkeit, festzustellen, ab welchem Baujahr die in den USA zugelassenen Kraftfahrzeuge mittlere Ethanolbeimischungen zu Benzin als Kraftstoff verwenden können (U.S. GOVERNMENT INFORMATION 2011: 4662f.). Aufgrund dieser Unsicherheit ist es notwendig, unterschiedliche Annahmen zu entwickeln und mit diesen weiter zu arbeiten. 
- Alle zugelassenen Kraftfahrzeuge in den USA ab dem Jahr 2017 vertragen E20 als Kraftstoff.

- Bis Ende 2023 zugelassene Kraftfahrzeuge vertragen keine höheren Ethanolbeimischungen als E20.

In beiden Annahmen wird davon ausgegangen, dass alle Kraftfahrzeuge E15 vertragen. Die Fahrzeuge, die vor 2001 zugelassen wurden und nur E10 als Kraftstoff verwenden können, werden aufgrund ihrer geringen Anzahl im Jahr 2023 nicht weiter betrachtet ${ }^{313}$.

Die erste Annahme „Hohe Ethanolverträglichkeit von Kraftfahrzeugen“ basiert auf einer Studie des EUROPEAN COMMISSION JOINT RESEARCH CENTRE, INSTIUTUT FOR ENERGY ET AL. (2011: 36), die für den europäischen Kraftfahrzeugmarkt das Jahr 2015 als frühstmöglichen Zeitpunkt der Verträglichkeit von E20 nennt. Da sich die Fahrzeuge in den Märkten der EU und der USA stark ähneln, ist die Einschätzung auf den Kraftfahrzeugmarkt der USA übertragbar. Die Projektion ist darüber hinaus realistisch, da Untersuchungen des NATIONAL RENEWABLE ENERGY LABORATORY (2009: xvif.) gezeigt haben, dass Fahrzeuge Baujahr 2004 in den USA zum Teil schon E20 als Kraftstoff nutzen können ${ }^{314}$. Die Annahme geht des Weiteren davon aus, dass die technischen Probleme an den Dichtungen und dem Verbrennungsmotor, die momentan noch die flächendeckende Nutzung von E25 verhindern, bis zum Jahr 2020 gelöst werden (WOOD MACKENZIE 2010: 102f.). Dies ist wahrscheinlich, denn durch die politisch vorgegebenen Verbrauchsmandate für Biokraftstoffe im RFS $2^{315}$ wird von den Automobilkonzernen erwartet, dass sie die genannten Probleme lösen, damit höhere Mengen Ethanol abgesetzt werden können (CONGRESSIONAL RESEARCH SERVICE 2010a: 3).

Die zweite Annahme "Geringe Ethanolverträglichkeit von Kraftfahrzeugen" basiert ebenfalls auf der Studie der Organisation EUROPEAN COMMISSION JOINT RE-

\footnotetext{
${ }^{313}$ Kraftfahrzeuge, die im Jahr 2000 oder früher zugelassen wurden und nur E10 vertragen, sind im Jahr 2023 mindestens 23 Jahre alt. Das durchschnittliche Alter der USamerikanischen Fahrzeuge liegt aktuell bei 10,8 Jahren (OAK RIDGE NATIONAL LABORATORY 2012: 3-1) und auch im Jahr 2023 wird ein Durchschnittsalter von 10 Jahren erwartet. Deshalb ist davon auszugehen, dass diese Kraftfahrzeuge aufgrund ihres Alters in der Mehrzahl nicht mehr für den öffentlichen Straßenverkehr zugelassen sind. Sie bilden nur einen sehr geringen Anteil der Automobilflotte im Jahr 2023 und sind somit zu vernachlässigen.

314 Alle Kraftfahrzeuge von VW, die in Europa ab dem Baujahr 2010 verkauft werden, können den Kraftstoff E20 verwenden (SEYFRIED 2012).

${ }^{315}$ Eine ausführliche Beschreibung des RFS 2 befindet sich in Kapitel 6.2.5.
} 
SEARCH CENTRE, INSTITUT FOR ENERGY ET AL. $(2011: 36)^{316}$, in der die Verwendung von E20 ab dem Jahr 2017 prognostiziert wird, da die gesamte Automobilindustrie die technischen Probleme am Verbrennungsmotor und an den Dichtungen bei der Nutzung von E20 nicht früher wird lösen können (WOOD MACKENZIE 2010: 102f.), bzw. die Automobilkonzerne erst 2017 bereit sein werden, Bauteile zu verwenden, die E20-tauglich sind. Eine weitere Verbesserung der Ethanolverträglichkeit der Kraftfahrzeuge, die die Verwendung von E25 ermöglichen würde, wird bis zum Jahr 2023 nicht erwartet (OAK RIDGE NATIONAL LABORATORY 2011: 2ff.).

Basierend auf der Annahme „Hohe Ethanolverträglichkeit von Kraftfahrzeugen“ (Schritt: 3) und unter Berücksichtigung der prognostizierten Anzahl an Fahrzeugen im Jahr 2023 sowie aus deren Altersstruktur (Schritt: 2) ergeben sich folgende Ergebnisse für das Jahr 2023 (Abbildung 86) ) $^{317}$ : 44,5 Millionen Kraftfahrzeuge bzw. 16\% der Kraftfahrzeugflotte können E25 als Kraftstoff verwenden. Weitere 67,5 Millionen Fahrzeuge (24,3\%) sind in der Lage, 20-prozentige Ethanolbeimischungen zu Benzin als Kraftstoff zu verwenden, und die restlichen 166 Millionen Kraftfahrzeuge - 59,7\% der Kraftfahrzeugflotte - sind E15-tauglich.

\footnotetext{
${ }^{316}$ In der Studie des EUROPEAN COMMISSION JOINT RESEARCH CENTRE, INSTITUT FOR ENERGY ET AL. (2011) wurden zwei Projektionen darüber gebildet, wie weit in Zukunft die europäische Kraftfahrzeugflotte mittlere Ethanolbeimischungen verträgt. Diese zwei unterschiedlichen Prognosen bilden die Grundlage der beiden Annahmen „Hohe Ethanolverträglichkeit von Kraftfahrzeugen“ und "Geringe Ethanolverträglichkeit von Kraftfahrzeugen“. ${ }^{317}$ Kraftfahrzeuge, die fähig sind, Ethanolbeimischungen von 25\% zu Benzin als Kraftstoff zu nutzen, sind ebenso in der Lage, auch geringere Ethanolbeimischungen zu Benzin $(20 \%, 15 \%$ und $10 \%$ ) als Kraftstoff zu verwenden.
} 


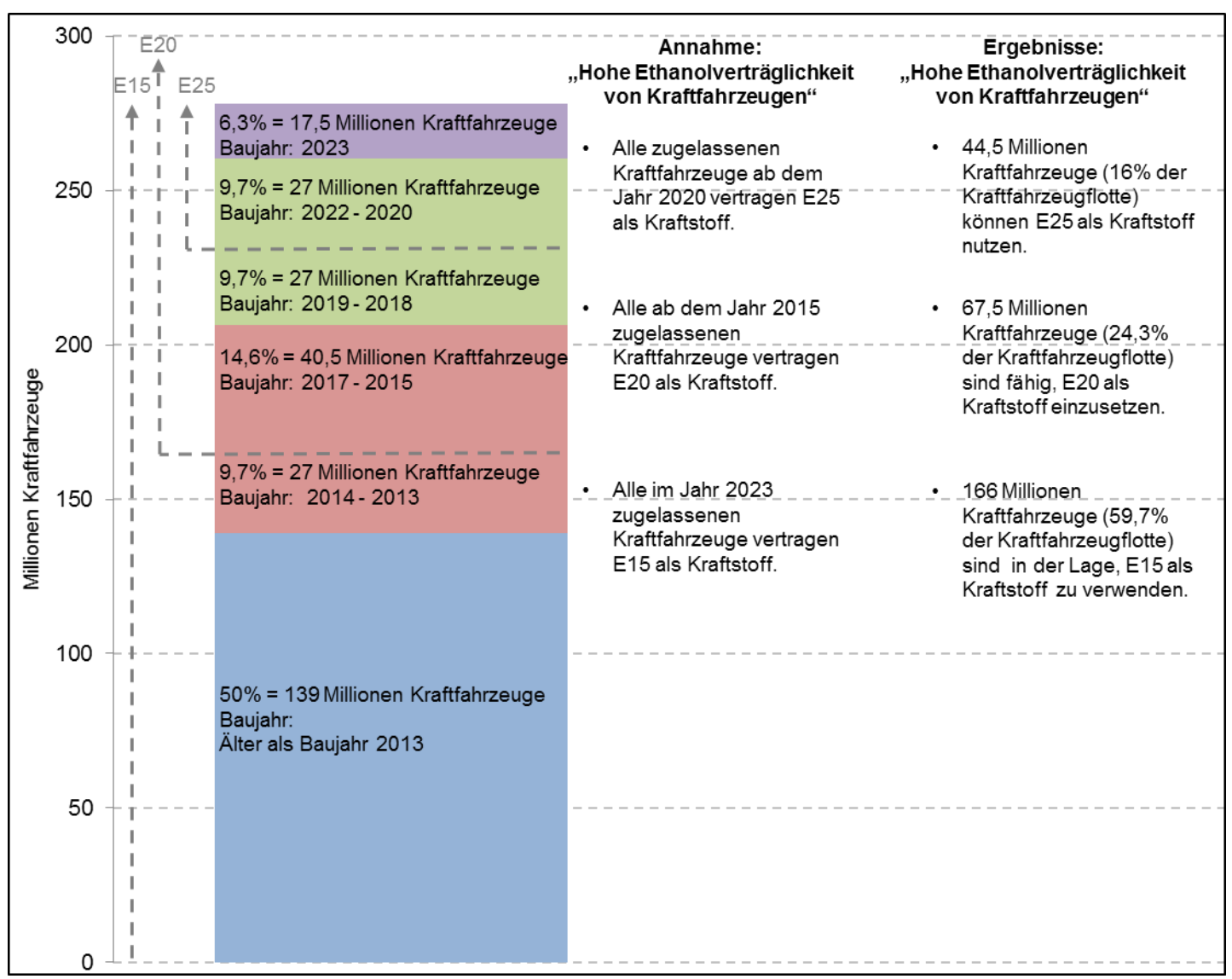

Abbildung 86: Darstellung der Ergebnisse der Annahme „Hohe Ethanolverträglichkeit von Kraftfahrzeugen" in den USA im Jahr 2023

Quelle: In Anlehnung an CENTER FOR AUTOMOTIVE RESEARCH 2011: 2ff.; WOOD MACKENZIE 2010: 144; U.S. ENERGY INFORMATION ADMINISTRATION 2012b: 169; OAK RIDGE NATIONAL LABORATORY 2012: 3-14; EUROPEAN COMMISSION JOINT RESEARCH CENTRE, INSTITUT FOR ENERGY ET AL. 2011: 36; EIGENE BERECHNUNG

Auf Grundlage der zweiten Annahme „Geringe Ethanolverträglichkeit von Kraftfahrzeugen" sowie der in Schritt zwei beschriebenen Altersstruktur der 278 Millionen Fahrzeuge im Jahr 2023 ergeben sich folgende Ergebnisse (Abbildung 87): 85,1 Millionen Kraftfahrzeuge - 30,6\% der gesamten Kraftfahrzeugflotte - sind in der Lage, den Kraftstoff E20 zu verwenden. Die anderen 192,9 Millionen Kraftfahrzeuge $(69,4 \%)$ sind E15-fähig. 


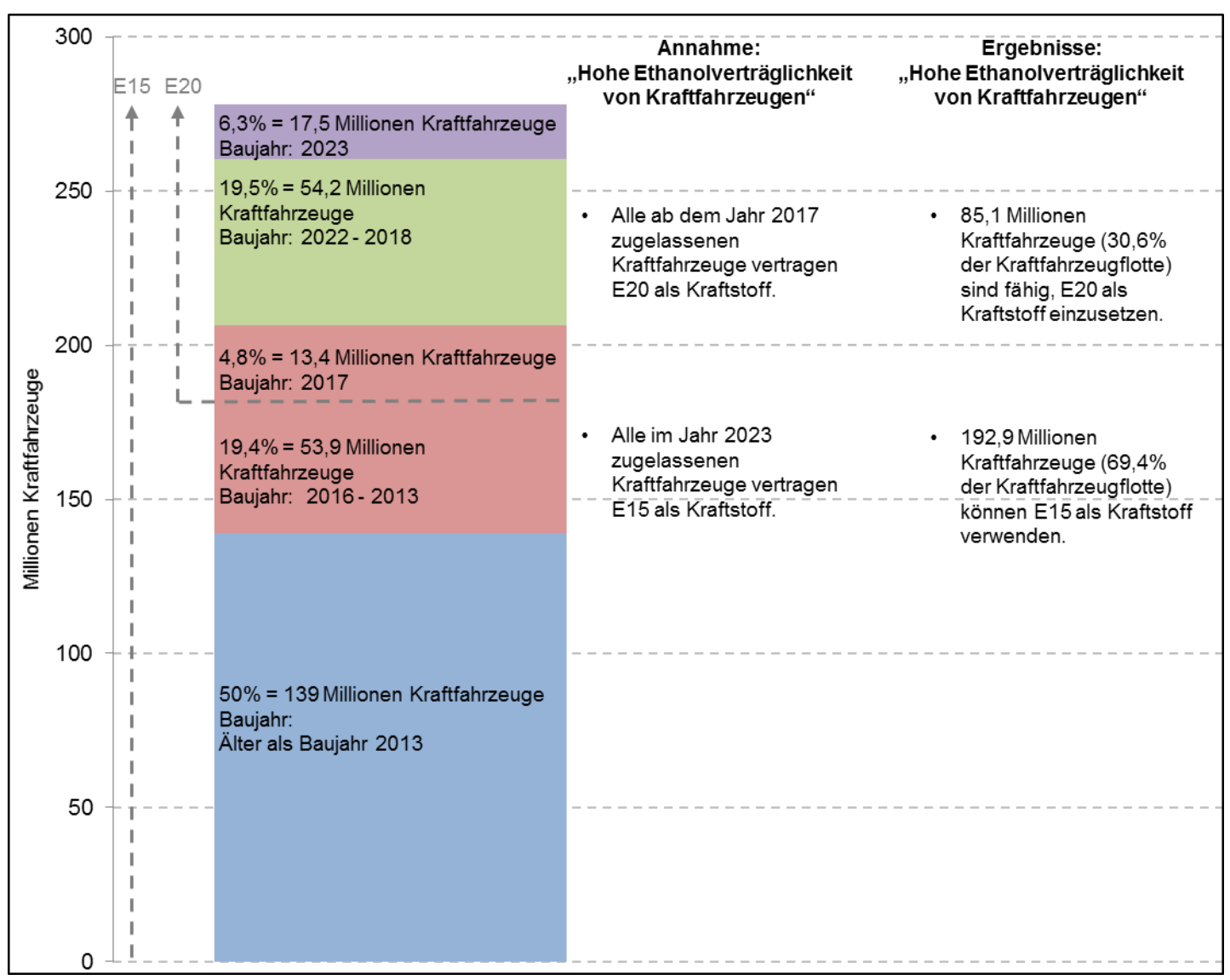

Abbildung 87: Darstellung der Ergebnisse der Annahme „Geringe Ethanolverträglichkeit von Kraftfahrzeugen" in den USA im Jahr 2023

Quelle: In Anlehnung an CENTER FOR AUTOMOTIVE RESEARCH 2011: 2ff.; WOOD MACKENZIE 2010: 144; U.S. ENERGY INFORMATION ADMINISTRATION 2012b: 169; OAK RIDGE NATIONAL LABORATORY 2012: 3-14; EUROPEAN COMMISSION JOINT RESEARCH CENTRE, INSTITUT FOR ENERGY ET AL. 2011: 36; EIGENE BERECHNUNG.

Die beiden Ergebnisse bilden die Grundlage der zwei Zukunftsprojektionen für das Jahr 2023, die in der „USA Szenario-Bildung und -Konsistenzprüfung“ weiter betrachtet werden:

Hohe Ethanolverträglichkeit von Kraftfahrzeugen

Beschreibung:

15A 16\% (44,5 Millionen) der Kraftfahrzeugflotte können E25 als Kraftstoff nutzen sowie 24,3\% (67,5 Millionen) der Kraftfahrzeugflotte sind E20-tauglich. Die restlichen 59,7\% (166 Millionen) der Fahrzeuge sind in der Lage, den Kraftstoff E15 zu verwenden.

\section{Geringe Ethanolverträglichkeit von Kraftfahrzeugen}

Beschreibung:

15B 30,6\% (85,1 Millionen) der Kraftfahrzeugflotte können E20 als Kraftstoff nutzen, die verbleibenden 69,4\% (192,9 Millionen) der Fahrzeuge sind fähig, E15 als Kraftstoff zu verwenden. 


\subsubsection{Biokraftstoff-Verbrauchsmandate - Renewable Fuel Standard 2 (RFS 2)}

Im RFS $2^{318}$ sind bis zum Jahr 2022 jährliche Mandate für Biokraftstoffe festgelegt, die im US-amerikanischen Transportbereich verbraucht werden müssen ${ }^{319}$. Der RFS 2 ist Bestandteil des „Energy Independence and Security Act of 2007“ (EISA) (U.S. GOVERNMENT INFORMATION 2007: 121 STAT. 1519). Die Erfüllung der Mandate wird von der EPA überwacht (CONGRESSIONAL RESEARCH SERVICE 2012: 2). Der RFS 2 ist die Fortsetzung des RFS 1, der durch den „Energy Policy Act“ im Jahr 2005 in Kraft getreten ist, jedoch keine Bedeutung mehr besitzt (CONGRESSIONAL RESEARCH SERVICE 2012: 1f.). Die jährlichen Verbrauchsmandate des RFS 2 steigen auf bis zu 136 Milliarden Liter im Jahr 2022 an und unterteilen sich in die „Conventional Biofuels“ und die „Advanced Biofuels“320. Die Gruppe der „Advanced Biofuels“ untergliedert sich des Weiteren in die Unterkategorien „Advanced: Cellulosic Biofuel“, „Advanced: Biomass-Based Diesel“ und „Advanced: Unspecified Biofuel“ (U.S. GOVERNMENT INFORMATION 2007: 121 STAT. 1519ff.). Abbildung 88 stellt die einzelnen Verbrauchsmandate grafisch dar ${ }^{321}$.

\footnotetext{
${ }^{318}$ Bei der Betrachtung des Schlüsselfaktors werden ausschließlich die jährlichen Verbrauchsmandate betrachtet. Nicht berücksichtigt werden die Treibhausgasreduktionswerte von Biokraftstoffen im Vergleich zu Fossilbrennstoff, die ebenfalls im RFS 2 beschrieben sind. Denn dieser Bestandteil des RFS 2 wurde als eigenständiger Faktor 23 in der Wirkungsanalyse betrachtet und besitzt lediglich einen „eher starken Einfluss“ auf den USamerikanischen Ethanolmarkt und wird daher nicht weiter im Prozess berücksichtigt (Kapitel 6.1.2.2).

${ }_{319}$ Die jährlich festgelegten Mandate beschreiben die politisch festgelegte Untergrenze des Biokraftstoffverbrauchs. Folglich dürfen auch größere Mengen Biokraftstoffe im USamerikanischen Transportbereich verwendet werden (CONGRESSIONAL RESEARCH SERVICE 2012: 2).

${ }^{320}$ Der Begriff „Advanced“ ist in diesem Zusammenhang kein technischer, sondern ein politischer Begriff. So werden Biokraftstoffe der ersten Generation beispielsweise aus Zuckerrohr und Sojabohnen ebenfalls der Gruppe „Advanced“ angerechnet (U.S. GOVERNMENT INFORMATION 2007: 121 STAT. 1519ff.).

${ }^{321}$ Die einzelnen Verbrauchsmandate des RFS 2 können jedoch jährlich von der EPA angepasst werden (CONGRESSIONAL RESEARCH SERVICE 2012: 3).
} 


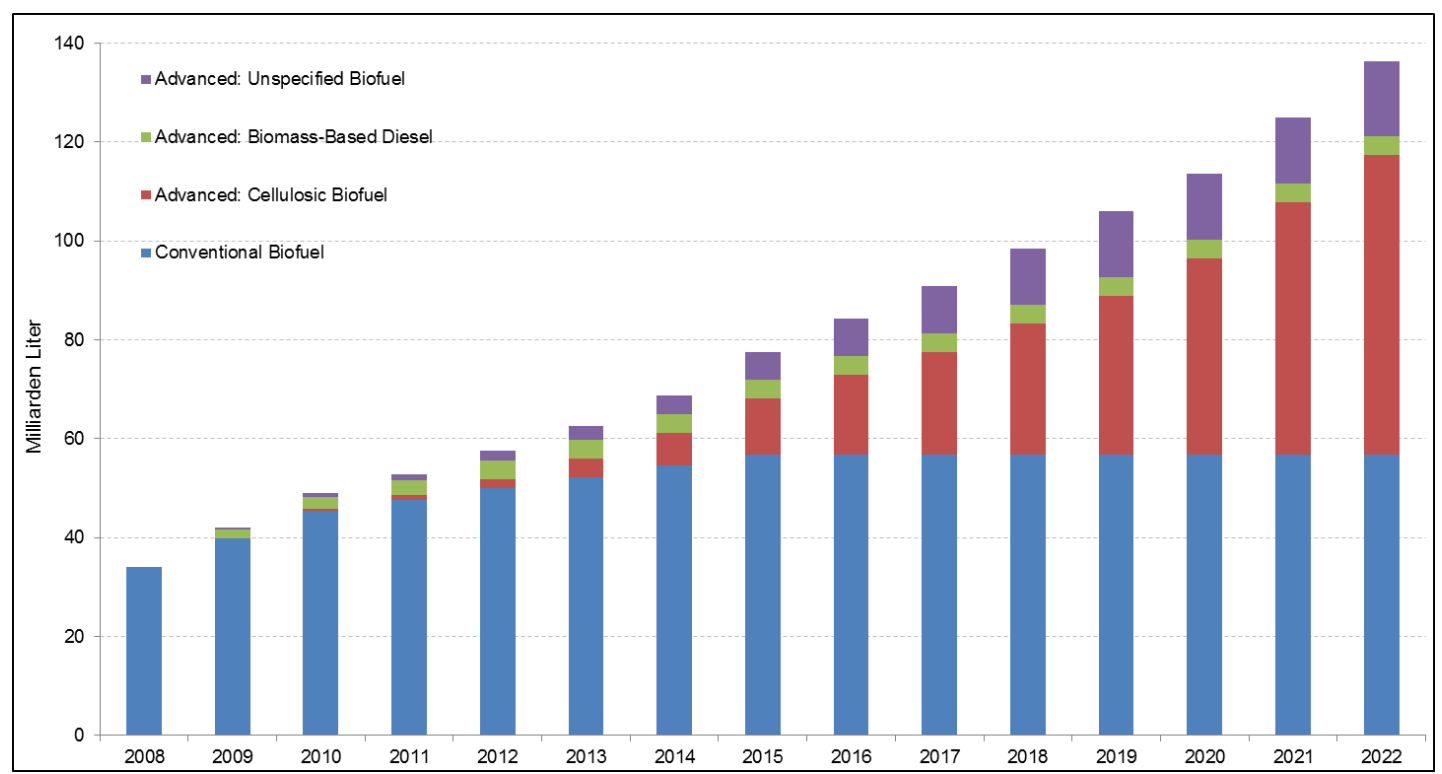

Abbildung 88: Biokraftstoffverbrauchsmandate des RFS 2 bis zum Jahr 2022

Quelle: In Anlehnung an U.S. GOVERNMENT INFORMATION 2007: 121 STAT. 1522; CONGRESSIONAL RESEARCH SERVICE 2012: 3

Die einzelnen Verbrauchsmandate besitzen im RFS 2 eine hierarchische Struktur. So können in der Gruppe „Advanced Biofuel“ Biokraftstoffe, die den Mandaten „Advanced: Cellulosic Biofuel“ und „Advanced: Biomass-Based Diesel“ angerechnet werden, auch das Mandat „Advanced: Unspecified Biofuel“ erfüllen (U.S. GOVERNMENT INFORMATION 2007: 121 STAT. 1521ff.; CONGRESSIONAL RESEARCH SERVICE 2012: 4). Dies zeigt, dass die Verbrauchsmandate „Advanced: Cellulosic Biofuel“ und „Advanced: Biomass-Based Diesel“ dem Mandat „Advanced: Unspecified Biofuel“ übergeordnet sind. Des Weiteren dürfen der Gruppe „Advanced Biofuel“ keine Biokraftstoffe angerechnet werden, die zur Gruppe der „Conventional Biofuels“ gehören (U.S. GOVERNMENT INFORMATION 2007: 121 STAT. 1519). Aufgrund dessen ist die Ethanolproduktion aus Mais ab dem Jahr 2015 auf maximal 57 Milliarden Liter beschränkt ${ }^{322}$ (U.S. GOVERNMENT INFORMATION 2007: 121 STAT. 1521ff.). Im Gegensatz dazu dürfen jedoch biogene Treibstoffe der Gruppe „Advanced Biofuel“ dem Verbrauchsmandat der „Conventional Biofuels“ angerechnet werden (CONGRESSIONAL RESEARCH SERVICE 2012: 5). Demzufolge ist die Gruppe „Advanced Biofuel“ dem Mandat „Conventional Biofuel" übergeordnet.

${ }^{322}$ Jegliche Biokraftstoffe aus dem Rohstoff Mais können ausschließlich dem Verbrauchsmandat der "Conventional Biofuels" angerechnet werden (U.S. GOVERNMENT INFORMATION 2007: 121 STAT. 1519ff.; CONGRESSIONAL RESEARCH SERVICE 2012: 4). 
Der RFS 2 beschreibt zwar die Verbrauchsmandate bis zum Jahr 2022, ist aber trotzdem seit dem Jahr 2011 hoch umstritten. Über eine Änderung wird intensiv dis-

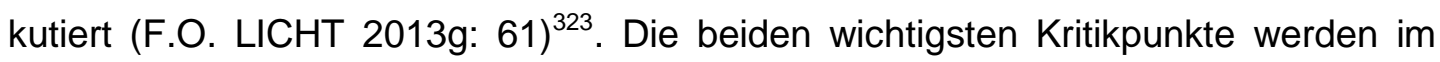
Folgenden ausführlich beschrieben. Der erste Kritikpunkt stellt die fehlende Produktion von Biokraftstoffen aus Zellulose dar, um das Verbrauchsmandat „Advanced: Cellulosic Biofuel“ erfüllen zu können. Zweitens ist es die „Blend Wall“-Problematik, die einen höheren Ethanolabsatz verhindert, sodass die Verbrauchsmandate des RFS 2 nicht erfüllt werden können.

Der RFS 2 beschreibt einen stetigen Anstieg des Mandats „Advanced: Cellulosic Biofuel“ von 0,378 Milliarden Litern im Jahr 2010 auf bis zu 61 Milliarden Liter im Jahr 2022 (Abbildung 88) (U.S. GOVERNMENT INFORMATION 2007: 121 STAT. 1523). Jedoch konnten die Verbrauchsmandate bereits in den Jahren 2010, 2011 und 2012 aufgrund fehlender Produktionskapazitäten nicht erfüllt werden und wurden von der EPA stark gekürzt (CONGRESSIONAL RESEARCH SERVICE 2012: 3; EPA 2010: 4; EPA 2011: 2): im Jahr 2010 von 0,378 Milliarden Litern auf 0,024 Milliarden Liter, im Jahr 2011 von 0,946 Milliarden Litern auf lediglich 0,025 Milliarden Liter und von 1,89 Milliarden Litern auf nur noch 0,032 Milliarden Liter im Jahr 2012 (CONGRESSIONAL RESEARCH SERVICE 2012: 3). Auch bis zum Jahr 2015 ist kein Anstieg der Produktionskapazitäten zu erwarten, da die ersten kommerziellen Anlagen nicht vor 2014 die Produktion aufnehmen werden ${ }^{324}$. Folglich wird die Differenz zwischen den geplanten Verbrauchsmandaten im RFS 2 (2013: 3,78 Milliarden Liter; 2014: 6,6 Milliarden Liter) und der tatsächlichen Produktion in Zukunft weiter zunehmen und die Kritiker des RFS 2 stärken. In Anbetracht einer derzeitigen (Jahr 2012) Produktion von lediglich 0,027 Milliarden Litern sind Zweifel angebracht, ob die gesetzten Ziele des RFS 2 hinsichtlich „Advanced: Cellulosic Biofuel“ (Jahr 2022: 61 Milliarden Liter) realistisch sind (OECD/FAO 2012: 224). Zur Erfüllung des Mandats wäre ein prozentuales jährliches Wachstum von 116\% notwendig; dies setzt den Bau von 605 Anlagen voraus, was mit enormen Investitionskosten verbunden

\footnotetext{
${ }^{323}$ Auch die Organisationen OECD/FAO (2012: 224) und U.S. ENERGY INFORMATION ADMINISTRATION (2012b: 97) betrachten in ihren Studien zur zukünftigen Entwicklung des Ethanolmarktes eine Senkung der Verbrauchsmandate des RFS 2.

${ }^{324}$ Folgende Unternehmen errichten momentan die ersten kommerziellen Anlagen zur Produktion von Zellulose-Ethanol in den USA:

- Abengoa Bioenergy baut eine Anlage zur jährlichen Produktion von 0,095 Milliarden Litern in Hugoton, Kansas (ABENGOA BIOENERGY 2012).

- POET und DSM arbeiten gemeinsam am „Project LIBERTY“, um eine 0,095-MilliardenLiter-Anlage in Emmetsburg, lowa, zu errichten (POET-DSM ADVANCED BIOFUELS 2012).

- DuPont Industrial Biosciences erbaut eine 0,1-Milliarden-Liter-Anlage in Nevada, lowa (DUPONT 2012).
} 
ist $^{325}$. Aus den genannten Gründen wird eine mögliche Kürzung des Mandats als realistisch betrachtet ${ }^{326}$.

Der zweite Grund ist die „Blend Wall“-Problematik. Der US-amerikanische Markt befindet sich derzeit an der E10-„Blend Wall“, welche ein weiteres Wachstum der Nachfrage verhindert (CONGRESSIONAL RESEARCH SERVICE 2010a: 5). Zukünftig wird die sinkende Benzinnachfrage in den USA (Kapitel 6.2.1) (U.S. ENERGY INFORMATION ADMINISTRATION 2012b: 98) den Absatz von Ethanol und damit die Erfüllung des RFS 2 noch erschweren, insbesondere da die jährlichen Verbrauchsmandate für Ethanol weiter ansteigen werden (Abbildung 88). Als der RFS 2 verabschiedet wurde, wurde der Rückgang der Benzinnachfrage in dieser Stärke nicht erwartet. Aufgrund dessen ist es möglich, dass die EPA die Verbrauchsmandate für Ethanol senkt, um sie der prognostizierten Benzinnachfrage anzupassen ${ }^{327}$. Auch die NACS (2012b: 14) ist der Auffassung, dass die „Blend Wall“-Problematik die EPA veranlassen könnte, die Verbrauchsmandate zu senken. Darüber hinaus ist schon seit 2010 ein weichender politischer Rückhalt für Biokraftstoffe und damit auch für den RFS 2 zu erkennen (F.O. LICHT 2012c: 319). Beispielhaft für diese Entwicklung ist die Entscheidung der Politiker, den „Volumetric Ethanol Excise Tax Credit" (VEETC) in Höhe von 0,45 US-\$/Gallone auslaufen zu lassen, was mit einem Wegfall des Ethanol-Importzolls in Höhe von 0,54 US\$/Gallone einherging (F.O. LICHT 2012c: 319; F.O. LICHT 2012f: 69f.). Auch der Druck der US-amerikanischen Nahrungsmittelindustrie und insbesondere der Ölindustrie, die eine Kürzung der Verbrauchsmandate des RFS 2 durchsetzen wollen, ist nicht zu unterschätzen (F.O. LICHT 2012c: 319; ETHANOL PRODUCER MAGAZINE 2012c; F.O. LICHT 2012g: 489f.).

\footnotetext{
${ }^{325}$ Bei einer durchschnittlichen Produktionskapazität einer Zellulose-Ethanolanlage von 0,1 Milliarden Litern - die drei derzeit im Bau befindlichen Anlagen von Abengoa Bioenergy, POET und DSM sowie DuPont Industrial Biosciences besitzen alle eine Produktionskapazität von jeweils rund 0,1 Milliarden Litern - wären 610 Anlagen notwendig, um eine Produktion von 61 Milliarden Litern Zellulose-Ethanol zu erreichen. Zum Aufbau von 610 Anlagen wird ein Investionsvolumen von rund 171 Milliarden US-\$ benötigt (die Berechnung basiert auf der Annahme, dass der Bau einer Anlage 280 Millionen US-\$ kosten wird) (BLOOMBERG NEW ENERGY FINANCE 2013: 2).

${ }^{326}$ Auch die OECD/FAO (2012: 92) hat große Zweifel hinsichtlich der Erfüllung des Verbrauchsmandats „Advanced: Cellulosic Biofuel“.

${ }^{327}$ Falls es zu keiner Anpassung der Verbrauchsmandate an die prognostizierte Entwicklung der Benzinnachfrage kommen sollte, so wäre die Erfüllung des RFS 2 nur möglich, indem alle Kraftfahrzeuge den Kraftstoff E30 tanken (Abbildung 73) oder es zu einer starken Steigerung des Verkaufs von E85 an FFV kommt. Der Verkauf von E30 an alle Kraftfahrzeuge ist jedoch nicht realistisch, da der Großteil der Fahrzeuge auch im Jahr 2022/2023 maximal E20 und/oder E15 wird tanken können (Kapitel 6.2.6). Des Weiteren ist auch ein starker Anstieg im FFV-Markt nicht wahrscheinlich. So erwartet die U.S. ENERGY INFORMATION ADMINISTRATION (2013f) lediglich eine Steigerung der Anzahl an FFV auf 18,2 Millionen bis zum Jahr 2023.
} 
Die US-amerikanische Politik hat verschiedene Möglichkeiten, die Verbrauchsmandate des RFS 2 anzupassen (OECD/FAO 2012: 96). Im Folgenden werden vier unterschiedliche Ausgestaltungen des RFS 2 (Option 1 - Option 4) für das Jahr 2023 dargestellt, die sich eindeutig voneinander unterscheiden und die grundlegenden möglichen Entwicklungen aufzeigen ${ }^{328}$. Da die EPA noch keine Verbrauchsmandate für das Jahr 2023 bestimmt hat, werden die Werte aus dem Jahr 2022 als Basis genutzt $^{329}$ (U.S. GOVERNMENT INFORMATION 2007: 121 STAT. 1522; CONGRESSIONAL RESEARCH SERVICE 2012: 2):

- Advanced: Unspecified Biofuel

- Advanced: Biomass-Based Diesel

- Advanced: Cellulosic Biofuel

- Conventional Biofuel
$=15$ Milliarden Liter

$=4$ Milliarden Liter

$=61$ Milliarden Liter

$=57$ Milliarden Liter

Die Abbildung 89 stellt die vier Optionen grafisch dar. Die einzelnen Optionen werden im folgenden Text einzeln beschrieben.

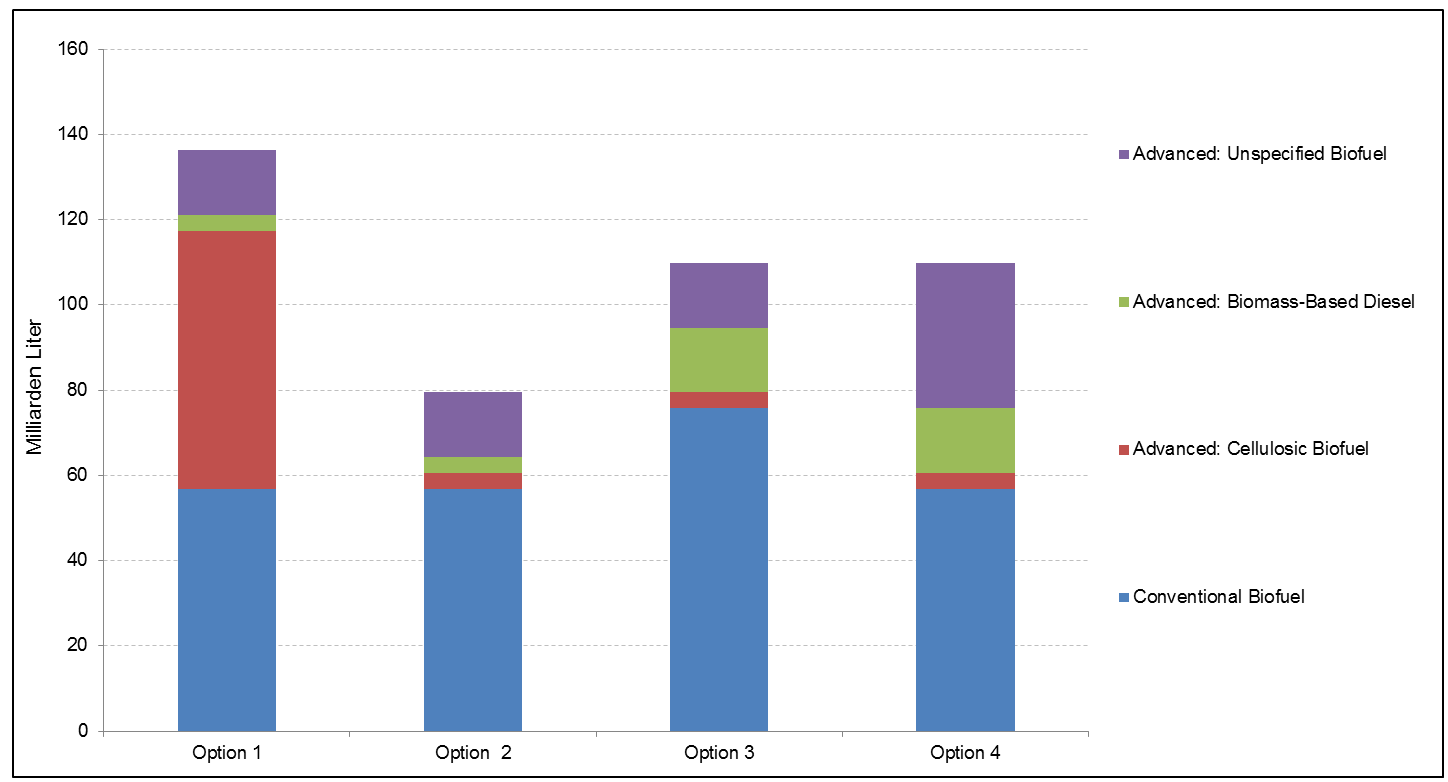

Abbildung 89: Verbrauchsmandate in Milliarden Litern im Jahr 2023 Option 1 - Option 4 Quelle: In Anlehnung an U.S. GOVERNMENT INFORMATION 2007: 121 STAT. 1522; OECD/FAO 2012: 97; EIGENE BERECHNUNG

${ }^{328}$ Die vier Optionen zur zukünftigen Entwicklung des RFS 2 wurden in Anlehnung an eine Studie der OECD/FAO (2012: 97) gebildet und intensiv mit Dr. Christoph Berg - F.O. Licht, Martin Mitchell - Clariant und Dr. Albrecht Schaper - Nordzucker AG diskutiert.

${ }^{329}$ Auch die U.S. ENERGY INFORMATION ADMINISTRATION (2012b: 97) prognostiziert, dass die Werte aus dem Jahr 2022 noch im Jahr 2023 Gültigkeit besitzen. 


\section{Option 1:}

Bei Option 1 kommt es zu keiner Veränderung des RFS 2 im Vergleich zur IstSituation. Voraussetzungen dieser Zukunftsprojektion sind, dass die USA zukünftig in der Lage sein werden, die benötigten Mengen an biogenem Kraftstoff aus dem Rohstoff Zellulose zu produzieren, um das Mandat „Advanced: Cellulosic Biofuel“ erfüllen zu können. Darüber hinaus besteht keine „Blend Wall“-Problematik, sodass ein Ethanolabsatz von 133 Milliarden Litern möglich ist. Folgende Verbrauchsmandate beschreiben Option 1:

- Advanced: Unspecified Biofuel

- Advanced: Biomass-Based Diesel

- Advanced: Cellulosic Biofuel

- Conventional Biofuel

$$
\begin{aligned}
& =15 \text { Milliarden Liter } \\
& =4 \text { Milliarden Liter } \\
& =61 \text { Milliarden Liter } \\
& =57 \text { Milliarden Liter }
\end{aligned}
$$

\section{Option 2:}

Basierend auf der Annahme, dass die USA auch zukünftig nicht in der Lage sein werden, das Verbrauchsmandat für „Advanced: Cellulosic Biofuel“ zu erfüllen, wird dieses von 61 Milliarden Litern auf nur noch 4 Milliarden Liter gekürzt. Infolge der Reduzierung verringern sich die Verbrauchsmandate für „Advanced Biofuel“ auf 23 Milliarden Liter sowie für den gesamten RFS 2 auf 80 Milliarden Liter. Einhergehend mit der Kürzung verringert sich auch die Problematik, die festgelegten Mandate im Transportbereich zu verbrauchen. Die folgenden Werte beschreiben Option 2:

- Advanced: Unspecified Biofuel

- Advanced: Biomass-Based Diesel

- Advanced: Cellulosic Biofuel

- Conventional Biofuel
$=15$ Milliarden Liter

$=4$ Milliarden Liter

= 4 Milliarden Liter

$=57$ Milliarden Liter 


\section{Option 3:}

Aus dem selben Grund wie bei Option 2 wird das Verbrauchsmandat für „Advanced: Cellulosic Biofuel“ um 57 Milliarden Liter gekürzt. Im Gegensatz zu Option 2 werden jedoch bei Option 3 die Mandate für „Conventional Biofuel“ und „Advanced: Biomass-Based Diesel“ von 57 Milliarden Litern auf 76 Milliarden Liter und von 4 Milliarden Litern auf 15 Milliarden Liter angehoben. Folglich sinkt der RFS 2 um 27 Milliarden Liter auf 110 Milliarden Liter, was die „Blend Wall“-Problematik mindert. Folgende Mandate beschreiben Option 3:

- Advanced: Unspecified Biofuel

- Advanced: Biomass-Based Diesel

- Advanced: Cellulosic Biofuel

- Conventional Biofuel
$=15$ Milliarden Liter

$=15$ Milliarden Liter

$=4$ Milliarden Liter

$=76$ Milliarden Liter

\section{Option 4:}

Wie auch schon bei Option 2 und bei Option 3 ist die Grundlage der vierten Option die Erwartung, dass die USA langfristig nicht fähig sein werden, das Verbrauchsmandat für „Advanced: Cellulosic Biofuel“ zu erfüllen. Infolgedessen wird das Mandat von 61 Milliarden Litern auf 4 Milliarden Liter gekürzt. Im Gegenzug werden jedoch die Mandate für „Advanced: Unspecified Biofuel“ und „Advanced: BiomassBased Diesel“ von 15 Milliarden Litern auf 34 Milliarden Liter und von 4 Milliarden Litern auf 15 Milliarden Liter angehoben. Dementsprechend verringert sich der RFS 2 um 27 Milliarden Liter auf nur noch 110 Milliarden Liter, was die „Blend Wall“Problematik mindert. Dieses Vorgehen - Absenkung des Mandats „Advanced: Cellulosic Biofuel“ und Erhöhung der Verbrauchsmandate „Advanced: Unspecified Biofuel“ und „Advanced: Biomass-Based Diese|“330 - wurde bereits von der EPA in den Jahren 2010, 2011 und 2012 aufgrund der zu geringen Produktion von „Advanced: Cellulosic Biofuel“ angewandt (CONGRESSIONAL RESEARCH SERVICE 2012: 3). Option 4 lässt sich anhand folgender Werte zusammenfassen:

- Advanced: Unspecified Biofuel

- Advanced: Biomass-Based Diesel

- Advanced: Cellulosic Biofuel

- Conventional Biofuel
$=34$ Milliarden Liter

$=15$ Milliarden Liter

$=4$ Milliarden Liter

$=57$ Milliarden Liter

\footnotetext{
${ }^{330}$ In Option 4 findet die Verschiebung der Verbrauchsmandate ausschließlich in der Gruppe der "Advanced Biofuels" statt. Das Verbrauchsmandat der "Conventional Biofuels" bleibt unverändert.
} 
Auf die Betrachtung einer weiteren Option, die einen Anstieg der Verbrauchsmandate im Vergleich zum derzeitigen RFS 2 darstellt, wurde bewusst verzichtet, da momentan kein Einfluss zu erkennen ist, der eine solche Entwicklung begründen würde. Auf Grundlage der Erläuterungen werden die folgenden vier Zukunftsprojektionen in der Studie weiter betrachtet:

Der RFS 2 bleibt unverändert bis zum Jahr 2023

Beschreibung:

Advanced: Unspecified Biofuel $=15$ Milliarden Liter, Advanced: Biomass-Based Diesel $=4$ Milliarden Liter, Advanced: Cellulosic Biofuel $=61$ Milliarden Liter und Conventional Biofuel = 57 Milliarden Liter

Starker Rückgang des RFS 2 aufgrund einer starken Kürzung des Mandats "Advanced: Cellulosic Biofuel“

22B Beschreibung:

Advanced: Unspecified Biofuel = 15 Milliarden Liter, Advanced: Biomass-Based Diesel $=4$ Milliarden Liter, Advanced: Cellulosic Biofuel $=4$ Milliarden Liter und Conventional Biofuel $=$ 57 Milliarden Liter

Rückgang des RFS 2 aufgrund einer starken Kürzung des Mandats „Advanced: Cellulosic Biofuel“ und geringer Steigerungen der Mandate für "Advanced: Biomass-Based Diesel“ und „Conventional Biofuel“

$22 \mathrm{C}$ Beschreibung:

Advanced: Unspecified Biofuel = 15 Milliarden Liter, Advanced: Biomass-Based Diesel = 15 Milliarden Liter, Advanced: Cellulosic Biofuel $=4$ Milliarden Liter und Conventional Biofuel = 76 Milliarden Liter

Rückgang des RFS 2 aufgrund einer starken Kürzung des Mandats „Advanced: Cellulosic Biofuel“ und geringer Steigerungen der Mandate für „Advanced: Biomass-Based Diesel“ und „Advanced: Unspecified Biofuel“ Beschreibung:

Advanced: Unspecified Biofuel $=34$ Milliarden Liter, Advanced: Biomass-Based Diesel $=15$ Milliarden Liter, Advanced: Cellulosic Biofuel $=4$ Milliarden Liter und Conventional Biofuel $=$ 57 Milliarden Liter

\subsubsection{Höhe der genehmigten prozentualen Beimischung von Ethanol zu Benzin}

Die maximal erlaubte Ethanolbeimischung zu Benzin wird in den USA von der EPA (United States Environmental Protection Agency) bestimmt (EPA 2012b; U.S. ENERGY INFORMATION ADMINISTRATION 2012h). Im Januar 2011 hob diese auf Antrag von Growth Energy ${ }^{331}$ und 54 US-amerikanischen Ethanolproduzenten die maximal erlaubte Beimischung von Ethanol zu Benzin für Kraftfahrzeuge vom Baujahr 2001 und jünger von 10\% vol (E10) auf 15\% vol (E15) an (U.S. GOVERNMENT INFORMATION 2011: 4662; EPA 2012b). Für Fahrzeuge vom Baujahr 2000 und älter, Motorräder sowie schwere Nutzfahrzeuge gilt diese Regelung jedoch nicht.

${ }^{331}$ Growth Energy ist eine Organisation, die die politischen Interessen der USamerikanischen Ethanolindustrie unter Leitung von Tom Buis vertritt (GROWTH ENERGY 2013). 
Diese dürfen weiterhin nur Benzin mit einem maximalen Ethanolanteil von $10 \%$ vol (E10) verwenden ${ }^{332}$ (U.S. GOVERNMENT INFORMATION 2011: 4662).

Dieser Faktor ist ein Bestandteil der „Blend Wall“-Problematik (Kapitel 6.1.2.2). Zur Überwindung der Problematik, zur Steigerung des US-amerikanischen Ethanolabsatzes und um die Verbrauchsmandate des RFS 2 erfüllen zu können, muss die EPA die erlaubte prozentuale Ethanolbeimischung zu Benzin anheben. Erfolgt dies nicht, so beschränkt der Schlüsselfaktor den Ethanolabsatz auf 77 Milliarden Liter im Jahr 2016, wodurch der RFS 2 in diesem und in den folgenden Jahren nicht erfüllt werden kann (Abbildung 90) (33/334. $^{3}$.

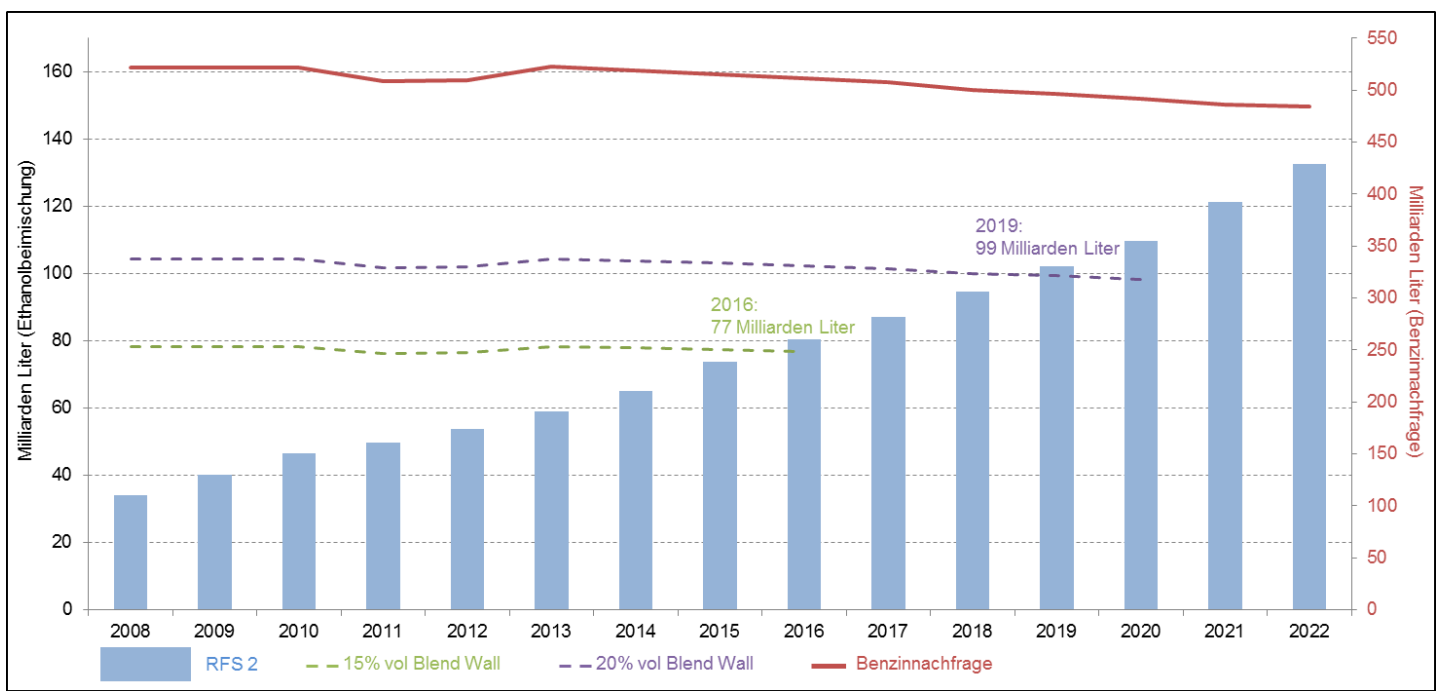

Abbildung 90: Ermittlung der „Blend Wall“ in Abhängigkeit von der maximal erlaubten Höhe der Ethanolbeimischung zu Benzin ${ }^{335}$

Quelle: In Anlehnung an U.S. GOVERNMENT INFORMATION 2007: 121 STAT. 1522; U.S. ENERGY INFORMATION ADMINISTRATION 2012b: 98; EIGENE BERECHNUNG

Im Folgenden werden zwei Zukunftsprojektionen beschrieben, die den Schlüsselfaktor im Jahr 2023 darstellen. In der ersten Zukunftsprojektion bleibt die von der EPA maximal erlaubte Ethanolbeimischung zu Benzin bei 15\% vol. Die Projektion ist realistisch, da sich der Kraftstoff E15 noch nicht auf dem US-amerikanischen Markt durchgesetzt hat und bisher nur an wenigen Tankstellen im Mittleren Westen der

\footnotetext{
${ }^{332}$ Der Kraftstoff E10 ist in den USA bereits seit 1978 zugelassen (U.S. GOVERNMENT INFORMATION 2011: 4662; U.S. ENERGY INFORMATION ADMINISTRATION 2012h). ${ }^{333}$ Die Berechnungen in Abbildung 90 basieren auf der Prognose der U.S. ENERGY INFORMATION ADMINISTRATION (2012b: 98), dass die US-amerikanische Benzinnachfrage bis zum Jahr 2022 sinken wird. Des Weiteren wird der Absatz von Ethanol als E85 an FFV nicht berücksichtigt. Der Einfluss der Schlüsselfaktoren 8 und 15, die ebenfalls in Zusammenhang mit der „Blend Wall“-Problematik stehen (Kapitel 6.1.2.2), wurden nicht berücksichtigt, um die Wirkung dieses Faktors auf den US-amerikanischen Ethanolmarkt isoliert darstellen zu können (CONGRESSIONAL RESEARCH SERVICE 2010a: 5).

${ }^{334}$ Auch die OECD/FAO (2012: 111) und der CONGRESSIONAL RESEARCH SERVICE (2010: 3) erwarten, dass bei einer maximal erlaubten Ethanolbeimischung von $15 \%$ vol zu Benzin im Jahr 2016 die „Blend Wall“ erreicht wird.

${ }_{335}$ In der Abbildung 90 ist das Verbrauchsmandat „Advanced: Biomass-Based Diesel“ nicht berücksichtigt, da ausschließlich die Beimischung von Ethanol zu Benzin betrachtet wird.
} 
USA verkauft wird (F.O. LICHT 2013c: 341; ETHANOL PRODUCER MAGAZINE 2013d). Infolgedessen besteht keine Notwendigkeit für die EPA, die Verträglichkeit von E20 als Kraftstoff zu testen und ihn gegebenenfalls zu genehmigen, da eine Erhöhung der Beimischung keine Veränderung des Marktes bewirken würde. Darüber hinaus spricht für die Beibehaltung des jetzigen Wertes, dass die Verbrauchsmandate des RFS 2 in Zukunft stark gesenkt werden könnten, wie in Projektion 22B (Kapitel 6.2.5) beschrieben, wodurch eine Erhöhung der genehmigten Ethanolbeimischung nicht mehr notwendig wäre, um die Verbrauchsmandate zu erfüllen ${ }^{336}$.

Die zweite Zukunftsprojektion betrachtet einen Anstieg der erlaubten maximalen Ethanolbeimischung zu Benzin auf 20\% vol im Jahr 2023. Diese Projektion ist relevant, da aufgrund der „Blend Wall“-Problematik die EPA die erlaubte Beimischungshöhe von Ethanol zu Benzin anheben müsste, um die Verbrauchsmandate zumindest zu einem höheren Anteil erfüllen zu können. Durch die Anhebung auf E20 würde erst im Jahr 2019 die „Blend Wall“ erreicht werden - und dies bei einem prognostizierten Ethanolabsatz von immerhin 99 Milliarden Litern (Abbildung 90). Darüber hinaus sind 30\% bis 40\% der Fahrzeuge in der Lage, im Jahr 2023 Benzin mit einem Ethanolanteil von mindestens $20 \%$ vol als Kraftstoff zu nutzen. Dies entspricht rund 100 Millionen Fahrzeugen ${ }^{337}$ und damit einer Marktgröße, für die es sich lohnt, die erlaubte maximale Ethanolbeimischung zu Benzin anzuheben ${ }^{338}$.

Ein höherer Anstieg als 20\% vol wird nicht betrachtet, da im Jahr 2023 nicht mehr als $16 \%$ der US-amerikanischen Kraftfahrzeuge in der Lage sein werden, Ethanolbeimischungen von über $20 \%$ vol als Kraftstoff zu verwenden (Kapitel 6.2.4) ${ }^{339}$. Basierend auf den Ausführungen werden die folgenden zwei Zukunftsprojektionen weiter betrachtet:

24A Die EPA erlaubt weiterhin eine Ethanolbeimischung zu Benzin in Höhe von $15 \%$ vol

24B Die EPA hebt die erlaubte Ethanolbeimischung zu Benzin auf 20\% vol an

\footnotetext{
${ }^{336}$ Auch die U.S. ENERGY INFORMATION ADMINISTRATION (2012b: 97) erwartet über das Jahr 2023 hinaus, dass die von der EPA erlaubte Obergrenze der Ethanolbeimischung $15 \%$ vol betragen wird.

${ }^{337}$ Eine ausführliche Betrachtung der Entwicklung der US-amerikanischen Kraftfahrzeugflotte befindet sich in Kapitel 6.2.4.

${ }_{338}$ Auch ALFSTAD (2008: 14) von der Forschungseinrichtung Brookhaven National Laboratory prognostiziert in seiner Studie eine Ethanolbeimischung von $20 \%$ vol zu Benzin.

${ }^{339}$ Dies bedeutet, dass die derzeitigen Verbrauchsmandate des RFS 2 nicht durch die alleinige Ethanolbeimischung zu Benzin erreicht werden können. Die Erfüllung der Mandate wäre jedoch durch einen starken Anstieg des Absatzes von E85 an FFV möglich, wobei allerdings zu beachten ist, dass der derzeitige E85-Absatz lediglich 0,146 Milliarden Liter beträgt (U.S. ENERGY INFORMATION ADMINISTRATION 2012j: 29).
} 


\subsubsection{Diskussion über die Energieunabhängigkeit der USA}

Die Diskussion und damit die gesellschaftliche Bedeutung des Themas Energieunabhängigkeit der USA betrifft ausschließlich den Energieträger Rohöl, da die USA bei den anderen Energieträgern - Erdgas, Kohle, erneuerbare Energien und Kernenergie - Selbstversorger sind und teilweise sogar Überschüsse exportieren (U.S. ENERGY INFORMATION ADMINISTRATION 2012e).

Das Thema Unabhängigkeit von Rohölimporten ist seit Jahrzehnten von Relevanz für die USA, da sie Rohöl in erheblichen Mengen importieren müssen, um ihren Verbrauch decken zu können. Die folgende Abbildung 91 zeigt die Entwicklung der Rohölversorgung der USA im Zeitraum von 1970 bis 2011.

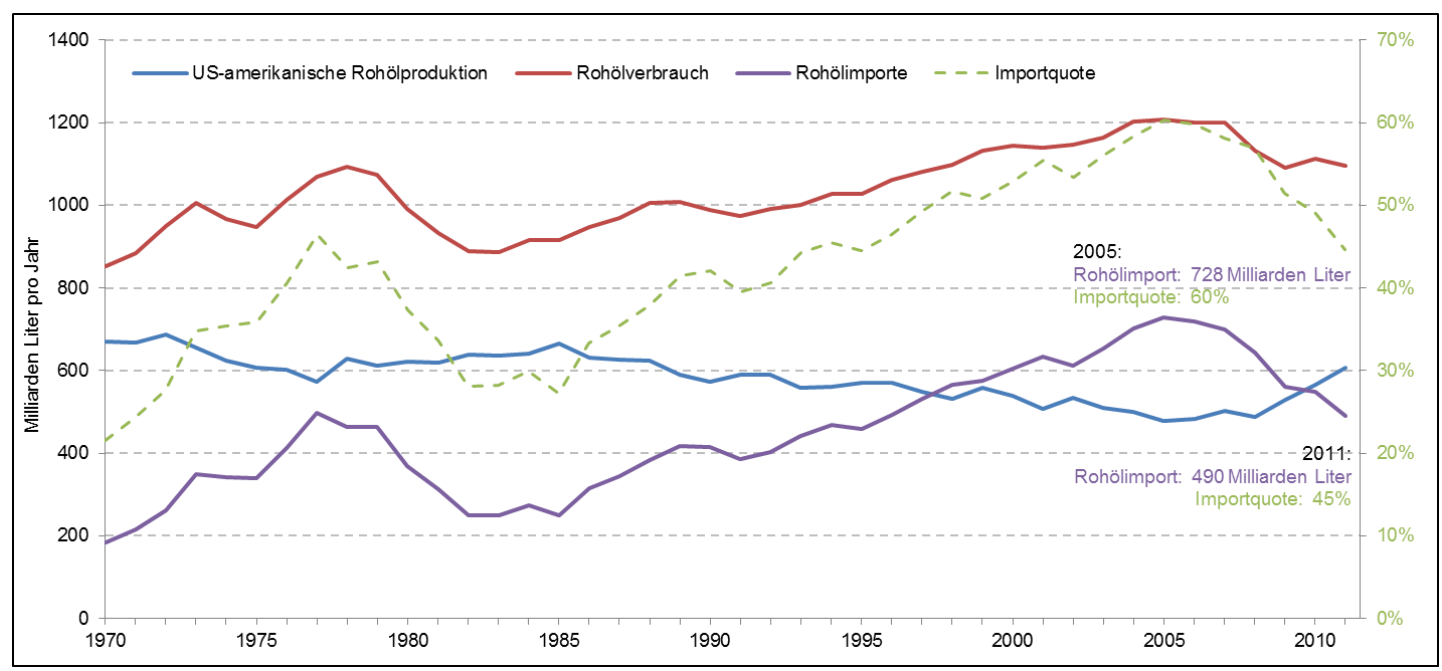

Abbildung 91: Entwicklung der US-amerikanischen Rohölversorgung im Zeitraum von 1970 bis 2011

Quelle: In Anlehnung an U.S. ENERGY INFORMATION ADMINISTRATION 2013a: 2; EIGENE BERECHNUNG

Wie in Abbildung 91 ersichtlich, stiegen die Rohölimporte im Zeitraum von Mitte der 1980er Jahre bis zum Jahr 2005 kontinuierlich bis auf 728 Milliarden Liter an, was einer Importquote von 60\% entspricht. Seitdem sind zwar die Rohölimporte gesunken, dennoch wurden im Jahr 2011 noch immer 45\% (490 Milliarden Liter) des USamerikanischen Rohölverbrauchs importiert.

Das Thema Energieunabhängigkeit hat für die US-amerikanische Gesellschaft eine hohe Bedeutung, da sie sich durch die Rohölexportstaaten erpressbar und in ihrer Souveränität und Sicherheit bedroht fühlt. Mithilfe von Äußerungen von USamerikanischen Präsidenten (Tabelle 18) kann dies gezeigt werden. 


\begin{tabular}{|c|c|c|c|}
\hline $\begin{array}{l}\text { US-amerikanischer } \\
\text { Präsident }\end{array}$ & Jahr & Zitat & $\begin{array}{l}\text { Rohölimporte } \\
\text { im Verhältnis } \\
\text { zum Verbrauch }\end{array}$ \\
\hline Gerald Ford & 1975 & $\begin{array}{l}\text { "I have set the following national energy goals to } \\
\text { assure that our future is as secure and productive } \\
\text { as our past: First, we must reduce oil imports by one } \\
\text { million barrels per day by the end of this year and by } \\
\text { two million barrels per day by the end of } 1977 \text {. Sec- } \\
\text { ond, we must end vulnerability to economic disrup- } \\
\text { tion by foreign suppliers by } 1985 . "\end{array}$ & $36 \%$ \\
\hline Jimmy Carter & 1979 & $\begin{array}{l}\text { „Beginning this moment, this nation will never use } \\
\text { more foreign oil than we did in } 1977-\text { never. From } \\
\text { now on, every new addition to our demand for ener- } \\
\text { gy will be met from our own production and our own } \\
\text { conservation. ... [F]or I am tonight setting the further } \\
\text { goal of cutting our dependence on foreign oil by one } \\
\text { half by the end of the next decade." }\end{array}$ & $43 \%$ \\
\hline Bill Clinton & 1995 & $\begin{array}{l}\text { „[T] he nation's growing reliance on imports of crude } \\
\text { oil and refined petroleum products threatens the } \\
\text { nation's security because they increase US vulner- } \\
\text { ability to oil supply interruptions.... [T] } \mathrm{The} \text { administra- } \\
\text { tion will continue its efforts to develop additional } \\
\text { cost-effective policies to enhance domestic energy } \\
\text { production and to revitalize the US petroleum indus- } \\
\text { try." }\end{array}$ & $45 \%$ \\
\hline George W. Bush & 2006 & $\begin{array}{l}\text { "America is addicted to oil, which is often imported } \\
\text { from unstable parts of the world.... By applying the } \\
\text { talent and technology of America, this country can } \\
\text { dramatically improve our environment, move be- } \\
\text { yond a petroleum-based economy, and make our } \\
\text { dependence on Middle East oil a thing of the past." }\end{array}$ & $60 \%$ \\
\hline Barack Obama & 2011 & $\begin{array}{l}\text { "The only way for America's energy supply to be } \\
\text { truly secure is by permanently reducing our de- } \\
\text { pendence on oil“. }\end{array}$ & $46 \%$ \\
\hline
\end{tabular}

Tabelle 18: Zitate von US-amerikanischen Präsidenten zum Thema Energieunabhängigkeit Quelle: In Anlehnung an BP 2008: 37ff.; RFA 2012a: 1; BEVILL 2011b; U.S. ENERGY INFORMATION ADMINISTRATION 2012b: 3

Die hohe Relevanz des Themas Unabhängigkeit von Rohölimporten wird des Weiteren dadurch bestätigt, dass die Unternehmen POET und DSM ihr Gemeinschaftsprojekt zum Bau einer kommerziellen Anlage zur Produktion von ZelluloseEthanol bewusst „Project LIBERTY“ nennen, um die Wichtigkeit des Projektes für die Befreiung der USA von Rohölimporten zu betonen (POET-DSM ADVANCED BIOFUELS 2012). Darüber hinaus zeigt die Verabschiedung des „Energy Independence and Security Act ${ }^{“ 340}$ die Bedeutung der Thematik, denn primäres Ziel des Acts ist es, eine höhere Unabhängigkeit von Rohölimporten zu erreichen, um somit die Sicherheit der USA zu erhöhen (U.S. GOVERNMENT INFORMATION 2007: 121 STAT. 1492).

Basierend auf den vorangegangenen Ausführungen wird die Referenzsituation als Ausgangspunkt zur Bildung der Zukunftsprojektionen für diesen Faktor wie folgt

${ }^{340}$ Der RFS 2 ist auch ein Bestandteil des „Energy Independence and Security Act” (U.S. GOVERNMENT INFORMATION 2007: 121 STAT. 1493) 
beschrieben: In den USA besitzt im Jahr 2012 das Thema Energieunabhängigkeit von Rohölimporten eine hohe gesellschaftliche Bedeutung.

Ausgehend von der Referenzsituation erfolgt die Betrachtung des Themas Energieunabhängigkeit bis zum Jahr 2023. In Abbildung 92 ist zu erkennen, dass die prognostizierten Rohölimporte bis zum Jahr 2023 auf 404 Milliarden Liter sinken, wodurch auch die Importquote auf 35\% schrumpft (U.S. ENERGY INFORMATION ADMINISTRATION 2013a: 3).

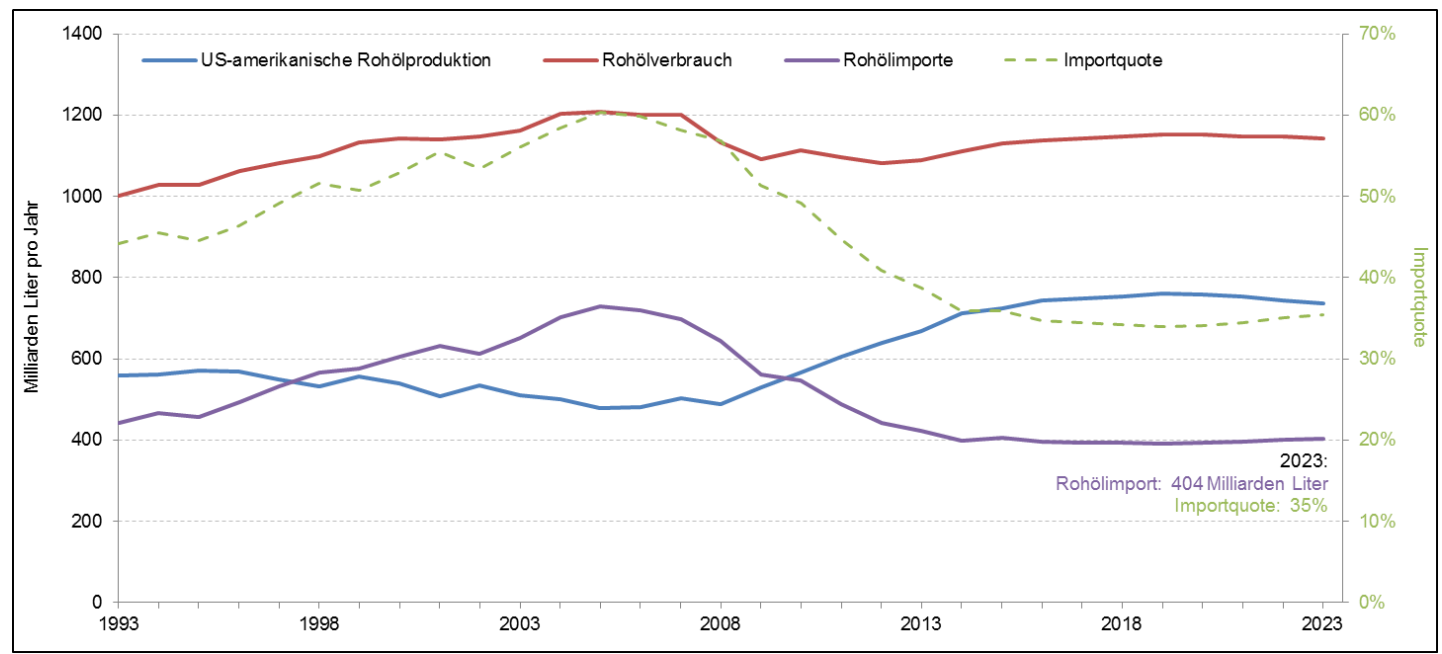

Abbildung 92: Entwicklung der US-amerikanischen Rohölversorgung im Zeitraum von 1993 bis 2023

Quelle: In Anlehnung an U.S. ENERGY INFORMATION ADMINISTRATION 2013a: 2; EIGENE BERECHNUNG

Der Rückgang der Rohölimporte ist bei einem leicht zunehmenden Rohölverbrauch auf die steigende Rohölproduktion in den USA zurückzuführen (U.S. ENERGY INFORMATION ADMINISTRATION 2012b: 3). Der Produktionsanstieg ist aufgrund des technischen Fortschritts möglich, der die Erschließung von Ölquellen im Golf von Mexiko und insbesondere die Förderung von Rohöl aus permeablem Sandstein und Schiefergestein ermöglicht ${ }^{341}$ (U.S. ENERGY INFORMATION ADMINISTRATION 2012b: 95). Trotz des erwarteten Rückgangs der Einfuhren gehören die USA in Zukunft neben der Europäischen Union und China zu den drei mit Abstand größten Rohölimporteuren der Welt (INTERNATIONAL ENERGY AGENCY 2011a: 92).

In Abbildung 93 sind die prozentualen nominalen Ausgaben für den Rohölverbrauch und gesondert für den Rohölimport als prozentualer Anteil am nominalen US-

${ }^{341}$ Die wichtigsten Gebiete zur Förderung von Rohöl aus Schiefergestein befinden sich in North Dakota (Bakken Formation), in Texas (Eagle Ford und Arabon/Bone Springs) und in Kalifornien (Monterey/Santos). Die U.S. ENERGY INFORMATION ADMINISTRATION (2011d) prognostiziert die Reserven auf insgesamt 3816 Milliarden Liter Rohöl, womit der US-amerikanische Markt, basierend auf einem jährlichen Rohölverbrauch von 1089 Milliarden Litern (Basisjahr 2011), 3,5 Jahre vollständig versorgt werden könnte (U.S. ENERGY INFORMATION ADMINISTRATION 2013a: 2). 
amerikanischen Bruttoinlandsprodukt (BIP) angegeben. Der prozentuale Anteil der Ausgaben für Rohöl am BIP sinkt von 5,89\% im Jahr 2011 auf nur noch 4,45\% im Jahr 2023. Bei den Rohölimporten wird eine identische Entwicklung prognostiziert: Auch diese sinken von 2,63\% im Jahr 2011 auf lediglich 1,57\% im Jahr 2023. Diese Entwicklung zeigt, dass der Einfluss der Ausgaben für Rohöl und Rohölimporte auf die US-amerikanische Wirtschaft sinkt, wodurch die wirtschaftliche Bedeutung des Themas Energieunabhängigkeit von Rohölimporten in Zukunft abnimmt.

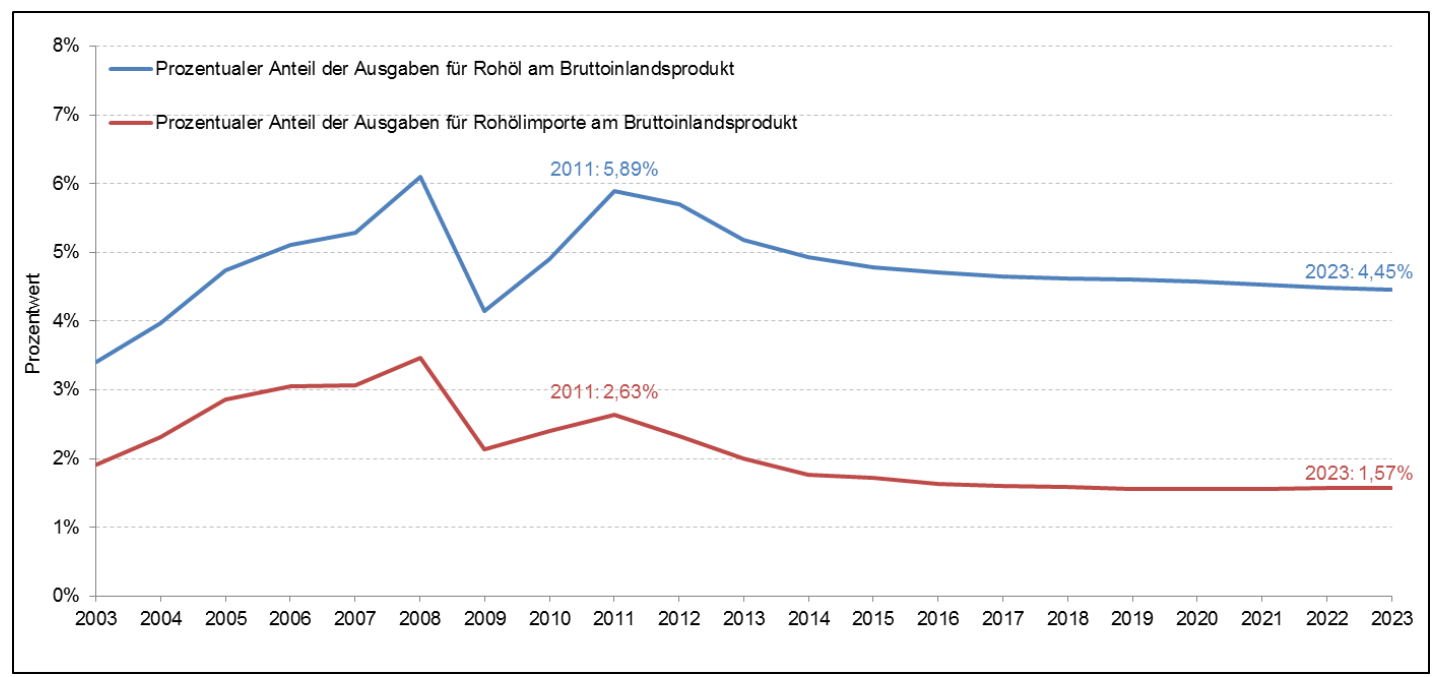

Abbildung 93: Entwicklung der prozentualen nominalen Ausgaben für Rohöl und Rohölimporte am BIP der USA

Quelle: In Anlehnung an U.S. ENERGY INFORMATION ADMINISTRATION 2013g; EIGENE BERECHNUNG

Ein weiterer wichtiger Faktor, der die gesellschaftliche Bedeutung der Thematik Unabhängigkeit von Rohölimporten senkt, ist das hohe Vorkommen an Schiefergas in den USA (U.S. ENERGY INFORMATION ADMINISTRATION 2012b: 93). Aufgrund des technischen Fortschritts ${ }^{342}$ sind die Erdgasreserven im Schiefergestein förderbar, was die USA zu einem Erdgasexporteur macht und den Preis für Erdgas in den USA stark senkt ${ }^{343}$ (U.S. ENERGY INFORMATION ADMINISTRATION 2012b: 3).

\footnotetext{
${ }^{342}$ Die Förderung von Schiefergas aus Schiefergestein erfolgt durch das Fracking. Bei diesem Verfahren wird unter hohem Druck ein Gemisch aus Sand, Wasser und Chemikalien in das Gestein gepresst, wodurch Risse entstehen, aus denen das Gas entweicht (HALLIBURTON 2013). Das Verfahren ist so erfolgreich, dass die U.S. ENERGY INFORMATION ADMINISTRATION (2012b: 93) für das Jahr 2023 prognostiziert, dass $41 \%$ der USamerikanischen Erdgasproduktion aus Schiefergestein erfolgt. Insgesamt werden die Reserven auf 750 Billionen Kubikfuß prognostiziert, wodurch die USA bei einem konstant bleibenden jährlichen Verbrauch von 25,2 Billionen Kubikfuß ihren Erdgasbedarf für 30 Jahre decken könnten (U.S. ENERGY INFORMATION ADMINISTRATION 2011d).

${ }^{343}$ Die Förderung von Schiefergas senkt zwar die gesellschaftliche Relevanz des Themas Unabhängigkeit von Rohöl. Eine Substitution von Rohöl durch Erdgas ist allerdings kaum möglich, da Erdgas nur minimal im wichtigsten Verbrauchsgebiet von Rohöl - dem Transportbereich - eingesetzt werden kann (U.S. ENERGY INFORMATION ADMINISTRATION 2012l).
} 
Infolge des prognostizierten Rückgangs der Rohölimporte, des geringeren Anteils der Ausgaben für Rohölimporte am US-amerikanischen BIP und der hohen Vorkommen an Schiefergas wird bis zum Jahr 2023 eine Abnahme der gesellschaftlichen Relevanz des Themas Unabhängigkeit von Rohölimporten prognostiziert. Da aber weiterhin erwartet wird, dass 35\% des Rohölverbrauchs im Jahr 2023 importiert werden, was einer absoluten Menge von 404 Milliarden Litern entspricht, wird das Thema auch in Zukunft von Bedeutung sein. Basierend auf den Ausführungen wird die folgende Zukunftsprojektion 28A in der „USA Szenario-Bildung und -Konsistenzprüfung" weiter betrachtet:

$28 \mathrm{~A}$

Energieunabhängigkeit ist in den USA im Jahr 2023 ein bedeutendes gesellschaftliches Thema, aber von geringerer Relevanz als im Jahr 2012

\subsubsection{Ethanolnachfrage}

Die US-amerikanische Ethanolnachfrage ist von 6 Milliarden Litern im Jahr 2000 auf 51,5 Milliarden Liter im Jahr 2012 angestiegen (Abbildung 94), was einem durchschnittlichen jährlichen Wachstum von 19,6\% entspricht. Allerdings ist zu beachten, dass das Wachstum infolge der „Blend Wall“-Problematik in den Jahren 2011 und 2012 deutlich geringer ausfiel als in den Vorjahren (F.O. LICHT 2013b: 213; BERG 2012c: 16f.). Mit einer Nachfrage in Höhe von 51,5 Milliarden Litern sind die USA der mit Abstand weltgrößte Ethanolverbraucher vor Brasilien (20,1 Milliarden Liter) und der EU27 (5,6 Milliarden Liter) (F. O. LICHT 2012f: 74; F.O. LICHT 2011a: 68).

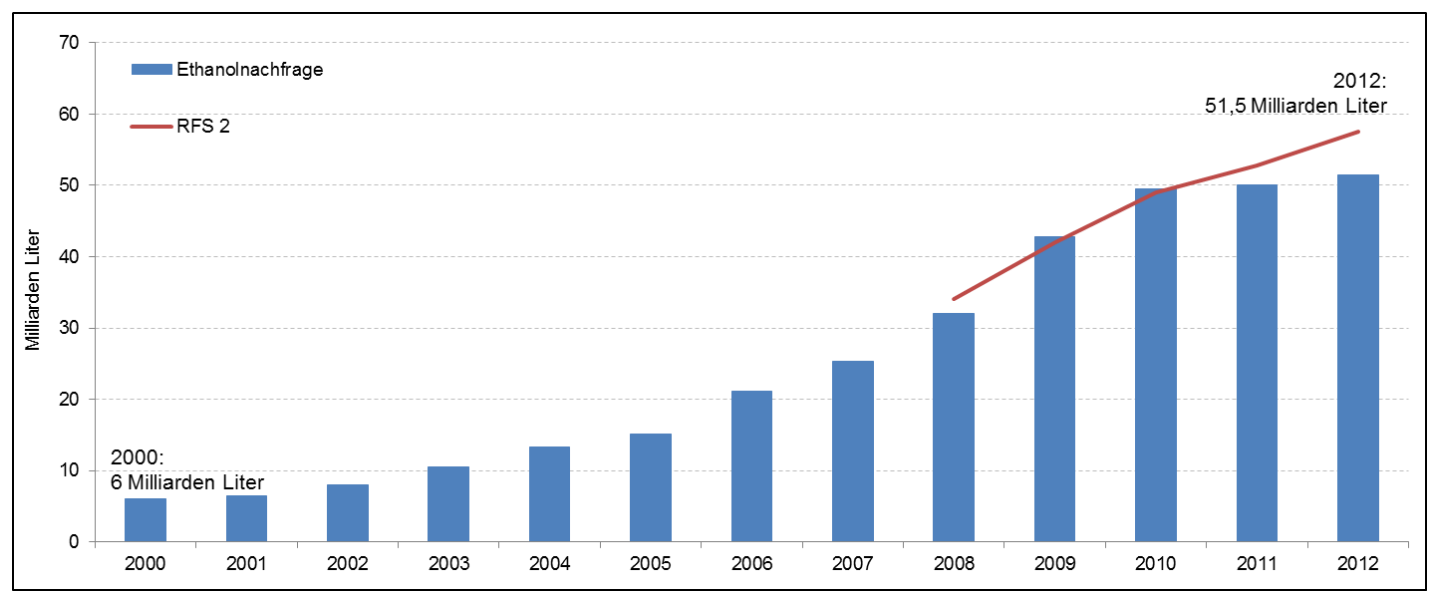

Abbildung 94: Historische Entwicklung der Ethanolnachfrage in den USA

Quelle: In Anlehnung an U.S. ENERGY INFORMATION ADMINISTRATION 2012b: 97; U.S. GOVERNMENT INFORMATION 2007: 121 STAT. 1522; BERG 2012c: 17; EIGENE BERECHNUNG

Entscheidend für das starke Wachstum der Ethanolnachfrage war der RFS 1 im Zeitraum von 2005 bis 2007 sowie der RFS 2 seit dem Jahr 2008 (U.S. ENERGY INFORMATION ADMINISTRATION 2011a: 81; OECD/FAO 2012: 92). Aus Abbil- 
dung 94 wird ersichtlich, dass sich die US-amerikanische Ethanolnachfrage in den Jahren von 2008 bis 2012 nahezu parallel zu den Verbrauchsmandaten des RFS 2 entwickelt hat. Allerdings konnten in den Jahren 2011 und 2012 die Verbrauchsmandate des RFS 2 aufgrund der bereits erwähnten „Blend Wall“-Problematik nicht erfüllt werden (F.O. LICHT 2013b: 213). Trotzdem wird auch in Zukunft eine Ethanolnachfrage entsprechend den Verbrauchsmandaten des RFS 2 erwartet. Da sich diese Arbeit ausschließlich auf die Entwicklung des Ethanolmarktes konzentriert, werden zur Ermittlung der Ethanolnachfrage im Jahr 2023 nur die Mandate „Conventional Biofuel“, „Advanced: Cellulosic Biofuel“ und „Unspecified Biofuel“ weiter betrachtet ${ }^{344}$. In der folgenden Abbildung 95 sind die unterschiedlichen Verbrauchsmandate "Option 1 bis 4“, die in Kapitel 6.2 .5 entwickelt wurden, dargestellt.

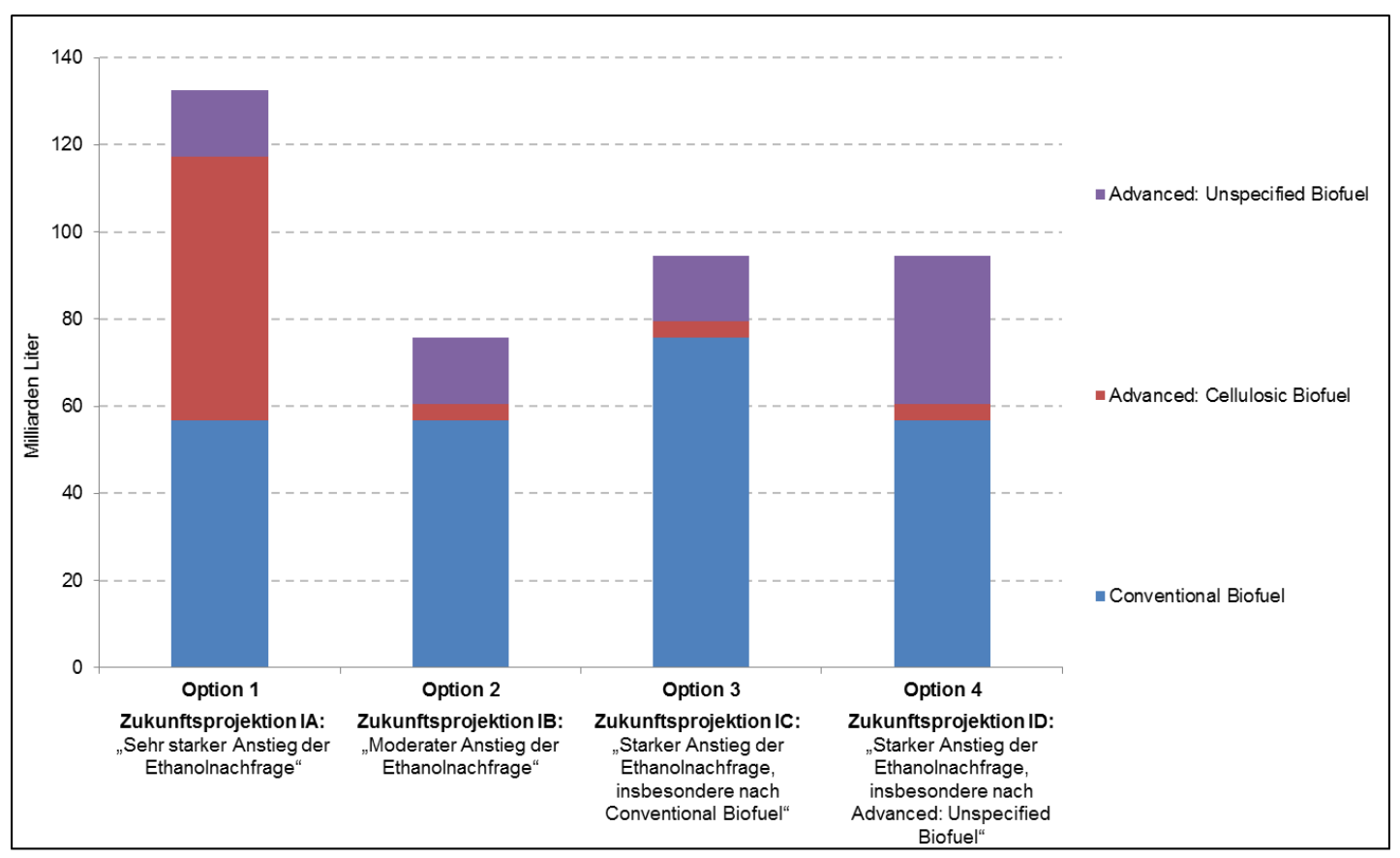

Abbildung 95: Prognostizierte Verbrauchsmandate des RFS 2 - Zukunftsprojektionen der Ethanolnachfrage

Quelle: EIGENE DARSTELLUNG

Basierend auf den vier Optionen werden die vier Zukunftsprojektionen des Schlüsselfaktors Ethanolnachfrage gebildet - „Sehr starker Anstieg der Ethanolnachfrage“, „Moderater Anstieg der Ethanolnachfrage“, „Starker Anstieg der Ethanolnachfrage, insbesondere nach Conventional Biofuel“ und „Starker Anstieg der Ethanolnachfrage, insbesondere nach Advanced: Unspecified Biofuel“ - und im dritten Kernschritt weiter betrachtet. Dabei ist zu beachten, dass die Ethanolnachfrage den prognostizierten Mandaten des RFS 2 entspricht.

${ }^{344}$ Das Verbrauchsmandat „Advanced: Biomass-Based Diesel“ wird nicht weiter betrachtet, da es die Beimischung von Biodiesel zu fossilem Diesel beschreibt (U.S. GOVERNMENT INFORMATION 2007: 121 STAT. 1519f.). 
Die vier Projektionen sind aus zwei Gründen als realistisch zu betrachten: (1) aufgrund der Übereinstimmung mit den Verbrauchsmandaten und (2), da die anerkannten Forschungsinstitute U.S. ENERGY INFORMATION ADMINISTRATION (2012b: 97), OECD/FAO (2012: 114), FAPRI (2013), Brookhaven National Laboratory (ALFSTAD 2008: A-3) und INTERNATIONAL ENERGY AGENCY (2012: 221) ebenfalls einen Anstieg der Ethanolnachfrage in den USA bis zum Jahr 2023 prognostizieren.

Basierend auf den vorangegangenen Erläuterungen werden die folgenden vier Zukunftsprojektionen weiter betrachtet:

IA

Sehr starker Anstieg der Ethanolnachfrage Beschreibung:

Advanced: Unspecified Biofuel $=15$ Milliarden Liter, Advanced: Cellulosic Biofuel $=61$

Milliarden Liter und Conventional Biofuel $=57$ Milliarden Liter

Moderater Anstieg der Ethanolnachfrage

IB

Beschreibung:

Advanced: Unspecified Biofuel $=15$ Milliarden Liter, Advanced: Cellulosic Biofuel $=4$

Milliarden Liter und Conventional Biofuel $=57$ Milliarden Liter

Starker Anstieg der Ethanolnachfrage, insbesondere nach Conventional Biofuel

IC

Beschreibung:

Advanced: Unspecified Biofuel $=15$ Milliarden Liter, Advanced: Cellulosic Biofuel $=4$ Milliarden Liter und Conventional Biofuel $=76$ Milliarden Liter

Starker Anstieg der Ethanolnachfrage, insbesondere nach Advanced: Unspecified Biofuel

ID Beschreibung:

Advanced: Unspecified Biofuel $=34$ Milliarden Liter, Advanced: Cellulosic Biofuel $=4$

Milliarden Liter und Conventional Biofuel $=57$ Milliarden Liter

\subsubsection{Ethanolproduktion}

Die Ethanolproduktion ist in den USA im Zeitraum von 2004 bis 2011 stetig von 13 Milliarden Litern auf 54 Milliarden Liter angestiegen (Abbildung 96), doch kam es im Jahr 2012 erstmals seit 16 Jahren zu einem Rückgang der Ethanolproduktion auf 52,5 Milliarden Liter. Diese Entwicklung ist auf drei Punkte zurückzuführen: (1) auf die „Blend Wall“-Problematik, welche einen weiteren Anstieg der USamerikanischen Ethanolnachfrage verhinderte, (2) auf die hohen Maispreise infolge der starken Dürre im Mittleren Westen der USA sowie (3) auf die deutlich geringeren Ethanolexporte in die EU27 und nach Brasilien (F.O. LICHT 2013b: 213; F.O. LICHT 2012f: 69; BERG 2012c: 17). Die US-amerikanische Ethanolproduktion ist in der Zeitspanne von 2004 bis 2012 jährlich um durchschnittlich 19\% angestiegen. 


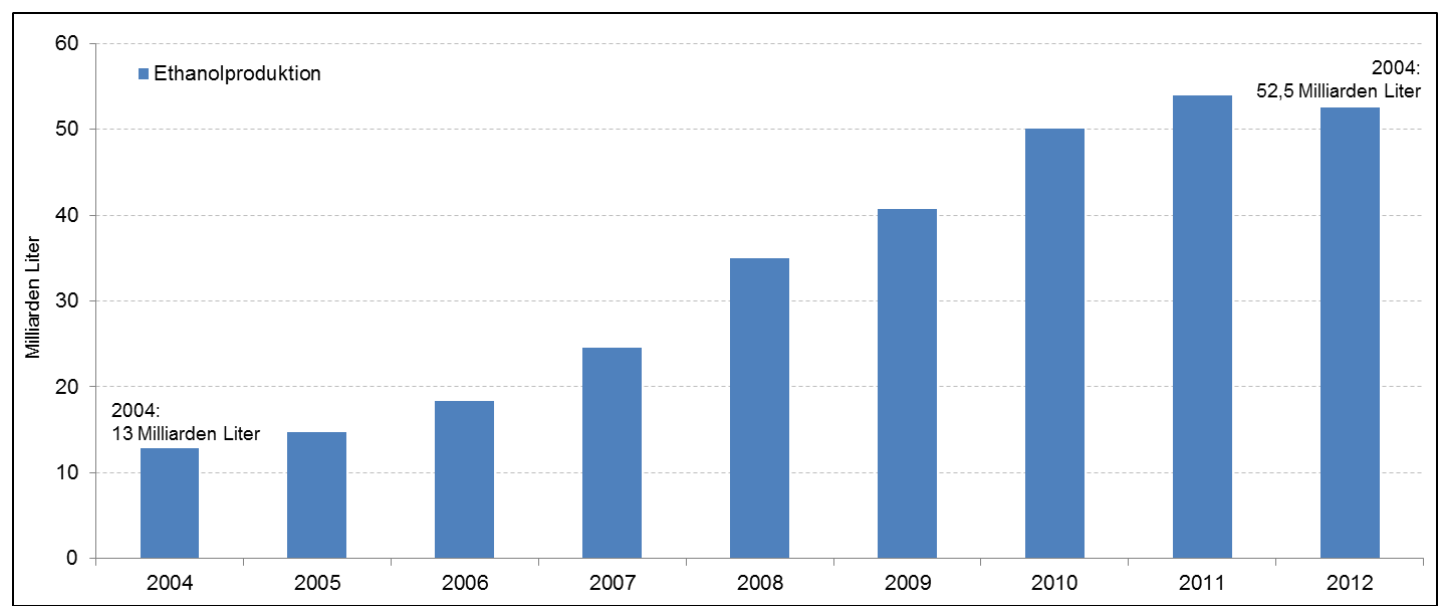

Abbildung 96: Historische Entwicklung der Ethanolproduktion in den USA Quelle: In Anlehnung an F.O. LICHT 2012f: 73; BERG 2012c: 17

Die Ethanolproduktion im Jahr 2012 in Höhe von 52,5 Milliarden Litern erfolgte durch 211 kommerzielle Anlagen, die fast ausschließlich Mais als Rohstoff verwendeten (Kapitel 6.2.3) (RFA 2012c). Die Herstellung von Zellulose-Ethanol ist dagegen von geringer Relevanz, da in den Jahren 2009 bis 2011 im Durchschnitt lediglich 0,027 Milliarden Liter Zellulose-Ethanol produziert wurden (OECD/FAO 2012: 224). Darüber hinaus wird es auch in den Jahren 2013 und 2014 zu keiner bedeutenden Steigerung der Produktion kommen, da die ersten kommerziellen Anlagen die Produktion nicht vor Ende 2014 aufnehmen werden ${ }^{345}$.

Zur Betrachtung der US-amerikanischen Ethanolproduktion im Jahr 2023 werden im Folgenden vier Zukunftsprojektionen dargestellt: (1) „Sehr starker Anstieg der Produktion von Zellulose-Ethanol“, (2) „Moderater Anstieg der Produktion von Unspecified-Ethanol ${ }^{346 ،, ~(3) ~ „ S t a r k e r ~ A n s t i e g ~ d e r ~ P r o d u k t i o n ~ v o n ~ M a i s-E t h a n o l “ ~ u n d ~(4) ~}$ „Starker Anstieg der Produktion von Unspecified-Ethanol“. Die Projektionen wurden sowohl auf Grundlage der prognostizierten Ethanolnachfrage (IA, IB, IC und ID) in Kapitel 6.2.8, als auch auf Grundlage der erwarteten Ethanolimporte und -exporte, dargestellt in Kapitel 6.2.10, gebildet.

${ }^{345}$ Die Unternehmen Abengoa Bioenergy, DuPont sowie ein Joint Venture von POET und DSM errichten momentan die ersten kommerziellen Anlagen zur Herstellung von ZelluloseEthanol in den USA (ABENGOA BIOENERGY 2012; DUPONT 2012; POET-DSM ADVAN-

${ }_{346}^{\text {CED BIOFUELS 2012). }}$ Der Begriff „Unspecified-Ethanol“ beschreibt Ethanol-Kraftstoffe, die nicht aus Mais und Zellulose produziert werden. Typische Rohstoffe sind Sorghum, Weizen, Zuckerrohr und Zuckerrübe. 
Sehr starker Anstieg der Produktion von Zellulose-Ethanol

Die Projektion basiert auf der Erfüllung der prognostizierten Ethanolnachfrage von Zukunftsprojektion „IA“. In der folgenden Abbildung 97 befinden sich links die Angaben zur Ethanolnachfrage und rechts zur Ethanolproduktion.

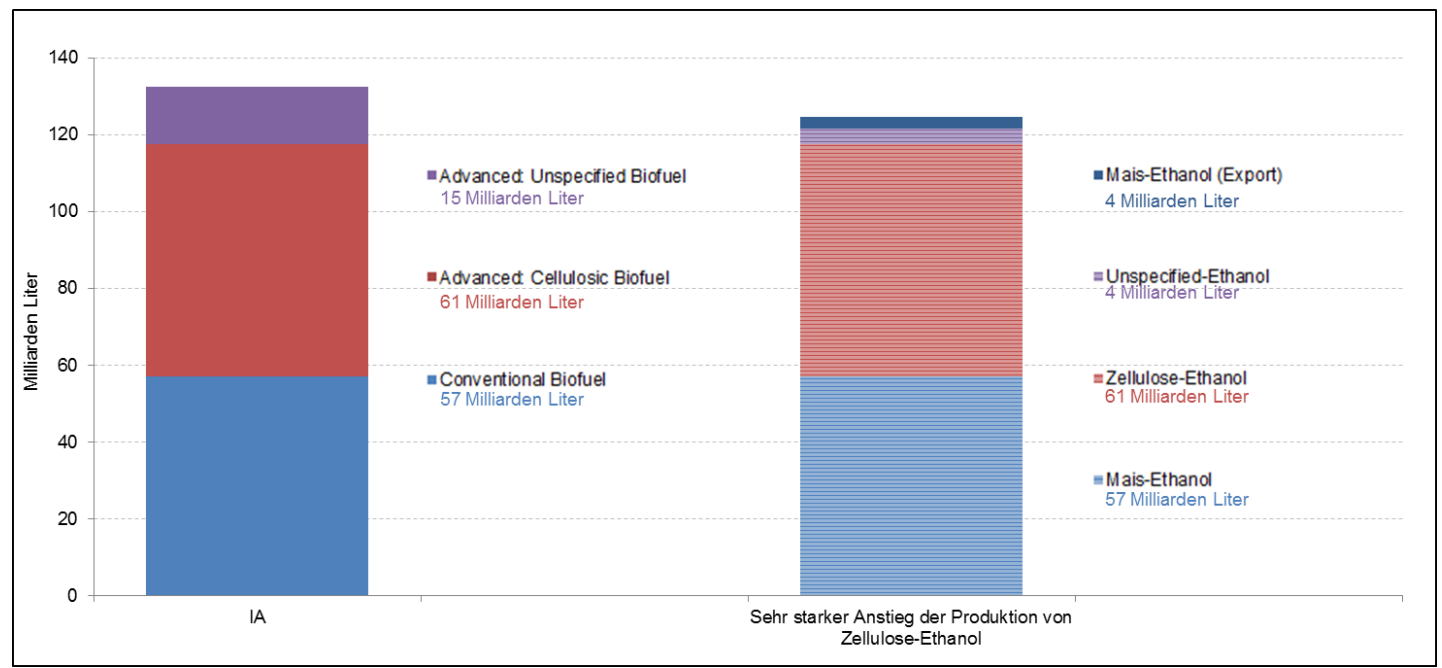

Abbildung 97: Zukunftsprojektion: „Sehr starker Anstieg der Produktion von ZelluloseEthanol“"

Quelle: EIGENE BERECHNUNG

Die Produktion von Ethanol zur Erfüllung der Nachfrage nach „Conventional Biofuel“ (57 Milliarden Liter) und „Advanced: Cellulosic Biofuel“ (61 Milliarden Liter) erfolgt in den USA vollständig durch die Produktion von Mais-Ethanol und ZelluloseEthanol. Bei einer derzeitigen Produktion von 52,5 Milliarden Litern Mais-Ethanol (BERG 2012c: 17) und bereits bestehenden Produktionskapazitäten in Höhe von 56 Milliarden Litern (RFA 2012c) ist eine zukünftige Produktion von 57 Milliarden Litern mit geringem zusätzlichem Aufwand möglich. Die Produktion von 61 Milliarden Litern Zellulose-Ethanol ${ }^{347}$ in den USA im Jahr 2023 bei einer momentanen Produktion von lediglich 0,027 Milliarden Litern ist ungleich schwerer zu realisieren (OECD/FAO 2012: 224). Die Prognose ist dennoch realistisch unter der Annahme, dass die ersten kommerziellen Anlagen im Jahr 2014 die Produktion aufnehmen werden. Dadurch kann bewiesen werden, dass eine wirtschaftlich rentable Produktion von Zellulose-Ethanol derzeit möglich ist, wodurch Investoren in den massiven Ausbau der Produktionskapazitäten bis zum Jahr 2023 investieren ${ }^{348}$.

${ }^{347}$ Die Produktion von 61 Milliarden Litern Zellulose-Ethanol im Jahr 2023 stellt die obere Grenze des Zukunftsraums dar. Ein noch stärkerer Anstieg der Produktion wird als nicht realistisch erachtet.

${ }^{348}$ Auch das Brookhaven National Laboratory prognostiziert einen starken Ausbau der Produktionskapazitäten von Zellulose-Ethanol ab dem Moment, in dem es möglich ist, den Biokraftstoff wirtschaftlich rentabel in kommerziellen Anlagen zu produzieren (ALFSTAD 2008: viii). 
Die Erfüllung der Nachfrage nach „Advanced: Unspecified Biofuel“ in Höhe von 15 Milliarden Litern erfolgt sowohl durch eine US-amerikanische Produktion von 4 Milliarden Litern Unspecified-Ethanol als auch durch brasilianische Importe von Zuckerrohr-Ethanol ${ }^{349}$ (11 Milliarden Liter). Die Herstellung von 4 Milliarden Litern Unspecified-Ethanol ist realistisch, da in den USA schon heute Projekte zur Ethanolproduktion aus Sorghum, Zuckerrohr und Zuckerrübe bestehen (U.S. GOVERNMENT INFORMATION 2007: 121 STAT. 1519; ETHANOL PRODUCER MAGAZINE 2012e; ETHANOL PRODUCER MAGAZINE 2012f). Des Weiteren wird eine Produktion von 4 Milliarden Litern Mais-Ethanol für den Exportmarkt erwartet, welche von der Menge her mit den Exporten aus den Jahren 2011 und 2012 vergleichbar ist ${ }^{350 / 351}$ (BERG 2012c: 17). Zusammengefasst ergibt sich eine Ethanolproduktion von 126 Milliarden Litern im Jahr 2023.

\section{Moderater Anstieg der Produktion von Unspecified-Ethanol}

Die im Folgenden dargestellte zweite Zukunftsprojektion des Schlüsselfaktors Ethanolproduktion basiert auf der Ethanolnachfrage, die in Zukunftsprojektion "IB“ beschrieben wurde (Kapitel 6.2.8). In Abbildung 98 sind sowohl die Werte der Ethanolproduktion als auch die der Ethanolnachfrage im Jahr 2023 veranschaulicht.

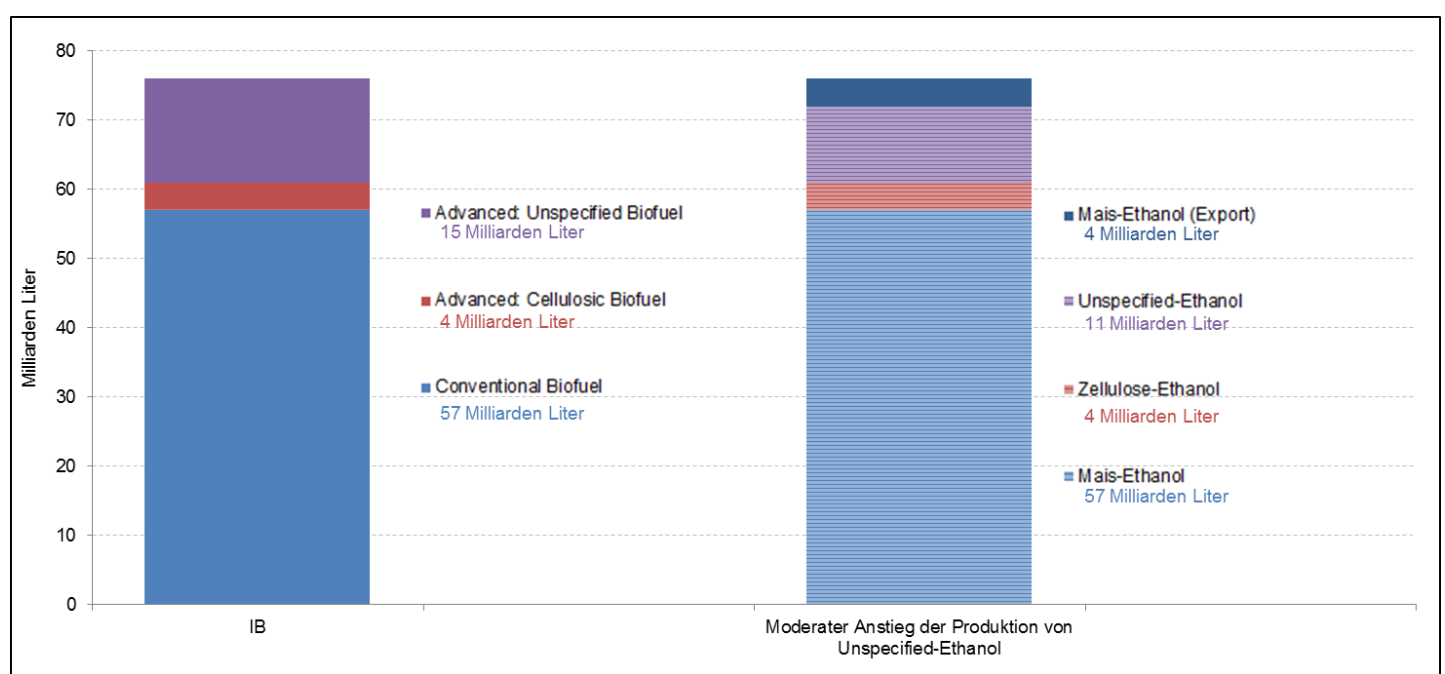

Abbildung 98: Zukunftsprojektion: „Moderater Anstieg der Produktion von UnspecifiedEthanol“"

Quelle: EIGENE BERECHNUNG

${ }^{349}$ Zuckerrohr-Ethanol aus Brasilien wird der Untergruppe „Advanced: Unspecified Biofuel" angerechnet (U.S. GOVERNMENT INFORMATION 2007: 121 STAT. 1519) und derzeit bereits importiert, um das Verbrauchsmandat „Advanced: Unspecified Biofuel“ zu erfüllen (F.O. LICHT 2012h: 193; F.O. LICHT 2011a: 65).

${ }^{350}$ Sowohl der Import von Zuckerrohr-Ethanol aus Brasilien als auch die Exporte von MaisEthanol werden im Kapitel „Ethanolhandel“ (Kapitel 6.2.10) genauer betrachtet.

${ }^{351}$ Aufgrund der Produktion von 57 Milliarden Litern für den US-amerikanischen Markt und 4 Milliarden Litern für den Exportmarkt beträgt die Gesamtproduktion von Mais-Ethanol 61 Milliarden Liter. 
Wie auch in der ersten Zukunftsprojektion wird die Nachfrage nach „Conventional Biofuel“ (57 Milliarden Liter) durch die US-amerikanische Produktion von MaisEthanol gedeckt. Die Menge des zur Erfüllung der Nachfrage nach „Advanced: CelIulosic Biofuel“ hergestellten Zellulose-Ethanols beträgt 4 Milliarden Liter ${ }^{352}$.

Die Höhe der Nachfrage nach „Advanced: Unspecified Biofuel“ liegt bei 15 Milliarden Litern und wird durch die US-amerikanische Produktion von 11 Milliarden Litern Unspecified-Ethanol sowie durch Importe von Zuckerrohr-Ethanol aus Brasilien (4 Milliarden Liter) befriedigt. Der moderate Produktionsanstieg auf 11 Milliarden Liter Unspecified-Ethanol basiert auf der Annahme, dass die USA im Jahr 2023 Unspecified-Ethanol zu - verglichen mit brasilianischem Zuckerrohr-Ethanol - konkurrenzfähigen Kosten produzieren können. Dies ist als realistisch zu betrachten, da, wie bereits in der ersten Zukunftsprojektion erläutert, schon heute Projekte zur Produktion des Kraftstoffs in den USA bestehen und die Ethanolproduktion, beispielsweise aus Weizen und Zuckerrüben, schon seit Jahren erfolgreich in der EU27 stattfindet (EUROPEAN COMMISSION JOINT RESEARCH CENTRE, INSTITUT FOR ENERGY ET AL. 2010: 74). Darüber hinaus wird auch wie in der ersten Prognose eine Produktion von 4 Milliarden Litern Mais-Ethanol für den Export prognostiziert. Aus der Addition der Werte ergibt sich für diese Projektion eine Ethanolproduktion von insgesamt 76 Milliarden Litern im Jahr 2023.

\section{Starker Anstieg der Produktion von Mais-Ethanol}

Die Ethanolproduktion, die in der dritten Zukunftsprojektion beschrieben wurde, dient der Erfüllung der Ethanolnachfrage in Projektion „IC“ (Kapitel 6.2.8). Die Werte der Ethanolnachfrage sowie der Ethanolproduktion sind in Abbildung 99 angegeben.

352 Aufgrund der intensiven Forschung im Bereich der Produktion von Zellulose-Ethanol (F.O. LICHT 2010: 452) und des Baus der ersten kommerziellen Anlagen ist eine Produktion von 4 Milliarden Litern im Jahr 2023 realistisch. 


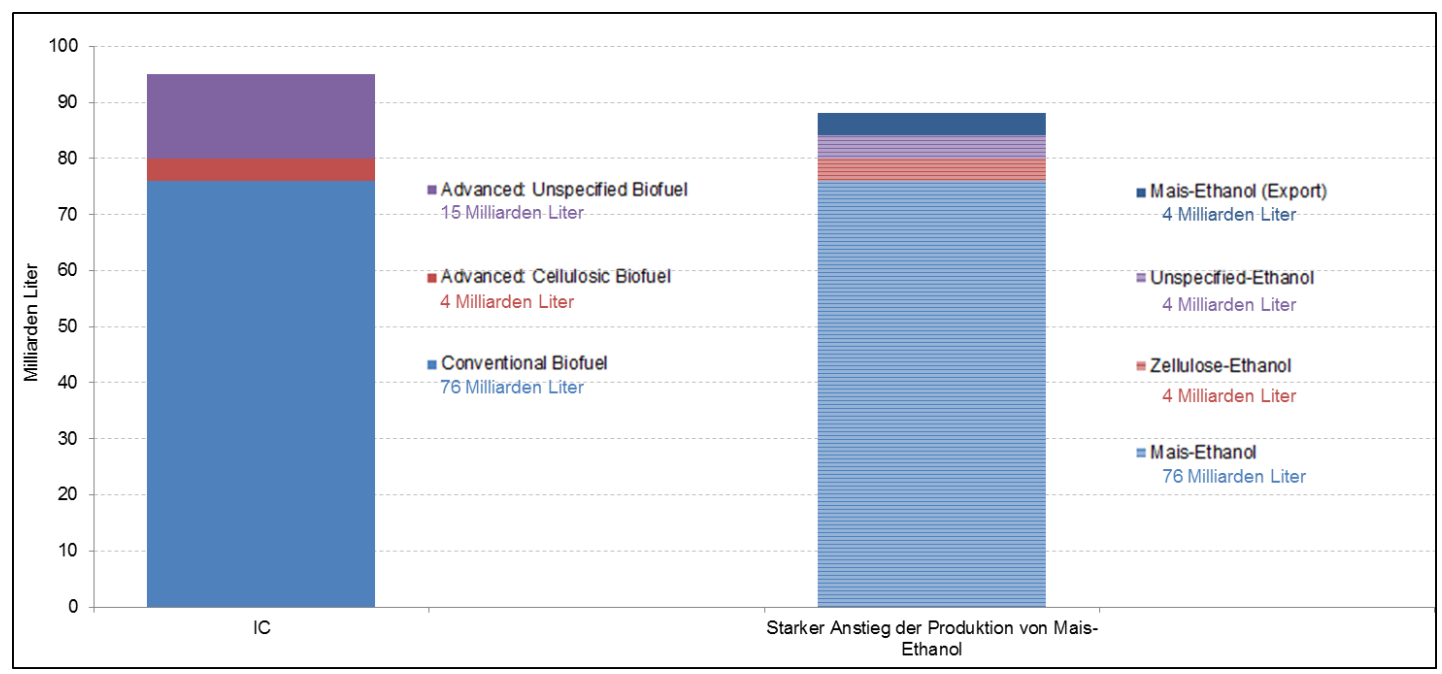

Abbildung 99: Zukunftsprojektion: „Starker Anstieg der Produktion von Mais-Ethanol“ Quelle: EIGENE BERECHNUNG

Diese Zukunftsprojektion unterscheidet sich im Vergleich zu den beiden ersten Prognosen durch den starken Anstieg der Ethanolproduktion aus Mais auf 80 Milliarden Liter im Jahr 2023, um die Nachfrage nach „Conventional Biofuel“ in Höhe von 76 Milliarden Litern und die Nachfrage des Exportmarktes (4 Milliarden Liter) befriedigen zu können. Die Produktion von 80 Milliarden Litern Mais-Ethanol ist realistisch, da die USA bereits Produktionskapazitäten von 56 Milliarden Litern besitzen (RFA 2012c) und in den letzten fünf Jahren gezeigt haben, wie schnell sie die Kapazitäten ausbauen können (Abbildung 96). Allein im Jahr 2010 sind die Produktionskapazitäten um fast 10 Milliarden Liter angestiegen (F.O. LICHT 2012f: 73).

Zur Erfüllung der Nachfrage nach „Advanced: Cellulosic Biofuel“ werden im Jahr 20234 Milliarden Liter Zellulose-Ethanol produziert. Darüber hinaus werden nochmals 4 Milliarden Liter Unspecified-Ethanol hergestellt. Zur vollständigen Deckung der Nachfrage nach „Advanced: Unspecified Biofuel“ in Höhe von 15 Milliarden Litern werden zusätzlich noch 11 Milliarden Liter Zuckerrohr-Ethanol aus Brasilien in die USA importiert. Insgesamt beträgt die Ethanolproduktion in der dritten Projektion 88 Milliarden Liter.

\section{Starker Anstieg der Produktion von Unspecified-Ethanol}

Die vierte Zukunftsprojektion orientiert sich an der Erfüllung der Ethanolnachfrage, die in Projektion „ID“ beschrieben wurde (Kapitel 6.2.8). Die Daten der Ethanolnachfrage und der Ethanolproduktion sind in Abbildung 100 dargestellt. 


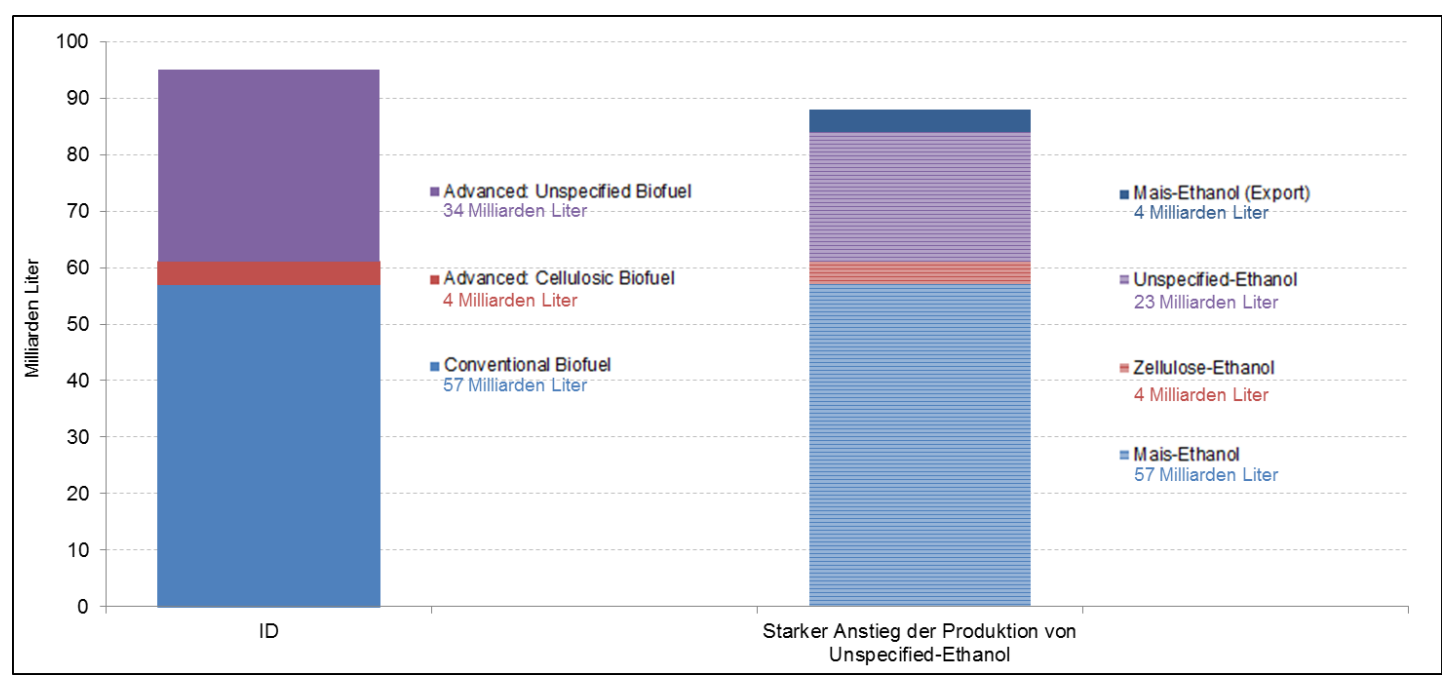

Abbildung 100: Zukunftsprojektion: „Starker Anstieg der Produktion von Unspecified-Ethanol“ Quelle: EIGENE BERECHNUNG

Im Jahr 2023 werden in den USA 57 Milliarden Liter Mais-Ethanol und 4 Milliarden Liter Zellulose-Ethanol hergestellt, um die Nachfrage nach „Conventional Biofuel“ und „Advanced: Cellulosic Biofuel“ befriedigen zu können.

Das Besondere bei dieser Projektion ist der starke Anstieg der Produktion von Unspecified-Ethanol auf 23 Milliarden Liter, um die Nachfrage nach „Advanced: Unspecified Biofuel“ in Höhe von 34 Milliarden Litern erfüllen zu können. Zwar werden ebenfalls 11 Milliarden Liter Zuckerrohr-Ethanol aus Brasilien importiert, doch basiert die Projektion auf der Annahme, dass die USA Unspecified-Ethanol zu konkurrenzfähigen Kosten produzieren kann. Eine Produktion von 23 Milliarden Litern ist realistisch, da Unternehmen bereits seit mehreren Jahren an der kommerziellen Produktion von Ethanol aus Sorghum, Zuckerrohr und Zuckerrübe in den USA forschen (U.S. GOVERNMENT INFORMATION 2007: 121 STAT. 1519; ETHANOL PRODUCER MAGAZINE 2012e; ETHANOL PRODUCER MAGAZINE 2012f.). Darüber hinaus ähnelt der Produktionsprozess zur Herstellung von Unspecified-Ethanol dem von Mais-Ethanol und ist damit deutlich einfacher und kostengünstiger als die Produktion von Zellulose-Ethanol (FNR 2009: 25). Des Weiteren erfolgt bereits seit mehreren Jahren in der EU27 eine erfolgreiche kommerzielle Ethanolproduktion aus Weizen und Zuckerrüben (EUROPEAN COMMISSION JOINT RESEARCH CENTRE, INSTITUT FOR ENERGY ET AL. 2010: 74).

Unter Berücksichtigung der Herstellung von 4 Milliarden Litern Mais-Ethanol für den Exportmarkt ergibt sich für diese Zukunftsprojektion eine Gesamtproduktion von 88 Milliarden Litern.

Die vier Zukunftsprojektionen der Ethanolproduktion sind realistisch. Einerseits aufgrund ihrer Herleitung aus der Ethanolnachfrage und andererseits, da auch führen- 
de Forschungsinstitute ${ }^{353}$ einen ähnlichen Anstieg der Ethanolproduktion im Zeitraum von 2012 bis 2025 erwarten. Die vier Zukunftsprojektionen werden in der „USA Szenario-Bildung und -Konsistenzprüfung“ weiter betrachtet:

IIA

Sehr starker Anstieg der Produktion von Zellulose-Ethanol

Beschreibung:

Mais-Ethanol $=57$ Milliarden Liter, Zellulose-Ethanol $=61$ Milliarden Liter, Unspecified-

Ethanol $=4$ Milliarden Liter und Mais-Ethanol (Export) $=4$ Milliarden Liter

IIB

Moderater Anstieg der Produktion von Unspecified-Ethanol

Beschreibung:

Mais-Ethanol $=57$ Milliarden Liter, Zellulose-Ethanol $=4$ Milliarden Liter, Unspecified-Ethanol

$=11$ Milliarden Liter und Mais-Ethanol (Export) $=4$ Milliarden Liter

Starker Anstieg der Produktion von Mais-Ethanol

IIC Beschreibung:

Mais-Ethanol $=76$ Milliarden Liter, Zellulose-Ethanol $=4$ Milliarden Liter, Unspecified-Ethanol

$=4$ Milliarden Liter und Mais-Ethanol (Export) $=4$ Milliarden Liter

Starker Anstieg der Produktion von Unspecified-Ethanol

IID Beschreibung:

Mais-Ethanol $=57$ Milliarden Liter, Zellulose-Ethanol $=4$ Milliarden Liter, Unspecified-Ethanol

$=23$ Milliarden Liter und Mais-Ethanol (Export) $=4$ Milliarden Liter

\subsubsection{Ethanolhandel}

Der US-amerikanische Ethanolmarkt hat sich von einem Importmarkt (2004 - 2009) zu einem Exportmarkt (2010 - 2012) gewandelt (Abbildung 101 $)^{354}$. So waren die USA in den Jahren 2011 und 2012 gar der weltgrößte Ethanolexporteur mit 5 bzw. 2,9 Milliarden Litern (F.O. LICHT 2012a: 301; BERG 2012c: 17$)^{355}$.

${ }^{353}$ Die Forschungsinstitute U.S. ENERGY INFORMATION ADMINISTRATION (2012b: 15), OECD/FAO (2012: 114), FAPRI (2013) und Brookhaven National Laboratory (ALFSTAD 2008: A-2) erwarten einen ähnlichen langfristigen Anstieg der US-amerikanischen Ethanolproduktion.

${ }^{554}$ Die genauen Werte des weltweiten Ethanolhandels sind schwer zu erfassen, da Ethanol nicht nur in Reinform gehandelt wird, sondern immer öfter in Mischungen mit Benzin. Bekanntestes Beispiel hierfür ist der Handel mit E90 (90\% Ethanol und 10\% Benzin) (F.O. LICHT 2012a: 301).

${ }^{355}$ Brasilien als zweitgrößter Exporteur hat im Jahr 20112 Milliarden Liter und im Jahr 2012 2,7 Milliarden Liter Ethanol exportiert (BERG 2012c: 16). 


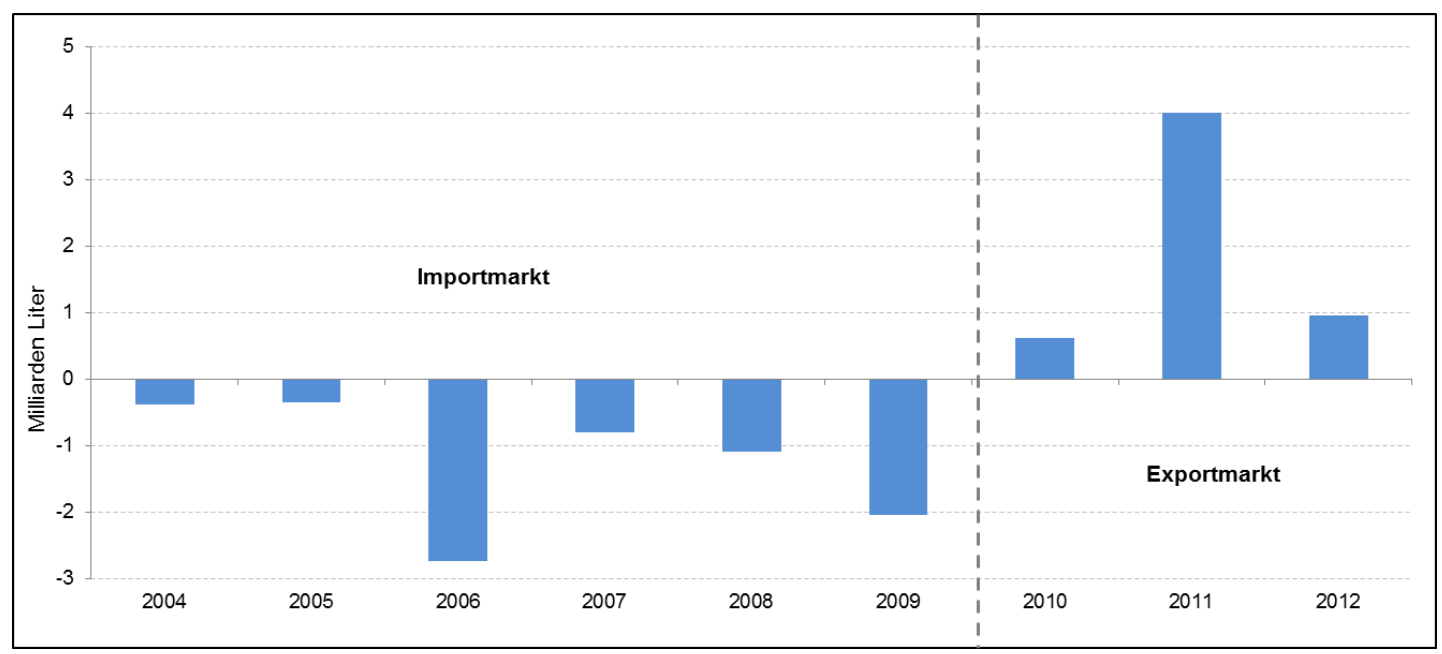

Abbildung 101: Entwicklung des Ethanolhandels in den USA im Zeitraum von 2004 bis $2012^{356}$

Quelle: Quelle: F.O. LICHT 2012f: 73f.; F.O. LICHT 2012a: 301ff.; BERG 2012c: 17; U.S. ENERGY INFORMATION ADMINISTRATION 2012b: 97; EIGENE BERECHNUNG

Diese Entwicklung zum Exportmarkt ist bis zum Jahr 2011 maßgeblich auf die folgenden drei Punkte zurückzuführen (F.O. LICHT 2012a: 302; F.O. LICHT 2012f: 73): (1) Infolge des starken Anstiegs der Produktion von Mais-Ethanol auf 54 Milliarden Liter im Jahr 2011 waren die USA aufgrund der bestehenden „Blend Wall“Problematik gezwungen, Ethanol zu exportieren, um die Überproduktion abzusetzen und damit einen Wertverfall des Ethanols in den USA zu verhindern. (2) Die USamerikanische Politik förderte den Ethanolexport durch den Volumetric Ethanol Excise Tax Credit (VEETC) in Höhe von 0,45 US-\$/Gallone und (3) Brasilien konnte die weltweiten Ethanolmärkte aufgrund des starken Rückgangs der brasilianischen Ethanolproduktion um 4,5 Milliarden Liter $^{357}$ nicht mehr ausreichend mit Ethanol versorgen und die USA übernahmen diese Aufgabe.

Im Jahr 2012 sind die USA zwar immer noch der größte Ethanolexporteur der Welt (2012: 2,9 Milliarden Liter), aber der Exportüberschuss ist von 4 Milliarden Litern im Jahr 2011 auf nur noch 1 Miliarde Liter geschrumpft. Verantwortlich für diesen Rückgang ist die geringere US-amerikanische Ethanolproduktion im Jahr $2012 \mathrm{im}$ Vergleich zum Vorjahr (2011: 54 Milliarden Liter; 2012: 52,5 Milliarden Liter) (F.O. LICHT 2012f: 73; BERG 2012c: 17), der Wegfall des VEETC zum Ende des Jahres 2011 (F.O. LICHT 2012a: 300; F.O. LICHT 2012f: 69) sowie die geringe Nachfrage nach Ethanolimporten aus der EU27 und Brasilien (F.O. LICHT 2012a: 300; BERG 2012c: 17). Darüber hinaus stiegen die Ethanolimporte in die USA infolge hoher Zuckerrohr-Ethanolimporte aus Brasilien auf 1,6 Milliarden Liter an (BERG 2012c:

${ }^{356}$ In Abbildung 101 wird die Differenz zwischen dem Ethanolexport (Minuend) und dem Ethanolimport (Subtrahend) dargestellt.

${ }^{357}$ Die brasilianische Ethanolproduktion ist von 25,5 Milliarden Litern im Jahr 2010 auf 21 Milliarden Liter im Jahr 2011 gesunken (F. O. LICHT 2012f: 74). 
17), sowohl um die Nachfrage nach „Advanced: Unspecified Biofuel“ (U.S. GOVERNMENT INFORMATION 2007: 121 STAT. 1519; OECD/FAO 2012: 94; F.O. LICHT 2012a: 301) als auch die Nachfrage nach Zuckerrohr-Ethanol in Kalifornien $^{358}$ (F.O. LICHT 2012h: 193; F.O. LICHT 2011a: 65) zu befriedigen. Außerdem gewannen Ethanolimporte zum Jahreswechsel 2011/2012 durch die Aufhebung des Importzollsatzes in Höhe von 0,54 US-\$ je Gallone an Konkurrenzfähigkeit gegenüber der US-amerikanischen Produktion ${ }^{359}$ (F.O. LICHT 2011a: 65).

In der folgenden Abbildung 102 sind die US-amerikanischen Ethanolexporte und -importe aus dem Jahr 2012 dargestellt. Die mit Abstand wichtigsten Exportmärkte sind Brasilien (Kapitel 6.1.1.2/Einflussfaktor 4), die EU27 (Kapitel 6.1.1.2/Einflussfaktor 4) und Kanada (F.O. LICHT 2012a: 302). Auf die Handelsbeziehungen zwischen den USA und Brasilien wird im Folgenden eingegangen.

\footnotetext{
${ }^{358}$ Kalifornien importiert Zuckerrohr-Ethanol aus Brasilien, um die $\mathrm{CO}_{2}$-Reduktionsziele des Low Carbon Fuels Standard (LCFS) sowie die Mandate des RFS 2 erfüllen zu können. Kalifornien kann Mais-Ethanol, welches im Mittleren Westen der USA produziert wird, nicht verwenden, da dieses den Berechnungen des California Air Resources Board (CARB) zufolge nicht die im LCFS vorgeschriebenen $\mathrm{CO}_{2}$-Einsparungen im Vergleich zu fossilem Kraftstoff erreicht. Zuckerrohr-Ethanol aus Brasilien erfüllt hingegen die $\mathrm{CO}_{2}$-Reduktionsvorgaben und wird aufgrund dessen in Kalifornien besonders nachgefragt (F.O. LICHT 2012h: 193). Eine genaue Beschreibung des LCFS und dessen Einfluss auf den US-amerikanischen Ethanolmarkt befinden sich in Kapitel 6.1.1.2/Einflussfaktor 25 .

${ }_{359}$ Der Importzoll für Ethanol wurde aufgehoben, da auch der Volumetric Ethanol Excise Tax Credit (VEETC) in Höhe von 0,45 US-\$/Gallone zum Ende des Jahres 2011 von der Politik nicht verlängert wurde (F.O. LICHT 2012a: 300; F.O. LICHT 2012f: 69).
} 




Abbildung 102: Ethanolexporte und -importe der USA im Jahr 2012

Quelle: In Anlehnung an U.S. ENERGY INFORMATION ADMINISTRATION (2013c); U.S. ENERGY INFORMATION ADMINISTRATION (2013d); U.S. ENERGY INFORMATION ADMINISTRATION (2013e)

Das Besondere beim Ethanolhandel zwischen den USA und Brasilien ist, dass dieser zweiseitig erfolgt. Auf der einen Seite importiert Brasilien Mais-Ethanol aus den USA, um die Ethanolnachfrage am Binnenmarkt, die sich aufgrund der gestiegenen Anzahl an FFV erhöht hat, befriedigen zu können. Gleichzeitig importieren aber auch die USA zur Erfüllung der Nachfrage nach „Advanced: Unspecified Biofuel" sowie für den kalifornischen Ethanolmarkt brasilianisches Zuckerrohr-Ethanol (F.O. LICHT 2012h: 193). Diese bizarre Situation des gegenseitigen Handels mit dem identischen Produkt ist das Ergebnis der US-amerikanischen Ethanolpolitik, da brasilianisches Zuckerrohr-Ethanol im US-amerikanischen Markt durch die Anrechnung als „Advanced: Unspecified Biofuel“ einen höheren politischen Wert im Vergleich zu Mais-Ethanol besitzt, welches lediglich der Erfüllung des Verbrauchsmandats "Conventional Biofuel“ dient (OECD/FAO 2012: 89; U.S. GOVERNMENT INFORMATION 2007: 121 STAT. 1519). 
Auch in Zukunft wird es zum zweiseitigen Handel zwischen den USA und Brasilien kommen, wie in den folgenden beiden Zukunftsprojektionen dargestellt, die den Schlüsselfaktor im Jahr 2023 beschreiben:

- Importüberschuss: Hohe Importe von Unspecified-Ethanol und moderate Exporte von Mais-Ethanol.

- Ausgeglichener Handel: Moderate Importe von Unspecified-Ethanol und moderate Exporte von Mais-Ethanol.

Der Ethanolhandel betrifft in beiden Zukunftsprojektionen nur die Ethanolarten Unspecified-Ethanol und Mais-Ethanol. Die Projektionen basieren auf der Annahme, dass die US-amerikanische Nachfrage nach „Advanced: Cellulosic Biofuel“ vollständig durch die US-amerikanische Zellulose-Ethanolproduktion gedeckt wird und auch keine Überproduktion an Zellulose-Ethanol erfolgt ${ }^{360}$.

Importüberschuss: Hohe Importe von Unspecified-Ethanol und moderate Exporte von Mais-Ethanol

In dieser Projektion werden 11 Milliarden Liter Unspecified-Ethanol in die USA importiert und 4 Milliarden Liter Mais-Ethanol exportiert. Die Importe von 11 Milliarden Litern Unspecified-Ethanol dienen der Erfüllung der Nachfrage nach „Advanced: Unspecified Biofuel“ in den Zukunftsprojektionen „IA“, „IC“ und „ID“. Bei den Importen handelt es sich fast ausschließlich um Zuckerrohr-Ethanol aus Brasilien, da der Kraftstoff dem Mandat „Advanced: Unspecified Biofuel“ angerechnet werden kann und somit in der Lage ist, die Nachfrage zu befriedigen (U.S. GOVERNMENT INFORMATION 2007: 121 STAT. 1519). Die Importe von 11 Milliarden Litern sind als realistisch zu betrachten, da Zuckerrohr-Ethanol aus Brasilien nach Einschätzung der OECD/FAO (2012: 94) der zukünftig günstigste Biokraftstoff sein wird, um die Nachfrage zu decken. Darüber hinaus besitzt Brasilien die landwirtschaftlichen und ökonomischen Voraussetzungen, um diese Mengen im Jahr 2023 zu produzieren und in die USA zu exportieren (OECD/FAO 2012: 99).

Des Weiteren wird ein Export von 4 Milliarden Litern Mais-Ethanol erwartet. Diese Menge ist vergleichbar mit den Exporten der Jahre 2011/2012 (BERG 2012c: 17), aber im Vergleich zur prognostizierten Mais-Ethanolproduktion von 61 Milliarden Liter (IIA, IIB und IID) bzw. 80 Milliarden Litern (IIC) im Jahr 2023 von nur untergeordneter Bedeutung. Der Export dient lediglich dazu, Überkapazitäten der US-

${ }^{360}$ Diese Annahme beruht auf der Einschätzung, dass die USA selbst die Nachfrage nach Zellulose-Ethanol erfüllen wollen, da sie die Technologie entwickelt haben und nicht von anderen Staaten abhängig sein wollen. Diese Annahme wurde intensiv mit Martin Mitchell von Clariant diskutiert und entwickelt. 
amerikanischen Produktion auf dem Weltmarkt abzusetzen. Eine gezielte Produktion von Mais-Ethanol für den Weltmarkt findet nicht statt. Von den Exporten werden große Mengen nach Brasilien geliefert, um dort die Ausfuhr von Zuckerrohr-Ethanol in die USA zu kompensieren und die aufgrund der wachsenden Flotte an FFV steigende brasilianische Binnennachfrage nach Ethanol zu decken (OECD/FAO 2012: 94f.).

Aus der Differenz von Ethanolimporten (11 Millarden Liter) und -exporten (4 Milliarden Liter) ergibt sich für diese Projektion ein Importüberschuss von 7 Milliarden Litern.

Ausgeglichener Handel: Moderate Importe von Unspecified-Ethanol und moderate Exporte von Mais-Ethanol

Im Gegensatz zur ersten werden in dieser Projektion nur 4 Milliarden Liter Unspecified-Ethanol importiert, um die Nachfrage nach „Advanced: Unspecified Biofuel“ in Zukunftsprojektion „IB“ erfüllen zu können. Auch bei diesen Importen handelt es sich um Zuckerrohr-Ethanol aus Brasilien. Des Weiteren wird ebenfalls ein Export von 4 Milliarden Litern Mais-Ethanol erwartet, von dem ein Teil nach Brasilien verkauft werden wird. Zusammenfassend ergibt sich für diese Prognose ein ausgeglichener Ethanolhandel.

Die Projektion ist realistisch, da die weltweiten brasilianischen Ethanolexporte im Jahr 2012 bereits 2,7 Milliarden Liter und im Jahr 2008 schon 5 Milliarden Liter betrugen (BERG 2012c: 16; F.O. LICHT 2012a: 304). Darüber hinaus wird kein stärkerer Anstieg des Exports von Mais-Ethanol als 4 Milliarden Liter erwartet, da, wie bereits erwähnt, der Exportmarkt für die USA lediglich die Aufgabe besitzt, eine Überproduktion abzusetzen. Die gezielte Produktion von Mais-Ethanol für den Exportmarkt wird in den Szenarien nicht betrachtet, da dieser Markt für die Ethanolproduzenten mit einem hohen Risiko verbunden ist, weil der weltweite Ethanolhandel stark von politischen Entscheidungen beeinflusst wird. Beispielhaft dafür ist die Neuregelung des Importzollsatzes für E90 in der EU27 im Jahr 2012 (Kapitel 5.2.5) (F.O. LICHT 2012a: 300f.).

Die beiden dargestellten Zukunftsprojektionen, die den Schlüsselfaktor im Jahr 2023 beschreiben, werden in der weiteren Studie berücksichtigt:

IIIA

Importüberschuss: Hohe Importe von Unspecified-Ethanol und moderate Exporte von Mais-Ethanol

Beschreibung:

Import Unspecified-Ethanol $=11$ Milliarden Liter; Export Mais-Ethanol $=4$ Milliarden Liter 
Ausgeglichener Handel: Moderate Importe von Unspecified-Ethanol und IIIB moderate Exporte von Mais-Ethanol

\subsubsection{Ethanolpreis}

Die folgende Abbildung 103 zeigt die Entwicklung des Ethanol-Weltmarktpreises im Zeitabschnitt von 2001 bis 2011 sowie den Verlauf des US-amerikanischen Ethanolpreises von 2009 bis $2011^{361}$.

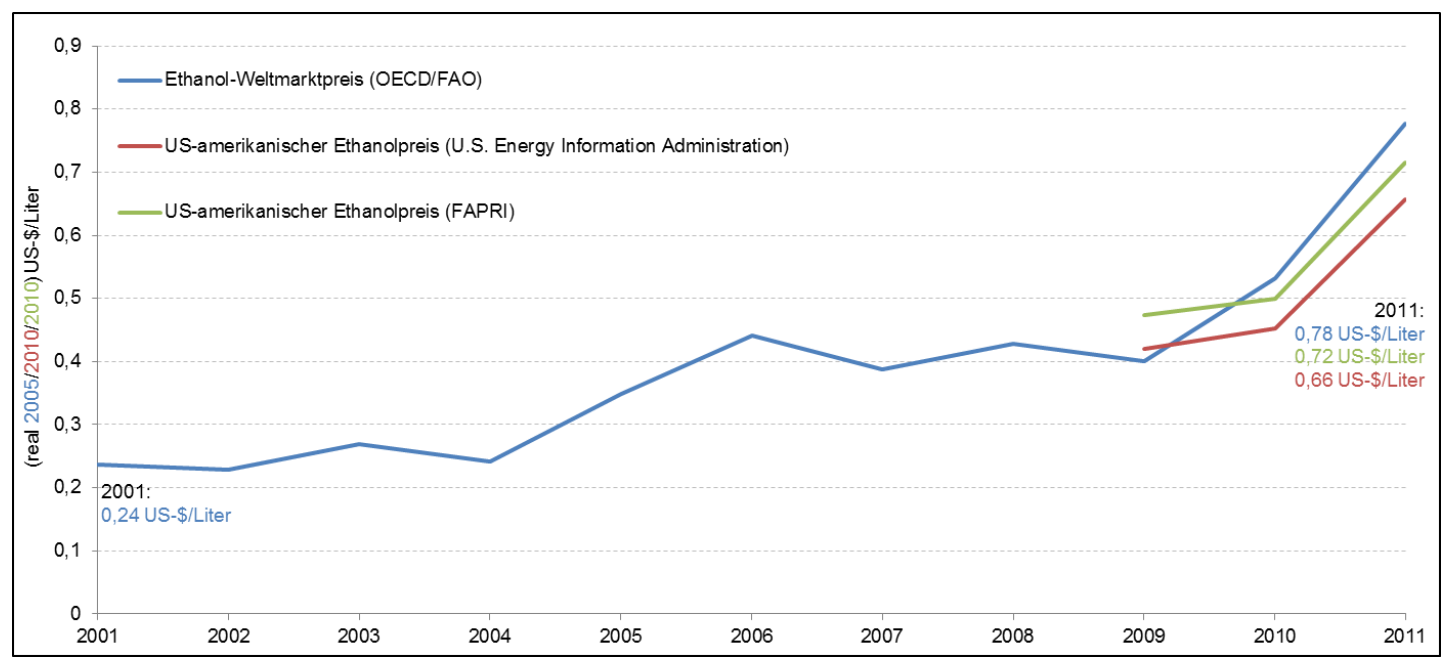

Abbildung 103: Entwicklung des (real 2005) Ethanol-Weltmarktpreises $(2001-2011)$ und des (real 2010) US-amerikanischen Ethanolpreises (2009 - 2011)

Quelle: In Anlehnung an OECD/FAO 2012: 89; U.S. ENERGY INFORMATION ADMINISTRATION 2012k; FAPRI 2012

Der (real 2005) Ethanol-Weltmarktpreis ist von 2001 bis 2009 stetig von 0,24 US\$/Liter auf 0,4 US-\$/Liter angestiegen. In den Jahren 2010 und 2011 hat sich der Weltmarktpreis sogar fast auf 0,78 US-\$/Liter verdoppelt. In den USA gab es in dem Zeitraum von 2009 bis 2011 eine sehr ähnliche Entwicklung, da auch dort der (real 2010) Ethanolpreis stark angestiegen ist. Diese Entwicklung des Welt- und USamerikanischen Ethanolpreises in den Jahren 2010 und 2011 ist maßgeblich auf die gestiegenen Rohstoffkosten (Kapitel 6.2.3), die geringe Steigerung der Ethanolpro-

${ }^{361}$ Die Ethanolpreise der Organisationen OECD/FAO, U.S. Energy Information Administration und FAPRI unterscheiden sich, da die OECD/FAO den Ethanol-Weltmarktpreis anhand des Preises für wasserfreies Ethanol ab Distillerie in Brasilien, Sao Paulo, angibt. Die U.S. Energy Information Administration und das FAPRI geben hingegen die Preisentwicklung mittels des US-amerikanischen Ethanolpreises wieder. Die Preise zwischen den beiden Einrichtungen unterscheiden sich dennoch, da das FAPRI den Ethanolpreis F.O.B. (Free On Board) in Omaha (Nebraska) und die U.S. Energy Information Administration den EthanolGroßhandelspreis (Ethanol Wholesale Price) angibt. Darüber hinaus beziehen sich die realen Ethanolpreise der U.S. Energy Information Administration und des FAPRI auf das Jahr 2010, die Preise der OECD/FAO jedoch auf das Jahr 2005. 
duktion in den USA ${ }^{362}$ und auf den Rückgang der Ethanolherstellung in Brasilien ${ }^{363}$ zurückzuführen. Darüber hinaus wirkte die unerwartet geringe Ethanolproduktion in Entwicklungsländern preiserhöhend (OECD/FAO 2012: 88).

Im folgenden Abschnitt wird die zukünftige Entwicklung des Ethanolpreises bis zum Jahr 2023 betrachtet. In Abbildung 104 sind die langfristigen prognostizierten Preise der Organisationen OECD/FAO, FAPRI und U.S. Energy Information Administration angegeben, die als Basis zur Ermittlung der Zukunftsprojektionen dienen.

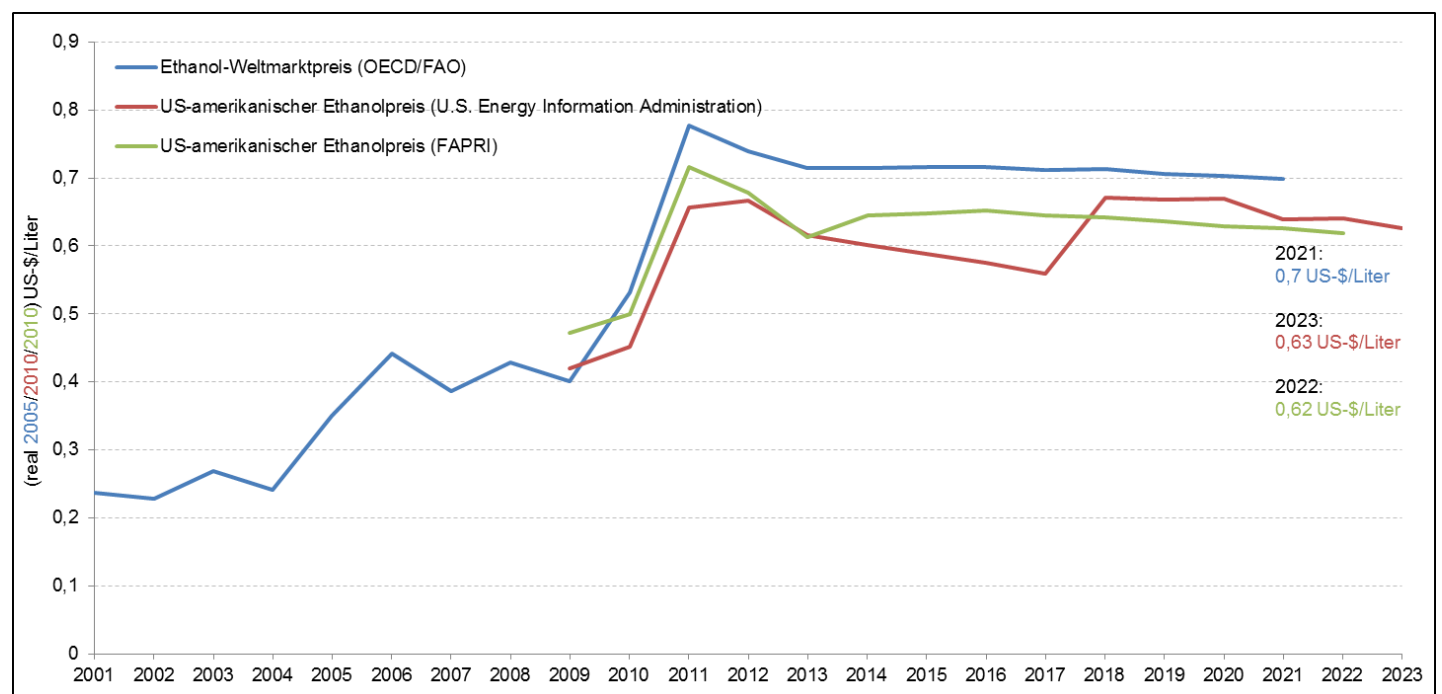

Abbildung 104: Zukünftige Entwicklung des (real 2005) Ethanol-Weltmarktpreises und des (real 2010) US-amerikanischen Ethanolpreises

Quelle: In Anlehnung an OECD/FAO 2012: 89; U.S. ENERGY INFORMATION ADMINISTRATION 2012k; FAPRI 2012

Alle drei Organisationen erwarten, folgend auf den starken Preisanstieg in den Jahren 2010 und 2011, einen leichten Rückgang des Ethanolpreises bis zum Jahr 2014. Langfristig bis zum Jahr 2023 prognostizieren die OECD/FAO, das U.S. Energy Information Administration und das FAPRI einen realen Ethanolpreis zwischen 0,62 US-\$/Liter und 0,7 US-\$/Liter, wobei der Weltmarktpreis über dem USamerikanischen Ethanolpreis liegen wird, wie es bereits in den Jahren 2010 und 2011 der Fall war. Die langfristig konstante Entwicklung des Ethanolpreises begründet die OECD/FAO (2012: 89) mit dem ebenfalls konstanten Verlauf der Mais- und Zuckerpreise (Kapitel 6.2.3). Des Weiteren wird nicht erwartet, dass der Ethanolpreis einem möglichen starken Anstieg des Rohölpreises folgen wird (OECD/FAO

\footnotetext{
${ }^{362}$ Die USA konnten das Produktionswachstum der vorherigen Perioden im Jahr 2011 nicht erreichen. Während die Produktion in den Jahren 2007 bis 2010 um durchschnittlich 8,5 Milliarden Liter anstieg, erreichten die USA im Jahr 2011 lediglich eine Steigerung der jährlichen Ethanolproduktion um 2 Milliarden Liter (Kapitel 6.2.9) (F.O. LICHT 2011a: 67).

${ }^{363}$ In Brasilien ist die Ethanolproduktion um 5,4 Milliarden Liter - von 28 Milliarden Litern im Jahr 2010 auf 22,6 Milliarden Liter im Jahr 2011 - zurückgegangen (F.O. LICHT 2011a: 67).
} 
2012: 89). Basierend auf der Projektion der U.S. ENERGY INFORMATION ADMINISTRATION (2012k) wird ein US-amerikanischer Ethanolpreis von (real 2010) 0,63 US-\$/Liter in der „USA Szenario-Bildung und -Konsistenzprüfung“ weiter betrachtet $^{364}$.

Darüber hinaus wird in der weiteren Studie noch ein zweiter, höherer USamerikanischer Ethanolpreis berücksichtigt. Dieser Ethanolpreis ergibt sich aus einer in Zukunftsprojektion IC (Kapitel 6.2.8) um 19 Milliarden Liter höheren Nachfrage nach Mais-Ethanol als momentan im RFS 2 beschrieben. Infolgedessen kommt es zu einer gesteigerten Mais-Ethanolproduktion (IIC/Kapitel 6.2.9), was die Nachfrage nach Mais steigert und zu einem Anstieg des Maispreises, von ursprünglich 180 US-\$/Tonne auf 209 US-\$/Tonne, führt (Kapitel 6.2.3/12aB). Diese Entwicklung wiederum begründet die höheren Produktionskosten und damit den Anstieg des USamerikanischen Ethanolpreises auf (real 2010) 0,7 US-\$/Liter ${ }^{365}$.

Basierend auf den vorangegangenen Erläuterungen werden die folgenden zwei Zukunftsprojektionen weiter betrachtet:

IVA | US-amerikanischer Ethanolpreis (real 2010) 0,63 US-\$/Liter

IVB | US-amerikanischer Ethanolpreis (real 2010) 0,7 US-\$/Liter

In der folgenden Abbildung 105 sind nochmals alle Zukunftsprojektionen der acht wirkenden und der vier beschreibenden Schlüsselfaktoren der „USA SzenarioPrognostik" zusammengefasst dargestellt.

\footnotetext{
${ }^{364}$ Es wird in der weiteren Studie die Prognose der U.S. ENERGY INFORMATION ADMINISTRATION (2012k) berücksichtigt, da in der Projektion der US-amerikanische Ethanolpreis und nicht der Weltmarktpreis betrachtet sowie ein Wert für das Jahr 2023 angegeben wird.

${ }^{365}$ Die Auswirkungen der höheren Rohstoffkosten auf den US-amerikanischen Ethanolpreis gehen aus der Studie der OECD/FAO (2012: 155f.) hervor.
} 


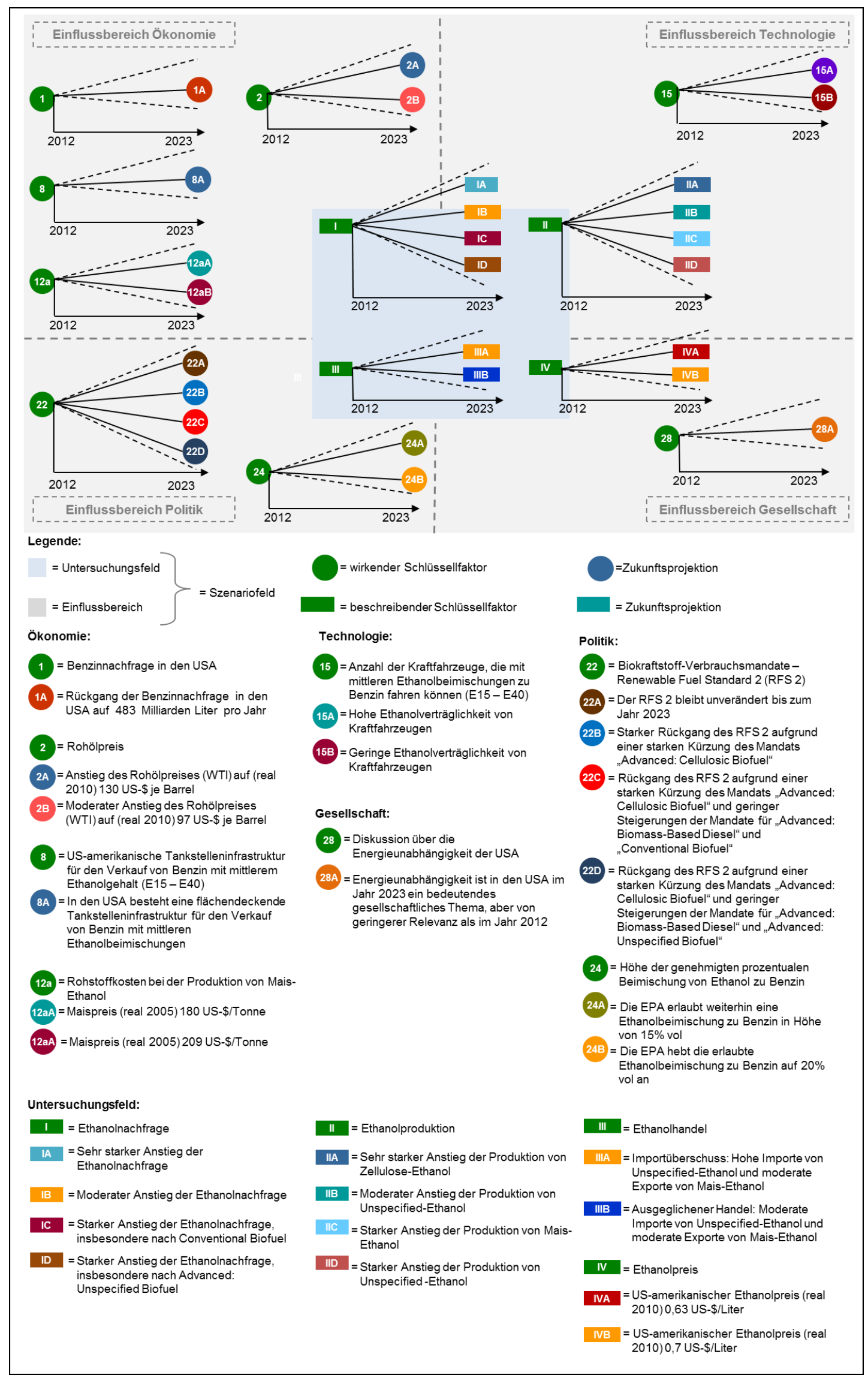

Abbildung 105: Zusammenfassung der Zukunftsprojektionen der wirkenden und beschreibenden Schlüsselfaktoren des Ethanolmarktes der USA

Quelle: EIGENE DARSTELLUNG 


\subsection{USA Szenario-Bildung und -Konsistenzprüfung}

Ziel des dritten Kernschritts ist es, aus den einzelnen wirkenden und beschreibenden Zukunftsprojektionen konsistente Szenarien zu erstellen, die den USamerikanischen Ethanolmarkt im Jahr 2023 beschreiben. Die Bildung der Szenarien erfolgt mittels des morphologischen Kastens in Kapitel 6.3.1. Daran anschließend werden die Szenarien anhand einer Expertenbefragung auf ihre Konsistenz hin überprüft. In Kapitel 6.3.2 ist die Durchführung und in Kapitel 6.3.3 die Auswertung der Konsistenzprüfung erklärt.

\subsubsection{Szenario-Bildung}

Die Bildung der unterschiedlichen Szenarien erfolgt durch den morphologischen Kasten in Abbildung 106. Die unterschiedlichen Zukunftsprojektionen sind im morphologischen Kasten entsprechend ihrer jeweiligen Zugehörigkeit zu einem Einflussbereich/Untersuchungsfeld angeordnet. Die Reihenfolge der Anordnung der Einflussbereiche entspricht der Stärke ihres Einflusses auf die zukünftige Entwicklung des US-amerikanischen Ethanolmarktes (Kapitel 6.1.2.2). Folglich befinden sich im oberen Abschnitt des Kastens die politischen und die ökonomischen Zukunftsprojektionen, gefolgt von jeweils einer technischen und einer gesellschaftlichen Zukunftsprojektion. Die Projektionen des Untersuchungsfelds befinden sich erst am Ende des morphologischen Kastens ${ }^{366}$, da deren Bestimmung von der Auswahl der wirkenden Zukunftsprojektionen abhängig ist. Jede Zukunftsprojektion ist durch ihre Kennzeichnung bestehend aus einer Nummer und einem Buchstaben eindeutig zu identifizieren. Die Verknüpfung der Projektionen zu den Szenarien erfolgt durch den Verfasser dieser Studie ${ }^{367}$. Im Folgenden sind die vier Szenarien „Unchanged RFS 2 - Strong Growth of Cellulosic Ethanol“, „Significant Reduction of the Consumption Mandate for ,Advanced: Cellulosic Biofuel““, „Corn Ethanol replaces Cellulosic Ethanol” und „Unspecified Ethanol replaces Cellulosic Ethanol” einzeln beschrieben ${ }^{368}$.

\footnotetext{
${ }^{366}$ In dem morphologischen Kasten (Abbildung 106) befinden sich, um die Übersichtlichkeit der Darstellung zu verbessern, nicht die Beschreibungen der Zukunftsprojektionen der Schlüsselfaktoren (I, II, III und IV). Die Beschreibungen dieser Zukunftsprojektionen sind am jeweiligen Ende der Kapitel 6.2.8 bis 6.2.11 ausformuliert.

${ }^{367}$ Die Szenarien wurden intensiv mit Dr. Christoph Berg - F.O. Licht, Martin Mitchell - Clariant, Dr. Albrecht Schaper - Nordzucker AG, Prof. Dr.-Ing. Achim Loewen - Hochschule für angewandte Wissenschaft und Kunst in Göttingen, Dr. Hinrich Harling und Dr. Beate Rother - KWS SAAT AG diskutiert.

${ }^{368}$ Damit die Experten, die zumeist aus den USA stammen, die Konsistenz der Szenarien problemlos beurteilen können, sind diese in englischer Sprache verfasst und die Daten in US-amerikanischen Einheiten angegeben.
} 


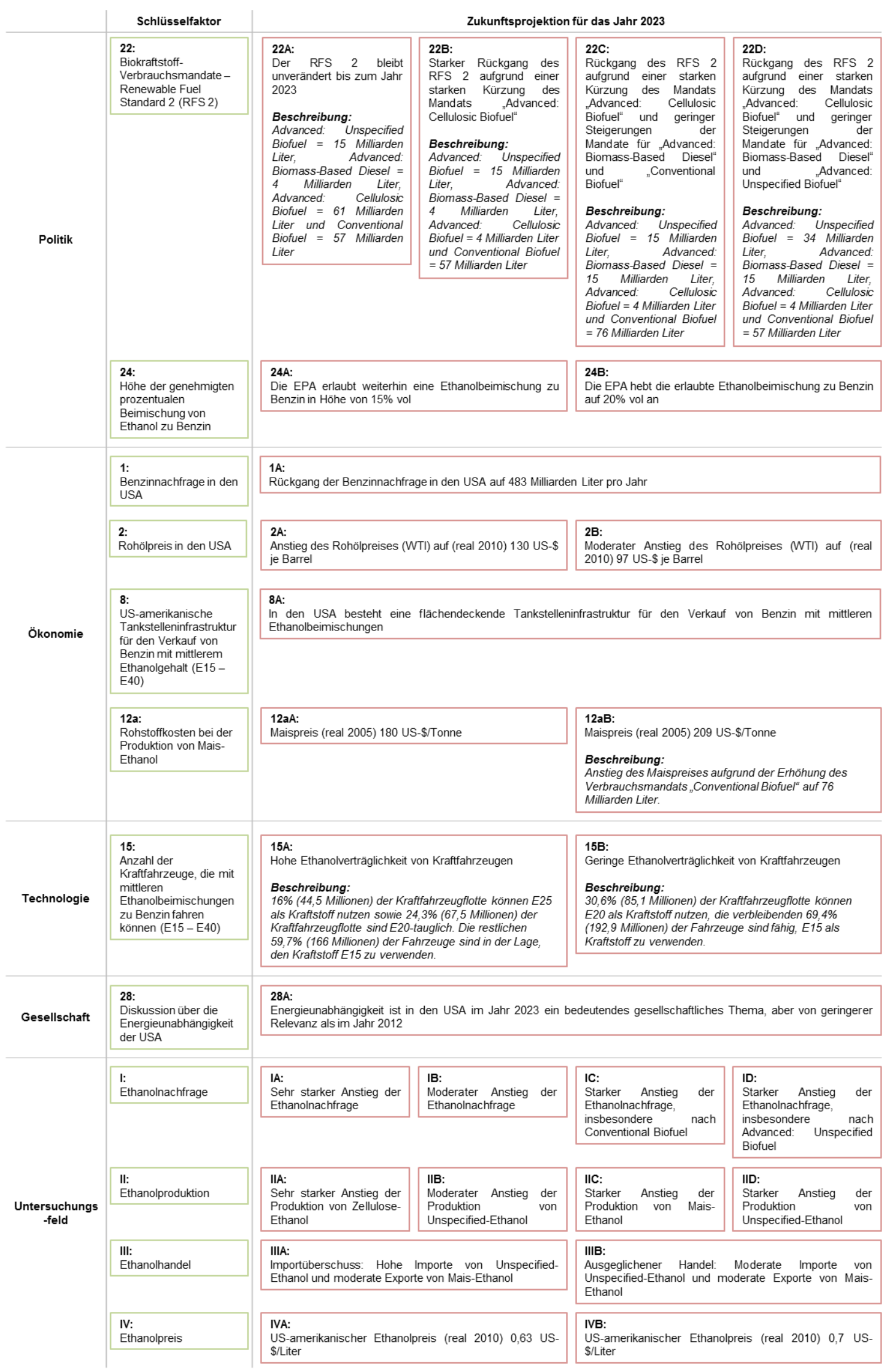

Abbildung 106: Morphologischer Kasten zur Bildung konsistenter Szenarien des USamerikanischen Ethanolmarktes im Jahr 2023

Quelle: EIGENE DARSTELLUNG 
Scenario 1: Unchanged RFS 2 - Strong Growth of Cellulosic Ethanol

Zukunftsprojektionen: 22A, 24B, 1A, 2A, 8A, 12aA, 15A, 28A, IA, IIA, IIIA, IVA

In the year 2023, after years with high levels of growth rates, the demand for fuel ethanol is around 35 billion gallons. The key driver for the expanding market is the unchanged consumption mandate for "Advanced: Cellulosic Biofuel" defined in the RFS 2.

The EPA released the following ethanol consumption mandates for the year 2023:

\begin{tabular}{l|c} 
Unit: billion gallons & \\
\hline Conventional Biofuel & 15 \\
\hline Advanced: Cellulosic Biofuel & 16 \\
\hline Advanced: Unspecified Biofuel & 4
\end{tabular}

The consumption mandate for "Conventional Biofuel" is fulfilled by the US-American production of about 16 billion gallons corn ethanol - of which around 1 billion gallons are exported to the world market. The industry is able to profitably produce corn ethanol for an ethanol price of circa (real 2010) US-\$2,4/gallon and for costs of corn of approximately (real 2005) US-\$4,6/bushel.

As a result of the US-American industry's ability to profitably produce cellulosic ethanol and the massive expansion of the production capacity, the USA satisfies the consumption mandate for "Advanced: Cellulosic Biofuel" by producing 16 billion galIons cellulosic ethanol.

In contrast to that, the mandate for "Advanced: Unspecified Biofuel" is mainly met by the import of 3 billion gallons sugar cane ethanol from Brazil. In the USA, only around 1 billion gallons are produced out of sugar beets, sorghum and wheat.

The USA is a net importer of 2 billion gallons ethanol, because of a total consumption of 35 billion gallons and a production of just 33 billion gallons.

Despite the declining US-American gasoline demand, the market is able to consume 35 billion gallons of ethanol, which can be explained by the following factors:

- The EPA increased the permitted maximum ethanol blends to gasoline to $20 \mathrm{vol} \%$

- All conventional cars in the USA can run on E15 and $40 \%$ of them are able to use ethanol blends of $20 \mathrm{vol} \%$ or even higher blends

- Strong increase of the US-American Flex Fuel Vehicle fleet

- Gasoline stations are able to sell intermediate and high ethanol blends nationwide

The WTI crude oil price has constantly risen to (real 2010) US-\$130/barrel until the year 2023. Although the topic "Energy Independence" is still vital in the year 2023, it is of less importance than in the year 2012. 
Scenario 2: Significant Reduction of the Consumption Mandate for "Advanced: CelIulosic Biofuel"

Zukunftsprojektionen: 22B, 24A, 1A, 2B, 8A, 12aA, 15B, 28A, IB, IIB, IIIB, IVA

In the year 2023, after years of moderate growth, the demand for fuel ethanol is around 20 billion gallons. However, the market has not grown stronger, because of the lacking production of cellulosic ethanol. Consequently, the EPA decided to significantly reduce the consumption mandate for "Advanced: Cellulosic Biofuel" from 16 billion gallons down to 1 billion gallons.

The EPA released the following consumption mandates for the year 2023:

\begin{tabular}{l|c} 
Unit: billion gallons & \\
\hline Conventional Biofuel & 15 \\
\hline Advanced: Cellulosic Biofuel & 1 \\
\hline Advanced: Unspecified Biofuel & 4
\end{tabular}

The consumption mandate for "Conventional Biofuel" is fulfilled by the US-American production of about 16 billion gallons corn ethanol - of which circa 1 billion gallons are exported to the world market. The industry is able to profitably produce corn ethanol for an ethanol price of around (real 2010) US-\$2,4/gallon and costs for corn of approximately (real 2005) US-\$4,6/bushel.

The USA meets the strongly reduced consumption mandate for "Advanced: Cellulosic Biofuel" by the production of 1 billion gallons cellulosic ethanol.

In order to fulfill the consumption mandate for "Advanced: Unspecified Biofuel", the USA imports only 1 billion gallons sugar cane ethanol from Brazil. The main part circa 3 billion gallons ethanol - is produced in the USA out of sugar beets, sorghum and wheat.

The USA has got an even balance of trade, because of the total consumption and production of 20 billion gallons ethanol.

Despite the decline in the US-American gasoline demand and the EPA's decision to keep the permitted maximum ethanol blends to gasoline at $15 \mathrm{vol} \%$, the USAmerican market is able to consume 20 billion gallons of ethanol. This can be explained by the fact that all cars are able to run on E15 and even $30 \%$ of these can use E20. In addition to that, all gasoline stations are capable of selling intermediate ethanol blends nationwide in the USA.

The WTI crude oil price has moderately risen up to (real 2010) US-\$97/barrel until the year 2023. The topic "Energy Independence" is still vital in 2023, but of less importance than in the year 2012. 


\section{Scenario 3: Corn Ethanol replaces Cellulosic Ethanol}

Zukunftsprojektionen: 22C, 24B, 1A, 2A, 8A, 12aB, 15A, 28A, IC, IIC, IIIA, IVB

After years of constant growth, there is a demand for fuel ethanol of around 25 billion gallons in the year 2023. The consumption mandate for "Conventional Biofuel" defined in the RFS 2 can be regarded as the key driver for the growing ethanol market.

The EPA raised the ethanol consumption mandate for "Conventional Biofuel" from initially 15 billion gallons up to 20 billion gallons in order to partially replace the lacking production of "Advanced: Cellulosic Biofuel".

The EPA released the following consumption mandates for the year 2023:

\begin{tabular}{l|c} 
Unit: billion gallons & \\
\hline Conventional Biofuel & 20 \\
\hline Advanced: Cellulosic Biofuel & 1 \\
\hline Advanced: Unspecified Biofuel & 4
\end{tabular}

The consumption mandate for "Conventional Biofuel" is met by the enormous USAmerican production of about 21 billion gallons corn ethanol - of which around 1 billion gallons are exported to the world market. The industry is able to profitably produce corn ethanol for an ethanol price of around (real 2010) US-\$2,65/gallon and of costs of corn of approximately (real 2005) US-\$5,3/bushel.

The USA fulfills the consumption mandate for "Advanced: Cellulosic Biofuel" by the production of 1 billion gallons cellulosic ethanol.

On the contrary, the mandate for "Advanced: Unspecified Biofuel" is mostly met by the import of around 3 billion gallons sugar cane ethanol from Brazil, while only about 1 billion gallons are produced in the USA out of sugar beets, sorghum and wheat.

As a result of the total ethanol consumption of 25 billion gallons and a production of just 23 billion gallons, the USA has a negative trade balance of 2 billion gallons.

Despite the decrease of the US-American gasoline demand, the market is nevertheless able to consume 25 billion gallons of ethanol. For the reason that the EPA permitted maximum ethanol blends to gasoline of $20 \mathrm{vol} \%$, all conventional cars on US roads are able to run on E15 and 40\% of the cars can use E20 or even higher ethanol blends. Moreover, gasoline stations are able to sell intermediate ethanol blends nationwide.

The WTI crude oil price has constantly risen to (real 2010) US-\$130/barrel in the year 2023. While there is still a vital debate about the issue of "Energy Independence" in 2023, it is of less importance than in the year 2012. 
Scenario 4: Unspecified Ethanol replaces Cellulosic Ethanol

Zukunftsprojektionen: 22D, 24B, 1A, 2A, 8A, 12aA, 15A, 28A, ID, IID, IIIA, IVA

After years of constant growth, the demand for fuel ethanol is around 25 billion gallons in the year 2023. The key driver for the growing market is the consumption mandate for "Advanced: Unspecified Biofuel" defined in the RFS 2.

The EPA raised the mandate for "Advanced: Unspecified Biofuel" from initially 4 billion gallons up to 9 billion gallons to partially replace the missing production of "Advanced: Cellulosic Biofuel".

The EPA released the following consumption mandates for the year 2023:

\begin{tabular}{l|c} 
Unit: billion gallons & \\
\hline Conventional Biofuel & 15 \\
\hline Advanced: Cellulosic Biofuel & 1 \\
\hline Advanced: Unspecified Biofuel & 9
\end{tabular}

In order to be able to meet the "Conventional Biofuel" consumption mandate, the USA produces a total of 16 billion gallons of corn ethanol - of which 1 billion gallons are exported to the world market. The ethanol industry is able to profitably produce corn ethanol for a price of around (real 2010) US-\$2,4/gallon and for costs of corn of circa (real 2005) US-\$4,6/bushel.

In order to fulfill the consumption mandate for "Advanced: Cellulosic Biofuel", the USA produces 1 billion gallons cellulosic ethanol.

The consumption mandate for "Advanced: Unspecified Biofuel" is fulfilled by the USAmerican production of 6 billion gallons ethanol out of sugar beets, sorghum and wheat and by imports of about 3 billion gallons sugar cane ethanol from Brazil.

As a consequence, the USA has got a negative trade balance of 2 billion gallons. This is due to a total ethanol consumption of 25 billion gallons and a production of only 23 billion gallons.

Despite the decreasing US-American gasoline demand, the market is able to consume 25 billion gallons of ethanol. For the reason that the EPA permitted maximum ethanol blends to gasoline of $20 \mathrm{vol} \%$, all conventional cars on US roads are able to run on E15 and $40 \%$ of the cars can use E20 or even higher ethanol blends. Moreover, gasoline stations are able to sell intermediate ethanol blends nationwide.

The WTI crude oil price has constantly risen to (real 2010) US-\$130/barrel in the year 2023. This issue "Energy Independence" is vital, but of less importance than in the year 2012. 


\subsubsection{Durchführung der Konsistenzprüfung}

Zur Überprüfung der Widerspruchsfreiheit der vier Szenarien erfolgte eine Konsistenzprüfung mittels eines standardisierten Onlinefragebogens. Da sich der Fragebogen zur Konsistenzprüfung der Szenarien für den Ethanolmarkt der EU27 bewährt hat, besitzt der Fragebogen für den Ethanolmarkt der USA (Anhang 4) die identische Struktur und gliedert sich dementsprechend in die folgenden drei Abschnitte:

1. Einführung in die Thematik

2. Konsistenzbeurteilung der Szenarien

3. Erhebung der persönlichen Daten der Probanden

Im ersten Abschnitt wurde den Probanden das Ziel der Dissertation sowie des Fragebogens beschrieben. Daran anschließend wurde erklärt, wie der Fragebogen zu beantworten ist, und explizit darauf hingewiesen, dass ausschließlich die Konsistenz der Szenarien bewertet werden soll.

Der wichtigste Bestandteil des Fragebogens befand sich im zweiten Abschnitt, in dem die Szenarien einzeln dargestellt wurden: (1) durch eine Zusammenfassung des Szenarios, (2) durch einen Link, um zu dem vollständig ausformulierten Szenario zu gelangen, und (3) durch eine Tabelle, in der die wichtigsten Informationen des Szenarios genannt wurden. Die Bewertung jedes Szenarios erfolgte anhand einer fünfstufigen Likert-Skala ${ }^{369}$ und eines Kommentarfeldes, in dem die Experten mögliche Inkonsistenzen im Szenario nennen und beschreiben konnten.

Zum Abschluss der Befragung wurden im dritten Abschnitt die Probanden nach dem Herkunftsland und der Branche des Unternehmens/der Organisation gefragt, für welche/s sie arbeiteten, sowie gebeten, ihre Kontaktdaten anzugeben, um ihnen die Ergebnisse der Erhebung zuschicken zu können.

Vor dem Beginn der Befragung wurde der Fragebogen durch Pretests ${ }^{370}$ überprüft. Da sich die Struktur des Fragebogens bereits als geeignet erwiesen hatte, lag der Schwerpunkt auf der Optimierung der Beschreibung der Szenarien und insbesondere auf den Tabellen, in denen die Kernpunkte der Szenarien genannt wurden.

${ }^{369}$ Die fünfstufige Likert-Skala besitzt folgende Abstufungen: „sehr gute Konsistenz“, „gute Konsistenz", „moderate Konsistenz", „schlechte Konsistenz" und "sehr schlechte Konsistenz". ${ }^{370} \mathrm{Im}$ Rahmen des Pretests wurde der Fragebogen beantwortet von und anschließend besprochen mit Dr. Christoph Berg - F.O. Licht, Martin Mitchell - Clariant, Dr. Albrecht Schaper - Nordzucker AG, Prof. Dr.-Ing. Achim Loewen - Hochschule für angewandte Wissenschaft und Kunst in Göttingen, Dr. Hinrich Harling und Dr. Beate Rother - KWS SAAT AG. 
An der Befragung haben nur Experten des US-amerikanischen Ethanolmarktes teilgenommen, da lediglich diese das Wissen besaßen, um die Konsistenz der Szenarien zu beurteilen. Sie beschäftigten sich hauptberuflich mit dem US-amerikanischen Ethanolmarkt und besetzten zum Teil Positionen in der Politik und in der Wirtschaft, in denen sie die Entwicklung des US-amerikanischen Ethanolmarktes bis zum Jahr 2023 beeinflussen konnten. Der Großteil der Probanden wurde bereits bei der Datenerhebung für die Wirkungsanalyse in Minneapolis (Minnesota) persönlich befragt und erklärte sich während der „F.O. Licht World Ethanol \& Biofuels“-Konferenz $2012^{371}$ bereit, an der Befragung teilzunehmen, oder wurde durch ein persönliches Anschreiben um die Teilnahme an der Befragung gebeten. Die Erhebung der Daten erfolgte von Mitte März bis Mitte April 2013 mithilfe der Software des Anbieters Onlineumfragen.com.

\subsubsection{Auswertung der Konsistenzprüfung}

Anhand der Auswertung der Konsistenzprüfung ist zu erkennen, ob die vier Szenarien (Kapitel 6.3.1) in sich konsistent sind oder auf Grundlage der Kommentare der befragten Experten überarbeitet werden müssen. Die Ergebnisse der Befragung sind valide, da jedes Szenario von mindestens 41 Experten des US-amerikanischen Ethanolmarktes beurteilt wurde. Die Experten sind für Unternehmen und Organisationen aus allen wichtigen Bereichen des US-amerikanischen Ethanolmarktes tätig (Abbildung 107) und stammen zum Großteil aus den USA (Abbildung 108).

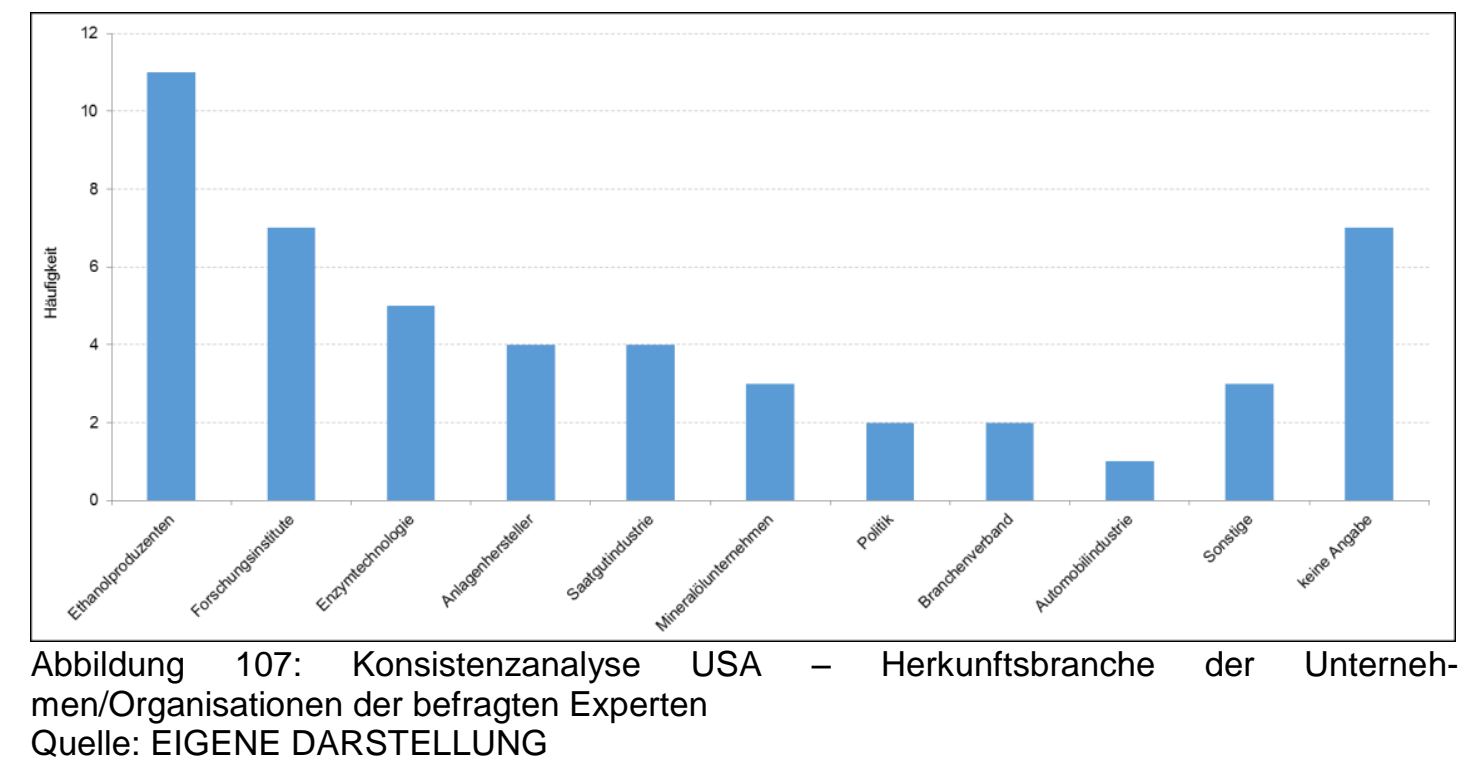

${ }^{371}$ Die Konferenz fand vom 5. bis zum 8. November 2012 in München statt. 


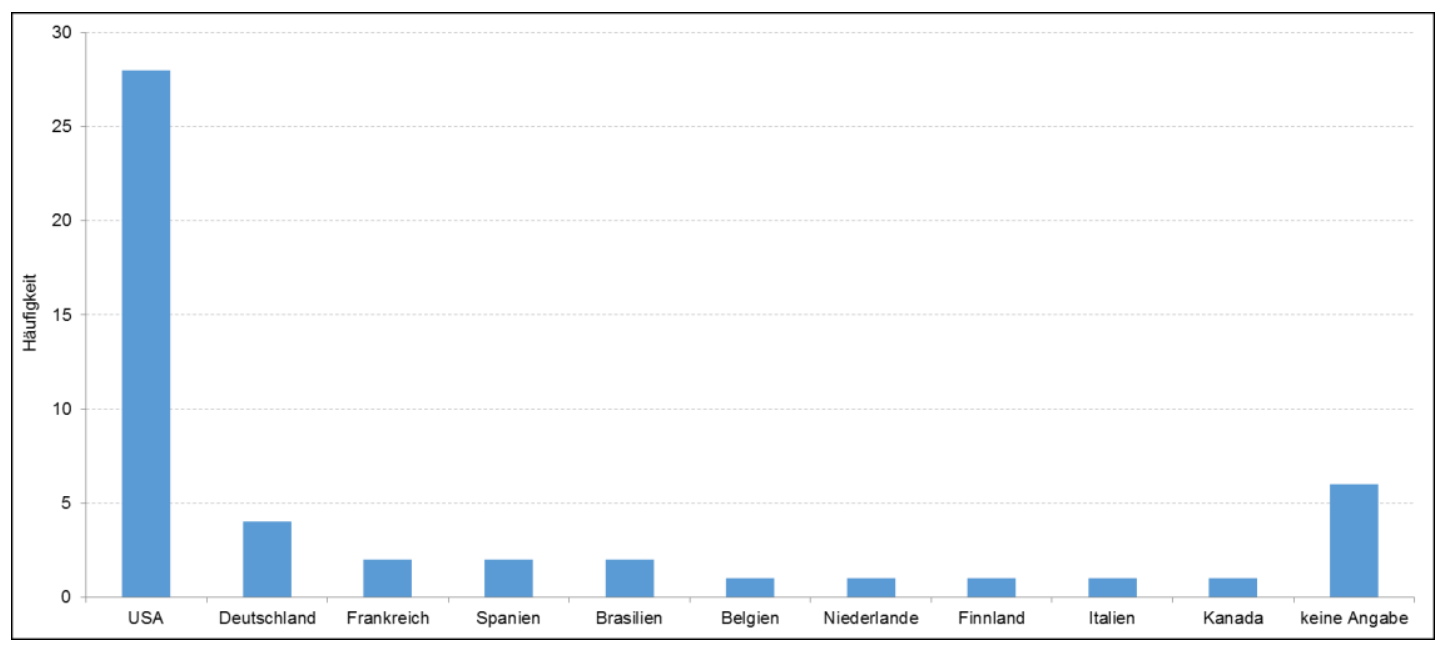

Abbildung 108: Konsistenzanalyse USA - Herkunftsland der Unternehmen/Organisationen der befragten Experten

Quelle: EIGENE DARSTELLUNG

Mithilfe der Beantwortung der folgenden zwei Fragen wird jedes Szenario einzeln analysiert:

1. Wie schätzen die Experten die Konsistenz des Szenarios ein?

2. Befindet sich im Szenario ein mögliches Konsistenzproblem, das von mehr als zwei Probanden genannt wurde ${ }^{372}$ ?

Ein Szenario bleibt unverändert, wenn dieses nach Auffassung der Experten eine „sehr gute Konsistenz“ oder eine „gute Konsistenz“ besitzt und nicht mehr als zwei Probanden auf dasselbe Konsistenzproblem im Szenario hingewiesen haben. Wird in der zweiten Frage trotz einer sehr guten oder guten Konsistenz von mindestens drei Experten das gleiche Konsistenzproblem beschrieben, so wird die mögliche Inkonsistenz in der Arbeit diskutiert, um daraufhin zu entscheiden, ob eine Veränderung des Szenarios notwendig ist. Besitzt ein Szenario in der ersten Frage nach Auffassung der Experten allerdings lediglich eine moderate, schlechte oder gar eine sehr schlechte Konsistenz, so wird das Szenario in der Studie nicht weiter betrachtet oder basierend auf den Anmerkungen der Probanden grundlegend überarbeitet.

\section{Szenario 1: Unchanged RFS 2 - Strong Growth of Cellulosic Ethanol}

Das Szenario besitzt nach Einschätzung von 49 Experten eine gute Konsistenz (Mittelwert: 1,86; Standardabweichung: 0,612). Da auch keine identischen Inkonsistenzen von mehr als zwei Probanden erkannt wurden, bleibt das Szenario unverändert.

${ }^{372}$ Bemerkungen zur Konsistenz, die nur ein- oder zweimal genannt wurden, werden aufgrund ihrer geringen Bedeutung bei mindestens 41 befragten Experten je Szenario nicht weiter betrachtet. 
Szenario 2: Significant Reduction of the Consumption Mandate for „Advanced: CelIulosic Biofuel“"

Das zweite Szenario besitzt mit einem Mittelwert von 1,76 (n: 45; Standardabweichung: 0,802) die beste Konsistenz der vier Szenarien. Trotzdem wird im Folgenden der Kernpunkt [2.1] „Moderate increase of the WTI crude oil price up to around (real 2010) US-\$ 97/barrel“ aufgrund der folgenden Kommentare von drei Experten diskutiert $^{373}$ :

- "Not sure I agree with your logic of $\$ 97$ WTI oil, when in the first scenario you had it at $\$ 130$, but less gasoline would be consumed because more ethanol would be produced (cellulosic). In scenario two you will be consuming more gasoline (assuming gasoline demand remains constant in question one and two), therefore WTI has to be higher in price."

- "To me it seems odd that the WTI price is lower in a scenario with less biofuels. I think it should be at least the same as in the previous scenario."

- The price for WTI is lower in scenario two (\$97) than in scenario one $(\$ 130)$. Better would be the same WTI price, since you have more ethanol being used in scenario one than in scenario two.

Die Probanden beschreiben in den Kommentaren keine Inkonsistenz innerhalb des Szenarios, sondern eine Unstimmigkeit zwischen den ersten beiden Szenarien. In Szenario 2 liegt der Preis für ein Barrel West Texas Intermediate (WTI) bei nur (real 2010) 97 US-\$, wohingegen der Rohölpreis im ersten Szenario (real 2010) 130 US\$/Barrel beträgt. Nach Auffassung der drei Experten sollte der Preis für ein Barrel WTI auch im zweiten Szenario mindestens (real 2010) 130 US-\$/Barrel betragen. Die Experten begründen dies wie folgt: In beiden Szenarien ist der Benzinverbrauch identisch, jedoch besteht im zweiten Szenario infolge der geringeren Ethanolbeimischung zu Benzin ein höherer Benzinbedarf, um die Nachfrage nach Kraftstoff decken zu können. Folglich müssen die US-amerikanischen Raffinerien im zweiten Szenario mehr Rohöl zur Benzinherstellung nachfragen als im ersten Szenario. Basierend auf der Annahme, dass das Angebot an Rohöl in beiden Szenarien identisch ist, widerspricht die höhere Rohölnachfrage im zweiten Szenario dem geringeren Rohölpreis von nur 97 US-\$/Barrel.

Die Experten haben in ihrer Argumentation Recht und auch eine Änderung des Szenarios wäre nicht falsch. Trotzdem bleibt das Szenario unverändert, denn der Rohölpreis (WTI) wird darüber hinaus stark von globalen Faktoren beeinflusst,

${ }^{373}$ Die Kommentare der Experten wurden zur besseren Lesbarkeit geringfügig sprachlich korrigiert, inhaltlich bleiben sie unverändert. 
wodurch die Wirkung der Substitution von Benzin durch Ethanol auf den Rohölpreis begrenzt ist. Dies ist insbesondere deshalb der Fall, da der Unterschied der Ethanolbeimischung zwischen den beiden Szenarien nur 57 Milliarden Liter beträgt und somit im Vergleich zur prognostizierten Rohölnachfrage in den USA im Jahr 2023 von 1147 Milliarden Litern von untergeordneter Bedeutung ist (U.S. ENERGY INFORMATION ADMINISTRATION 2013h). Die U.S. ENERGY INFORMATION ADMINISTRATION (2012b: 23) nennt als wichtigste Einflussgrößen in Bezug auf den Rohölpreis die folgenden globalen Faktoren: (1) das Angebot an Rohöl und anderen Treibstoffen ${ }^{374}$ von Nicht-OPEC-Staaten, (2) Investionen der OPEC in die zukünftige Rohölförderung und Entscheidungen der OPEC über Rohölfördermengen, (3) die wirtschaftliche Konkurrenzfähigkeit anderer Treibstoffe im Vergleich zu Rohöl und (4) die weltweite Nachfrage nach Rohöl und anderen Treibstoffen. Ethanol-Kraftstoff in den USA ist zwar ein Bestandteil der „anderen Treibstoffe“, aber eben nicht von globaler Bedeutung, sodass trotz der geringeren Substitution von Benzin durch Ethanol im zweiten Szenario ein Rohölpreis von (real 2010) 97 US-\$/Barrel, wie von der INTERNATIONAL ENERGY AGENCY (2011a: 61f.) prognostiziert, realistisch ist.

\section{Szenario 3: Corn Ethanol replaces Cellulosic Ethanol}

Das dritte Szenario besitzt mit einem Mittelwert von 2,00 eine gute Konsistenz ( $n: 43$; Standardabweichung: 0,873). Aus diesem Grunde und da keine Konsistenzprobleme innerhalb des Szenarios mehr als zwei Mal genannt wurden, bleibt das Szenario unverändert.

\section{Szenario 4: Unspecified Ethanol replaces Cellulosic Ethanol}

Es wird keine Veränderung am Szenario vorgenommen, da dieses nach Einschätzung von 41 Experten mit einem Mittelwert von 1,98 und einer Standardabweichung von 0,851 eine gute Konsistenz besitzt und keine Inkonsistenz mehr als zwei Mal genannt wurde.

\subsection{Ergebnisse der Betrachtung des Ethanolmarktes der USA}

Zu Beginn dieser Arbeit (Kapitel 1.1) wurden zwei Ziele definiert: Erstens sollten jene Faktoren identifiziert werden, die den Ethanolmarkt bis zum Jahr 2023 entscheidend beeinflussen werden, und zweitens galt es, unterschiedliche Szenarien

\footnotetext{
${ }^{374}$ Unter dem Begriff „andere Treibstoffe“ fasst die U.S. Energy Information Administration die folgenden Kraftstoffe zusammen: Biokraftstoffe, Bitumen (Ölsand), Coal-to-Liquid (CtL), Biomass-to-Liquid (BtL), Gas-to-Liquid (GtL), Extra-Schweröl und Ölschiefer.
} 
zu erstellen, die den US-amerikanischen Ethanolmarkt im Jahr 2023 beschreiben. Beide Ziele wurden erfüllt.

Wie bereits in der Vergangenheit wird der US-amerikanische Ethanolmarkt auch bis zum Jahr 2023 stark von politischen Faktoren bestimmt sein ${ }^{375}$. Inbesondere Entscheidungen hinsichtlich der Höhe der Verbrauchsmandate im RFS 2 (Einflussfaktor 22) und der von der EPA erlaubten prozentualen Ethanolbeimischung zu Benzin (Einflussfaktor 24) werden den Markt maßgeblich beeinflussen. Darüber hinaus wird die „Blend Wall“-Problematik entscheidend sein, denn alle vier Faktoren ${ }^{376}$, die im Zusammenhang mit der Problematik stehen, befinden sich unter den ersten sieben Faktoren mit dem stärksten Einfluss auf den US-amerikanischen Ethanolmarkt. Von vergleichbarer Bedeutung für die Zukunft des Marktes sind außerdem noch die Entwicklung des Maispreises (Einflussfaktor 12a) und die des Rohölpreises (Einflussfaktor 2). Des Weiteren ist die gesellschaftliche Bedeutung des Themas der Energieunabhängigkeit der USA (Einflussfaktor 28) zu betrachten, um frühzeitig zu erkennen, zu welchem der folgenden vier Szenarien sich der Ethanolmarkt der USA hin entwickeln wird.

Jedes Szenario wird im Folgenden mittels seiner wichtigsten Kernpunkte beschrieben. Im Anschluss wird das Spezifische der einzelnen Szenarien herausgestellt, um sie eindeutig voneinander abzugrenzen ${ }^{377}$.

\section{Szenario 1: Unchanged RFS 2 - Strong Growth of Cellulosic Ethanol Beschreibung:}

Nach Jahren des starken Wachstums beträgt die US-amerikanische Ethanolnachfrage 133 Milliarden Liter. Entscheidender Treiber des starken Wachstums sind die Verbrauchsmandate des RFS 2, die unverändert bleiben. Die EPA fordert die Erfüllung der folgenden Mandate im Jahr 2023:

\begin{tabular}{l|c} 
Einheit: Milliarden Liter & \\
\hdashline Conventional Biofuel & 57 \\
\hline Advanced: Cellulosic Biofuel & 61 \\
\hline Advanced: Unspecified Biofuel & 15
\end{tabular}

${ }^{375}$ Eine genaue Beschreibung der Wirkungsstärke der einzelnen Einflussfaktoren befindet sich in Kapitel 6.1.2.2.

${ }^{376}$ Die folgenden vier Einflussfaktoren stehen in direkter Verbindung zur "Blend Wall"Problematik: 24: „Höhe der genehmigten prozentualen Beimischung von Ethanol zu Benzin“, 1: „Benzinnachfrage in den USA“, 8: „US-amerikanische Tankstelleninfrastruktur für den Verkauf von Benzin mit mittlerem Ethanolgehalt (E15 - E40)“ und 15: „Anzahl der Kraftfahrzeuge, die mit mittleren Ethanolbeimischungen zu Benzin (E15 - E40) fahren können“.

${ }^{377}$ In Kapitel 6.3.1 sind die einzelnen Szenarien ausführlich beschrieben. 
Die Nachfrage nach 57 Milliarden Litern „Conventional Biofuel“ wird durch die USamerikanische Produktion von 61 Milliarden Litern Mais-Ethanol erfüllt - 4 Milliarden Liter werden exportiert. Darüber hinaus sind die USA aufgrund eines massiven Ausbaus der Produktionskapazitäten zur Herstellung von Zellulose-Ethanol in der Lage, die Nachfrage nach 61 Milliarden Litern „Advanced: Cellulosic Biofuel“ vollständig zu befriedigen. Im Gegensatz dazu wird die Nachfrage nach 15 Milliarden Litern „Advanced: Unspecified Biofuel“ maßgeblich durch den Import von 11 Milliarden Litern Zuckerrohr-Ethanol aus Brasilien gedeckt. In den USA beträgt die Produktion von Unspecified-Ethanol aus Sorghum, Weizen und Zuckerrüben lediglich 4 Milliarden Liter.

Trotz des Rückgangs der US-amerikanischen Benzinnachfrage ist der Markt fähig, 133 Milliarden Liter Ethanol-Kraftstoff zu verbrauchen. Dies ist auf die folgenden Faktoren zurückzuführen: (1) Die EPA erlaubt eine Ethanolbeimischung von $20 \%$ vol zu Benzin, (2) alle Kraftfahrzeuge sind in der Lage, E15 als Kraftstoff zu nutzen, und sogar 40\% der Fahrzeugflotte können E20 als Kraftstoff verwenden, (3) es kommt zu einem starken Anstieg der FFV-Flotte und (4) die Tankstellen können mittlere und hohe Ethanolbeimischungen zu Benzin in den gesamten USA verkaufen.

\section{Abgrenzung:}

In diesem Szenario kommt es zu einem sehr starken Marktwachstum, wodurch die Jahre 2011 und 2012, in denen der Ethanolmarkt deutlich langsamer gewachsen ist, eine Ausnahme darstellen. Besonders stark ist das Wachstum im Bereich ZelluloseEthanol, da die Produktion von derzeit lediglich 0,027 Milliarden Litern (OECD/FAO 2012: 224) auf 61 Milliarden Liter ansteigt und damit größer sein wird als die momentane Mais-Ethanolproduktion in den USA. Um diese Entwicklung zu erreichen, muss es bis zum Jahr 2023 zu einem enormen Wachstum kommen, welches mit der Entwicklung des US-amerikanischen Mais-Ethanolmarktes in den Jahren 2008 bis 2010 vergleichbar ist. Voraussetzung der Entwicklung ist die starke Unterstützung der US-amerikanischen Politik, die trotz starker Kritik der Mineralöl- und der Nahrungsmittelindustrie den RFS 2 unverändert lässt. Darüber hinaus besteht keine „Blend Wall“-Problematik, sodass der Ethanolabsatz von 133 Milliarden Litern möglich ist. Das Besondere an diesem Szenario hinsichtlich der "Blend Wall“Problematik im Gegensatz zu den anderen drei Szenarien ist der starke Anstieg der FFV-Flotte in den USA, ohne den ein Absatz von 133 Milliarden Litern EthanolKraftstoff unmöglich wäre. 
Dieses Szenario beschreibt die optimale Entwicklung des US-amerikanischen Ethanolmarktes aus Sicht der Ethanolindustrie und insbesondere jener Unternehmen, die an der Entwicklung von Zellulose-Ethanol arbeiten.

Szenario 2: Significant Reduction of the Consumption Mandate for "Advanced: CelIulosic Biofuel“

\section{Beschreibung:}

Nach Jahren des moderaten Wachstums beträgt die Ethanolnachfrage im Jahr 2023 76 Milliarden Liter. Der Ethanolmarkt ist nicht stärker gewachsen, da die EPA das Mandat für „Advanced: Cellulosic Biofuel“ von 61 Milliarden Litern auf 4 Milliarden Liter gekürzt hat. Die EPA veröffentlicht die folgenden Verbrauchsmandate im Jahr 2023:

\begin{tabular}{l|c} 
Einheit: Milliarden Liter & \\
\hline Conventional Biofuel & 57 \\
\hline Advanced: Cellulosic Biofuel & 4 \\
\hline Advanced: Unspecified Biofuel & 15
\end{tabular}

Die Nachfrage nach „Conventional Biofuel“ (57 Miliarden Liter) und „Advanced: Cellulosic Biofuel“ (4 Milliarden Liter) wird durch die US-amerikanische Produktion von 61 Milliarden Litern Mais-Ethanol - 4 Milliarden Liter werden exportiert - sowie 4 Milliarden Litern Zellulose-Ethanol erfüllt. Durch die Produktion von 11 Milliarden Litern Unspecified-Ethanol aus Sorghum, Weizen und Zuckerrüben sowie den Import von 4 Milliarden Litern Zuckerrohr-Ethanol aus Brasilien wird die Nachfrage nach „Advanced: Unspecified Ethanol“ in Höhe von 15 Milliarden Litern befriedigt. Darüber hinaus ist trotz des Rückgangs der Benzinnachfrage der Absatz von 76 Milliarden Litern Ethanol-Kraftstoff möglich, da die Tankstellen fähig sind, mittlere Ethanolbeimischungen zu Benzin zu verkaufen.

\section{Abgrenzung:}

Im Vergleich zu den anderen Szenarien und dem Zeitraum von 2008 bis 2010 fällt das Wachstum des Ethanolmarktes in diesem Szenario deutlich geringer aus. Grund dafür ist die Kürzung des Mandats „Advanced: Cellulosic Biofuel“ um 57 Milliarden Liter auf lediglich 4 Milliarden Liter. Dies zeigt, dass die Ethanolindustrie nur noch eine geringe politische Unterstützung besitzt und die Mineralölindustrie sich mit ihrem Ziel der Kürzung des RFS 2 durchgesetzt hat. Der Markt erreicht aber durch die Lösung der „Blend Wall“-Problematik dennoch nur ein geringes Marktwachstum. Aufgrund der geringeren Ethanolnachfrage im Vergleich zu den anderen 
drei Szenarien ist es ausreichend, dass die Tankstellen Benzin mit mittleren Ethanolbeimischungen verkaufen können, um die „Blend Wall“-Problematik zu verhindern.

Die USA besitzen des Weiteren in diesem Szenario einen ausgeglichenen Ethanolhandel. In den anderen drei Szenarien ergibt sich infolge von Importen in Höhe von 11 Milliarden Litern Zuckerrohr-Ethanol aus Brasilien und Exporten von nur 4 Milliarden Litern Mais-Ethanol ein Importüberschuss von 7 Milliarden Litern. Trotz des ausgeglichenen Ethanolhandels hat die US-amerikanische Ethanolindustrie in diesem Szenario nur verloren, da der RFS 2 um 57 Milliarden Liter „Advanced: Cellulosic Biofuel" gekürzt wurde. Besonders kritisch ist diese Entwicklung für Unternehmen, die über Jahre in die Entwicklung von Zellulose-Ethanol investiert haben.

\section{Szenario 3: Corn Ethanol replaces Cellulosic Ethanol}

Beschreibung:

Nach Jahren des konstanten Wachstums beträgt die Ethanolnachfrage im Jahr 202395 Milliarden Liter. Der wesentliche Wachstumstreiber war die Anhebung des Verbrauchsmandats „Conventional Biofuel“ von 57 Milliarden Litern auf 76 Milliarden Liter, während im Gegenzug das Mandat „Advanced: Cellulosic Biofuel“ stark gekürzt wurde. Die EPA fordert die Erfüllung der folgenden Verbrauchsmandate im Jahr 2023:

\begin{tabular}{l|c} 
Einheit: Milliarden Liter & \\
\hline Conventional Biofuel & 76 \\
\hline Advanced: Cellulosic Biofuel & 4 \\
\hline Advanced: Unspecified Biofuel & 15
\end{tabular}

Die hohe Nachfrage nach 76 Milliarden Litern „Conventional Biofuel“ wird durch die US-amerikanische Produktion von 80 Milliarden Litern Mais-Ethanol, von denen 4 Milliarden Liter exportiert werden, befriedigt. Mithilfe der Produktion von 4 Milliarden Litern Zellulose-Ethanol wird die Nachfrage nach „Advanced: Cellulosic Biofuel“ erfüllt. Im Gegensatz dazu wird die Nachfrage nach „Advanced: Unspecified Biofuel“ (15 Milliarden Liter) maßgeblich durch den Import von 11 Milliarden Litern Zuckerrohr-Ethanol aus Brasilien gedeckt. Die US-amerikanische Produktion von Unspecified-Ethanol aus Sorghum, Weizen und Zuckerrüben beträgt lediglich 4 Milliarden Liter.

Trotz des Rückgangs der US-amerikanischen Benzinnachfrage ist der Absatz von 95 Milliarden Litern Ethanol-Kraftstoff möglich, was auf drei Aspekte zurückzuführen ist: (1) auf die von der EPA erlaubten Ethanolbeimischung zu Benzin in Höhe von 20\% 
vol, (2) auf die Fähigkeit aller Kraftfahrzeuge, E15, und die Fähigkeit von 40\% der Fahrzeugflotte, auch E20 als Kraftstoff zu verwenden, und (3) darauf, dass die Tankstellen in den USA Benzin mit mittleren Ethanolgehalten verkaufen können.

\section{Abgrenzung:}

In dem betrachteten Szenario kommt es zu einem konstanten Wachstum des Ethanolmarktes. Doch die starke Entwicklung des Marktes wie im Zeitraum von 2008 bis 2010 wird nicht wieder erreicht. Das konstante Wachstum ist auf die Anhebung des Mandats „Conventional Biofuel“ um 19 Milliarden Liter auf 76 Milliarden Liter zurückzuführen. Allerdings erfolgt dies als Ausgleich zur starken Kürzung des Verbrauchsmandats „Advanced: Cellulosic Biofuel“ um 57 Milliarden Liter. Folglich gehen der US-amerikanischen Ethanolindustrie Mandate in Höhe von 38 Milliarden Litern verloren. Die Anhebung des Mandats für "Conventional Biofuels“ ist jedoch ein großer Erfolg für die Mais-Ethanolindustrie im Corn Belt der USA und ein klares Zeichen der politischen Unterstützung für Mais-Ethanol. Darüber hinaus ist die Kürzung der Mandate um 38 Milliarden Liter ein Erfolg für die Mineralölindustrie, die Steigerung der Mais-Ethanolproduktion allerdings eine deutliche Niederlage für die Nahrungsmittel- und Veredlungsindustrie, die eine Kürzung der Produktion von Mais-Ethanol zum Ziel hat.

Des Weiteren ist der Absatz von 95 Milliarden Litern möglich, da keine „Blend Wall“Problematik mehr besteht. Jedoch ist darauf hinzuweisen, dass durch die starke Kürzung des Mandats „Advanced: Cellulosic Biofuel“ ein Großteil des Marktes für die US-amerikanische Zellulose-Ethanolindustrie wegbricht.

\section{Szenario 4: Unspecified Ethanol replaces Cellulosic Ethanol Beschreibung:}

Im Jahr 2023 beträgt die Ethanolnachfrage nach Jahren des konstanten Wachstums 95 Milliarden Liter. Entscheidender Wachstumstreiber war die Anhebung des Verbrauchsmandats „Advanced: Unspecified Biofuel“ um 19 Milliarden Liter auf 34 Milliarden Liter, um die starke Reduzierung des Mandats „Advanced: Cellulosic Biofuel" teilweise auszugleichen. Die EPA veröffentlicht die folgenden Verbrauchsmandate im Jahr 2023:

\begin{tabular}{l|c} 
Einheit: Milliarden Liter & \\
\hline Conventional Biofuel & 57 \\
\hline Advanced: Cellulosic Biofuel & 4 \\
\hline Advanced: Unspecified Biofuel & 34
\end{tabular}


Die Nachfrage nach „Conventional Biofuel“ (57 Milliarden Liter) und „Advanced: Cellulosic Biofuel“ (4 Milliarden Liter) wird durch die US-amerikanische Produktion von 61 Milliarden Litern Mais-Ethanol - 4 Milliarden Liter werden exportiert - sowie 4 Milliarden Litern Zellulose-Ethanol gedeckt. Die Produktion von 23 Milliarden Litern Unspecified-Ethanol aus Sorghum, Weizen und Zuckerrüben dient, ebenso wie der Import von 11 Milliarden Litern Zuckerrohr-Ethanol aus Brasilien, der Erfüllung der Nachfrage nach 34 Milliarden Litern „Advanced: Unspecified Biofuel“.

Der Absatz von 95 Milliarden Litern Ethanol-Kraftstoff ist trotz der Reduktion der USamerikanischen Benzinnachfrage aufgrund der folgenden drei Faktoren möglich: (1) Aufgrund der Entscheidung der EPA, die erlaubte Ethanolbeimischung zu Benzin auf $20 \%$ vol anzuheben, (2) können $40 \%$ der Kraftfahrzeugflotte mindestens E20 und die restlichen Fahrzeuge E15 als Kraftstoff verwenden und (3) besitzen die Tankstellen in den USA die Fähigkeit, Benzin mit mittleren Ethanolbeimischungen zu verkaufen.

\section{Abgrenzung:}

Die Szenarien 3 und 4 sind sich zum Teil ähnlich: In beiden Szenarien kommt es zu einem konstanten Wachstum sowohl der Ethanolnachfrage (95 Milliarden Liter) als auch der Ethanolproduktion (88 Milliarden Liter). Darüber hinaus besteht aus den identischen Gründen trotz des Rückgangs der Benzinnachfrage keine „Blend Wall“Problematik und das Mandat „Advanced: Cellulosic Biofuel“ wird von ursprünglich 61 Milliarden Litern auf nur noch 4 Milliarden Liter reduziert.

Der entscheidende Unterschied zwischen den beiden Szenarien besteht darin, dass das Verbrauchsmandat „Advanced: Unspecified Biofuel“ im vierten Szenario um 19 Milliarden Liter auf 34 Milliarden Liter ansteigt und das Mandat „Conventional Biofuel“ unverändert bleibt. Infolgedessen kommt es zu einem starken Anstieg der Produktion von Unspecified-Ethanol aus Sorghum, Weizen und Zuckerrüben auf 23 Milliarden Liter. Von dieser Entwicklung werden insbesondere die ländlichen Gebiete außerhalb des Corn-Belts profitieren, in denen Sorghum, Weizen und Zuckerrüben angebaut werden, aber auch die gesamte Ethanolindustrie, da die Politiker dieser Regionen sich nun ebenfalls auf nationaler Ebene für die Interessen der Ethanol-Kraftstoffindustrie einsetzen werden.

Die vier Szenarien bilden einen breiten Zukunftsraum ab, in dem sowohl ein sehr starkes Wachstum („Unchanged RFS 2 - Strong Growth of Cellulosic Ethanol“) als auch ein nur moderates Wachstum („Significant Reduction of the Consumption Mandate for 'Advanced: Cellulosic Biofuel“") des US-amerikanischen Ethanolmark- 
tes prognostiziert werden. Darüber hinaus beschreiben die Szenarien 1, 3 und 4 die USA als Ethanolimportmarkt, wohingegen Szenario 2 einen ausgeglichenen Ethanolhandel erwartet (Abbildung 109). Die Szenarien stimmen darin überein, dass die bereits bestehende Mais-Ethanolproduktion erhalten bleibt und sogar leicht (Szenario 1, 2 und 4) bis stark (Szenario 3) ansteigt. Außerdem wird in allen Szenarien erwartet, dass die „Blend Wall“-Problematik behoben wird, um ein weiteres Wachstum des Marktes zu ermöglichen.

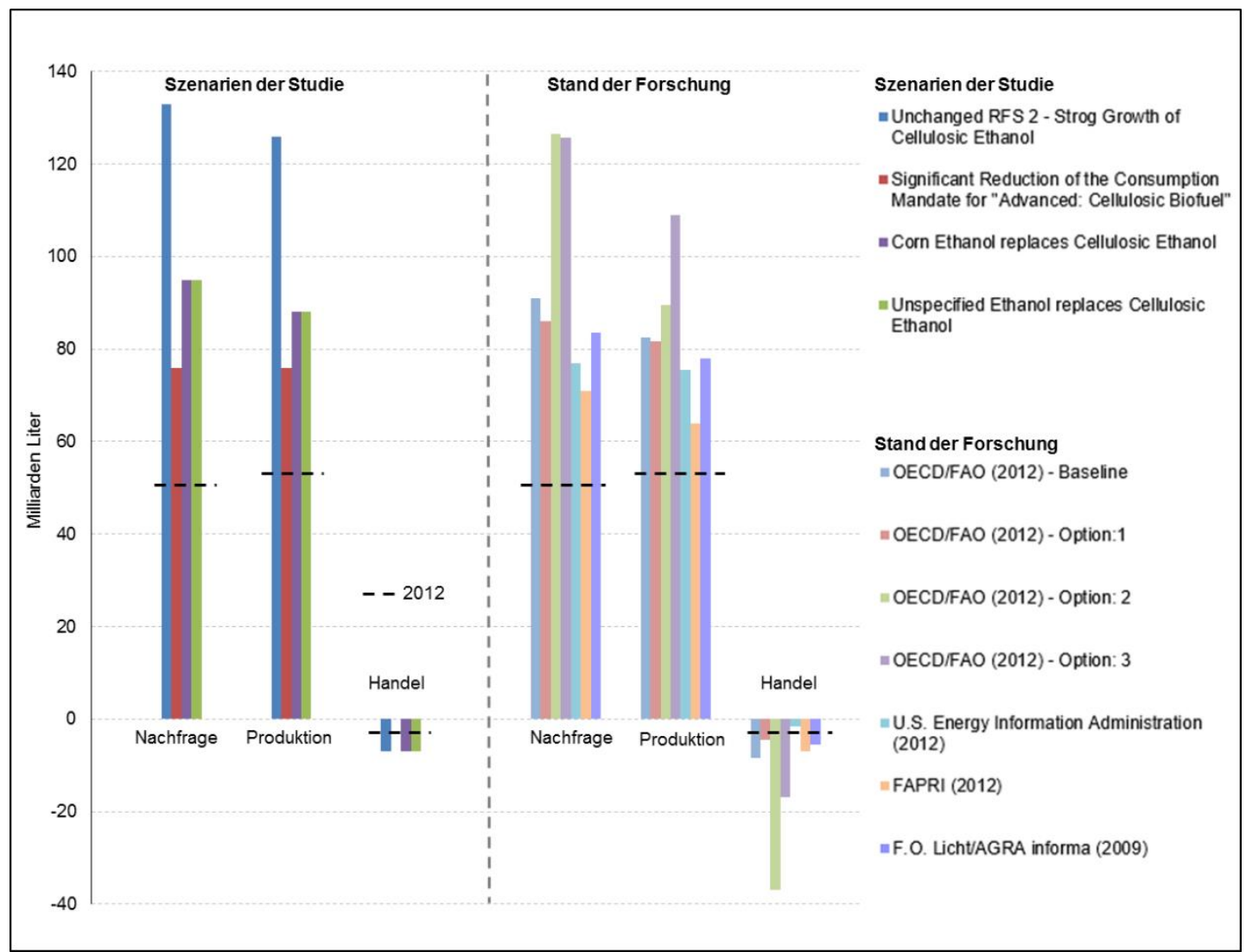

Abbildung 109: USA: Vergleich der Szenarien der Studie mit dem Stand der Forschung ${ }^{3 / 8}$ Quelle: In Anlehnung an OECD/FAO 2012: 87ff.; U.S. ENERGY INFORMATION ADMINISTRATION 2012b: 97ff.; FAPRI 2012: 1ff.; F.O. LICHT/AGRA INFORMA 2009: 133ff; EIGENE BERECHNUNG

Bei einem Vergleich der Szenarien mit dem Stand der Forschung ist zu erkennen, dass in Anbetracht des langfristigen Zeithorizonts sehr ähnliche Entwicklungen prognostiziert werden und die dargestellten Zukunftsräume somit übereinstimmen. Darüber hinaus wird im Vergleich zur Ist-Situation (Jahr 2012) übereinstimmend ein Anstieg sowohl der Ethanolnachfrage als auch der Ethanolproduktion erwartet. Der einzige deutliche Unterschied zwischen den Szenarien und dem Stand der Forschung betrifft den Ethanolhandel, bei dem die OECD/FAO (2012: 114) in Zukunftsprojektion „Option 2“ einen Importüberschuss von 37 Milliarden Litern prog-

${ }^{378}$ Die Prognosen im Stand der Forschung beschreiben den US-amerikanischen Ethanolmarkt in den Jahren 2020/2021. Einzige Ausnahme ist die Projektion der U.S. Energy Information Administration, die den Markt im Jahr 2025 darstellt. 
nostiziert, wohingegen in den Szenarien der Studie lediglich ein Importüberschuss von maximal 7 Milliarden Litern ${ }^{379}$ erwartet wird (Abbildung 109). In den Szenarien der vorliegenden Untersuchung wurde bewusst kein höherer Importüberschuss berücksichtigt. Außerdem wird dieser Bestandteil der Projektion der OECD/FAO (2012: 114) als nicht realistisch erachtet, da diese auf einem Ethanolimport von 51 Milliarden Litern Zuckerrohr-Ethanol aus Brasilien basiert. Brasilien wird in den nächsten zehn Jahren nicht 51 Milliarden Liter Ethanol in die USA exportieren können, da die brasilianische Ethanolproduktion im Jahr 2012 lediglich 20,5 Milliarden Liter betrug (F.O. LICHT 2012f: 74) und Brasilien bisher nie mehr als 5 Milliarden Liter Ethanol exportierte (F.O. LICHT 2012a: 304). Darüber hinaus wird Brasilien selbst einen hohen Ethanolbedarf haben, um die wachsende Flotte an FFV mit Kraftstoff zu versorgen (OECD/FAO 2012: 94f.). Des Weiteren ist zu bedenken, dass der Ausbau der Produktionskapazitäten zur Herstellung von Zuckerrohr-Ethanol in den Jahren 2008 bis 2012 deutlich langsamer als geplant erfolgte (F.O. LICHT 2013d: 294f).

${ }^{379}$ Der Importüberschuss von 7 Milliarden Litern ergibt sich aus einem Ethanolimport von 11 Milliarden Litern Zuckerrohr-Ethanol aus Brasilien und dem Export von 4 Milliarden Litern Mais-Ethanol. 
Aus den Ergebnissen der Untersuchung, die in Kapitel 7.1 zusammengefasst werden, leiten sich in Kapitel 7.2 Handlungsempfehlungen für die Ethanolindustrie ab. Zum Abschluss der Dissertation werden in Kapitel 7.3 Ansätze zur weiteren Forschung aufgezeigt.

\subsection{Zusammenfassung der Untersuchung}

Anlass der Untersuchung war die Erkenntnis, dass die etablierten Forschungsinstitute trotz der derzeitig starken Verunsicherung der Märkte und eines Rückgangs der Ethanolproduktion in der EU27 und den USA im Jahr 2012 ein weiteres Wachstum des Ethanolmarktes in beiden Regionen prognostizieren (F.O. LICHT 2012f: 74).

Die Verunsicherung des Ethanolmarktes der EU27 ist auf eine mögliche Änderung der politischen Förderung von Biokraftstoffen zurückzuführen. Durch politische Maßnahmen soll die momentan von der ersten Generation geprägte europäische Ethanolindustrie (EUROPEAN COMMISSION JOINT RESEARCH CENTRE, INSTITUT FOR ENERGY ET AL. 2010: 74) langfristig zu einem Markt, der von Biokraftstoffen der zweiten Generation dominiert wird, gewandelt werden.

Die momentane Verunsicherung des US-amerikanischen Marktes wird durch die „Blend Wall“-Problematik und durch eine mögliche Absenkung der Mandate des RFS 2 verursacht. Darüber hinaus ist ein merklich sinkender politischer Rückhalt für Biokraftstoffe zu erkennen (F.O. LICHT 2012c: 319). Dies verunsichert den Markt stark, da der RFS 2 der entscheidende Wachstumstreiber des US-amerikanischen Ethanolmarktes ist.

Aus diesem Spannungsfeld zwischen den Prognosen der Forschungsinstitute, die ein weiteres Wachstum der Märkte erwarten, und der gegenwärtigen Verunsicherung des Ethanolmarktes der EU27 und der USA ergaben sich die beiden Ziele dieser Arbeit: (1) die Faktoren für den Ethanol-Kraftstoffmarkt der EU27 und der USA zu identifizieren, die den jeweiligen Markt bis zum Jahr 2023 entscheidend beeinflussen werden, und (2) unterschiedliche Szenarien zu bilden, die die Märkte im Jahr 2023 beschreiben ${ }^{380}$.

${ }^{380}$ Der Ethanolmarkt in der EU27 und den USA wird einzeln, aber nicht isoliert voneinander betrachtet. Dies bedeutet, dass die Bestimmung der Faktoren und die Bildung der Szenarien für die beiden Märkte getrennt voneinander erfolgt, der wechselseitige Einfluss der Märkte jedoch berücksichtigt wird. 
Die Erfüllung der beiden genannten Ziele der Studie erfolgte mithilfe eines optimierten Verfahrens der induktiven Szenario-Technik ${ }^{381}$. Diese Prognosemethode gliedert sich in die drei aufeinander aufbauenden Kernschritte „Szenariofeld-Analyse“, „Szenario-Prognostik“ und „Szenario-Bildung und -Konsistenzprüfung“. Nach Durchführung des ersten Kernschritts, bei dem eine Befragung von Experten des Ethanolmarktes der EU27 und der USA vorgenommen wurde, konnten die Schlüsselfaktoren bestimmt werden. Diese sind die Faktoren, die den jeweiligen Ethanolmarkt bis zum Jahr 2023 am stärksten beeinflussen werden. Für den Ethanolmarkt der EU27 wird ersichtlich, dass der Markt - wie bereits in der Vergangenheit - auch in Zukunft maßgeblich von politischen Entscheidungen beeinflusst sein wird. Um die zukünftige Entwicklung des Marktes frühzeitig zu erkennen, ist es von besonderer Bedeutung, das Thema „Politische Förderung von Ethanol“ zu betrachten, da dieses die drei Faktoren ${ }^{382}$ mit dem stärksten Einfluss auf den Ethanolmarkt der EU27 vereint.

Auch der US-amerikanische Ethanolmarkt wird in Zukunft erheblich durch die Politik beeinflusst werden. Von besonderer Relevanz sind politische Faktoren, die die zukünftige Ausgestaltung des RFS 2 und die von der EPA erlaubte Höhe der prozentualen Ethanolbeimischung zu Benzin betreffen. Darüber hinaus wird auch weiterhin die „Blend Wall“-Problematik von entscheidender Bedeutung sein.

Basierend auf den Ergebnissen der "Szenariofeld-Analyse“ werden mittels der Kernschritte "Szenario-Prognostik“ und "Szenario-Bildung und -Konsistenzprüfung" die Szenarien erstellt, die die Ethanolmärkte im Jahr 2023 beschreiben. Zur Überprüfung der Widerspruchsfreiheit der Szenarien wurden diese mithilfe eines standardisierten Fragebogens von Experten auf ihre Konsistenz hin überprüft. Die Szenarien beschreiben Entwicklungen des Marktes, die sich eindeutig voneinander unterscheiden und somit den realistischen Zukunftsraum im Jahr 2023 darstellen. Im Folgenden werden die Szenarien des Ethanolmarktes der EU27 und der USA kurz dargestellt:

Das erste Szenario des Ethanolmarktes der EU27 „Strong Growth of First Generation Ethanol" beschreibt ein weiterhin starkes Wachstum des europäischen Ethanolmarktes der ersten Generation (Nachfrage: 14,5 Milliarden Liter; Produktion: 13

\footnotetext{
${ }^{381}$ In Kapitel 4 ist das optimierte Verfahren der induktiven Szenario-Technik ausführlich beschrieben.

382 Das Thema „Politische Förderung von Ethanol“ besteht aus den wirkenden Einflussfaktoren 19b: „Die maximal erlaubte prozentuale Beimischung von Ethanol zu Benzin“, 18a: „Der politisch geforderte prozentuale Anteil von erneuerbaren Energien am Gesamtenergieverbrauch im Verkehrsbereich“ und 20: „Bestimmungen der Mitgliedsstaaten der EU27 zur Förderung von Ethanol-Kraftstoff".
} 
Milliarden Liter). Ein Wandel des Biokraftstoffmarktes zur zweiten Generation, wie von der europäischen Politik gefordert, findet in dem Szenario nicht statt. Stattdessen kommt es zu einem Umdenken der Politik und der Bereitschaft, weiterhin biogene Treibstoffe der ersten Generation zu fördern. Im zweiten Szenario „5\% Cap for First Generation Ethanol“ kommt es hingegen nur noch zu einem geringen Marktwachstum der ersten Generation (Nachfrage: 7,5 Milliarden Liter; Produktion: 6 Milliarden Liter). Grund dafür ist die politische Entscheidung, die Förderung von Biokraftstoffen der ersten Generation zu beschränken und anstelle dessen Biokraftstoffe der zweiten Generation stärker zu fördern, um einen Wandel des Marktes hin zur zweiten Generation zu erreichen. Allerdings kommt es trotz der politischen Förderung zu keinem Wachstum des Ethanolmarktes der zweiten Generation, da die Herstellungskosten zu hoch sind und die Produktionskapazitäten fehlen. Die dritte Projektion "Growth of Cellulosic Ethanol“ beschreibt den erfolgreichen Wandel des Marktes hin zur zweiten Generation durch die ausschließliche Förderung von Ethanol der zweiten Generation sowie den Aufbau von Produktionskapazitäten zur Herstellung von Zellulose-Ethanol. Da Biokraftstoffe der zweiten Generation dem 10\%Ziel mehrfach angerechnet werden, sind deutlich geringere Mengen ausreichend, um das Ziel zu erreichen, wodurch es zu einem Schrumpfen des Marktes kommt (Nachfrage: 3 Milliarden Liter; Produktion: 3 Milliarden Liter). Das vierte und letzte Szenario „No Import Tariff“ unterscheidet sich von den ersten drei, da sich in diesem Szenario die politische Förderung von Biokraftstoffen ausschließlich am Ziel der maximalen Treibhausgasreduktion orientiert. Infolgedessen kommt es zu einem geringen Anstieg der Ethanolnachfrage auf 7,5 Milliarden Liter und zu hohen Importen von Zuckerrohr-Ethanol aus Brasilien (4,5 Milliarden Liter), da dieser Kraftstoff eine sehr gute $\mathrm{CO}_{2}$-Bilanz besitzt. Die Herstellung von Ethanol der ersten Generation findet in der EU27 nicht mehr statt; dafür steigt die Produktion von ZelluloseEthanol auf 3 Milliarden Liter an.

Im ersten Szenario des US-amerikanischen Ethanolmarktes „Unchanged RFS 2 Strong Growth of Cellulosic Ethanol“ kommt es zu einem sehr starken Wachstum des Ethanolmarktes (Nachfrage: 133 Milliarden Liter; Produktion: 126 Milliarden Liter), welches insbesondere durch den Anstieg der Nachfrage nach und der Produktion von Zellulose-Ethanol getragen wird. Entscheidender Treiber dieser Entwicklung sind die Mandate des RFS 2, die unverändert bleiben. Im zweiten Szenario „Significant Reduction of the Consumption Mandate for 'Advanced: Cellulosic Biofuel“" wächst der Ethanolmarkt (Nachfrage: 76 Milliarden Liter; Produktion: 76 Milliarden Liter) im Gegensatz zum ersten Szenario nur noch moderat, da das Ver- 
brauchsmandat für Biokraftstoffe, welche aus Zellulose, Hemizellulose und Lignin produziert werden, um 57 Milliarden Liter auf nur noch 4 Milliarden Liter gekürzt wird. Ebenso wie im zweiten Szenario wird auch im dritten Szenario „Corn Ethanol replaces Cellulosic Ethanol“ das Mandat für biogene Kraftstoffe aus Zellulose, Hemizellulose und Lignin stark gekürzt. Allerdings wird im Gegenzug das Mandat für Mais-Ethanol um 19 Milliarden Liter angehoben, wodurch es zu einem konstanten Wachstum des Ethanolmarktes (Nachfrage: 95 Milliarden Liter; Produktion: 88 Milliarden Liter), vor allem von Mais-Ethanol, kommt. Auch im vierten Szenario „Unspecified Ethanol replaces Cellulosic Ethanol“ wird das Mandat für Kraftstoffe aus Zellulose, Hemizellulose und Lignin stark reduziert. Zum Ausgleich wird aber das Mandat für Biokraftstoffe aus Sorghum, Weizen und Zuckerrüben um 19 Milliarden Liter aufgestockt. So kommt es zu einem konstanten Marktwachstum insbesondere von Ethanol, das aus diesen Rohstoffen hergestellt wird (Nachfrage: 95 Milliarden Liter; Produktion: 88 Milliarden Liter). Infolgedessen werden die US-amerikanischen Regionen, in denen diese Kulturen angebaut werden, stark von der Ethanolindustrie profitieren.

In Abbildung 110 sind nochmals alle Szenarien des Ethanolmarktes der EU27 und der USA anhand der Ethanolnachfrage, der Ethanolproduktion und des Ethanolhandels dargestellt.

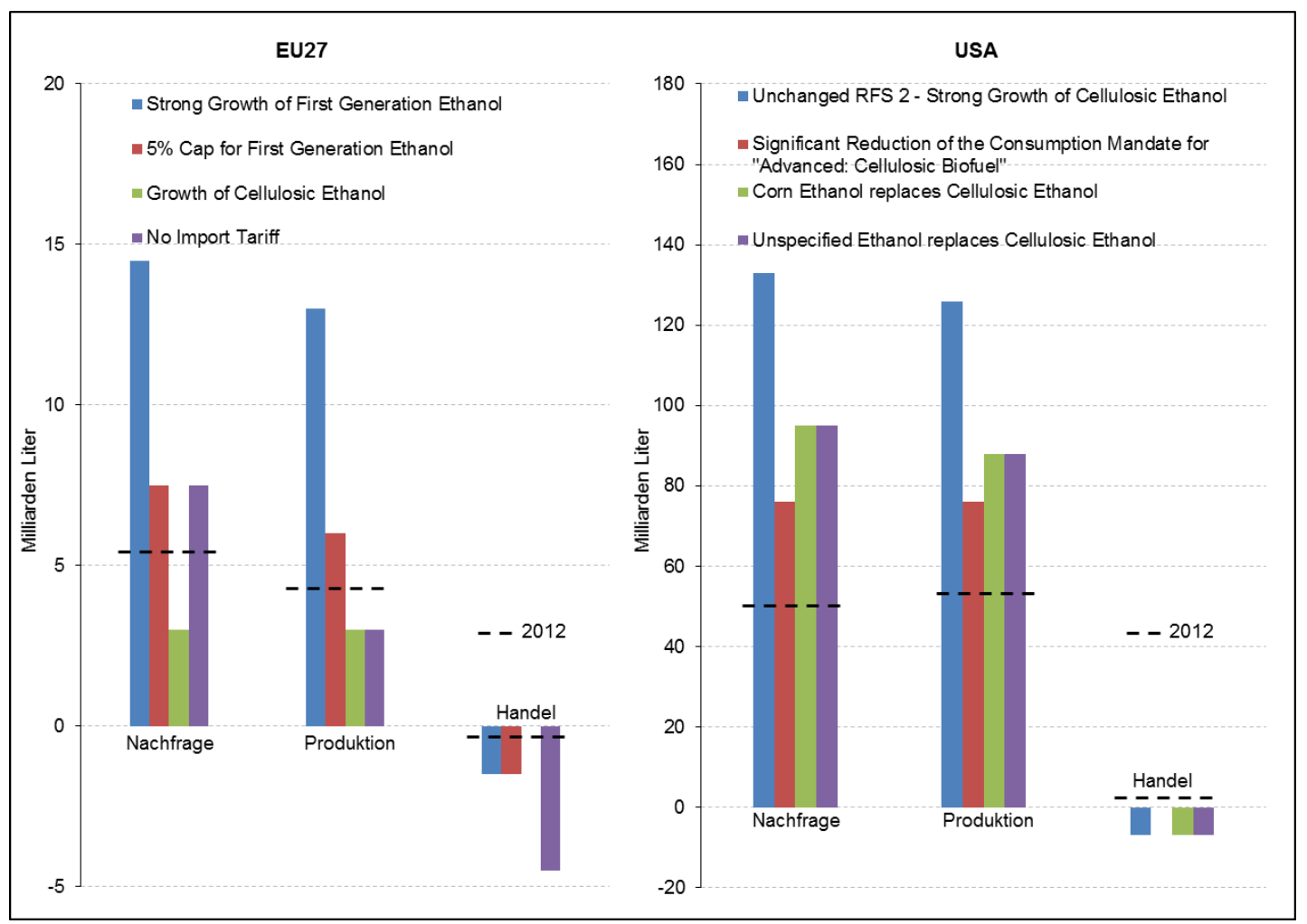

Abbildung 110: Szenarien des Ethanolmarktes der EU27 und der USA Quelle: EIGENE BERECHNUNG 
Aus Abbildung 110 wird ersichtlich, dass die Szenarien, die den Ethanolmarkt der EU27 im Jahr 2023 beschreiben, einen im Verhältnis zur Größe des Marktes sehr breiten Zukunftsraum aufzeigen. So wird sowohl ein starker Anstieg als auch ein Schrumpfen des Marktes bis zum Jahr 2023 prognostiziert. Dieser breite Zukunftsraum ergibt sich, da die Politik intensiv über einen Wandel des Ethanolmarktes von Kraftstoffen der ersten hin zu denen der zweiten Generation diskutiert, wodurch die bereits bestehende Ethanolindustrie der ersten Generation in ihrer Existenz bedroht ist.

In den USA befindet sich die zukünftige Ausgestaltung des RFS 2 im Zentrum der Debatte. Im Gegensatz zur EU27 wird aber die bereits bestehende Ethanolindustrie nicht in Frage gestellt, wodurch der Zukunftsraum, im Verhältnis zur Marktgröße, deutlich kleiner ist und alle Szenarien ein zukünttiges Wachstum des Marktes beschreiben.

\subsection{Handlungsempfehlungen für die Ethanolwirtschaft}

Unternehmen erarbeiten im Rahmen ihrer strategischen Planung Strategien, um auf Marktentwicklungen vorbereitet zu sein. Zur Bildung dieser bedarf es sowohl einer Unternehmens- als auch einer Umfeldanalyse. Ein Bestandteil der Umfeldanalyse ist die Untersuchung der zukünftigen Entwicklung des Makroumfeldes ${ }^{383}$ (JOHNSON ET AL. 2011: 78). Unternehmen, die im Ethanolmarkt der EU27 und/oder der USA tätig sind, können zur Analyse die in dieser Studie entwickelten Szenarien verwenden (Abbildung 111). Darüber hinaus wird neben der „SzenarioTechnik“ auch die „PEST-Analyse“ ${ }^{384}$ zur Untersuchung des Makroumfeldes verwendet (JOHNSON ET AL. 2011: 80). Dieses Instrument wurde bereits in dem optimierten Verfahren der Szenario-Technik durch die Bestimmung der wirkenden Einflussfaktoren angewandt ${ }^{385}$ (Kapitel 5.1.1.2 und 6.1.1.2). Aufgrund der hohen Anzahl an erkannten wirkenden Einflussfaktoren ist es ratsam, dass sich die Unternehmen auf die wirkenden Schlüsselfaktoren konzentrieren, da diese die wesentli-

\footnotetext{
${ }^{383}$ Das Makroumfeld betrachtet die grundlegenden Umfeldfaktoren, die die Entwicklung des zu untersuchenden Marktes beeinflussen (JOHNSON ET AL. 2011: 78).

384 In der „PEST-Analyse“ werden die wichtigsten Faktoren genannt, die die zukünftige Entwicklung des Makroumfeldes beeinflussen. Die Faktoren werden entsprechend der englischen Abkürzung PEST in die Bereiche Political, Economical, Social und Technological unterteilt (JOHNSON ET AL. 2011: 80).

${ }_{385}$ Das in dieser Studie zur Anwendung gekommene Verfahren "SEPT formula" (Social, Economical, Political, Technological) von WILSON (1998: 87) entspricht der „PESTAnalyse" lediglich die Bezeichnung ist eine andere.
} 
chen Veränderungen des Ethanolmarktes der EU27 und der USA beschreiben (Kapitel 5.1.2.2 und 6.1.2.2).

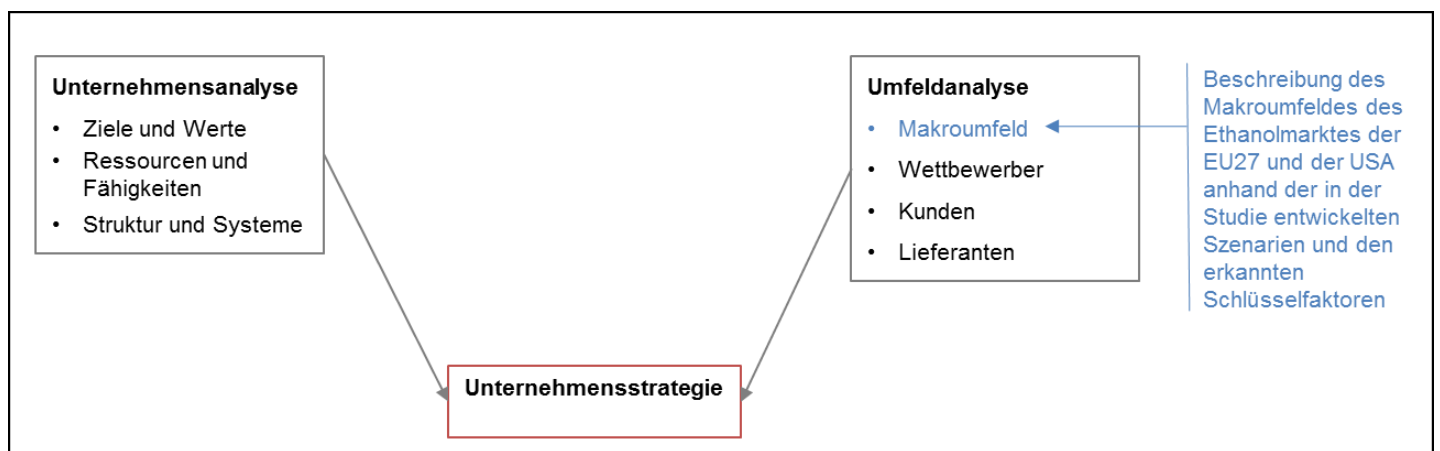

Abbildung 111: Die optimierte Szenario-Technik als Instrument zur Beschreibung des Makroumfeldes des Ethanolmarktes der EU27 und der USA

Quelle: GRANT/NIPPA 2006: 34; JOHNSON ET AL. 2011: 79; STEINMANN/SCHREYÖGG 2005: 172

Für Unternehmen, die im Ethanolmarkt der EU27 tätig sind, wäre es fatal, davon auszugehen, dass es zu einem weiteren starken Wachstum des Marktes kommen wird, wie von den etablierten Forschungsinstituten prognostiziert (Kapitel 2.3). Die Unternehmen sollten alle vier in der Studie gebildeten Szenarien in ihrer strategischen Planung berücksichtigen. Dabei sollten sie insbesondere den Schlüsselfaktor „Politische Förderung von Ethanol“ betrachten, um frühzeitig zu erkennen, in Richtung welches der vier Szenarien sich der Markt entwickelt. Insbesondere Unternehmen, die im Ethanolmarkt der ersten Generation tätig sind, müssen die politischen Entscheidungen beobachten, um rechtzeitig zu bemerken, ob es zu einem Wandel des Marktes von der ersten hin zur zweiten Generation kommt.

Auch die Ethanolexporteure in Brasilien und den USA sollten die Entwicklung der vier Szenarien betrachten. Wenn es zu einer ausschließlichen politischen Förderung von Biokraftstoffen der zweiten Generation käme, bestünde in der EU27 keine Nachfrage mehr nach Zuckerrohr- und Mais-Ethanolimporten. Folglich müssten die Exporteure neue Märkte erschließen, damit der Rückgang des Exports in die EU27 kompensiert werden könnte.

Die Szenarien dienen außerdem der Unterstützung der Ethanolindustrie der zweiten Generation, denn anhand der Szenarien ist das Marktpotenzial von ZelluloseEthanol in der EU27 im Jahr 2023 zu erkennen. Diese Information ist essentiell, um die Wirtschaftlichkeit von langfristigen Investionen, beispielsweise in die Entwicklung neuer Enzyme zur Aufspaltung von Zellulose bei der Produktion von ZelluloseEthanol, zu ermitteln. 
Im Folgenden werden beispielhaft für ein Unternehmen, das Ethanol der ersten Generation aus Weizen in der EU $27^{386}$ produziert, Handlungsoptionen beschrieben. Diese orientieren sich an der in den vier Szenarien dargestellten Entwicklung des Ethanolmarktes der EU27 (Kapitel 5.3.1). Bei den Handlungsempfehlungen handelt es sich lediglich um Vorschläge, da nur das Makroumfeld und nicht die anderen Bereiche des Umfeldes (Wettbewerber, Lieferanten und Kunden) und auch nicht das Unternehmen selbst analysiert wurden (Abbildung 111).

\section{Szenario 1: Strong Growth of First Generation Ethanol}

Aufgrund des starken Wachstums der Ethanolproduktion der ersten Generation auf 13 Milliarden Liter im Jahr 2023, infolge der politischen Unterstützung für Biokraftstoffe der ersten Generation, sollte das Unternehmen seine Produktionskapazitäten ausbauen. Das könnte beispielsweise durch eine Erweiterung der bestehenden Anlagen, dem Bau neuer Anlagen oder dem Erwerb von Anlagen von Wettbewerbern geschehen. Darüber hinaus ist darauf zu achten, dass sowohl die bereits bestehenden Anlagen als auch mögliche neue Anlagen die zukünftig politisch geforderten prozentualen Treibhausgasreduktionswerte im Vergleich zu fossilem Kraftstoff erfüllen. Da es in diesem Szenario zu keinem Wandel des Marktes von der ersten hin zur zweiten Generation kommt, besteht keine Notwendigkeit in die Produktion von Zellulose-Ethanol zu investieren.

\section{Szenario 2: 5\% Cap for First Generation Ethanol}

Die Ethanolproduktion der EU27 wird nur moderat auf 6 Milliarden Liter bis zum Jahr 2023 ansteigen. Grund dafür ist die politische Entscheidung der 5-prozentigen Begrenzung der Anrechnung von Biokraftstoffen der ersten Generation auf das 10\%Ziel. Das Unternehmen sollte deshalb darauf achten, seinen Marktanteil zu halten und nicht in neue Anlagen der ersten Generation investieren, da bereits Produktionskapazitäten von 8 Milliarden Litern bestehen (USDA FOREIGN AGRICULTURAL SERVICE 2012: 11). Wie im ersten Szenario ist auch hier relevant, dass die bereits bestehenden Anlagen im Jahr 2023 die politisch geforderte $\mathrm{CO}_{2}$-Reduktion im Vergleich zu fossilem Kraftstoff erreichen. Im diesem Szenario erfolgt zwar noch keine kommerzielle Produktion von Zellulose-Ethanol in der EU27, trotzdem sollte das Unternehmen, um langfristig wachsen zu können, in die Produktion von Zellulose-Ethanol investieren, da die Politik einen Wandel des Marktes anstrebt und Zellu-

\footnotetext{
${ }^{386}$ Betrachtet wird ein Unternehmen, das Ethanol der ersten Generation aus Weizen produziert, da dies der am häufigsten verwendete Rohstoff in der EU27 ist (EUROPEAN COMMISSION JOINT RESEARCH CENTRE, INSTITUT FOR ENERGY ET AL. 2010: 74).
} 
lose-Ethanol durch die vierfache Anrechnung seines Energiegehalts auf das 10\%Ziel stark fördern wird.

\section{Szenario 3: Growth of Cellulosic Ethanol}

Da im Jahr 2023 ausschließlich Biokraftstoffe der zweiten Generation politisch gefördert werden, besteht in Zukunft in der EU27 keine Nachfrage mehr nach EthanolKraftstoff der ersten Generation. Das Unternehmen sollte deshalb rechtzeitig neue Märkte erschließen, um bestehen zu können, beispielsweise durch einen Umbau der Produktionsanlagen, um Zellulose-Ethanol herzustellen, oder durch den Export von Ethanol-Kraftstoff auf den Weltmarkt. Doch die Produktion müsste am Weltmarkt konkurrenzfähig sein im Vergleich zu US-amerikanischem Mais-Ethanol und brasilianischem Zuckerrohr-Ethanol, welches derzeit noch zu deutlich geringeren Kosten produziert wird (BERG 2012d: 2; HENNIGES 2007: 55ff.). Das Unternehmen sollte darüber hinaus auch einen Marktaustritt in Betracht ziehen, um eventuelle Verluste so gering wie möglich zu halten.

\section{Szenario 4: No Import Tariff}

Aufgrund der Anhebung der politisch geforderten $\mathrm{CO}_{2}$-Reduktion im Vergleich $\mathrm{zu}$ fossilem Kraftstoff und der Entscheidung, einen ILUC-Faktor in der Berechnung der $\mathrm{CO}_{2}$-Emissionen bei der Herstellung von Ethanol im Jahr 2023 zu berücksichtigen, ist die Erfüllung der politische geforderten Treibhausgasreduktion bei der Produktion von Weizen-Ethanol nicht mehr möglich. Infolgedessen entfällt die politische Förderung und es besteht keine Nachfrage mehr nach Weizen-Ethanol in der EU27. Folglich sollte das Unternehmen wie in Szenario 3 frühzeitig neue Absatzmärkte erschließen, um auf dem Markt bestehen zu können. Möglich wäre der Umbau der Produktionsanlage, um Zellulose-Ethanol herzustellen, oder der Export des WeizenEthanols in Märkte, die geringere Anforderungen an die $\mathrm{CO}_{2}$-Reduktion stellen. Auch für dieses Szenario sollten Strategien entwickelt werden, um einen Marktaustritt vorzubereiten.

In den USA muss die Ethanolindustrie auf die zukünftige Ausgestaltung des RFS 2 achten und sich darauf einstellen, dass das Mandat für Biokraftstoffe, welche aus Zellulose, Hemizellulose und Lignin produziert werden, stark gekürzt werden könnte. Insbesondere Unternehmen, die seit Jahren intensiv an der Entwicklung der kommerziellen Produktion von Zellulose-Ethanol arbeiten, müssen diese mögliche Veränderung des RFS 2 in ihrer strategischen Planung berücksichtigen. In Anbetracht einer möglichen starken Kürzung des genannten Mandats könnte es im Gegenzug 
aber auch zu einer Anhebung entweder des Mandats für Mais-Ethanol oder für Ethanol aus Sorghum, Weizen und Zuckerrüben kommen, sodass sich für diese Ethanolbereiche ein hohes Wachstumspotential ergibt. Die US-amerikanische Ethanolindustrie kann des Weiteren davon ausgehen, dass das Mandat für Mais-Ethanol nicht gesenkt wird und somit die bereits bestehenden Kapazitäten zur Herstellung auch in Zukunft genutzt werden. Darüber hinaus wird erwartet, dass die „Blend Wall“-Problematik an Relevanz verliert und ein weiteres Marktwachstum zulässt.

Wie bereits für den Ethanolmarkt der EU27 dargestellt, werden im Folgenden für ein Unternehmen, das im Corn Belt der USA Mais-Ethanol ${ }^{387}$ produziert, Handlungsoptionen beschrieben, die sich an der in den vier Szenarien dargestellten Entwicklung des US-amerikanischen Ethanolmarktes orientieren. Wichtig zu erwähnen ist, dass es sich lediglich um Empfehlungen handelt, die als Denkanstoß für die strategische Planung zu verstehen sind.

\section{Szenario 1: Unchanged RFS 2 - Strong Growth of Cellulosic Ethanol}

Es kommt zu einem starken Wachstum des Ethanolmarktes bis zum Jahr 2023, da der RFS 2 unverändert bleibt. Besonders stark ist das Wachstum im Bereich Zellulose-Ethanol, da die Produktion von derzeit 0,027 Milliarden Litern auf 61 Milliarden Liter ansteigt (OECD/FAO 2012: 224). Das Unternehmen sollte diese Entwicklung nutzen und in die Produktion von Zellulose-Ethanol investieren. Von weiteren Investionen im Bereich Mais-Ethanol ist hingegen abzuraten. Die US-amerikanische Produktion wird nicht über 61 Milliarden Liter ansteigen, weil die heimische Nachfrage ab dem Jahr 2015 auf 57 Milliarden Liter beschränkt ist und schon heute Produktionskapazitäten von 56 Milliarden Litern bestehen (U.S. GOVERNENT INFORMATION 2007: 121 STAT. 1522; RFA 2012c).

Szenario 2: Significant Reduction of the Consumption Mandate for "Advanced: CelIulosic Biofuel"

Der Ethanolmarkt wird bis zum Jahr 2023 nur moderat wachsen, da das Mandat für Biokraftstoffe, produziert aus Zellulose, Hemizellulose und Lignin, von 61 Milliarden Litern auf 4 Milliarden Liter gekürzt wird. Aus diesem Grund besteht ein nur geringes Wachstum in der Produktion von Zellulose-Ethanol. Des Weiteren wird auch kein starker Anstieg in der Herstellung von Mais-Ethanol erwartet, da der RFS 2 die Bin-

${ }^{387}$ Betrachtet wird ein Unternehmen aus dem Corn Belt, das Mais als Rohstoff zur Ethanolproduktion verwendet, da momentan die US-amerikanische Ethanolproduktion fast nur im Corn Belt und fast nur aus dem Rohstoff Mais erfolgt (U.S. ENERGY INFORMATION ADMINISTRATION 2013b: 1; U.S. ENERGY INFORMATION ADMINISTRATION 2012j: 6ff.). 
nennachfrage ab dem Jahr 2015 auf 57 Milliarden Liter begrenzt. Die beste Möglichkeit für ein weiteres Wachstum ergibt sich für das Unternehmen aus der Produktion von Ethanol aus Sorghum, Weizen und Zuckerrüben, da ein Produktionsanstieg auf 11 Milliarden Liter prognostiziert wird. Das Unternehmen sollte von dieser Entwicklung durch den Bau neuer Anlagen oder die Erweiterung der bereits bestehenden Anlagen profitieren, um sowohl Mais als auch Sorghum und Weizen als Rohstoff zu nutzen. Dies ist möglich, da die Produktionsprozesse sehr ähnlich sind. So hat das Unternehmen die Möglichkeit, in Abhängigkeit vom Ethanolpreis und den jeweiligen Rohstoffkosten den Produktionsprozess deckungsbeitragsoptimierend anzupassen. Darüber hinaus wird der starke Einfluss des Maispreises auf die Wirtschaftlichkeit des Unternehmens gesenkt.

\section{Szenario 3: Corn Ethanol replaces Cellulosic Ethanol}

Trotz der Kürzung des Mandats für Biokraftstoffe, produziert aus Zellulose, Hemizellulose und Lignin, auf nur noch 4 Milliarden Liter kommt es zu einem konstanten Marktwachstum, da das Verbrauchsmandat für Mais-Ethanol angehoben wird. Infolgedessen steigt die US-amerikanische Mais-Ethanolproduktion auf 80 Milliarden Liter an. Das betrachtete Unternehmen sollte Nutzen aus dieser Entwicklung ziehen und in den Ausbau seiner Produktionskapazitäten investieren. Das könnte z.B. durch die Vergrößerung der bereits bestehenden Anlagen, den Bau neuer Anlagen oder die Übernahme bestehender Anlagen geschehen.

\section{Szenario 4: Unspecified Ethanol replaces Cellulosic Ethanol}

Es kommt zu einem konstanten Marktwachstum, da das Mandat für Biokraftstoffe, hergestellt aus Sorghum, Weizen und Zuckerrüben, angehoben wird, um die starke Reduzierung des Mandats für biogene Treibstoffe, produziert aus Zellulose, Hemizellulose und Lignin, auszugleichen. Aufgrund dieser politischen Entscheidung steigt die Produktion von Ethanol aus Sorghum, Weizen und Zuckerrüben auf 23 Milliarden Liter an. Das Unternehmen sollte diese Entwicklung nutzen, um weiter zu wachsen und seine Abhängigkeit von der alleinigen Produktion von Mais-Ethanol zu senken. Da der Produktionsprozess zur Herstellung von Ethanol aus Sorghum und Weizen ähnlich der Herstellung von Mais-Ethanol ist, könnten bestehende Anlagen erweitert werden, um alle drei Rohstoffe zu verwenden. Dies gäbe dem Unternehmen die Möglichkeit, in Abhängigkeit von den Marktpreisen für Ethanol und den Rohstoffkosten den Produktionsprozess gewinnoptimierend anzupassen. Darüber hinaus könnten auch neue Produktionsanlagen in dem jeweiligen Anbaugebiet der als Rohstoff verwendeten Kultur errichtet werden. 
Zusammenfassend ist zu erkennen, dass sich die Ethanolindustrie in den USA und insbesondere in der EU27 im Rahmen ihrer strategischen Planung sehr flexibel aufstellen muss, um bis zum Jahr 2023 erfolgreich auf dem Markt bestehen zu können. Die Unternehmen sollten für die langfristige Sicherung des Unternehmenserfolgs falls nicht bereits vorhanden - den Aufbau eines strategischen Planungsprozesses in Betracht ziehen, da der Ethanolmarkt der EU27 und der USA von einer hohen Unsicherheit geprägt ist und maßgeblich von politischen Entscheidungen beeinflusst wird.

\subsection{Ansätze für die weitere Forschung}

Die Untersuchung der langfristigen Entwicklung des Ethanolmarktes der EU27 und der USA sollte in regelmäßigen Abständen wiederholt werden, da sich die Märkte in Richtungen verändern können, die zum jetzigen Zeitpunkt nicht erkennbar sind und deshalb in den Szenarien nicht berücksichtigt wurden. Darüber hinaus sollte eine erneute Betrachtung der Märkte nach entscheidenden Veränderungen erfolgen.

Im Ethanolmarkt der EU27 kommt es zu einer maßgeblichen Veränderung, wenn die neuen Rahmenbedingungen zur politischen Förderung von Biokraftstoffen in der EU27 verabschiedet sind. Derzeit wird, basierend auf den Vorschlägen von Energiekommissar Günther Oettinger und Klimakommissarin Connie Hedegard vom 17. Oktober 2012, intensiv im Europaparlament und im Ministerrat über die zukünftige Ausgestaltung der Förderung diskutiert. Allerdings wird vor Anfang 2015 mit keiner Einigung gerechnet, da die Meinungen sowohl im Europaparlament ${ }^{388}$ als auch im Ministerrat ${ }^{389}$ noch weit auseinandergehen (F.O. LICHT 2013h: 83; F.O. LICHT 2013g: 62; AGRA-EUROPE 2013b: EU-NACHRICHTEN 1).

\footnotetext{
${ }^{388}$ Das Europaparlament hat sich zwar im September 2013 darauf geeinigt, dem Vorschlag der Kommissare Günther Oettinger und Connie Hedegard in abgeschwächter Form zu folgen, trotzdem beschleunigt dies nicht den Prozess der Festlegung von neuen politischen Rahmenbedingungen zur Förderung von Biokraftstoffen in der EU27, da der französischen Berichterstatterin Corinne Lepage ein Mandat für die direkte Aufnahme von Verhandlungen mit den Ministerrat nicht erteilt wurde. Stattdessen geht das Thema in eine Zweite Lesung was den Vorgang deutlich verzögert, insbesondere da Ende Mai 2014 ein neues Europäisches Parlament gewählt wird (F.O. LICHT 2013g: 61; F.O. LICHT 2013h: 83; AGRAEUROPE 2013a: EU-NACHRICHTEN 1).

${ }^{389}$ Mitte Dezember 2013 legte die litauische Ratspräsidentschaft den Energieministern der europäischen Mitgliedsstaaten einen Kompromissvorschlag zur zukünftigen Ausgestaltung der Förderung von Biokraftstoffen in der EU27 vor. Dieser wurde - trotz starker Unterstützung von Günther Oettinger - jedoch abgelehnt, da er für einige Mitgliedsstaaten einen zu starken (Polen und Ungarn) und für andere einen zu geringen (Italien, Dänemark und Niederlande) Wandel des Marktes hin zu Biokraftstofffen der zweiten Generation beschreibt (AGRA-EUROPE 2013b: EU-NACHRICHTEN 1).
} 
Die Zukunft des US-amerikanischen Ethanolmarktes sollte erneut untersucht werden, wenn die EPA einen neuen RFS genehmigt hat. Bereits seit zwei Jahren wird das Thema diskutiert, eine Änderung ist aber nicht vor Ende 2014/Anfang $2015 \mathrm{zu}$ erwarten (F.O. LICHT 2013g: 62).

Darüber hinaus könnte der Faktor „Tank-oder-Teller-Diskussion“ bei einer erneuten zeitnahen Untersuchung der langfristigen Entwicklung des Ethanolmarktes der EU27 und der USA eine größere Bedeutung besitzen. In dieser Studie wurde der Faktor sowohl im Ethanolmarkt der EU27 (Kapitel 5.1.2.2/Einflussfaktor 23) als auch in dem der USA (Kapitel 6.1.2.2/Einflussfaktor 26) berücksichtigt, gehörte aber nicht zu den Schlüsselfaktoren ${ }^{390}$. Dies könnte darauf zurückzuführen sein, dass die Durchführung der Wirkungsanalyse für den Ethanolmarkt der EU27 und der USA im Mai und Anfang Juni des Jahres 2012 erfolgte und damit kurz vor der intensiv geführten „Tank-oder-Teller-Diskussion“ im Sommer und Herbst des gleichen Jahres. Durch die Berücksichtigung auch dieses Faktors könnte ein umfangreicheres Zukunftsbild des Marktes entstehen. Die möglichen politischen Konsequenzen der Diskussion sind aber in den Zukunftsprojektionen der politischen Schlüsselfaktoren bereits berücksichtigt und damit in die Szenarien integriert.

Aufgrund des starken Einflusses politischer Entscheidungen auf die Entwicklung des Ethanolmarktes der EU27 und der USA (Kapitel 5.1.2.2/6.1.2.2) und der derzeit intensiv geführten Diskussion über die zukünftige Ausgestaltung der politischen Förderung von Biokraftstoffen in beiden Märkten (F.O. LICHT 2013g: 61f.; F.O. LICHT 2013h: 82f.) ist es interessant zu erforschen, was die politische Entscheidungsfindung beeinflusst. Im Zentrum der Untersuchung sollte sowohl der Einfluss von gesellschaftlichen Diskussionen als auch die Wirkung von Lobbyaktivitäten stehen. Beispielhaft für gesellschaftliche Themen sind die „Tank-oder-Teller-Diskussion“ und die Diskussion über den Nutzen von Ethanol zur Förderung der Energieunabhängigkeit. Die Bedeutung dieser beiden Diskussionen für den Ethanolmarkt der EU27 und der USA wurde bereits in den Kapiteln 5.1.1.2 (Einflussfaktor 23 und 25) und Kapitel 6.1.1.2 (Einflussfaktor 26 und 28) beschrieben.

Bei der Analyse des Einflusses von Lobbyaktivitäten in der EU27 gilt es zwei Seiten zu betrachten: (1) die Interessensvertretungen der europäischen Ethanolproduzen-

\footnotetext{
${ }^{390}$ Im Ethanolmarkt der EU27 und der USA besitzt die „Tank-oder-Teller-Diskussion“ nach Einschätzung der befragten Experten lediglich einen moderaten Einfluss auf die zukünftige Entwicklung des jeweiligen Marktes.
} 
ten $^{391}$ und die Vertretungen der Landwirte ${ }^{392}$. Sie setzen sich für die europäische Ethanolindustrie ein, weil die Ethanolproduktion den Absatz landwirtschaftlicher Produkte steigert. (2) Die Lobbyarbeit der Ethanol-kritischen Organisationen ${ }^{393}$, die ein Ende der Nutzung von biogenen Treibstoffen oder eine strikte Begrenzung der Verwendung fordern. Unabhängig von den beiden genannten Gruppen, sollte auch der Einfluss der Mineralölindustrie und der Automobilindustrie betrachtet werden. Beide Branchen besitzen die Mittel und Ressourcen, um die politischen Entscheidungen über die zukünftige Ausgestaltung der Förderung von Biokraftstoffen in ihrem Sinne zu beeinflussen.

Auch im Ethanolmarkt der USA stehen sich zwei Lobbygruppen gegenüber. Auf der einen Seite die Ethanolbefürworter, vertreten durch die Ethanolindustrie ${ }^{394}$ und die Landwirte im Corn Belt: Sie fordern ein weiteres Wachstum des Ethanolmarktes, da die Ethanolindustrie zu den wichtigsten Abnehmern von Mais gehört ${ }^{395}$. Auf der anderen Seite stehen die US-amerikanische Mineralöl- und Veredelungsindustrie, die ein Schrumpfen des Ethanolmarktes zum Ziel haben (F.O. LICHT 2012c: 319; F.O. LICHT 2012g: 489f.). Die Mineralölindustrie verfolgt dieses Ziel, da Ethanol fossiles Benzin substituiert, wodurch die ohnehin abnehmende Benzinnachfrage durch die Verwendung von Ethanol noch stärker sinkt ${ }^{396}$ (U.S. ENERGY INFORMATION ADMINISTRATION 2012b: 98). Die tierische Veredelungsindustrie, die wie die Ethanolindustrie Mais als Rohstoff verwendet, erhofft sich durch ein Schrumpfen des Ethanolmarktes, eine Minderung der Nachfrage nach Mais, um ihren Rohstoff kostengünstiger beziehen zu können ${ }^{397 / 398}$.

Forschungsbedarf besteht des Weiteren in der Betrachtung der Entwicklung des Ethanolmarktes der EU27 und der USA über das Jahr 2023 hinaus. Notwendig ist

\footnotetext{
${ }^{391}$ Wichtigster Vertreter der europäischen Ethanolproduzenten ist die Organisation „ePURE“, die mit 21 Mitgliedern 90\% der europäischen Ethanolherstellung vertritt (EPURE 2013).

392 Die Interessen der Landwirte werden durch den "Deutschen Bauernverband“ und "COPA COGECA“ repräsentiert.

${ }^{393}$ Als Biokraftstoff ablehnende Organisationen mit politischem Einfluss sind beispielsweise „Oxfam", der „Naturschutzbund Deutschland e.V.“ (NABU) und der „Bund für Umwelt und Naturschutz Deutschland“" (BUND) zu nennen (AGRA-EUROPE 2013b: EU-NACHRICHTEN 1).

${ }^{394}$ Die US-amerikanische Ethanolindustrie wird durch die Organisationen „Renewable Fuels Association“, „Growth Energy“ und „American Coalition for Ethanol“ politisch repräsentiert.

${ }^{395} \mathrm{Im}$ Jahr 2012 wurden 42\% der US-amerikanischen Maisernte zur Ethanolherstellung verwendet (U.S. DEPARTMENT OF ENERGY 2013b). Vertreten werden die politischen Interessen der Landwirte beispielsweise durch die "National Corn Growers Association“.

${ }^{396}$ Repräsentiert wird die einflussreiche US-amerikanische Mineralölindustrie durch das "American Petroleum Institute“.

${ }^{397}$ Bedeutender Vertreter der Veredlungsindustrie ist z.B. das "National Chicken Council“.

${ }^{398}$ Im Jahr 2008 forderte der texanische Governeur Rick Perry auf Druck der texanischen Veredlungsindustrie die Aufhebung des RFS 2 (F.O. LICHT 2012g: 490).
} 
dies, da die Ethanolwirtschaft, um langfristige Entscheidungen treffen zu können, beispielsweise über den Bau einer Produktionsanlage zur Herstellung von ZelluloseEthanol, einen Zeitraum von über zehn Jahren betrachten muss, weil die Anlagen eine Nutzungsdauer von mindestens 15 Jahren haben ${ }^{399}$. Neben der zeitlichen Erweiterung der Untersuchung besteht auch Forschungsbedarf hinsichtlich der geographischen Ausweitung des Untersuchungsfeldes. Im Fokus steht dabei der brasilianische Markt als zweitgrößter Produzent und Konsument von Ethanol (F.O. LICHT 2012f: 74). Dieser Markt ist auch deshalb von Bedeutung, da er in den Szenarien des Ethanolmarktes der EU27 (Kapitel 5.2.10) und der USA (Kapitel 6.2.10) der wichtigste Ethanolexporteur ist und dadurch beide Märkte beeinflusst. Ebenso ist auch die Betrachtung anderer Biokraftstoffmärkte von Belang für die Forschung, beispielsweise durch die Untersuchung des BtL- oder Biobutanolmarktes in der EU27 und den USA. Das Interessante an diesen Märkten ist, dass beide biogenen Treibstoffe Ethanol-Kraftstoff substituieren können ${ }^{400}$.

Da sich diese Studie, wie in der Einleitung erläutert (Kapitel 1.1), ausschließlich auf den Ethanol-Kraftstoffmarkt konzentiert, besteht ebenfalls weiterer Forschungsbedarf in der Untersuchung der zukünftigen Entwicklung des Ethanolmarktes in der chemischen Industrie und der Nahrungsmittelindustrie.

Zum Abschluss der Betrachtung der weiteren Forschung gilt es, auf die Untersuchung der Wirkung der Szenarien auf andere Märkte hinzuweisen. Von besonderer Wichtigkeit sind der Agrarmarkt der EU27 und insbesondere jener der USA. Aufgrund der in den Szenarien prognostizierten Größe des US-amerikanischen Ethanolmarktes steht besonders dieser im Zentrum der Betrachtung. So wurden bereits im Jahr 2011 40\% (124 Millionen Tonnen) der US-amerikanischen Maisernte zur Ethanolproduktion verwendet, was entscheidend den Anbau von landwirtschaftlichen Kulturen in den USA beeinflusst hat (USDA 2013b). Auch in Zukunft wird es einen starken Einfluss auf den Agrarmarkt geben, die Art und Stärke des Einflusses variiert allerdings zwischen den Szenarien.

\footnotetext{
${ }^{399}$ Das AGRICULTURAL MARKETING RESOURCE CENTER (2013) betrachtet in seinen Berechnungen eine Nutzungsdauer von 15 Jahren für Ethanolanlagen in den USA. HENNIGES (2007: 268ff.) Rechnungen basieren auf einer Nutzungsdauer von 20 Jahren für Ethanolanlagen in der EU27 und den USA.

${ }^{400}$ Die biogenen Kraftstoffe BtL und Biobutanol wurden in der Untersuchung des Ethanolmarktes der EU27 (Kapitel 5.1.1.2/Einflussfaktor 16/Einflussfaktor 17) und der USA (Kapitel 6.1.1.2/Einflussfaktor 20/Einflussfaktor 21) berücksichtigt. Die befragten Experten erkennen jedoch keinen bedeutenden Einfluss auf einen der beiden Ethanolmärkte.
} 
Basierend auf der umfangreichen Analyse des Ethanolmarktes der EU27 und der USA mittels des Verfahrens der optimierten Szenario-Technik ist festzustellen, dass sich beide Märkte aufgrund von politischen Entscheidungen maßgeblich bis zum Jahr 2023 verändern werden. In Richtung welches der jeweils vier Szenarien - darauf können wir gespannt sein. 


\section{Literaturverzeichnis}

ABENGOA BIOENERGY (2012): Abengoa Bioenergy Biomass of Kansas, URL: http://www.abengoabioenergy.com/web/en/acerca_de/oficinas_e_instalacionin /bioetanol/eeuu/kansas/index.html, Abrufdatum: 31.10.2012.

acatech (Deutsche Akademie der Technikwissenschaften, 2012): Biotechnologische Energieumwandlung in Deutschland - Stand, Kontext, Perspektiven, URL:

http://www.acatech.de/fileadmin/user_upload/Baumstruktur_nach_Website/Ac atech/root/de/Publikationen/Stellungnahmen/120516_acatech_POSITION_BE _WEB.pdf, Abrufdatum: 03.09.2013.

ACEA (European Automobile Manufacturers' Association, 2012a): Can all Cars Run on Biofuels?, URL: http://www.acea.be/collection/fuels_faq/, Abrufdatum: 30.08.2012.

ACEA (European Automobile Manufacturers' Association, 2012b): Lead-Time is Essential: Car's Concept and Production Phase take up to 12 Years, URL: http://www.acea.be/news/news_detail/lead_time_is_essential_cars_concept_a nd_production_phase_take_up_to_12_year/, Abrufdatum: 02.09.2012.

ACEA (European Automobile Manufacturers' Association, 2012c): What do Car Manufacturers think about Biofuels?, URL:http://www.acea.be/collection/fuels_faq/, Abrufdatum: 22.08.2012.

ACEA (European Automobile Manufacturers' Association, 2011a): The Automobile Industry Pocket Guide, URL: http://www.acea.be/images/uploads/files/20110921_Pocket_Guide_3rd_editie d.pdf, Abrufdatum: 23.08.2012.

ACEA (European Automobile Manufacturers' Association, 2011b): EU Economic Report, URL: http://www.acea.be/images/uploads/files/20110927_ER_1105_2011_I_Q4.pdf, Abrufdatum: 23.08.2012. 
Agra-Europe (2013a): Europaparlament über Biokraftstoffe gespalten, in: AgraEurope EU-Nachrichten, Jahrgang. 13, Nr. 38, S. 1.

Agra-Europe (2013b): EU-Energieminister finden keinen Kompromiss zu Biokraftstoffen, in: Agra-Europe EU-Nachrichten, Jahrgang. 13, Nr. 51, S. 1.

Agra-Europe (2012): Brüssel plant Deckel für Biokraftstoffe, in: Agra-Europe EUNachrichten, Jahrgang. 12, Nr. 38, S. 9 - 10.

Agricultural Marketing Resource Center (2013): Seasonality of Corn Ethanol Profitability, URL:

http://www.agmrc.org/renewable_energy/ethanol/seasonality-of-corn-ethanolprofitability/, Abrufdatum: 12.08.2013.

Agricultural Marketing Resource Center (2012): Seasonality of corn ethanol profitability, URL: http://www.agmrc.org/renewable_energy/ethanol/seasonality-ofcorn-ethanol-profitability/, Abrufdatum: 18.11.2013.

Alfstad, T. (2008): Scenario Analysis of Global Biofuel Markets, URL: http://www.bnl.gov/isd/documents/43476.pdf, Abrufdatum: 23.07.2011.

Al-Riffai, P./ Dimaranan, B./ Laborde, D. (2010): Global Trade and Environmental Impact Study of the EU Biofuels Mandate, URL: http://trade.ec.europa.eu/doclib/docs/2010/march/tradoc_145954.pdf, Abrufdatum: 12.09.2012.

Axson, D.A.J. (2011): Szenario Planning: Navigating Through Today's Uncertain World, in: Journal of Accountancy, March 2011, S. 22 - 27.

Aye, C.H. (2009): Die Zukunft der deutschen öffentlichen Apotheken - Eine Szenario-Analyse bis zum Jahr 2020, Hamburg.

Backhaus, K./ Erichson, B./ Plinke, W./ Weiber, R. (2006): Multivariate Analysemethoden - Eine anwendungsorientierte Einführung, 11. Auflage, Berlin/Heidelberg/NewYork. 
BDB $^{e}$ (Bundesverband der deutschen Bioethanolwirtschaft e.V., 2013): Aufgaben und Ziele, URL: http://www.bdbe.de/der-verband/biokraftstoff/\#, Abrufdatum: 28.01.2014.

Becker, A. (2011): Impacts of European biofuel policies on global biofuel and agricultural markets, URL: http://hss.ulb.uni-bonn.de/2011/2650/2650.pdf, Abrufdatum: 30.05.2013.

Belgium National Renewable Energy Action Plan 2010: National Renewable Energy Action Plan pursuant to Directive 2009/28/EC, URL: http://ec.europa.eu/energy/renewables/action_plan_en.htm, Abrufdatum: 03.09.2013.

Berg, C. (2012a): Persönliches Gespräch mit Dr. Christoph Berg am 25.04.2012 in Ratzeburg.

Berg, C. (2012b): Persönliches Gespräch mit Dr. Christoph Berg am 05.12.2012 in Ratzeburg.

Berg, C. (2012c): World Ethanol The Next 10 Years (will there be that many?), Vortrag im Rahmen des Kongresses „WORLD ETHANOL \& BIOFUELS 2012“, 06.11.2012, München.

Berg, C. (2012d): World Biofuels 2012, Vortrag im Rahmen des Kongresses „World Biofuels 2012“, 09.05.2012, Sevilla.

Beta Renewables (2013): The world's first plant for the production of second generation biofuels has been opened in Northern Italy, URL:

http://www.betarenewables.com/site/newsDetail/id/6, Abrufdatum: 22.10.2013.

Bevill, K. (2011a): Shell steps into biofuel production through Brazil joint venture, URL: https://ethanolproducer.com/articles/7994/enter-shell, Abrufdatum: 17.03.2012.

Bevill, K. (2011b): Obama: Stop hitting snooze button on energy security, URL: http://ethanolproducer.com/articles/7627/obama-stop-hitting-snooze-buttonon-energy-security, Abrufdatum: 08.10.2012. 
Biofuels Platform (2012): Production of Bioethanol in the EU, URL:

http://www.biofuels-platform.ch/en/infos/eu-bioethanol.php, Abrufdatum: 26.09.2012.

Blasche, U.G. (2006): Die Szenariotechnik als Modell für komplexe Probleme. Mit Unsicherheiten leben lernen, in: Wilms, F.E.P. (Hrsg.): Szenariotechnik, Bern, S. $61-92$.

Bloomberg (2013): Raízen to Spend \$102 Million on Brazil Cellulosic Ethanol Plant, URL: http://www.bloomberg.com/news/2013-03-13/raizen-to-spend-102million-on-brazil-cellulosic-ethanol-plant.html, Abrufdatum: 19.11.2013.

Bloomberg (2012a): Ethanol-Blended Fuel Is Confusing Consumers, AAA Says, URL: http://www.bloomberg.com/news/2012-11-30/ethanol-blended-gasolinegrade-may-damage-car-engines-aaa-says.html, Abrufdatum: 12.12.2012.

Bloomberg (2012b): Butamax Taps Fagen for First Ethanol-to-Butanol Conversion Plant, URL: http://www.bloomberg.com/news/2012-04-19/butamax-taps-fagenfor-first-ethanol-to-butanol-conversion-plant.html, Abrufdatum: 27.11.2013.

Bloomberg New Energy Finance (2013): Cellulosic Ethanol Heads For Cost Competitiveness by 2016, URL: http://bnef.com/Downloads/pressreleases/284/pdffile/, Abrufdatum: 01.10.2013.

Bloomberg New Energy Finance (2010): Next-generation ethanol and biochemicals: what's in it for Europe?, URL: http://www.bioenergy.novozymes.com/files/documents/BNEF_report_nextgen eration_biofuels.pdf, Abrufdatum: 27.05.2011.

BP (2008): US Energy in Context: Data \& Analysis of US Energy Supply, Production \& Consumption, URL: http://www.bp.com/liveassets/bp_internet/us/bp_us_english/STAGING/local_a ssets/downloads/e/USEnergyinContext_lores_complete1215.pdf, Abrufdatum: 08.10.2012. 
Bradfield, R./ Wright, G./ Burt, G./ Cairns, G/ Heijden, K. Van Der (2005): The origins and evolution of scenario techniques in long range business planning, in: Futures 37, Glasgow, S. $795-812$.

Brockhoff, K. (1977): Prognoseverfahren für die Unternehmensplanung, Wiesbaden.

Bundesanstalt für Landwirtschaft und Ernährung 2013: KN-Code, URL: http://www.ble.de/SharedDocs/GlossarEintraege/K/KN-Code.html, Abrufdatum: 18.10.2013.

Bundesministerium für Wirtschaft, Familie und Jugend: Nationaler Aktionsplan 2010 für erneuerbare Energie für Österreich (NREAP-AT) - gemäß der Richtlinie 2009/28/EG des Europäischen Parlaments und des Rates, URL: http://ec.europa.eu/energy/renewables/action_plan_en.htm, Abrufdatum: 03.09.2013.

Bundesrepublik Deutschland (2009): Nationaler Aktionsplan für erneuerbare Energie gemäß der Richtlinie 2009/28/EG zur Förderung der Nutzung von Energie aus erneuerbaren Quellen, URL:

http://ec.europa.eu/energy/renewables/action_plan_en.htm, Abrufdatum: 03.09. 2013.

Butamax Advanced Biofuels (2013): Butamax Advanced Biofuels LLC fact sheet, URL: http://www.butamax.com/Portals/0/pdf/butamax_advanced_biofuels_Ilc_fact_s heet.pdf, Abrufdatum: 13.08.2013.

California Air Resources Board (2007): Subarticle 7. Low Carbon Fuel Standard, URL: http://www.arb.ca.gov/fuels/lcfs/CleanFinalRegOrder112612.pdf, Abrufdatum: 12.11.2013.

California Energy Commission (2013): Low Carbon Fuel Standard, URL: http://www.energy.ca.gov/low_carbon_fuel_standard/, Abrufdatum: 12.11.2013. 
California Energy Commission (2007): Carbon Intensity Lookup Table for Gasoline and Fuels that Substitute for Gasoline, URL:

http://www.arb.ca.gov/fuels/lcfs/lu_tables_11282012.pdf, Abrufdatum:

12.11.2013.

Center for Automotive Research (2011): The U.S. Automotive Market and Industry in 2025, URL: http://www.cargroup.org/assets/files/ami.pdf, Abrufdatum: 17.10.2012.

Center for Climate and Energy Solutions (2013): Low Carbon Fuel Standard, URL: http://www.c2es.org/us-states-regions/policy-maps/low-carbon-fuelstandard, Abrufdatum: 12.11.2013.

Chatham House (2013): Sustainability and Cost Considerations of Renewable Transport Fuels in the United Kingdom, URL: http://www.v8register.net/FilesDHT/Chatham\%20House\%20Report\%20Renewable\%20Transport\%20Fuels\%20130306.pdf, Abrufdatum: 02.10.2013.

Chau, K.T./ Wong, Y.S. (2002): Overview of power management in hybrid electric vehicles, in: Energy Conversion and Management, Vol. 43, S. 1953 - 1968.

Clariant (2012): Clariant startet Biokraftstoff der Zukunft, URL: http://www.clariant.ch/C12576850036A6E9/A33B719348F4A059C1257A4404 4CCC02/\$FILE/20120720_Clariant_startet_Biokraftstoff_der_Zukunft.pdf, Abrufdatum: 31.10.2012.

Congressional Research Service (2012): Renewable Fuel Standard (RFS): Overview and Issues, URL: https://opencrs.com/document/R40155/, Abrufdatum: 24.03.2012.

Congressional Research Service (2010a): Intermediate-Level Blends of Ethanol in Gasoline, and the Ethanol "Blend Wall", URL: http://www.fas.org/sgp/crs/misc/R40445.pdf, Abrufdatum: 18.10.2012. 
Congressional Research Service (2010b): Agriculture-Based Biofuels: Overview and Emerging Issues, URL:

http://digital.library.unt.edu/ark:/67531/metadc29593/m1/1/high_res_d/R41282 _20100ct14.pdf, Abrufdatum: 20.10.2012.

Congressional Research Service (2010c): Calculation of Lifecycle Greenhouse Gas Emissions for the Renewable Fuel Standard (RFS), URL: http://cnie.org/NLE/CRSreports/10Apr/R40460.pdf, Abrufdatum: 25.11.2013.

COPA COGECA (2014): Die starke Vertretung der europäischen Landwirte und ihrer Genosssenschaften, URL: http://www.copacogeca.be/CogecaHistory.aspx, Abrufdatum: 28.01.2014.

Cornelius, P./ Putte, A. Van de/ Romani, M. (2005): Three Decades of Scenario Planning in Shell, in: California Management Review, Vol. 48, No. 1, S. 92 109.

CropEnergies AG (2013a): Geschäftsbericht 2012/13, URL:

http://www.cropenergies.com/de/investorrelations/Finanzberichte/CEGeschaeftsbericht-2013-05-07-DE.pdf, Abrufdatum: 12.08.2013.

CropEnergies AG (2013b): CropEnergies übernimmt britischen Bioethanolhersteller Ensus, URL: http://www.cropenergies.com/Pdf/de/Presse/Press_releases/Pressemitteilung _19_07_2013.pdf, Abrufdatum: 12.08.2013.

CropEnergies AG (2013c): Präsentation von der Hauptversammlung 30. Juli 2013, URL: http://www.cropenergies.com/de/investorrelations/Praesentationen/2013-0726-HV-Praesentation.pdf, Abrufdatum: 14.08.2013.

CropEnergies AG (2012): Bioethanolanlage Zeitz, URL: http://www.cropenergies.com/de/Downloads/Broschueren/CE-Zeitz-2012.pdf, Abrufdatum: 13.10.2013. 
Czech Republik Ministry of Industry and Trade (2012): National Renewable Energy Action Plan of the Czech Republic, URL:

http://ec.europa.eu/energy/renewables/action_plan_en.htm, Abrufdatum: 03.09.2013.

De La Torre Ugarte, D.G./ Burton, B.C./ Jensen, K. (2007): Sixty Billion Gallons by 2030: Economic and Agricultural Impacts of Ethanol and Biodiesel Expansion, in: American Journal of Agricultural Economics, Vol. 89, Issue 5, S.1290 $-1295$.

DUPONT (2012): About DUPONT Biofuel Solutions, URL:

http://www.ddce.com/about/history.html, Abrufdatum: 31.10.2012.

Ecofys/ Agra CEAS/ Chalmers University/ IIASA/ Winrock (2011): Biofuels Baseline 2008, URL:

http://www.ecofys.com/files/files/ecofys_2011_biofuels_baseline(2008).pdf, Abrufdatum: 24.03.2012.

EFOA (The European Fuel Oxygenates Association, 2013): What are the advantages of fuel ether blending compared to ethanol blending?, URL: http://www.efoa.eu/en/faq/fuel-ethers---benefits-and-uses/what-are-theadvantages-of-fuel-ether-blending-compared-to-ethanol-blending.aspx, Abrufdatum: 08.08.2013.

Environmental Protection Agency (2012): Fuel Economy Leaders: 2012 Model Year, Washington D.C., URL: http://www.epa.gov/fueleconomy/overallhigh.htm, Abrufdatum: 20.03.2012.

Environmental Protection Agency/ Department of Transportation (2012): 2017 and Later Model Year Light-Duty Vehicle Greenhouse Gas Emissions and Corporate Average Fuel Economy Standards, in: Federal Register, Vol. 77, No. 199, S. $62623-63200$.

EPA (United States Environmental Protection Agency, 2012a): Fuels and Fuel Additives. E15: Frequently Asked Questions, URL: http://www.epa.gov/otaq/regs/fuels/additive/e15/e15-faq.htm, Abrufdatum: 17.12.2012. 
EPA (United States Environmental Protection Agency, 2012b): E15 (a blend of gasoline and ethanol), URL: http://www.epa.gov/otaq/regs/fuels/additive/e15/, Abrufdatum: 11.12.2012.

EPA (United States Environmental Protection Agency, 2011): EPA Finalizes 2012 Renewable Fuel Standards, URL: http://www.epa.gov/otaq/fuels/renewablefuels/documents/420f11044.pdf, Abrufdatum: 30.11.2012.

EPA (United States Environmental Protection Agency, 2010): EPA Finalizes Regulations for the National Renewable Fuel Standard Program for 2010 and Beyond, URL: http://www.epa.gov/oms/renewablefuels/420f07019.pdf, Abrufdatum: 30.11.2012.

ePURE (European Renewable Ethanol, 2013): Who We Are, URL: http://www.epure.org/who-we-are, Abrufdatum: 28.01.2014.

ePURE (European Renewable Ethanol, 2010): Position Paper Indirect land use change impacts of biofuels, URL: http://www.epure.org/sites/default/files/publication/fr7f000001-0ffd-9570.pdf, Abrufdatum: 24.09.2013.

Ernst \& Young (2011): Biofuels and indirect land use change. The case for mitigation, URL: http://www.epure.org/pdf/443ea6c089-be92-7321.pdf, Abrufdatum: 14.09.2012.

Ethanol Producer Magazine (2013a): Project Liberty construction on target for early 2014 startup, URL:

http://www.ethanolproducer.com/articles/9937/project-liberty-construction-ontarget-for-early-2014-startup, Abrufdatum: 13.08.2013.

Ethanol Producer Magazine (2013b): EPA proposes 2014 renewable fuels standards, URL: http://ethanolproducer.com/articles/10476/epa-proposes-2014renewable-fuels-standards, Abrufdatum: 19.11.2013. 
Ethanol Producer Magazine (2013c): EPA proposes 2014 renewable fuels standards, URL: http://ethanolproducer.com/articles/10476/epa-proposes-2014renewable-fuels-standards, Abrufdatum: 20.11.2013.

Ethanol Producer Magazine (2013d): Murphy USA opens first Arkansas E15 station, URL: http://ethanolproducer.com/articles/10560/murphy-usa-opens-firstarkansas-e15-station, Abrufdatum: 18.12.2013.

Ethanol Producer Magazine (2012a): EU to alter RED to require advanced, cellulosic biofuels, URL: http://ethanolproducer.com/articles/9136/eu-to-alter-redto-require-advanced-cellulosic-biofuels, Abrufdatum: 25.09.2012.

Ethanol Producer Magazine (2012b): GM, Ford announce E15 compatibility with new models, URL: http://ethanolproducer.com/articles/9195/gm-fordannounce-e15-compatibility-with-new-models, Abrufdatum: 23.10.2012.

Ethanol Producer Magazine (2012c): The Battle for the RFS, URL: http://www.ethanolproducer.com/articles/8857/the-battle-for-the-rfs, Abrufdatum: 20.06.2012.

Ethanol Producer Magazine (2012d): Dinneen tells ethanol industry to brace for brutal RFS fight, URL: http://www.ethanolproducer.com/articles/8844/dinneentells-ethanol-industry-to-brace-for-brutal-rfs-fight, Abrufdatum: 20.06.2012.

Ethanol Producer Magazine (2012e): Enery Beets: Who will Leap First?, URL: http://www.ethanolproducer.com/articles/8707/energy-beets-who-will-leap-first, Abrufdatum: 25.01.2013.

Ethanol Producer Magazine (2012f): Sweet Sorghum, Other Sugar Crops Show Promise, URL: http://www.ethanolproducer.com/articles/8856/sweet-sorghumother-sugar-crops-show-promise, Abrufdatum: 25.01.2013.

Ethanol Producer Magazine (2012g): lowa ethanol plants signal intent to produce butanol, URL: http://ethanolproducer.com/articles/8753/iowa-ethanol-plantssignal-intent-to-produce-butanol, Abrufdatum: 27.11.2013. 
Ethanol Producer Magazine (2012h): Fuel Ethanol Workshop: 87\% of ethanol producers to attend, URL: http://ethanolproducer.com/articles/8821/fuelethanol-workshop-87-of-ethanol-producers-to-attend, Abrufdatum: 02.12.2013.

EUROOBSERV'ER (2013): Biofuels Barometer, URL: http://www.energiesrenouvelables.org/observ-er/stat_baro/observ/baro216_en.pdf, Abrufdatum: 12.08.2013.

European BEST project (2010): BioEthanol for Sustainable Transport, URL: https://www.eve.es/ecomovil/pdf/BEST_FinalReport.pdf, Abrufdatum: 14.03.2012.

European Commission (2013a): EU energy in figures - Statistical Pocketbook 2013, URL: http://ec.europa.eu/energy/publications/doc/2013_pocketbook.pdf, Abrufdatum: 16.08.2013.

European Commission (2013b): Renewable energy - Action Plans \& Forecasts, URL: http://ec.europa.eu/energy/renewables/action_plan_en.htm, Abrufdatum: 20.09.2013.

European Commission (2012a): EU energy in figures - Statistical Pocketbook 2012, URL:

http://ec.europa.eu/energy/publications/doc/2012_energy_figures.pdf, Abrufdatum: 17.08.2012.

European Commission (2012b): Commission Staff Working Document NER300 Moving towards a low carbon economy and boosting innovation, growth and employment across the EU, URL:

http://ec.europa.eu/clima/news/docs/2012071201_swd_ner300.pdf, Abrufdatum: 03.09.2013.

\section{European Commission Directorate - General for Energy (2009): EU energy} trends to 2030, URL:

http://ec.europa.eu/energy/observatory/trends_2030/doc/trends_to_2030_upu pda_2009.pdf, Abrufdatum: 09.08.2012. 
European Commission Directorate - General for Research Sustainable Energy Systems (2006): Biofuels in the European Union - A vision for 2030 and beyond, URL:

ftp://ftp.cordis.europa.eu/pub/fp7/energy/docs/biofuels_vision_2030_en.pdf, Abrufdatum: 21.05.2013.

European Commission Joint Research Centre, Institute for Energy (2011): Estimate of GHG emissions from global land use change scenarios, URL: http://iet.jrc.ec.europa.eu/sites/default/files/documents/scientific_publications/2 011/technical_note_eu24817.pdf, Abrufdatum: 14.09.2012.

European Commission Joint Research Centre, Institute for Energy (2010): Indirect Land Use Change from increased biofuels demand, Comparison of models and results for marginal biofuels production from different feedstocks, URL: http://ec.europa.eu/energy/renewables/consultations/doc/public_consultation_i luc/study_4_iluc_modelling_comparison.pdf, Abrufdatum: 14.09.2012.

\section{European Commission Joint Research Centre, Institute for Energy/ EUCAR/}

CONCAWE (2011): EU renewable energy targets in 2020: Analysis of scenarios for transport, URL:

http://ies.jrc.ec.europa.eu/uploads/jec/JECBiofuels\%20Report_2011_PRINT.p df, Abrufdatum: 24.07.2011.

\section{European Commission Joint Research Centre, Institute for Energy/ EUCAR/} CONCAWE (2010): Overview of Results, URL:

http://ies.jrc.ec.europa.eu/uploads/jec/JEC\%20Biofuels\%20Programme.pdf, Abrufdatum: 30.08.2012

European Commission Joint Research Centre, Institute for Prospective Technological Studies (2010a): Impacts of the EU Biofuel Target on Agricultural Markets and Land use - A Comparative Modelling Assessment, URL: http://ipts.jrc.ec.europa.eu/publications/pub.cfm?id=3439, Abrufdatum: 08.08.2011. 


\section{European Commission Joint Research Centre, Institute for Prospective Tech-}

nological Studies (2010b): Plug-in Hybrid and Battery Electric Vehicles Market penetration scenarios of electric drive vehicles, URL: http://ftp.jrc.es/EURdoc/JRC58748_TN.pdf, Abrufdatum: 22.03.2012.

Europäische Kommission (2013): Kumulierte monatliche Rohöleinfuhren (Mengen und Preise) nach EU-Ländern und Drittländern, URL: http://ec.europa.eu/energy/observatory/oil/doc/import/coi/crude-oil-imports.zip, Abrufdatum: 16.08.2013.

Europäische Kommission (2012): Vorschlag für eine Richtlinie des Europäischen Parlaments und des Rates zur Änderung der Richtlinie 98/70/EG über die Qualität von Otto- und Dieselkraftstoffen und zur Änderung der Richtlinie 2009/28/EG zur Förderung der Nutzung von Energie aus erneuerbaren Quellen, URL:

http://ec.europa.eu/energy/renewables/biofuels/doc/biofuels/com_2012_0595_ de.pdf, Abrufdatum: 18.10.2012.

Europäische Kommission (2011): Verordnung (EU) Nr. 1006/2011 DER KOMMISSION, URL: http://eurlex.europa.eu/LexUriServ/LexUriServ.do?uri=OJ:L:2011:282:0001:0912:DE:P DF, Abrufdatum: 03.03.2012.

Europäische Kommission (2010): Energie 2020. Eine Strategie für wettbewerbsfähige, nachhaltige und sichere Energie, URL: http://eurlex.europa.eu/LexUriServ/LexUriServ.do?uri=COM:2010:0639:FIN:DE:PDF, Abrufdatum: 19.09.2012.

Europäische Kommission eurostat (2012): Personenkraftwagen, nach Antriebsenergie des Motors, URL: http://appsso.eurostat.ec.europa.eu/nui/setupModifyTableLayout.do, Abrufdatum: 25.08.2012.

Europäische Union (2014): Kroatien, URL: http://europa.eu/abouteu/countries/member-countries/croatia/index_de.htm, Abrufdatum: 08.02.2014. 
European Biofuels (2013a): Biobutanol, URL: http://www.biofuelstp.eu/butanol.html, Abrufdatum: 20.08.2013.

European Biofuels (2013b): Biomass to Liquids (BtL), URL:

http://www.biofuelstp.eu/btl.html, Abrufdatum: 03.09.2013.

Eurostat (2012): Versorgung, Umwandlung, Verbrauch - erneuerbare Energien (Biotreibstoff) - Jährliche Daten, URL:

http://appsso.eurostat.ec.europa.eu/nui/print.do?print=true, Abrufdatum:

27.09.2012.

FAPRI (Food and Agricultural Policy Research Institute, 2012): FAPRI-ISU 2012 World Agricultural Outlook, URL: http://www.fapri.iastate.edu/outlook/2012/, Abrufdatum: 21.01.2013.

FAPRI (Food and Agricultural Policy Research Institute, 2011a): FAPRI-ISU 2011 World Agricultural Outlook, URL:

http://www.fapri.iastate.edu/outlook/2011/tables/1_wheat.pdf, Abrufdatum: 09.09.2012.

FAPRI (Food and Agricultural Policy Research Institute, 2011b): FAPRI-ISU 2011 World Agricultural Outlook, URL:

http://www.fapri.iastate.edu/outlook/2011/tables/2_grain.pdf, Abrufdatum: 10.09.2012.

FAPRI (Food and Agricultural Policy Research Institute, 2011c): FAPRI-ISU 2011 World Agricultural Outlook, URL:

http://www.fapri.iastate.edu/outlook/2011/tables/5_biofuels.pdf, Abrufdatum: 26.09.2012.

Fink, A. (2011): Intensiv-Workshop Szenario-Management vom 16.06. bis zum 17.06.2011 in Hohenkammer.

Fink, A./ Siebe, A. (2006): Handbuch Zukunftsmanagement - Werkzeuge der strategischen Planung und Früherkennung, Frankfurt am Main. 
Fink, A./ Schlake, O./ Siebe, A. (2002): Erfolg durch Szenario-Management - Prinzip und Werkzeuge der strategischen Vorausschau, Frankfurt am Main.

Fink, A./ Schlake, O./ Siebe, A. (2000): Wie Sie mit Szenarien die Zukunft vorausdenken, in: Harvard Business Manager (Hrsg.): Sonderdruck aus Heft 2, I. Quartal, S. 2 - 13.

Finkbeiner, M. (2013): Indirekte Landnutzungsänderungen in Ökobilanzen - wissenschaftliche Belastbarkeit und Übereinstimmung mit internationalen Standards, URL: http://www.ufop.de/index.php/download_file/view/1820/570/, Abrufdatum: 24.09.2013.

Finland Ministry of Employment and the Economy Energy Department (2010): Finland's national action plan for promoting energy from renewable sources pursuant to Directive 2009/28/EC, URL: http://ec.europa.eu/energy/renewables/action_plan_en.htm, Abrufdatum: 03.09.2013.

FNR (Fachagentur Nachwachsende Rohstoffe e.V., 2009): Biokraftstoffe - Eine vergleichende Analyse, Gülzow.

F.O. Licht (2013a): Global Cellulosic Biofuels Capacity could Hit 1 BLN Litre Mark in 2015, in: World Ethanol \& Biofuels Report, Vol.11, No. 12, S. 236 - 240.

F.O. Licht (2013b): Crunch Time for the US Ethanol Industry?, in: World Ethanol \& Biofuels Report, Vol.11, No. 11, S. $212-215$.

F.O. Licht (2013c): World Ethanol Production Continues on Consolidation Course, in: World Ethanol \& Biofuels Report, Vol.11, No. 17, S. 340 - 343.

F.O. Licht (2013d): "Dialogue of the Deaf" Coming to an End in Brazil, in: World Ethanol \& Biofuels Report, Vol. 11, No. 15, S. $294-298$.

F.O. Licht (2013e): Blending Demand for Fuel Ethanol Recovers in June, in: World Ethanol \& Biofuels Report, Vol. 11, No. 24, S. 500. 
F.O. Licht (2013f): World Ethanol Trade to Critically Depend on US Demand, in: World Ethanol \& Biofuels Report, Vol. 11, No. 19, S. 386 - 391.

F.O. Licht (2013g): World Ethanol Production Shows Signs of Recovery, in: World Ethanol \& Biofuels Report, Vol. 12, No. 4, S. $60-63$.

F.O. Licht (2013h): Structural Change in the EU's Ethanol Industry, in: World Ethanol \& Biofuels Report, Vol. 12, No. 5, S. $82-85$.

F.O. Licht (2012a): 2012 World Ethanol Trade May Shrink After 2011 Record, in: World Ethanol \& Biofuels Report, Vol.10, No. 15, S. $300-304$.

F.O. Licht (2012b): The World of Biofuels in 2012, in: World Ethanol \& Biofuels Report, Vol. 10, No. 9, S.168-172.

F.O. Licht (2012c): Recovery in World Ethanol Production Critically Depends on Brazil, in: World Ethanol \& Biofuels Report, Vol. 10, No. 16, S.318-323.

F.O. Licht (2012d): European Union: Member State Legislations for Biofuels, in: World Ethanol \& Biofuels Report, Vol. 10, No. 20, S. $410-411$.

F.O. Licht (2012e): Commission Proposal Seeks to Limit Use of Food-Based Biofuels, in: World Ethanol \& Biofuels Report, Vol. 11, No. 2, S.27 - 28.

F.O. Licht (2012f): World Ethanol Production may see only Lacklustre Recovery in 2013, in: World Ethanol \& Biofuels Report, Vol. 11, No. 4, S.73 - 74.

F.O. Licht (2012g): To Waive or Not to Waive - The RFS Under Siege, in: World Ethanol \& Biofuels Report, Vol. 10, No. 24, S. 488 - 490.

F.O. Licht (2012h): Picking up the Pieces the US Ethanol Industry in 2012, in: World Ethanol \& Biofuels Report, Vol. 10, No. 10, S.190 - 194.

F.O. Licht (2011a): World Ethanol Production Falls In 2011 - Next Years's Rebound May Be Modest, in: World Ethanol \& Biofuels Report, Vol. 10, No. 4, S. $63-67$. 
F.O. Licht (2011b): EU Tightens Import Rules For Ethanol, in: World Ethanol \& Biofuels Report, Vol. 10, No. 5, S. $86-88$.

F.O. Licht (2011c): ETBE - A Product on the Decline, in: World Ethanol \& Biofuels Report, Vol. 10, No. 8, S. $148-150$.

F.O. Licht (2011d): US Ethanol Producers Extend their Lead in World Ranking, Vol. 9, No. 19 , S. $352-356$.

F.O. Licht (2010): Still the Promising Option? Cellulosic Ethanol in the EU and the USA, in: World Ethanol \& Biofuels Report, Vol. 8, No. 22, S. 450 - 455.

F.O. Licht (2009): World Ethanol Industry May be in for Recovery in 2010, in: World Ethanol \& Biofuels Report, Vol.8, No.4, S. $67-71$.

F.O. Licht/ AGRA informa (2009): World Ethanol Markets - The Outlook to 2020, Ratzeburg.

Frankfurter Allgemeine Zeitung (2012): Tank oder Teller Deutschland "vermaist", URL: http://www.faz.net/aktuell/politik/inland/tank-oder-teller-deutschlandvermaist-11860119.html, Abrufdatum: 26.10.2012.

Fraunhofer ISI/ NEA/ TRT/ TML/ IWW/ IPTS/ TNO (Frauenhofer Institute Systems and Innovation Research/ Transport Research and Training/ Trasporti e Territorio SRL/ Transport \& Mobility/ Institute for Economic Policy Research University of Karlsruhe/ Institute for Prospective Technological Studies European Commission/ The Netherlands Organisation for Applied Scientific Research, 2010): iTREN-2030 Integrated transport and energy baseline until 2030, URL: http://www.isi.fraunhofer.de/isimedia/docs/n/en/publikationen/iTREN_2030_D6_Final_Report.pdf?WSESSIW SES=a2ae5cccbc583256410081e5a795c112, Abrufdatum: 24.08.2012.

Frerichs, W./ Kübler, K. (1980): Gesamtwirtschaftliche Prognoseverfahren, München.

Garson, D. (2012): Testing Statistical Assumptions, URL:

http://www.statisticalassociates.com/assumptions.pdf, Abrufdatum: 16.09.2013. 
Gausemeier, J./ Plass, C./ Wenzelmann, C. (2009): Zukunftsorientierte Unternehmensgestaltung - Strategien, Geschäftsprozesse und IT-Systeme für die Produktion von morgen, München.

Gausemeier, J./ Fink, A./ Schlake, O. (1995): Szenario-Management: Planen und Führen mit Szenarien, München.

Georgoff, D.M./ Murdick, R.G. (1986): Manager's Guide to Forecasting: How to choose the best technique, or combination of techniques, to help solve your particular forecasting dilemma, in: Harvard Business Review (Hrsg.): Accurate Business Forecasting, Boston, S. $1-11$.

Geschka, H./ Hell, W./ Schwarz-Geschka, M. (2006): Zukunft der Mobilität. Ein umfassendes Szenarioprojekt, in: Wilms, F.E.P. (Hrsg.): Szenariotechnik, Bern, S. $241-263$.

Geschka, H./ Hammer, R. (1986): Die Szenario-Technik in der strategischen Unternehmensplanung, in: Hahn, D./ Taylor, B. (Hrsg.): Strategische Unternehmensplanung, 4. Auflage, Heidelberg, S. $238-263$.

Geschka, H./ Reibnitz, U. von (1986): Die Szenario-Technik - Ein Instrument der Zukunftsanalyse und der strategischen Planung, in: Töpfer, A./ Afheldt, H. (Hrsg.): Praxis der strategischen Unternehmensplanung, 2. Auflage, Stuttgart, S. $125-170$.

Gevo (2013): Company Overview, URL: http://gevo.com/about/company-overview/, Abrufdatum: 21.08.2013.

\section{Gobierno de Espana Ministerio de Industria, Turismo Y Comercio (2010):}

Spain's National Renewable Energy Action Plan 2011-2020, URL: http://ec.europa.eu/energy/renewables/action_plan_en.htm, Abrufdatum: 03.09.2013.

Götze, U. (1993): Szenario-Technik in der strategischen Unternehmensplanung, 2. Auflage, Wiesbaden. 
Götze, U. (2006): Cross-Impact-Analyse zur Bildung und Auswertung von Szenarien, in: Wilms, F.E.P. (Hrsg.): Szenariotechnik, Bern, S. $145-181$.

Graf, H.G. (2000): Globale Szenarien - Megatrends im weltweiten Kräftespiel, Frankfurt am Main.

Grant, R. M./ Nippa, M. (2006): Strategisches Management: Analyse, Entwicklung und Implementierung von Unternehmensstrategien, 5. Auflage, München.

Greece Ministry of Environment Energy \& Climate Change (2010): National Renewable Energy Action Plan in the Scope of Directive 2009/28/EC, URL: http://ec.europa.eu/energy/renewables/action_plan_en.htm, Abrufdatum: 03.09.2013.

Growth Energy (2013): Growth Energy Leadership, URL: http://www.growthenergy.org/about-growth-energy/structuremembers/leadership-board-of-directors/, Abrufdatum: 04.01.2013.

GRUPPO MOSSI \& GHISOLFI (2012): Beta Renewables and Novozymes to form strategic partnership in the cellulosic biofuel market, URL: http://www.gruppomg.com/news.php?newsid=33, Abrufdatum: 31.10.2012.

Halliburton (2013): Hydraulic Fracturing 101, URL:

http://www.halliburton.com/public/projects/pubsdata/hydraulic_fracturing/fractu ring_101.html, Abrufdatum: 06.12.2013.

Hees, F./ Michulitz, C./ Richert, A. (2006): Szenarien in Aus- und Weiterbildung, in: Wilms, F.E.P. (Hrsg.): Szenariotechnik, Bern, S. $291-304$.

Heinecke, A. (2006): Die Anwendung induktiver Verfahren in der Szenario-Technik, in: Wilms, F.E.P. (Hrsg.): Szenariotechnik, Bern, S. $183-213$.

Henniges, O. (2007): Die Bioethanolproduktion - Wettbewerbsfähigkeit in Deutschland unter Berücksichtigung der internationalen Konkurrenz, 2. Auflage, Lohmar/Köln. 
Henniges, O./ Zeddies, J. (2006): Bioenergy in Europe: Experiences and Prospects, in: IFPRI (Hrsg.): Bioenergy and Agriculture Promises and Challenges, Focus 14, Brief 9 of 12.

Hentze, H./ Müller, K. D./ Schlicksupp, H. (1989): Praxis der Managementtechniken, München.

Herzhoff, M. (2004): Szenario-Technik in der chemischen Industrie - Untersuchung von Software-Tools am Beispiel einer Studie zum Markt für Flammschutzmittel im Jahr 2010 und der praktischen Bedeutung der Szenario-Technik, Berlin.

Hungary (2010): Hungary's Renewable Energy Utilisation Action Plan - on trends in the use of renewable energy sources until 2020, URL:

http://ec.europa.eu/energy/renewables/action_plan_en.htm, Abrufdatum: 03.09.2013.

Huss, W.R./ Honton, E.J. (1987): Scenario Planning - What Style Should You Use?, in: Long Range Planning, Vol. 20, S. $21-29$.

ICCT (The International Council on Clean Transportation, 2012): European Vehicle Market Statistics - Pocketbook 2012, URL: http://www.theicct.org/sites/default/files/publications/Pocketbook_LowRes_wit hNotes-1.pdf, Abrufdatum: 22.07.2013.

ICF International/ Ecologic Institute (2011): An overview of Electric Vehicles on the market and in development, Delft, URL: http://ec.europa.eu/clima/policies/transport/vehicles/docs/d1_en.pdf, Abrufdatum: 21.03.2012.

IER/ RWI/ ZEW (Institut für Energiewirtschaft und Rationelle Energieanwendung/ Rheinisch-Westfälisches Institut für Wirtschaftsforschung/ Zentrum für Europäische Wirtschaftsforschung, 2010): Die Entwicklung der Energiemärkte bis 2030, URL: http://www.ier.unistuttgart.de/publikationen/energieprognose2009/Energieprognose_2009_Hau ptbericht.pdf, Abrufdatum: 31.07.2013. 
ifeu (Institut für Energie und Umweltforschung Heidelberg GmbH, 2008): Bioenergie aus Getreide und Zuckerrübe: Energie- und Treibhausgasbilanzen, URL: http://www.ifeu.org/landwirtschaft/pdf/IFEU_Bioenergie_LAB.pdf, Abrufdatum: 12.08.2013.

ifeu (Institut für Energie und Umweltforschung Heidelberg GmbH, 2004): $\mathrm{CO}_{2}$ neutrale Wege zukünftiger Mobilität durch Biokraftstoffe - Eine Bestandsaufnahme, Heidelberg.

IFPRI (Institut für Energie und Umweltforschung Heidelberg GmbH, 2012):

Ethanol Trade Policy and Global Biofuel Mandates, URL:

http://ageconsearch.umn.edu/bitstream/126869/2/ICAE\%20Brazil\%20BF\%20fi nal\%20sub.pdf, Abrufdatum: 12.09.2012.

IFPRI (Institut für Energie und Umweltforschung Heidelberg GmbH, 2011): Assessing the Land Use Change Consequences of European Biofuel Policies, URL: http://trade.ec.europa.eu/doclib/docs/2011/october/tradoc_148289.pdf, Abrufdatum: 14.09.2012.

Industry Week (2012): Brazil to Boost Sugarcane Output for Ethanol, URL: http://www.industryweek.com/articles/brazil_to_boost_sugarcane_output_for_ ethanol_26685.aspx?ShowAll=1, Abrufdatum: 17.03.2012.

International Energy Agency (2012): World Energy Outlook 2012, Paris.

International Energy Agency (2011a): World Energy Outlook 2011, URL: http://han.sub.uni-goettingen.de/han/91597/www.oecdilibrary.org/docserver/download/fulltext/6111241e.pdf ?expires $=1345226531 \& \mathrm{i}$ $\mathrm{d}=\mathrm{id} \&$ accname $=$ oid009539\&checksum $=E 9650$ A7A27C4FB9CF7C56BF5D6B C7217, Abrufdatum: 03.08.2012.

International Energy Agency (2011b): Technology Roadmap Biofuels for Transport, URL: http://www.iea.org/papers/2011/biofuels_roadmap.pdf, Abrufdatum: 22.09.2011. 
International Energy Agency (2010a): World Energy Outlook 2010, URL:

http://han.sub.uni-goettingen.de/han/91597/www.oecd-

ilibrary.org/energy/world-energy-outlook_20725302, Abrufdatum: 07.07.2011.

International Energy Agency (2010b): Sustainable Production of Second-

Generation Biofuels - Potential and perspectives in major economics and developing countries, URL:

http://www.iea.org/publications/freepublications/publication/second_generation biofuels.pdf, Abrufdatum: 27.05.2013.

International Energy Agency (2009): Medium-Term Oil Market Report, URL:

http://omrpublic.iea.org/omrarchive/mtomr2009.pdf, Abrufdatum: 07.07.2011.

International Energy Agency Bioenergy (2007): Market Evalution: Fuel Ethanol, URL: http://www.bioenergytrade.org/reports/archive/reports-2007-2009.html, Abrufdatum: 09.03.2012.

International Sugar Organization (2011): Sugar, Fuel Ethanol and Crude Oil Prices, URL: http://www.unctad.info/upload/GCF2011/doc/A1A3/gcf2011_A2_Jolly.en.pdf, Abrufdatum: 04.10.2012.

lowa State University (2007): The ethanol outlook for Brazil and the United States and implications for livestock, in: INTERNATIONAL SUGAR JOURNAL, Vol. 109, No. 1299 , S. $174-177$.

Ireland (2010): National Renewable Energy Action Plan, URL:

http://ec.europa.eu/energy/renewables/action_plan_en.htm, Abrufdatum: 03.09.2013.

Italian Ministry for Economic Development (2010): Italian National Renewable Energy Action Plan, URL:

http://ec.europa.eu/energy/renewables/action_plan_en.htm, Abrufdatum: 03.09.2013.

Jessen, H. (2012): By the numbers - Trends and patterns in the ethanol industry, in: Ethanol Producer Magazine, April 2012, S. 34 - 39. 
Johnson, G./ Scholes, K./ Whittington, R. (2011): Strategisches Management: Eine Einführung - Analyse, Entscheidung und Umsetzung, 9. Auflage, München.

Kahn, H./ Wiener, A.J. (1968): The Year 2000 - A Framework for Speculation on the next Thirty-Three Years, 4. printing, New York.

Kastenhuber, M. (2007): Bioethanol - Kraftstoff der Zukunft? Ganzheitliche Analyse und empirische Erhebung über die Zukunftschancen des Kraftstoffs Bioethanol, Hamburg.

KiOR (2013): Home, URL: http://www.kior.com/, Abrufdatum: 27.11.2013.

Klein, G./ Graf, H.G./ Schöllhorn, A. (2006): Entscheidungsvorbereitung mit Szenarien im Team-Dialog, in: Wilms, F.E.P. (Hrsg.): Szenariotechnik, S. 351 379.

Klima- OG Energiministeriet (2010): National Action Plan - For renewable energy in Denmark, URL: http://ec.europa.eu/energy/renewables/action_plan_en.htm, Abrufdatum: 03.09.2013.

Kosow, H./ Gaßner, R. (2008): Methoden der Zukunfts- und Szenarioanalyse Überblick, Bewertung und Auswahlkriterien, in: Institut für Zukunftsstudien und Technologiebewertung (Hrsg.): Werkstatt Bericht Nr. 103, Berlin.

\section{Le Gouvernment du Grand-Duche de Luxembourg Ministère de l'économie et} du Commerce extérieur (2010): Luxembourg action plan for renewable energy, URL: http://ec.europa.eu/energy/renewables/action_plan_en.htm, Abrufdatum: 03.09.2013.

Lithuania (2010): National Renewable Energy Action Plan, URL: http://ec.europa.eu/energy/renewables/action_plan_en.htm, Abrufdatum: 03.09.2013.

LMC INTERNATIONAL (2011): Ethanol in Europe, in: Commodity Market Development in Europe - Outlook, URL: http://ftp.jrc.es/EURdoc/JRC67918.pdf, Abrufdatum: 01.11.2012. 
Majandus- ja Kommunikatsiooniministeerium (2010): National Renewable Energy Action Plan Estonia, URL:

http://ec.europa.eu/energy/renewables/action_plan_en.htm, Abrufdatum:

03.09.2013.

Malta (2011): Template For National Renewable Energy Action Plans, URL:

http://ec.europa.eu/energy/renewables/action_plan_en.htm, Abrufdatum:

03.09.2013.

Massachusetts Institute of Technology (2007): Units \& Conversions Fact Sheet, URL:

http://www.mitenergyclub.org/assets/2008/11/15/Units_ConvFactors.MIT_Ene rgyClub_Factsheet.v8.pdf, Abrufdatum: 14.08.2013.

Mercer, D. (1995): Scenarios Made Easy, in: Long Range Planning, Vol. 28, Nr. 4, S. $81-86$.

Meyer-Schönherr, M. (1992): Szenario-Technik als Instrument der strategischen Planung, Berlin.

Millett, S.M./ Honton, E.J. (1991): A Managers's Guide to Technology Forecasting and Strategy Analysis Methods, Columbus.

Ministère de l'Écologie, de l'Énergie, du Développement durable et de la Mer (2009): National action plan for the promotion of renewable energies 20092020, URL : http://ec.europa.eu/energy/renewables/action_plan_en.htm, Abrufdatum: 03.09.2013.

Ministry of Economy and Construction of the Slovak Republic (2010): National Renewable Energy Action Plan, URL: http://ec.europa.eu/energy/renewables/action_plan_en.htm, Abrufdatum: 03.09.2013.

Mißler-Behr, M. (1993): Methoden der Szenarioanalyse, Wiesbaden.

Mißler-Behr, M. (2006): Auf der Suche nach Zukunftsbildern - Eine Regelbasis zur Szenarienauswahl, in: Wilms, F.E.P. (Hrsg.): Szenariotechnik, S. $215-239$. 
Monbiot, G. (2007): If we want to save the planet, we need a five-year freeze on biofuels, URL:

http://www.guardian.co.uk/commentisfree/2007/mar/27/comment.food, Abrufdatum: 19.03.2012.

NACS (The Association for Convenience \& Fuel Retailing, 2012a): NACS Retail Fuels Report 2012, URL:

http://www.nacsonline.com/NACS/Resources/campaigns/GasPrices_2012/Do cuments/2012NACSFuelsReport_full.pdf, Abrufdatum: 17.12.2012.

NACS (The Association for Convenience \& Fuel Retailing, 2012b): The Future of Fuels: An Analysis of Future Energy Trends and Potential Retail Market Opportunities, URL: http://www.nacsonline.com/NACS/Resources/campaigns/GasPrices_2012/Do cuments/FutureofFuelsReport_2012.pdf, Abrufdatum: 17.12.2012.

NACS (The Association for Convenience \& Fuel Retailing, 2011): Fueling America: Key Facts and Figures, URL: http://www.nacsonline.com/NACS/Resources/campaigns/GasPrices_2011/Do cuments/GasPriceKit2011.pdf, Abrufdatum: 13.12.2012.

National Renewable Energy Laboratory (2013): Production Facilities Ethanol, URL: http://maps.nrel.gov/transatlas, Abrufdatum: 15.11.2013.

National Renewable Energy Laboratory (2009): Effects of Intermediate Ethanol Blends on Legacy Vehicles and Small Non-Road Engines, Report 1 - Updated, URL: http://www.nrel.gov/docs/fy09osti/43543.pdf, Abrufdatum: 13.12.2012.

National Renewable Energy Laboratory (2007): Research Advances Cellulosic Ethanol, URL: http://www.nrel.gov/biomass/pdfs/40742.pdf, Abrufdatum: 10.09.2013.

National Renewable Energy Laboratory (2006): Cost-Benefit Analysis of Plug-In Hybrid Electric Vehicle Technology, URL: http://www.nrel.gov/vehiclesandfuels/vsa/pdfs/40485.pdf, Abrufdatum: 22.03.2012. 
NCGA (National Corn Growers Association, 2012): Corn Rooted in Human History. 2012 World of Corn, URL:

http://www.ncga.com/uploads/useruploads/woc_2012.pdf, Abrufdatum:

22.03.2012.

Netherlands (2010): National renewable energy action plan, URL:

http://ec.europa.eu/energy/renewables/action_plan_en.htm, Abrufdatum:

03.09.2013.

Oak Ridge National Laboratory (2012): Transportation Energy Data Book Edition 31, URL: http://cta.ornl.gov/data/tedb31/Edition31_Full_Doc.pdf, Abrufdatum: 17.10.2012.

Oak Ridge National Laboratory (2011): Intermediate Ethanol Blends Infrastructure Materials Compatibility Study: Elastomers, Metals, and Sealants, URL: http://info.ornl.gov/sites/publications/files/Pub27766.pdf, Abrufdatum: 23.10.2012.

Oak Ridge National Laboratory (2007): Fuel Economy and Emissions of the Ethanol-Optimized Saab 9-5 Bipower, URL:

http://www1.eere.energy.gov/biomass/pdfs/analysis_saab2007.pdf, Abrufdatum: 13.12.2012.

OECD (Organisation for Economic Co-operation and Development, 2012): Die OECD in Zahlen und Fakten 2011-2012, URL: http://www.oecdilibrary.org/economics/die-oecd-in-zahlen-und-fakten-20112012_9789264125476-de, Abrufdatum: 19.08.2012.

OECD/ FAO (Organisation for Economic Co-operation and Development/ Food and Agriculture Organization of the United Nations, 2012): OECD-FAO Agricultural Outlook 2012-2021, URL: http://www.oecd-ilibrary.org/agricultureand-food/oecd-fao-agricultural-outlook-2012_agr_outlook-2012-en, Abrufdatum: 02.08.2012. 
OECD/ FAO (Organisation for Economic Co-operation and Development/ Food and Agriculture Organization of the United Nations, 2011): OECD-FAO Agricultural Outlook 2011-2020, URL: http://dx.doi.org/10.1787/agr_outlook2011-en, Abrufdatum: 20.07.2011.

OPEC (Organization of the Petroleum Exporting Countries, 2012): World Oil Outlook, URL:

http://www.opec.org/opec_web/static_files_project/media/downloads/publicatio ns/WOO2012.pdf, Abrufdatum: 23.01.2013.

OPEC (Organization of the Petroleum Exporting Countries, 2011): World Oil Outlook, URL:

http://www.opec.org/opec_web/static_files_project/media/downloads/publicatip ub/WOO_2011.pdf, Abrufdatum: 10.08.2012.

OPEC (Organization of the Petroleum Exporting Countries, 2010): World Oil Outlook, URL: http://www.opec.org/opec_web/en/publications/340.htm, Abrufdatum: 08.07.2011.

Osec (2009): Automobilindustrie Europa. Aktuelle und künftige Entwicklung der Europäischen Automobilindustrie, URL:

http://www.osec.ch/sites/default/files/bbf_europe_automotive_industry_2009.p df, Abrufdatum: 02.09.2012.

Pasternack, G. (2006): Die wirtschaftlichen Aussichten der ostdeutschen Braunkohlenwirtschaft bis zum Jahr 2020 - Eine Szenario-Analyse, Hamburg.

POET (2013): Cellulosic Ethanol, URL: http://www.poet.com/cellulosic, Abrufdatum: 18.11.2013.

POET (2009): POET joins Magellan Midstream Partners to Assess Dedicated Ethanol Pipeline, URL: http://www.poet.com/pr/poet-joins-magellan-midstreampartners-to-assess-dedicated-ethanol-pip, Abrufdatum: 15.11.2013.

POET-DSM Advanced Biofuels (2012): Project LIBERTY - A Decade of Cellulosic Research, URL: http://www.projectliberty.com/about/, Abrufdatum: 08.12.2012. 
Poland Minister of Economy (2010): National Renewable Energy Action Plan, URL: http://ec.europa.eu/energy/renewables/action_plan_en.htm, Abrufdatum: 03.09.2013.

Portugal (2010): National Renewable Energy Action Plan, URL:

http://ec.europa.eu/energy/renewables/action_plan_en.htm, Abrufdatum: 03.09.2013.

Raízen (2013): About us, URL: http://www.raizen.com.br/en/raizen/about-us, Abrufdatum: 13.08.2013.

Reibnitz, U. von (1992): Szenario-Technik - Instrumente für die unternehmerische und persönliche Erfolgsplanung, 2. Auflage, Wiesbaden.

Republic of Bulgaria Ministry of Economy, Energy and Tourism (2010): National Renewable Energy Action Plan - drawn up in accordance with the template for national renewable energy action plans as set out in Directive 2009/28/EC of the European Parliament and of the Council, URL: http://ec.europa.eu/energy/renewables/action_plan_en.htm, Abrufdatum: 03.09.2013.

Republic of Cyprus Ministry of Commerce, Industry and Tourism (2010): Reply of the Republic of Cyprus to a letter from the Directorate-General for Energy of the European Commission relating to clarification questions about the National Renewable Energy Action Plan 2010-2020, URL:

http://ec.europa.eu/energy/renewables/action_plan_en.htm, Abrufdatum: 03.09.2013.

Republic of Latvia (2010): National Renewable Energy Action Plan for implementing Directive 2009/28/EC of the European Parliament and of the Council of 23 April 2009, URL: http://ec.europa.eu/energy/renewables/action_plan_en.htm, Abrufdatum: 03.09.2013.

RFA (Renewable Fuels Association, 2012a): Oil Dependence: A National Threat, URL: http://ethanolrfa.3cdn.net/99805e3d3d287bdf33_uqm6bw403.pdf, Abrufdatum: 08.10.2012. 
RFA (Renewable Fuels Association, 2012b): The New Fuel: E15, URL: http://www.ethanolrfa.org/pages/E15, Abrufdatum: 17.10.2012.

RFA (Renewable Fuels Association, 2012c): Biorefinery Locations, URL: http://www.ethanolrfa.org/bio-refinery-locations/, Abrufdatum: 07.01.2013.

RFA (Renewable Fuels Association, 2012d): Vortrag im Rahmen des Kongresses „WORLD ETHANOL \& BIOFUELS 2012“, 06.11.2012, München.

Richtlinie 2009/28/EG (2009) des Europäischen Parlaments und des Rates vom 23. April 2009 zur Förderung der Nutzung von Energie aus erneuerbaren Quellen und zur Änderung und anschließenden Aufhebung der Richtlinien 2001/77/EG und 2003/30/EG.

Richtlinie 2009/30/EG (2009) des Europäischen Parlaments und des Rates vom 23. April 2009 zur Änderung der Richtlinie 98/70/EG im Hinblick auf die Spezifikationen für Otto-, Diesel- und Gasölkraftstoffe und die Einführung eines Systems zur Überwachung und Verringerung der Treibhausgasemissionen sowie zur Änderung der Richtlinie 1999/32/EG des Rates im Hinblick auf die Spezifikationen für von Binnenschiffen gebrauchte Kraftstoffe und zur Aufhebung der Richtlinie 93/12/EWG.

Richtlinie 2003/30/EG (2003) des Europäischen Parlaments und des Rates vom 8. Mai 2003 zur Förderung der Verwendung von Biokraftstoffen oder anderen erneuerbaren Kraftstoffen im Verkehrssektor.

Richtlinie 2003/96/EG (2003) des Rates vom 27. Oktober 2003 zur Restrukturierung der gemeinschaftlichen Rahmenvorschriften zur Besteuerung von Energieerzeugnissen und elektrischen Strom.

\section{RITA (Research and Innovative Technology Administration Bureau of Trans-} portation Statistics, 2012): Table 4-7: Domestic Demand for Gasoline (Million gallons) by Mode, URL: http://www.bts.gov/publications/national_transportation_statistics/html/table_0 4_07.html, Abrufdatum: 28.11.2012. 
Ritchey, T. (2011): General Morphological Analysis: A general method for nonquantified modelling, URL: http://www.swemorph.com/pdf/gma.pdf, Abrufdatum: 07.12.2011.

Romania (2010): National Renewable Energy Action Plan, URL: http://ec.europa.eu/energy/renewables/action_plan_en.htm, Abrufdatum: 03.09.2013.

Schlicksupp, H. (2004): Ideenfindung, 6. Auflage, Würzburg.

Schlicksupp, H. (1989): Innovation, Kreativität und Ideenfindung, 3. Auflage, Würzburg.

Seyfried, F. (2012): Aussage beim Vortrag „Alternative fuels for cars opportunities and challenges“ im Rahmen des Kongresses „WORLD ETHANOL \& BIOFUELS 2012“, 08.11.2012, München.

Shell (2013): Raízen, URL: http://www.shell.com/global/environmentsociety/environment/climate-change/biofuels-alternative-energiestransport/biofuels/raizen.html, Abrufdatum: 23.07. 2013.

Shell (2009): Shell Pkw-Szenarien bis 2030. Fakten, Trends und Handlungsoptionen für nachhaltige Auto-Mobilität, URL: http://wwwstatic.shell.com/static/deu/downloads/publications_2009_shell_mobility_scens cena.pdf, Abrufdatum: 24.08.2012.

Slovenia (2010): National Renewable Energy Action Plan 2010-2020, URL: http://ec.europa.eu/energy/renewables/action_plan_en.htm, Abrufdatum: 03.09.2013.

Steinmann, H./ Schreyögg, G. (2005): Management: Grundlagen der Unternehmensführung Konzepte - Funktionen - Fallstudien, 6. Auflage, Wiesbaden.

Sun, Y./ Cheng, J. (2002): Hydrolysis of lignocellulosic materials for ethanol production: a review, in: Bioresource Technology, Vol. 83, S. 1 - 11. 
Sweden Regeringskansliet (2010): The Swedish National Action Plan for the promotion of the use of renewable energy in accordance with Directive 2009/28/EC and the Commission Decision of 30.06.2009, URL: http://ec.europa.eu/energy/renewables/action_plan_en.htm, Abrufdatum: 03.09.2013.

Technology Review (2007): Konkurrenz für Ethanol, URL: http://www.heise.de/tr/artikel/Konkurrenz-fuer-Ethanol-279671.html, Abrufdatum: 20.08.2013.

The Wall Street Journal (2012): Auto Sales, URL:

http://online.wsj.com/mdc/public/page/2_3022-autosales.html, Abrufdatum: 23.10.2012.

The White House (2013a): Our Environment, URL: http://www.whitehouse.gov/energy/our-environment, Abrufdatum: 20.11.2013.

The White House (2013b): Develop and Secure America's Energy Resources, URL: http://www.whitehouse.gov/energy/securing-american-energy, Abrufdatum: 21.11.2013.

Thomasson, M. (2011): Examining the Short and Long Term Prospects for Ethanol Exports, Vortrag im Rahmen des Kongresses "WORLD ETHANOL \& BIOFUELS 2011", 07.11.2011, Barcelona.

Transportation Research Board (2011): Policy Options for Reducing Energy Use and Greenhouse Gas Emissions from U.S. Transportation, URL: http://www.nap.edu/catalog.php?record_id=13194, Abrufdatum: 28.11.2012.

UFOP (Union zur Förderung von Oel- und Proteinpflanzen e. V., 2011): Internationale Biodiesel-Märkte Produktions- und Handelsentwicklungen, URL: http://www.ufop.de/files/6713/3879/6224/EV_Ecofys-UFOP_dt_2012.pdf, Abrufdatum: 29.07.2013.

United Kingdom (2010): National Renewable Energy Action Plan for the United Kingdom, URL: http://ec.europa.eu/energy/renewables/action_plan_en.htm, Abrufdatum: 03.09.2013. 
United States Census Bureau (2013): U.S. Population Density (By Counties), URL: http://www.census.gov/dmd/www/pdf/512popdn.pdf, Abrufdatum: 22.11. 2013.

University of Nebraska (2007): Understanding Ethanol Plant Economics: Will Boom Turn Bust?, URL:

http://www.ianrpubs.unl.edu/live/ec849/build/ec849.pdf, Abrufdatum:

01.10.2013.

USDA (United States Department of Agriculture, 2013a): World Corn Trade, URL: http://www.ers.usda.gov/topics/crops/corn/trade.aspx\#world, Abrufdatum: 19.12.2013.

USDA (United States Department of Agriculture, 2013b): U.S. domestic corn use, URL: http://www.ers.usda.gov/topics/crops/corn/background.aspx, Abrufdatum: 14.01.2014.

USDA (United States Department of Agriculture, 2012): USDA Agricultural Projections to 2021, URL: http://www.usda.gov/oce/commodity/archive_projections/USDAAgriculturalPrU SDAAgric2021.pdf, Abrufdatum: 07.09.2012.

USDA (United States Department of Agriculture, 2008): U.S. Biobased Products Market Potential and Projections Through 2025, URL: http://www.usda.gov/oce/reports/energy/BiobasedReport2008.pdf, Abrufdatum: 19.09.2011.

USDA Foreign Agricultural Service (2012): EU Biofuels Annual 2012, URL: http://gain.fas.usda.gov/Recent\%20GAIN\%20Publications/Biofuels\%20AnnuA n_The\%20Hague_EU-27_6-25-2012.pdf, Abrufdatum: 12.09.2012.

USDA Foreign Agricultural Service (2011): EU-27 Annual Biofuels Report, URL:http://gain.fas.usda.gov/Recent\%20GAIN\%20Publications/Biofuels\%20A nnual_The\%20Hague_EU-27_6-11-2010.pdf, Abrufdatum: 01.03.2011.

U.S. Department of Energy (2013a): Ethanol Feedstocks, URL:

http://www.afdc.energy.gov/fuels/ethanol_feedstocks.html, Abrufdatum: 07.01.2013. 
U.S. Department of Energy (2013b): U.S. Total Corn Production and Corn Used for Fuel Ethanol Production, URL:

http://www.afdc.energy.gov/data/\#tab/all/data_set/10339, Abrufdatum: 25.06.2013.

U.S. Department of Energy (2013c): Biobutanol, URL:

http://www.afdc.energy.gov/fuels/emerging_biobutanol.html, Abrufdatum: 20.08.2013.

U.S. Department of Energy (2013d): xTL Fuels, URL:

http://www.afdc.energy.gov/fuels/emerging_xtl_fuels.html, Abrufdatum: 03.09.2013.

U.S. Department of Energy (2013e): Ethanol Production and Distribution, URL: http://www.afdc.energy.gov/fuels/ethanol_production.html, Abrufdatum: 15.11.2013.

U.S. Department of Energy (2013f): Ethanol Benefits and Considerations, URL: http://www.afdc.energy.gov/fuels/ethanol_benefits.html, Abrufdatum: 22.11.2013.

U.S. Department of Energy (2013g): Light-Duty Vehicle Search, URL: http://www.afdc.energy.gov/vehicles/search/light/\#pane=autos\&pane_page=\% 3Fautos\%255Bcategory_id\%255D\%3D\%26autos\%255Bfuel_id\%255D\%3D10 002\%26autos\%255Bmanufacturer_id\%255D\%3D\%26autos\%255Bmodel_yea rs\%255D\%255B\%255D\%3D2015\%26autos\%255Bmodel_years\%255D\%255 B\%255D\%3D2014\%26autos\%255Bmodel_years\%255D\%255B\%255D\%3D2 013\%26autos\%255Bmodel_years\%255D\%255B\%255D\%3D2012\%26autos\% 255Bmodel_years\%255D\%255B\%255D\%3D2011\%26page\%3D2, Abrufdatum: 28.11.2013.

U.S. Department of Energy (2013h): Volumetric Ethanol Excise Tax Credit (VEETC), URL: http://www.afdc.energy.gov/laws/law/US/399, Abrufdatum: 21.01.2014. 
U.S. Department of Energy (2012a): Alternative Fuels Data Center, URL: http://www.afdc.energy.gov/fuels/ethanol_feedstocks.html, Abrufdatum: 04.09.2012.

U.S. Department of Energy (2012b): Blender Pump Dispensers, URL: http://www.afdc.energy.gov/technology_bulletin_0210.html, Abrufdatum: 13.12.2012.

U.S. Department of Energy/ Oak Ridge National Laboratory (2011): Biomass Energy Data Book, URL: http://cta.ornl.gov/bedb/pdf/BEDB4_Full_Doc.pdf, Abrufdatum: 08.10.2012.

U.S. Department of Transportation (2012a): National Transportation Statistics, URL:

http://www.bts.gov/publications/national_transportation_statistics/pdf/entire.pdf, Abrufdatum: 15.10.2012.

U.S. Department of Transportation (2012b): Light Duty Vehicle, Short Wheel Base and Motorcycle Fuel Consumption and Travel, URL: http://www.bts.gov/publications/national_transportation_statistics/html/table_0 4_11.html, Abrufdatum: 16.10.2012.

U.S. Energy Information Administration (2013a): Annual Energy Outlook 2013 with projections to 2040 , URL: http://www.eia.gov/forecasts/aeo/pdf/0383(2013).pdf, Abrufdatum: 06.06.2013.

U.S. Energy Information Administration (2013b): Ethanol production capacity little changed in the past year, URL: http://www.eia.gov/todayinenergy/detail.cfm?id=11331, Abrufdatum: 25.06.2013.

U.S. Energy Information Administration (2013c): U.S. Imports from Brazil of Fuel Ethanol, URL: http://www.eia.gov/dnav/pet/hist/LeafHandler.ashx?n=PET\&s=MFEIM_NUSNBR_1\&f=A, Abrufdatum: 12.11.2013. 
U.S. Energy Information Administration (2013d): U.S. Exports to Brazil of Fuel Ethanol, URL:

http://www.eia.gov/dnav/pet/hist/LeafHandler.ashx?n=pet\&s=m_epooxe_eex_ nus-nbr_mbbl\&f=a, Abrufdatum: 14.11.2013.

U.S. Energy Information Administration (2013e): Exports by Destination, URL: http://www.eia.gov/dnav/pet/pet_move_expc_a_EPOOXE_EEX_mbbl_a.htm, Abrufdatum: 14.11.2013.

U.S. Energy Information Administration (2013f): Light-Duty Vehicle Stock by Technology Type, Reference case, URL: http://www.eia.gov/oiaf/aeo/tablebrowser/\#release=AEO2013\&subject=15AEO2013\&table $=49-A E O 2013 \&$ region $=0-0$ \&cases $=$ ref2013- $d 102312 a$, Abrufdatum: 28.11.2013.

U.S. Energy Information Administration (2013g): Oil/Liquids, URL: http://www.eia.gov/forecasts/aeo/source_oil.cfm, Abrufdatum: 06.12.2013.

U.S. Energy Information Administration (2013h): Oil/Liquids from Market Trends, URL: http://www.eia.gov/forecasts/aeo/source_oil_all.cfm\#developing_nations, Abrufdatum: 30.12.2013.

U.S. Energy Information Administration (2012a): Frequently Asked Questions. What are the differences between various types of crude oil prices, URL: http://www.eia.gov/tools/faqs/faq.cfm?id=11\&t=5, Abrufdatum: 17.08.2012.

U.S. Energy Information Administration (2012b): Annual Energy Outlook 2012 with Projections to 2025, URL: http://www.eia.gov/forecasts/aeo/pdf/0383.pdf, Abrufdatum: 07.08.2012.

U.S. Energy Information Administration (2012c): What drives crude oil prices?, URL:

http://www.eia.gov/finance/markets/reports_presentations/eia_what_drives_cc rud_oil_prices.pdf, Abrufdatum: 15.08.2012. 
U.S. Energy Information Administration (2012d): Petroleum \& Other Liquids, URL:

http://www.eia.gov/dnav/pet/hist/LeafHandler.ashx?n=PET\&s=RWTC\&f=W, Abrufdatum: 19.08.2012.

U.S. Energy Information Administration (2012e): Total Energy Flow 2011, URL: http://www.eia.gov/totalenergy/data/annual/pdf/sec1_3.pdf, Abrufdatum: 11.10.2012.

U.S. Energy Information Administration (2012f): AEO2013 Early Release Overview, URL: http://www.eia.gov/forecasts/aeo/er/pdf/0383er(2013).pdf, Abrufdatum: 10.12.2012.

U.S. Energy Information Administration (2012g): Transportation from Market Trends, URL: http://www.eia.gov/forecasts/aeo/sector_transportation_all.cfm, Abrufdatum: 10.12.2012.

U.S. Energy Information Administration (2012h): Legislation and regulations 6 . EPA approval of E15 waiver, URL: http://www.eia.gov/forecasts/aeo/LR_epa_waiver.cfm, Abrufdatum: 11.12.2012.

U.S. Energy Information Administration (2012i): Access to alternative transportation fuel stations varies across the lower 48 states, URL: http://www.eia.gov/todayinenergy/detail.cfm?id=6050\#tabs_AltTransportFuelSt ations-4, Abrufdatum: 12.12.2012.

U.S. Energy Information Administration (2012j): Biofuels Issues and Trends, URL: http://www.eia.gov/biofuels/issuestrends/pdf/bit.pdf, Abrufdatum: 07.01.2013.

U.S. Energy Information Administration (2012k): Petroleum Product Prices, URL: http://www.eia.gov/oiaf/aeo/tablebrowser/\#release=AEO2012\&subject=3AEO2012\&table=12-AEO2012\&region=0-0\&cases=ref2012-d020112c, Abrufdatum: 20.02.2013. 
U.S. Energy Information Administration (2012I): Primary Energy Consumption by Source and Sector, URL:

http://www.eia.gov/totalenergy/data/annual/pecss_diagram.cfm, Abrufdatum:

07.12.2013.

U.S. Energy Information Administration (2011a): Annual Energy Outlook 2011 with Projections to 2035, URL: http://www.eia.gov/forecasts/aeo/, Abrufdatum: 07.07.2011.

U.S. Energy Information Administration (2011b): International Energy Outlook 2011, URL: http://www.eia.gov/forecasts/ieo/pdf/0484.pdf, Abrufdatum: 06.08.2012.

U.S. Energy Information Administration (2011c): Gasoline with higher ethanol content getting closer to U.S. drivers' fuel tanks, URL: http://www.eia.gov/todayinenergy/detail.cfm?id=4230, Abrufdatum: 11.12.2012.

U.S. Energy Information Administration (2011d): Review of Emerging Resources: U.S. Shale Gas and Shale Oil Plays, URL: http://www.eia.gov/analysis/studies/usshalegas/, Abrufdatum: 06.12.2013.

U.S. Energy Information Administration (2007): Biofuels in the U.S. Transportation Sector, URL: http://www.eia.gov/oiaf/aeo/otheranalysis/bts.html, Abrufdatum: 01.08.2011.

U.S. Government Information (2011): Federal Register, Vol. 76, No. 17, URL: http://www.cftc.gov/ucm/groups/public/@Irfederalregister/documents/file/20121154a.pdf, Abrufdatum: 16.10.2012.

U.S. Government Information (2007): Energy Independence and Security Act of 2007, URL: http://www.gpo.gov/fdsys/pkg/PLAW-110publ140/pdf/PLAW110publ140.pdf, Abrufdatum: 10.02.2012.

VDA (Verband der Automobilindustrie, 2011): Elektromobilität - Eine Alternative zum ÖI, URL:

http://www.vda.de/de/publikationen/publikationen_downloads/detail.php?id=96 9, Abrufdatum: 08.03.2012. 
Velte, D./ Araguas, J.P.L. de/ Nielson, O./ Jörß, W. (2004): The EurEnDel Scenarios Europe's Energy System by 2030, URL:

http://www.cgee.org.br/atividades/redirKori/365, Abrufdatum: 07.12.2011.

Verity, J. (2003): Scenario planning as a strategy technique, in: European Business Journal, Vol. 5, Issue 4, S.185 - 195.

Vester, F. (2003): Die Kunst vernetzt zu denken Ideen und Werkzeuge für einen neuen Umgang mit Komplexität - Ein Bericht an den Club of Rome, 3. Auflage, München.

Weber, K. (1990): Wirtschaftsprognostik, München.

Wilms, F.E.P. (2006a): Szenariotechnik, Bern.

Wilms, F.E.P. (2006b): Szenarien sind Systeme, in: Wilms, F.E.P. (Hrsg.): Szenariotechnik, Bern, S. $39-60$.

Wilson, I. (1998): Mental Maps of the Future: An Intuitive Logics Approach to Sceanrio Planning, in: Fahey, L. /Randell, R.M. (Hrsg.): Learning from the Future competitive foresight Scenarios, Kanada, S. 81 - 108.

Wood Mackenzie (2013): Biofuels and European fossil fuel supply - Current practice and future challenges, Vortrag im Rahmen des Kongresses „European Biodiesel 2013", 13.06.2013, Lissabon.

Wood Mackenzie (2010): Impact of the use of biofuels on oil refining and fuels specifications; URL:

http://ec.europa.eu/energy/oil/studies/doc/2011_06_impact_biofuels.pdf, Abrufdatum: 08.08.2012.

World Bank Development Prospects Group (2012): Commodity Price Forecast Update, URL: http://siteresources.worldbank.org/INTPROSPECTS/Resources/3349341304428586133/Price_Forecast.pdf, Abrufdatum: 08.09.2012. 
World Bank Development Prospects Group (2010): Placing the 2006/08 Commodity Price Boom into Perspective, URL: http://wwwwds.worldbank.org/servlet/WDSContentServer/WDSP/IB/2010/07/21/0001583 49_20100721110120/Rendered/PDF/WPS5371.pdf, Abrufdatum: 19.03.2012.

World Bank Development Prospects Group (2008): A Note on Rising Food Prices, URL: http://wwwwds.worldbank.org/servlet/WDSContentServer/WDSP/IB/2008/07/28/0000204 39_20080728103002/Rendered/PDF/WP468.pdf, Abrufdatum: 19.03.2012.

World Watch Institute (2006): Biofuels for Transportation - Global Potential and Implications for Sustainable Agriculture and Energy in the $21^{\text {st }}$ Century. Extended Summary, URL: https://www.worldwatch.org/system/files/EBF038.pdf, Abrufdatum: 13.03.2012.

Zerres, M. P. (1988): Szenario - Ein Filmdreh der Zukunft, in: Franke, R./ Zerres, M.P. (Hrsg.): Planungstechniken Instrumente für zukunftsorientierte Unternehmensführung, Frankfurt am Main, S. $67-82$.

Zwicky, F. (1989): Morphologische Forschung - Wesen und Wandel materieller und geistiger struktureller Zusammenhänge, 2. Auflage, Glarus. 


\section{Anhang}

Anhang 1: Beschreibung der Studien zum Stand der Forschung

Anhang 2: Fragebogen zur Durchführung der EU27-Wirkungsanalyse

Anhang 3: Fragebogen zur EU27-Konsistenzprüfung

Anhang 4: Fragebogen zur Durchführung der USA-Wirkungsanalyse

363

Anhang 5: Fragebogen zur USA-Konsistenzprüfung

369 


\section{Anhang 1: Beschreibung der Studien zum Stand der Forschung}

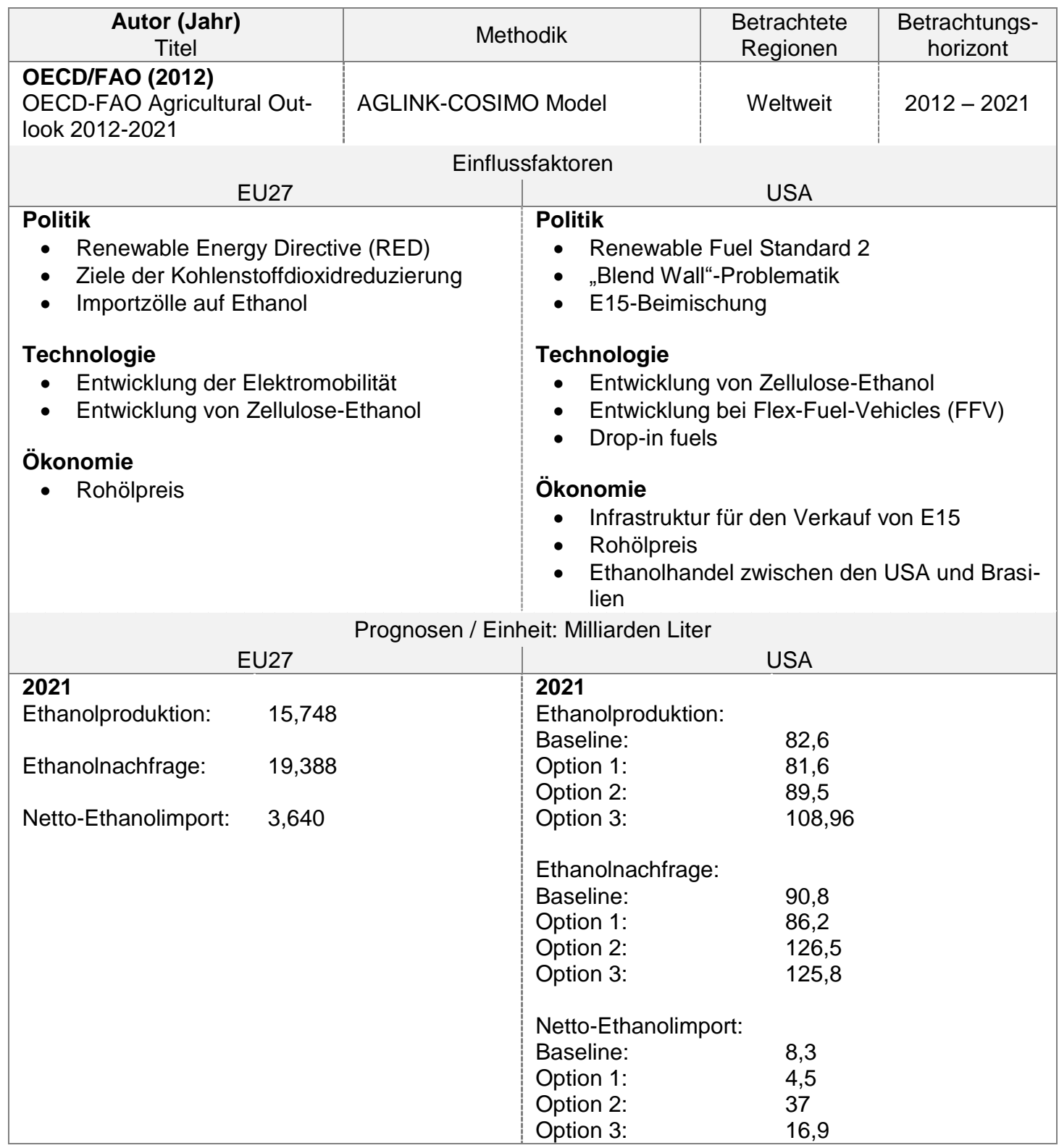

Quelle: In Anlehnung an OECD/FAO 2012: $87 \mathrm{ff}$.

\begin{tabular}{|c|c|c|c|}
\hline $\begin{array}{c}\text { Autor (Jahr) } \\
\text { Titel }\end{array}$ & Methodik & $\begin{array}{l}\text { Betrachtete } \\
\text { Regionen }\end{array}$ & $\begin{array}{l}\text { Betrachtungs- } \\
\text { horizont }\end{array}$ \\
\hline $\begin{array}{l}\text { U.S. Energy Information } \\
\text { Administration (2012) } \\
\text { Annual Energy Outlook } 2012 \\
\text { with Projections to } 2035\end{array}$ & $\begin{array}{l}\text { U.S. Energy Information Admi- } \\
\text { nistration National Energy Mo- } \\
\text { deling System }\end{array}$ & USA & $2011-2035$ \\
\hline \multicolumn{4}{|c|}{ Einflussfaktoren } \\
\hline \multirow[t]{2}{*}{ EU27 } & \multicolumn{2}{|c|}{$\begin{array}{ll} & \text { USA } \\
\text { Politik }\end{array}$} & \\
\hline & & \multicolumn{2}{|c|}{$\begin{array}{l}\text { Politik } \\
\text { - Renewable Fuel Standard } 2 \text { (RFS 2) } \\
\text { - } \text { "Blend Wall“-Problematik } \\
\text { Technologie } \\
\text { - Entwicklung bei Flex-Fuel-Vehicles (FFV) } \\
\text { - Nutzung von E85 } \\
\text { - E15-Beimischung }\end{array}$} \\
\hline
\end{tabular}




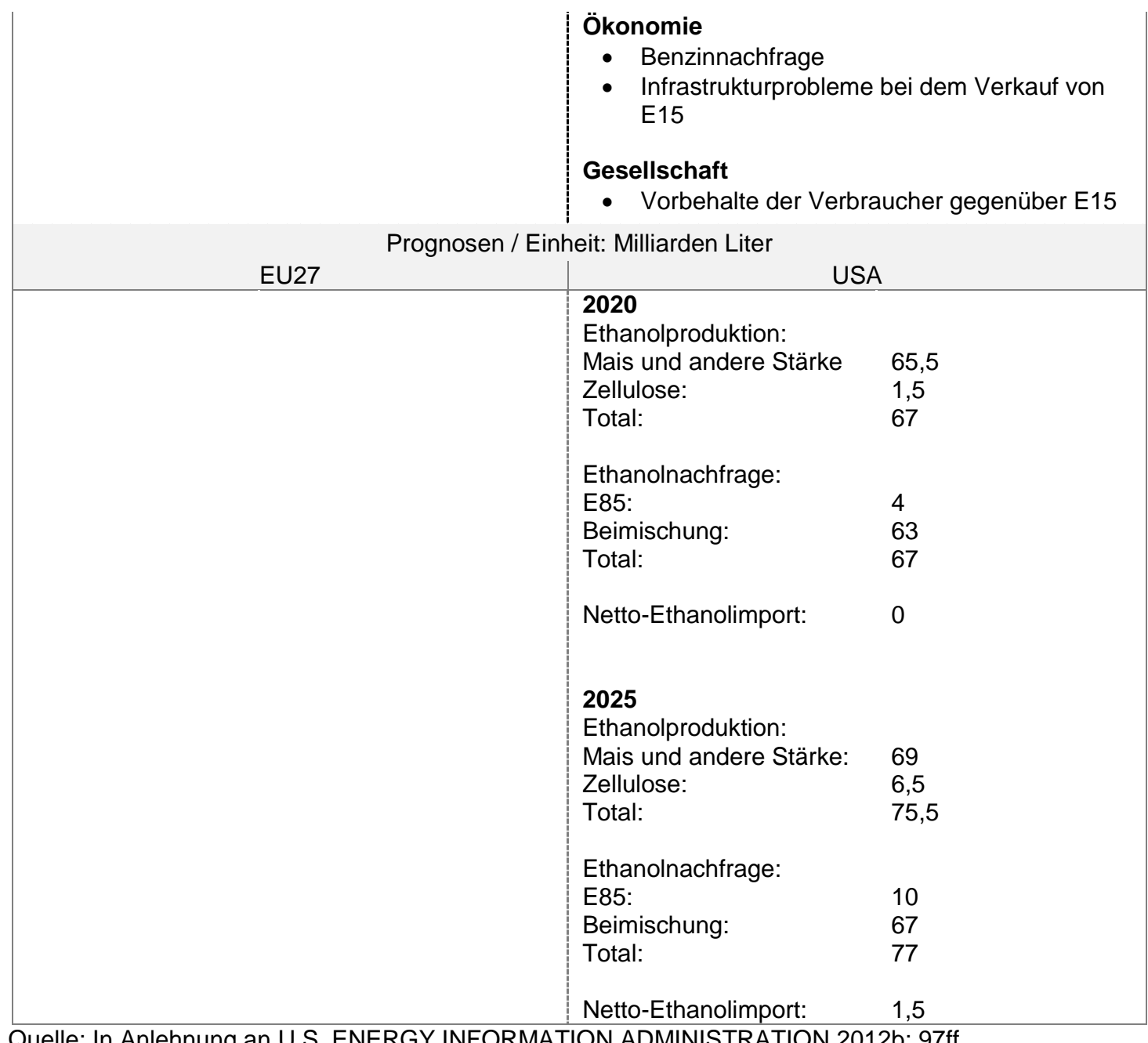

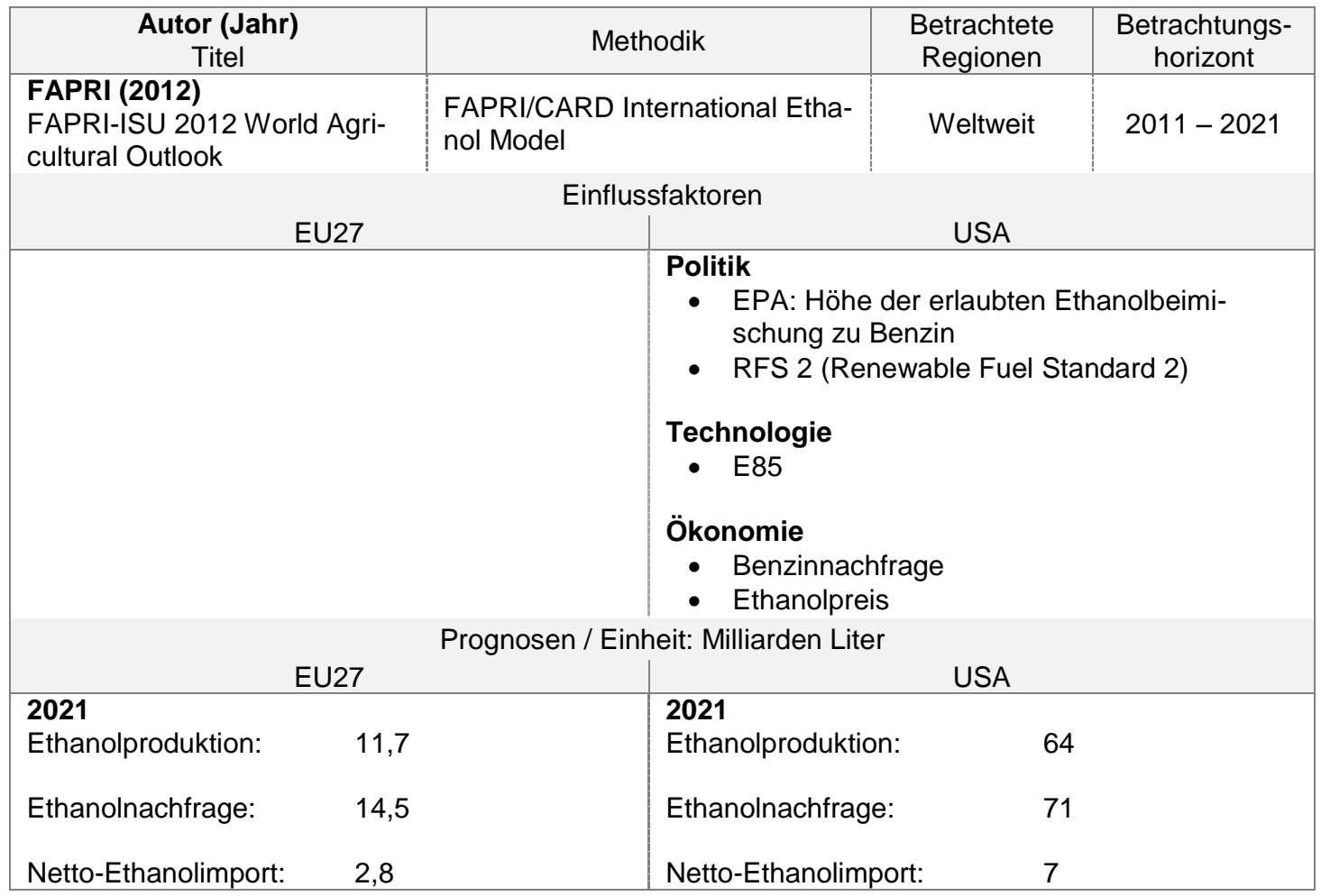

Quelle: In Anlehnung an FAPRI 2012: $1 \mathrm{ff}$. 


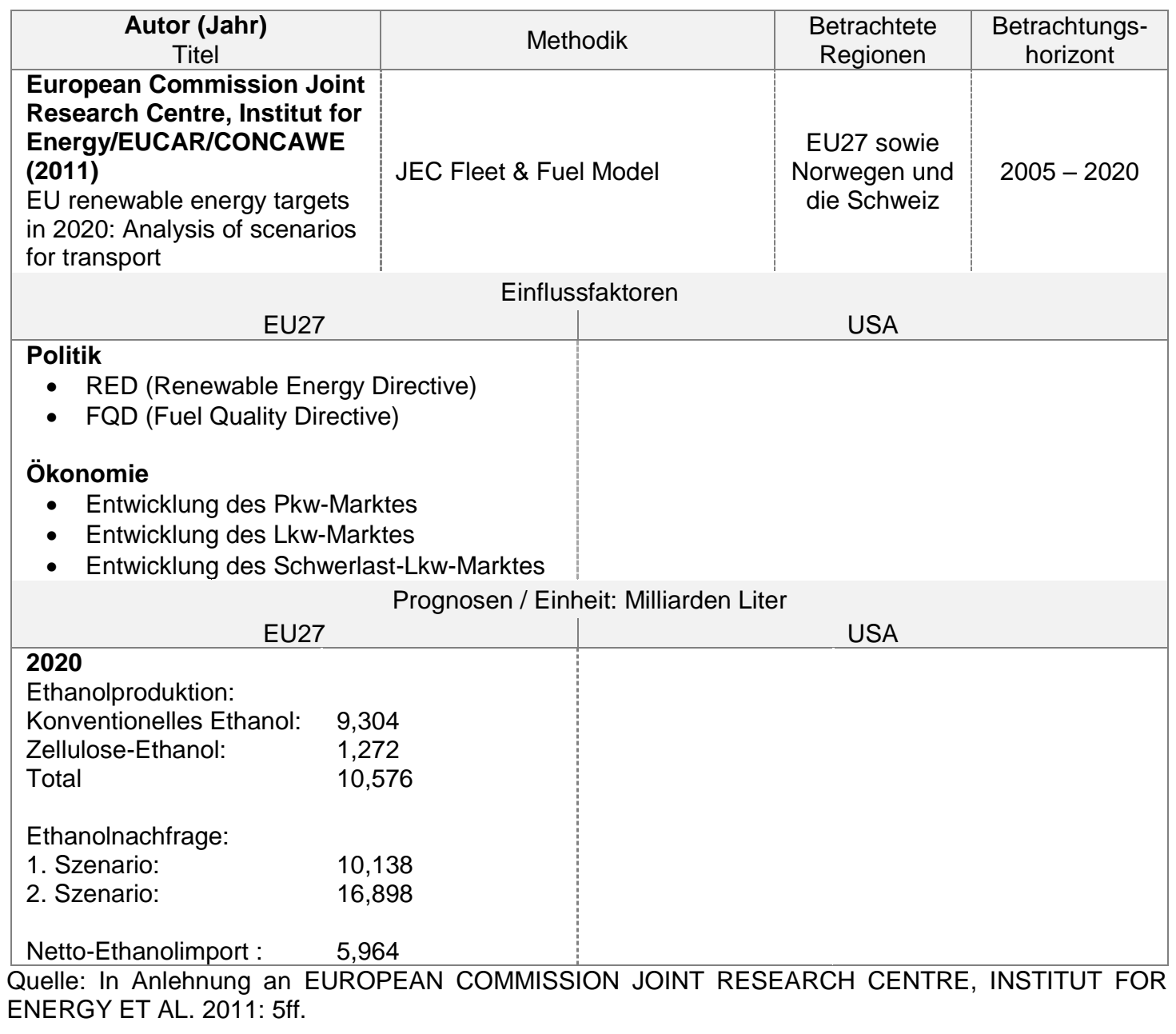

\begin{tabular}{|c|c|c|c|}
\hline $\begin{array}{c}\text { Autor (Jahr) } \\
\text { Titel }\end{array}$ & Methodik & $\begin{array}{l}\text { Betrachtete } \\
\text { Regionen }\end{array}$ & $\begin{array}{c}\text { Betrachtungs- } \\
\text { horizont }\end{array}$ \\
\hline $\begin{array}{l}\text { Joint Research Centre } \\
(\mathbf{2 0 1 0 )} \\
\text { Impacts of the EU Biofuel } \\
\text { Target on Agricultural Markets } \\
\text { and Land Use }\end{array}$ & $\begin{array}{l}\text { Verwendete Modelle in der Ana- } \\
\text { lyse: AGLINK-COSIMO, ESIM } \\
\text { and CAPRI }\end{array}$ & EU & $2010-2020$ \\
\hline \multicolumn{4}{|c|}{ Einflussfaktoren } \\
\hline \multicolumn{2}{|l|}{ EU27 } & USA & \\
\hline $\begin{array}{l}\text { Politik } \\
\text { - Steuernachlass bei Ethanolbeimischung } \\
\text { - Ethanol-Beimischungsquote } \\
\text { - Ethanol Importzölle }\end{array}$ & Ibeimischung & & \\
\hline \multicolumn{4}{|c|}{$\begin{array}{l}\text { Ökonomie } \\
\text { • Entwicklung des Bruttoinlandsproduktes } \\
\text { - Rohölpreis } \\
\text { - Wechselkurs (€/US-\$) } \\
\text { - Benzinverbrauch }\end{array}$} \\
\hline \multicolumn{4}{|l|}{$\begin{array}{l}\text { Gesellschaft } \\
\text { - EU-Bevölkerung }\end{array}$} \\
\hline \multicolumn{4}{|c|}{ Prognosen / Einheit: Milliarden Liter } \\
\hline & \multicolumn{3}{|c|}{ USA } \\
\hline $\begin{array}{l}\text { 2020/AGLINK-COSIMO } \\
\text { Baseline Szenario } \\
\text { Ethanolproduktion: } \\
\text { Erste Generation: }\end{array}$ & & & \\
\hline
\end{tabular}




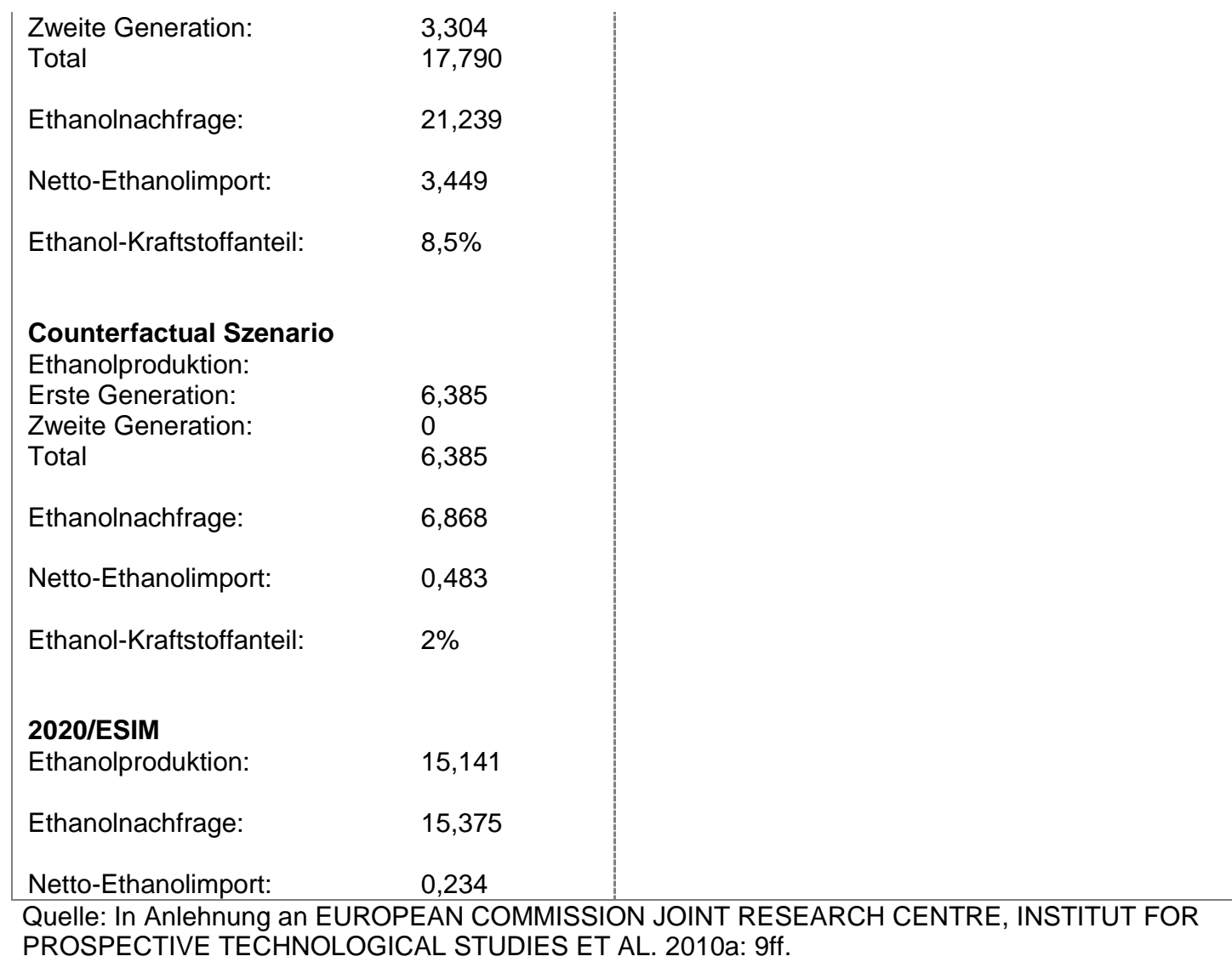

\begin{tabular}{|c|c|c|c|c|}
\hline $\begin{array}{c}\text { Autor (Jahr) } \\
\text { Titel }\end{array}$ & \multicolumn{2}{|c|}{ Methodik } & $\begin{array}{c}\text { Betrachtete } \\
\text { Regionen }\end{array}$ & $\begin{array}{c}\text { Betrachtungs- } \\
\text { horizont }\end{array}$ \\
\hline $\begin{array}{l}\text { F.O. Licht/AGRA informa } \\
\text { (2009) } \\
\text { World Ethanol Markets - The } \\
\text { Outlook to } 2020\end{array}$ & \multicolumn{2}{|c|}{$\begin{array}{l}\text { In der Veröffentlichung nicht } \\
\text { genannt }\end{array}$} & Weltweit & $2008-2020$ \\
\hline \multicolumn{5}{|c|}{ Einflussfaktoren } \\
\hline \multicolumn{2}{|l|}{ EU27 } & \multicolumn{3}{|c|}{ USA } \\
\hline \multicolumn{2}{|c|}{$\begin{array}{l}\text { Politik } \\
\text { - RED (Renewable Energy Directive) } \\
\text { - Ethnaol-Importzölle } \\
\text { Technologie } \\
\text { - Entwicklunng im Bereich Zellulose-Ethanol } \\
\text { Ökonomie } \\
\text { - Rohölpreis } \\
\text { - Rohstoffkosten } \\
\text { Gesellschanolimporte aus Brasilien } \\
\text { - Debatte über Klimawandel } \\
\text { - Tank-oder-Teller-Diskussion }\end{array}$} & \multicolumn{3}{|c|}{$\begin{array}{l}\text { Politik } \\
\text { - Energy Independence and Security Act } \\
\text { - } \text { Farm Bill } 2008 \\
\text { CBI (Caribbean Basin Initiative) trade } \\
\text { agreement } \\
\text { Technologie } \\
\text { - Entwicklung im Bereich Zellulose-Ethanol } \\
\text { - Transportfähigkeit des Ethanols } \\
\text { Ökonomie } \\
\text { - Rohölpreis } \\
\text { - Rohstoffkosten } \\
\text { - Ethanolimporte aus Brasilien } \\
\text { Gesellschaft } \\
\text { - Diskussion über die Abhängigkeit von Ener- } \\
\text { - } \text { gieimporten } \\
\text { Tank-oder-Teller-Diskussion }\end{array}$} \\
\hline \multicolumn{5}{|c|}{ Prognosen / Einheit: Milliarden Liter } \\
\hline \multicolumn{2}{|l|}{ EU27 } & \\
\hline
\end{tabular}




\begin{tabular}{|ll|ll|}
\begin{tabular}{|ll|ll|}
$\mathbf{2 0 2 0}$ & $\mathbf{2 0 2 0}$ & \\
Ethanolproduktion: & 10,965 & Ethanolproduktion: & 78 \\
Ethanolnachfrage: & 14,2 & Ethanolnachfrage: & 83,5 \\
Netto-Ethanolimport : & 3,4 & Netto-Ethanolimport: & 5,5 \\
\hline
\end{tabular} \\
Quelle: In Anlehnung an F.O. LICHT/AGRA INFORMA 2009: 133
\end{tabular}






\begin{tabular}{|ll|ll|} 
Total: & 20,028 & Total: & 71,6 \\
Ethanolnachfrage: & & Ethanolnachfrage: & \\
Getreide-Ethanol: & 12,030 & Getreide-Ethanol: & 56,781 \\
Zucker-Ethanol: & 1,086 & Zucker-Ethanol: & 11,053 \\
Zellulose-Ethanol: & 15,842 & Zellulose-Ethanol: & 21,357 \\
Total: & 28,958 & Total: & 89,191 \\
& & & \\
Netto-Ethanolimport: & & Netto-Ethanolimport: & \\
Getreide-Ethanol: & 0 & Getreide-Ethanol: & 0 \\
Zucker-Ethanol: & 1,086 & Zucker-Ethanol: & 11,053 \\
Zellulose-Ethanol: & 7,843 & Zellulose-Ethanol: & 6,537 \\
Total: & 8,929 & Total: & 17,590 \\
\hline
\end{tabular}

Quelle: In Anlehnung an ALFSTAD 2008: $1 \mathrm{ff}$.

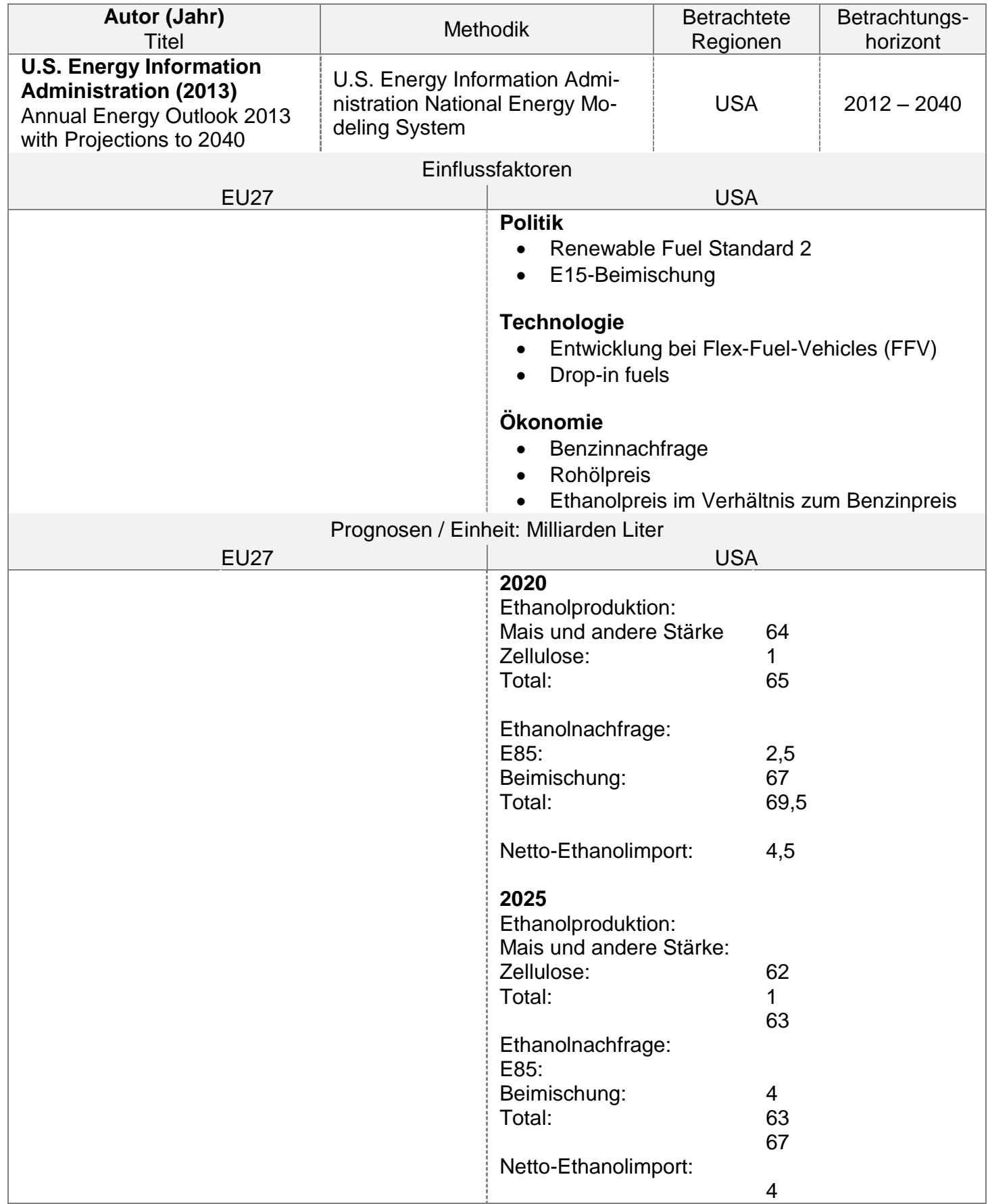

Quelle: In Anlehnung an U.S. ENERGY INFORMATION ADMINISTRATION 2013: 83ff. 


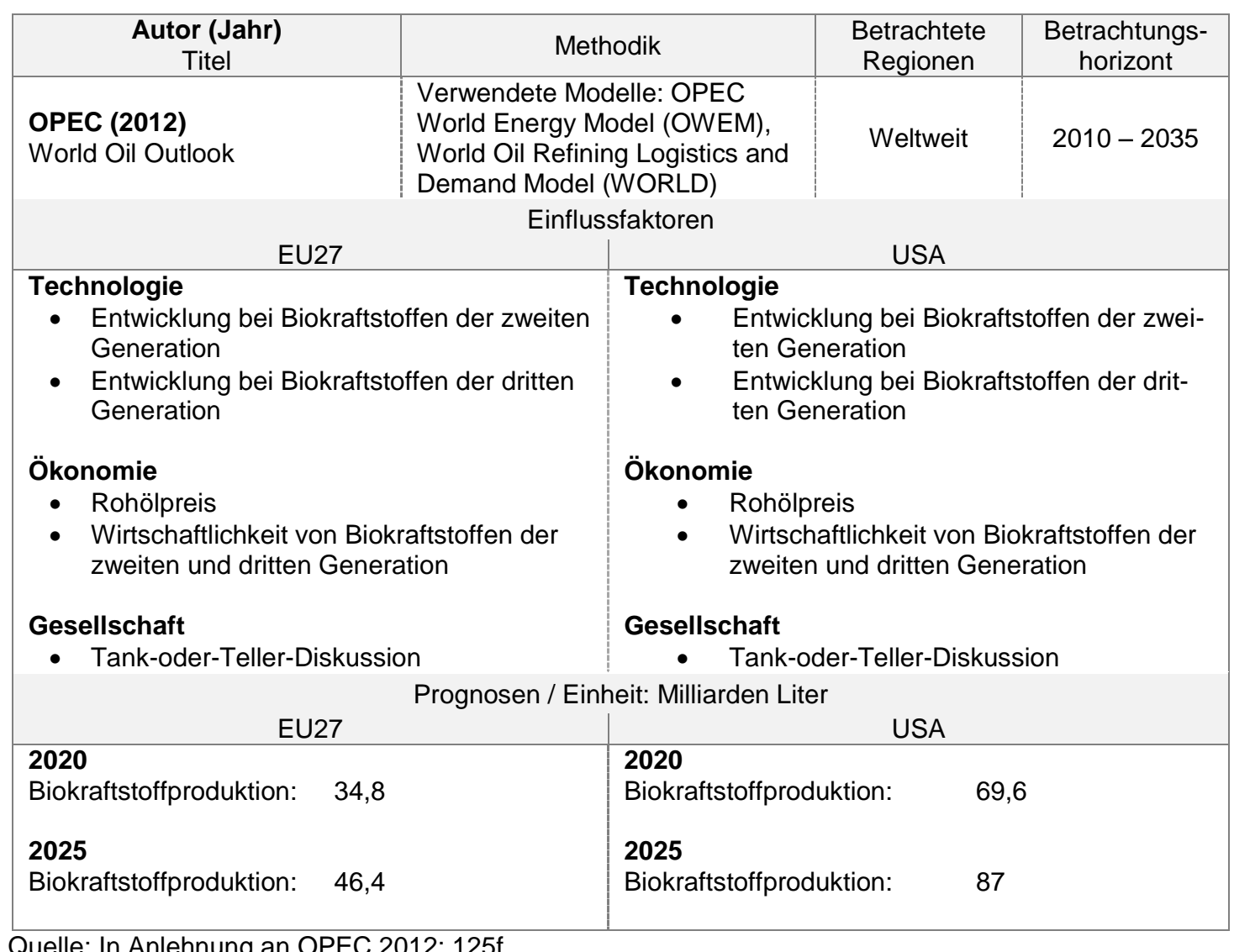

\begin{tabular}{|c|c|c|c|c|}
\hline $\begin{array}{l}\text { Autor (Jahr) } \\
\text { Titel }\end{array}$ & \multicolumn{2}{|c|}{ Methodik } & $\begin{array}{l}\text { Betrachtete } \\
\text { Regionen }\end{array}$ & $\begin{array}{l}\text { Betrachtungs- } \\
\text { horizont }\end{array}$ \\
\hline $\begin{array}{l}\text { International Energy Agency } \\
\text { (2012) } \\
\text { World Energy Outlook } 2012\end{array}$ & \multicolumn{2}{|c|}{$\begin{array}{l}\text { IEA's World Energy Model } \\
\text { (WEM) }\end{array}$} & Weltweit & $2010-2035$ \\
\hline \multicolumn{5}{|c|}{ Einflussfaktoren } \\
\hline \multicolumn{2}{|l|}{ EU27 } & \multicolumn{3}{|c|}{ USA } \\
\hline \multicolumn{2}{|c|}{$\begin{array}{l}\text { Politik } \\
\text { - } 10 \% \text {-Ziel } \\
\text { - Diskussion über das Thema Indirect Land } \\
\quad \text { Use Change (ILUC) }\end{array}$} & \multicolumn{3}{|c|}{$\begin{array}{l}\text { Politik } \\
\text { - Renewable Fuel Standard } 2 \\
\text { - E15-Beimischung }\end{array}$} \\
\hline \multicolumn{2}{|c|}{$\begin{array}{l}\text { Technologie } \\
\text { - } \mathrm{CO}_{2} \text {-Einsparung von Biokraftstoffen } \\
\text { - Entwicklung von Biokraftstoffen der zwei- } \\
\text { ten Generation }\end{array}$} & \multicolumn{3}{|c|}{$\begin{array}{l}\text { Technologie } \\
\text { - } \mathrm{CO}_{2} \text {-Einsparung von Biokraftstoffen } \\
\text { - Entwicklung von Biokraftstoffen der zweiten } \\
\text { Generation }\end{array}$} \\
\hline \multicolumn{2}{|c|}{$\begin{array}{l}\text { Ökonomie } \\
\text { - Benzinnachfrage } \\
\text { - } \text { Finanzielle Unterstützung für Biokraftstoffe } \\
\quad \text { der zweiten Generation }\end{array}$} & \multicolumn{3}{|c|}{$\begin{array}{l}\text { Ökonomie } \\
\text { • Benzinnachfrage } \\
\text { • } \text { Finanzielle Unterstützung für Biokraftstoffe } \\
\text { der zweiten Generation }\end{array}$} \\
\hline \multicolumn{2}{|c|}{$\begin{array}{l}\text { Gesellschaft } \\
\text { - Vorbehalte der Verbraucher gegenüber } \\
\quad \text { höheren Ethanolbeimischungen zu Benzin }\end{array}$} & \multicolumn{3}{|c|}{$\begin{array}{l}\text { Gesellschaft } \\
\bullet \quad \text { Energieunabhängigkeit }\end{array}$} \\
\hline \multicolumn{5}{|c|}{ Prognosen / Einheit: Milliarden Liter } \\
\hline \multicolumn{2}{|l|}{ EU27 } & \multicolumn{3}{|c|}{ USA } \\
\hline \multicolumn{2}{|l|}{$\begin{array}{l}2035 \\
\text { Ethanolproduktion: } \quad 11,6\end{array}$} & \multicolumn{3}{|c|}{$\begin{array}{l}2035 \\
\text { Ethanolproduktion: } \quad 81,2\end{array}$} \\
\hline
\end{tabular}




\begin{tabular}{|c|c|c|c|c|}
\hline $\begin{array}{c}\text { Autor (Jahr) } \\
\text { Titel }\end{array}$ & \multicolumn{2}{|c|}{ Methodik } & $\begin{array}{l}\text { Betrachtete } \\
\text { Regionen }\end{array}$ & $\begin{array}{c}\text { Betrachtungs } \\
\text { horizont }\end{array}$ \\
\hline $\begin{array}{l}\text { OECD/FAO (2011) } \\
\text { OECD-FAO Agricultural Out- } \\
\text { look 2011-2020 }\end{array}$ & \multicolumn{2}{|c|}{$\begin{array}{l}\text { OECD's Aglink Model erweitert } \\
\text { durch FAO's Cosimo Model }\end{array}$} & Weltweit & $2011-2020$ \\
\hline \multicolumn{5}{|c|}{ Einflussfaktoren } \\
\hline \multicolumn{2}{|l|}{ EU27 } & \multicolumn{3}{|c|}{ USA } \\
\hline \multicolumn{2}{|c|}{$\begin{array}{l}\text { Politik } \\
\text { - RED (Renewable Energy Directive) } \\
\text { - Ziele der Kohlenstoffdioxidreduzierung } \\
\text { Technologie } \\
\text { - Entwicklung bei den Elektro- und Hybrid- } \\
\text { - } \text { fahrzeugen } \\
\text { Treibstoffe der 2. Generation }\end{array}$} & \multicolumn{3}{|c|}{$\begin{array}{l}\text { Politik } \\
\text { - Ethanol Importzölle } \\
\text { Technologie } \\
\text { - Treibstoffe der 2. Generation } \\
\text { Ökonomie } \\
\text { - Rohölpreis }\end{array}$} \\
\hline \multicolumn{5}{|c|}{$\begin{array}{l}\text { Ökonomie } \\
\text { • Benzinnachfrage }\end{array}$} \\
\hline \multicolumn{5}{|c|}{ Prognosen / Einheit: Milliarden Liter } \\
\hline \multicolumn{2}{|l|}{ EU27 } & \multicolumn{3}{|c|}{ USA } \\
\hline $\begin{array}{l}2020 \\
\text { Ethanolproduktion: }\end{array}$ & 16,3 & \multicolumn{2}{|c|}{$\begin{array}{l}\mathbf{2 0 2 0} \\
\text { Ethanolproduktion: } \\
\text { Mais-Ethanol: } \\
\text { Zellulose Ethanol: } \\
\text { Total: }\end{array}$} & $\begin{array}{l}59,6 \\
4,3 \\
63,9\end{array}$ \\
\hline $\begin{array}{l}\text { Ethanolnachfrage: } \\
\text { Ethanolnachfrage im }\end{array}$ & 8,7 & \multirow{3}{*}{\multicolumn{2}{|c|}{$\begin{array}{l}\text { Ethanolnachfrage: } \\
\text { Ethanolnachfrage im Kraftstoffbe- } \\
\text { reich: } \\
\text { Netto-Ethanolimport: }\end{array}$}} & 73,4 \\
\hline Kraftstoffbereich: & \multirow{2}{*}{$\begin{array}{l}6,1 \\
2,4\end{array}$} & & & 70,5 \\
\hline Netto-Ethanolimport: & & & & 9,5 \\
\hline $\begin{array}{l}\text { Ethanol-Kraftstoffanteil } \\
\text { Energieanteil: } \\
\text { Volumenanteil: }\end{array}$ & $\begin{array}{l}2 \% \\
.8 \%\end{array}$ & \multicolumn{2}{|c|}{$\begin{array}{l}\text { Ethanol-Kraftsto } \\
\text { Energieanteil: } \\
\text { Volumenanteil: }\end{array}$} & $\begin{array}{l}8,4 \% \\
12,1 \%\end{array}$ \\
\hline
\end{tabular}

Quelle: In Anlehnung an OECD/FAO 2011: 77ff.

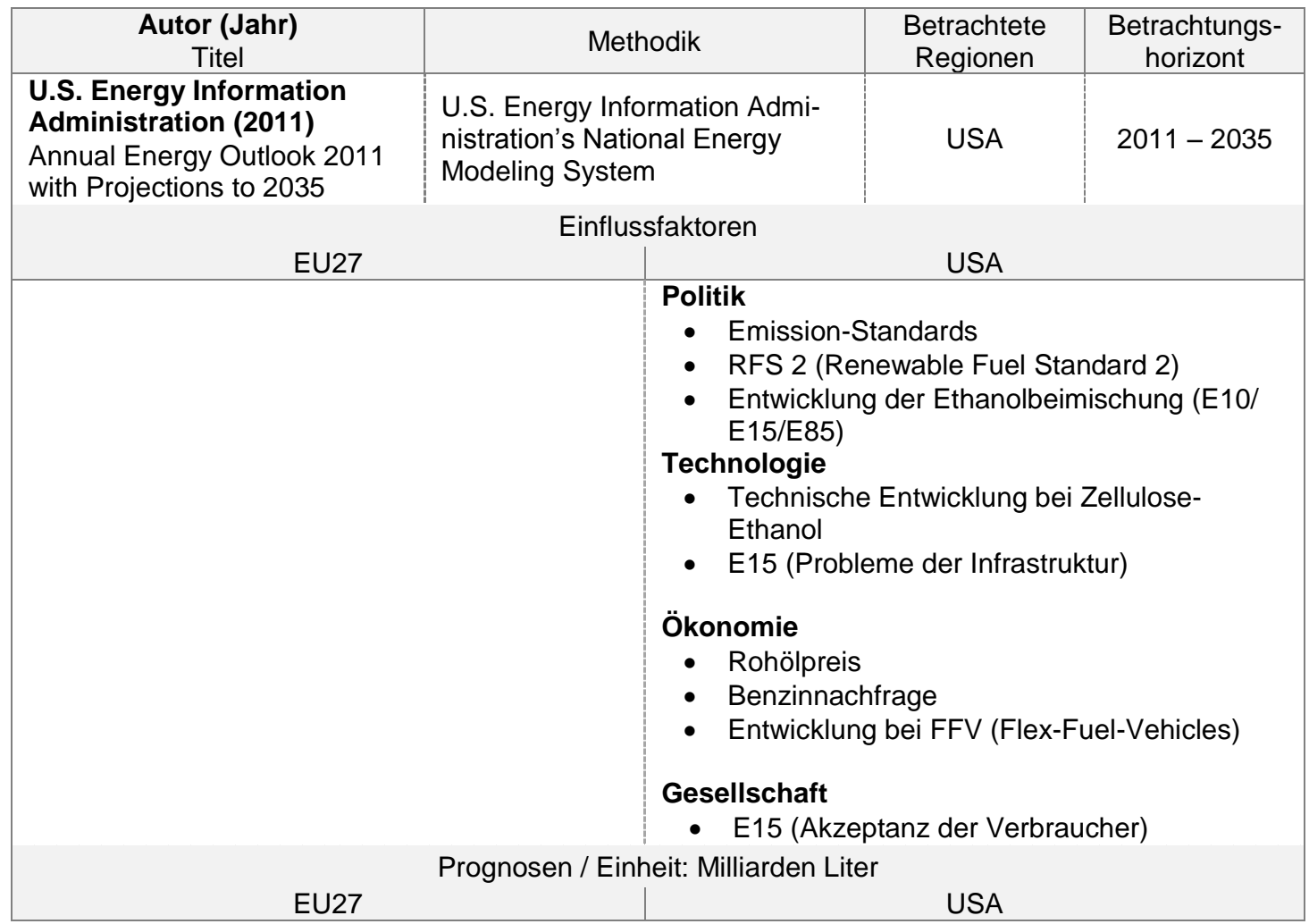


Ethanolproduktion:

Mais und andere Stärke

70,132

Zellulose:

Total:

Ethanolnachfrage:

E85:

Beimischung:

Total:

Netto-Ethanolimport:

2025

Ethanolproduktion:

Mais und andere Stärke:

Zellulose:

Total:

Ethanolnachfrage:

E85:

Beimischung:

Total:

Quelle: In Anlehnung an U.S. ENERGY INFORMATION ADMINISTRATION 2011a: 23ff.

\begin{tabular}{|c|c|c|c|c|}
\hline $\begin{array}{c}\text { Autor (Jahr) } \\
\text { Titel }\end{array}$ & \multicolumn{2}{|c|}{ Methodik } & $\begin{array}{l}\text { Betrachtete } \\
\text { Regionen }\end{array}$ & $\begin{array}{l}\text { Betrachtungs- } \\
\text { horizont }\end{array}$ \\
\hline $\begin{array}{l}\text { International Energy Agency } \\
\text { (2010) } \\
\text { World Energy Outlook } 2010\end{array}$ & \multicolumn{2}{|c|}{$\begin{array}{l}\text { IEA's World Energy Model } \\
\text { (WEM) }\end{array}$} & Weltweit & $2010-2035$ \\
\hline \multicolumn{5}{|c|}{ ktoren } \\
\hline \multicolumn{2}{|l|}{ EU27 } & \multicolumn{3}{|c|}{ USA } \\
\hline $\begin{array}{l}\text { Politik } \\
\text { - } \quad \text { RED (Renewable Energy } \\
\text { - } \text { FQD (Fuel Quality Directive } \\
\text { - } \text { Ethanol-Beimischungsquot } \\
\text { - } \text { Kohlenstoffdioxid-Handel } \\
\text { - } \quad \text { Berücksichtigung von indire } \\
\text { zungsänderungen in der B } \\
\text { Nachhaltigkeit der Ethanolp } \\
\text { Technologie } \\
\text { - Entwicklung der Elektromo } \\
\text { - Entwicklung bei Wassersto } \\
\text { - Nachhaltigkeit der Ethanolp } \\
\text { - Dritte Generation der Biokr } \\
\text { - Produktionskosten } \\
\text { Ökonomie } \\
\text { - Wirtschaftliche Entwicklung } \\
\text { - Rohölpreis } \\
\text { - } \quad \text { Benzinnachfrage } \\
\text { Gesellschaft } \\
\text { - Tank-oder-Teller-Diskussio }\end{array}$ & $\begin{array}{l}\text { irective) } \\
\text { kten Landnut- } \\
\text { rechnung der } \\
\text { roduktion } \\
\text { ilität } \\
\text { rf } \\
\text { aftstoffe }\end{array}$ & $\begin{array}{cc}\text { Politik } \\
\text { - } & \text { RFS } \\
\text { - } & \text { Stel } \\
\text { - } & \text { Koh } \\
\text { - } & \text { Gen } \\
\text { Technolo } \\
\text { - Entv } \\
\text { - Entv } \\
\text { - Entv } \\
\text { - Nac } \\
\text { - Dritt } \\
\text { - Proc } \\
\text { Ökonomi } \\
\text { - Wirt } \\
\text { - Roh } \\
\text { - Ben } \\
\text { Gesellsc } \\
\text { - Stre } \\
\text { - Tnd }\end{array}$ & $\begin{array}{l}\text { able Fuels Sta } \\
\text { ass bei Ethano } \\
\text { nischungsquot } \\
\text { ioxid-Handel } \\
\text { Beimischungs } \\
\text { bei Advanced } \\
\text { in der Elektron } \\
\text { bei Wassersto } \\
\text { it der Ethanolf } \\
\text { ation der Biokr } \\
\text { osten } \\
\text { he Entwicklung } \\
\text { rage } \\
\text { h Energiesiche } \\
\text { ingigkeit } \\
\text { eller-Diskussic }\end{array}$ & $\begin{array}{l}\text { ard) } \\
\text { imischung } \\
\text { ote (E15) } \\
\text { fuels } \\
\text { ilität } \\
\text { duktion } \\
\text { stoffe } \\
\text { eit }\end{array}$ \\
\hline \multicolumn{5}{|c|}{ eit: Milliarden Liter } \\
\hline \multicolumn{2}{|l|}{ EU27 } & \multicolumn{3}{|c|}{ USA } \\
\hline
\end{tabular}


Biokraftstoffnachfrage

New Policies Scenario:

51,689

Current Policies Scenario:

49,7

450 Scenario:

\section{5}

Biokraftstoffnachfrage

New Policies Scenario:

Current Policies Scenario:

123,258

450 Scenario:

Quelle: In Anlehnung an INTERNATIONAL ENERGY AGENCY 2010a: 355ff.



Quelle: In Anlehnung an BLOOMBERG NEW ENERGY FINANCE 2010: 1ff

\begin{tabular}{|c|c|c|c|c|}
\hline $\begin{array}{c}\text { Autor (Jahr) } \\
\text { Titel }\end{array}$ & \multicolumn{2}{|c|}{ Methodik } & $\begin{array}{l}\text { Betrachtete } \\
\text { Regionen }\end{array}$ & $\begin{array}{l}\text { Betrachtungs- } \\
\text { horizont }\end{array}$ \\
\hline $\begin{array}{l}\text { OPEC (2010) } \\
\text { World oil Outlook }\end{array}$ & \multicolumn{2}{|c|}{$\begin{array}{l}\text { Verwendete Modelle: OPEC } \\
\text { World Energy Model (OWEM), } \\
\text { World Oil Refining Logistics and } \\
\text { Demand Model (WORLD) }\end{array}$} & Weltweit & $2009-2030$ \\
\hline \multicolumn{5}{|c|}{ Einflussfaktoren } \\
\hline \multicolumn{2}{|c|}{ EU27 } & \multicolumn{3}{|c|}{ USA } \\
\hline \multicolumn{2}{|c|}{$\begin{array}{l}\text { Politik } \\
\text { - Zertifizierung der Biokraftstoffe }\end{array}$} & \multicolumn{3}{|c|}{$\begin{array}{l}\text { Politik } \\
\text { - Anhebung der erlaubten Ethanolbeimischung } \\
\text { auf } 15 \% \text { (E15) }\end{array}$} \\
\hline
\end{tabular}




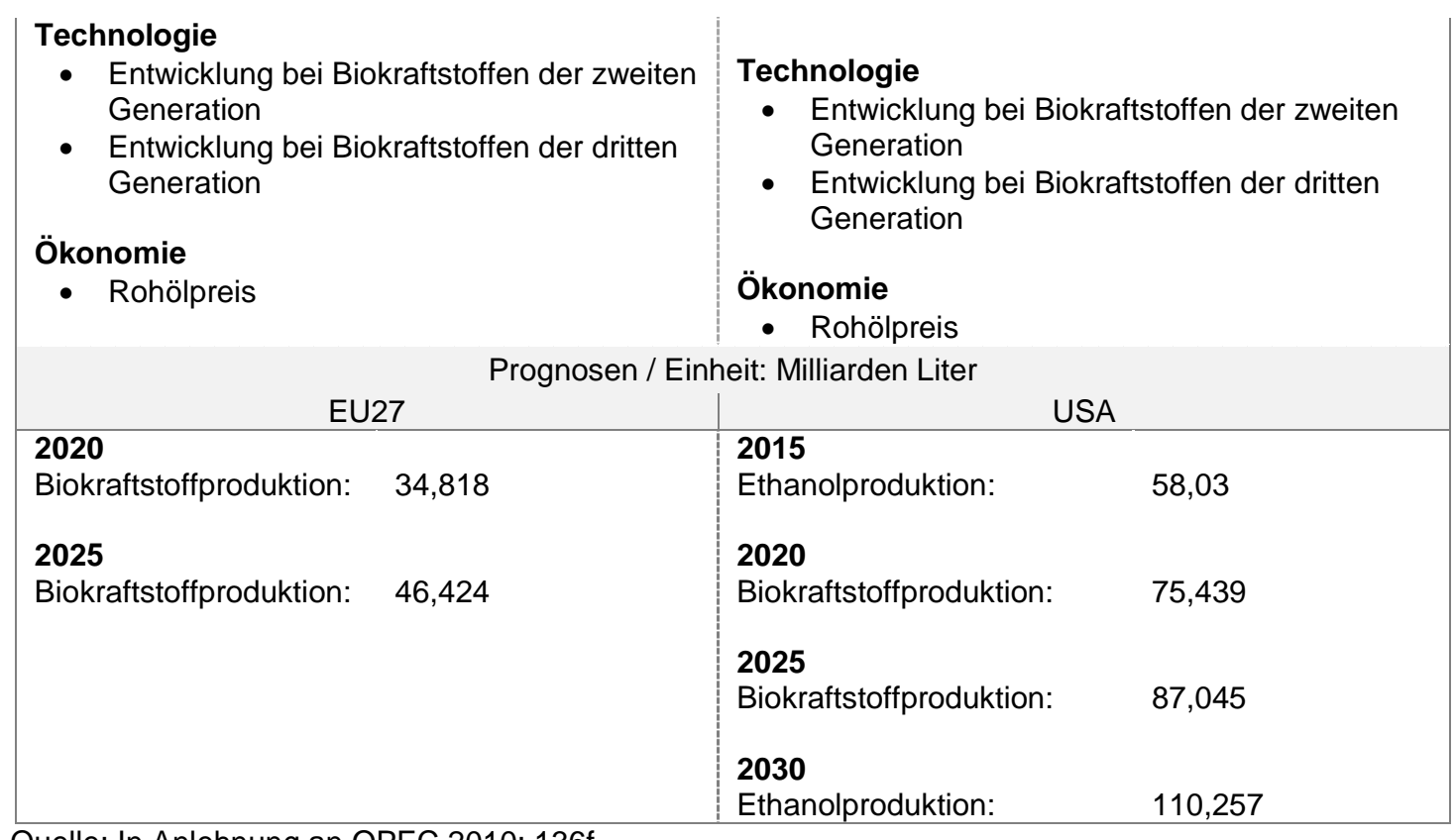

Quelle: In Anlehnung an OPEC 2010: $136 f$.

\begin{tabular}{|c|c|c|c|c|}
\hline $\begin{array}{l}\text { Autor (Jahr) } \\
\text { Titel }\end{array}$ & \multicolumn{2}{|c|}{ Methodik } & $\begin{array}{l}\text { Betrachtete } \\
\text { Regionen }\end{array}$ & $\begin{array}{l}\text { Betrachtungs- } \\
\text { horizont }\end{array}$ \\
\hline $\begin{array}{l}\text { International Energy Agency } \\
\text { (2009) } \\
\text { Medium-Term Oil Market } \\
\text { Report }\end{array}$ & \multicolumn{2}{|c|}{$\begin{array}{l}\text { IEA's World Energy Model } \\
\text { (WEM) }\end{array}$} & Weltweit & $2008-2014$ \\
\hline \multicolumn{5}{|c|}{ Einflussfaktoren } \\
\hline \multicolumn{2}{|l|}{ EU27 } & \multicolumn{3}{|c|}{ USA } \\
\hline \multicolumn{2}{|c|}{$\begin{array}{l}\text { Politik } \\
\text { - EU-Zielvorgaben und deren Umsetzung } \\
\text { bei Biokraftstoffen } \\
\text { - EU-Handelsbeschränkungen bei Ethanol- } \\
\text { importen }\end{array}$} & \multicolumn{3}{|c|}{$\begin{array}{l}\text { Politik } \\
\text { - Ethanol-Importzölle } \\
\text { - Politische Ziele der Kohlenstoffdioxidreduzie- } \\
\text { rung } \\
\text { - } 15 \% \text {-Ethanolbeimischung Entscheidung der } \\
\\
\text { EPA (Environmental Protection Agency) }\end{array}$} \\
\hline $\begin{array}{l}\text { Ökonomie } \\
\text { - Rohölpreis } \\
\text { - Rohstoffkosten der Herste }\end{array}$ & ung & \multicolumn{3}{|c|}{$\begin{array}{l}\text { Technologie } \\
\text { - Produktionskosten der Ethanolherstellung }\end{array}$} \\
\hline \multicolumn{2}{|c|}{$\begin{array}{l}\text { Gesellschaft } \\
\text { - Tank-oder-Teller-Diskussion } \\
\text { - Diskussion über die Nachhaltigkeit von } \\
\text { Ethanol }\end{array}$} & \multicolumn{3}{|c|}{$\begin{array}{l}\text { Ökonomie } \\
\text { - } \text { Maispreise } \\
\text { - } \text { Ethanolimporte aus Brasilien } \\
\text { - } \text { Entwicklung bei Flex-Fuel-Vehicles } \\
\text { Gesellschaft } \\
\text { - Diskussion über die Nachhaltigkeit von } \\
\text { Ethanol }\end{array}$} \\
\hline \multicolumn{5}{|c|}{ Prognosen / Einheit: Milliarden Liter } \\
\hline \multicolumn{2}{|l|}{ EU27 } & \multicolumn{3}{|c|}{ USA } \\
\hline \multicolumn{2}{|l|}{$\begin{array}{l}2014 \\
\text { Ethanolproduktion: }\end{array}$} & \multicolumn{3}{|c|}{$\begin{array}{l}2014 \\
\text { Ethanolproduktion: } \quad 46,076\end{array}$} \\
\hline
\end{tabular}

Quelle: In Anlehnung an INTERNATIONAL ENERGY AGENCY 2009: 68ff. 


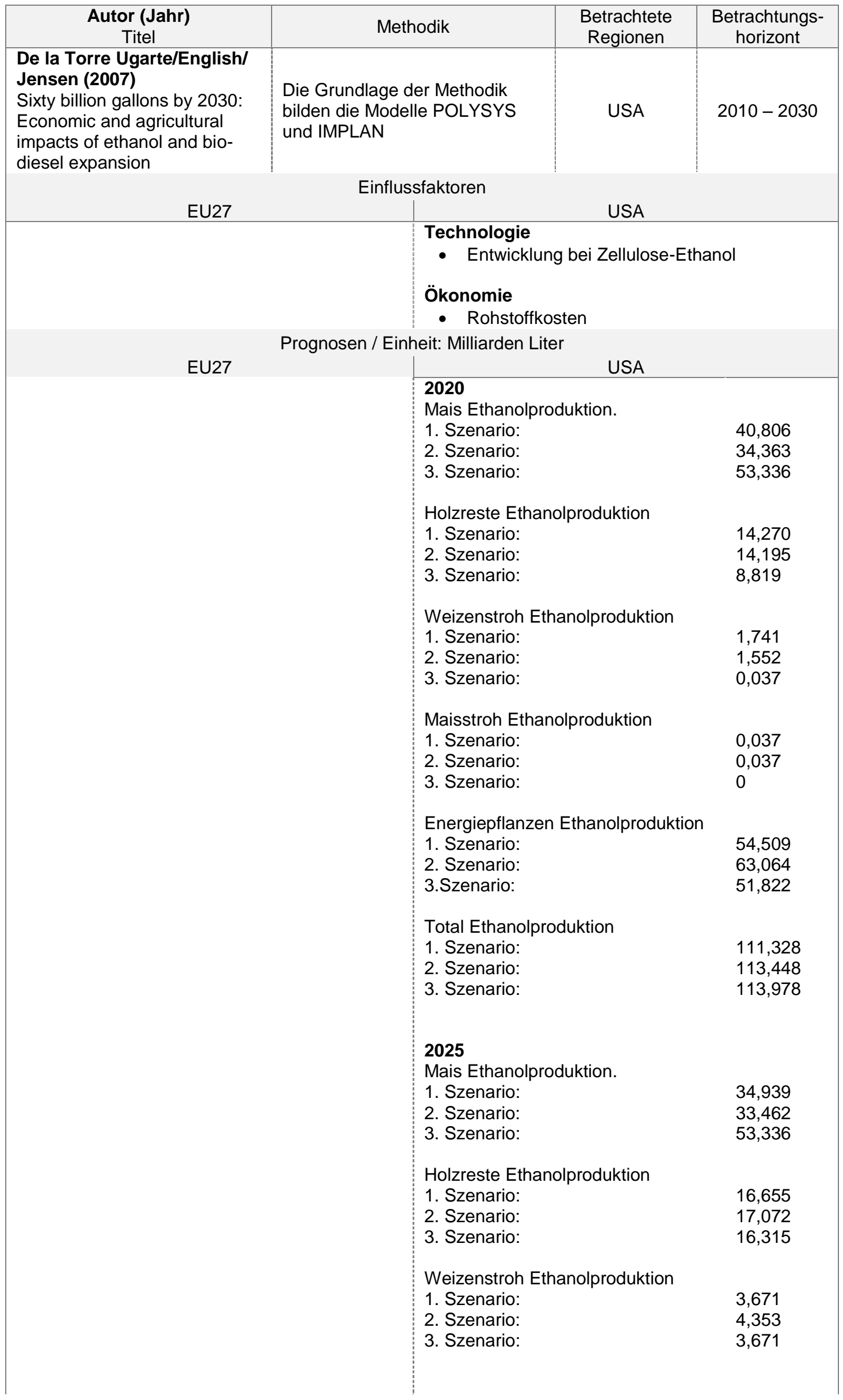









\section{Anhang 2: Fragebogen zur Durchführung der EU27-Wirkungsanalyse}

Dear Sir or Madam,

My name is Tillmann Anschütz and as a graduate student at the University of Göttingen, Germany - Department of Agricultural Economics and Rural Development - I am currently working on my doctoral thesis on the subject of "Ethanol Markets in the EU and in the USA in the Year 2023". I am academically accompanied by Prof. Dr. Ludwig Theuvsen.

The objective of my study is to develop scenarios for the Ethanol Markets in the EU and in the USA in the Year 2023. The following core questions will be answered in the dissertation:

1.

What are the essential factors that influence the Ethanol Markets in the EU and in the USA?

2. How are these factors connected to each other?

3. How will the Ethanol Market look like in the EU and in the USA in the Year $2023 ?$

At the moment, I am working on the first question for the Ethanol Market of the EU. In order to answer this question, the 28 factors listed in the questionnaire that affect the Ethanol Market of the European Union need to be rated.

I kindly ask you as an expert of the Ethanol Market to rate how strongly each factor influences the future of the Ethanol Market of the EU until 2023.

To evaluate each factor, please use the rating scale below:

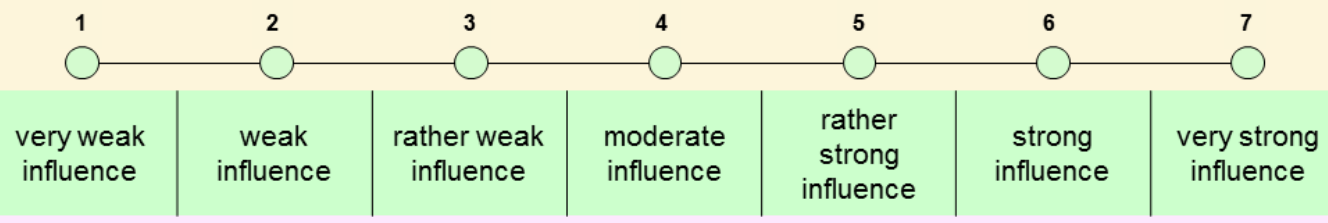

In case you feel insecure rating a factor, please leave the rating scale blank.

Of course, all answers will be kept anonymously.

Thank you very much for your time and your support.

T. Anschint $z$

Tillmann Anschütz 


\section{Economics [EU] GÖTTINGEN}

\section{\begin{tabular}{c|c|c|c|c|c|c}
$\begin{array}{c}1=\text { very weak } \\
\text { influence }\end{array}$ & $\begin{array}{c}2=\text { weak } \\
\text { influence }\end{array}$ & $\begin{array}{c}3=\text { rather weak } \\
\text { influence }\end{array}$ & $\begin{array}{c}4 \text { = moderate } \\
\text { influence }\end{array}$ & $\begin{array}{c}5=\text { rather } \\
\text { strong influence }\end{array}$ & $\begin{array}{c}6=\text { strong } \\
\text { influence }\end{array}$ & $\begin{array}{c}7=\text { very strong } \\
\text { influence }\end{array}$
\end{tabular}}

\begin{tabular}{l|l|}
\hline 1. & Factor: \\
\hline Evaluation: \\
\hline
\end{tabular}

\section{Gasoline demand in the EU}

12

2.

Factor:

Evaluation

Crude oil price in the EU

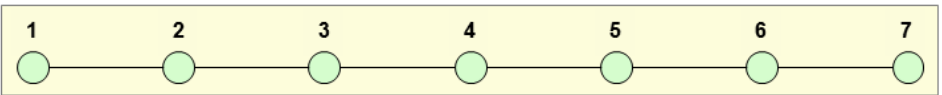

3. Factor:

Evaluation:

Ethanol imports from Brazil into the EU

12

4.

\begin{tabular}{l|} 
Factor: \\
\hline Evaluation:
\end{tabular}

Ethanol imports from the USA into the EU

1

5.

Factor:

Evaluation:
Ethanol imports from countries with duty-free access to the EU

1

6.

\begin{tabular}{|l|}
\hline Factor: \\
\hline Evaluation: \\
\hline
\end{tabular}

Market for commercially valuable by-products of the ethanol production in the EU

1

\begin{tabular}{|l|l|}
\hline 7. & Factor: \\
\hline Evaluation: \\
\hline
\end{tabular}

Demand for ETBE (Ethyl-tertiary butyl ether) in the EU

\begin{tabular}{|ccccccc|}
\hline 1 & 2 & 3 & 4 & 5 & 6 & 7 \\
& & & & & & \\
\hline
\end{tabular}




\section{Economics [EU]

\begin{tabular}{c|c|c|c|c|c|c}
$\begin{array}{c}1 \text { = very weak } \\
\text { influence }\end{array}$ & $\begin{array}{c}2=\text { weak } \\
\text { influence }\end{array}$ & $\begin{array}{c}3=\text { rather weak } \\
\text { influence }\end{array}$ & $\begin{array}{c}4=\text { moderate } \\
\text { influence }\end{array}$ & $\begin{array}{c}5=\text { rather } \\
\text { strong influence }\end{array}$ & $\begin{array}{c}6=\text { strong } \\
\text { influence }\end{array}$ & $\begin{array}{c}7=\text { very strong } \\
\text { influence }\end{array}$
\end{tabular}

\begin{tabular}{l|l|}
\hline 8. & Factor: \\
\hline Evaluation: \\
\hline
\end{tabular}

Vertical strategic partnerships between companies in the ethanol market of the EU

$\begin{array}{lllllll}1 & 2 & 3 & 4 & 5 & 6 & 7 \\ & & & & & & \end{array}$

9.

\begin{tabular}{|l|}
\hline Factor: \\
\hline \hline Description: \\
\hline Evaluation: \\
\hline
\end{tabular}

Production costs for first generation ethanol in the EU - without feedstock costs

The feedstock costs are not included in the production costs since they are considered separately in Factor 11.



10.

\begin{tabular}{|l|}
\hline Factor: \\
\hline Description: \\
\hline
\end{tabular}

Evaluation:
Production costs for cellulosic ethanol in the EU - without feedstock costs

The feedstock costs are not included in the production costs since they are considered separately in Factor 12

$\begin{array}{lllllll}1 & 2 & 3 & 5 & 6 & 7 \\ & & & & & & \end{array}$

\begin{tabular}{l|l|}
\hline 11. & Factor: \\
\hline Evaluation: \\
\hline
\end{tabular}

\section{Feedstock costs for first generation ethanol in the EU}

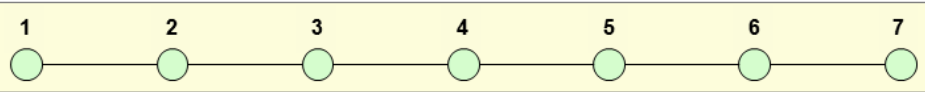

12. Factor:

Evaluation:
Feedstock costs for cellulosic ethanol in the EU

1

Further comments concerning Economics: 


\section{Technology [EU]}

\begin{tabular}{|c|c|c|c|c|c|c|c|}
\hline \multicolumn{2}{|c|}{$\begin{array}{l}1=\text { very weak } \\
\text { influence }\end{array}$} & $\begin{array}{l}2=\text { weak } \\
\text { influence }\end{array}$ & $\begin{array}{c}3=\text { rather weak } \\
\text { influence }\end{array}$ & $\begin{array}{l}4=\text { moderate } \\
\text { influence }\end{array}$ & $\begin{array}{c}5=\text { rather } \\
\text { strong influence }\end{array}$ & $\begin{array}{l}6=\text { strong } \\
\text { influence }\end{array}$ & $\begin{array}{l}7=\text { very strong } \\
\text { influence }\end{array}$ \\
\hline 13. & & & \multicolumn{5}{|c|}{ Number of Flexible Fuel Vehicles in the EU } \\
\hline & \multicolumn{2}{|c|}{ Evaluation: } & 1 & 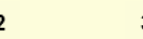 & 4 & 5 & 6 \\
\hline
\end{tabular}

14. Factor:

Evaluation:

Number of conventional gasoline vehicles in the EU able to run on intermediate ethanol blends (E15 - E40)

1

15.

Factor:

Number of Electric Drive Vehicles in the EU

Description

Evaluation:

Electric Drive Vehicles are Battery Electric Vehicles and Hybrid Electric Vehicles.

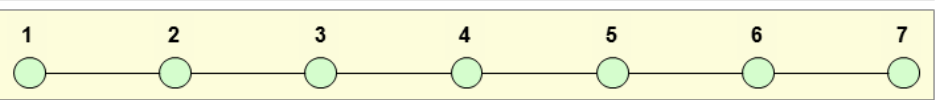

16. Factor:

Evaluation:

Enzymes technology for cellulosic ethanol

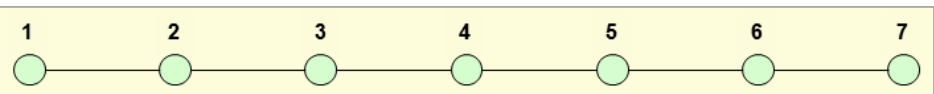

17. Factor:

Evaluation:

GHG balance of first generation ethanol produced in the EU

12

18. Factor:

Evaluation:
GHG balance of cellulosic ethanol produced in the EU

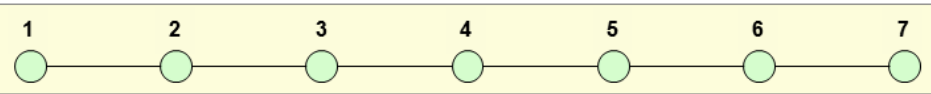

19. Factor:

Evaluation
Biomass to Liquid (BtL) capable of substituting fuel ethanol in the EU

1

\begin{tabular}{l|l|}
\hline 20. & Factor: \\
\hline Evaluation: \\
\hline
\end{tabular}

Further comments concerning Technology: 


\section{Politics [EU]}

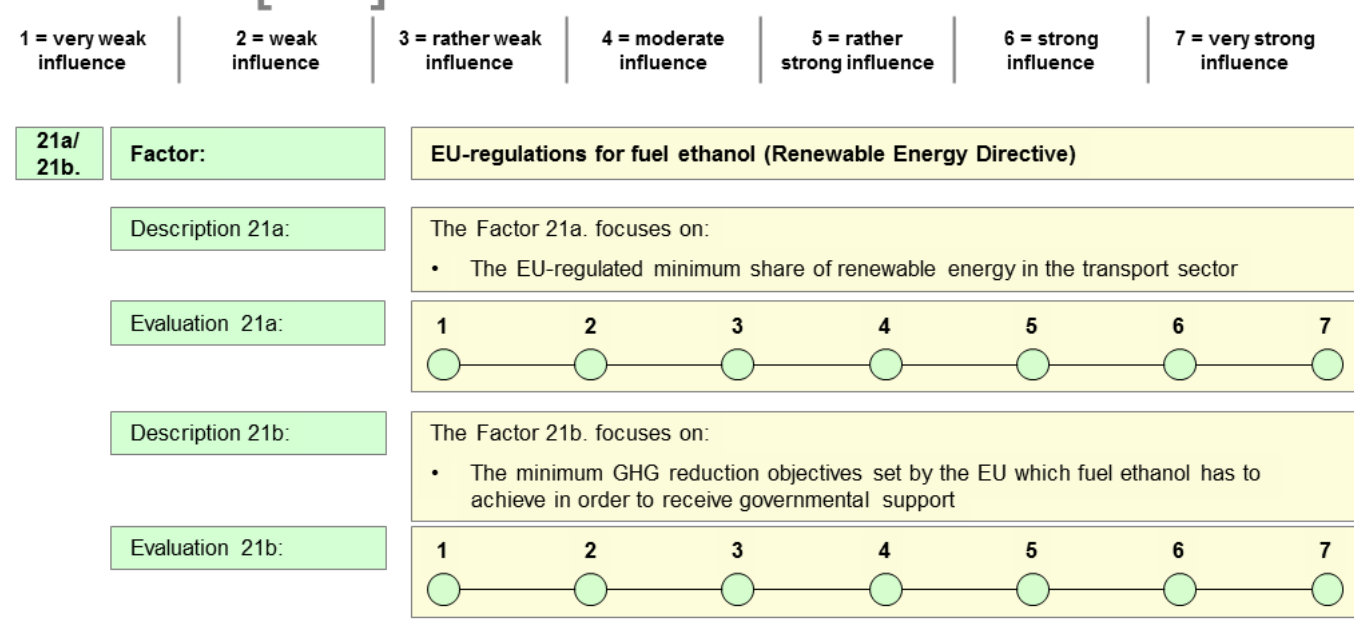

\begin{tabular}{|l|l|}
\hline $\begin{array}{l}\text { 22al } \\
\text { 22b. }\end{array}$ & Factor: \\
& Description 22a: \\
& Evaluation 22a: \\
& Description 22b: \\
& \\
\hline Evaluation 22b: \\
\hline 23. \\
\hline
\end{tabular}

\section{EU-regulations for EU road transport fuel (Fuel Quality Directive)}

The factor 22a. focuses on:

- The GHG reduction objective for EU road transport fuel

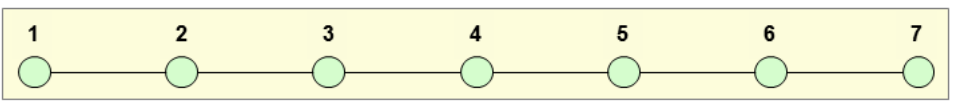

The factor 22b. focuses on:

- The legally obligated maximum blending share of ethanol to gasoline

1

\begin{tabular}{|l|l|}
\hline 24. & Factor: \\
\hline & Evaluation: \\
\hline
\end{tabular}

Regulations of the EU member states for fuel ethanol in the transport sector

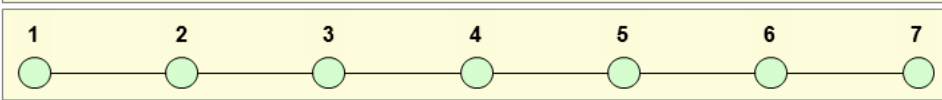

\section{EU regulations concerning ILUC (Indirect Land Use Change)}

$\begin{array}{lllllll}1 & 2 & 4 & 5 & 6 & 7\end{array}$

\begin{tabular}{l|l|}
\hline 25. & Factor: \\
\hline Evaluation: \\
\hline
\end{tabular}

Customs regulations for ethanol imports into the EU

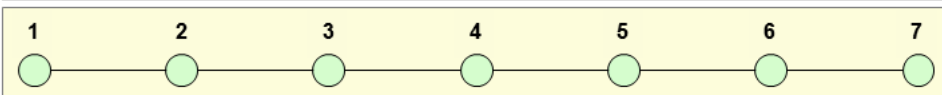

Further comments concerning Politics: 
Society [EU]

GEORG-AUGUST-UNIVERSITÄT GÖTTINGEN

\begin{tabular}{c|c|c|c|c|c|c}
$\begin{array}{c}\text { 1=very weak } \\
\text { influence }\end{array}$ & $\begin{array}{c}2=\text { weak } \\
\text { influence }\end{array}$ & $\begin{array}{c}3=\text { rather weak } \\
\text { influence }\end{array}$ & $\begin{array}{c}4=\text { moderate } \\
\text { influence }\end{array}$ & $\begin{array}{c}5=\text { rather } \\
\text { strong influence }\end{array}$ & $\begin{array}{c}6=\text { strong } \\
\text { influence }\end{array}$ & $\begin{array}{c}7=\text { very strong } \\
\text { influence }\end{array}$
\end{tabular}

26.

\begin{tabular}{l}
\hline Factor: \\
\hline Description: \\
\hline Evaluation: \\
\hline
\end{tabular}

Food vs. Fuel Discussion in the EU

The factor exclusively concentrates on the debate in the EU and not on the scientific reasoning behind the food vs. fuel discussion.

1

27.

\begin{tabular}{|l|}
\hline Factor: \\
\hline \hline Description: \\
\hline
\end{tabular}

Evaluation:
Discussion in the EU about the effects of ethanol on the environment

The factor exclusively concentrates on the debate in the $\mathrm{EU}$ about the effects of ethanol on the environment. Thus, the factor does not consider the scientific reasoning behind the debate.

1

28.

\begin{tabular}{|l|}
\hline Factor: \\
\hline Description: \\
\hline Evaluation: \\
\hline
\end{tabular}

Debate in the EU about the effects of ethanol on the energy independence of the EU

Only the debate in the EU about the effects of ethanol on EU energy independence is of interest and not, if the arguments brought forward are backed by scientific findings.

1

Further comments concerning Society:

Country of origin of your company/ organization:

Industry sector of your company/ organization: Ethanol Production $\bigcirc \quad$ Enzymes Technology $\bigcirc \mid$ Plant Engineer $\bigcirc$ \begin{tabular}{l|l|l|l} 
Petroleum Company $\bigcirc$ & Research Institute $\bigcirc$ & Industry Association $\bigcirc \quad$ Other:
\end{tabular}

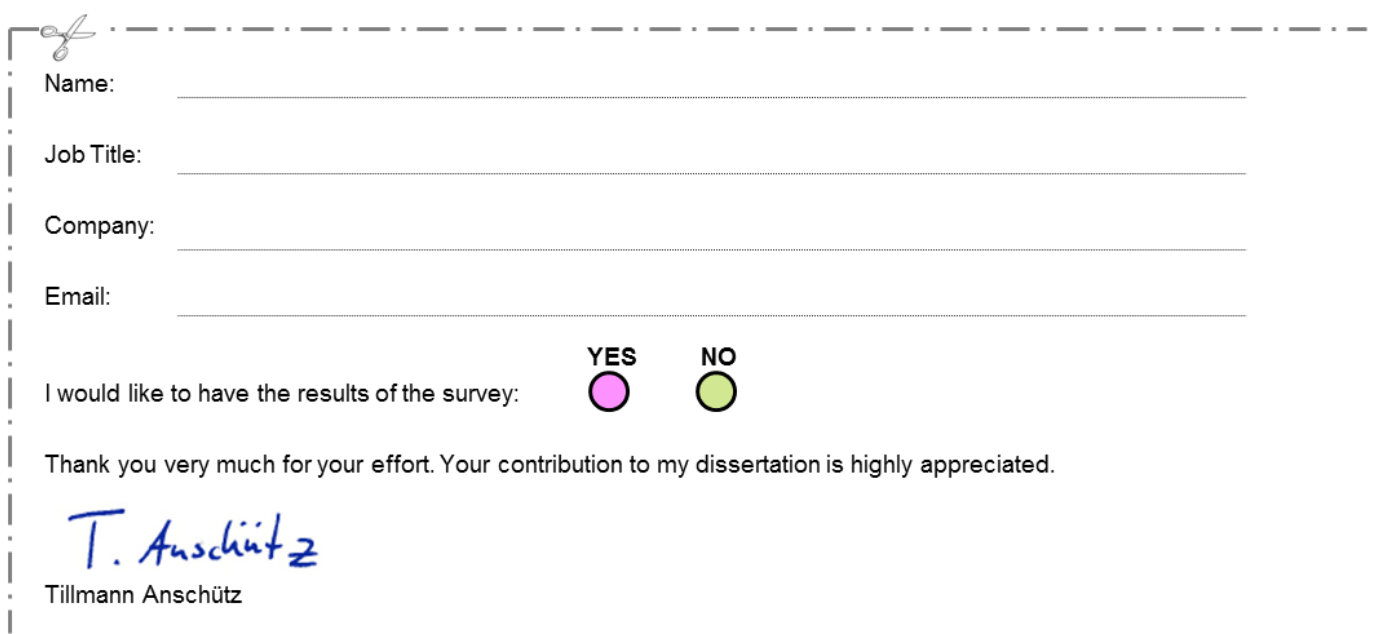




\section{Anhang 3: Fragebogen zur EU27-Konsistenzprüfung}

Dear Sir or Madam,

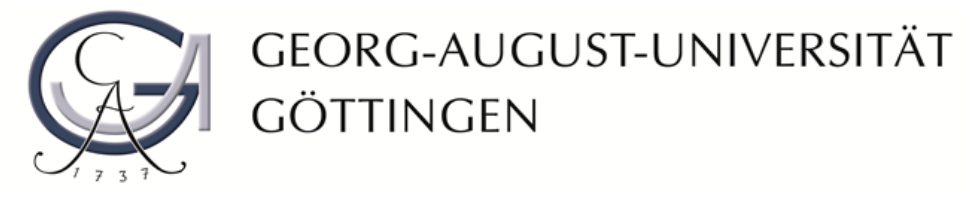

It is the objective of my study to develop scenarios for the fuel ethanol market of the EU 27 in the year 2023. Based on extensive research, I developed four different scenarios. I would like to ask you to support me by rating the consistency of the four scenarios described on the following pages.

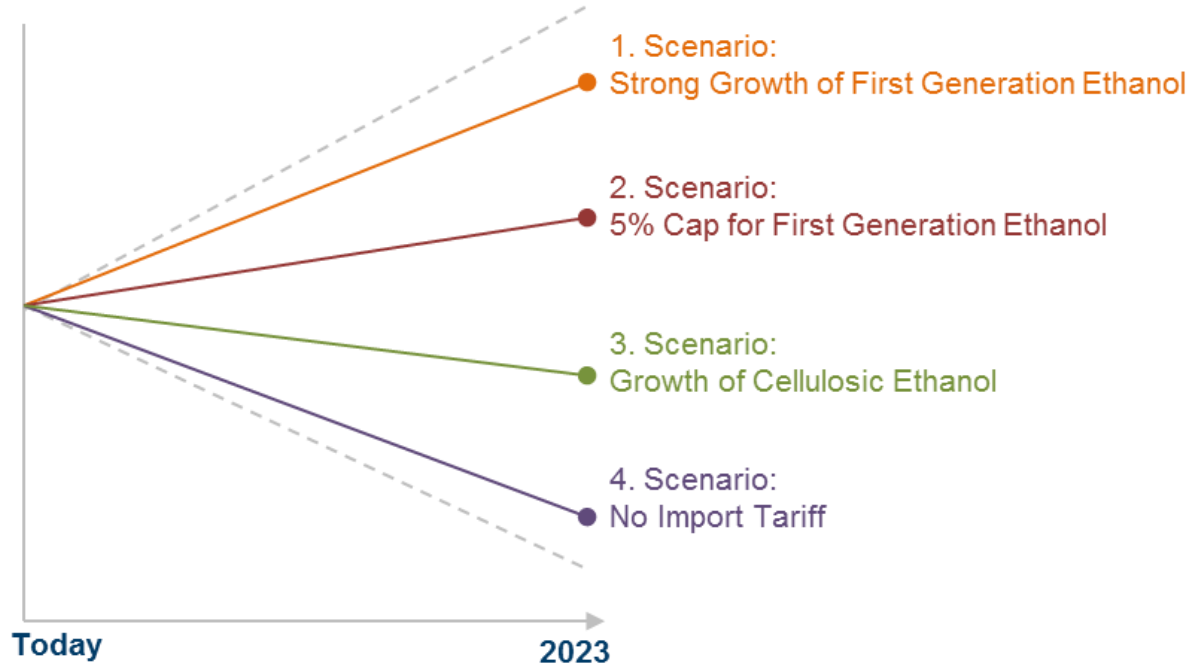

The scenarios $1-4$ describe the fuel ethanol market of the EU 27 in the year 2023. Each scenario is briefly described and the main components of each scenario are named. When rating the consistency of the scenarios, please keep in mind that the actual figures in the scenarios shall not be regarded as the decisive information. Rather essential are the trends of the future development that are pointed out in the scenarios. Moreover, please concentrate solely on the consistency of the scenarios and not whether the scenarios are realistic. Consistency means that the components of each scenario are coherent and do not contradict one another.

Furthermore, please be open minded since scenarios are innovative and indicate new market developments. In case you feel unsure rating a scenario, please leave the rating scale blank. It will not take you more than 10-15 minutes to complete the questionnaire and of course, all answers will be kept anonymously.

Your support of my dissertation is highly appreciated.

Sincerely yours

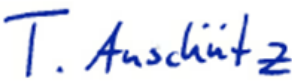

Tillmann Anschütz 
Scenario 1:

Strong Growth of First Generation Ethanol

In the year 2023, after years of constant growth, the demand for fuel ethanol is around 14.5 billion liters in the EU $27-13$ billion liters are produced in the EU 27 as first generation ethanol and 1.5 billion liters are imported. The strong political support for biofuels is the key driver for the growing market. The GHG reduction mandates were raised but the ILUC-Factor is irrelevant. However, the ethanol producers are able to meet the GHG requirements. Moreover, all cars can use E10 and $54 \%$ of them are able to run on E20. The crude oil price constantly rose, while the gasoline demand decreased continually until the year 2023. The ethanol producers are able to profitably produce first generation ethanol.

\section{Please use this link to get to a more detailed description of the scenario}

Scenario Components:

\begin{tabular}{|c|c|c|c|c|c|}
\hline Politics & $\begin{array}{l}{[1.1]} \\
{[1.2]} \\
{[1.3]} \\
{[1.4]} \\
{[1.6]}\end{array}$ & \multicolumn{4}{|c|}{$\begin{array}{l}\text { Objective: } 10 \% \text { of the energy used in the transport sector derive from renewable } \\
\text { sources } \\
\text { No cap for the use of first generation biofuels } \\
\text { Political support for second generation biofuels } \\
\text { Stricter GHG regulations for ethanol } \\
\text { The ILUC-Factor is irrelevant } \\
\text { Tariffs on ethanol imports into the EU } 27\end{array}$} \\
\hline Economics & $\begin{array}{l}{[2.1]} \\
{[2.2]} \\
{[2.3]}\end{array}$ & $\begin{array}{l}\text { Increase of th } \\
\text { Constant dec } \\
\text { Price for whe }\end{array}$ & $\begin{array}{l}\text { I crude oil price } \\
\text { of the gasoline }\end{array}$ & $\begin{array}{l}\text { around (real } 20 \\
\text { and in the EU } 27 \\
S-\$ / \text { ton }\end{array}$ & 130 US-\$/ barrel \\
\hline Technology & [3.1] & Conventional & line vehicles ha & igh ethanol con & oility \\
\hline $\begin{array}{l}\text { Descriptive } \\
\text { Factors }\end{array}$ & $\begin{array}{l}{[4.1]} \\
{[4.2]} \\
{[4.3]} \\
{[4.4]} \\
{[4.5]}\end{array}$ & $\begin{array}{l}\text { Strong increa } \\
\text { billion liters } \\
\text { No significant } \\
\text { Strong increa } \\
\text { Ethanol impor } \\
\text { Ethanol mark }\end{array}$ & $\begin{array}{l}\text { the production } \\
\text { uction of cellulo } \\
\text { the demand for } \\
\text { around } 1.5 \text { billic } \\
\text { e of about (rea }\end{array}$ & $\begin{array}{l}\text { generation eth } \\
\text { hanol } \\
\text { thanol up to ab } \\
75 \text { US-\$/ hl }\end{array}$ & up to around 13 \\
\hline $\begin{array}{l}\text { Evaluation of } \\
\text { Consistency m }\end{array}$ & $\begin{array}{l}\text { e Con } \\
\text { ans tha }\end{array}$ & $\begin{array}{l}\text { stency: } \\
\text { he componen }\end{array}$ & scenario are & rent and do not & adict one another. \\
\hline $\begin{array}{l}\text { Very Goo } \\
\text { Consisten }\end{array}$ & & $\begin{array}{c}\text { Good } \\
\text { Consistency }\end{array}$ & $\begin{array}{c}\text { Moderate } \\
\text { Consistency }\end{array}$ & $\begin{array}{c}\text { Poor } \\
\text { Consistency }\end{array}$ & $\begin{array}{c}\mathbf{5} \\
\text { Very Poor } \\
\text { Consistency }\end{array}$ \\
\hline $\begin{array}{l}\text { If you think } \\
\text { to name them }\end{array}$ & $\begin{array}{l}\text { Donen } \\
d \text { to } e\end{array}$ & $\begin{array}{l}\text { of the scenar } \\
\text { lain why you }\end{array}$ & $\begin{array}{l}\text { hot consistent } \\
\text { them as incon }\end{array}$ & ne another, ple & e the box below \\
\hline
\end{tabular}


Scenario 2:

5\% Cap for First Generation Ethanol

In the year 2023, the demand for fuel ethanol is around 7.5 billion liters in the EU $27-6$ billion liters are produced domestically and 1.5 billion liters are imported. The ethanol market has not grown further due to the political cap to count no more than $5 \%$ of first generation biofuel towards the $10 \%$ goal. Although cellulosic ethanol receives strong political support, there is not any significant production. The GHG reduction mandates were raised but the ILUC-Factor is irrelevant. Nevertheless, the ethanol producers are able to meet the GHG requirements. All cars in the EU 27 are able to run on E10, but they are not capable of using higher blends. The crude oil price rose moderately, while the gasoline demand decreased continually until the year 2023 . The ethanol producers are able to profitably produce first generation ethanol.

\section{Please use this link to get to a more detailed description of the scenario}

\section{Scenario Components:}

\begin{tabular}{|c|c|c|}
\hline \multirow{6}{*}{ Politics } & [1.1] & $\begin{array}{l}\text { Objective: } 10 \% \text { of the energy used in the transport sector derive from renewable } \\
\text { sources }\end{array}$ \\
\hline & [1.2] & $5 \%$ cap for the use of first generation biofuels in the EU 27 \\
\hline & [1.3] & Strong political support for cellulosic ethanol \\
\hline & [1.4] & Stricter GHG regulations for ethanol \\
\hline & [1.5] & The ILUC-Factor is irrelevant \\
\hline & [1.6] & Tariffs on ethanol imports into the EU 27 \\
\hline \multirow{3}{*}{ Economics } & [2.1] & $\begin{array}{l}\text { Moderate increase of the WTI crude oil price up to around (real 2010) } 97 \text { US-\$/ } \\
\text { barrel }\end{array}$ \\
\hline & [2.2] & Constant decrease of the gasoline demand in the EU 27 \\
\hline & [2.3] & Price for wheat about (real 2005) 206 US-\$/ ton \\
\hline Technology & [3.1] & All conventional gasoline vehicles are able to run on E10 \\
\hline \multirow{5}{*}{$\begin{array}{l}\text { Descriptive } \\
\text { Factors }\end{array}$} & [4.1] & $\begin{array}{l}\text { Moderate increase of the production of first generation ethanol up to around } 6 \\
\text { billion liters }\end{array}$ \\
\hline & [4.2] & No significant production of cellulosic ethanol \\
\hline & [4.3] & Moderate increase of the demand for fuel ethanol up to about 7.5 billion liters \\
\hline & [4.4] & Ethanol imports of around 1.5 billion liters \\
\hline & [4.5] & Ethanol market price of about (real 2005) 75 US-\$/ hl \\
\hline
\end{tabular}

Evaluation of the Consistency:

Consistency means that the components of the scenario are coherent and do not contradict one another.

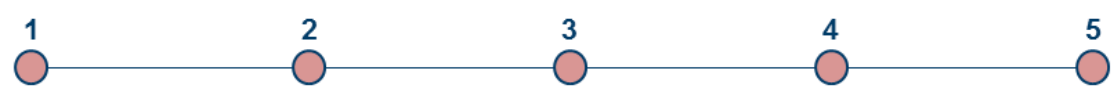

\begin{tabular}{|c|c|c|c|c|}
\hline $\begin{array}{c}\text { Very Good } \\
\text { Consistency }\end{array}$ & $\begin{array}{c}\text { Good } \\
\text { Consistency }\end{array}$ & $\begin{array}{c}\text { Moderate } \\
\text { Consistency }\end{array}$ & $\begin{array}{c}\text { Poor } \\
\text { Consistency }\end{array}$ & $\begin{array}{c}\text { Very Poor } \\
\text { Consistency }\end{array}$ \\
\hline
\end{tabular}

If you think components of the scenario are not consistent with one another, please use the box below to name them and to explain why you regard them as inconsistent. 
Scenario 3:

\section{Growth of Cellulosic Ethanol}

In the year 2023, politics exclusively support second generation biofuels to achieve the $10 \%$ goal. Cellulosic ethanol gets multiple counted towards the $10 \%$ goal and substitutes first generation fuel ethanol. The demand for cellulosic ethanol is around 3 billion liters and gets completely supplied by the production in the EU 27. Furthermore, unlike cellulosic ethanol producers, most first generation ethanol producers are not capable of achieving the politically required $\mathrm{CO}_{2}$ savings due to the stricter $\mathrm{GHG}$ reduction targets and the consideration of an ILUC-Factor. All cars in the EU 27 are able to run on E10 but they are not capable of using higher blends. The crude oil price rose while the gasoline demand decreased continually until the year 2023. The EU 27 ethanol producers are able to profitably produce cellulosic ethanol because of the high cellulosic ethanol market price.

\section{Please use this link to get to a more detailed description of the scenario}

Scenario Components:



If you think components of the scenario are not consistent with one another, please use the box below to name them and to explain why you regard them as inconsistent. 


\section{Scenario 4:}

No Import Tariff

In the year 2023, the import tariff for fuel ethanol had been abolished and Brazil uses this situation by exporting around 4.5 billion liters cane ethanol to the EU 27. Furthermore, unlike Brazilian ethanol, most first generation ethanol produced in the EU 27 is not capable of achieving the politically required $\mathrm{CO}_{2}$ savings due to the stricter GHG reduction targets and the consideration of an ILUC-Factor. Cellulosic ethanol produced in the EU 27 is able to fulfill the GHG targets and gets strongly politically supported by multiple counting the energy content towards the $10 \%$ goal. The EU 27 production of cellulosic ethanol is around 3 billion liters and is needed to meet the demand for fuel ethanol of around 7.5 billion liters. All cars in the EU 27 are able to run on E10, but they are not capable of using higher blends. The crude oil price rose while the gasoline demand decreased continually until the year 2023. Furthermore, in contrast to first generation ethanol, EU 27 producers of cellulosic ethanol are able to economically produce cellulosic ethanol because of the higher market price compared to the competitive cane ethanol price.

\section{Please use this link to get to a more detailed description of the scenario:}

\section{Scenario Components:}

\begin{tabular}{|c|c|c|c|c|c|}
\hline \multirow{5}{*}{ Politics } & [1.1] & \multicolumn{4}{|c|}{$\begin{array}{l}\text { Objective: } 10 \% \text { of the energy used in the transport sector derive from renewable } \\
\text { sources }\end{array}$} \\
\hline & [1.2] & \multicolumn{4}{|c|}{ Strong political support for cellulosic ethanol } \\
\hline & [1.3] & \multicolumn{4}{|c|}{ Stricter GHG regulations for ethanol } \\
\hline & [1.4] & \multicolumn{4}{|c|}{ Consideration of a raw material dependent ILUC-Fact } \\
\hline & [1.5] & \multicolumn{4}{|c|}{ Duty-free import of ethanol into the EU 27} \\
\hline Economics & $\begin{array}{l}{[2.1]} \\
{[2.2]} \\
{[2.3]}\end{array}$ & \multicolumn{4}{|c|}{$\begin{array}{l}\text { Increase of the WTI crude oil price up to around (real 2010) } 130 \text { US-\$/ barrel } \\
\text { Constant decrease of the gasoline demand in the EU } 27 \\
\text { Price for wheat about (real 2005) } 206 \text { US-\$/ ton }\end{array}$} \\
\hline Technology & [3.1] & \multicolumn{4}{|c|}{ All conventional gasoline vehicles are able to run on E10 } \\
\hline $\begin{array}{l}\text { Descriptive } \\
\text { Factors }\end{array}$ & $\begin{array}{l}{[4.1]} \\
{[4.2]} \\
{[4.3]} \\
{[4.4]} \\
{[4.5]}\end{array}$ & $\begin{array}{l}\text { No EU } 27 \text { pro } \\
\text { the cellulosic } \\
\text { Moderate inc } \\
\text { Ethanol impo } \\
\text { Cane ethano } \\
\text { The cellulosic }\end{array}$ & $\begin{array}{l}\text { on of first gener } \\
\text { ol production u } \\
4.5 \text { billion liters } \\
\text { of the ethanol price of arou } \\
\text { anol market pric }\end{array}$ & $\begin{array}{l}\text { ethanol for the } \\
\text { round } 3 \text { billion } \\
\text { d up to about } \\
\text { al 2005) } 65 \text { US } \\
\text { gher than the }\end{array}$ & $\begin{array}{l}\text { harket, but increase } \\
\text { lion liters }\end{array}$ \\
\hline \multicolumn{6}{|c|}{ Evaluation of the Consistency: } \\
\hline \multicolumn{6}{|c|}{ Consistency means that the components of the scenario are coherent and do not contradict one another. } \\
\hline $\begin{array}{l}\text { Very Goo } \\
\text { Consisten }\end{array}$ & & $\begin{array}{c}\text { Good } \\
\text { Consistency }\end{array}$ & $\begin{array}{c}\text { Moderate } \\
\text { Consistency }\end{array}$ & $\begin{array}{c}\text { Poor } \\
\text { Consistency }\end{array}$ & $\begin{array}{l}\text { Very Poor } \\
\text { Consistency }\end{array}$ \\
\hline
\end{tabular}

If you think components of the scenario are not consistent with one another, please use the box below to name them and to explain why you regard them as inconsistent. 


\section{Personal Data:}

Country of origin of your company/ organization

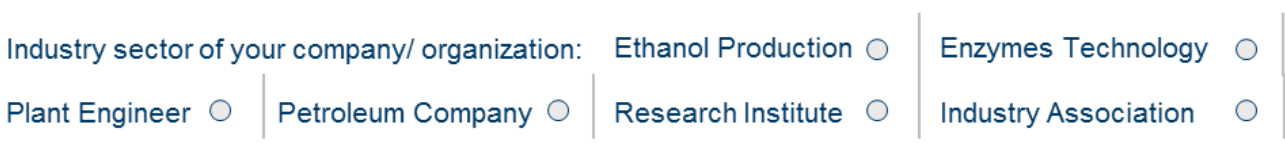
Other:

Name:

Job Title:

Company:

Email:

I would like to have the scenarios and the results of the survey: $\overbrace{}^{\text {YES }}$ NO

Thank you very much for your participation. Your contribution to my dissertation is highly appreciated.

T. Anschintz

Tillmann Anschütz 


\section{Anhang 4: Fragebogen zur Durchführung der USA-Wirkungsanalyse}

\section{Dear Sir or Madam,}

My name is Tillmann Anschütz and as a graduate student at the University of Göttingen, Germany - Department of Agricultural Economics and Rural Development - I am currently working on my doctoral thesis on the subject of "Ethanol Markets in the EU and in the USA in the year 2023 ". I am academically accompanied by Prof. Dr. Ludwig Theuvsen.

The objective of my study is to develop scenarios for the Ethanol Markets in the EU and in the USA in the year 2023. The following core questions will be answered in the dissertation:

1. What are the essential factors that influence the Ethanol Markets in the EU and in the USA?

2. How are these factors connected to each other?

3. How will the Ethanol Markets look like in the EU and in the USA in the year 2023?

At the moment, I am working on the first question for the Ethanol Market of the USA. In order to answer this question, the 31 factors listed in the questionnaire that affect the Ethanol Market of the USA need to be rated.

I kindly ask you as an expert of the Ethanol Market to rate how strongly each factor influences the future of the Ethanol Market of the USA until 2023.

To evaluate each factor, please use the rating scale below:

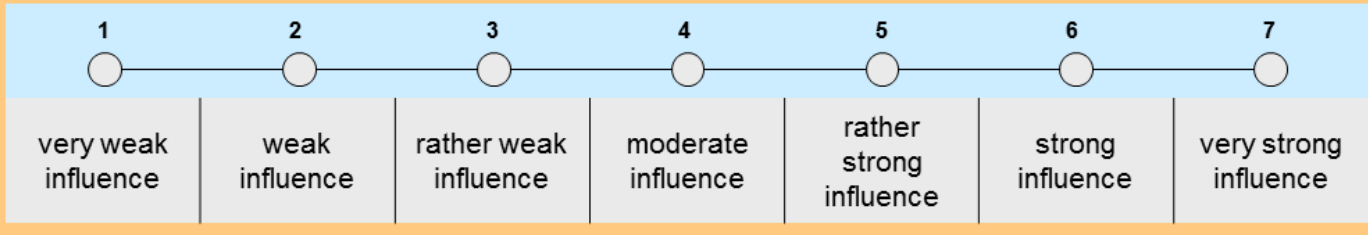

In case you feel unsure rating a factor, please leave the rating scale blank.

Of course, all answers will be kept anonymously.

Thank you very much for your time and your support.

T. Anscluntz

Tillmann Anschütz 


\section{Economics [USA]}

\begin{tabular}{|c|c|c|c|c|c|c|c|}
\hline \multicolumn{2}{|c|}{$\begin{array}{c}1 \text { = very weak } \\
\text { influence }\end{array}$} & $\begin{array}{l}2=\text { weak } \\
\text { influence }\end{array}$ & $\begin{array}{c}3=\text { rather weak } \\
\text { influence }\end{array}$ & $\begin{array}{c}4=\text { moderate } \\
\text { influence }\end{array}$ & $\begin{array}{c}5=\text { rather strong } \\
\text { influence }\end{array}$ & $\begin{array}{l}6=\text { strong } \\
\text { influence }\end{array}$ & $\begin{array}{c}7 \text { = very strong } \\
\text { influence }\end{array}$ \\
\hline \multirow[t]{2}{*}{1.} & \multicolumn{2}{|c|}{ Factor: } & \multicolumn{5}{|c|}{ Gasoline demand in the USA } \\
\hline & \multicolumn{2}{|c|}{ Evaluation: } & 1 & 3 & 4 & 5 & 7 \\
\hline
\end{tabular}

\begin{tabular}{|l|l|}
\hline 2. & Factor: \\
\hline & Evaluation: \\
\hline
\end{tabular}



\begin{tabular}{l|l|}
\hline 3. & Factor: \\
\hline \hline Evaluation: \\
\hline
\end{tabular}

Ethanol imports from Brazil into the USA



4. Factor:

Evaluation:

Ethanol exports from the USA to Brazil

1

\begin{tabular}{l|l|}
\hline 5. & Factor: \\
\hline Evaluation: \\
\hline
\end{tabular}

Ethanol exports from the USA to the EU

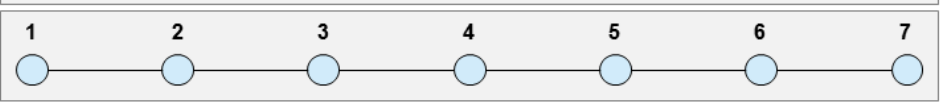

$\begin{aligned} 6 . & \text { Factor: } \\ & \text { Description: } \\ & \text { Evaluation: }\end{aligned}$

Ethanol exports from the USA to markets abroad - excluding the Brazilian and the EU ethanol market

The US-American ethanol exports to the Brazilian and EU ethanol market are not included since they are considered separately in the factors 4 and 5 .

1

\begin{tabular}{l|l|}
\hline 7. & Factor: \\
\hline & Evaluation: \\
\hline
\end{tabular}

US-American ethanol transport infrastructure from the production sites to the ethanol markets at the east and west coast

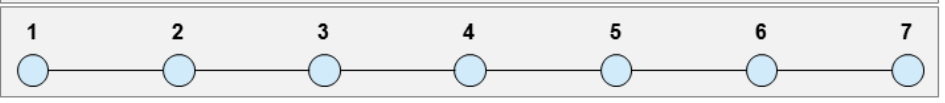

\begin{tabular}{l|l|}
\hline 8. & Factor: \\
\hline & Evaluation: \\
\hline
\end{tabular}

US-American end consumer distribution infrastructure for intermediate (E15 - E40) ethanol blends

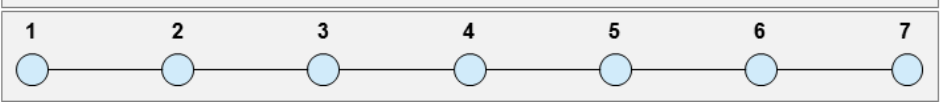




\section{Economics [USA]}

\begin{tabular}{c|c|c|c|c|c|c|c|c}
$\begin{array}{c}1=\text { very weak } \\
\text { influence }\end{array}$ & $\begin{array}{c}2=\text { weak } \\
\text { influence }\end{array}$ & $\begin{array}{c}3=\text { rather weak } \\
\text { influence }\end{array}$ & $\begin{array}{c}4=\text { moderate } \\
\text { influence }\end{array}$ & $\begin{array}{c}5=\text { rather strong } \\
\text { influence }\end{array}$ & $\begin{array}{c}6=\text { strong } \\
\text { influence }\end{array}$ & $\begin{array}{c}7=\text { very strong } \\
\text { influence }\end{array}$
\end{tabular}

9. Factor:

Evaluation:

US-American end consumer distribution infrastructure for high (E85) ethanol blends

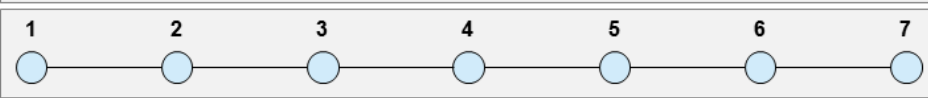

10.

Factor:

Market price for DDGS (Dried Distillers Grains and Solubles) in the USA

Evaluation:

12

11.

\begin{tabular}{l} 
Factor: \\
\hline Evaluation:
\end{tabular}

Vertical strategic partnerships between companies in the ethanol market of the USA

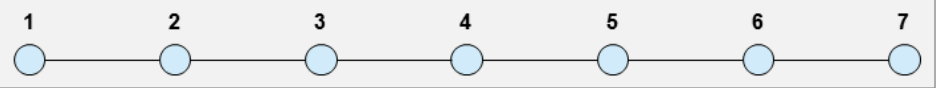

12.

Factor:

Description:

Production costs for corn ethanol in the USA - without feedstock costs

The feedstock costs are not included in the production costs since they are considered separately in factor 14

Evaluation

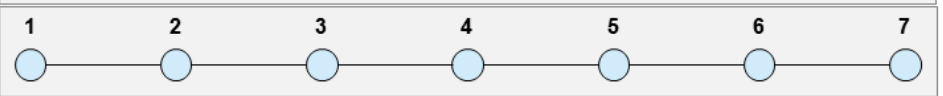

13.

\begin{tabular}{|l|}
\hline Factor: \\
\hline Description: \\
\hline
\end{tabular}

Production costs for cellulosic ethanol in the USA - without feedstock costs

The feedstock costs are not included in the production costs since they are considered separately in factor 15

Evaluation

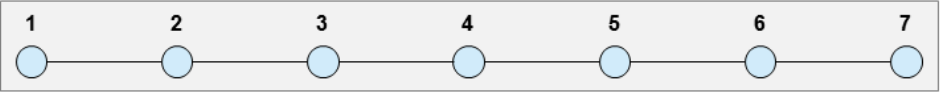

14.

\begin{tabular}{l|} 
Factor: \\
\hline Evaluation:
\end{tabular}

Feedstock costs for corn ethanol in the USA

$\begin{array}{lllllll}1 & 2 & 3 & 5 & 6 & 7 \\ & & & & & & \end{array}$

15.

\begin{tabular}{|l|}
\hline Factor: \\
\hline Evaluation: \\
\hline
\end{tabular}

Feedstock costs for cellulosic ethanol in the USA

$\begin{array}{lllllll}1 & 2 & 3 & 4 & 5 & 6 & 7 \\ & & & & & & \\ & & & & & & \end{array}$

Further comments concerning Economics: 


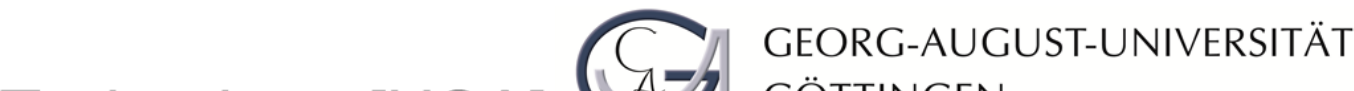 Technology [USA] An GÖTTINGEN}

\begin{tabular}{|c|c|c|c|c|c|c|}
\hline $\begin{array}{l}1 \text { = very weak } \\
\text { influence }\end{array}$ & $\begin{array}{l}2=\text { weak } \\
\text { influence }\end{array}$ & $\begin{array}{c}3=\text { rather weak } \\
\text { influence }\end{array}$ & $\begin{array}{l}4=\text { moderate } \\
\text { influence }\end{array}$ & $\begin{array}{c}5=\begin{array}{c}\text { rather strong } \\
\text { influence }\end{array}\end{array}$ & $\begin{array}{l}6=\text { strong } \\
\text { influence }\end{array}$ & $\begin{array}{c}7 \text { = very strong } \\
\text { influence }\end{array}$ \\
\hline \multirow[t]{2}{*}{16.} & Factor: & \multicolumn{5}{|c|}{ Number of Flexible Fuel Vehicles in the USA } \\
\hline & Evaluation: & 1 & 3 & 4 & 5 & 7 \\
\hline \multirow[t]{2}{*}{17.} & Factor: & \multicolumn{5}{|c|}{$\begin{array}{l}\text { Number of conventional gasoline vehicles in the USA able to run on intermediate } \\
(E 15-E 40) \text { ethanol blends }\end{array}$} \\
\hline & Evaluation: & 1 & 3 & 4 & 5 & 7 \\
\hline
\end{tabular}

\begin{tabular}{l|l|}
\hline 18. & Factor: \\
\hline Description: \\
\hline Evaluation: \\
\hline
\end{tabular}

\section{Number of Electric Drive Vehicles in the USA}

Electric Drive Vehicles are Battery Electric Vehicles and Hybrid Electric Vehicles.

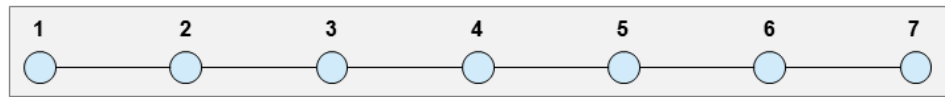

\begin{tabular}{l|l|}
\hline 19. & Factor: \\
\hline \hline Evaluation: \\
\hline
\end{tabular}

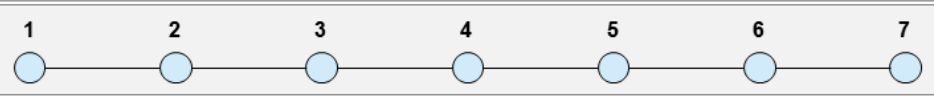

\begin{tabular}{l|l|}
\hline 20. & Factor: \\
\hline Evaluation: \\
\hline
\end{tabular}

GHG balance of corn ethanol produced in the USA

$\begin{array}{lllllll}1 & 2 & 4 & 5 & 6 & 7 \\ & & & & & & \end{array}$

\begin{tabular}{l|l|}
\hline 21. & Factor: \\
\hline & Evaluation: \\
\hline
\end{tabular}

GHG balance of cellulosic ethanol produced in the USA

\begin{tabular}{l|l|}
\hline 22. & Factor: \\
\hline & Evaluation: \\
\hline
\end{tabular}

Biomass to Liquid (BtL) capable of substituting fuel ethanol in the USA

\begin{tabular}{l|l|}
\hline 23. & Factor: \\
\hline & Evaluation: \\
\hline
\end{tabular}

\begin{tabular}{|lllllll}
\hline \multicolumn{3}{|c|}{ Biobutanol capable of substituting fuel ethanol in the USA } \\
\hline
\end{tabular}

Further comments concerning Technology: 


\section{Politics [USA]}

\begin{tabular}{c|c|c|c|c|c|c|c|c}
$\begin{array}{c}1=\text { very weak } \\
\text { influence }\end{array}$ & $\begin{array}{c}2=\text { weak } \\
\text { influence }\end{array}$ & $\begin{array}{c}3=\text { rather weak } \\
\text { influence }\end{array}$ & $\begin{array}{c}4=\text { moderate } \\
\text { influence }\end{array}$ & $\begin{array}{c}5=\text { rather strong } \\
\text { influence }\end{array}$ & $\begin{array}{c}6=\text { strong } \\
\text { influence }\end{array}$ & $\begin{array}{c}7=\text { very strong } \\
\text { influence }\end{array}$
\end{tabular}

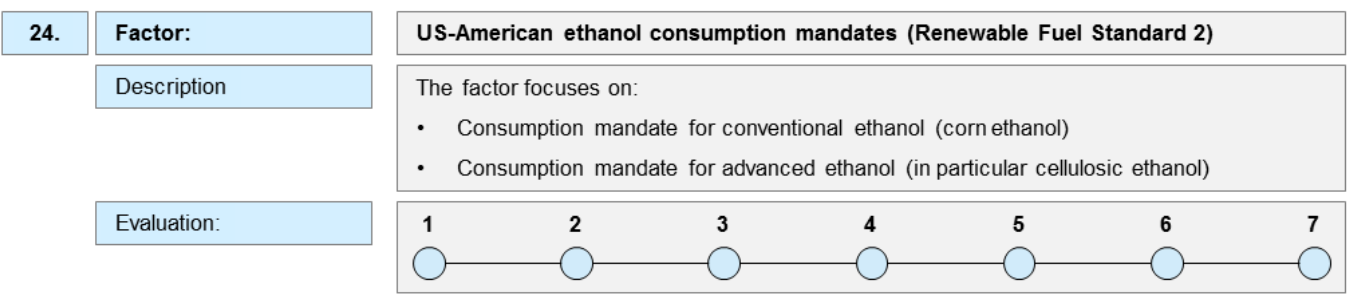

\begin{tabular}{|c|c|c|}
\hline 25. & Factor: & US-American GHG reduction specifications for ethanol (Renewable Fuel Standard 2) \\
\hline & Description: & $\begin{array}{l}\text { The factor focuses on: } \\
\text { - } \text { GHG reduction specification for conventional ethanol (corn ethanol) } \\
\text { - GHG reduction specification for advanced ethanol (in particular cellulosic ethanol) }\end{array}$ \\
\hline & Evaluation: & 1 \\
\hline
\end{tabular}

26. Factor:
\[ \text { Evaluation: } \]

The legally permitted maximum ethanol blends to gasoline as regulated by the US Environmental Protection Agency

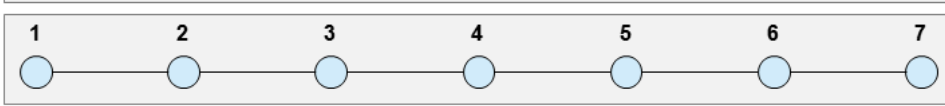

\begin{tabular}{l|l|}
\hline 27. & Factor: \\
\hline Evaluation: \\
\hline
\end{tabular}

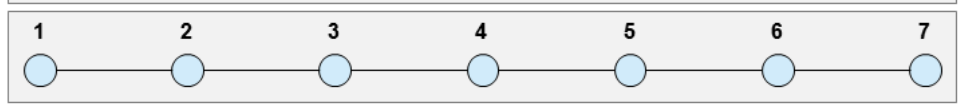

Further comments concerning Politics: 


\section{Society [USA]}

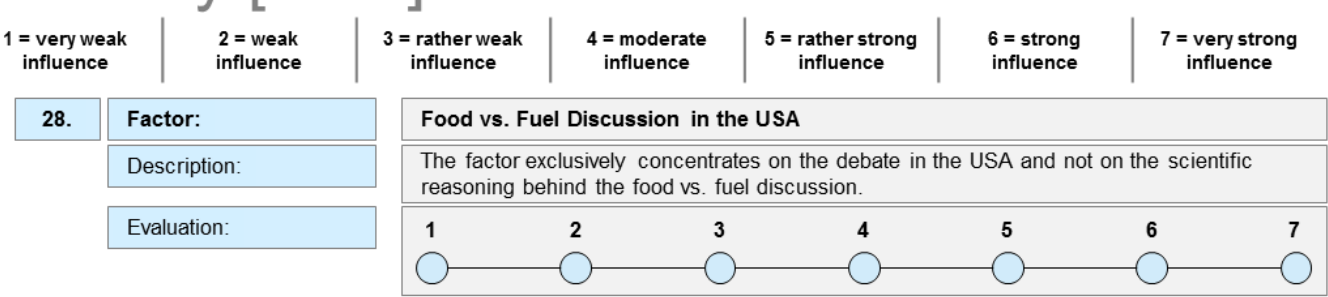

29.

\begin{tabular}{l|} 
Factor: \\
\hline Description:
\end{tabular}

\section{Discussion in the USA about the effects of ethanol on the environment}

The factor exclusively concentrates on the debate in the USA about the effects of ethano on the environment. Thus, the factor does not consider the scientific reasoning behind the debate

Evaluation:

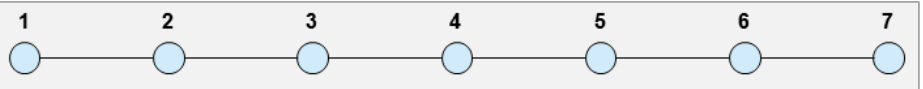

30.

\begin{tabular}{|l|}
\hline Factor: \\
\hline Description:
\end{tabular}

Debate in the USA about the effects of ethanol on US-American energy independence

Only the debate in the USA about the effects of ethanol on US-American energy

independence is of interest and not whether the arguments brought forward are backed by scientific findings.

Evaluation:

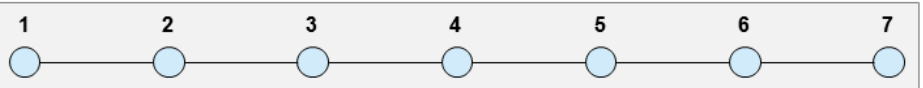

31.

\begin{tabular}{|l|}
\hline Factor: \\
\hline Description: \\
\hline Evaluation: \\
\hline
\end{tabular}

Discussion in the USA about the effects of ethanol on the US-American economy

The factor focuses on the discussion in the USA about the effects of ethanol on the USAmerican economy and not on the scientific reasoning behind the debate.

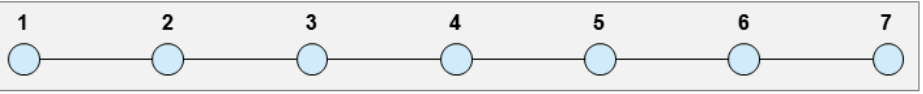

Further comments concerning Society:

Country of origin of your company/ organization:

Industry sector of your company/ organization: Ethanol Production $\bigcirc \quad$ Enzymes Technology $\bigcirc \quad$ Plant Engineer $\bigcirc$

\begin{tabular}{l|l|l|l} 
Petroleum Company $\bigcirc$ & Research Institute $\bigcirc$ & Industry association $\bigcirc$ & Other:
\end{tabular}

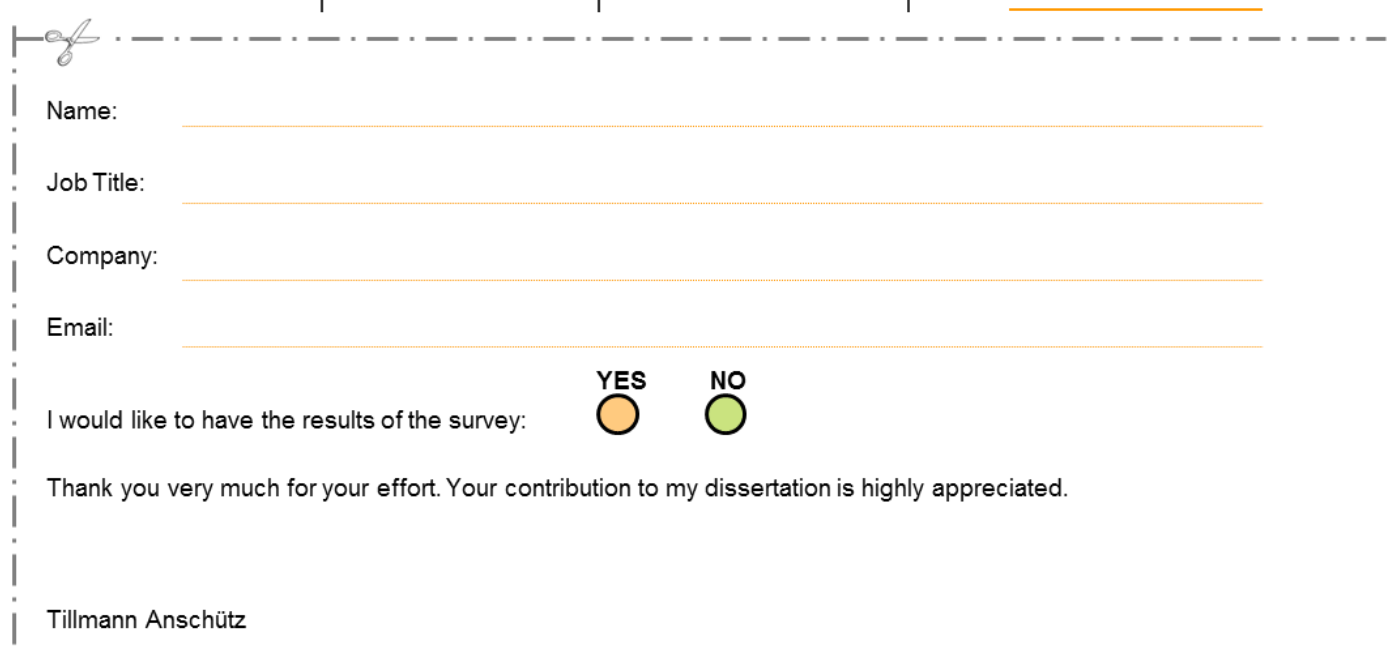




\section{Anhang 5: Fragebogen zur USA-Konsistenzprüfung}

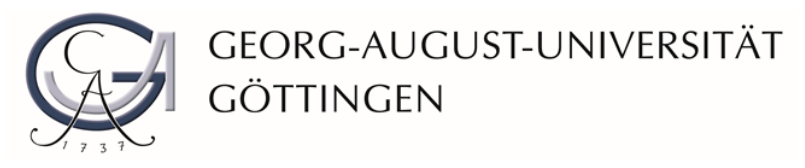

Dear Sir or Madam,

The objective of my study to develop scenarios for the fuel ethanol market of the USA in the year 2023. Based on extensive research, I developed four different scenarios. I would like to ask you to support me by rating the consistency of the four scenarios described on the following pages.

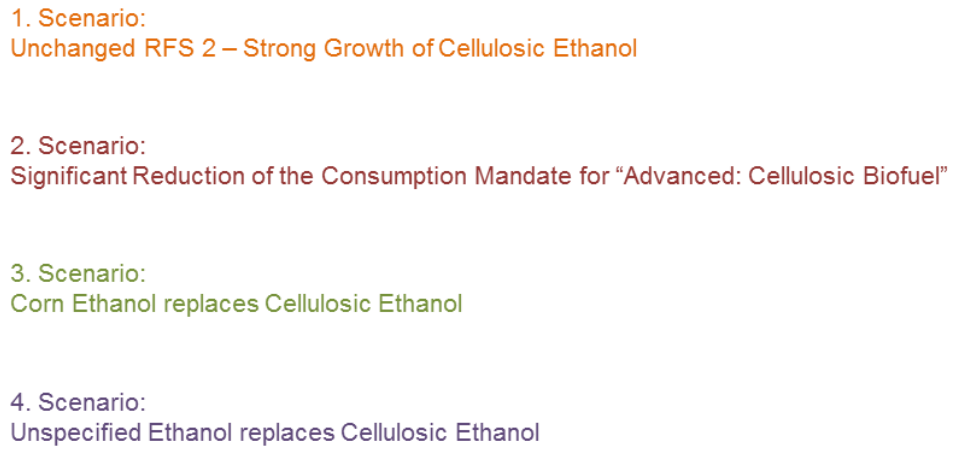

The scenarios $1-4$ describe the fuel ethanol market of the USA in the year 2023 . Since the scenarios exclusively concentrate on the ethanol market, the biodiesel market will be disregarded.

Each scenario is presented to you individually. You can find a short description and a link to a more detailed explanation of the scenario at the top of each page. Below this, for further explanation the components the scenario consists of are listed. When rating the consistency of the scenarios, please be reminded that the actual figures in the scenarios shall not be regarded as the decisive information. Essential are the trends of the future development that are pointed out in the scenarios. Moreover, please concentrate solely on the consistency of the scenarios and not whether the scenarios are realistic. Consistency means that the components of the scenario are coherent within themselves and do not contradict one another. Furthermore, please be open minded since scenarios are innovative and indicate new market developments. In case you feel unsure rating a scenario, please leave the rating scale blank. It will not take you more than 10-15 minutes to complete the questionnaire and of course, all answers will be kept anonymously. Please scroll down to get to the first scenario.

Your support of my dissertation is highly appreciated.

$$
\frac{\text { Sincerely yours }}{\text { T. Ansclintz }}
$$

Tillmann Anschütz 
Scenario 1:

Unchanged RFS 2 - Strong Growth of Cellulosic Ethanol

In the year 2023, the total demand for fuel ethanol is around 35 billion gallons. The key driver for the expanding market is the unchanged consumption mandate for "Advanced: Cellulosic Biofuel".

The EPA released the following ethanol consumption mandates for the year 2023 :

\begin{tabular}{l|c} 
Unit: billion gallons & \\
\hline Conventional Biofuel & 15 \\
\hline Advanced: Cellulosic Biofuel & 16 \\
\hline Advanced: Unspecified Biofuel & 4
\end{tabular}

The consumption mandate for "Conventional Biofuel" is fulfilled by the US-American production of about 16 billion gallons of corn ethanol - of which around 1 billion gallons are exported. By producing 16 billion gallons cellulosic ethanol, the USA satisfies the consumption mandate for "Advanced: Cellulosic Biofuels". In contrast to that, the mandate for "Advanced: Unspecified Biofuel" is met by the import of 3 billion gallons sugar cane ethanol from Brazil as only around 1 billion gallons are produced in the USA

The crude oil price has constantly risen and despite the declining gasoline demand, the USA is able to consume 35 billion gallons of ethanol in the year 2023.

For further explanation of the scenario use the link below or see "Scenario Components".

Please use this link to get to a more detailed description of the scenario

Scenario Components:



If you think components of the scenario are not consistent with one another, please use the box below to name them and to explain why you regard them as inconsistent. 
Scenario 2:

Significant Reduction of the Consumption Mandate for "Advanced: Cellulosic Biofuel"

In the year 2023, the demand for fuel ethanol is around 20 billion gallons. The market has not grown stronger, due to the EPA's decision to reduce the mandate for "Advanced: Cellulosic Biofuel" down to 1 billion gallons.

The EPA released the following consumption mandates for the year 2023:

\begin{tabular}{l|c} 
Unit: billion gallons & \\
\hline Conventional Biofuel & 15 \\
\hline Advanced: Cellulosic Biofuel & 1 \\
\hline Advanced: Unspecified Biofuel & 4
\end{tabular}

The consumption mandate for "Conventional Biofuel" is fulfilled by the US-American production of about 16 billion gallons corn ethanol of which about 1 billion gallons are exported. The USA meets the reduced consumption mandate for "Advanced: Cellulosic Biofuel" by the production of around 1 billion gallons cellulosic ethanol. To fulfill the consumption mandate for "Advanced: Unspecified Biofuel", the USA imports 1 billion gallons sugar cane ethanol from Brazil. The main part - circa 3 billion gallons - is produced in the USA.

The WTI crude oil price has risen moderately and despite the decline in the US-American gasoline demand, the US-American market is able to consume 20 billion gallons of ethanol.

For further explanation of the scenario, please use the link below or see "Scenario Components".

\section{Please use this link to get to a more detailed description of the scenario}

\section{Scenario Components:}

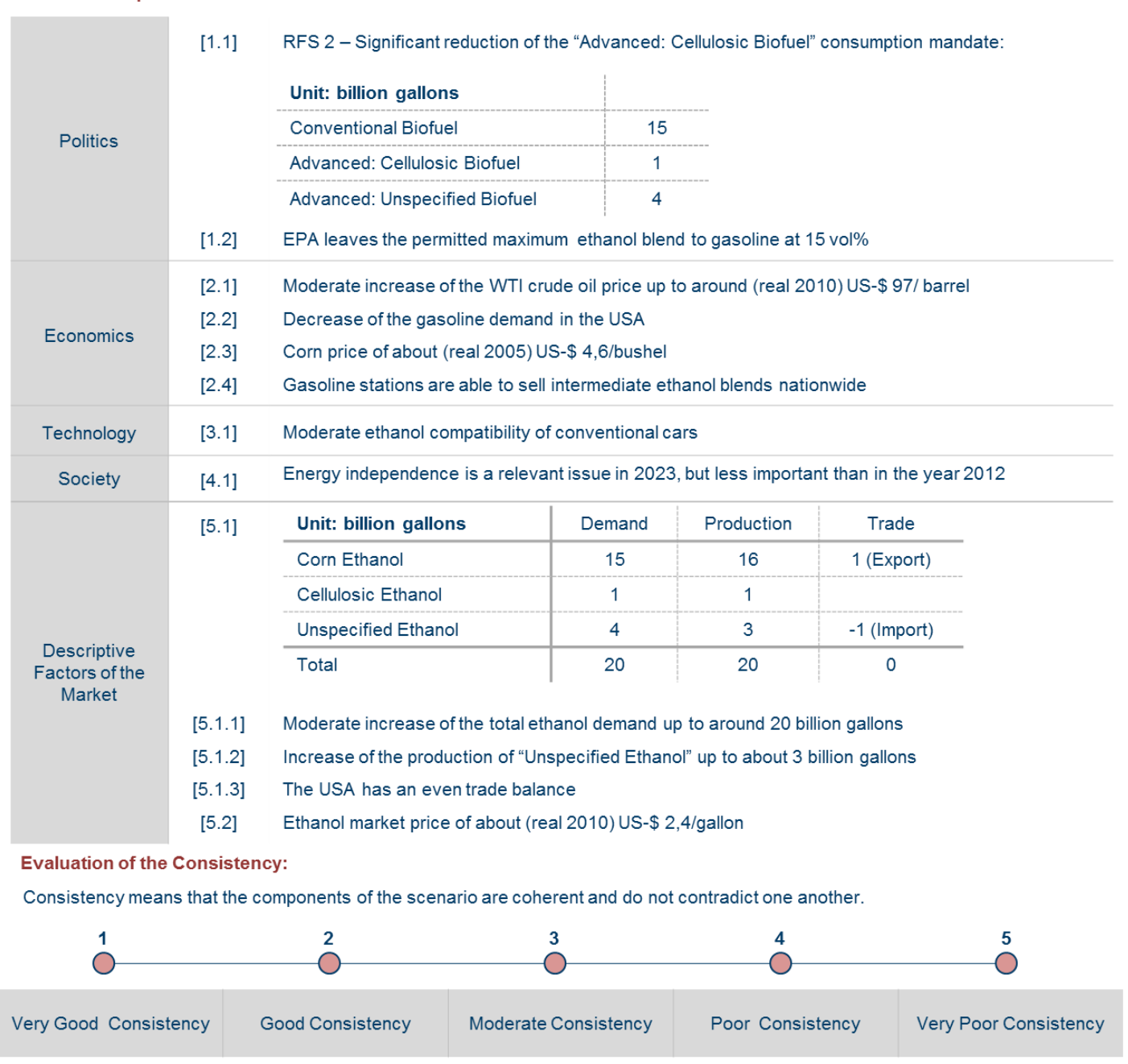

If you think components of the scenario are not consistent with one another, please use the box below to name them and to explain why you regard them as inconsistent. 
Scenario 3:

Corn Ethanol replaces Cellulosic Ethanol

There is a demand for fuel ethanol around 25 billion gallons in the year 2023. The consumption mandate for "Conventional Biofuel" can be regarded as the key driver for the growing ethanol market.

The EPA raised the mandate for "Conventional Biofuel" up to 20 billion gallons in order to partially replace the lacking production of "Advanced: Cellulosic Biofuel".

The EPA released the following consumption mandates for the year 2023

\begin{tabular}{l|c} 
Unit: billion gallons & \\
\hline Conventional Biofuel & 20 \\
\hline Advanced: Cellulosic Biofuel & 1 \\
\hline Advanced: Unspecified Biofuel & 4
\end{tabular}

The consumption mandate for "Conventional Biofuel" is met by the US-American production of about 21 billion gallons corn ethanol - of which around 1 billion gallons are exported. The USA fulfills the mandate for "Advanced: Cellulosic Biofuel" by the production of 1 billion gallons cellulosic ethanol. The mandate for "Advanced: Unspecified Biofuel" is mostly met by the import of around 3 billion gallons sugar cane ethanol from Brazil, while only about 1 billion gallons are produced in the USA.

The WTI crude oil price has constantly risen and despite the decrease of the gasoline demand, the US-American market is able to consume 25 billion gallons of ethanol.

For further information on the scenario, please use the link below or see "Scenario Components".

\section{Please use this link to get to a more detailed description of the scenario}

\section{Scenario Components:}

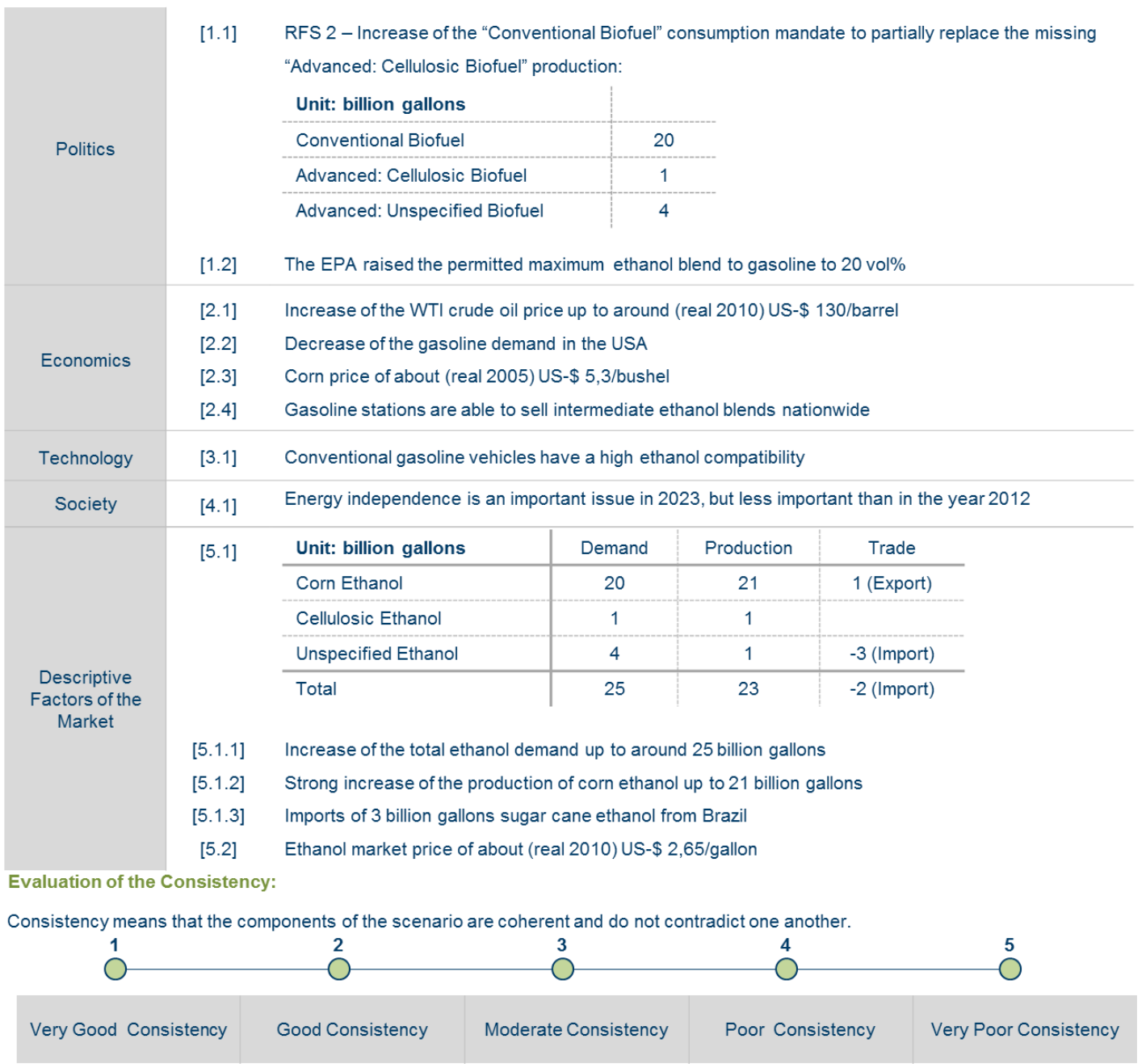

If you think components of the scenario are not consistent with one another, please use the box below to name them and to explain why you regard them as inconsistent. 
Scenario 4:

Unspecified Ethanol replaces Cellulosic Ethanol

There is a demand for fuel ethanol of around 25 billion gallons in the year 2023. The key driver for the growing market is the consumption mandate for "Advanced: Unspecified Biofuel".

The EPA raised the mandate for "Advanced: Unspecified Biofuel" to 9 billion gallons to partially replace the missing production of "Advanced: Cellulosic Biofuel".

The EPA released the following consumption mandates for the year 2023 :

\begin{tabular}{l|c} 
Unit: billion gallons & \\
\hline Conventional Biofuel & 15 \\
\hline Advanced: Cellulosic Biofuel & 1 \\
\hline Advanced: Unspecified Biofuel & 9
\end{tabular}

In order to meet the "Conventional Biofuel" mandate, the USA produces a total of 16 billion gallons of corn ethanol - of which 1 billion gallons are exported to the world ethanol market. By producing 1 billion gallons cellulosic ethanol, the USA is able to fulfill the consumption mandate for "Advanced: Cellulosic Biofuel". The mandate for "Advanced: Unspecified Biofuel" is fulfilled by the USAmerican production of 6 billion gallons ethanol out of sugar beets, sorghum and wheat and by imports of 3 billion gallons sugar cane ethanol from Brazil.

The WTI crude oil price has constantly risen and despite the decrease of the US-American gasoline demand, the market is able to consume 25 billion gallons of ethanol.

For further information on the scenario, please use the link below or see "Scenario Components"

Please use this link to get to a more detailed description of the scenario

Scenario Components:

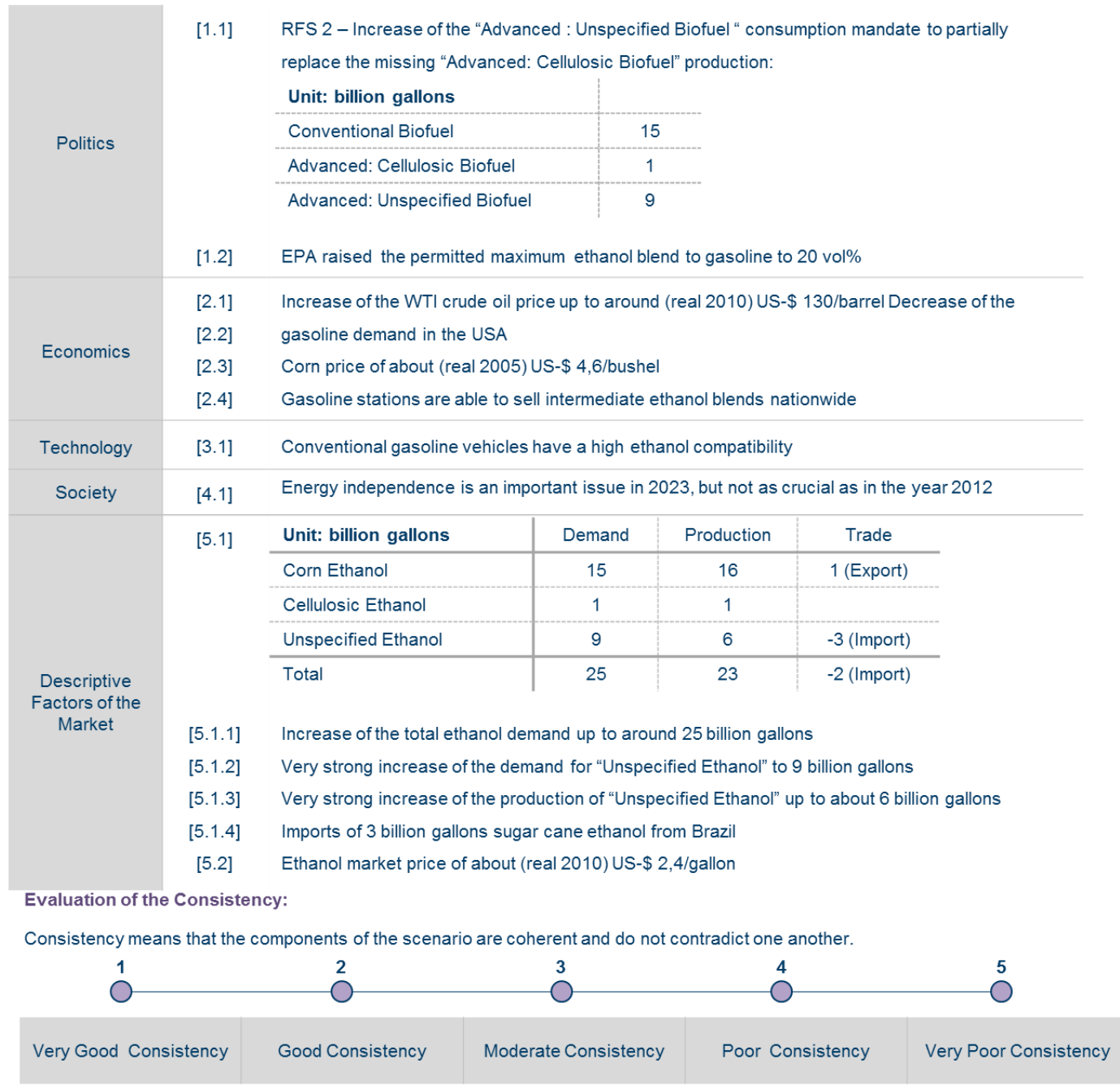

If you think components of the scenario are not consistent with one another, please use the box below to name them and to explain why you regard them as inconsistent. 


\section{Personal Data:}

Country of origin of your company/ organization:

\begin{tabular}{|c|c|c|c|}
\hline Industry sector of $y$ & ur company/ organization: & Ethanol Production & Enzymes Technology \\
\hline er $\bigcirc$ & Petroleum Company $\bigcirc$ & Research Institute & Industry Association \\
\hline
\end{tabular}

Other:

Name:

Job Title:

Company:

Email:

YES NO

I would like to have the scenarios and the results of the survey:

Thank you very much for your participation. Your contribution to my dissertation is highly appreciated.

T. Ansclintz

Tillmann Anschütz 